\title{
A facility to Search for Hidden Particles at the CERN SPS: the SHiP physics case
}

\author{
Sergey Alekhin, ${ }^{1,2}$ Wolfgang Altmannshofer, ${ }^{3}$ Takehiko Asaka, ${ }^{4}$ Brian Batell, ${ }^{5}$ \\ Fedor Bezrukov, ${ }^{6,7}$ Kyrylo Bondarenko, ${ }^{8}$ Alexey Boyarsky ${ }^{8}{ }^{8}$ Nathaniel Craig, ${ }^{9}$ \\ Ki-Young Choi, ${ }^{10}$ Cristóbal Corral, ${ }^{11}$ David Curtin, ${ }^{12}$ Sacha Davidson, ${ }^{13,14}$ André de Gouvêa, ${ }^{15}$ \\ Stefano Dell'Oro, ${ }^{16}$ Patrick deNiverville, ${ }^{17}$ P. S. Bhupal Dev, ${ }^{18}$ Herbi Dreiner, ${ }^{19}$ \\ Marco Drewes, ${ }^{20}$ Shintaro Eijima, ${ }^{21}$ Rouven Essig, ${ }^{22}$ Anthony Fradette, ${ }^{17}$ Björn Garbrecht, ${ }^{20}$ \\ Belen Gavela, ${ }^{23}$ Gian F. Giudice, ${ }^{5}$ Dmitry Gorbunov, ${ }^{24,25}$ Stefania Gori, ${ }^{3}$ \\ Christophe Grojean $\$,{ }^{26,27}$ Mark D. Goodsell, ${ }^{28,29}$ Alberto Guffanti, ${ }^{30}$ Thomas Hambye, ${ }^{31}$ \\ Steen H. Hansen, ${ }^{32}$ Juan Carlos Helo, ${ }^{11}$ Pilar Hernandez, ${ }^{33}$ Alejandro Ibarra, ${ }^{20}$ \\ Artem Ivashko, ${ }^{8,34}$ Eder Izaguirre, ${ }^{3}$ Joerg Jaeckel $\$,{ }^{35}$ Yu Seon Jeong, ${ }^{36}$ Felix Kahlhoefer, ${ }^{27}$ \\ Yonatan Kahn, ${ }^{37}$ Andrey Katz, ${ }^{5,38,39}$ Choong Sun Kim, ${ }^{36}$ Sergey Kovalenko, ${ }^{11}$ \\ Gordan Krnjaic, ${ }^{3}$ Valery E. Lyubovitskij, ${ }^{40,41,42}$ Simone Marcocci, ${ }^{16}$ Matthew Mccullough, ${ }^{5}$ \\ David McKeen, ${ }^{43}$ Guenakh Mitselmakher , ${ }^{44}$ Sven-Olaf Moch, ${ }^{45}$ Rabindra N. Mohapatra, ${ }^{46}$ \\ David E. Morrissey, ${ }^{47}$ Maksym Ovchynnikov, ${ }^{34}$ Emmanuel Paschos, ${ }^{48}$ Apostolos Pilaftsis, ${ }^{18}$ \\ Maxim Pospelov $\$,{ }^{3,17}$ Mary Hall Reno, ${ }^{49}$ Andreas Ringwald, ${ }^{27}$ Adam Ritz, ${ }^{17}$ \\ Leszek Roszkowski, ${ }^{50}$ Valery Rubakov, ${ }^{24}$ Oleg Ruchayskiy ${ }^{\star 21}$ Jessie Shelton, ${ }^{51}$ \\ Ingo Schienbein, ${ }^{52}$ Daniel Schmeier, ${ }^{19}$ Kai Schmidt-Hoberg, ${ }^{27}$ Pedro Schwaller, ${ }^{5}$ \\ Goran Senjanovic, ${ }^{53,54}$ Osamu Seto, ${ }^{55}$ Mikhail Shaposhnikov ${ }^{\star}, \S,{ }^{21}$ Brian Shuve, ${ }^{3}$ \\ Robert Shrock, ${ }^{56}$ Lesya Shchutska ${ }^{4},{ }^{44}$ Michael Spannowsky, ${ }^{57}$ Andy Spray, ${ }^{58}$ Florian Staub, ${ }^{5}$ \\ Daniel Stolarski, ${ }^{5}$ Matt Strassler, ${ }^{39}$ Vladimir Tello, ${ }^{53}$ Francesco Tramontano $§, 59,60$ \\ Anurag Tripathi, ${ }^{59}$ Sean Tulin, ${ }^{61}$ Francesco Vissani, ${ }^{16,62}$ Martin W. Winkler, ${ }^{63}$ \\ Kathryn M. Zurek ${ }^{64,65}$
}

\begin{abstract}
This paper describes the physics case for a new fixed target facility at CERN SPS. The SHiP (Search for Hidden Particles) experiment is intended to hunt for new physics in the largely unexplored domain of very weakly interacting particles with masses below the Fermi scale, inaccessible to the LHC experiments, and to study tau neutrino physics. The same proton beam setup can be used later to look for decays of tau-leptons with lepton flavour number non-conservation, $\tau \rightarrow 3 \mu$ and to search for weakly-interacting sub-GeV dark matter candidates. We discuss the evidence for physics beyond the Standard Model and describe interactions between new particles and four different portals - scalars, vectors, fermions or axion-like particles. We discuss motivations for different models, manifesting themselves via these interactions, and how they can be probed with the SHiP experiment and present several case studies. The prospects to search for relatively light SUSY and composite particles at SHiP are also discussed. We demonstrate that the SHiP experiment has a unique potential to discover new physics and can directly probe a number of solutions of beyond the Standard Model puzzles, such as neutrino masses, baryon asymmetry of the Universe, dark matter, and inflation.
\end{abstract}

\footnotetext{
^Editor of the paper

$\S$ Convener of the Chapter
} 
${ }^{1}$ Deutsches Elektronensynchrotron DESY, Platanenallee 6, D-15738 Zeuthen, Germany

${ }^{2}$ Institute for High Energy Physics, 142281 Protvino, Moscow region, Russia

${ }^{3}$ Perimeter Institute for Theoretical Physics, 31 Caroline St. N, Waterloo, Ontario, Canada

${ }^{4}$ Department of Physics, Niigata University, Niigata 950-2181, Japan

${ }^{5}$ Theory Division, Physics Department, CERN, CH-1211 Geneva 23, Switzerland

${ }^{6}$ Physics Department, University of Connecticut, Storrs, CT 06269-3046, USA

${ }^{7}$ RIKEN-BNL Research Center, Brookhaven National Laboratory, Upton, NY 11973, USA

${ }^{8}$ Instituut-Lorentz for Theoretical Physics, Universiteit Leiden, Niels Bohrweg 2, Leiden, The Netherlands

${ }^{9}$ Department of Physics, University of California, Santa Barbara, CA 93106, USA

${ }^{10}$ Korea Astronomy and Space Science Institute,Daejon 305-348, Republic of Korea

${ }^{11}$ Departamento de Física, Universidad Técnica Federico Santa María and Centro Científico Tecnológico de Valparaíso, Casilla 110-V, Valparaíso, Chile

${ }^{12}$ Maryland Center for Fundamental Physics, University of Maryland, College Park, MD 20742, USA

${ }^{13}$ IPNL, CNRS/IN2P3, 4 rue E. Fermi, Université Lyon 1,69622 Villeurbanne cedex, France

${ }^{14}$ Université de Lyon, F-69622, Lyon, France

${ }^{15}$ Northwestern University, Department of Physics 8 Astronomy, Evanston, IL 60208-3112, USA

${ }^{16}$ Gran Sasso Science Institute, Viale Crispi 7, 67100 L'Aquila, Italy

${ }^{17}$ Department of Physics and Astronomy, University of Victoria, Victoria, BC, V8P 5C2, Canada

${ }^{18}$ Consortium for Fundamental Physics, School of Physics and Astronomy, University of Manchester, Manchester M13 9PL, United Kingdom

${ }^{19}$ Bethe Center for Theoretical Physics $\&$ Physikalisches Institut der Universität Bonn, 53115 Bonn, Germany

${ }^{20}$ Physik-Department, Technische Universität München, James-Franck-Straße, 85748 Garching, Germany

${ }^{21}$ Ecole Polytechnique Fédérale de Lausanne, FSB/ITP/LPPC, BSP, CH-1015, Lausanne, Switzerland

${ }^{22}$ C. N. Yang Institute for Theoretical Physics, Stony Brook University, Stony Brook, NY 11794, USA

${ }^{23}$ Departamento de Física Teórica and Instituto de Física Teórica, IFT-UAM/CSIC, Universidad Autónoma de Madrid, Cantoblanco, 28049, Madrid, Spain

${ }^{24}$ Institute for Nuclear Research of the Russian Academy of Sciences, Moscow 117312, Russia

${ }^{25}$ Moscow Institute of Physics and Technology, Dolgoprudny 141700, Russia

${ }^{26}$ ICREA at IFAE, Universitat Autònoma de Barcelona, E-08193 Bellaterra, Spain

${ }^{27}$ Deutsches Elektronen-Synchroton (DESY), Notkestrasse 85, D-22607 Hamburg, Germany

${ }^{28}$ Sorbonne Universités, UPMC Univ Paris 06, UMR 7589, LPTHE, F-75005, Paris, France

${ }^{29}$ CNRS, UMR 7589, LPTHE, F-75005, Paris, France

${ }^{30}$ Niels Bohr International Academy and Discovery Center, Niels Bohr Institute, University of Copenhagen, Blegdamsvej 17, DK-2100 Copenhagen, Denmark

${ }^{31}$ Service de Physique Théorique, Université Libre de Bruxelles,Bld du Triomphe, CP225, 1050 Brussels, Belgium

${ }^{32}$ Dark Cosmology Centre, Niels Bohr Institute, University of Copenhagen, Juliane Maries Vej 30,2100 Copenhagen, Denmark

${ }^{33}$ Instituto de Física Corpuscular (IFIC), CSIC-Universitat de València Apartado de Correos 22085,E-46071 Valencia, Spain

${ }^{34}$ Department of Physics, Kiev National Taras Shevchenko University, Glushkov str. 2 building 6, Kiev, 03022, Ukraine

${ }^{35}$ Institut für theoretische Physik, Universität Heidelberg, Philosophenweg 16, 69120 Heidelberg, Germany

${ }^{36}$ Department of Physics and IPAP, Yonsei University, Seoul 120-749, Korea

${ }^{37}$ Massachusetts Institute of Technology, Cambridge, MA 02139, USA

${ }^{38}$ Université de Genève, Department of Theoretical Physics and Center for Astroparticle Physics (CAP), 24 quai E. Ansermet, CH-1211 Geneva 4, Switzerland

${ }^{39}$ Department of Physics, Harvard University, Cambridge, MA 02138, USA

${ }^{40}$ Institut für Theoretische Physik, Universität Tübingen, Kepler Center for Astro and Particle Physics, Auf der Morgenstelle 14, D-72076 Tübingen, Germany

${ }^{41}$ Department of Physics, Tomsk State University, 634050 Tomsk, Russia

${ }^{42}$ Mathematical Physics Department, Tomsk Polytechnic University, Lenin avenue 30, 634050 Tomsk, Russia

${ }^{43}$ Department of Physics, University of Washington, Seattle, Washington 98195, USA

${ }^{44}$ University of Florida, Gainesville, USA

${ }^{45}$ II. Institut für Theoretische Physik, Universität Hamburg, Luruper Chaussee 149, D-22761 Hamburg, Germany

${ }^{46}$ Maryland Center for Fundamental Physics and Department of Physics, University of Maryland, College Park, Maryland 20742, USA

${ }^{47}$ TRIUMF, 4004 Wesbrook Mall, Vancouver, BC V6T 2A3, Canada 
${ }^{48}$ Department of Physics, Technical University of Dortmund, D-44221, Dortmund, Germany

${ }^{49}$ University of Iowa, Iowa City, Iowa, 52242, USA

${ }^{50}$ National Centre for Nuclear Research, Hoza 69, 00-681 Warsaw, Poland

${ }^{51} 1110$ West Green Street Urbana, IL 61801, Dept of Physics, University of Illinois at Urbana-Champaign, USA

${ }^{52}$ LPSC, Université Grenoble-Alpes, CNRS/IN2P3, 53 avenue des Martyrs, 38026 Grenoble, France

${ }^{53}$ Theory Group, Gran Sasso Science Institute, Viale Crispi 7, 67100 L'Aquila, Italy

${ }^{54}$ ICTP, Trieste, Italy

${ }^{55}$ Department of Life Science and Technology,Hokkai-Gakuen University,Sapporo 062-8605, Japan

${ }^{56}$ C. N. Yang Institute for Theoretical Physics, Stony Brook University Stony Brook, NY 11794 USA

${ }^{57}$ Institute for Particle Physics Phenomenology, Department of Physics, Durham University, Durham DH1 3LE, United Kingdom

${ }^{58}$ ARC Centre of Excellence for Particle Physics at the Terascale, School of Physics, The University of Melbourne, Victoria 3010, Australia

${ }^{59}$ INFN, sezione di Napoli, Complesso di Monte Sant'Angelo, via Cintia, I-80126, Napoli, Italy

${ }^{60}$ Universitá di Napoli "Federico II", Complesso di Monte Sant'Angelo, via Cintia, I-80126, Napoli, Italy

${ }^{61}$ Department of Physics and Astronomy, York University 4700 Keele Street, Toronto, Ontario, M3J 1 P3, Canada

${ }^{62}$ INFN, Laboratori Nazionali del Gran Sasso, Assergi, L'Aquila, Italy

${ }^{63}$ Bethe Center for Theoretical Physics and Physikalisches Institut der Universität Bonn Nussallee 12, 53115 Bonn, Germany

64 Theory Group Lawrence Berkeley National Laboratory, Berkeley, CA 94709, USA

${ }^{65}$ Berkeley Center for Theoretical Physics University of California, Berkeley, CA 94709, USA 


\section{Contents}

1 Introduction $\quad 8$

2 Vector portal 11

2.1 Classification of vector portals 11

$\begin{array}{lll}2.1 .1 & \text { Kinetic mixing } & 12\end{array}$

2.1.2 Anomaly-free gauge groups $\left(B-L, L_{\mu}-L_{\tau}\right.$ etc) 13

$\begin{array}{lll}2.1 .3 & \text { Other froms of vector portals. } & 14\end{array}$

$\begin{array}{lll}2.1 .4 & \text { Chern-Simons portal } & 15\end{array}$

$\begin{array}{lll}2.2 & \text { Matter states charged under new } U(1) & 17\end{array}$

$\begin{array}{lll}2.2 .1 & \text { Higgs mechanism in the dark sector } & 17\end{array}$

$\begin{array}{ll}2.2 .2 \text { Supersymmetric } U(1)^{\prime} \text { models } & 18\end{array}$

$\begin{array}{lll}2.3 & \text { Physics motivation for light mass (less than weak scale) vector particles } & 18\end{array}$

$\begin{array}{lll}\text { 2.3.1 Putative solution to the muon } g-2 \text { discrepancy } & 19\end{array}$

2.3.2 Mediator of interaction with DM and possible connection to astrophysical positron excess 20

2.3.3 Self-intereaction of dark matter via light mediators 23

2.4 Main features of vector portal phenomenology. 24

2.4.1 Decay rates, modes, branchings, $c \tau$ for dark photon 24

$\begin{array}{lll}2.4 .2 & \text { Other vector candidates } & 26\end{array}$

2.4.3 Higgsstrahlung process for $U(1)^{\prime}$ and delayed decays of $h^{\prime}$. 26

2.5 Summary of the existing constraints on light vector and light DM states 27

2.5.1 Current status of experimental constraints on exotic vector states 27

2.5.2 Production and detection of light vector portal DM 28

$\begin{array}{lll}\text { 2.5.3 Cosmological and astrophysical constraints on vector portals } & 29\end{array}$

2.6 Case studies for SHiP 30

2.6.1 Production and detection of kinetically mixed dark photons and baryonic vectors. $\quad 30$

2.6.2 Production and detection of other unstable particles $\left(h^{\prime}, \mathrm{HNL}\right)$ through the vector portal 31

2.6.3 Possible sensitivity to light DM through SHiP neutrino detector. 31

2.7 Conclusions 32

3 Scalar portal 33

3.1 The scalar sector of the Standard Model and Beyond 33

3.1.1 Scalar portal effective Lagrangian 33

3.1.2 Hidden Valleys 34

$\begin{array}{lll}3.1 .3 & \text { Light scalars in supersymmetry } & 38\end{array}$

$\begin{array}{lll}\text { 3.1.3.1 Singlet extensions } & 38\end{array}$ 
3.1.3.2 Additional Abelian gauge groups 38

3.1.3.3 Models with $R$-parity violation $\quad 39$

3.2 Linear scalar portals: Higgs-scalar mixing $\quad 39$

3.2.1 Existing experimental limits $\quad 41$

3.2.2 What SHiP can do 42

$3.3 \mathcal{Z}_{2}$ scalar portals: pair-production of light hidden particles and exotic Higgs decays 44

3.3.1 Probing Exotic Higgs Decays at SHiP 45

3.3.2 What SHiP can do 46

3.3.2.1 Hidden sector scalars 46

3.3.2.2 Hidden sector fermions and vectors 49

3.4 Pseudoscalar portals $\quad 49$

3.5 Scalar portals and Dark Matter $\quad 52$

3.5.1 Scalar as a mediator between DM and the SM $\quad 52$

3.5.2 Scalar as a DM candidate 53

$\begin{array}{lll}3.6 & \text { Dark pions } & 56\end{array}$

3.6.1 The model and scales $\quad 56$

3.6.2 Dark pion lifetime and decay modes $\quad 56$

$\begin{array}{lll}\text { 3.6.3 What SHiP could do? } & 57\end{array}$

$\begin{array}{lll}3.7 & \text { Scalar portals and inflation } & 57\end{array}$

$\begin{array}{lll}3.7 .1 & \text { Light inflatons } & 57\end{array}$

4 Neutrino portal $\quad 62$

4.1 Heavy neutral leptons $\quad 62$

4.2 Active neutrino phenomenology 65

4.2.1 Three-flavour neutrino oscillations. A theoretical overview 65

4.2.2 Present experimental status of neutrino masses and mixings 66

$\begin{array}{lll}\text { 4.2.3 } & \text { Short-Baseline neutrino anomalies } & 67\end{array}$

$\begin{array}{lll}\text { 4.2.4 Future neutrino experiments } & 68\end{array}$

$\begin{array}{lll}4.3 & \text { HNLs and neutrino masses } & 69\end{array}$

$\begin{array}{lll}\text { 4.3.1 Seesaw formula } & 69\end{array}$

$\begin{array}{lll}\text { 4.3.2 Seesaw scales } & 71\end{array}$

$\begin{array}{lll}\text { 4.3.2.1 GUT-scale seesaw } & 72\end{array}$

$\begin{array}{lll}\text { 4.3.2.2 TeV-scale seesaw } & 72\end{array}$

4.3.2.3 GeV-scale seesaw $\quad 74$

$\begin{array}{lll}\text { 4.3.2.4 eV-scale Seesaw } & 75\end{array}$

$\begin{array}{lll}\text { 4.3.3 Beyond the minimal seesaw model } & 77\end{array}$

$\begin{array}{lll}\text { 4.3.3.1 Left-right symmetric models } & 77\end{array}$

4.3.3.2 Left-right symmetric models with GeV-scale HNLs 84

4.3.3.3 Inverse seesaw and GeV scale singlet fermions $\quad 85$

4.3.3.4 Seesaw I vs inverse seesaw singlet neutrinos at SHiP $\quad 86$

4.3.4 Possible origins of the keV-MeV-GeV scale of HNL masses 87

4.4 Direct HNL searches $\quad 88$

4.4.1 Direct signatures of HNL: kink searches; peak searches 88

4.4.2 Direct signatures of HNL: fixed target experiments 89

4.4.3 Direct Signatures of HNL: Collider Searches 91

$\begin{array}{lll}4.5 & \text { Indirect HNL probes } & 96\end{array}$

4.5.1 Neutrinoless double beta decay for non-seesaw HNL 96

4.5.2 Neutrinoless double beta decay for two seesaw HNLs 97

$\begin{array}{lll}\text { 4.5.3 Charged lepton flavour violating processes } & 100\end{array}$ 
4.5.4 HNL and primordial nucleosynthesis 103

4.6 HNL and baryon asymmetry of the Universe 104

$\begin{array}{lll}\text { 4.6.1 Sakharov conditions. Leptogenesis } & 104\end{array}$

$\begin{array}{lll}\text { 4.6.2 Thermal leptogenesis } & 106\end{array}$

$\begin{array}{lll}\text { 4.6.3 Resonant Leptogenesis } & 108\end{array}$

$\begin{array}{ll}\text { 4.6.4 Leptogenesis via HNL oscillations } & 109\end{array}$

4.6.4.1 Leptogenesis via oscillations with two HNLs 111

4.6.4.2 Leptogenesis via oscillations with three HNLs 112

4.7 HNL and dark matter 116

4.7.1 Bounds on HNL as dark matter. No assumptions on production mechanism 117

4.7.2 Bounds on DM HNL if produced via mixing with active neutrinos only 117

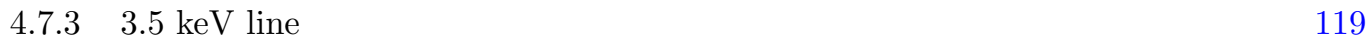

$4.8 \nu \mathrm{MSM} \quad 120$

4.8.1 Lepton asymmetry and dark matter production in the model with $3 \mathrm{HNL} \quad 121$

4.8.2 Lepton number violation in the $\nu$ MSM 123

4.8.3 Inflation, vacuum stability, dark energy and naturalness in the $\nu$ MSM 124

$\begin{array}{lll}4.9 & \text { Conclusions } & 127\end{array}$

5 ALPs and other PNGBs at SHiP 128

$\begin{array}{llr}5.1 & \text { ALPs and why they are interesting } & 128\end{array}$

$\begin{array}{lll}5.1 .1 & \text { ALP origins } & 129\end{array}$

5.1.2 Connection to Dark Matter 130

5.2 Interactions, phenomenological features and existing limits 130

$\begin{array}{lll}5.3 & \text { ALPs coupled to two gauge bosons } & 131\end{array}$

5.3.1 Prospects for SHiP 132

5.4 ALPs coupled to SM fermions 132

5.4.1 Interactions, phenomenological features and existing limits 132 5.4.1.1 ALP-pion mixing $\quad 133$

5.4.1.2 Effective flavour-changing interactions 133

$\begin{array}{lll}\text { 5.4.1.3 } & \text { ALP-mediated rare decays } & 134\end{array}$

5.4.2 Prospects at SHiP 135

$\begin{array}{lll}5.5 & \text { Concluding remark } & 136\end{array}$

6 SUSY 138

$\begin{array}{lll}6.1 & \text { Introduction } & 138\end{array}$

6.2 A Very Light Supersymmetric Neutralino and R-Parity Violation 139

$\begin{array}{lll}\text { 6.2.1 } & \text { Motivation for a very light neutralino } & 139\end{array}$

$\begin{array}{lll}6.2 .2 & \text { R-parity Violation } & 140\end{array}$

6.2.3 Finding Neutralinos at SHiP via R-Parity violation 140

6.2.4 Comparison with Previous Bounds 142

6.2.5 Concluding remarks 142

6.3 Light particles from the SUSY breaking sector 143

6.3.1 Origin of light sgoldstinos $\quad 143$

6.3.2 Sgoldstino couplings and phenomenology 144

$\begin{array}{lll}\text { 6.3.3 Sgoldstinos at SHiP } & 146\end{array}$

$\begin{array}{ll}\text { 6.3.4 Concluding remarks } & 148\end{array}$

$\begin{array}{lll}6.4 & \text { Light Dirac gauginos } & 148\end{array}$

$\begin{array}{lll}\text { 6.4.1 Origins of Pseudo-Dirac fermions } & 148\end{array}$

6.4.2 Effective model, phenomenological features 149 
$\begin{array}{lll}\text { 6.4.3 Origin of the effective model } & 150\end{array}$

6.4.3.1 Z portal 150

6.4.3.2 Higgs portal 151

$\begin{array}{ll}\text { 6.4.3.3 Sfermion portal } & 151\end{array}$

$\begin{array}{lll}6.4 .4 & \text { Detection at SHiP } & 151\end{array}$

6.4.4.1 Decays 151

6.4.4.2 Production $\quad 152$

6.4.5 Concluding remarks 152

6.5 SUSY vector portal I: Hidden Photinos 153

$\begin{array}{lll}6.5 .1 & \text { Motivation } & 153\end{array}$

$\begin{array}{lll}6.5 .2 & \text { Features } & 154\end{array}$

$\begin{array}{lll}\text { 6.5.3 } & \text { Existing bounds } & 154\end{array}$

6.5.3.1 Direct bounds 154

6.5.3.2 Cosmological bounds $\quad 155$

6.5.4 What SHiP can do $\quad 155$

$\begin{array}{lll}\text { 6.5.4.1 R-parity conserving photinos } & 155\end{array}$

$\begin{array}{lll}\text { 6.5.4.2 R-parity-violating photinos } & 156\end{array}$

$\begin{array}{lll}\text { 6.5.5 Concluding remarks } & 156\end{array}$

6.6 SUSY vector portal II: Novel Hidden Photon decays 156

$\begin{array}{lll}\text { 6.6.1 Setup } & 156\end{array}$

$\begin{array}{lll}\text { 6.6.2 SHiP Sensitivity } & 157\end{array}$

$\begin{array}{ll}\text { 6.6.3 Concluding remarks } & 157\end{array}$

$\begin{array}{lll}6.7 & \text { Axinos and saxions, ALPinos and sALPs } & 158\end{array}$

$\begin{array}{lll}\text { 6.7.1 Motivation } & 158\end{array}$

6.7.2 Phenomenology of saxions and axinos and possibilities at SHiP 158

$\begin{array}{lll}\text { 6.7.2.1 Saxions } & 158\end{array}$

6.7.2.2 Axino LSP with R-parity breaking 159

6.7.2.3 Axinos with R-parity conservation 159

$\begin{array}{lll}\text { 6.7.3 Concluding remarks } & 160\end{array}$

6.8 Additional Possibilities 161

6.8.1 Pair production of light neutralinos and decay to gravitino 161

6.8.2 Flavor violating production of light neutralinos $\quad 161$

6.9 SUSY at SHiP: Final remarks 162

7 Tau neutrino physics and other precision measurements in SHiP 164

$\begin{array}{lll}7.1 & \text { Tau neutrino physics } & 165\end{array}$

$\begin{array}{lll}\text { 7.1.1 } & \text { Flux of tau neutrinos } & 167\end{array}$

$\begin{array}{lll}7.1 .2 & \text { Expected sensitivity } & 171\end{array}$

$\begin{array}{lll}7.2 & \text { Deep inelastic muon and electron neutrino scattering } & 177\end{array}$

$\begin{array}{lll}\text { 7.2.1 } & \text { Status of perturbative QCD calculations } & 177\end{array}$

7.2.2 Strangeness from heavy-quark DIS in CC interactions 180

7.2.3 Nuclear effects in $\nu N$ DIS and global analyses of nuclear PDFs 182

7.2.4 $\alpha_{S}$ measurement via Gross-Llewellin Smith sum rule 185

7.2.5 Precise Ratios for Neutrino Nucleon Interactions $\quad 186$

$\begin{array}{lll}7.3 & \text { Limit on Tau neutrino magnetic moment } & 188\end{array}$

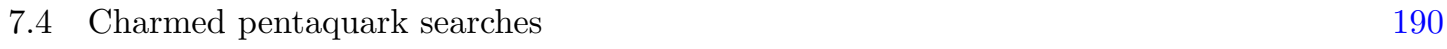

$\begin{array}{lll}7.5 & \text { Summary } & 191\end{array}$ 
8.4 Relation to two-body LVF decays of Z boson, neutral pseudoscalar and vector mesons 194

$\begin{array}{ll}\text { 8.5 Current and future experimental sensitivities } & 196\end{array}$

$\begin{array}{ll}\text { 8.6 Proposal for a fixed-target facility } & 196\end{array}$

9 Conclusion $r$

$\begin{array}{lr}\text { A The SHiP experiment } & 201\end{array}$

B Notations $r$

$\begin{array}{ll}\text { B.1 Abbreviations: } & 204\end{array}$

\section{Acknowledgement}

We are extremely grateful to Walter Bonivento, Annarita Buonaura, Geraldine Conti, Hans Dijkstra, Giovanni De Lellis, Antonia Di Crescenzo, Andrei Golutvin, Elena Graverini, Richard Jacobsson, Gaia Lanfranchi, Thomas Ruf, Nicola Serra, Barbara Storaci, Daniel Treille for their invaluable contributions during all stages of work on this document and in particular for their help with models' sensitivity estimates.

We are grateful to all the members of SHiP for their dedicated work to make this experiment possible. 


\section{Chapter 1}

\section{Introduction}

The Standard Model of elementary particle physics has provided a consistent description of Nature's fundamental constituents and their interactions. Its predictions have been tested and confirmed by numerous experiments. The Large Hadron Collider's runs at 7 and $8 \mathrm{TeV}$ culminated in the discovery of a Higgs boson-like particle with the mass of about $126 \mathrm{GeV}$ - the last critical Standard Model component [1-5]. Thus for the first time we are in the situation when all the particles, needed to explain the results of all previous accelerator experiments have been found. At the same time, no significant deviations from the Standard Model were found in direct or in indirect searches for new physics (see e.g. the summary of the recent search results in [6-25] and most up-to-date information at [26-29]). For this particular value of the Higgs mass it is possible that the Standard Model remains mathematically consistent and valid as an effective field theory up to a very high energy scale, possibly all the way to the scale of quantum gravity, the Planck scale [30-32].

However, it is clear that the SM is not a complete theory. It fails to explain a number of observed phenomena in particle physics, astrophysics and cosmology. These major unsolved challenges are commonly known as "beyond the Standard Model" problems:

$\triangleright$ Neutrino masses and oscillations: what makes neutrinos disappear and then re-appear in a different form? Why do neutrinos have mass?

$\triangleright$ Baryon asymmetry of the Universe (BAU): what mechanism created the tiny matterantimatter imbalance in the early Universe?

$\triangleright$ Dark Matter (DM) : what is the most prevalent kind of matter in our Universe?

$\triangleright$ Cosmological inflation: What drives the accelerated expansion of the universe during the early stages of its evolution?

$\triangleright$ Dark Energy: What drives the accelerated expansion of the universe during the present stage of its evolution?

Some yet unknown particles or interactions would be needed to explain these puzzles and to answer these questions. But in that case, why haven't they yet been observed?

One possible answer is that the hypothetical particles are heavy and require even higher collision energy to be observed, the so-called "energy frontier" research. Major particle physics experiments of the last few decades, including LEP and LHC at CERN, and Tevatron in the US, have followed this path.

Another possibility is that our inability to observe new particles lies not in their heavy mass, but rather in their extremely feeble interactions. If true, this would imply that a different approach to detect them should be used: an experiment needs to cross the "intensity frontier", rather than the "energy frontier" (Figure 1.1).

An example when a part of beyond-the-Standard Model phenomena mentioned above is resolved by introducing relatively light new particles only is given by the $\nu \mathrm{MSM}$ (discussed in Section 4.8). 


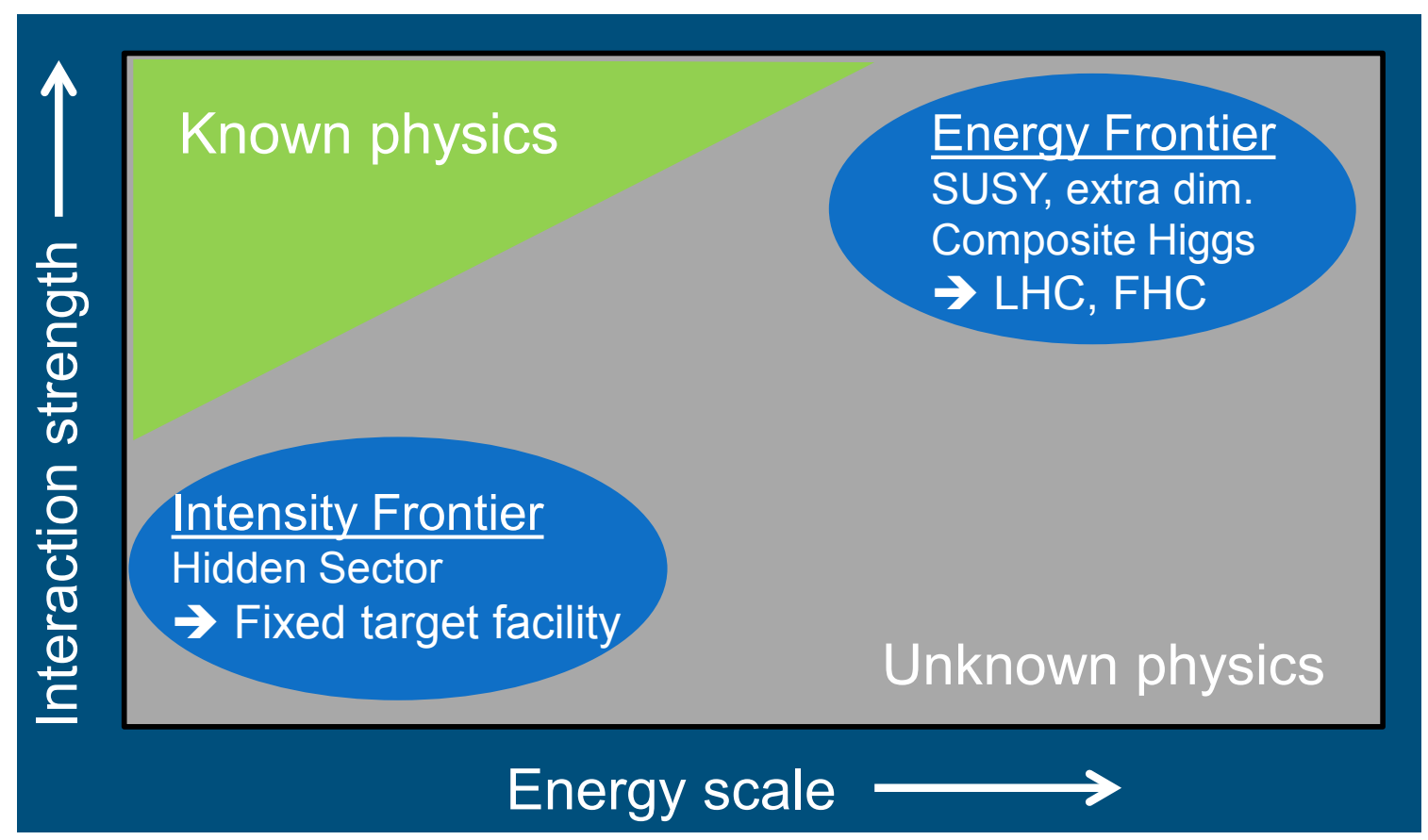

Figure 1.1: New physics that can be explored at intensity frontier experiments and its complimentarily with the energy frontier

Alternatively, some of the new particles, responsible for the resolution of the BSM puzzles, can be heavy or do not interact directly with the SM sector. These "hidden sectors" may nevertheless be accessible to the intensity frontier experiments via few sufficiently light particles, which are coupled to the Standard Model sectors either via renormalizable interactions with small dimensionless coupling constants ("portals") or by higher-dimensional operators suppressed by the dimensionful couplings $\Lambda^{-n}$, corresponding to a new energy scale of the hidden sector.

For the Standard Model, renormalizable portals can be classified into the following 3 types, depending on the mass dimension of the SM singlet operator.

Dimension $\mathrm{GeV}^{2}$, Vector portal: new particles are Abelian fields, $A_{\mu}^{\prime}$ with the field strength $F_{\mu \nu}^{\prime}$, that couple to the hypercharge field $F_{Y}^{\mu \nu}$ via

$$
\mathcal{L}_{\text {Vector portal }}=\epsilon F_{\mu \nu}^{\prime} F_{Y}^{\mu \nu}
$$

where $\epsilon$ is a dimensionless coupling characterising the mixing between the new vector field with the $Z$-boson and the photon. The phenomenology of the vector portal is discussed in Section 2.

Dimension $\mathrm{GeV}^{2}$, Scalar portal: new particles are neutral singlet scalars, $S_{i}$ that couple to the square of the Higgs field $|\Phi|^{2}$ :

$$
\mathcal{L}_{\text {Scalar portal }}=\left(\lambda_{i} S_{i}^{2}+g_{i} S_{i}\right)\left(\Phi^{\dagger} \Phi\right),
$$

where $\lambda_{i}$ are dimensionless and $g_{i}$ are dimensionful couplings. The phenomenology of the scalar portal is discussed in Section 3.

Dimension $\mathrm{GeV}^{\frac{5}{2}}$, Neutrino portal: the singlet operators $\left(\bar{L}_{\alpha} \cdot \tilde{\Phi}\right)$ couple to new neutral singlet fermions $N_{I}$

$$
\mathcal{L}_{\text {Neutrino portal }}=F_{\alpha I}\left(\bar{L}_{\alpha} \cdot \tilde{\Phi}\right) N_{I} .
$$


Here $L_{\alpha}$ is one of the $S U(2)$ lepton doublets, and $\tilde{\Phi}_{a}=\epsilon_{a b} \Phi_{b}, F_{\alpha I}$ are dimensionless Yukawa couplings, for other notations see Appendix B. The phenomenology of the neutrino portal will be discussed in Section 4.

Of course, higher dimensional, non-renormalizable couplings of new particles to the SM operators are also possible. An important example is provided by pseudo-scalar axion-like particles A, that couple to a dimension 4 two photon operator (Section 5) via

$$
\mathcal{L}_{\mathrm{A}}=\frac{A}{4 f_{A}} \epsilon^{\mu \nu \lambda \rho} F_{\mu \nu} F_{\lambda \rho} .
$$

Yet another example is a Chern-Simons like (parity odd) interaction of electroweak gauge bosons with a new vector field $V_{\mu}, \quad[33]$ :

$$
\mathcal{L}_{\mathrm{CS}}=\epsilon^{\mu \nu \lambda \rho} c_{Y} V_{\mu}\left(\Phi^{\dagger} D_{\nu} \Phi\right)\left(F_{Y}\right)_{\lambda \rho}+\ldots
$$

(see Section 2.1.4 for details).

The goal of this paper is demonstrate the capability of high intensity proton fixed target experiments to discover relatively light new particles. In particular, we will show that such experiments can probe an interesting parameter space for a number of BSM models representing the portals described above. This will potentially allow for direct experimental checks of the mechanisms of matter-antimatter asymmetry of the Universe, the origin of neutrino masses, and the particle physics nature of dark matter.

This paper was prepared together with the companion document, Technical Proposal [34], that describes a concrete experiment, $\mathrm{SHiP}$ (Search for Hidden Particles) ${ }^{1}$ that was proposed in 2013 [35]. Both documents together are submitted to the SPS and PS experiments Committee (SPSC) at CERN. Therefore, in this paper we use the characteristics of the SHiP experiment (summarised in Appendix A) when estimating the potential to detect new particles. This document gives an overview of the physics, while [34] provides sensitivity estimates for selected models.

In addition we describe the sensitivity of the SHiP facility to discover new interactions between the known Standard Model particles by searching for rare processes such as $\tau \rightarrow 3 \mu$ decays, and to study the physics of the $\tau$-neutrino sector (detect $\bar{\nu}_{\tau}$, measure cross-sections and form-factors, etc.).

\footnotetext{
${ }^{1}$ http://ship.web.cern.ch
} 


\section{Chapter 2}

\section{Vector portal}

We review the main features and physics motivations behind vector portals between the Standard Model (SM) and dark sectors. Several case studies for the SHiP experiment are presented.

\subsection{Classification of vector portals}

The gauge structure of the Standard Model, the celebrated $S U(3) \times S U(2) \times U(1)$ combination, is a minimal choice compatible with the chiral nature of fermions and spontaneous electroweak symmetry breaking that renders the weak bosons and fermions massive. Establishing this structure, through a combination of theoretical and experimental efforts, is one of the major achievements of $20^{\text {th }}$ century physics.

It is possible that the gauge structure of the SM descends from a larger gauge group, as is the case in Grand Unified Theories, or GUTs. In that case one expects that at least several of the new vector states are very heavy, e.g. $m_{V} \sim 10^{16} \mathrm{GeV}$, well beyond the direct reach of accelerators. It is also possible that the SM is accompanied by additional gauge structures that allow for (sub-) TeV gauge bosons, as is the case of multiple $U(1)$ 's, $S U(3) \times S U(2) \times[U(1)]^{n}[36]$. The high-energy LHC experiments place very strong bounds on the possible existence of new vector states associated with new $U(1)$ gauge groups, provided that the coupling of such vector states to the SM is sizable $[37,38]$. An alternative possibility, relatively light vector states (e.g. in the $\mathrm{GeV}$ mass range) with small couplings to the SM, is poorly constrained by the LHC experiments and represents instead an attractive physics target for many experiments at the intensity frontier [39].

The fixed target experiment SHiP proposed at the CERN SPS [35] is a powerful tool for studying extensions of the SM based on new vector particles. This chapter discusses the physics motivations and phenomenology for such particles, provides a classification of vector portals, and points to several promising models that can be probed with proton fixed target (and beam dump) experiments. The specific examples identified as attractive physics opportunities for SHiP to be discussed in this chapter are:

- Kinetically mixed dark photons in the GeV mass range with mixing angles $\epsilon \sim O\left(10^{-6}\right)$, as well as gauge bosons coupled to baryons, $V^{(B)}$.

- Signatures of "dark Higgs" states produced through the vector portal and the dark Higgsstralhung process.

- Signatures of heavy neutral leptons (HNL) produced via the vector portal.

- Signatures of sub-GeV (meta)stable states (e.g. light dark matter) via its production and subsequent scattering via the vector portal in a neutrino-like detector. 
Throghout this chapter, we denote the new vector state as $V_{\mu}$, or simply $V$. We will also often employ a superscript to indicate the SM current coupled to the new vector state, e.g. $V^{(B-L)}, V^{(B)}$ etc. Furthermore, the new (beyond the SM) states that couple to $V_{\mu}$ will be generically called $\chi$, while the new dark Higgs states associated with $U(1)^{\prime}$ group will be called $h^{\prime}$.

\subsubsection{Kinetic mixing}

Consider a QED-like theory with one (or several) extra vector particle(s), coupled to the electromagnetic current. A mass term, or in general a mass matrix for the vector states, is protected against additive renormalization due to the conservation of the electromagnetic current. If the mass matrix for such vector states has a zero determinant, $\operatorname{det}\left(M_{V}^{2}\right)=0$, then the theory contains one massless vector, to be identified with a photon, and several massive vector states.

This is the model of 'para-photons', introduced by Okun in early 1980s [40], that can be reformulated in an equivalent language using the kinetic mixing portal. Following Holdom [41], one writes a QED-like theory with two $U(1)$ groups, supplemented by the cross term in the kinetic Lagrangian and a mass term for one of the vector fields,

$$
\mathcal{L}=\mathcal{L}_{\psi, A}+\mathcal{L}_{\chi, A^{\prime}}-\frac{\epsilon}{2} F_{\mu \nu} F_{\mu \nu}^{\prime}+\frac{1}{2} m_{A^{\prime}}^{2}\left(A_{\mu}^{\prime}\right)^{2}
$$

$\mathcal{L}_{\psi, A}$ and $\mathcal{L}_{\chi, A^{\prime}}$ are the standard QED-type Lagrangians,

$$
\begin{array}{r}
\mathcal{L}_{\psi, A}=-\frac{1}{4} F_{\mu \nu}^{2}+\bar{\psi}\left[\gamma_{\mu}\left(i \partial_{\mu}-e A_{\mu}\right)-m_{\psi}\right] \psi, \\
\mathcal{L}_{\chi, A^{\prime}}=-\frac{1}{4}\left(F_{\mu \nu}^{\prime}\right)^{2}+\bar{\chi}\left[\gamma_{\mu}\left(i \partial_{\mu}-g^{\prime} A_{\mu}^{\prime}\right)-m_{\chi}\right] \chi,
\end{array}
$$

with $F_{\mu \nu}$ and $F_{\mu \nu}^{\prime}$ standing for the field strength tensors. States $\psi$ represent the QED electron fields while states $\chi$ are charged under the "dark" $U(1)^{\prime}$. In the limit of $\epsilon \rightarrow 0$, the two sectors become completely decoupled. In eq. (2.1.1), the mass term for $A^{\prime}$ explicitly breaks the second $U(1)$ but is protected from additive renormalization and hence is technically natural ${ }^{1}$. Using the equations of motion, $\partial_{\mu} F_{\mu \nu}=e J_{\nu}^{\mathrm{EM}}$, the interaction term can be rewritten as

$$
-\frac{\epsilon}{2} F_{\mu \nu} F_{\mu \nu}^{\prime}=A_{\mu}^{\prime} \times(e \epsilon) J_{\mu}^{\mathrm{EM}}
$$

showing that the new vector particle couples to the electromagnetic current with strength, reduced by a small factor $\epsilon$. The generalization of (2.1.1) to the SM is straightforward, by subsituting the QED $U(1)$ with the hypercharge $U(1)$ of the SM.

There are a multitude of notations and names referring to one and the same model. We shall refer to the $A^{\prime}$ state as the "dark photon". It can also be denoted as $V^{(Y)}$, a vector state coupled to the hypercharge current. We choose to call the mixing angle $\epsilon$, and throughout this chapter assume $\epsilon \ll 1$. In contrast, one does not have to assume a smallness of $g^{\prime}$ coupling, which can be comparable to the gauge couplings of the SM, $g^{\prime} \sim g_{\mathrm{SM}}$.

Although the model of this type is an exceedingly simple and minimal extension of the SM, one can already learn a number of instructive features:

1. The mixing parameter $\epsilon$ is dimensionless, and therefore can retain information about loops of charged particles at some heavy scale $M$ without power-like decoupling. In the simplest example, a new fermionic field charged under both $U(1)$ 's will generate an additional contribution to the mixing angle that scales as $\Delta \epsilon \sim g^{\prime} e /\left(12 \pi^{2}\right) \times \log \left(\Lambda_{U V}^{2} / M\right)^{2}$. Alternatively, the

${ }^{1}$ When breaking of $U(1)^{\prime}$ is triggered by a Higgs mechanism, there can be an additional "gauge hierarchy" issue related to the naturalness of the $h^{\prime}$ mass term. 


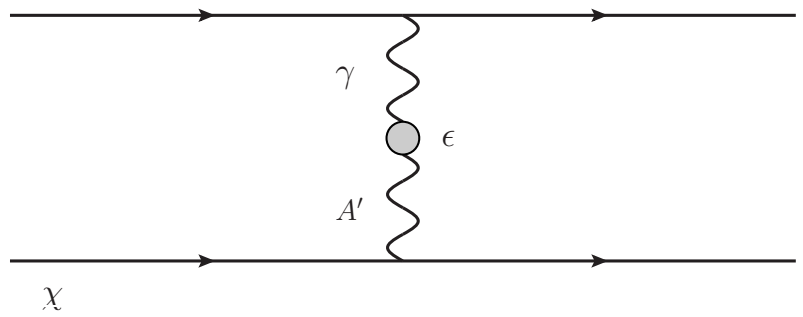

Figure 2.1: The interaction through the exchange by a mixed $\gamma-A^{\prime}$ propagator between the SM particles and particles $\chi$ charged under new $U(1)^{\prime}$ group. In the limit of $m_{A^{\prime}} \rightarrow 0$ the apparent electromagentic charge of $\chi$ is $e \epsilon$.

mixing $\epsilon$ can be generated at two or higher loop order, so that one can entertain a wide range of mixing angles.

2. If both groups are unbroken, $m_{V} \rightarrow 0$, the states $\chi$ are "millicharged particles" with electric charge $q_{\chi}=e \epsilon$. For $m_{V} \neq 0$, at $\left|q^{2}\right|<m_{V}^{2}$, the particles $\chi$ can be thought of as neutral particles with a non-vanishing electric charge radius, $r_{\chi}^{2} \simeq 6 \epsilon m_{V}^{-2}$. The diagram describing the basic interaction between the two sectors is shown in Fig. 2.1.

3. If there are no states charged under $U(1)^{\prime}$ (or they are very heavy), and $m_{V}$ is taken to be zero, then the two sectors decouple even at non-zero $\epsilon$. This leads to the suppression of all interactions for a dark photon inside a medium. If $m_{V}$ becomes smaller than the characteristic plasma frequency all processes with emission or absorption of dark photons decouple as $\sim m_{V}^{2}$ $[42]$.

4. The new vector boson interacting with the SM via the electromagnetic current conserves several approximate symmetries of the SM, including parity, flavour, and $C P$. Moreover, $A^{\prime}$ does not couple directly to neutrinos. As a consequence of these two features, the interaction strength due to the exchange of $A^{\prime}$ can be taken to be stronger than that of weak interactions, $(e \epsilon)^{2} / m_{A^{\prime}}^{2} ;\left(e \epsilon g^{\prime}\right) / m_{A^{\prime}}^{2} \gg G_{F}$. This property proves very useful in constructing light dark matter models with the use of vector portal.

Although this model was known to theorists and well-studied over the years (e.g. Refs. [43, 44]), there has been a revival of scenarios involving a kinetically-mixed $A^{\prime}$ during the last decade. Much of this activity has been in response to various astrophysical anomalies which can be interpreted as a sign of dark matter interacting with the SM through a kinetically mixing vector. Renewed interest in dark photons has triggered new analyses of past or existing experiments [45-54], and generated proposals for new dedicated experiments, which are currently at various stages of implementation [55-58]. In this chapter, we will demonstrate that the SHiP proposal is capable of probing new domains of the parameter space for this model, with and without light dark matter.

\subsubsection{Anomaly-free gauge groups $\left(B-L, L_{\mu}-L_{\tau}\right.$ etc)}

The kinetically-mixed portal described above represents the simplest way to couple a new vector particle to the SM, without charging any of the SM fields under the new gauge group. There is also an interesting alternative route in which certain combinations of the SM fields are charged under the new $U(1)^{\prime}$.

The most prominent example of this type is $V^{(B-L)}$, which, provided the SM is supplemented with three right-handed neutrinos, is anomaly free. While the multitude of scales for the mass 
of $B-L$ gauge boson is possible, the value of the coupling is quite constrained by the fact that neutrinos acquire a new interaction with electrons and baryons, so that broadly speaking we require $\left(g_{(B-L)}\right)^{2} / m_{V^{(B-L)}}^{2}<G_{F}$.

The only model-building complication that arises in this construction is related to neutrino masses. One can consider Dirac masses for the neutrinos, in which case this problem does not exist. On the other hand, the Majorana masses of right-handed $(\mathrm{RH})$ neutrinos, and more generally the effective operator of dimension five that generates light neutrino masses, $(L H)^{2}$, are incompatible with $B-L$ gauge symmetry. One can solve this problem with additional model ingredients. For example, the right-handed neutrino mass can be associated with the condensation of an additional Higgs field, $\Phi$, that has a charge -2 under this gauge group. so that the mass term in the Lagrangian $y_{N} N N \Phi$ is gauge-invariant. Then the ratio of masses of the $U(1)_{B-L}$ gauge boson and the righthanded neutrino mass would scale as

$$
\frac{m_{V^{(B-L)}}}{m_{N}} \sim \frac{g^{(B-L)}}{y_{N}} .
$$

It is possible then that the masses of the $\mathrm{RH}$ neutrinos and the $B-L$ gauge boson can be comparable, and the lightness of $V^{(B-L)}$ may in turn imply a relative lightness of $N$. This will be important for the phenomenological signals of the $B-L$ interaction in fixed target experiments.

Individual lepton flavours can also be gauged in specific anomaly-free combinations. Thus, the $B-3 L_{i}$ combinations, where $i$ is an individual flavour, have been considered in the past, and their phenomenology is very similar to the $B-L$ case discussed above. One specific group, based on the $L_{\mu}-L_{\tau}$ combination, is an exception: since neither electrons nor quarks are charged under this group it is very difficult to constrain experimentally, and the strength of the new interaction can be comparable with the weak strength. For example, $\left(g_{\left(L_{\mu}-L_{\tau}\right)}\right)^{2} / m_{V}^{2} \sim G_{F}$ is not excluded. There is an extensive theoretical literature dedicated to such symmetries, see e.g. Refs. [59-64].

\subsubsection{Other froms of vector portals.}

The examples of vector portals described above are special in that they are UV complete and do not require new physics at the weak scale. In this subsection we discuss other possibilities that require additional steps for UV completion.

One example with a distinct phenomenology is based on gauged baryon number, $U(1)_{B},[65-68]$. This symmetry is anomalous (in particular there are mixed electroweak- $B$ anomalies) and therefore a gauged $U(1)_{B}$ construction requires a UV completion. Such a completion can be obtained, for instance, with new electroweak charged chiral fermions that cancel the anomaly and obtain a weak scale mass through spontaneous symmetry breaking. Therefore, from the low-energy point of view, the $U(1)_{B}$ portal is legitimate, leading to rather distinct "leptophobic" phenomenology.

Individual quark flavours can also be gauged in anomaly-free combinations. For example, gauging two quark flavour $q_{1}-q_{2}$ in a manner similar to $L_{\mu}-L_{\tau}$ is possible. The difficulty lies with quark mass sector, where additional Higgses must be introduced in order to generate mass terms consistent with the gauge groups of the SM and of the new $U(1)$. While this may be possible from a model-building point of view, one has to address the rather severe phenomenological challenge of new tree-level flavour-changing neutral currents.

Finally, even anomalous gauge groups can be considered as low energy effective field theories. In general, even in the presence of gauge anomalies gauge invariance can be restored at the expense of non-renormalizability of the theory [69]. This leads to an associated energy scale at which one loses calculability and requires a UV completion. For our purposes, it is sufficient to assume that this scale is at the weak scale or above and then consider the effective theory of vector particles, under the assumption that some UV completion may be found. 
On pure phenomenological grounds, one can classify the possible vector couplings to the SM fields using the framework of the effective Lagrangian where for simplicity we assume the minimal flavour violation $(\mathrm{MFV})$ ansatz. The vector interactions of the MFV type [70] are given by the following combinations of the SM fields, Yukawa matrices, and unknown coefficients $a_{I}$ and $b_{I J}$ :

$$
\begin{aligned}
\mathcal{L}_{\text {int }} & =V_{\mu} \bar{Q} \gamma_{\mu}\left(a_{Q} \mathbf{1}+b_{Q U} Y_{U}^{\dagger} Y_{U}+b_{Q D} Y_{D}^{\dagger} Y_{D}+\ldots\right) Q \\
& +V_{\mu} \bar{U} \gamma_{\mu}\left(a_{U} \mathbf{1}+b_{U U} Y_{U} Y_{U}^{\dagger}+\ldots\right) U \\
& +V_{\mu} \bar{D} \gamma_{\mu}\left(a_{D} \mathbf{1}+b_{D D} Y_{D} Y_{D}^{\dagger}+\ldots\right) D \\
& +V_{\mu} \bar{L} \gamma_{\mu}\left(a_{L} \mathbf{1}+b_{L E} Y_{E}^{\dagger} Y_{E}+\ldots\right) L \\
& +V_{\mu} \bar{E} \gamma_{\mu}\left(a_{E} \mathbf{1}+b_{E E} Y_{E} Y_{E}^{\dagger}+\ldots\right) E .
\end{aligned}
$$

At this point, it is more appropriate to think of $V_{\mu}$ as of "ordinary" Maxwell-Proca field, rather than a gauge boson. Written in this form, it is easy to see that the mass $m_{V}$ is not protected at loop level, and indeed will receive additive corrections proportional to the cutoff scale. Within effective field theories, the coefficients $a_{I}$ and $b_{I J}$ cannot be fixed from first principles, but instead can be constrained directly from experiment. Their smallness guarantees that the cutoff can be taken at the $\mathrm{TeV}$ scale or higher.

So far we have primarily discussed dimension four vector portals, but the non-renormalizable part of (2.1.5) essentially descends from higher-dimensional operators. It is clear that at $\operatorname{dim}>4$, one can construct many new forms of the higher-dimensional operators. For example, one can have a fully gauge invariant dipole portal,

$$
\mathcal{L}_{\text {dipole }}=\sum_{i j} \bar{f}_{i}\left(1 \times \mu_{i j}+\gamma_{5} \times d_{i j}\right) \sigma_{\alpha \beta} f_{j} V_{\alpha \beta}+(\text { h.c. }) .
$$

Here, $f_{i}$ represents different $\mathrm{SM}$ fermions, $V_{\alpha \beta}$ is the field strength of the exotic vector state, and $\mu_{i j}$ and $d_{i j}$ are complex-valued Wilson coefficients. This generalizes the dipole operator discussed in [71]. Due to the explicit gauge invariance, the mass of the vector boson $V$ does not receive corrections from (2.1.6) and can be small. Also, the $S U(2) \times U(1)$ properties of the SM fermions implies that the actual dimension of such operators is six, with $\mu$ and $d$ scaling as $\sim v_{E W} / \Lambda_{\mathrm{UV}}^{2}$. The phenomenology of this portal, including the production and decay of dipole-coupled vectors, has not been studied in any detail in the literature. We note in passing that the production and decay of $V$ via (2.1.6) can look similar to the case of the axion-like particle, $\left(\partial_{\mu} a\right) \bar{f}_{i} \gamma_{\mu} f$, due to the same scaling with the momentum of the exotic particle.

\subsubsection{Chern-Simons portal}

Motivation. Another way to couple a new vector particle $V_{\mu}$ to the SM is given by the so-called effective Chern-Simons interaction (or the Chern-Simons portal):

$$
\mathcal{L}_{c s}=c_{Z} \epsilon^{\mu \nu \lambda \rho} Z_{\mu} V_{\nu} \partial_{\lambda} Z_{\rho}+c_{\gamma} \epsilon^{\mu \nu \lambda \rho} Z_{\mu} V_{\nu} \partial_{\lambda} A_{\rho}+c_{W} \epsilon^{\mu \nu \lambda \rho} W_{\mu} V_{\nu} \partial_{\lambda} W_{\rho}
$$

where $A_{\mu}, Z_{\mu}, W_{\mu}$ stand for the photon, $W^{ \pm}$and $Z$-boson fields, and $c_{Z}, c_{\gamma}, c_{W}$ are some dimensionless coefficients. In terms of fields $A, Z$ and $W$ the operators (2.1.7) are "dimension 4 operators". The $S U(2)$ gauge invariant form of these interaction (where $Z_{\mu}=\Phi^{\dagger} D_{\mu} \Phi$, etc.) requires, however, to consider them as higher dimension operators (dimension-6):

$$
\mathcal{L}_{c s}=\frac{1}{\Lambda_{Y}^{2}} \epsilon^{\mu \nu \lambda \rho} \Phi^{\dagger} D_{\mu} \Phi V_{\nu} F_{Y}^{\lambda \rho}+\frac{1}{\Lambda_{\mathrm{SU}(2)}^{2}} \epsilon^{\mu \nu \lambda \rho} \Phi F_{W}^{\lambda \rho} D_{\mu} \Phi^{\dagger} V_{\nu}, \ldots
$$


where $F_{Y}$ and $F_{W}$ is the hypercharge and the $\mathrm{SU}(2)$ field strengths correspondingly and the $\Lambda$ 's are new scales that depend on the origin of the effective operators (2.1.8). The coefficients in (2.1.7) are expressed as the ratios of these scales to the Higgs vev: $c_{\gamma}=\cos \theta_{W} \frac{v^{2}}{\Lambda_{Y}^{2}}, c_{Z}=\sin \theta_{W} \frac{v^{2}}{\Lambda_{Y}^{2}}$ and $c_{W}=\frac{v^{2}}{\Lambda_{\mathrm{su}(2)}^{2}}$ which fixes $c_{Z} / c_{\gamma}=\tan \theta_{W}$. It is possible however to write an operator of dimension 8 that would also lead to $V Z \partial Z$ term. Therefore in what follows we consider $c_{Z}$ and $c_{\gamma}$ as two independent dimensionless parameters.

Such generalized Chern-Simons interactions appear in various models (see e.g. [33, 72-81]). They can appear, for example, if a mixed gauge anomaly with respect to the SM gauge field and a new gauge symmetry related to $V$, is cancelled in a non-trivial way between new chiral heavy particles. This is similar to the way the $U(1)_{Y} \times S U(2)^{2}$ and $U(1)_{Y}^{3}$ gauge anomalies are cancelled between quark and leptonic sectors. In this respect, the Chern-Simons interaction is similar to the so-called D'Hoker-Farhi interaction [82, 83], describing the contribution of the top quark at the energies below its mass (but above the masses of all other SM fermions). As without the top quark the gauge current in the SM would not be conserved, there is a contribution at $E<m_{\text {top }}$ that is not suppressed by the mass of the top (in fact, does not depend on it at all). The interactions (2.1.7) are analogs of the DF term, but involving also a new vector field $V$. If the UV model that leads to the effective interactions (2.1.8) becomes ill-defined as $|\Phi| \rightarrow 0$ (see e.g. [33]), one can write $\Lambda_{Y}^{2} \rightarrow \frac{|\Phi|^{2} \cos \theta_{W}}{c_{\gamma}}$ (similarly for $\left.\Lambda_{\mathrm{SU}(2)}^{2}\right)$ and the terms (2.1.7) become true operators of dimension4. Therefore, similarly to the case of kinetic mixing, the "non-decoupling" of the anomalies can be viewed as an additional window into the deep UV physics. Other mechanisms leading to the effective Chern-Simons interaction can appear in the models with extra dimensions, models with "anomaly inflow", string theory inspired models.

Existing bounds. The bounds on $c_{Z}, c_{W}, c_{\gamma}$ in the range of masses $m_{V}<$ few GeV come from the possible contributions of the Chern-Simons interactions to the $Z$ or $W$ total width. This contributions is dominated by the longitudinal component of $V$-boson:

$$
\Gamma(Z \rightarrow \gamma V)=\frac{c_{\gamma}^{2} \cos \theta_{W} M_{W}}{96 \pi}\left(\frac{M_{Z}^{2}}{m_{V}^{2}}+1\right) \quad ; \quad \Gamma\left(W^{+} \rightarrow X u \bar{d}\right) \approx \frac{c_{W}^{2} \alpha_{W} M_{W}}{432 \pi^{2}} \frac{M_{W}^{2}}{m_{V}^{2}}
$$

where $\alpha_{W}$ is the weak coupling constant and $\theta_{W}$ is the Weinberg's angle. Similar formula exists for $\Gamma\left(Z \rightarrow Z^{*} X\right)$. We are interested in the $V$ bosons that can travel $c \tau_{V} \gg 60 \mathrm{~m}$ (decay volume of the SHiP detector) and having mass $m_{V}<5 \mathrm{GeV}$. For $m_{V} \ll M_{Z}$ the bounds go $c_{V}^{2} / m_{V}^{2}<$ const where $c_{V}$ is one of the $c_{\gamma}, c_{Z}$ or $c_{W}$ constants. These bounds are roughly at the level $c_{Z}^{2}, c_{W}^{2} \lesssim$ $10^{-3}\left(\frac{m_{V}}{1 \mathrm{GeV}}\right)^{2}$. In case of $c_{\gamma}$ a significantly stronger bound comes from the measuring of the single photon events at LEP [84]. There the branching at the level $\mathrm{Br}<10^{-6}$ was established for photons with the mass above $15 \mathrm{GeV}$. This leads to the strong bound $c_{\gamma}^{2} \lesssim 10^{-9}\left(\frac{m_{V}}{1 \mathrm{GeV}}\right)^{2}$

The main production modes of $V_{\mu}$ depending on each of the three terms in (2.1.7) are listed below:

- For $m_{V}<m_{D}$ the dominant source of production is via weak decays of the mesons (such as $D^{ \pm} \rightarrow W^{*} \stackrel{\text { CS }}{\rightarrow} \rho^{ \pm}+V$ or $D \rightarrow W^{*} \stackrel{\text { CS }}{\rightarrow} \ell+\bar{\nu}+V$ where $\rho^{ \pm}$is the vector meson, $\ell$ is one of the leptons $(e, \mu)$ and $\bar{\nu}$ is the corresponding flavour of neutrino. These processes are controlled by the $c_{W}$ constant (the matrix element for the process $D^{ \pm} \rightarrow W^{*} \stackrel{\mathrm{CS}}{\rightarrow} \pi^{ \pm}+V$ is zero due to antisymmetric nature of the vertex $(2.1 .7))$

- At masses $m_{V}<m_{J / \psi}$ channels of production via $Z$ bosons $J / \psi \rightarrow Z^{*} \rightarrow \gamma+V$ (controlled by the $c_{\gamma}$ constant) or $J / \psi \rightarrow Z^{*} \rightarrow Z+V$ (controlled by the $c_{Z}$ constant) with the subsequent decay of $Z$ to a fermion-antifermion pair. This is the main channel for $m_{D}<m_{V}<m_{J / \psi}$ or in the situations when $c_{W} \ll c_{Z}, c_{\gamma}$. 


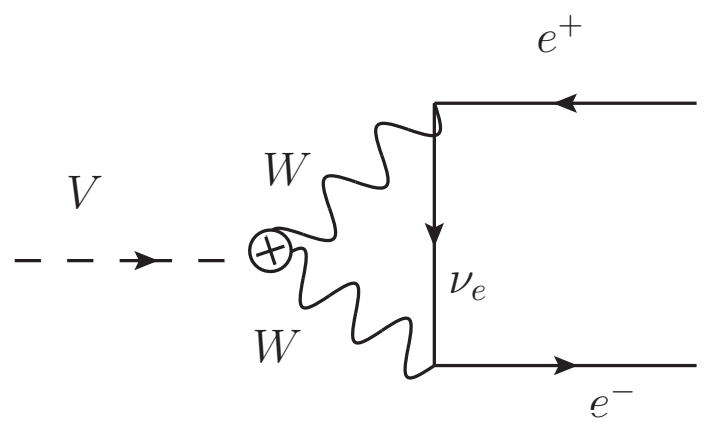

Figure 2.2: Loop decay of $V$ boson to a pair of fermions, mediated by the $c_{W}$ term in the ChernSimons interaction (2.1.7). A similar process with $c_{Z}$ coupling and two $Z$ bosons running in the loop also exist.

- Additionally, for $m_{V}$ below the masses of light electromagnetically decaying mesons $\pi^{0}, \eta, \omega$ new channels open, governed by the $c_{\gamma}$ vertex: $\pi^{0} \rightarrow \gamma \gamma^{*}$ with the off-shell photon $\gamma^{*}$ decaying to $V$ and a fermion-antifermion pair.

The decay modes of $V$ boson again depend on the relative size of $c_{\gamma}, c_{Z}, c_{W}$ coefficients.

- Naively, the decay via $c_{\gamma}$ vertex is only singly weak suppressed and therefore should dominate. There are many interesting experimental signatures for decays $V \rightarrow \gamma h^{0}$ where $h^{0}$ is a neutral meson $\left(\pi^{0}, \eta, \rho^{0}\right)$. The same vertex governs the decay modess $V \rightarrow \gamma+f+\bar{f}$, suppressed by the extra phase space factor). However, due to the strong bounds from LEP on the $c_{\gamma}$ the number of events due to $c_{\gamma}$ vertex is expected to be well below 1 at all masses, even for maximally efficient production (maximal values of $c_{W}, c_{Z}$ constants allowed based on the LEP results).

- The decay via $c_{W}$ or $c_{Z}$ are double weak suppressed. Among the tree-level processes the dominant one is $V \rightarrow \pi+\rho$ (where $\pi$ is a pseudo-scalar meson and $\rho$ is a vector meson. (Decay of $V$ to two pions is impossible due to the antisymmetric structure of the interaction (2.1.7)). Additional 3 -body decays are strongly suppressed by the phase-space factor. However, a loop-mediated decay $V \rightarrow f+\bar{f}$ (Fig. 2.2) dominates over the tree-level processes due to "compensation" of one of the $W$ or $Z$ propagators in the loop.

The resulting number of "detector events" can be as large as few thousands (via the loop-mediated process producing a pair of fermions) for masses $\mathcal{O}(1) \mathrm{GeV}$ and $c_{Z}, c_{W}$ of the order of their maximal values, allowed from the LEP bounds.

\subsection{Matter states charged under new $U(1)$}

The new vector portal is likely to be accompanied by new states charged under $U(1)^{\prime}$. In this section we review different theoretical constructions behind the finite mass for the new vector bosons and discuss the possibility that the new $U(1)^{\prime}$ sector is supersymmetric.

\subsubsection{Higgs mechanism in the dark sector}

There are several theoretical ways of breaking the $U(1)^{\prime}$ symmetry. In all UV-complete models, the new vector particle couples to the conserved current, which allows for the introduction of a "hard" or "Stuckelberg" mass term, $\mathcal{L}_{m}=\frac{1}{2} m_{V}^{2} V_{\mu}^{2}$. This represents the most minimal possibility. If, however, the additional gauge symmetry is broken in the same way as in the SM, a new Higgs field in the dark sector will be introduced. 
The "Higgsing" of the $U(1)^{\prime}$ introduces extra interactions in the dark sector. Consider the simplest possibility in which the new scalar field is charged under $U(1)^{\prime}$ [85],

$$
\mathcal{L}_{\phi}=\left|D_{\mu} \phi\right|^{2}-\lambda|\phi|^{4}+\mu^{2}|\phi|^{2},
$$

where $D_{\mu}$ is the covariant derivative. After the spontaneous symmetry breaking, $\phi=\left(v^{\prime}+h^{\prime}\right) / \sqrt{2}$, the vector field becomes massive with $m_{V}=g^{\prime} v^{\prime}$. Besides the mass terms, several interaction terms appear:

$$
\mathcal{L}_{h^{\prime}}=\frac{1}{2} m_{V}^{2} V_{\mu}^{2}+\frac{1}{2}\left(\partial_{\mu} h^{\prime}\right)^{2}-\frac{1}{2} m_{h^{\prime}}^{2} h^{\prime 2}+\frac{m_{V}^{2}}{v^{\prime}} h^{\prime} V_{\mu}^{2}+\frac{m_{V}^{2}}{v^{\prime 2}} h^{\prime 2} V_{\mu}^{2}-\frac{m_{h^{\prime}}^{2}}{2 v^{\prime}} h^{\prime 3}-\frac{m_{h^{\prime}}^{2}}{8 v^{\prime 2}} h^{\prime 4},
$$

where $m_{h^{\prime}}$ is the mass for the dark Higgs particle. Depending on the relative sizes of the quartic coupling $\lambda$ and the square of the gauge coupling $g^{\prime 2}$ the dark Higgs can be lighter or heavier than the vector. This will become important for the potential signals of dark sector as $m_{h^{\prime}} \leq m_{V}$ opens the possibility for a macroscopic decay length of the dark Higgs [85, 86].

A more complicated Higgs sector can lead to an additional important effect for a dark photon beyond the kinetic mixing. In particular, a mass mixing between $V$ and the SM $Z$ boson becomes possible [87], opening a host of additional phenomenological consequences such as parity violation mediated by $V$ exchange and the coupling of $V$ to the SM neutrinos.

\subsubsection{Supersymmetric $U(1)^{\prime}$ models}

Supersymmetric extensions of vector portal models are also well-studied. For a SUSY version of the dark photon model, see e.g. [88-91]. One can imagine a variety of scenarios vis-a-vis the origin of supersymmetry breaking that could lead to different mass patterns between the observable and dark sectors. One of the most interesting possibilities includes a scenario with an approximately supersymmetric dark sector in which the breaking of supersymmetry in the $U(1)^{\prime}$ sector is mediated by the vector portal coupling from the SM. In the simplest scenarios of this kind, one expects the following relations to hold true:

$$
m_{A^{\prime}}^{2} \sim \epsilon^{1 / 2} M_{Z} ; \quad m_{A^{\prime}}=m_{h^{\prime}} .
$$

The first relation arises because of the induced $D$-term in the $U(1)^{\prime}$ sector that scales as $\sim \epsilon$ [90]. The second relation is the consequence of the minimal Higgs sector in the SUSY version of the dark photon model [91]. Equal masses for $h^{\prime}$ and $A^{\prime}$ particles forbid the decays of $h^{\prime}$ to $A^{\prime}$ and prolong the lifetime of $h^{\prime}$, thus making supersymmetric dark Higgses an interesting target for the studies at SHiP. More details on supersymmetric scenarios in conjunction with the light vector portal can be found in the SUSY chapter of this white paper, Chapter 6.

\subsection{Physics motivation for light mass (less than weak scale) vector par- ticles}

The interest in vector portals comes from several sources. The appearance of such light vector portals is quite common in top-down constructions, such string-inspired models and GUT theories. At the same time, several variants of the vector portal models, especially with relatively light mediators, have been invoked as a remedy for a number of observational anomalies, both in particle physics and astrophysics. This section reviews a subset of the problems that vector portal models help to resolve. 


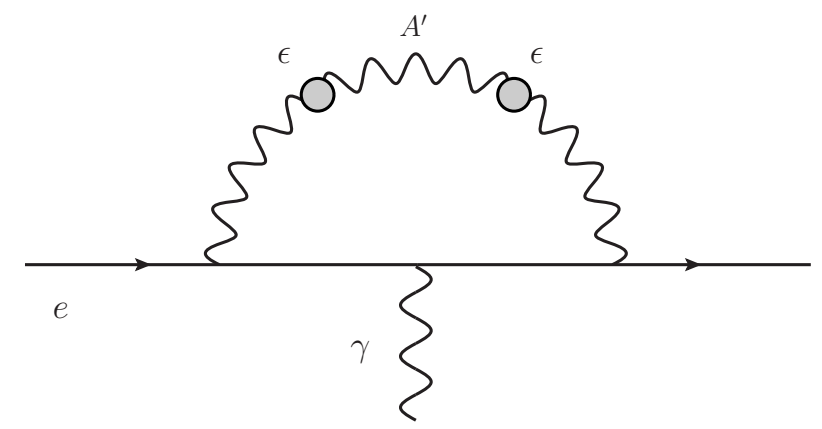

Figure 2.3: One-loop correction to the muon anomalous magnetic moment due to the exchange of the dark photon.

\subsubsection{Putative solution to the muon $g-2$ discrepancy}

The persistent discrepancy between the measured muon anomalous magnetic moment and the SM prediction at the level of $\sim 3 \sigma$ [92] has generated significant experimental and theoretical activity aiming for a possible explanation. The intense scrutiny of the SM contributions has not produced any obvious candidate for an extra contribution $\Delta a_{\mu} \sim+3 \times 10^{-9}$ that would cover a theoretical shortfall and match the observed value. Among the new physics explanations for this discrepancy are weak scale solutions [93], as well as possible new contributions from light and very weakly coupled new particles (see, e.g., [47, 86, 94]). With the LHC continuously squeezing the available parameter space for the weak-scale $g-2$-relevant new physics, solutions with light particles appear as an attractive alternative.

It is easy to see that a light vector particle coupled to muons via the vector portal provides an upward correction to $g-2$. In most models the new vector particle does not have an axial-vector coupling to charged leptons, and a simple one-loop diagram, Fig. 2.3, gives a positive correction to the magnetic anomaly,

$$
a_{l}^{V}=\frac{\alpha}{2 \pi}\left(\frac{g^{\prime}}{e}\right)^{2} \times \int_{0}^{1} d z \frac{2 m_{l}^{2} z(1-z)^{2}}{m_{l}^{2}(1-z)^{2}+m_{V}^{2} z}=\frac{\alpha}{2 \pi}\left(\frac{g^{\prime}}{e}\right)^{2} \times\left\{\begin{array}{c}
1 \text { for } m_{l} \gg m_{V}, \\
2 m_{l}^{2} /\left(3 m_{V}^{2}\right) \text { for } m_{l} \ll m_{V} .
\end{array}\right.
$$

In this expression $g^{\prime} / e$ is the strength of the $V_{\mu}$ coupling to the muon vector current in units of electric charge. For the kinetically-mixed dark photon $A^{\prime}$, we have $g^{\prime} / e=\epsilon$. For the choice of $\epsilon \sim$ few $\times 10^{-3}$ at $m_{V} \sim m_{\mu}$, the new contribution brings theory and experiment into agreement. Since 2008, significant experimental advances have been made towards testing this possibility, while at the same time various theoretical extensions of the simplest kinetic mixing portal explanation have been proposed. The following picture has emerged:

- The minimal dark photon model, with no light particles charged under $U(1)^{\prime}$ is excluded (or at least very close to being excluded) by a complementary array of experiments. The most difficult part of the parameter space, the vicinity of $m_{A^{\prime}} \sim 30 \mathrm{MeV}$, has finally been ruled out only recently as a solution to the $g-2$ puzzle, $[52,54]$.

- A slightly extended model of the dark photon can still offer a solution to the $g-2$ discrepancy. For example, if $A^{\prime} \rightarrow \chi \bar{\chi}$ in the dark sector the visible $A^{\prime} \rightarrow e^{-} e^{+}$decays will be diluted. In any case, it appears that $m_{A^{\prime}}<200 \mathrm{MeV}$ is required [95].

- Finally, the least constrained model is based on the gauged $L_{\mu}-L_{\tau}$ vector portal $[61,62,64]$, and the vector mass below $m_{V} \sim 400 \mathrm{MeV}$ can still be considered as a potential solution to 


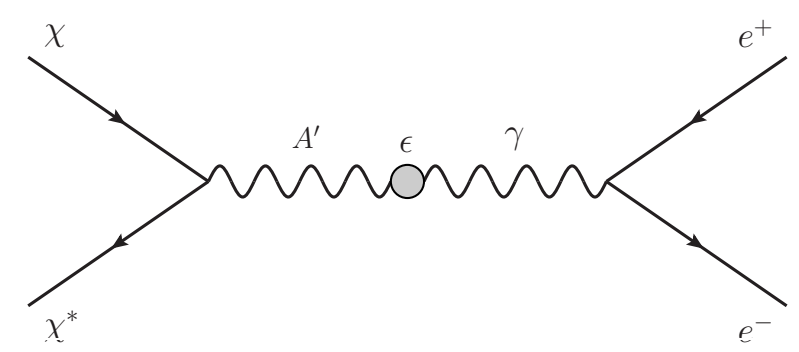

Figure 2.4: Light $\left(m_{\chi} \sim\right.$ few $\left.\mathrm{MeV}\right)$ scalar dark matter annihilating to electron-positron pairs due to the mixed $\gamma-A^{\prime}$ propagator. The annihilation occurs in the $p$-wave.

the muon $g-2$ discrepancy [96, 97].

To summarize, a light vector particle remains an attractive solution to the muon $g-2$ discrepancy, and more experimental work is required to exclude this possibility in a model-independent way.

\subsubsection{Mediator of interaction with DM and possible connection to astrophysical positron excess}

Vector portals offer a means to connect the SM to dark matter. In the last few years, the direct searches for dark matter have intensified, paralleled by broad investigations of the theoretical opportunities for dark matter. The weakly interacting massive particle (WIMP) paradigm offers perhaps the largest number of opportunities for the experimental discovery of dark matter via its non-gravitational interaction. In the standard WIMP paradigm, known from 1970s [98, 99], the correct cosmological abundance of dark matter is achieved via its self annihilation at high temperatures, $T \sim m_{\chi}$, where $m_{\chi}$ is the WIMP mass. Simple calculations show that the required WIMP abundance is achieved if

$$
\sigma_{\text {annih }}(v / c) \sim 1 \mathrm{pbn} \Longrightarrow \Omega_{\mathrm{DM}} \simeq 0.25
$$

where $v / c$ is the approximate relative velocity at the time of annihilation. The nature of the interaction responsible for the self-annihilation of WIMPs to the SM states is important. It sets the size of the self-annihilation cross section and ultimately the abundance of WIMP dark matter. If the interactions are mediated by forces that have weak strength and operate through the exchange of weak scale particles, then for small and large masses one expects the following scaling with the WIMP mass,

$$
\sigma(v / c) \propto\left\{\begin{array}{c}
G_{F}^{2} m_{\chi}^{2} \text { for } m_{\chi} \ll M_{W}, \\
1 / m_{\chi}^{2} \text { for } m_{\chi} \gg M_{W} .
\end{array} \quad \Longrightarrow \quad \text { few } \mathrm{GeV}<m_{\chi}<\text { few } \mathrm{TeV}\right.
$$

This famously determines the so-called "Lee-Weinberg window", or the mass range for the DM under the assumption of weak-scale mediators. According to this logic thermal relic MeV-GeV scale dark matter is disfavored.

The crucial assumption in the argument above is the link between the weak scale and the mass of the mediator particles. As was argued in the previous sections certain vector portals do allow interaction strengths in excess of $G_{F}$. This in turn opens the door to the construction of rather natural models of light dark matter, which can be made as light as a few $\mathrm{MeV}$ [100]. It is important to note that such light WIMPs are challenging to probe via direct scattering of galactic DM particles on atoms [101], and therefore alternative strategies aimed covering this mass range must be developed. 


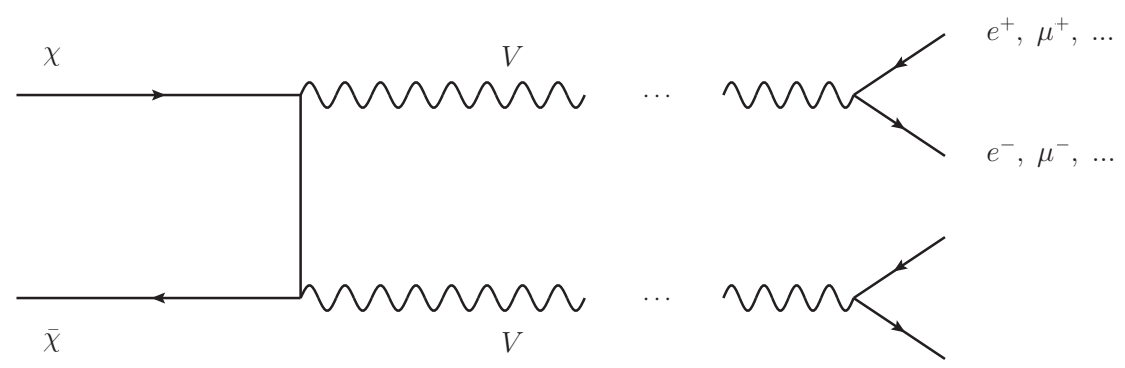

Figure 2.5: Sequential annihilation of heavy WIMP dark matter, first to light metastable mediators, with their subsequent decay to light SM states.

On the phenomenological side, the light dark matter can be behind an unexpectedly strong emission of $511 \mathrm{keV}$ photons from the galactic bulge, as observed by SPI/INTEGRAL [102]. It is presently unclear whether new physics needs to be invoked for the explanation of such emission, and we refer readers to the on-going discussions in the literature [103]. Nonetheless, a dark-matterrelated origin of $511 \mathrm{keV}$ excess, in which the non-relativistic or semi-relativistic positrons are products of DM annihilation or decay, is possible [104]. For example, scalar dark matter charged under the new $U(1)^{\prime}$ with a mass in the $m_{\chi} \sim$ few $\mathrm{MeV}$ range can pass all the existing constraints [100], and supply the requisite source for positrons. Direct calculations in the model where mediation of the SM-DM interaction occurs due to the dark photon, as in Fig. 2.4, gives the annihilation crosssection in the form

$$
\sigma_{\operatorname{annih}}(v / c) \simeq \frac{8 \pi \alpha \alpha_{D} \epsilon^{2}\left(m_{\chi}^{2}+2 m_{e}^{2}\right) v^{2}}{3\left(m_{A^{\prime}}^{2}-4 m_{\chi}^{2}\right)^{2}} \sqrt{1-m_{e}^{2} / m_{\chi}^{2}}
$$

Here $\alpha_{D}=\left(g^{\prime}\right)^{2} /(4 \pi)$, and $m_{\chi} \gg m_{e}$ is assumed. The extra factor of the squared relative velocity in this formula is indicative of the $p$-wave annihilation, and is what ultimately allows this model to escape strong constraints on light dark matter annihilation imposed by the accurate measurements of CMB anisotropies. The least constrained region of the parameter space corresponds to very light mediators, $m_{A^{\prime}}<100 \mathrm{MeV}$, and $2 m_{\chi}<m_{A^{\prime}}$. With this choice of parameters, $\sigma_{\text {annih }}(v / c)$ can be significantly larger than 1 pbn, making MeV-scale dark matter possible.

Another prominent astrophysical anomaly that has generated a great deal of speculation as a possible sign of dark matter is the observation of the rise in the fractional positron flux as a function of energy. In 2008, the results of PAMELA satellite experiment $[105,106]$ indicated that the fractions of galactic anti-proton flux, $n_{\bar{p}} /\left(n_{p}+n_{\bar{p}}\right)$, as a function of energy, behaves according to the fiducial expectations from the astrophysical modelling of the cosmic ray origin and propagation. In contrast, the corresponding fraction of positrons, $n_{\bar{e}} /\left(n_{e}+n_{\bar{e}}\right)$, exhibits a significant upturn above $E>10 \mathrm{GeV}$, prompting speculations about the necessity of additional primary sources of energetic positrons. This measurement was independently confirmed through FERMI-LAT observations [107], and brought to the new level of accuracy by the AMS-2 experiment [108]. The annihilation of heavy dark matter with $m_{\chi}>M_{W}$ could be a theoretically attractive source of such positrons. Yet the simplest WIMP models do not fit the positron excess because of two problems. The required annihilation rate capable of supplying the positron excess is above the WIMP freeze-out annihilation rate by $\sim$ two orders of magnitude. In addition, models where the final state annihilation products are heavy SM particles $(b, t, W, Z, h)$ will necessarily produce antiprotons and are therefore are tightly constrained by $n_{\bar{p}} /\left(n_{p}+n_{\bar{p}}\right)$.

It was soon realized that these problems can be rather efficiently circumvented if the heavy WIMP dark matter is interacting with the SM via relatively light mediators [109, 110], and the 
$\mathrm{DM} \rightarrow \mathrm{SM}$ annihilation occurs via an intermediate stage of light mediators as in Fig. 2.5. In particular, for the light vector mediator one finds that:

- The WIMP dark matter abundance is regulated via the $\chi \bar{\chi} \rightarrow V V \rightarrow \mathrm{SM}$ annihilation process. If $m_{V}$ is sufficiently light, then the $v \sim 0.3 c$ and $v \sim 10^{-3} c$ annihilation regimes (freeze-out vs galactic environment) can be markedly different. The existence of the dark-force-induced attraction between WIMP and anti-WIMP particles creates a Coulomb (or Sommerfeld) type enhancement of the cross-section, providing an extra factor $S \sim \pi \alpha_{D} / v \gg 1$ at small velocities. The galactic annihilation can be further enhanced by the resonant states in the $\chi-\bar{\chi}$ system. This solves the problem for required enhancement of the annihilation cross section in the galactic environment.

- The fraction of leptons in the final states can be significanly enhanced. Indeed, for $m_{V}<2 m_{p}$, the antiprotons cannot be generated in the decay of mediators $V$. The annihilation branching to photons is also significantly reduced if $\pi^{0}$ cannot be easily generated in the decay products.

- Finally, the two-stage annihilation process, $\chi \bar{\chi} \rightarrow V V$, followed by decay $V \rightarrow \mathrm{SM}$, allows hiding the WIMP dark matter from direct searches. This is because the coupling to the SM (e.g. $\epsilon$ for dark photons) is not constrained by the annihilation cross section, and can be taken almost arbitrarily small [111].

This theoretical construction proves that galactic positron excess could come from the annihilation of WIMP dark matter (there are no show stoppers), although at this point it is impossible to argue in favor of the dark matter explanation vs competing astrophysical mechanisms (see, e.g. $[112,113])$. These models do come under additional constraints from the absence of broad excesses in $\gamma$-ray fluxes [114], and more recently from the absence of DM-induced distortion of the CMB anisotropies as reported by Planck collaboration (Planck collaboration, to appear). While these constraints are very important, they cannot rule out the theoretical construction above in a modelindependent way. Thus, it is important to constrain the DM-SM mediators directly from terrestrial experiments, and for the broad ranges of possible couplings.

Vector portals can also open a window to non-thermal dark matter, particularly in the case of sterile neutrino dark matter, $N$ [115]. Sterile neutrinos mix with Standard Model (SM) neutrinos, and therefore any new vector interactions involving SM neutrinos, such as gauged lepton number symmetries, indirectly couple to sterile neutrinos as well. This can modify the sterile neutrinos' cosmological abundance, and the existence of new vector interactions coupled to SM leptons also provides a new phenomenological probe of theories with sterile neutrino dark matter. In the minimal model where the sterile neutrinos interact with the SM only via mixing (the neutrino minimal SM, $\nu \mathrm{MSM}), N$ production is entirely determined by the $N$ mass and its mixing with a lepton doublet $L_{\alpha}$, given by $\sin \theta_{\alpha}$. Production of $N$ proceeds by electroweak production of SM neutrinos, which subsequently oscillate into $N$; this is known as the Dodelson-Widrow mechanism [116]. Currently, the combination of constraints on warm DM and X-rays completely rules out the Dodelson-Widrow mechanism as being the sole explanation for the DM abundance. Alternate mechanisms have also been proposed to enhance the production rate of $N$, such as via large lepton asymmetry and/or the decays of new singlet scalars in the inflation or Higgs sectors [117-121].

New vector mediators $V$ coupled to SM neutrinos also produce a larger abundance of $N$ by analogy with the Dodelson-Widrow mechanism [115]: the $V$ particle results in a higher scattering rate of $N_{1}$ in the early universe, and consequently a larger production rate of $N$ through oscillation. This is true even if the $V$ has no coupling to $N$ prior to electroweak symmetry breaking. If the new interaction occurs in the neutral current only, it does not contribute to $N \rightarrow \nu_{\alpha} \gamma$ decay and can be safe from X-ray constraints. Since the new interactions exclusively couple to SM particles, the vector portal provides a direct connection between sterile neutrino cosmology and the phenomenology of 
new vector states. The sterile neutrino abundance as a function of $M_{N}$, mixing angle, $M_{V}$, and $g^{\prime}$ is computed in Ref. [115], taking into account all finite-temperature corrections and oscillation damping effects associated with both the electroweak interactions and the new vector state $V$. New vector portal interactions with $M_{V} \sim \mathrm{MeV}-\mathrm{GeV}$ and $g^{\prime} \sim 10^{-6}-10^{-2}$ are consistent with dark matter with masses 1-100 keV $N$, as well as all cosmological constraints and X-ray bounds on $\sin \theta_{\alpha}$. This gives a concrete window for phenomenological probes of $V$.

\subsubsection{Self-intereaction of dark matter via light mediators}

Finally a noteworthy motivation for the light vector portal particles come from the host of observational questions related to the dark matter sub-structure and their possible resolution via self-interaction of DM particles. The astrophysical structure of galaxies and clusters of galaxies can be dramatically affected by scattering interactions between dark matter particles [122, 123]. This effect, known as dark matter self-interactions, is motivated based on two outstanding issues in our understanding of structure formation.

- Core-cusp problem: Observations indicate that dwarf galaxies exhibit reduced central densities with flat profiles (cores) [124-128], as opposed to steeper density profiles (cusps) predicted by collisionless DM-only simulations [129]. More recently, dark matter cores have been found in larger systems as well, including low surface brightness spiral galaxies [130-133] and clusters [134].

- Too-big-to-fail problem: Collisionless DM-only simulations predict that MW-like halos should contain a population of $\mathcal{O}(10)$ subhalos that are too massive to host any of the known most massive classical dwarfs, as inferred by stellar kinematics [135, 136]. Satellite populations of the Andromeda Galaxy and the field of the Local Group also exhibit a dearth of massive satellites expected from collisionless DM-only simulations [137, 138].

These discrepancies may be explained if DM particles within halos are not collisionless, as is usually assumed, but instead undergo elastic scattering with other DM particles. DM self-interactions lead to thermodynamic heat transport from the hotter outer halo to the colder inner halo, thereby reducing the central halo density as DM particles get heated and escape. Additionally, the formation of cores in satellite galaxies can modify their stellar kinematics, resolving the mismatch between the most massive satellites observed in the MW and those found in simulations. Recent simulations of self-interacting dark matter have shown that a self-interaction cross section per unit mass of

$$
\sigma / m_{\chi} \gtrsim 1 \mathrm{~cm}^{2} / \mathrm{g} \sim 2 \times 10^{-24} \mathrm{~cm}^{2} / \mathrm{GeV}
$$

can resolve the core-cusp and too-big-to-fail problems on dwarf scales [139-142].

On the other hand, these discrepancies may simply point to the inadequacy of using DM-only simulations to make inferences about the real Universe containing both DM and baryons. A variety of mechanisms have been proposed to generate cores in DM halos through gravitational feedback from baryons, including supernovae in gas-rich dwarfs [143, 144], environmental interactions between satellite galaxies and the baryonic disk of the larger host galaxy [145], and active galactic nuclei in clusters [146].

Although the origin of these discrepancies remains an open and hotly-debated question, selfinteracting dark matter remains an appealing alternative to the WIMP paradigm that can be tested in both astrophysical and terrestrial contexts. Self-interactions are present for WIMP dark matter. However, the self-interaction cross section for WIMPs is set by the weak scale, with $\sigma \sim 10^{-36} \mathrm{~cm}^{2}$ and $m_{\chi} \sim 100 \mathrm{GeV}$, giving the figure of merit $\sigma / m_{\chi} \sim 10^{-38} \mathrm{~cm}^{2} / \mathrm{GeV}$ that is too small by fourteen orders of magnitude. Therefore, self-interacting dark matter requires the existence of new dark mediator particles that are much lighter than the weak scale. 
The minimal model for self-interacting dark matter consists of a dark matter particle $\chi$ (taken to be a fermion) and a mediator particle, scalar $\phi$ or vector $V$, with interaction [147-154]

$$
\mathcal{L}_{\text {int }}=\left\{\begin{array}{ll}
g_{\chi} \bar{\chi} \gamma^{\mu} \chi V_{\mu} & \text { vector mediator } \\
g_{\chi} \bar{\chi} \chi \phi & \text { scalar mediator }
\end{array} .\right.
$$

The coupling $g_{\chi}$ can be related to a "dark" fine structure constant $\alpha_{D}=g_{\chi}^{2} /(4 \pi)$. In the small velocity limit, the Born self-interaction cross section is ${ }^{2}$

$$
\sigma=4 \pi \alpha_{D}^{2} m_{\chi}^{2} / m_{V}^{4} \approx 6 \times 10^{-23} \mathrm{~cm}^{2}\left(\frac{\alpha_{D}}{0.01}\right)^{2}\left(\frac{m_{\chi}}{10 \mathrm{GeV}}\right)^{2}\left(\frac{30 \mathrm{MeV}}{m_{V}}\right)^{4}
$$

independent of whether mediator is scalar or vector. If the self-interaction cross section is large enough to explain small-scale astrophysical observations, the mediator particle must have mass much lighter than the weak scale, typically in the range [154]

$$
m_{V} \sim \mathrm{MeV}-\mathrm{GeV},
$$

over a wide range of DM mass $\left(m_{\chi} \sim \mathrm{MeV}-\mathrm{TeV}\right)$ and coupling $\left(\alpha_{D} \sim 10^{-4}-10^{-1}\right)$.

The existence of new force in the dark sector at the $\mathrm{MeV}-\mathrm{GeV}$ scale provides a new motivation for direct searches at high-luminosity colliders. Self-interactions and small scale structure anomalies do not by themselves require that light mediator couples directly to the Standard Model. However, without such a coupling, and in the absence of additional light states there will be a population of mediator particles that dominates the DM mass budget over self-interacting $\chi$ particles [155]. Thus, they must decay to SM states, and, requiring that decay occurs before Big Bang Nucleosynthesis to avoid excessive entropy injection, there is minimal value on the coupling between $V$ (or $\phi$ ) and SM particles that depends on specific model realization. Typically, a vector mediator coupling through the kinetic mixing portal must have $\epsilon \gtrsim 10^{-10}$, while a scalar mediator $\phi$ coupling by mixing with the Higgs boson must have a mixing angle larger than around $10^{-5}$. On the other hand, these mixing parameters can be much larger and indeed close to the current experimental limits.

\subsection{Main features of vector portal phenomenology.}

In this section we review the main phenomenological features of light vector states coupled to the SM vector currents and light matter states (dark Higgses, HNLs etc) that may be associated with them.

\subsubsection{Decay rates, modes, branchings, $c \tau$ for dark photon}

The phenomenology of massive dark photons with $m_{V}>2 m_{e}$ is quite straightforward. The decay rates of the $A^{\prime}$ to the SM states can be easily calculated for any point in the parameter space $\left\{m_{A^{\prime}}, \epsilon\right\}$. More generally, the biggest uncertainty in this scenario is related to the question of the existence of new states $\chi$ charged under $U(1)^{\prime}$, which would open additional dark decay channels. In general, the decay width is given by

$$
\Gamma_{A^{\prime}}=\sum_{l} \Gamma_{A^{\prime} \rightarrow l^{+} l^{-}}+\sum_{\text {hadrons }} \Gamma_{A^{\prime} \rightarrow \text { hadrons }}+\sum_{\chi} \Gamma_{A^{\prime} \rightarrow \chi \bar{\chi}}
$$

\footnotetext{
${ }^{2}$ In general, Eq. (2.3.7) breaks down for $\alpha_{\chi} m_{\chi} / m_{\phi} \gtrsim 1$, where nonperturbative Sommerfeld-type effects become important, or for $m_{\chi} v / m_{V} \gtrsim 1$, where $\sigma$ can no longer be represented by a contact interaction. In these regimes, a numerical solution for $\sigma$ has been performed $[149,154]$.
} 
The decays to leptons and new "dark states" $\chi$ are elementary to handle,

$$
\begin{gathered}
\Gamma_{A^{\prime} \rightarrow l^{+} l^{-}}=\frac{1}{3} \epsilon^{2} \alpha m_{V}\left(1+\frac{2 m_{l}^{2}}{m_{V}^{2}}\right) \sqrt{1-\frac{4 m_{l}^{2}}{m_{V}^{2}}}, \\
\Gamma_{A^{\prime} \rightarrow \chi \bar{\chi}}=\frac{1}{3} \alpha_{D^{\prime} m_{V}}\left(1+\frac{2 m_{\chi}^{2}}{m_{V}^{2}}\right) \sqrt{1-\frac{4 m_{\chi}^{2}}{m_{V}^{2}}},
\end{gathered}
$$

while the expressions for the decays to hadrons can be extracted from the well-known spectral function of virtual photon transition, $\gamma^{*} \rightarrow$ hadrons,

$$
\Gamma_{V \rightarrow \text { hadrons }}=\frac{1}{3} \alpha \epsilon^{2} m_{V} \sqrt{1-\frac{4 m_{\mu}^{2}}{m_{V}^{2}}}\left(1+\frac{2 m_{\mu}^{2}}{m_{V}^{2}}\right) R\left(s=m_{V}^{2}\right) .
$$

The $R$-ratio can be found in [156]. If dark states are kinematically accessible, then "visible" vs "dark" decay widths scale as $\sim \alpha \epsilon^{2} / \alpha_{D}$.

If the decays to dark states are non-existent or subdominant, $\Gamma_{A^{\prime} \rightarrow S M} \gg \Gamma_{A^{\prime} \rightarrow \chi \bar{\chi}}$, then the SM decay channels are not diluted by dark decays. This is the most studied and most constrained case. Making a specific choice of parameters, one can see that in the absence of dark decays, (boosted) dark photons can propagate over macroscopic distances. Choosing $m_{A^{\prime}}=200 \mathrm{MeV}$ for simplicity, one determines that

$$
c \tau_{A^{\prime}} \gamma \sim 40 \mathrm{~m} \times\left(\frac{10^{-6}}{\epsilon}\right)^{2} \times \frac{\gamma}{100} .
$$

Thus, this range of mixing angles is of special interest for the SHiP-like experiments.

The production of vector states is a more complicated process than their decays, especially if they are produced in $p$ collisions with a target. So far, several production channels have been identified:

1. The dark photons, or more generically, light vectors states can be produced in meson decays, which in turn are copiously produced in $p$-on-target collisions. For light vector masses, by far the most important process is $\pi \rightarrow \gamma V$, which is suppressed by $\epsilon^{2}$, but not $\alpha$, since the main decay of $\pi^{0}$ is electromagnetic. In the literature, $\eta, \rho / \omega$, and $\Delta$ sources of $A^{\prime}$ have been discussed [157]. The weak decays of $K, D$ mesons play a subdominant role in the production of dark photons

2. The (quasi)elestic scattering of incident protons on nucleons in the target can lead to the production of vector states via bremsstrahlung process $p p \rightarrow p p V[158,159]$. This type of process is quite similar to the production of vector states from the electrons in collision with nuclei, but is more difficult to handle due to the non-elementary nature of nucleons.

3. For large $m_{V}$, it is expected that the direct perturbative QCD production of vector states via the underlying $q+\bar{q} \rightarrow V ; q+g \rightarrow q+V$ processes should become dominant. Some of the QCD production cross sections have been calculated in Ref. [160].

It is fair to say that at this point there are no universally accepted calculational tools available for the computation of production rates and distributions of extra vector states in $p p$ and $p n$ collisions over a wide range of incident energies and for all values of masses $m_{V}$. We expect that in the future more theoretical work will be done to fill the needs of the SHiP experiment.

The opposite regime in which dark decays are dominant, which occurs for $2 m_{\chi}<m_{A^{\prime}}$ and $\alpha_{D} \gg \alpha \epsilon^{2}$, is also of considerable interest. In that case, the only model-independent signature is the missing energy decays of $A^{\prime}$. If, however, $\chi$-states do not fragment further into additional light 
dark states, they can participate in the scattering on the SM particles via the exchange of the mixed $A-A^{\prime}$ propagator, Fig. 2.1. The rate for the scattering on electrons [157] is given by

$$
\frac{d \sigma_{e \chi \rightarrow e \chi}}{d E_{f}}=\frac{\alpha_{D} \epsilon^{2}}{\alpha} \times \frac{8 \pi \alpha^{2} m_{e}\left(1-E_{f} / E\right)}{\left(m_{A^{\prime}}^{2}+2 m_{e} E_{f}\right)^{2}},
$$

where $E_{f}$ is the electron recoil energy, and assuming $E_{f} \gg m_{e}$. This scattering process, as well as scattering on nucleons and nuclei, can be viewed as the search channels for light dark matter $[157,161,162]$. The treatment of the light dark matter-nucleon scattering can be complicated as it may occur in a variety of dynamical regimes, as evident from recent literature [163-165].

\subsubsection{Other vector candidates}

The phenomenology of other $U(1)^{\prime}$ gauge symmetries and their associated vector bosons are in principle straightforward to handle. Many gross features are similar to the case of the kinetically mixed dark photon. In addition, if the gauge symmetry involves lepton number or any individual lepton flavors $L_{i}$ it is guaranteed that there exists a new decay channel to neutrinos, $V \rightarrow \nu \bar{\nu}$. When the right-handed neutrinos are lighter than the vector boson an additional decay channel $V \rightarrow N \bar{N}$ opens up, where $N$ denotes the right-handed neutrino, or more generally a HNL,

$$
\Gamma_{V \rightarrow N \bar{N}}=\frac{1}{6} \frac{\left(g^{\prime}\right)^{2}}{4 \pi} m_{V}\left(1-\frac{m_{N}^{2}}{m_{V}^{2}}\right) \sqrt{1-\frac{4 m_{N}^{2}}{m_{V}^{2}}}
$$

Thus, the vector portals based on leptonic symmetries may provide a new source of right-handed neutrinos in the SHiP experiment. Compared to the previous chapters, the signal of HNL charged under light $U(1)^{\prime}$ will scale as production $\times$ decay $\sim\left(g^{\prime}\right)^{2} \times|U|^{2}$, as opposed to the minimal HNL case with $|U|^{4}$ scaling, where $U$ is the HNL mixing angle.

Another interesting candidate that can give distinct signatures at $\mathrm{SHiP}$ is the vector boson of the baryonic $U(1)^{B}$. If the kinetic mixing parameter for the $V^{(B)}$ gauge boson is small the decays of the baryonic vector may be dominated by the underlying $\frac{1}{3} V_{\mu}^{(B)} g_{(B)} \bar{q} \gamma_{\mu} q$ interaction. In the mass range $m_{B}<1 \mathrm{GeV}$ the main decay channels are hadronic, and because of the symmetries of the underlying current they are $V \rightarrow \gamma \pi^{0}$ and $V \rightarrow \pi^{+} \pi^{-} \pi^{0}$. These decay channels are additionally suppressed because of their loop origin $\left(\gamma \pi^{0}\right)$ and multiplicity of the final state $\left(\pi^{+} \pi^{-} \pi^{0}\right)$. Using results of Refs. $[67,68]$, one can estimate the propagation length of baryonic vector with $m_{V}=200 \mathrm{MeV}$ as

$$
\left.c \tau_{V^{(B)}} \gamma\right|_{\epsilon=0} \sim 70 \mathrm{~m} \times\left(\frac{10^{-4}}{g_{(B)}}\right)^{2} \times \frac{\gamma}{100} .
$$

(A non-zero kinetic mixing angle in excess of $10^{-6}$ would shorten this decay length.)

\subsubsection{Higgsstrahlung process for $U(1)^{\prime}$ and delayed decays of $h^{\prime}$.}

If the mixing angle is in ballpark of $\epsilon \sim 10^{-3}$ the lifetime of dark photon is very short and the decay length is microscopic as long as $m_{V}>2 m_{e}$. However, it does not mean that such a theory cannot have long-lived particles. One candidate is the long-lived dark Higgs particle that can be produced in association with $V$ [85]. In the dark photon model, if $m_{h^{\prime}} \leq m_{A^{\prime}}$ the decays of $h^{\prime}$ are suppressed, as $h^{\prime} \rightarrow A_{\mathrm{on}-\text { shell }}^{\prime}+X_{\mathrm{SM}}$ channels become kinematically forbidden. As a consequence the decays occur via two off-shell $A^{\prime}$, either via loop mechanism or at tree level [85], with the decay width scaling as $\Gamma_{h^{\prime}} \sim \epsilon^{4}$. Taking for concreteness $m_{A^{\prime}} \sim m_{h^{\prime}} \sim 2 \mathrm{GeV}$, one can estimate the decay 
rate of $h^{\prime}$ to two vector mesons ( $\rho$ or $\omega$ ), and deduce the ensuing decay length,

$$
c \tau_{h^{\prime}} \gamma \sim 10^{7} \mathrm{~m} \times\left(\frac{10^{-3}}{\epsilon}\right)^{4} \times\left(\frac{\alpha}{\alpha_{D}}\right) \times \frac{\gamma}{10} .
$$

One can see that the decay length can be very long compard to the size of the beam dump experiments, leading to the additional suppression of the signal.

The production of energetic $h^{\prime}$ can be described by the underlying $q \bar{q} \rightarrow h^{\prime} A^{\prime}$ annihilation process that has the cross section [85],

$$
\sigma_{q \bar{q} \rightarrow h^{\prime} A^{\prime}} \simeq 0.2 \mathrm{fb} \times Q_{q}^{2}\left(\frac{\alpha_{D}}{\alpha}\right)\left(\frac{\epsilon^{2}}{10^{-6}}\right) \frac{(10 \mathrm{GeV})^{2}}{\hat{s}},
$$

where $Q_{q}$ is the quark charge in units of $e$. In case of a more complicated Higgs sector, the rates can be enhanced by the production of the on-shell $A^{\prime}$ decaying to a pair of non-identical $h^{\prime}$ bosons $[166,167]$.

\subsection{Summary of the existing constraints on light vector and light DM states}

In the last few years, there has been a concerted effort to explore the parameter space of the dark photon model, both with and without dark matter. There are also a number of experimental studies searching for other particles coupled to the SM via vector portal. Here we summarize these developments.

\subsubsection{Current status of experimental constraints on exotic vector states}

Fig. 2.6 summarizes the existing constraints on the dark photon model, under the assumption that the dark decays are absent or subdominant. One can clearly see that the direct constraints can be subdivided into two main categories: those that come from the prompt decays of the dark photons, and those that are derived from the delayed decays of dark photons inside the detectors placed some distance behind beam dumps. The combination of all constraints covers the region of parameter space motivated by the solution to the muon $g-2$ discrepancy. Future electron-on-target experiments such as HPS [39] will add sensitivity in $\epsilon \sim 10^{-3}-10^{-5}$ and $m_{A^{\prime}} \leq$ few $100 \mathrm{MeV}$ window that is comparatively less constrained.

It is important to note that the excluded regions corresponding to $\epsilon \sim 10^{-6}$ and masses in excess of $m_{\pi}$ come from relatively old experiments, CHARM and E137, which are proton and electron beam dump experiments, respectively. (The shape of these regions can be understood as follows: above the upper boundary of that region the lifetime $c \tau_{A^{\prime}} \gamma$ is too short for the sufficient number of dark photons to reach the detector. Below the lower boundary, the size of $\epsilon$ is too small to produce and detect a sufficient number of $A^{\prime}$.) It is clear that the improvement on the number of protons-on-target and shorter distance to the detector, such as in the SHiP proposal, will improve the sensitivity to $A^{\prime}$ around the CHARM/E137 region.

Other models of light vectors have received comparatively less attention. The constraints on $B-L$ vector portal have been assessed in [168], with additional constraint that the RH neutrinos are very light. The sensitivity of $\phi, \eta, \eta^{\prime}$ meson decays to the baryonic vector $V^{(B)}$ was derived in [67]. The full analysis of constraints on $\left\{\alpha_{(B)}, m_{V}\right\}$ plane has not been performed yet.

Some cases of other exotic particles produced in association with $V$ have been constrained in experiment. BaBar studies have placed limits on dark Higgsstrahlung [169], by exploiting $A^{\prime} h^{\prime}$ production with subsequent decays of $h^{\prime}$ to $2 A^{\prime}$ and eventually to pairs of charged SM particles. The 


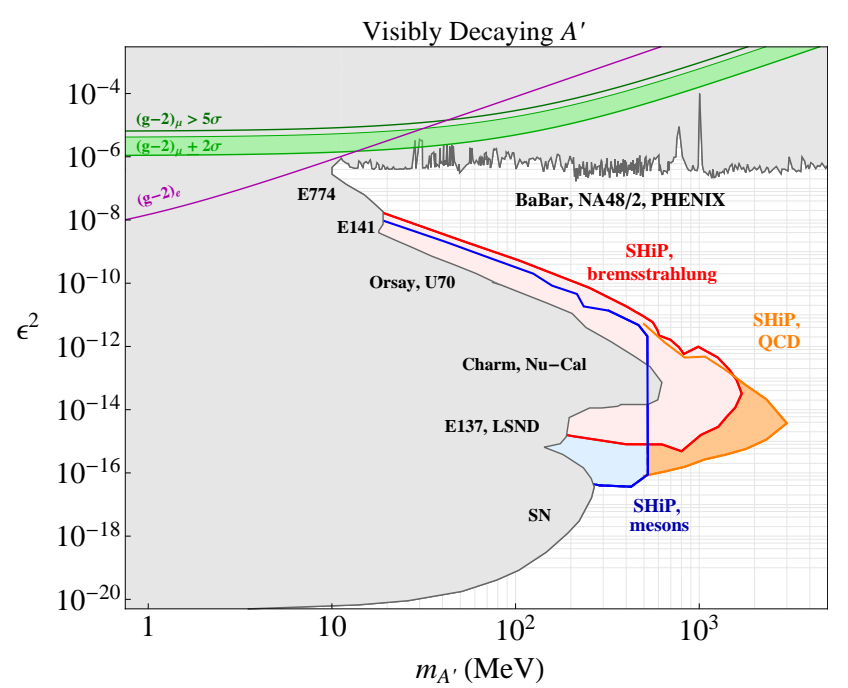

Figure 2.6: Summary of constraints on the dark photon model. The limits at $\epsilon \sim 10^{-7} ; m_{A^{\prime}}>200$ $\mathrm{MeV}$ range come from old experiments, and can be improved with SHiP. The $g-2$ region of interest is shown as a green band. The projected SHiP sensitivity contour is derived using three modes of production: mesons, bremsstrahlung, and QCD production.

ensuing constraints are quite strong (reaching down to $\epsilon \sim$ few $\times 10^{-4}$ at $\alpha_{D} \sim \alpha$ ), but applicable only to $m_{h^{\prime}}>2 m_{A^{\prime}}$ region of parameter space. Another study at KLOE [170] have searched for missing energy signature from $h^{\prime}$ decays outside of the detector, and reached the constraints at the level of $\epsilon \sim$ few $\times 10^{-3}$. Constraints on the most motivated case, $m_{h^{\prime}} \simeq m_{A^{\prime}}$, are more difficult to obtain because they involve stable $h^{\prime}$ on the scale of the detector.

\subsubsection{Production and detection of light vector portal DM}

New constraints on vector portals occur when direct production of light dark matter states $\chi$ opens up. The missing energy constraints on dark photons derived from $e^{+} e^{-}$colliders were analyzed in [95]. Invisible decays of $A^{\prime}$ are usually harder to detect, except $K^{+} \rightarrow \pi^{+} A^{\prime} \rightarrow \pi^{+}+$missing energy, where the competing SM process, $K^{+} \rightarrow \pi^{+} \nu \bar{\nu}$ is extremely suppressed [86]. Also, fixed targets experiments sensitive to the missing energy decays of vector states have been proposed recently $[171,172]$.

A rather systematic study of the detection of light dark matter produced via the dark photon portal has been performed in a number of papers [157, 162, 173, 174]. The most stringent constraints follow from the highest POT experiment, LSND, provided that the dark matter is within kinematic reach. A typical detection scheme in the proton beam dump experiments is built on the following chain of events:

$$
p p \rightarrow \pi^{0}+X, \quad \pi^{0} \rightarrow V \gamma, \quad V \rightarrow \chi \bar{\chi}, \quad \chi \text { scattering on electrons/nuclei }
$$

These results significantly constrain, but do not fully rule out, MeV-scale dark matter models, suggested as a candidate explanation of the $511 \mathrm{keV}$ excess from the galactic bulge. Currently, the MiniBooNE collaboration is conducting a dedicated search for such states in a beam dump mode run [175]. The summary of the existing constraints on light dark matter produced via vector portal is given in Fig. 2.7. Similar constraints were also derived for light dark matter coupled to the $\mathrm{SM}$ via the baryonic vector portal [68]. It is important to emphasize that these constraints cover the low mass region of parameter space inaccessible to traditional underground direct detection 

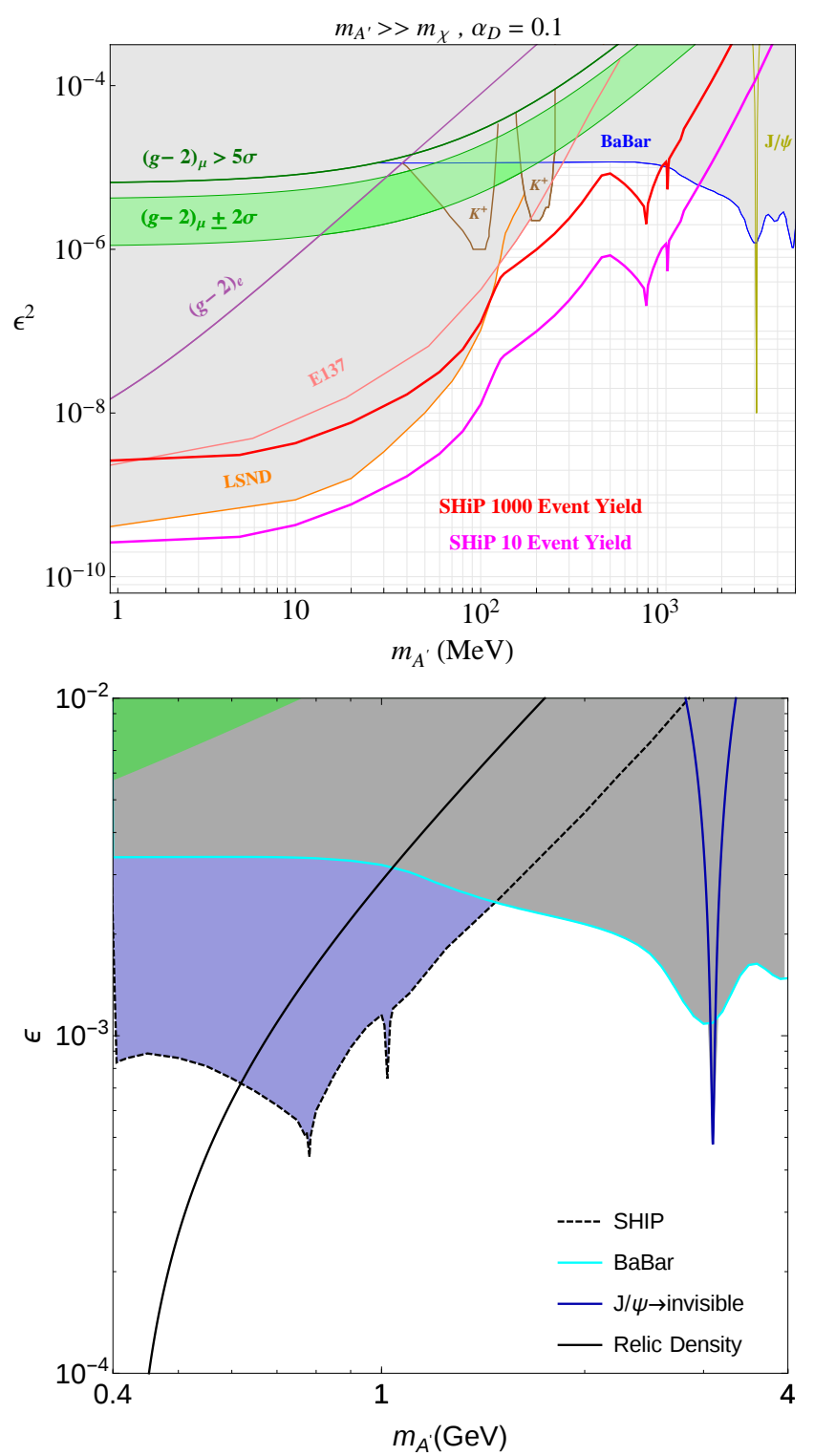

Figure 2.7: Summary of the constraints on the scattering of the light dark matter produced in $e$ and $p$ collisions with fixed targets. Upper pannel, small dark matter mass $m_{\chi}$ and $\alpha_{D}=0.1$, with SHiP sensitivity contours assuming 10 and 1000 electron-scattering events; Lower pannel is for $m_{\chi}=200 \mathrm{MeV}$. The SHiP sensitivty with 10 events is shown as a blue region.

experiments.

\subsubsection{Cosmological and astrophysical constraints on vector portals}

The question of the astrophysical and cosmological constraints on light particles associated with the vector portal is very important. The consistency of deuterium and helium abundance predictions with observations require that the new particles decay or significantly reduce their energy density at the time prior to BBN, or more specifically, before the neutron-proton interconversion freeze-out. If coupling constants in the dark sector $\left(\epsilon, \alpha_{D}, \ldots\right)$ are sizable, this requirement translates into $m_{V, \chi}>$ few $\mathrm{MeV}[176,177]$. Recent measurements of the radiation energy density at the time of the CMB, parametrized by $N_{e f f}$, also agrees very well with the SM prediction of 3.04, reinforcing the BBN 
bound. Models that have extra forces acting on neutrinos, such as $B-L$, are also constrained by cosmology. A stronger-than-weak force between SM neutrinos and electrons would keep neutrinos in equilibrium for longer, and raise their overall energy density. If RH neutrinos are very light and also charged under $B-L$, this would impose very stringent constraints on mediators of $B-L$ force [168]. However, if the $B-L$ is broken via a suitable Higgs mechanism, the RH neutrinos can be made heavy and unstable, which would significantly weaken constraints on $\left\{m_{V}, g_{(B-L)}\right\}$ parameter space.

Late annihilation of WIMP dark matter into the SM channels other than neutrinos is constrained by the distortions of the observed pattern in the CMB anisotropies. This rules out the $s$-wave annihilating WIMPs with masses less than $\sim 10 \mathrm{GeV}$. However, the scalar dark matter annihilates via a vector mediator in the $p$-wave which significantly lessens all constraints from the CMB. Alternatively, light fermionic dark matter may exist in a particle-antiparticle asymmetric state, and be prevented from annihilation at the CMB decoupling epoch that way.

Finally, significant restrictions on parameter space of light particles, including those discussed in this chapter, are imposed by stellar astrophysics. If the masses are in excess of few $\mathrm{MeV}$, only supernovae will provide significant bounds [178]. The dark photon parameter space is limited by the possibility of it being produced in the SN, and decaying outside of the explosive zone, providing a disruptive energy transport mechanism. This imposes strong bounds on the kinetic mixing parameter $\epsilon$ and vector masses below $200 \mathrm{MeV}$. The constraint from supernovae, Fig. 2.6, partially overlaps with the beam dump constraints. Light dark matter is also constrained by the same mechanism in $\mathrm{SN}$, if it has a possibility to escape the explosive zone. In addition, light particles are constrained if they possess a significant coupling to neutrinos, thus degrading their energy below the ones observed in the explosion of SN 1987a. The latter rules out MeV-scale WIMPs with significant coupling to neutrinos [179].

To conclude, astrophysics and cosmology cannot resolutely rule out the existence of light - few $\mathrm{MeV}$ and heavier - particles coupled to the SM via the vector portal. Specifically, SHiP has unique sensitivity to a variety of new particles with masses on the order of a few $100 \mathrm{MeV}$ and heavier, and lifetimes below $1 \mathrm{~s}$, which are not constrained by cosmological or astrophysical considerations.

\subsection{Case studies for SHiP}

Here we describe preliminary sensitivity studies for several benchmark vector portal models. Estimates performed for this chapter use basic assumptions about SHiP geometry and normalize the event yield on $10^{20}$ protons on target (POT).

\subsubsection{Production and detection of kinetically mixed dark photons and baryonic vec- tors.}

As stated previously, there exist only partial calculations of the production of vector particles at SHiP. To derive sensitivity to dark photons, we use the results of the three channels identified earlier (meson decays, bremsstrahlung, and direct QCD production). Note that bremsstrahlung production must be accompanied by a form factor suppression, if the mass of the dark photon is above the typical QCD scale. Using the results of recent estimates in Ref. [159], and supplementing it with our own estimates of the QCD production (for $m_{V} \gtrsim 1 \mathrm{GeV}$ region) in the contour shown in red, in Fig. 2.6. The QCD production is simulated using MadGraph5 [180], with the renormalization and factorization scales fixed to $m_{A^{\prime}}$. We have extended the QCD estimate to $m_{A^{\prime}}$ as low as 500 $\mathrm{MeV}$, although we note that in this range there are significant renormalization and factorization scale uncertainties. The detectable level of signal events was set to $N=3$. 
As one can see, new territories on $\left\{m_{A^{\prime}}, \epsilon\right\}$ parameter space are going to be covered by SHiP, up to $m_{A^{\prime}} \sim 3 \mathrm{GeV}$. If the values of $\epsilon \sim 10^{-7}$ are thought to be generated by loops of charged particles, this region corresponds to the two- or three-loop mediation mechanism.

These estimates can be extended to the case of the baryonic vector, when the coupling to the baryon current dominates over the kinetic mixing effect. In the $m_{V}>\mathrm{GeV}$ mass domain there is no accidental extra longevity of $V^{(B)}$, and one can use the approximate translation from the dark photon case, $\left\{m_{A^{\prime}}, \epsilon\right\} \rightarrow\left\{V^{(B)}, \alpha^{(B)} / \alpha\right\}$. Below GeV masses there are no efficient hadronic decays compared to the dark photon case, and as a result the SHiP experiment will be sensitive to larger values of the parameter space relative to the dark photon case. A detailed exploration of this parameter space and comparison to the SHiP sensitivity, with the derivation of bounds from past experiments should be performed in future work.

\subsubsection{Production and detection of other unstable particles ( $h^{\prime}$, HNL) through the vector portal}

The parameter space of these models is much wider, $\left\{m_{A^{\prime}}, m_{h^{\prime}}, \epsilon, \alpha_{D}\right\}$ for the dark Higgs, and $\left\{m_{V}, m_{N}, \theta, \alpha_{D}\right\}$ for the HNL (Here $\theta$ is the value of the HNL mixing angle, also called $U$ in this report.) Scanning the parameter space in this case represents a significant challenge. Instead, we opt for exploring the sensitivity of SHiP to some representative points on the parameter space.

For the case of dark Higgs-strahlung production of $h^{\prime}$ with its subsequent decay registered by SHiP detector, we choose the point $m_{h^{\prime}}=m_{A^{\prime}}=2 \mathrm{GeV}$. In that case $h^{\prime}$ is fairly long-lived (eq. (2.4.9)) while the production occurs at minimum virtuality of $4 \mathrm{GeV}^{2}$, where perturbative QCD can be employed. The total number of events, seen as multiple $\pi$ mesons from the decay of $h^{\prime}$, can be estimated for SHiP as

$$
N_{\text {events }} \sim O(10) \times\left(\frac{\epsilon}{10^{-3}}\right)^{6} \times \frac{\alpha^{\prime}}{\alpha} .
$$

This limit is competitive with other constraints (e.g. the missing energy signal at B-factories), and is indeed much better than sensitivity that can be achieved at MINOS or T2K.

The production of HNLs charged under the vector force is another interesting case to consider. Here we take $q \bar{q} \rightarrow V \rightarrow N N \rightarrow N$ decay sequence of processes, where the last chain is the weak decay of $N$, suppressed by the small mixing angle $\theta$. Taking for definitiveness the $B-L$ portal with $m_{V}=2 \mathrm{GeV}$ mass mediator, and $m_{N}=0.5 \mathrm{GeV}$, we arrive at the following estimate of the total event rate,

$$
N_{\text {events }} \sim O\left(10^{3}\right) \times\left(\frac{\alpha_{B-L}}{10^{-8}}\right) \times \frac{10^{-1} \mathrm{~s}}{\tau_{N}} .
$$

As it is easy to see, this is a very large rate, reaching down to extremely small values of $\alpha_{B-L}$, and see-saw ballpark for the lifetimes of HNLs. Thus, vector portals provide a very efficient mechanism for producing and detecting HNL at SHiP.

\subsubsection{Possible sensitivity to light DM through SHiP neutrino detector.}

Due to the planned neutrino detector at the SHiP facility, the experiment will potentially have a significant reach to light dark matter production in the target, with its subsequent scattering in the neutrino detector. To estimate the sensitivity we assume a model of kinetic mixing mediation, and calculate the number of electron scattering events in a 10 ton detector. A proper calculation would have to include the hadronic contributions from deep-inelastic scattering analogous to Ref. [164], which is expected to significantly increase the total event rate. For now, we take into account the scattering on electrons. We normalize the sensitivty to 10 and 1000 candidate events. It remains to be seen if ten events can be separated from the weak interaction background of regular neutrinos, but it can be attempted using kinematic differences between neutrinos scattering (via heavy mediator), and light dark matter scattering (via a relatively light $A^{\prime}$.) The resulting sensitivity contour are 
shown in Fig. 6, bottom pannel, for a $200 \mathrm{MeV}$ mass of dark matter particle. Even if the backrounds would not be reduced below 1000 events, the sensitivty is quite considerable and compares well with previous experiments. Moreover, if one switches from $A^{\prime}$ to a leptophobic mediator, such as $V^{(B)}$, the constraints from lepton-driven experiments (BaBar and E137) will be significantly relaxed, and SHiP will produce a strong additional reach to LSND and MiniBooNE.

\subsection{Conclusions}

The vector portals from SM to dark sectors are well-motivated, and have been in the focus of both experimental and theoretical communities in the last five-to-ten years. We have summarized the main features of vector portal phenomenology, exemplified (but not limited to!) by the case of the dark photon $A^{\prime}$. Preliminary assessment of the sensitivity shows that the planned SHiP facility will increase the reach in terms of the probed parameter space to many models of vector portals, both through the decay and scattering signatures. 


\section{Chapter 3}

\section{Scalar portal}

\subsection{The scalar sector of the Standard Model and Beyond}

The recent discovery at the CERN LHC [2, 4] of the Higgs boson [181-184] provides strong evidence that fundamental scalar bosons exist in nature. It is thus a timely and well-motivated task to search for further light scalar or pseudoscalar particles. The presence of such additional states is predicted in many extensions of the Standard Model (SM) Higgs sector. In particular if there are additional states which are singlet under the SM gauge group, they can be light and often have highly suppressed couplings to SM particles. Alternatively, such states can arise as pseudo-NambuGoldstone bosons (PNGB) of a spontaneously broken symmetry. Hidden sectors with mass scales at or below the electroweak scale are motivated by many outstanding questions in particle physics such as baryogenesis (see e.g. [185] for a recent review), dark matter [111, 186, 187], and the hierarchy problem [188-192], and therefore represent a generic expectation for physics beyond the SM [193].

There are already stringent experimental constraints on the mass $m_{S}$ of hidden scalars and their coupling to SM particles $g_{\star}$. Nevertheless, wide regions in the $\left(m_{S}, g_{\star}\right)$ plane are still unexplored. The SHiP experiment will give new insights especially for the mass region below $10 \mathrm{GeV}$.

\subsubsection{Scalar portal effective Lagrangian}

The most general renormalisable Lagrangian including the SM and an additional (CP-even) singlet scalar reads

$$
\mathcal{L}=\mathcal{L}_{\mathrm{SM}}+\frac{1}{2} \partial_{\mu} S \partial^{\mu} S+\left(\alpha_{1} S+\alpha S^{2}\right)\left(H^{\dagger} H\right)+\lambda_{2} S^{2}+\lambda_{3} S^{3}+\lambda_{4} S^{4},
$$

with the scalar self couplings $\lambda_{2,3,4}$ and the portal couplings $\alpha_{1}, \alpha$ to the SM Higgs doublet $H$. The SM Lagrangian is denoted by $\mathcal{L}_{\mathrm{SM}}$.

This Lagrangian constitutes a very well-motivated portal into hidden sectors for a variety of reasons. First and foremost, the operator $|H|^{2}$ is the lowest-dimension gauge- and Lorentz-singlet that can be constructed from SM fields, and is thus one of only a few possible candidates to mediate the leading interactions between the SM and hidden-sector matter that is neutral under all SM gauge groups (see e.g. [186, 193-196]). For a hidden sector made of scalar fields, the portal operators appearing in Eq. (3.1.1) are renormalizable, meaning that if the interactions between the extra scalar and the Higgs boson were generated at very high scales such as the GUT, or even Planck scale, then they may remain as a relevant interaction down to the energy scales probed by experiments today. And also, as it is believed that dark matter is neutral under all SM gauge interactions, and the Higgs portal can provide a bridge between the SM and neutral sectors, it is 
natural to speculate that scalars coupled through the Higgs portal may play a role in dark sector physics, or perhaps themselves constitute the dark matter within the present day Universe. ${ }^{1}$

The physics of the scalar portal model differs substantially whether the linear coupling $\alpha_{1}$ is present or whether it is forbidden by an exact $\mathcal{Z}_{2}$ symmetry. In the latter case, the new particles must be pair-produced, as discussed in Section 3.3. In the former case, that we shall treat in detail in Section 3.2, the scalar $S$ mixes with the Higgs boson after electroweak symmetry breaking and this mixing induces a coupling of $S$ to all SM particles. Note that when $S$ acquires a vacuum expectation value and spontaneously breaks the $\mathcal{Z}_{2}$ symmetry, then a linear mixing between the scalar and the Higgs boson is also induced.

\subsubsection{Hidden Valleys}

The Hidden Valley scenario [193] is a central motivation for SHiP, because it commonly predicts one or more long-lived particles - of low mass and zero charge, and potentially of any spin and parity. The scenario encompasses a broad class of models with a low mass hidden sector, called a "Hidden Valley." The Hidden Valley is coupled to the Standard Model either through a heavy mediator (such as a $Z^{\prime}$, Higgs, or a heavy fermion or scalar like a leptoquark or neutralino), through a light weakly coupled mediator (such as a kinetically mixed U(1) or a light scalar with small couplings to SM states), or through a loop of heavy particles (such as quirks or a particle/sparticle pair). The states in the hidden sector may have a wide variety of dynamics, ranging from new strong interactions [193] (and dual descriptions thereof) to supersymmetry [197]. There may be multiple gauge groups in the hidden sector, as well as stable states that could be the dark matter.

Hidden Valleys have inspired the construction of low mass dark matter sectors. This includes $\mathrm{MeV}[89,111,198]$ and, later, $\mathrm{GeV}$ dark sectors [109] with dark forces having a Sommerfeld enhancement. They are a natural conceptual framework for Asymmetric Dark Matter [199]. The Dark pions model discussed in Section 3.6 provides a concrete realization of such models. Hidden Valleys also arise in the context of "dark" solutions to naturalness, also known as "neutral naturalness" models, such as the Twin Higgs [188] and folded supersymmetry [189], where the top partner does not carry Standard Model color. Although the hidden sector of the original Twin Higgs did not give directly observable signals, variations [200] do generate observable long-lived particles in much of their parameter space.

One of the initial interests in the Hidden Valley was for decay of heavy mediators (e.g. a $Z^{\prime}$ or the Higgs) into low-mass Hidden Valley states that are charged under a dark confining gauge group. As a result of the confinement, the dark states hadronize, and because of their low mass, a high multiplicity results. A figure depicting the process is shown in Fig. 3.1. The states that are produced may be either stable or long lived. Those that decay but have long lifetimes on detector scales will give rise to displaced vertices. Many such displaced vertices may appear in a given event due to the high multiplicities and different bound states that result from the hadronization (each of which may have its own lifetime). An example of a Hidden Valley bound state decaying to the Standard Model is shown in Fig. 3.2. Other particles, stabilized by a flavor or other global symmetry, may be good dark matter candidates.

The $\mathcal{Z}_{2}$ scalar portal model of Section 3.3 is an explicit (and minimal) realization of the Hidden Valley scenario with the Higgs boson being the heavy mediator that is dominantly responsible for (pair-)production of the low-mass hidden sector states. In more general models, the light hidden sector states can of course also be produced singly in different kinematical configurations.

Supersymmetry also can play an important role in Hidden Valleys. It was first pointed out in $[89,197]$ that a supersymmetric hidden sector could lead to extended cascade decays, as the

\footnotetext{
${ }^{1}$ We will also consider light scalar masses however that for $m_{S} \ll m_{h}$ some tuning is required to keep $S$ light as the Higgs portal operator generates a weak-scale mass for $S$ with $\mathcal{O}(1)$ couplings. This tuning is implicitly assumed throughout.
} 


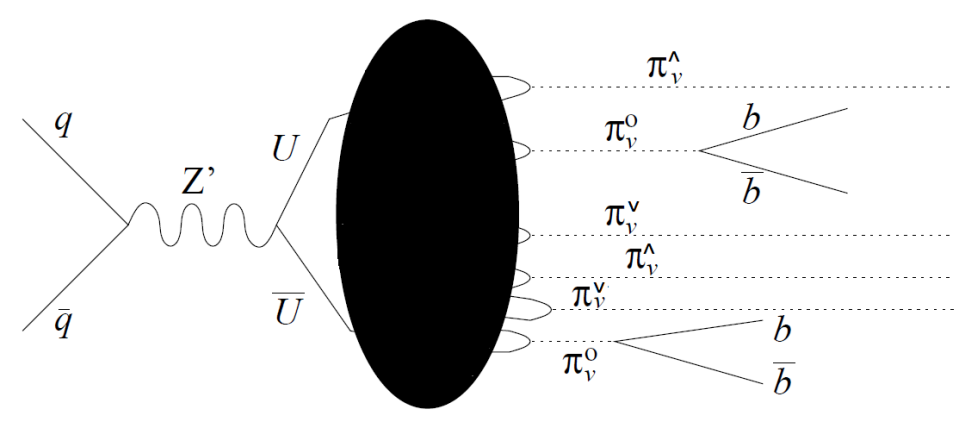

Figure 3.1: An example of a process in a confining Hidden Valley. In a proton-proton collision a resonance $Z^{\prime}$ is produced, which in turn decays to particles $U, \bar{U}$ in the Hidden Valley. Hidden strong interactions generate confinement and hidden hadrons result, some of which decay through an off-shell $Z$ or $Z^{\prime}$ to Standard Model particles, potentially with macroscopic decay lengths.

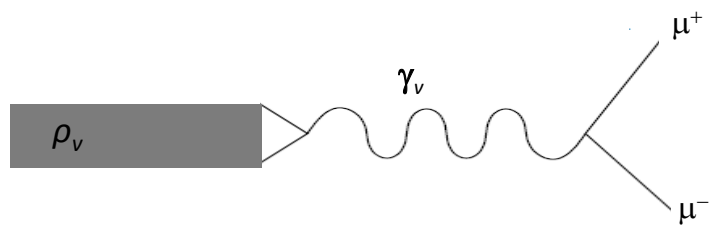

Figure 3.2: A Hidden Valley vector meson, whether fundamental or composite, may decay to Standard Model leptons or quarks. Here a composite $\rho$-meson of a hidden confining group mixes with a dark photon, which in turn can decay to Standard Model muons via its mixing with the ordinary photon.

lightest ordinary supersymmetric particle decays into the hidden sector. Such a process is shown in Fig. 3.3. This can lead both to displaced vertices, a higher number of final states and reduced missing energy. (Similar effects can arise in other solutions to the naturalness puzzle, such as extra dimensions, if they have new global symmetries.) The implications for a model of dark matter was studied in detail in [201].

Hidden Valleys subsequently provided the framework for thinking about how to construct dark matter from low mass hidden sectors. Two early examples occurred for the case of $\mathrm{MeV}$ dark matter. The idea was to construct a dark sector with a dark photon to which dark matter annihilates [111]. If this sector is supersymmetric and the dark photon kinetically mixes with the visible one [89], such a light sector is naturally light. As in [197] the lightest ordinary supersymmetric particle decays into the hidden sector. These models provided the tools for the kinetically mixed dark photon which was the basis of the models of [109, 202]. The dark photon is one of the classes of models that SHiP is searching for.

Once one considers a complex dark sector, many new avenues open up for new processes, such as 


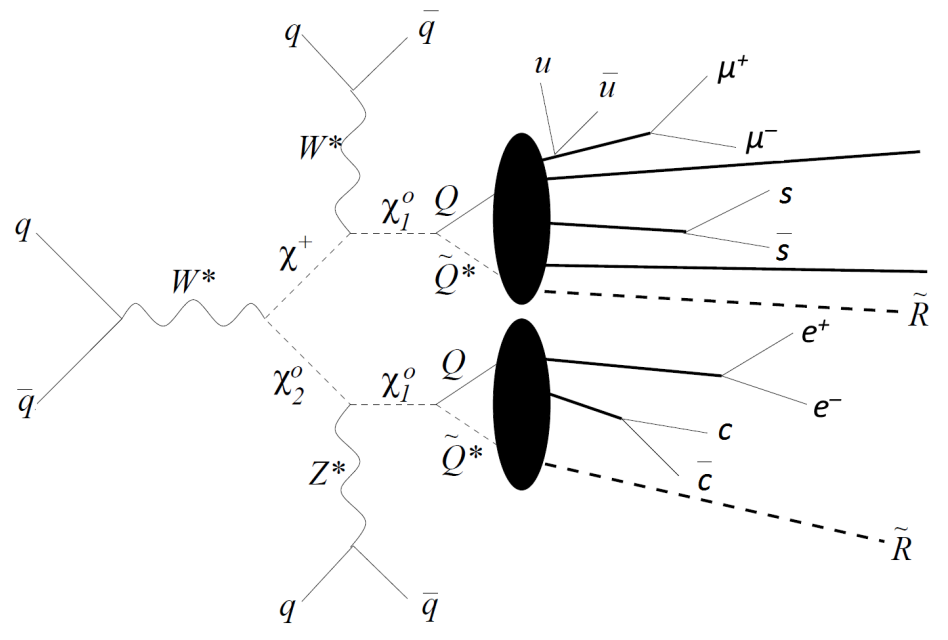

Figure 3.3: An example of a process in a supersymmetric Hidden Valley. At left is a typical supersymmetric event, which in minimal supersymmetry would terminate with two stable neutralinos $\chi^{0}$. Instead these decay to Hidden Valley particles, some of which decay to Standard Model quarks or leptons. The $\tilde{R}$ is the lightest R-parity-odd particle. The decays of the $\chi^{0}$ and/or one or more Hidden Valley particles may be displaced.

dark baryogengesis [203], see Fig. 3.4. SHiP may be able to detect the dark Higgs states responsible for a dark phase transition to generate the particle-anti-particle asymmetry in the Universe. These processes appear naturally within the content of Asymmetric Dark Matter.

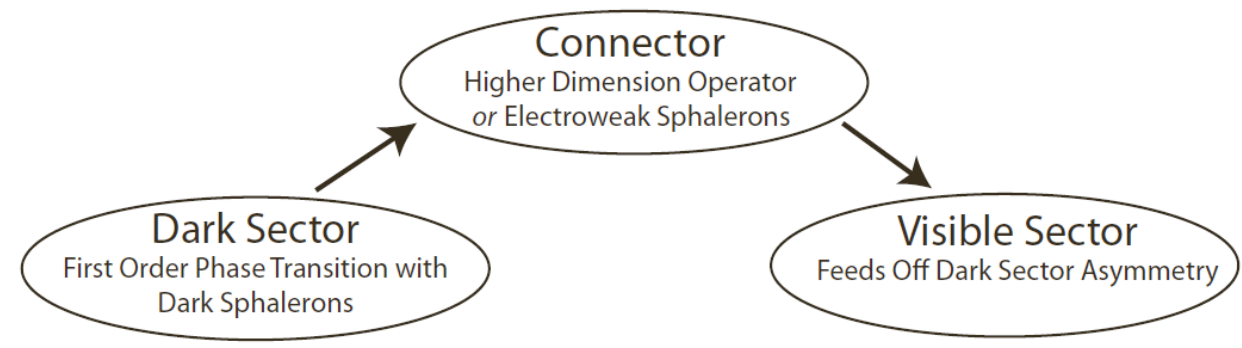

Figure 3.4: In a Hidden Valley with asymmetric dark matter, the dark baryon asymmetry created in a first-order phase transition in a Hidden Valley can be transferred to the Standard Model sector [203].

Dark solutions to naturalness typically have Hidden Valleys and often have observable longlived particles. Such models [188, 200, 204-206] require (1) mixing of visible and twin Higgses (allowing the Higgs boson(s) to mediate between the hidden sector and the visible sector) and (2) new gauge groups, at least one of which typically confines and generates hadrons that can decay via Higgs mixing [207]. This can lead to new phenomena such as shown in Fig. 3.5. Dark photons with very small kinetic mixing, and therefore long lifetimes, are also possible in these models. The full range of signals, including the role of dark photons and extra Goldstone modes in production rates, has as yet been little explored.

It is important to emphasize that the phenomenology of Hidden Valleys is often not captured by a simplified model of a single new long-lived particle. In models with multiple hidden particles, the long-lived particles observable at SHiP may not be directly produced. As suggested in Figs. 3.1, 


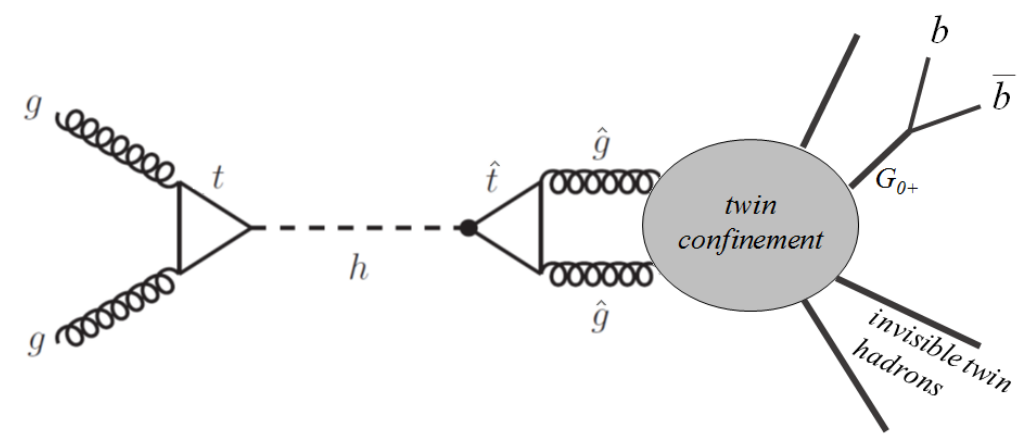

Figure 3.5: Example of a Twin Higgs collider event [200]. The SM-like Higgs $h$ decays through a loop of twin tops into a pair of twin gluons $\hat{g}$, which subsequently hadronize to produce various twin glueballs $G$. These glueballs states decay to Standard Model fermions with a wide range of lifetimes.

3.3 and 3.5, they may instead may be daughters of the originally produced hidden particle(s), generated by prompt decays, showering or hadronization [193]. This has three important and general consequences.

- Since the produced particle is not the observed long-lived particle, the coupling determining the production rate need not be correlated with the coupling that determines the lifetime. This potentially allows enhanced rates at SHiP, and different kinematics, compared to one-particle simplified models.

- Multiple particles may be produced in decays (or other dynamics, such as hadronization) leading to two or even more displaced vertices in a single SHiP event.

- To the extent they rely on one-particle simplified models, widely quoted limits from previous experiments on these long-lived particles are substantially altered or inapplicable.

Taken together, these possibilities broaden both the reach of SHiP and the classes of phenomena for which it should search. In addition to the possibility of multiple displaced vertices per event (which in some cases may be very closely spaced), one might also encounter vertices with $>2$ tracks (e.g. $\mu^{+} \mu^{-} \pi^{+} \pi^{-}$), or vertices with accompanying missing energy (so that the tracks do not form a resonance).

At the LHC the long-lived particles are usually not produced directly, but emerge in cascade decays of heavy mediators. Because its centre of mass energy is merely $28 \mathrm{GeV}$, SHiP cannot produced directly EW scale mediators between the hadronic sector and the Hidden Valleys. And production from highly off-shell mediator is also expected not to be copious (see Section 3.3 for an explicit example of Higgs portal hidden valley). However, if the hidden valley is endowed with very light particles, production of these new states at the SHiP will be possible. Probably the most promising scenario would be light hidden particles with the same quantum numbers as the mediator. In this case these particles can be singly produced at SHiP due to their tiny (but nonvanishing) coupling to the hadrons. These particles can also be produced in rare decays of B- and D-mesons, which is especially important for the mediators which predominantly couple to the heavy generations of quarks (e.g. the Higgs boson). Pair production of sufficiently light particles is also possible at $\mathrm{SHiP}$, for instance in exotic decays of $\mathrm{B}$ - and $\mathrm{D}$-mesons (for an explicit example, see 
Section 3.3) or in off-shell dark-vector production. However, pair production does not mean the final state will contain two Hidden Valley particles only, since the produced particles may undergo non-trivial Hidden Valley dynamics, in which they decay, shower or hadronize into a more complex final state with different particles than those originally produced.

The tremendous variety of phenomena that can arise in Hidden Valleys, combined with the potential importance of Hidden Valleys in the greatest problems in particle physics already mentioned above, as dark matter, naturalness, baryogenesis, and others, helps motivate a program of experiments looking for dark sector particles. Since lifetimes of Hidden Valley particles are often macroscopically long, an experiment such as SHiP forms an essential part of that program.

\subsubsection{Light scalars in supersymmetry}

Light scalars with masses below $50 \mathrm{GeV}$ and weak couplings to $\mathrm{SM}$ are not possible in the minimalsupersymetric standard model (MSSM) given current experimental constraints. Such scenarios are however realised in well-motivated extensions of the MSSM.

\subsubsection{Singlet extensions}

The simplest extension of the MSSM consists in adding one chiral singlet superfield. The corresponding superpotential reads

$$
\mathcal{W}_{\mathrm{GNMSSM}}=\mathcal{W}_{\mathrm{MSSM}}+\lambda S H_{u} H_{d}+\xi_{s} S+\frac{1}{2} \mu_{s} S^{2}+\frac{1}{3} \kappa S^{3} .
$$

This model, known as the GNMSSM, follows from an underlying $\mathcal{Z}_{4}^{R}$ symmetry which is broken down to $R$-parity [208-211]. It has been shown to be consistent with grand unification [209] and, unlike the scale-invariant singlet extension with a $\mathcal{Z}_{3}$, (the NMSSM), does not suffer from a domain wall or tadpole problem.

In the limit $\xi_{s}, \mu_{s}^{2} \ll v^{2}, \lambda<\mu_{s} / v$ and $\lambda \kappa<\mu_{s}^{3} / v^{3}$, the GNMSSM can naturally accomodate a light scalar with mass $m_{s} \sim \mu_{s}$ [212]. Here $v$ denotes the electroweak vev and we assumed that the scale of the singlet soft terms does not exceed $m_{s}$. The mixing of the light scalar and the SM Higgs is mostly controlled by the $\lambda$-coupling. Light singlet scalars often come together with light singlinos in a similar mass range. These are good candidates for the dark matter in our Universe. We are going to discuss this later in more detail.

\subsubsection{Additional Abelian gauge groups}

Models with additional Abelian gauge groups not only introduce an extra gauge boson. Often also additional scalars are added which are needed to break the new $U(1)$. There is a wide range of $U(1)$ extensions of the MSSM, see for instance Ref. [213]. In the case that the new gauge couplings are assumed to be of similar size as the SM ones, there are limits on the mass of the $Z^{\prime}$ of about $2.5 \mathrm{TeV}$. However, the new scalars can still be signficantly lighter than that. This can happen for instance in the B-L-SSM [214, 215]. In this model the MSSM particle content is extended by three right-handed neutrino superfields $\nu^{R}$ to cancel the $B-L$ gauge anomalies and two bileptons $\eta, \eta^{\prime}$ to break the $B-L$. The new terms in the superpotential are

$$
\mathcal{W}_{\mathrm{B}-\mathrm{L}-\mathrm{SSM}}=\mathcal{W}_{\mathrm{MSSM}}+Y_{\nu} l \nu^{R} H_{u}+Y_{x} \nu^{R} \eta \nu^{R}+\mu^{\prime} \eta \eta^{\prime}
$$

The bilepton mass eigenstates have similar features as the Higgs fields: one usually becomes heavy, while the other one remains light. The mass of the lighter state can be as low as a few GeV which is not spoiled by radiative corrections. The only mixing between the bileptons and the Higgs doublets at tree-level is generated by gauge-kinetic mixing [216]. Therefore, this mixing is naturally small. In these models several new dark matter candidate arise compared to the MSSM. The LSP can 
be a light neutralino which is mainly a gaugino of the new sector or a bileptino. However, also sneutrino LSPs are possible. These are mainly right-handed states and therefore not in conflict with direct detection limits. For both possibilites light singlets could play a crucial part in explaining the relic density: for neutralinos there can be resonance effects, while sneutrinos always annihilate very efficiently into light bileptons if this is kinematically allowed [217].

\subsubsection{Models with $R$-parity violation}

If $R$-parity is broken dynamically like in the $\mu \nu \mathrm{SSM}[218,219]$

$$
\mathcal{W}_{\mu \nu \mathrm{SSM}}=\mathcal{W}_{\mathrm{MSSM}}+Y_{\nu} H_{u} l \nu^{R}+\lambda \nu^{R} H_{u} H_{d}+\frac{1}{3}\left(\nu_{R}\right)^{3}
$$

there is a mixing between the Higgs doublets and the sneutrinos. If three generations of righthanded neutrino superfields are assumed, actually three, potentially different, couplings $\lambda_{i}$ between the Higgs doublets and singlets are present in contrast to other singlet extensions. This model shares many features with singlet extensions without $R$-parity violation and one finds also regions with light singlets which are consistent with all observations today [220].

\subsection{Linear scalar portals: Higgs-scalar mixing}

We first focuss our attention to scalar portal models with a linear mixing between the light scalar $S$ and the Higgs boson:

$$
\mathcal{L}=\mathcal{L}_{\mathrm{SM}}+\frac{1}{2} \partial_{\mu} S \partial^{\mu} S+\left(\alpha_{1} S+\alpha S^{2}\right)\left(H^{\dagger} H\right)+\lambda_{2} S^{2}+\lambda_{3} S^{3}+\lambda_{4} S^{4},
$$

with $\alpha_{1} \neq 0$. Notice that a $\mathcal{Z}_{2}$ symmetric model also effectively induces a linear mixing between the scalar $S$ and the Higgs boson if $S$ develops a vacuum expectation value: $\alpha_{1}=4 \alpha\langle S\rangle$. Cosmological considerations restrict the range of phenomenogically viable values of the portal coupling $\alpha$, see Section 3.7.

A low-energy effective Lagrangian of the form (3.2.1) is naturally obtained in the supersymmetric models discussed previously. In the supersymmetric case, an additional singlet fermion and singlet pseudoscalar enter the theory. The possible role of the singlet fermion as a dark matter candidate is discussed in the next section. However, there are also other interesting aspects of light singlets.

Especially, if the singlets are lighter than mesons like $\Upsilon$ or $B$ stringent bounds arise as we will see. In that case the mesons can decay into on-shell singlets. These singlets not necessarily escape the detector but could further decay into SM final states. Hence, the partial widths of singlets into leptons or lighter mesons are very important.

Assuming that the mass of the scalar is substantially below the electroweak scale, the Higgs boson can be integrated out. The low-energy effective Lagrangian reads

$$
\mathcal{L}=\mathcal{L}_{\mathrm{SM}}-\frac{g_{\star} m_{f}}{v} S \bar{f} f+\mathcal{L}_{\text {self }},
$$

where $v \simeq 246 \mathrm{GeV}$ denotes the electroweak vev and $f$ stands for a SM fermion with mass $m_{f}$. Self interactions of $S$ are contained in $\mathcal{L}_{\text {self }}$. The effective coupling $g_{\star}$ originates from the mixing of the light scalar with the SM Higgs. For instance, in the limit of small mixing and for light singlet masses, $m_{S} \ll m_{h}$, the mixing angle is given by

$$
g_{\star}=\sin \theta \simeq \theta \simeq \frac{\alpha_{1} v}{m_{h}^{2}} .
$$


Both the production mechanisms and decay pattern (branching ratios) are exactly the same as would be for the SM Higgs boson of the same mass. The production cross sections and the decay rates are only overall suppressed by $g_{\star}^{2}$ as compared to the Higgs boson case. For detailed study see [221]. Note, that calculation of the decay parameters is complicated for the mass around $2 \mathrm{GeV}$ due to emergence of a large number of hadronic resonances. The decay branching ratios and lifetime (which is inversely proportional to the squared mixing angle) in the mass interval relevant for SHiP are presented in Fig. 3.6.
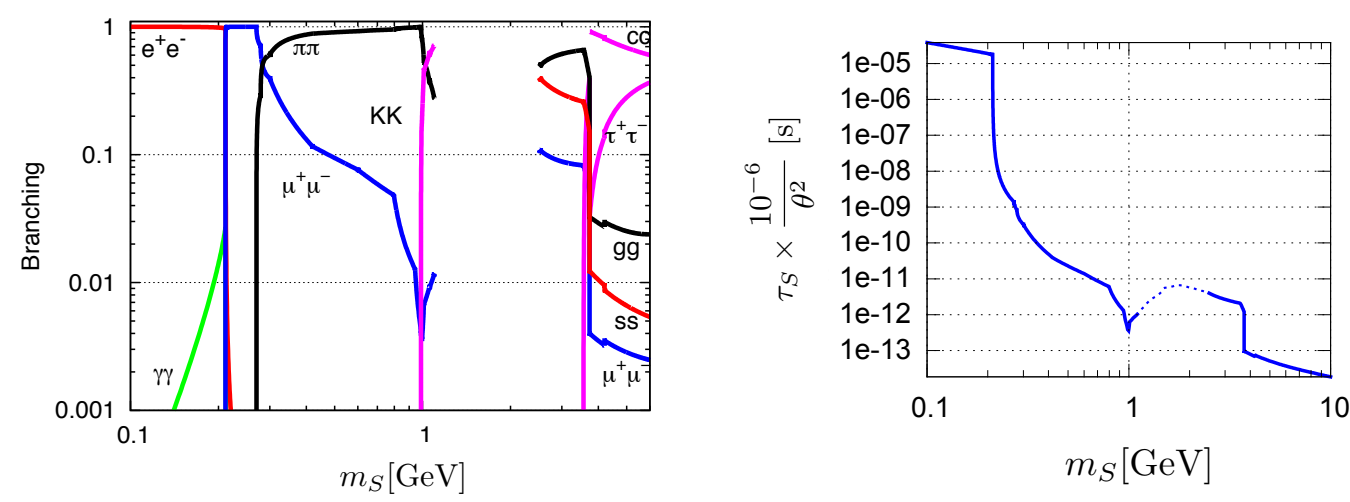

Figure 3.6: Left panel: decay branching ratios function of the scalar mass $m_{S}$. Reproduced from Ref. [222]. Right panel: scalar lifetime as a function of its mass $m_{S}$ for constant mixing angle with the Higgs boson $\theta^{2}=10^{-6}$. Lifetime for other mixing angles is obtained by the simple rescaling of the graph.

The mixing angle determines the decay length of $S$, which should be on the order of the distance between the target and the detector for $S$ to be detectable. If there are no additional light states, $S$ will decay to SM particles. If $m_{S}$ is below the electron threshold, i.e. $m_{S}<2 m_{e}$, $S$ can still decay to two photons. Above the electron threshold, but below the hadronic threshold, i.e. $2 m_{e}<m_{S}<2 m_{\pi}$, the scalar can decay to electrons and/or muons (see Fig. 3.7), with a width given by

$$
\Gamma(S \rightarrow \ell \bar{\ell})=\frac{g_{\star}^{2} m_{\ell}^{2} m_{S}}{8 \pi v^{2}}\left(1-\frac{4 m_{\ell}^{2}}{m_{S}^{2}}\right)^{3 / 2} .
$$

As the scalar coupling is proportionally to the mass of the fermion, the lifetime of the scalar can be macroscopic for different characteristic values of the mixing angle depending on whether it is heavier or lighter than twice the muon mass,

$$
c \tau_{S} \simeq 50 \mathrm{~m} \times \begin{cases}\left(\frac{0.02}{g_{\star}}\right)^{2}\left(\frac{50 \mathrm{MeV}}{m_{S}}\right) & \text { for } \quad m_{S}<2 m_{\mu}, \\ \left(\frac{5 \times 10^{-5}}{g_{\star}}\right)^{2}\left(\frac{250 \mathrm{MeV}}{m_{S}}\right) & \text { for } \quad m_{S}>2 m_{\mu} .\end{cases}
$$

For even heavier scalars, additional hadronic decay channels are open, although there are significant theoretical uncertainties in the computation of their rates [223-226]. The spectator model [227] 

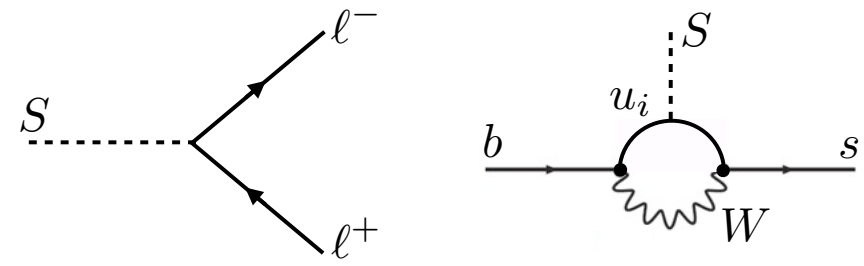

Figure 3.7: Left: The decay of the singlet to leptons, $S \rightarrow \ell^{+} \ell^{-}$. Right: The $b-s-S$ flavor-changing penguin, leading to production of $S$ via decays such as $B \rightarrow K S$.

provides a good estimates of ratio of the partial widths of the scalar $S$ :

$$
\begin{gathered}
\Gamma_{\mu^{+} \mu^{-}}: \Gamma_{\pi \pi}: \Gamma_{K K}: \Gamma_{\eta \eta}: \Gamma_{D D}: \Gamma_{\tau^{+} \tau^{-}}: \Gamma_{g g} \\
=m_{\mu}^{2} \beta_{\mu}^{3}: 3\left(m_{u}^{2}+m_{d}^{2}\right) \beta_{\pi}^{3}: 3 \frac{9}{13} m_{s}^{2} \beta_{K}^{3}: 3 \frac{4}{13} m_{s}^{2} \beta_{\eta}^{3}: \\
m_{c}^{2} \beta_{D}^{3}: m_{\tau}^{2} \beta_{\tau}^{3}:\left(\frac{\alpha_{s} m_{S}}{3 \pi}\right)^{2}\left(6-2 \beta_{\pi}^{3}-\beta_{K}^{3}\right) .
\end{gathered}
$$

with $\Gamma_{X Y} \equiv \Gamma(S \rightarrow X Y)$ and $\beta_{\mu}=\sqrt{1-4 m_{\mu}^{2} / m_{S}^{2}} \Theta\left(m_{S}-2 m_{\mu}\right)$ where we use the step-function $\Theta$.

One may expect a sizable branching ratio to dimuon pairs. Therefore, provided these scalars can be produced in sufficient quantity, one can search for the striking signal of a dilepton pair in the SHiP detector.

Another aspect of light singlets is that they open new decay channels for the SM Higgs, see Section 3.3. The process $h \rightarrow S S$ can trigger multi-lepton final states for which strong limits come from LEP searches. These limits can be translated into limits on the mixing angles between the doublet and singlet scalars [228]. Here we will mainly concentrate on the mass range $m_{S}<10 \mathrm{GeV}$, where the light scalar can be produced in meson decays. In this case the searches for rare decays typically give stronger constraints on which we shall focus in the following.

\subsubsection{Existing experimental limits}

There are stringent constraints on new light states coupling to SM particles. Of particular interest in this context are experimental searches for rare meson decays, because the presence of a new light scalar mediator $S$ will in general lead to a large enhancement in the rates of flavour-changing processes such as $K \rightarrow \pi S, D \rightarrow \pi S$ or $B \rightarrow K S$ [229, 230].

These transitions are induced through the flavor changing penguins, e.g., $b-s-S$ (where $s$ is the strange quark) for $B \rightarrow K S$ decays, etc., as shown in Fig. 3.7. Upon integrating out the $W$ boson and top quark, we can write the effective $b-s-S$ coupling as [231]

$$
\mathcal{L} \supset \frac{3 \sqrt{2} G_{F} m_{t}^{2} V_{t s}^{*} V_{t b}}{16 \pi^{2}} \frac{m_{b}}{v} g_{\star} S \bar{s}_{L} b_{R}+\text { h.c. }
$$

From Eq. (3.2.7), it is straightforward to compute the branching ratios for $B \rightarrow K S$, and we refer the reader to $[229,231]$ for explicit formulae. Similar computations can be performed for the decays of Kaons to $S$.

Flavour observables therefore provide an unique opportunity to constrain the interactions of the dark sector with SM particles via a light mediators. In Fig. 3.8 we show current constraints on a light scalar particle with Yukawa-like couplings to SM states. These bounds come from a variety of rare decays of $\Upsilon$ and $B$ mesons as well as from kaons. In addition the bound due to CHARM, 


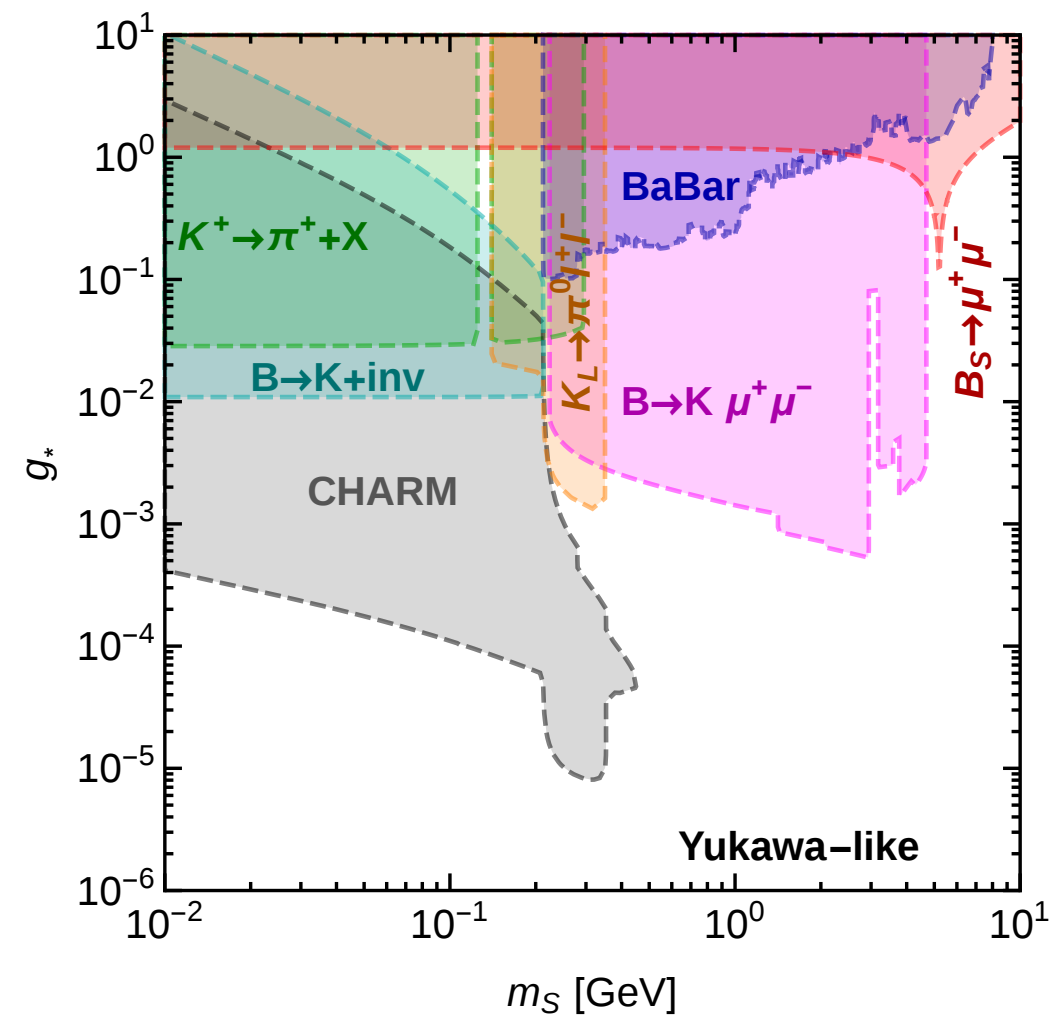

Figure 3.8: Currently excluded parameter space for a light scalar with Yukawa-like couplings.

a previous proton fixed target experiment, is shown. The treatment of the experimental contraints in order to arrive at these bounds has been identical to the treatment in [232] with the difference that pseudoscalars ${ }^{2}$ have been considered there. In practice this means that the flavour changing couplings as well as the branching ratios and total width had to be adapted to the scalar case.

\subsubsection{What SHiP can do}

The main production mechanism for light scalars with Yukawa-like couplings at SHiP comes from B-meson and kaon decays. Note that although very light scalars are predominantly produced via kaon decays due to the larger production cross section of kaons, SHiP is designed such that kaons will typically be stopped in the target before decaying, so that the fraction of scalars emitted in the direction of the detector is much smaller. We estimate the fraction of kaons which decay before absorption and therefore contribute to the production of scalars boosted towards the detector to be $0.2 \%$.

To estimate the number of scalars produced in kaon and B-meson decays we first estimate the total number of kaons and B-mesons produced, using $N_{B, K}=N_{\mathrm{PoT}} \sigma_{B, K} / \sigma_{p N}$ with $\sigma_{p N}$ the total cross section for proton nucleon collisions and $N_{\mathrm{PoT}}=2 \cdot 10^{20}$ the total number of protons on target for SHiP. We take $\sigma_{p N} \sim 10 \mathrm{mb}$ and assume $\sigma_{K}=20 \mathrm{mb}$ and $\sigma_{B}=3.6 \mathrm{nb}$, such that in total about $8 \cdot 10^{17}$ kaons and $7 \cdot 10^{13} \mathrm{~B}$ mesons will be produced.

The number of scalars produced in B-meson decays is then simply given by $N_{S}=N_{B} \times B R(B \rightarrow$

${ }^{2}$ Pseudoscalars are considered in Chapter 5 where one can also find some more details on the employed procedure. Comparing Figs. 3.9 and 5.2 we find that the difference in parity has only a subdominant effect. 


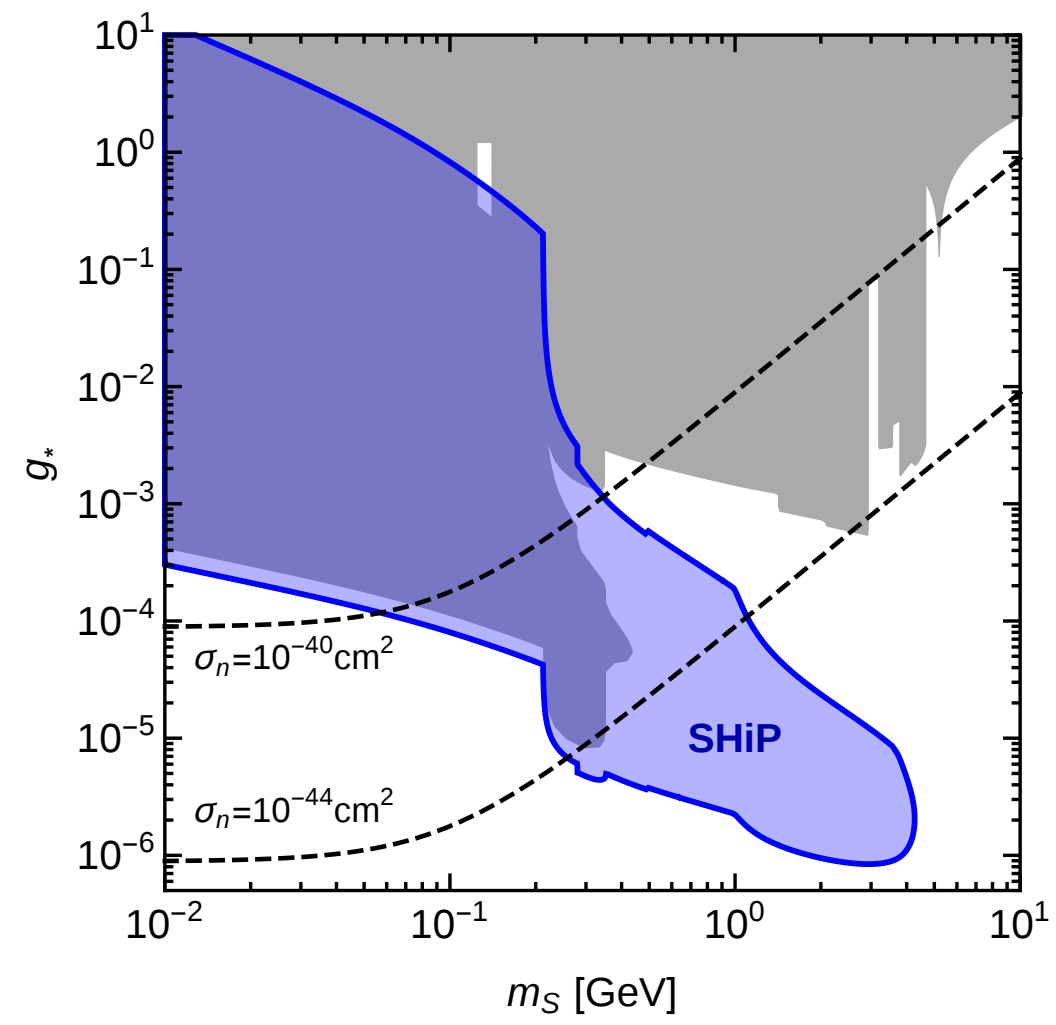

Figure 3.9: Projected sensitivity of SHiP for a scalar $S$ with Yukawa-like couplings to all SM fermions, in comparison to the existing bounds. The decays $S \rightarrow e e, \mu \mu, \pi \pi$ and $K K$ are considered. Both $S$ production from $B$ and $K$ decays are considered with the assumption that all $B$ mesons but only $0.2 \%$ of the kaons decay before being stopped. Also shown are contours of constant DM nucleon cross section, where we assumed that $S$ acts as the mediator between DM and nucleons (see Section 3.5 for details).

$\left.S+X_{s}\right)[221,230]$ and similarly for the kaons. ${ }^{3}$ Here $B \rightarrow S+X_{s}$ is the inclusive decay, where $X_{s}$ can be any strange meson and the decay rate reads [233]

$$
\Gamma\left(B \rightarrow S+X_{s}\right)=\frac{1}{8 \pi} \frac{\left(m_{b}^{2}-m_{S}^{2}\right)^{2}}{m_{b}^{3}}\left|h_{s b}^{S}\right|^{2},
$$

with $h_{s b}^{S}$ the scalar component of the induced flavour changing coupling.

The number of decays in the detector region can then be calculated by multiplying the number $N_{S}$ of scalars produced with the probability that the scalar decays inside the detector. In addition the branching ratio of the scalar into observable final states has to be taken into account. We take these states to be $\gamma \gamma, e^{+} e^{-}, \mu^{+} \mu^{-}, \pi \pi$ and $K K$. If the detector is placed at a distance $l$ (to be taken equal to $70 \mathrm{~m}$ ) and has a length $\Delta l$ (to be taken equal to $55 \mathrm{~m}$ ), the number of events in the detector region is

$$
N_{\text {det }} \sim N_{S}\left[\exp \left(-\frac{l}{\gamma \beta c \tau_{S}}\right)-\exp \left(-\frac{l+\Delta l}{\gamma \beta c \tau_{S}}\right)\right],
$$

where $\tau_{S}=\Gamma_{S}^{-1}$ is the lifetime of the scalar and $\gamma=E_{S} / m_{S}$ is its relativistic gamma factor.

${ }^{3}$ We make the assumption here that the $B$-mesons still have a sufficiently large boost that the majority of the scalars produced in the decays will travel in the direction of the detector. 
We take $E_{S} \sim 25 \mathrm{GeV}$ (see Ref. [234]) and assume that SHiP will observe no background events, so that it can rule out all parameters that predict $N_{\text {det }}>3$. The projected exclusion region (together with the current bounds on light scalars from above) is shown in Fig. 3.9. The shape of the exclusion region can be understood from the requirement that the scalar should neither decay too quickly nor too slowly in order to give an observable signal. The kaon decays dominate below the $m_{K}-m_{\pi}$ threshold while the $B$ decays dominate above this threshold. Despite the huge kaon production cross-section, the impact of the kaons in the SHiP sensitivity is largely reduced by the fact that most of them are stopped in the target before decaying.

Assuming that $S$ mediates the interactions between DM and nucleons, see Section 3.5, we can compare the expected sensitivity of SHiP with the sensitivity of DM direct detection experiments. Current LUX bounds reside in the range $\sigma_{n}<10^{-44} \mathrm{~cm}^{2}-10^{-40} \mathrm{~cm}^{2}$ for DM masses $m_{\chi} \simeq 5-10 \mathrm{GeV}$.

As can be seen in Fig. 3.9, SHiP is expected to probe a regime of scalar masses and couplings currently not accessible to any accelerator or DM experiment.

\section{3 $\mathcal{Z}_{2}$ scalar portals: pair-production of light hidden particles and exotic Higgs decays}

We now turn our attention to scalar portals where the scalar $S$ does not develop a vacuum expectation value and cannot mix with the Higgs boson:

$$
\mathcal{L}=\mathcal{L}_{\mathrm{SM}}+\frac{1}{2} \partial_{\mu} S \partial^{\mu} S+\alpha S^{2}\left(H^{\dagger} H\right)+\lambda_{2} S^{2}+\lambda_{4} S^{4} .
$$

It is natural that the hidden/dark sector will also be composed of additional fermions and gauge bosons that can also couple to the SM Higgs bosons with higher-dimensional portals:

$$
\Delta \mathcal{L}=\frac{1}{\Lambda}|H|^{2} \bar{\psi} \psi
$$

for a BSM fermion $\psi$ and

$$
\Delta \mathcal{L}=\frac{1}{\Lambda^{2}} m_{Z_{D}}^{2}|H|^{2} Z_{D \mu} Z_{D}^{\mu},
$$

for a gauge boson after dark symmetry breaking.

This $\mathcal{Z}_{2}$ scalar portal models are an explicit (and minimal) realization of the Hidden Valley scenarios discussed in Section 3.1.2 with the Higgs boson being the heavy mediator that is dominantly responsible for (pair-)production of the low-mass hidden sector states.

In the presence of BSM states below the Higgs mass, such Higgs portal couplings will generally mediate exotic decays of the $125 \mathrm{GeV}$ Higgs boson into BSM states. Due to the small width of the SM Higgs boson, these decays can easily have $\mathcal{O}(10 \%)$ branching fractions; see [235] for an overview of Higgs portal couplings and a discussion of the signature space in related models.

The $28 \mathrm{GeV}$ center-of-mass energy at SHiP is much too low to produce on-shell Higgs bosons. However, sensitivity to exotic Higgs decays arises in two ways: production of exotic decay final states through an off-shell higgs, or single on-shell production of light BSM states that inherit SM couplings through mixing. In each case, the main signal of Higgs-portal-coupled hidden sectors at $\mathrm{SHiP}$ is the production of light BSM states in the decay of heavy mesons, mediated by interactions of the form (3.3.1)-(3.3.3). The resulting signatures depend in detail on the spectrum and decays of the new BSM states.

As discussed in Section 3.2, when the BSM scalar mixes with the Higgs boson, it can be singly produced at SHiP, and decays back to the SM through the same Higgs portal coupling that governs its production. This also arises when a light pseudo-scalar mixes with the $C P$-odd component of a two-Higgs doublet model. In more general cases the BSM states decay back to the SM via 
a different coupling, as in the case of dark vector bosons $Z_{D}[41,43,88]$, or they may decay to additional BSM state(s), as in the Hidden Valley models [193, 197, 207, 236-238], see Section 3.1.2. Hidden sector cascades can result in long-lived particles in many ways. The original state may be long-lived, thanks to approximate symmetries and/or near-degeneracies in the hidden sector, and thus decay within the SHiP detector to an additional BSM state plus one or more SM particles. Alternatively, the original particle may decay promptly within the hidden sector to new states that are much less strongly coupled to the SM than their parent.

To keep our discussion general, in the present section we consider the production of BSM states through the Higgs portal couplings of Eqs. (3.3.1)-(3.3.3). We assume that the new states do not mix with SM states and thus must be pair-produced, and that their decay is governed by additional physics beyond the Higgs portal coupling. This serves as a useful means of estimating the discovery reach of SHiP to generic low-mass hidden sectors, and placing SHiP in comparison to other probes of the Higgs portal such as the LHC.

\subsubsection{Probing Exotic Higgs Decays at SHiP}

For simplicity, we consider the production of a single SM singlet $X$, which may be a real scalar or pseudo-scalar, a Dirac fermion, or a dark photon. The interactions of Eqs. (3.3.1)-(3.3.3) then yield exotic decays of the Higgs boson

$$
h \rightarrow X X,
$$

and mediate pair production of $X$ at SHiP through off-shell $h$ exchange. After electroweak symmetry breaking, the relevant interaction terms in the Lagrangian are

$$
\Delta \mathcal{L}= \begin{cases}g_{h X X} v h X X & (\text { scalar }), \\ g_{h X X} h \bar{X} X & (\text { fermion }), \\ g_{h X X} \frac{m_{X}^{2}}{v} h X_{\mu} X^{\mu} & \text { (gauge boson) }\end{cases}
$$

all of which give $\operatorname{Br}(h \rightarrow X X) \sim 0.1$ for $g_{h X X} \sim 10^{-2}$. In order to generate visible signals at SHiP, the hidden sector $X$ particles must have decays involving at least one SM particle. We make no assumptions about how this occurs, but express all our results in terms of the proper decay length $c \tau$ of $X$ into SM particles. Decays to hidden sector particles are also possible, but we ignore this complication for the sake of this signal estimate, expressing the number of $X$ particles decaying in the SHiP (or LHC) detector as

$$
N_{X}=N_{X}\left(m_{X}, c \tau_{X}\right) .
$$

This minimal set of theoretical assumptions allows us to compare SHiP's discovery potential for low-mass hidden sectors to that of a high-energy collider like the LHC in a relatively modelindependent manner. In parameterizing the couplings $g_{h X X}$ separately from the lifetime of $X$, we implicitly assume that different couplings are responsible for production and decay of the hiddensector $X$ particles (though of course one can interpret our results for the case where both arise from a single coupling). This allows us to investigate the case where SHiP is most sensitive - that of long-lived particles with appreciable production cross sections. There are many well-motivated theories where this scenario is realized.

A well-known example is the broken $U(1)$ extension of the SM, where mixing between the dark Higgs $s$ and the SM-like Higgs leads to $h^{(*)} \rightarrow Z_{D} Z_{D}$ production as well as $s^{(*)} \rightarrow Z_{D} Z_{D}$, while kinetic mixing between the dark $U(1)$ and hypercharge allows the $Z_{D}$ to decay to SM fermions. ${ }^{4}$ The ability of high-energy colliders to probe this scenario has been explored in [239], which demonstrated reach for extremely small values of the kinetic mixing parameter $\epsilon$. Our results below will be good

\footnotetext{
${ }^{4}$ The discovery potential of SHiP to the pure kinetic mixing case has been explored in [159].
} 


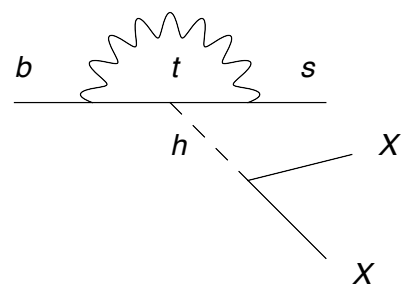

Figure 3.10: Feynman diagram responsible for the production of $X$ through exotic decays of B mesons, $B \rightarrow K X X$.

estimates for this hidden sector when both the dark scalar and dark photon have similar masses $\lesssim \mathrm{GeV}$. This Higgsed $U(1)$ theory is a simple and minimal example of a multi-particle hidden sector. More general hidden valley models [193, 197, 207, 236-238], see Section 3.1.2, may contain a plethora of light states with a common mass scale, giving rise to rich phenomenology with possibly long-lived hidden-sector particles. For instance, a confining hidden sector could generate hidden mesons which, as in QCD, can be very long-lived owing to (e.g.) approximate flavor symmetries. The scalar Higgs portal coupling of Eq. (3.1.1) could pair-produce light hidden-sector mesons, which could eventually decay to (e.g.) lighter mesons via an off-shell $h$, or back to SM particles through non-renormalizable interactions. This is an example of a scenario to which SHiP has excellent discovery potential, for a range of masses and decay lengths which we will establish below.

\subsubsection{What SHiP can do}

In this section we estimate the number of produced $X$ particles that decay within the detector volume of SHiP and compare it to the corresponding signal yield at the LHC. This will reveal regions of parameter space where SHiP has superior discovery potential. All signal rates scale linearly with $\operatorname{Br}(h \rightarrow X X)$ in the limit where this branching ratio is small.

\subsubsection{Hidden sector scalars}

For $m_{X} \lesssim 2 \mathrm{GeV}$, the dominant $X$ production mechanism at SHiP is through the decays $B \rightarrow$ $K^{(*)} X X$. This process occurs through the penguin-like diagram of Fig. 3.10. The large top Yukawa coupling partially offsets the loop suppression, and the diagram is no more CKM-suppressed than the dominant SM $b$ decay. This makes rare $B$ decays a prime place to discover new light states $X[240,241]$.

The branching ratio for $B \rightarrow K^{(*)} X X$ has been calculated in [240]. In our notation, it is

$$
\operatorname{Br}\left(B^{+} \rightarrow K^{+(*)} X X\right) \approx\left(1.2 \times 10^{-4}\right) g_{h X X}^{2} F\left(m_{X}\right)
$$

where $F\left(m_{X}\right)$ is $\approx 1$ for $m_{X}=0.1 \mathrm{GeV}$ and $\approx 0.65$ for $m_{X}=1 \mathrm{GeV}[242] .{ }^{5}$ Isospin predicts a similar exotic branching ratio for $B^{0}, \bar{B}^{0}$ mesons as well. To estimate the production of $X$ particles at SHiP, we simulate $p p \rightarrow b \bar{b}$ in MadGraph [243], extract the $B^{ \pm}, B^{0}$, and $\bar{B}^{0}$ mesons from each event, and decay them to the three-body final state $K^{(*)} X X$ according to phase space. This allows us to compute the distribution of boosts of $X$ in the lab frame as a function of its mass. For the purposes of this estimate we take the SHiP detector to be $50 \mathrm{~m}$ long, with a circular $5 \mathrm{~m}$ diameter cross section starting at a distance of $60 \mathrm{~m}$ from the target. We then define the geometric acceptance $\epsilon_{\text {geom }}$ as the fraction of $X$ particles with momentum at an angle $\theta<\theta_{\max }=2.5 / 60$ from the beam

\footnotetext{
${ }^{5}$ The small $(<10 \%)$ differences between $B^{+} \rightarrow K^{+} X X$ and $B^{+} \rightarrow K^{+*} X X$ can be safely neglected in our estimations.
} 
axis. The boost distribution of all surviving $X$ particles is then convolved with the probability of decaying within the SHiP detector. We estimate this probability via

$$
P_{\text {decay }}\left(L_{1}, L_{2}\right)=\exp \left(-\frac{L_{1}}{b c \tau_{X}}\right)-\exp \left(-\frac{L_{2}}{b c \tau_{X}}\right)
$$

where $c \tau_{X}$ is the proper decay length, $b=\left|\vec{p}_{X}\right| / m_{X}, L_{1}=50 \mathrm{~m}$, and $L_{2}=110 \mathrm{~m}$. The exponential dependence means that detector geometry sensitively affects the number of observed signal events. For the luminosity of the fixed-target experiment we use

$$
\mathcal{L}_{\mathrm{SHiP}}=N_{\mathrm{POT}} \cdot n_{\text {tungsten }} \cdot L_{\mathrm{eff}} \approx 2.3 \times 10^{8} \mathrm{fb}^{-1},
$$

where $N_{\text {POT }}=2 \times 10^{20}$, and $n_{\text {tungsten }} \approx 1.15 \times 10^{25} \mathrm{~cm}^{-3}$ is the number density of protons in Tungsten. The effective target length is taken to be $L_{\text {eff }} \approx 1 \mathrm{~m}$. These approximations will give signal yields to within a factor of $\sim 2$. The number of $X$ particles decaying in the SHiP detector is then given by

$$
N_{X}=\mathcal{L} \cdot \sigma_{p p \rightarrow b b} \cdot \operatorname{Br}\left(B^{+} \rightarrow K^{+(*)} X X\right) \cdot\left\langle n_{B}\right\rangle \cdot 2 \cdot \epsilon_{\text {geom }} \cdot P_{\text {decay }}
$$

where $\left\langle n_{B}\right\rangle$ is the average number of $B^{ \pm}, B^{0}$, and $\bar{B}^{0}$ mesons per event.

A corresponding signal may also be observed in exotic decays of $B$ mesons at the LHC. We estimate the event yield at LHC analogously to the yield at SHiP, generating $p p \rightarrow b \bar{b}$ events in MadGraph and decaying $B_{d}$ mesons according to three-body phase space to estimate the boost distribution. We again use the Madgraph cross-section for $b \bar{b}$ production; neglecting $K$-factors is consistent within the $\mathcal{O}(1)$ precision of our estimates. We estimate the potentially visible signal to be those $X$ 's which decay within the tracker at the LHC multipurpose experiments, a length of approximately $1 \mathrm{~m}$, so that $P_{\text {decay }}^{\mathrm{LHC}}$ is given by Eq. (3.3.8) with $L_{1}=0$ and $L_{2}=1 \mathrm{~m}$. The fraction of $X$ particles with $|\eta|<2.5$ constitutes the geometric acceptance $\epsilon_{\text {geom }}^{\mathrm{LHC}}$ of the LHC.

When $X$ is too heavy to be pair produced in $B$ meson decay, gluon-fusion production through $p p \rightarrow h^{*} \rightarrow X X$ remains a possible production mechanism. This is to be compared to the signal yield from exotic Higgs decays in gluon fusion at the LHC. The exotic partial width of a Higgs to two scalars is given at tree-level by

$$
\Gamma_{h \rightarrow X X}=\frac{1}{8 \pi} \frac{g_{h X X}^{2} v^{2}}{m_{h}} \sqrt{1-4 \frac{m_{X}^{2}}{m_{h}^{2}}} .
$$

The LHC signal is greatly enhanced by the small Higgs width, while the SHiP signal is greatly suppressed by the off-shell Higgs propagator. We estimate the signal yields using the complex scalar + dark vector extension MadGraph model of [239] to simulate gluon fusion production of two scalars at $\sqrt{s}=14 \mathrm{TeV}$ for the LHC and at $\sqrt{s}=28 \mathrm{GeV}$ for SHiP. As before, we select $X$ particles within the respective geometric acceptances of both detectors, and estimate signal yields by convolving the resulting boost distribution with the probability that an $X$ with given proper decay length will decay within the detector volume. This yields for the LHC

$$
N_{X}^{\mathrm{LHC}}=\mathcal{L} \cdot \sigma_{p p \rightarrow h} \cdot \operatorname{Br}(h \rightarrow X X) \cdot 2 \cdot \epsilon_{\text {geometric }}^{\mathrm{LHC}} \cdot P_{\text {decay }}^{\mathrm{LHC}}
$$

where $\mathcal{L}$ is the integrated luminosity. For the cross section $\sigma_{p p \rightarrow h}$ we use the $14 \mathrm{TeV}$ result given by the Higgs cross section working group [244]. Meanwhile the SHiP yield is

$$
N_{X}^{\mathrm{SHiP}}=\mathcal{L}_{\mathrm{SHiP}} \cdot \sigma_{p p \rightarrow X X} \cdot 2 \cdot \epsilon_{\text {geometric }}^{\mathrm{SHiP}} \cdot P_{\text {decay }}^{\mathrm{SHiP}},
$$



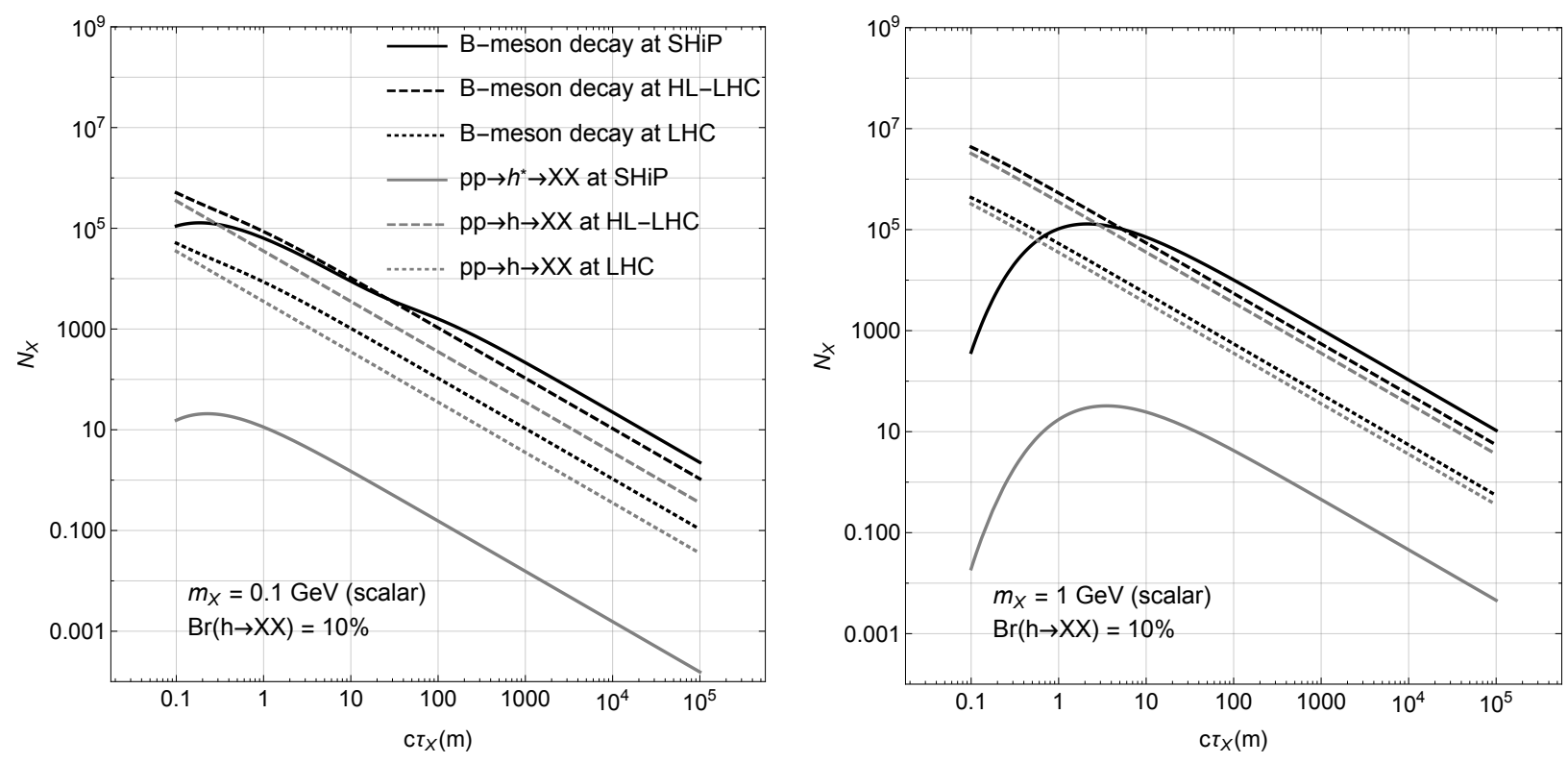

Figure 3.11: Estimated number of $0.1 \mathrm{GeV}$ (left) and $1 \mathrm{GeV}$ (right) $X$ (pseudo-)scalar particles, produced via an off-shell $h^{*}$ in either gluon fusion or $B$-meson decay, that decay within the SHiP and LHC detector volumes. For the LHC and HL-LHC, 300 and $3000 \mathrm{fb}^{-1}$ of luminosity was assumed. All event rates are proportional to $\operatorname{Br}(h \rightarrow X X)$ (up to $\mathrm{Br}^{2}$ corrections), which is set to $10 \%$ in this figure. SHiP has superior signal yield to the LHC and has greater sensitivity to long-lived particles than does the HL-LHC. Combined with the likely superior detection efficiency of the dedicated fixed-target experiment, this shows that SHiP is very well-suited to discovering long-lived hidden-sector (pseudo-)scalars with masses $\lesssim 2 \mathrm{GeV}$.

where $\mathcal{L}_{\mathrm{SHiP}}$ is the SHiP luminosity, and the cross section $\sigma_{p p \rightarrow X X}$ is obtained from Madgraph and can be easily rescaled as a function of $\operatorname{Br}(h \rightarrow X X)$. We find that when $X$ is too heavy to be produced in rare $B$ decays, the number of $X$ particles decaying in the detector is at least three orders of magnitude larger at the LHC than at SHiP, even for the best-case decay length.

Figure 3.11 shows the estimated signal yields as a function of decay length for $m_{X}=0.1$ and $1 \mathrm{GeV}$ and $\operatorname{Br}(h \rightarrow X X)=10 \%$, which will not be excluded by the LHC. Note that the number of $X$-particles decaying within the detector volume at SHiP is within an $\mathcal{O}(1)$ factor of the HL-LHC ${ }^{6}$, and will far exceed decays observed at the $14 \mathrm{TeV}$ LHC with $300 \mathrm{fb}^{-1}$. Furthermore, depending on the way that $X$ decays, the detection efficiency in the high-background environment of the LHC may be significantly lower than at the dedicated SHiP experiment which is designed to look for such signals. This makes SHiP very well suited to detecting hidden-sector scalars and pseudoscalars coupled through the Higgs portal, provided they have a mass of $\lesssim \mathrm{GeV}$ and a decay length of $c \tau_{X} \gtrsim 0.1 \mathrm{~m}$.

Precision Higgs couplings measurements at the HL-LHC and ILC will be sensitive to any nonSM decays with branching ratios larger than $\mathcal{O}(1 \%)$ and $\mathcal{O}(0.1 \%)[245,246]$. Since signal yield scales approximately linearly with exotic Higgs branching ratios, Fig. 3.11 shows that there is potential for sizable SHiP and HL-LHC signals from $h \rightarrow X X$ even for branching ratios of $\sim 10^{-5}$.

\footnotetext{
${ }^{6}$ This coincidence is due to the number of $B$-hadrons produced at the HL-LHC being within an order of magnitude of the number produced at SHiP, since with SHiP's design parameters, the typical number of Xs decaying within the region of interest at LHC is also coincidentally within roughly an order of magnitude of those decaying in SHiP's detector. This result thus depends sensitively on SHiP's detector geometry.
} 


\subsubsection{Hidden sector fermions and vectors}

The sensitivity of SHiP to exotic fermions coupled via Eq. (3.3.2) is less striking. For a given exotic Higgs decay branching fraction, the branching ratio of $B \rightarrow K \bar{\psi} \psi$ is suppressed by $(p / v)^{2}$ relative to the scalar case, where $p=p_{B}-p_{K}$ is the momentum transfer in the decay. In this case exotic Higgs decays at the LHC will prove to be a better probe of this coupling.

The coupling of the Higgs to dark vector bosons via Eq. (3.3.3) alone leads to even more stringent suppressions of the $B \rightarrow K X X$ branching fraction, as the branching ratio scales like $\left(m_{Z_{D}} / m_{h}\right)^{4}$ compared to the scalar case. However, if the dark sector includes a scalar $S$ that is responsible for generating the dark vector's mass, then Higgs- $S$ mixing naturally generates the interactions of Eq. (3.3.3) while also allowing for pair production of $Z_{D}$ through an off-shell $S$. This process occurs at the same order in the small Higgs- $S$ mixing as the off-shell Higgs-portal, and is kinematically unsuppressed when $m_{S} \sim m_{Z_{D}}$, which is a natural expectation. (Compare to $m_{Z} \sim m_{W} \sim m_{h}$ in the SM.) In this case, our results for the (pseudo-)scalar case may be approximately applied to dark vectors as well, meaning SHiP can have superior sensitivity to the LHC.

\subsection{Pseudoscalar portals}

Adding a second Higgs doublet to the SM Higgs sector is one of the simplest extensions of the Standard Model Higgs sector compatible with the observed value of the $\rho$-parameter near unity. Such extensions are required by many well-motivated theories, most notably supersymmetry [247] and axion solutions to the strong CP problem [248, 249]. Unacceptably large tree-level flavor changing neutral currents may be avoided by requiring that all fermions with a given set of quantum numbers couple to only one Higgs doublet. This defines four types of two-Higgs-doublet models (2HDMs): Type I, where all fermion species couple to one Higgs doublet, $\mathrm{H}_{2}$; Type II, where downtype quarks and leptons couple to one Higgs doublet $H_{1}$ and up-type quarks to another, $H_{2}$; Type III, where quarks couple to $H_{2}$ and leptons to $H_{1}$; and Type IV, where down-type quarks couple to $H_{1}$ and up-type quarks and leptons couple to $H_{2}$. In these $2 \mathrm{HDMs}$, the couplings of Higgs bosons to the fermions and electroweak bosons of the SM are controlled by two mixing angles, $\alpha$ and $\beta$, as reviewed in e.g. [235, 250-252].

Many models further extend this Higgs sector to include a complex SM singlet scalar $S$, which can only have renormalizable couplings to the Higgs doublets. In particular, the next-to-minimal supersymmetric SM (NMSSM) adds a singlet chiral superfield to the field content of the MSSM in order to dynamically generate the $\mu$ term in the MSSM superpotential; see [253] for a review. The presence of the singlet allows for additional quartic and cubic couplings in the SUSY Higgs potential, which can open new parameter space for electroweak baryogenesis (as reviewed in [185]) and add tree-level contributions to the Higgs mass [254-256]. In more generality, the presence of an additional Higgs doublet allows an important extension to the Higgs portal mechanism, where SM singlet pseudoscalars $a$ can obtain couplings to SM states through mixing with the pseudoscalar $A^{0}$ in the extended Higgs sector, and thereby opening up a new potential class of interactions between the SM and low-mass hidden sectors ${ }^{7}$. For definiteness, we will focus on NMSSM-like models, but similar results apply in other types of singlet-extended 2HDMs.

The scalar potential in the NMSSM is determined by the superpotential

$$
W=\lambda S H_{u} H_{d}+\frac{\kappa}{3} S^{3}
$$

${ }^{7}$ This has sometimes been called the "axion portal" [257]. In this section we focus on a particular model motivated by the NMSSM. The more generic case of a pseudoscalar axion-like particle is discussed in Chapter 5 . 
and the soft supersymmetry-breaking terms

$$
V_{\text {soft }}=m_{u}^{2}\left|H_{u}\right|^{2}+m_{d}^{2}\left|H_{d}\right|^{2}+m_{S}^{2}|S|^{2}+\left(-\lambda A_{\lambda} S H_{u} H_{d}+\frac{1}{3} \kappa A_{\kappa} S^{3}+\text { h.c. }\right),
$$

together with the $D$-term potential for $H_{u}$ and $H_{d}$. The resulting potential has two important global symmetry limits, both of which yield a light pseudoscalar when the global symmetry is spontaneously broken by the vacuum expectation values of the Higgs fields.

When $A_{\lambda}, A_{\kappa} \rightarrow 0$, the scalar potential has a global $U(1)_{R}$ symmetry where $S \rightarrow e^{i \alpha} S$, $H_{u} \rightarrow e^{i \alpha} H_{u}$, and $H_{d} \rightarrow e^{i \alpha} H_{d}$ [258-260]. In this limit, the interaction that controls the mixing between the imaginary part of $S$ with the $C P$-odd boson $A^{0}$ in the MSSM Higgs sector is

$$
\delta \mathcal{L}=-|\kappa|^{2}\left(S^{2} H_{u}^{*} H_{d}^{*}+\text { h.c. }\right)
$$

When instead the $S$ self-couplings vanish, $\kappa, A_{\kappa} \rightarrow 0$, the potential realizes a global $U(1)_{P Q}$ symmetry, $H_{u} \rightarrow e^{i \alpha} H_{u}, H_{d} \rightarrow e^{i \alpha} H_{d}$, and $S \rightarrow e^{-2 i \alpha} S$ [261-264]. Here the pseudoscalar mixing is controlled by the interaction

$$
\delta \mathcal{L}=-\lambda A_{\lambda}\left(S H_{u} H_{d}+\text { h.c. }\right)
$$

In both cases the light axion obtains a small mass from small explicit breaking of the global symmetry. The light axion that appears in the spectrum has couplings to SM states governed by the mixing angle between $\operatorname{Im} S$ and $A^{0}$,

$$
\tan \theta=\frac{v}{\sqrt{2} f_{a}} \sin 2 \beta \times\left\{\begin{array}{cl}
2 & \mathrm{PQ} \\
-1 & \mathrm{R}
\end{array}\right.
$$

Below we will discuss SHiP's prospects for discovering $R$-axions of the specific model introduced above (in Chapter 5 we discuss the more general case of axion-like particles). It is worth noting that in the $P Q$ limit, the mostly singlet-like scalar $s$ and singlino $\tilde{s}$ are also light [262, 265, 266], and could present additional discovery opportunities for SHiP.

Light pseudoscalars can be produced in meson decays, most notably in $B \rightarrow K^{(*)} a$. These $b \rightarrow s$ transitions are mediated by the penguin-like diagrams shown in the left panel of Fig. 3.13 [231, 267269]. The contribution of the charged Higgs loop to the full decay amplitude is important for obtaining a finite result, and consequently the $B \rightarrow K a$ branching ratio depends on the charged Higgs mass $m_{H^{+}}$as well as the couplings of $a$. The effective operator [267-269] that mediates the meson decay is

$$
\mathcal{O}=\frac{g^{3} V_{t s}^{*} V_{t b}}{128 \pi^{2}} \frac{m_{t}^{2}}{m_{W}^{3}}\left(X_{1} \cot \beta+X_{2} \cot ^{3} \beta\right) \partial_{\mu} a \bar{s} \gamma^{\mu} P_{L} b,
$$

where $X_{1}, X_{2}$ are functions of $m_{H+}, m_{t}$, and $m_{W}$. Here we estimate SHiP's sensitivity to $a$ 's produced in $B$ decays; for sufficiently light $a$ 's, additional sensitivity may be possible from analogous exotic $K$ decays. We generate $b \bar{b}$ events in MadGraph [243] and shower and hadronize them in Pythia. We use MadGraph's leading order result for the $b \bar{b}$ production cross-section at SHiP. Subsequently we extract the resulting $B^{0}, \bar{B}^{0}$, and $B^{ \pm}$mesons and decay them to $K^{(*)} a$ final states according to phase space. We take the branching ratio $\operatorname{Br}\left(B \rightarrow K^{(*)} a\right)$ to be the same for all $B_{d}$ mesons, and use hadronic form factors from [242]. We show $\operatorname{Br}(B \rightarrow K a)$ as a function of $m_{H+}$ and $f_{a}$ in Fig. 3.12, for fixed $m_{a}=300 \mathrm{MeV} ; \operatorname{Br}\left(B \rightarrow K^{*} a\right)$ is similar. Note that the branching fraction vanishes along a line in these plots, where the two terms in parentheses in Eq. (3.4.6) exactly cancel. Note also that the $a$ production cross-section decreases with increasing tan $\beta$, following the decreasing coupling of the $a$ to the top quark. 

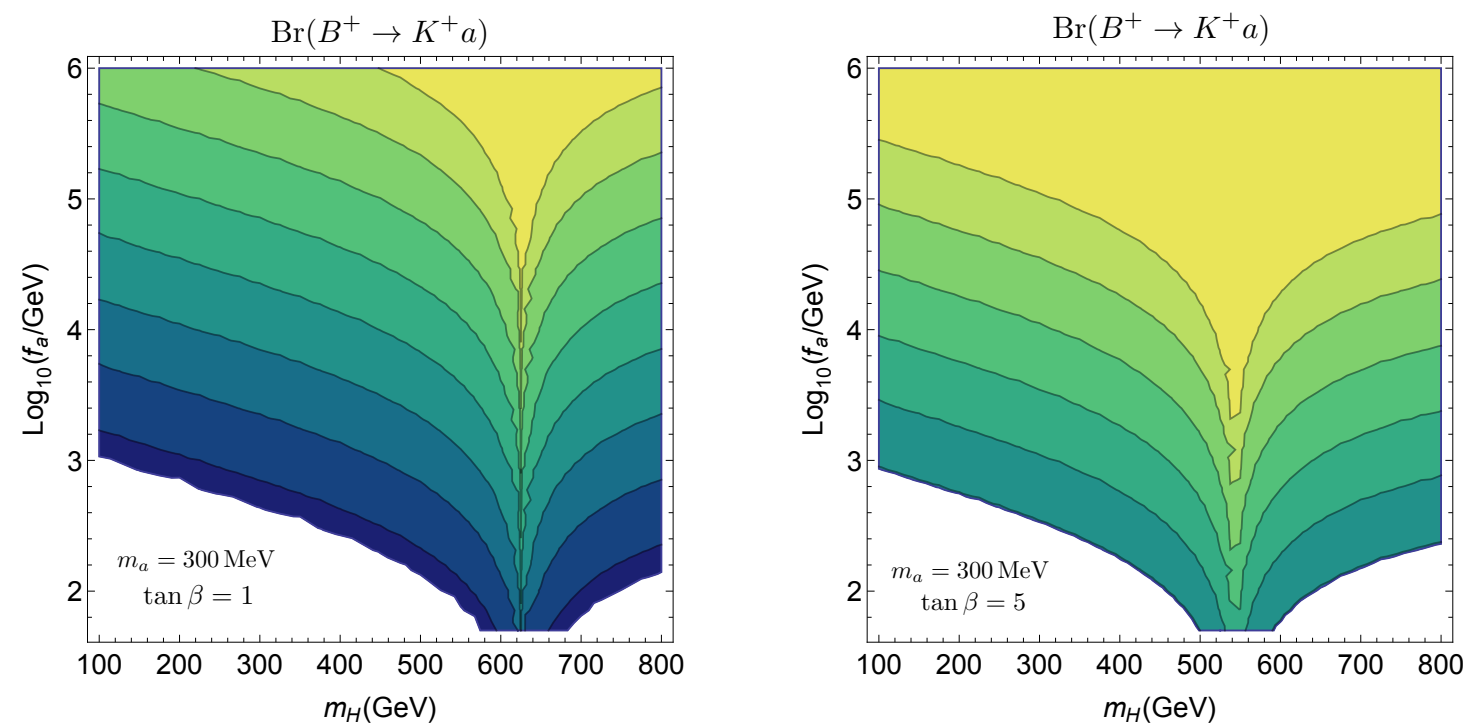

Figure 3.12: The branching ratio $\operatorname{Br}\left(B^{+} \rightarrow K^{+} a\right)$, for $m_{a}=300 \mathrm{MeV}$ and $\tan \beta=1$ (left) and $\tan \beta=5$ (right). Contours range from $10^{-13}$ (yellow) to $10^{-5}$ (dark blue) in logarithmic increments of $10^{-1}$.

We estimate the number of decays within the SHiP detector as follows: we take the SHiP detector to have a circular cross section $5 \mathrm{~m}$ in diameter starting at a distance of $60 \mathrm{~m}$ from the target, and estimate the geometric acceptance as the fraction of as with momentum at an angle $\theta<\theta_{\max }=2.5 / 60$ from the beam axis. The boost distribution of the surviving $a$ 's is then convolved with the probability of decaying within the SHiP detector, which is approximately

$$
P_{\text {decay }}\left(L_{1}, L_{2}\right)=\exp \left(-\frac{L_{1}}{b c \tau}\right)-\exp \left(-\frac{L_{2}}{b c \tau}\right)
$$

where $c \tau$ is the proper decay length, $b=\left|\vec{p}_{a}\right| / m_{a}=\beta_{a} \gamma_{a}, L_{1}=50 \mathrm{~m}$, and $L_{2}=110 \mathrm{~m}$. For the luminosity we assume $2 \times 10^{20}$ protons on a tungsten target with an effective target length of 1 meter.

We consider pseudo-scalars decaying to pairs of light leptons, for which the partial width is

$$
\Gamma(a \rightarrow \ell \ell)=m_{a} \frac{\sin ^{4} \beta}{16 \pi} \frac{m_{\ell}^{2}}{f_{a}^{2}} \sqrt{1-\frac{4 m_{\ell}^{2}}{m_{a}^{2}}} .
$$

For pseudoscalars with $m_{a} \gtrsim 400 \mathrm{MeV}$, the decay $a \rightarrow 3 \pi$ opens up, and becomes increasingly important at higher masses, along with other hadronic channels. For simplicity we consider leptonic decays only, and correspondingly restrict our attention to $m_{a}<500 \mathrm{MeV}$. Neglected hadronic decay channels will decrease the pseudoscalar lifetime at a given mass, which in turn will slightly increase the maximum value of $f_{a}$ that can be probed. We estimate SHiP's reach for axions by requiring at least three events where an axion decays within SHiP's detector. Our results are shown in the right panel of Fig. 3.13 for $m_{H+}=200 \mathrm{GeV}$. Increasing values of the charged Higgs mass will slightly decrease the upper boundary of the region of sensitivity, thanks to the corresponding decrease in $\operatorname{Br}(B \rightarrow K a)$ shown in Fig. 3.12. SHiP thus has sensitivity to a very large region within singlet parameter space, with reach extending to pseudoscalars much more weakly coupled to the Standard Model than those that can be tested at BaBar [270] or the LHC [271]. 

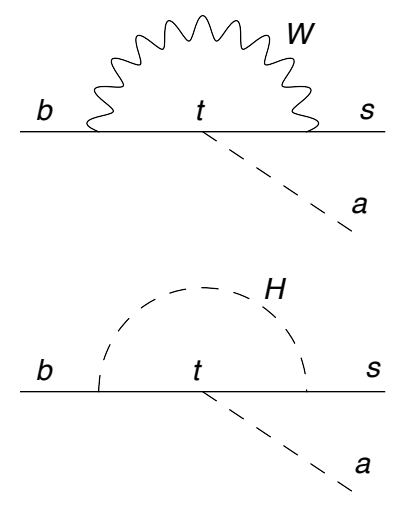

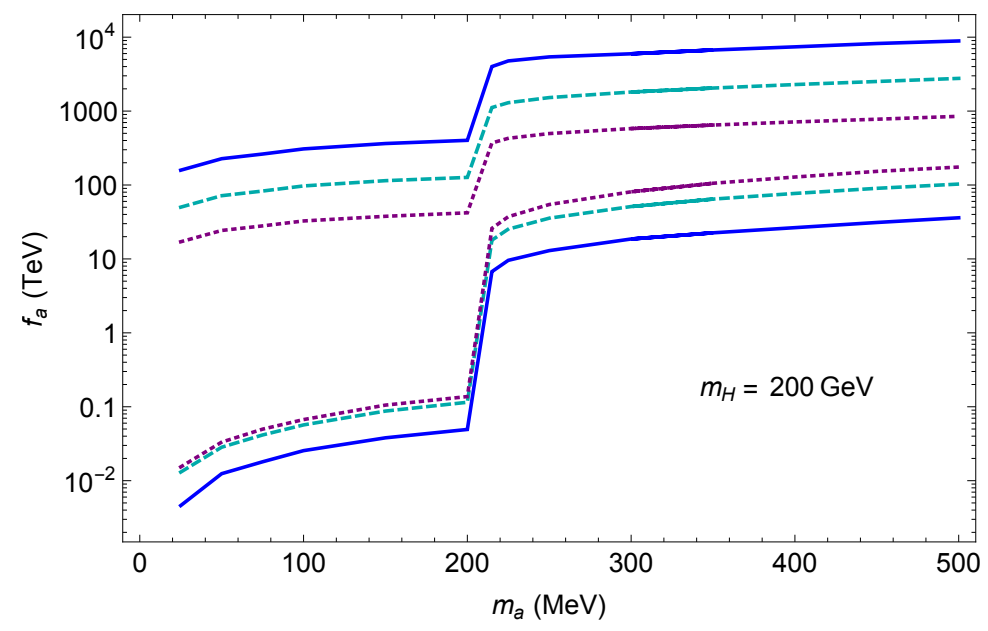

Figure 3.13: Left: Feynman diagrams governing the production of light pseudoscalars in $B$ decays. Right: Estimated SHiP sensitivity to pseudoscalars in the $m_{a}-f_{a}$ plane, for $\tan \beta=1$ (blue, solid), $\tan \beta=5$ (cyan, dashed), and $\tan \beta=15$ (purple, dotted). The charged Higgs mass has been fixed to $m_{H^{+}}=200 \mathrm{GeV}$. For each value of $\tan \beta$ the region between the two lines yields at least three events where pseudoscalars decay within the detector according to the estimated event yield described in the text.

\subsection{Scalar portals and Dark Matter}

Light scalars may certainly provide an interesting connection to the puzzle of dark matter (DM). It is quite conceivable that dark matter resides in the form of a SM singlet particle which is protected against decay by a (discrete) symmetry. In this case, a light scalar could mediate the interactions between DM and the SM (see Section 3.5.1) or even constitute the DM abundance itself if is a stable particle as a result of a discrete symmetry (see Section 3.5.2).

\subsubsection{Scalar as a mediator between DM and the SM}

The Lagrangian for the minimal model reads

$$
\mathcal{L}=\mathcal{L}_{\mathrm{SM}}-\frac{g_{\star} m_{f}}{v} S \bar{f} f-\frac{1}{2} \kappa S \bar{\chi} \chi,
$$

where $\chi$ denotes the dark matter particle which we assume to be a Majorana fermion, while $S$ stands for the mediator. The coupling of the scalar to SM fermions $f$ arises from mixing with the SM Higgs as discussed in Section 3.2.

In the hot early universe, the dark matter fermions are in thermal equilibrium due to their interactions with the SM bath. DM pairs can directly annihilate into SM particles via an intermediate scalar $S$. Alternatively, if kinematically allowed, they can annihilate into pairs of scalars, which subsequently decay to SM particles. As far as the experimental constraints on the scalar $S$ are concerned, only its interactions with the SM are relevant. Indeed, the coupling $g_{\star}$ is subject to strong bounds and, hence, processes involving this coupling must be suppressed. Therefore, we shall assume that the second class of processes dominates. The annihilation cross section for $\chi \chi \rightarrow S S$ can be estimated as [212]

$$
\sigma v_{\mathrm{rel}} \simeq \sigma_{1} v_{\mathrm{rel}}^{2}=\frac{\kappa^{4} m_{\chi}}{24 \pi} \sqrt{m_{\chi}^{2}-m_{S}^{2}} \frac{9 m_{\chi}^{4}-8 m_{\chi}^{2} m_{S}^{2}+2 m_{S}^{4}}{\left(2 m_{\chi}^{2}-m_{S}^{2}\right)^{4}} v_{\mathrm{rel}}^{2},
$$




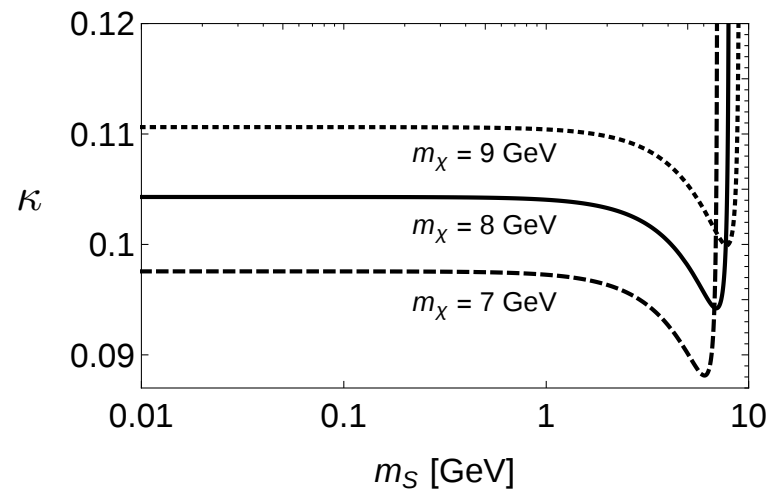

Figure 3.14: Relic density as a function of the coupling $\kappa$ and the mediator mass $m_{S}$ for $m_{\chi}=$ $7,8,9 \mathrm{GeV}$.

where $v_{\text {rel }}$ denotes the relative velocity between two dark matter particles. Notice that this process is p-wave suppressed due to the $\mathrm{CP}$ properties of the initial and final state particles. Imposing that the relic density of $\chi$ matches the observed dark matter density $\Omega_{\chi} h^{2}=0.1199$ [272], we can determine $\kappa$. More specifically for $p$-wave suppressed annihilations and $m_{\chi}=5-10 \mathrm{GeV}$ the DM relic density is achieved for $\sigma_{1} \simeq 1.6 \cdot 10^{-25} \mathrm{~cm}^{3} / \mathrm{s}$ ( see e.g. [273]). The coupling $\kappa$ which yields the correct relic density for a given mass $m_{S}$ is shown in Fig. 3.14. Unless there is a strong phase space suppression, the result only weakly depends on the dark matter mass. For $m_{\chi} \simeq 5-10 \mathrm{GeV}$, we find $\kappa \simeq 0.1$.

Direct dark matter detection experiments search for the coherent scattering of DM particles on nuclei. In the simple model, we presented, a sizeable (spin-independent) DM nucleon cross section $\sigma_{n}$ can arise via exchange of the scalar mediator. We find

$$
\sigma_{n} \simeq 10^{-40} \mathrm{~cm}^{2}\left(\frac{\kappa}{0.1}\right)^{2}\left(\frac{g_{\star}}{0.01}\right)^{2}\left(\frac{\mathrm{GeV}}{m_{S}}\right)^{4},
$$

which is valid for mediator masses larger than the momentum transfer in the scattering, i.e. $m_{S} \gtrsim$ $100 \mathrm{MeV}$ (somewhat depending on the target material). For smaller masses $1 / m_{S}^{4}$ has to be replaced by $1 /\left(q^{2}+m_{S}^{2}\right)^{2}$. Notice that the cross section is strongly enhanced for small mediator masses due to the $m_{S}^{-4}$ dependence and becomes constant for mediator masses smaller than the momentum transfer.

Imposing the correct dark matter relic density, we can directly relate the coupling $g_{\star}$ to the DM nucleon cross section. Hence, in this simplistic dark matter model, the most recent constraints on $\sigma_{n}$ from the LUX experiment can be translated into limits on $g_{\star}$. In Fig. 3.9, we have depicted contours of constant $\sigma_{n}$ as a function of the mediator mass. We assumed $\kappa=0.1$ as expected for $m_{\chi}=5-10 \mathrm{GeV}$ (see above). The LUX constraints depend strongly on the DM mass. The two contours of $\sigma_{n}=10^{-40} \mathrm{~cm}^{2}$ and $\sigma_{n}=10^{-44} \mathrm{~cm}^{2}$ shown in the figure correspond to the current LUX limit at $m_{\chi} \simeq 5 \mathrm{GeV}$ and $m_{\chi} \simeq 10 \mathrm{GeV}$ respectively.

Finally we note that pseudoscalars discussed in Chapter 5 can also act as messengers to the DM sector. Direct detection limits are weaker in this case.

\subsubsection{Scalar as a DM candidate}

We now want to speculate that perhaps the scalar $S$ constitutes the dark matter within the present day Universe, $S$ being a stable particle as a result of a $\mathcal{Z}_{2}$ symmetry in his interactions. We shall 


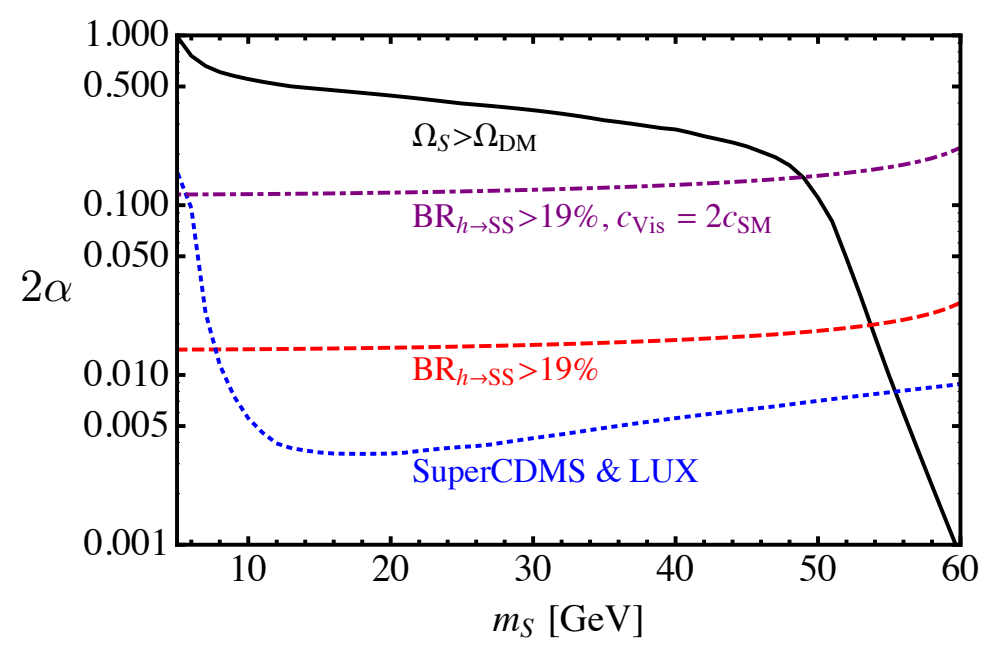

Figure 3.15: The Higgs portal coupling required to saturate the observed DM density is shown in black. The region below the black line overproduces dark matter, and thus to agree with cosmological observations an additional late-time dilution of the DM density is required, or $S$ must be made unstable by breaking the $Z_{2}$ stabilization symmetry. The strongest direct detection constraints from LUX [275] and SuperCDMS [276] are also shown. The LHC constraints on additional Higgs decays $h \rightarrow S S$ are shown in dashed red. Any region above this line is in tension with the LHC Higgs observations. The Higgs constraints may be weakened somewhat if it is assumed that Higgs couplings to SM states are increased, to counteract the reduction in signal from invisible decays. The dot-dashed purple line shows the result of assume a doubling of SM Higgs couplings.

now consider this possibility in more detail and follow closely the recent review [274]. The scalar portal Lagrangian that we consider reduces to

$$
\mathcal{L} \supset \alpha S^{2}|H|^{2}
$$

In the early Universe the scalar $S$ will be in thermal equilibrium with SM particles due to the Higgs portal coupling. This coupling enables $S$-annihilations to occur through an s-channel Higgs into SM states such as light fermions, $S S \rightarrow h^{*} \rightarrow \bar{f} f$, vector bosons $S S \rightarrow h^{*} \rightarrow V V$, and Higgs pairs $S S \rightarrow h h$. As the Universe cools and expands these annihilations become less frequent and eventually 'freeze-out', such that the co-moving density of dark matter remains constant. Taking these effects into account the model becomes very predictive and it is possible to calculate the relic density of dark matter. The Higgs portal coupling required to generate the observed dark matter density is shown in Fig. 3.15. Unfortunately there is no simple formula for the relic abundance in the region near the Higgs pole $\left(m_{S}>50 \mathrm{GeV}\right.$ in Fig. 3.15) or in the region where certain annihilations occur close to a mass threshold, such as around $m_{S} \sim 8 \mathrm{GeV}$ for annihilation into bottom quarks. However in the intermediate region $10 \lesssim m_{S} \lesssim 50 \mathrm{GeV}$ the relic abundance scales approximately as the inverse of the squared coupling, i.e. $\Omega h^{2}(30 \mathrm{GeV}) \sim 0.11\left(\frac{0.18}{\alpha}\right)^{2}$.

This dark matter could be detected in Direct Detection experiments by searching for dark matter scattering on nuclei, which in this case occurs through a t-channel Higgs boson. The cross section for Higgs portal dark matter with spin-independent scattering on a nucleon of mass $m_{n}$ is $[274]$

$$
\sigma_{S I}=\frac{\alpha^{2} f_{N}^{2}}{\pi} \frac{\mu^{2} m_{n}^{2}}{m_{h}^{2} m_{S}^{2}}
$$


where $\mu$ is the reduced mass of the nucleon and dark matter and $f_{N} \approx 0.29$ [277] is the Higgsnucleon coupling. In Fig. 3.15 we plot constraints from the LUX experiment [275] and also at the very low mass range from a dedicated SuperCDMS analysis [276]. The plotted constraints assume the DM abundance is equal to the observed abundance $\left(\Omega_{D M} h^{2}=0.11\right)$. In the excluded region the model actually leads to a DM abundance which is too large, thus the exclusions are conservative in assuming that the dark matter abundance has been diluted after freeze-out to realize the observed abundance. If the model is taken at face value, without assuming some dilution, then regions of parameter space below $m_{S} \lesssim 56 \mathrm{GeV}$ which are excluded by direct detection are also excluded by cosmological observations. It is clear that these constraints are in significant tension with a Higgs portal dark matter candidate.

There are additional constraints from the LHC. If $2 m_{S}<m_{h}$ then the Higgs boson may decay into the neutral scalar with a decay width given by Eq. (3.3.11) with $g_{h} X X=\alpha$. By comparison, the decay width of the Higgs boson is predicted to be $\Gamma_{h}=4.07 \mathrm{MeV}$ in the SM. If it is assumed that the Higgs couplings to all SM states are precisely as predicted in the SM then LHC observations of the Higgs boson already place strong bounds on the invisible decay width of the Higgs boson, placing the branching ratio at $\mathrm{BR}_{S}<19 \%$ [278]. This then translates into a bound on the parameter space, as shown in Fig. 3.15 .

As can be seen from Fig. 3.15 these constraints already rule out the simplest thermal Higgs portal dark matter candidate of mass $m_{S} \lesssim 55 \mathrm{GeV}$. However this does not imply that the Higgs portal is not relevant for dark matter, and simple modifications of the model may allow for a wide variety of dark matter models. A comprehensive review of the fixed target phenomenology is given in $[157,230]$.

Here we will consider a model based on the dark matter 'Freeze-In' mechanism [279]. It may be that the $\mathcal{Z}_{2}$ symmetry which stabilizes the scalar $S$ is broken by some small amount through operators that couple the scalar to the Higgs and also potentially to other stable neutral dark matter candidates $\psi$. The relevant Lagrangian is now

$$
\mathcal{L} \supset \alpha_{1} S|H|^{2}+\alpha S^{2}|H|^{2}+\kappa S \bar{\psi} \psi .
$$

As the couplings $\alpha_{1}$ and $\kappa$ break the $\mathcal{Z}_{2}$ symmetry they can be technically very small, with $\alpha_{1} \ll m_{h}$ while the quartic coupling respects the $\mathcal{Z}_{2}$ symmetry and can still be $\mathcal{O}\left(10^{-2}\right)$ while remaining consistent with known bounds. If the re-heat temperature of the Universe after inflation is above the weak scale then the $\mathcal{Z}_{2}$-symmetric scalar quartic coupling will maintain thermal equilibrium between the Standard Model particles and the scalar $S$. However, if the Yukawa coupling $\kappa$ between the scalar and the dark matter candidate $\psi$ is very small, $\kappa \sim 10^{-12}$, then in the early Universe $\psi$ is out of equilibrium with $S$ and all other fields. Interestingly this class of model realizes the so-called 'Freeze-In' mechanism and the infrequent production of $\psi$ in the early Universe from its feeble coupling to $S$ can realize the observed dark matter abundance for $\psi$.

As discussed in the previous sections, once produced the scalar may also decay into Standard Model states due to the trilinear scalar coupling $\alpha_{1}$. This model motivates $\alpha_{1}$ to be small thus it is likely that the dominant production mechanism would be pair production due to the quartic coupling followed by decay due to $\alpha_{1}$. The phenomenology of a light scalar with a small mixing with the Higgs has been discussed in Sections 3.2 and 3.3 and the phenomenology of this model would be similar to these cases with the exception that if $m_{\psi}<m_{S} / 2$ there may also be decays to the new dark matter candidates $S \rightarrow \bar{\psi} \psi$. These additional decay channels may suppress the observable decays after the scalar has been produced. Throughout we will assume $\kappa \sim 10^{-12}$ such that these decays are greatly subdominant, and the full decay width is given approximately by

$$
\Gamma_{s}\left(m_{S}\right)=g_{\star}^{2} \Gamma_{h}\left(m_{S}\right),
$$


where $\Gamma_{h}\left(m_{S}\right)$ is the decay width of the SM Higgs boson at mass $m_{S}$, and $g_{\star} \simeq \alpha_{1} v / m_{h}^{2}$ is the small mixing angle between the Higgs boson and the scalar $S$, see Eq. (3.2.3).

Thus we see that this model uses the Higgs portal coupling in order to realize a model of 'Freeze-In' dark matter and motivates a scalar which is long-lived due to small breaking of a $\mathcal{Z}_{2}$ symmetry. In this case, as $S$ is unstable and does not constitute the dark matter, the constraints on $S$ from the thermal abundance and direct detection limits shown in Fig. 3.15 no longer apply, and only the invisible Higgs width constraint is important

\subsection{Dark pions}

Composite dark matter $(\mathrm{DM})$ models are a viable alternative to the WIMP paradigm. A popular class of models (see e.g. [280-282]) has a dark sector that is QCD like, with stable baryonic resonances as DM candidates which carry a conserved DM number, see Section 3.1.2. In those models the lightest bound states are often the pseudo-Goldstone bosons of chiral symmetry breaking in the dark sector, so called dark pions. These dark pions are not necessarily stable, since they do not carry DM charge. Instead they may decay back to SM particles in a variety of ways, depending on how the dark sector communicates with the SM.

\subsubsection{The model and scales}

For definiteness, consider an extension of the SM gauge group to $G=G_{\mathrm{SM}} \times S U\left(N_{d}\right)$. For simplicity we will restrict ourselves to $N_{d}=3$, although this is of limited importance in the following. Furthermore we introduce $N_{f D}$ dark quarks $q_{D}$ which are fundamentals of $S U\left(N_{d}\right)$ and neutral with respect to $G_{\mathrm{SM}}$. For $N_{f D}$ small enough, the $S U\left(N_{d}\right)$ gauge theory will be confining in the infrared, at a scale $\Lambda_{D}$. Motivated by the idea of asymmetric DM [283, 284], we take $\Lambda_{D}$ to be in the few to $10 \mathrm{~s}$ of $\mathrm{GeV}$ range. ${ }^{8}$

We assume that the dark sector communicates with the SM only through mediators $X$ with masses $M_{X} \gg \Lambda_{D}$, in the spirit of the Hidden Valley models [193] described in Section 3.1.2. Absence of direct and indirect evidence for new physics also suggests $M_{X} \gg v$, where $v=246 \mathrm{GeV}$ is the weak scale. The two kinds of mediators we will consider in the following are A) a $Z^{\prime}$ that couples to dark quarks and some combination of SM fermions with a coupling strength $g^{\prime}$ and B) a bi-fundamental scalar $\Phi$ which carries QCD and $S U\left(N_{d}\right)$ charges, and which has Yukawa couplings of the form $y_{i j} \Phi \bar{q}_{D, i} q_{\mathrm{SM}, \mathrm{j}}$.

\subsubsection{Dark pion lifetime and decay modes}

Integrating out the mediator, we obtain dimension six couplings between the dark quarks and SM fermions:

$$
\mathcal{L}_{\text {eff }}=\frac{c_{\text {eff }}^{2}}{M_{X}^{2}}\left(\bar{q}_{D} \Gamma q_{D}\right)\left(\bar{f}_{\mathrm{SM}} \Gamma f_{\mathrm{SM}}\right),
$$

with some Dirac structure $\Gamma$. This allows a decay of dark pions into SM fermion pairs. For $\Gamma=\gamma_{\mu}$, the decay width goes as [282, 285]

$$
\Gamma\left(\pi_{D} \rightarrow \bar{f} f\right)=\frac{c_{\mathrm{eff}}^{4} g_{f} f_{\pi D}^{2} m_{f}^{2}}{32 \pi M_{X}^{4}} m_{\pi D},
$$

\footnotetext{
${ }^{8}$ For models that predict this, see e.g. [282].
} 
where $f_{\pi D}$ and $m_{\pi D}$ are the dark pion decay constant and mass, $g_{f}$ is the number of degrees of freedom of $f$, and we neglect a phase space factor. This corresponds to a lifetime $c \tau_{0}$ of

$$
c \tau_{0}=2 \mathrm{~m} \times \frac{1}{c_{\mathrm{eff}}^{4} g_{f}} \times \frac{(\mathrm{GeV})^{3}}{f_{\pi D}^{2} m_{\pi D}} \times \frac{(0.1 \mathrm{GeV})^{2}}{m_{f}^{2}} \times \frac{M_{X}^{4}}{(\mathrm{TeV})^{4}} .
$$

In analogy with QCD we will consider $m_{\pi D} \sim f_{\pi D} \sim \Lambda_{D} /(4 \pi)$. The dominant decay channels depend on the flavour structure of the couplings, and on the dark pion masses. In the case where on-shell decays to pairs of SM fermions are not kinematically accessible (e.g. if there are no couplings to leptons), the dominant decay can be into photon pairs, in analogy with neutral pion decays in the SM. In any case there will be a region of parameter space where the dark pion lifetime is of order meters or longer, such that their decays could be observed in the SHIP detector.

\subsubsection{What SHiP could do?}

There are two contributions to dark pion production at SHIP. First, the effective operator (3.6.1) leads to direct production of dark quark pairs, provided there is a nonzero coupling to first or second generation SM quarks. The dark quarks will shower and hadronize, producing several dark pions in the process. The production cross section can be calculated easily, the dark parton shower can be simulated using a modified version of PYTHIA 8 [285, 286].

In addition, dark pions can also be produced from decays of SM mesons, provided they are sufficiently light. If sufficient phase space is available, multiple dark pions may be produced. The two production modes probe different combinations of the couplings of the dark quarks to SM quarks.

There are several regions of parameter space where dark pion lifetimes are such that they will reach the SHIP detector before decaying. Possible decay modes include decays to di-photons, to SM hadrons and also to pairs of SM leptons. Therefore the SHIP experiment will be able to place limits on dark pion production cross sections as a function of the lifetime.

On the theory side, it will be necessary to better understand the allowed parameter space, in particular taking into account constraints from flavour physics. This will allow to place bounds on specific models of dark pions and on the mediator scale. Furthermore simulations of the production of dark pions will be needed to obtain their kinematic distributions, which affect detector efficiencies and the laboratory lifetime.

\subsection{Scalar portals and inflation}

In this section we are going to give an example of a portal model, based on the linear scalar portal of Section 3.2, that provides a connection of the inflationary physics and particle physics, leading to a light inflaton particle whose cosmologically interesting parameter region can be constrained by the SHIP experiment.

\subsubsection{Light inflatons}

The visible part of our expanding Universe seems to be spatially flat, homogeneous and isotropic. These facts are quite puzzling given the history of the Universe, where radiation and matter domination stages play a very important role. At these stages of expansion the scale factor $a(t)$ grows as $a(t) \propto t^{\alpha}$ with $\alpha<1$, and hence the physical distances $l \propto a(t)$ grow slower than the size of the causally connected region (dubbed cosmological horizon), $l_{H} \propto t$. Inside a volume of size $l_{H}$ the physical processes can be correlated (e.g. recombination happens simultaneously everywhere in this region), but separate horizon volumes are causally disconnected, starting from the moment of Big Bang. This implies, in particular, that the plasma temperatures can be expected to differ 
significantly in these volumes, while, in reality, in the visible Universe it is the same with per mille accuracy. There are other illustrations of the problems we have started with, disguised under the names of flatness, entropy, initial perturbations problems, see e.g. [287] for detailed discussion.

The most widely accepted solution is an early-time inflationary stage preceding the hot Universe. At that stage the Universe experiences almost exponential (or at least accelerated) expansion, which makes it flat, homogeneous and isotropic. Usually a scalar field (dubbed inflaton) is present in the model, whose dynamics is responsible for the rapid expansion. Quite remarkably, the amplitude of quantum fluctuations of this field (or one of the possibly accompanying scalar fields) gets frozen as the wavelength stretches over horizon. Thus, when the inflation finally terminates, they form classical gaussian fluctuations of the scalar field, which later (after the Universe's reheating, etc.) source the matter spatial inhomogeneities and scalar curvature perturbations. With properly chosen model parameters, these inhomogeneities nicely describe the cosmic microwave background radiation (CMB) anisotropy and serve as seeds of the large scale structure formation in the late Universe. The parameters of these perturbations can be deduced from CMB observations [288].

An implementation of the inflationary stage requires some modification of the SM and/or General Relativity. If the new scalar degree of freedom $S$ needed to drive inflation is introduced into the particle physics sector, it must couple to the SM fields to assure the post-inflationary reheating of the Universe (i.e. production of the SM particles in the early Universe, which subsequently thermalize) and successful transfer of the inflaton fluctuations into adiabatic matter perturbations. It is natural to expect that the inflaton and the Higgs boson will interact via the renormalizable scalar portal operator

$$
\mathcal{L}_{\text {int }}=\alpha S^{2} H^{\dagger} H
$$

The dimensionless parameter $\alpha$ is bounded from above and from below [289]. It cannot be very small, otherwise the reheating is inefficient, i.e. the reheating temperature $T_{\text {reh }}$ is too small. The widely recognized limit for the reheating is about several $\mathrm{MeV}$ is related to the onset of Big Bang (primordial) Nucleosynthesis. Yet many models would require having $T_{\text {reh }}$ above the electroweak scale, to obtain dark matter abundance and baryogenesis (e.g., for the model (3.7.2) this implies $\alpha \gtrsim 0.7 \times 10^{-11}[289]$ ). Further, $\alpha$ cannot be large, (say $\alpha \lesssim \sqrt{0.1 \beta} \sim 10^{-7}$ [222], for the particular model (3.7.2)) otherwise the quantum corrections, originating from (3.7.1), change the inflaton potential in such a way that the primordial density perturbations generated at inflation become large and strongly scale dependent, what is incompatible with observations.

The upper and lower limits on $\alpha$ are rather general and relevant for any inflationary model. If only a renormalizable model is accepted, then only quadratic, cubic and quartic self-couplings are allowed in the inflaton potential. The former two contain dimensionful parameters with reference energy scale (determined by normalizing the perturbation spectrum to CMB anisotropy) much above the electromagnetic scale, which would contribute to the gauge hierarchy problem, lifting up the SM Higgs boson mass (for discussion see e.g. [290, 291]). It seems preferable to avoid introducing a new high energy scale in particle physics, and hence no new heavy degrees of freedom. Therefore, models with light inflaton are justified, and below we consider a minimal example of this type.

The Lagrangian of the model is a generalization of the scalar portal model considered in Section 3.2 with an explicit non-minimal interaction of the scalar field $S$ to gravity

$$
\mathcal{L}_{S \mathrm{SM}}=\mathcal{L}_{\mathrm{SM}}+\frac{\left(\partial_{\mu} S\right)^{2}}{2}+\frac{m_{S}^{2} S^{2}}{2}-\frac{\beta S^{4}}{4}-\lambda\left(H^{\dagger} H-\frac{\alpha}{\lambda} S^{2}\right)^{2}-\frac{M_{P}^{2}+\xi S^{2}}{2} R,
$$

where the first term is the SM Lagrangian except of the Brout-Englert-Higgs (BEH) field potential, $R$ is the scalar curvature, $M_{P}$ is the reduced Planck mass, and the dimensionless parameters taking positive values are $\lambda, \beta, \alpha, \xi$, for the Higgs boson selfcoupling, inflaton selfcoupling, Higgs-inflaton portal, and the inflaton non-minimal coupling to gravity, respectively. The last term is required by 
renormalization of scalar field in the external gravitational background (of course, gravity itself is still non-renormalizable). Similar term may be written for the BEH as well, we ignore it because, while in perturbative regime, it changes neither inflation ${ }^{9}$, nor low-energy phenomenology of the model.

Only one dimensionful parameter, $m_{S}$, is introduced in the particle physics part of (3.7.2). It determines the mass scale of all the particles, and therefore it should be approximately of the order of the electroweak scale. This assumption is made for simplification of the theory and has roots in the attempts to have a scale invariant fundamental theory (for example, one can consider the inflaton as a messenger of scale symmetry breaking from some hidden sector). Indeed, while at (trans)planckian values of the fields the inflaton self-interaction leads to the inflationary stage ${ }^{10}$. The primordial perturbations depend on the values of $\beta$ and $\xi$. The requirement of the normalization of the scalar density perturbations defines $\beta$ approximately as ${ }^{11}$

$$
\beta=\frac{3 \pi^{2} \Delta_{\mathcal{R}}^{2}}{2} \frac{(1+6 \xi)(1+6 \xi+8(N+1) \xi)^{2}}{(1+8(N+1) \xi)(N+1)^{3}},
$$

see Fig. 3.16. Then the observed inflationary parameters can be parametrised by $\xi$ [222]. These predictions are given in Fig. 3.16 together with the latest observational constraints [288, 296]. Note, that if measurement of $r$ is made, then both parameters $\xi$ and $\beta$ become defined uniquely.

At small field values, the inflaton field gains non-zero vacuum expectation value $\langle S\rangle=m_{S} / \sqrt{\beta}$ that spontaneously breaks the $\mathcal{Z}_{2}$ symmetry $S \rightarrow-S$, and in turn generates a vacuum expectation value for the SM Higgs [221],

$$
v=\langle S\rangle \sqrt{\frac{2 \alpha}{\lambda}}=m_{S} \sqrt{\frac{2 \alpha}{\lambda \beta}} \approx 246 \mathrm{GeV} .
$$

Two scalar excitations about the vacuum have masses

$$
m_{h}=\sqrt{2 \lambda} v \approx 126 \mathrm{GeV} \text { and } m_{\chi}=m_{h} \sqrt{\frac{\beta}{2 \alpha}} .
$$

Since $\beta$ is determined by cosmology, the inflaton mass is defined by mixing $\alpha$ responsible for postinflationary reheating of the Universe. This coupling is bounded from above and below as explained right after Eq. (3.7.2), that yields upper and lower limits on the inflaton mass. It happens to be naturally light, say for $\xi=0$ it is confined between about $270 \mathrm{MeV}$ and $1.8 \mathrm{GeV}$ [221].

As already explained in Section 3.2, the vev of $S$ induces, after electroweak symmetry breaking, a linear mixing between the Higgs boson and the inflaton. In other words the massive states $h$ and $\chi$ with masses $m_{h}$ and $m_{\chi}$ are slightly rotated as compared to the "flavor" states $H$ and $S$. This mixing defines the phenomenology of light inflaton $\chi$, which can be produced in scattering of the SM particles and can decay into SM particles, as presented in detail in Section 3.2. The lower bound on inflaton mass $m_{\chi} \gtrsim 270 \mathrm{MeV}$ comes from the analysis [221] of published data of the CHARM experiment [234], where the inflaton might be produced in kaon decays after the beam target. For a heavier inflaton the best production mechanism is 2-body decay of the B-mesons with branching

\footnotetext{
${ }^{9}$ At large non-minimal coupling for the Higgs field, $\xi_{H} \gg 1$, the BEH field can lead to the inflationary stage on its own, the mechanism is called Higgs inflation [292]. See also [293-295] for analysis of the models with several fields with large non-minimal coupling.

${ }^{10}$ The inflation develops along the valley $\lambda H^{\dagger} H=\alpha S^{2}$, so the term in brackets in (3.7.2) is nullified and contributes nothing to the energy density at inflation.

${ }^{11}$ this equation has a typo in [222].
} 

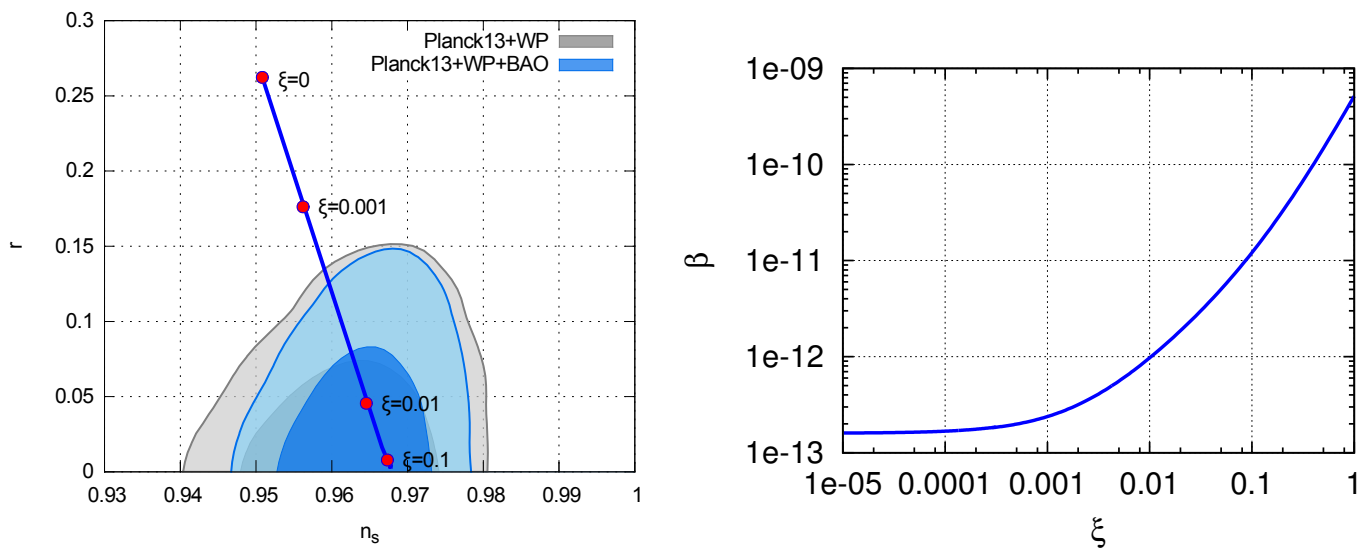

Figure 3.16: Left panel: Cosmologically allowed regions (68\% and $95 \%$ confidence level) in (scalar tilt $n_{s}-1$, tensor-to-scalar power ratio $r$ ) parameter space [288], and the light inflaton model predictions for various values of $\xi$. Right panel: Relation between the $\xi$ and $\beta$ parameters determined from the observed normalization of the primordial density perturbations. Parameter $\beta$ is in turn related to the Higgs-inflaton mixing. Reproduced from Ref. [222].

ratios $[221,222]$

$$
\operatorname{Br}(B \rightarrow \chi K) \simeq 4.8 \times 10^{-6} \times\left(1-\frac{m_{\chi}^{2}}{m_{b}^{2}}\right)^{2}\left(\frac{\theta^{2}}{10^{-6}}\right)
$$

Thus light inflaton can be searched for at $B$-factories, like LHCb and Belle II.

The relevant region of the model parameter space is presented in Fig. 3.17. Following the
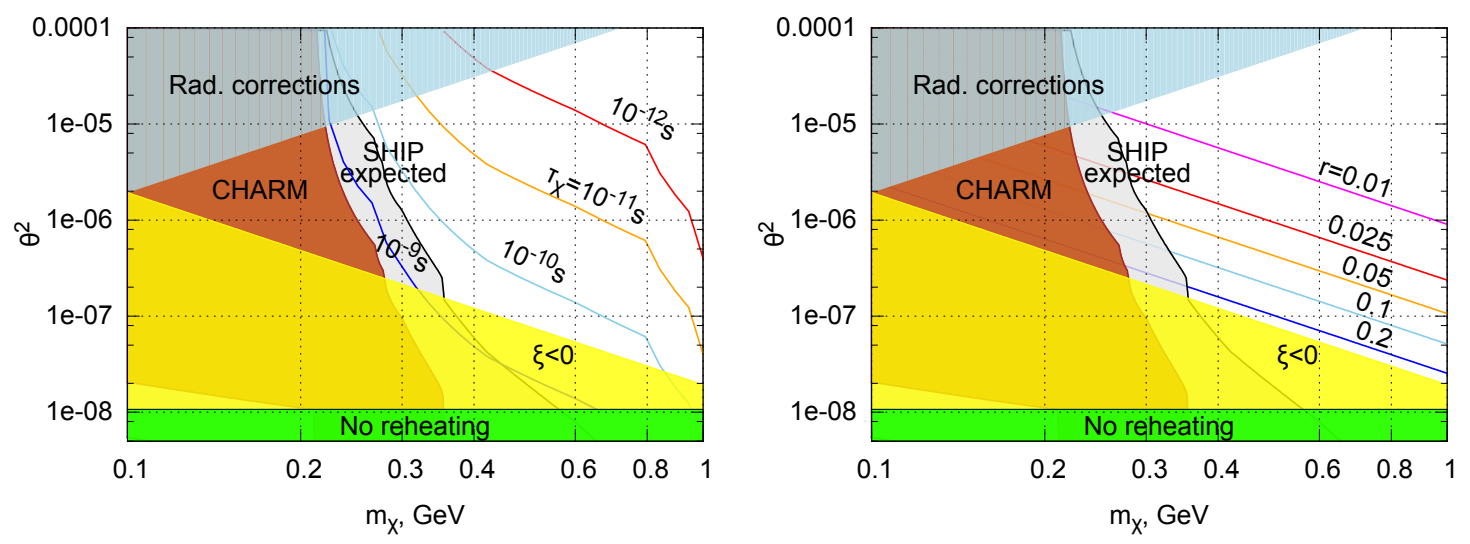

Figure 3.17: Allowed (blank) and forbidden (shaded) regions of model parameter space. The gray shaded region is the estimate of the SHIP sensitivity. Left plot: lines of constant inflaton lifetime are superimposed. Right plot: lines of constant tensor-to-scalar ratio $r$ are superimposed.

discussion of Section 3.2, it is clear that, for a fixed-target experiment like SHiP, the interesting region of the model parameter space is that where the Higgs-inflaton mixing $\theta$ is not very small (so that the production rate is sizable), and the decay length is not very short (which in turn requires that the mass is not too large). So, thought the latter is (much) smaller than the distance from the target to the detector, $\sim 100 \mathrm{~m}$, the inflaton production rate is sufficiently large to compete with the exponential suppression of the inflaton flux in the detector. The detailed description of 
the bounds indicated in Fig. 3.17 can be found in [221, 222]. The estimate of the SHIP sensitivity is performed similar to the analysis of the CHARM in [221], but with the distance to the detector equal to $50 \mathrm{~m}$. Note, that the main constraint in this region comes from the decay exponent, so change in the experiment luminosity introduces only minor improvement.

The model (3.7.2) may be supplemented with three sterile neutrinos $N_{I}, I=1,2,3$ of type I seesaw model, see Section 4.3.1, so that the inflaton coupling provides the mechanism of their production in the early Universe and the inflaton vev gives them violating lepton number Majorana masses. In particular, for the $\nu$ MSM discussed in Section 4.8, the inflaton can help to populate the Universe with dark matter particles [119], which are the lightest of the three sterile neutrinos $N_{I}$. Remarkably, this mechanism is consistent with an explanation of the anomalous $3.5 \mathrm{keV}$ line observed by X-ray orbital telescopes [297, 298] as a radiative decay of $7 \mathrm{keV}$ sterile neutrino dark matter [299]. It is worth noting, that in case the DM line is confirmed and tensor-to-scalar ratio $r$ is defined by CMB observations, all the parameters in the model (3.7.2) become fixed from cosmology, and definite predictions for the particle physics search can be made.

Finally, we should note that the assumption of the specific pattern of the scale symmetry breaking, made in (3.7.2), may be modified by introducing the Higgs mass term $H^{\dagger} H$, inflaton cubic selfcoupling $S^{3}$, and inflaton-Higgs coupling $S H^{\dagger} H$. In this case wider region of parameters $m_{\chi}$ and $\theta$ may be allowed from cosmological requirements. One still expects that the masses of the particles would be in the electroweak region, the inflaton decay pattern will coincide with that of the Higgs boson, and the Higgs-inflaton mixing angle will be of the similar order, however the exact relation with cosmology would depend on the three new parameters. These possibilities may be addressed by future studies of scale invariant extensions of the SM. 


\section{Chapter 4}

\section{Neutrino portal}

\subsection{Heavy neutral leptons}

The neutrino portal is defined as coupling of one or several fermions neutral with respect to the Standard Model gauge interactions $N_{I}(I=1,2, \ldots \mathcal{N})$ to the gauge-invariant operator $\left(\bar{L}_{\alpha} \cdot \tilde{\Phi}\right)$ :

$$
\mathcal{L}_{\text {Neutrino portal }}=F_{\alpha I}\left(\bar{L}_{\alpha} \cdot \tilde{\Phi}\right) N_{I}+\text { h.c. }
$$

where $F_{\alpha I}$ is a dimensionless Yukawa coupling (complex in general), $L_{\alpha}$ is the left lepton doublet ( $\alpha$ is the flavour index $\alpha=\{e, \mu, \tau\}$ ); $\Phi$ is the Higgs doublet and $\tilde{\Phi}_{a}=\epsilon_{a b} \Phi_{b}$. Only the right-chiral components of the fermions $N_{I}$ couples to the Standard Model sector. ${ }^{1}$

In the Higgs phase $\Phi$ can be replaced by its vev and therefore $\tilde{\Phi}=\frac{1}{\sqrt{2}}\left(\begin{array}{l}v \\ 0\end{array}\right)$, the term (4.1.1) leads to the quadratic mixing of neutrinos with the new particles $N_{I}$. From a phenomenological point of view, particles $N_{I}$ can be produced instead of neutrinos in any final state where kinematically allowed (see Fig. 4.2 below) and therefore they are often called heavy (right-handed) neutrinos or sterile neutrinos (owning to their lack of the SM charges as opposed to ordinary or active neutrinos) or gauge-singlet fermions or heavy neutral leptons (HNLs). If $\mathcal{N}=3$ each SM neutrino gets its right-chiral counterpart as all other fermions of the Standard Model have and the three generations structure of the SM is retained, see Fig. 4.1. The fermions $N_{I}$ can have any mass $M_{I}$, independent of the value of the Yukawa constants $F_{\alpha I}$ in (4.1.1) (see below and in the Section 4.3 for the discussion of possible origins of neutrino masses).

If the masses $M_{I}$ are sufficiently light, they can be searched in accelerator experiments (as discussed below in Sec. 4.4, see also [313] and "Heavy Neutral Leptons" section of PDG [156]). The existing searches use a model-independent phenomenological approach, assuming that only a single HNL is kinematically accessible, while any other HNLs, if present in the theory, are sufficiently heavy and do not affect the analysis. Thus, there are only two free parameters to be constrained in this approach: the mass $M_{I}$ of the relevant HNL and its interaction with an active neutrino of flavour $\alpha$ governed by the Yukawa coupling $F_{\alpha I}$. In the absence of a signal, upper limits are set on the mixing parameter $\left|F_{\alpha I}\right|^{2}$ as a function of the HNL mass $M_{I}$ for a given flavor $\alpha$, usually

\footnotetext{
${ }^{1}$ In this chapter we follow the logic based on a model-independent portal interaction (4.1.1) in the bottom-up fashion, starting from the simplest phenomenology of HNLs and then discussing the possible roles HNLs can play in different models. Historically, the motivation for considering right-handed neutrinos was coming from ideas on leftright symmetry, spontaneous parity violation and grand unification [300-309]. The chiral electroweak group $S U(2)_{L} \times$ $U(1)$ was proposed to be extended to the left-right symmetric one $S U(2)_{L} \times S U(2)_{R} \times U(1)_{B-L}$, necessitating to complement the fermionic content of the theory by N's. The smallness of active neutrino masses was a result of what is currently known as the see-saw mechanism [304-309]: the heavy Majorana N's lead to light $\nu$. A great bit of phenomenology of HNL's, was studied already in 1980s in Refs. [310-312].
} 


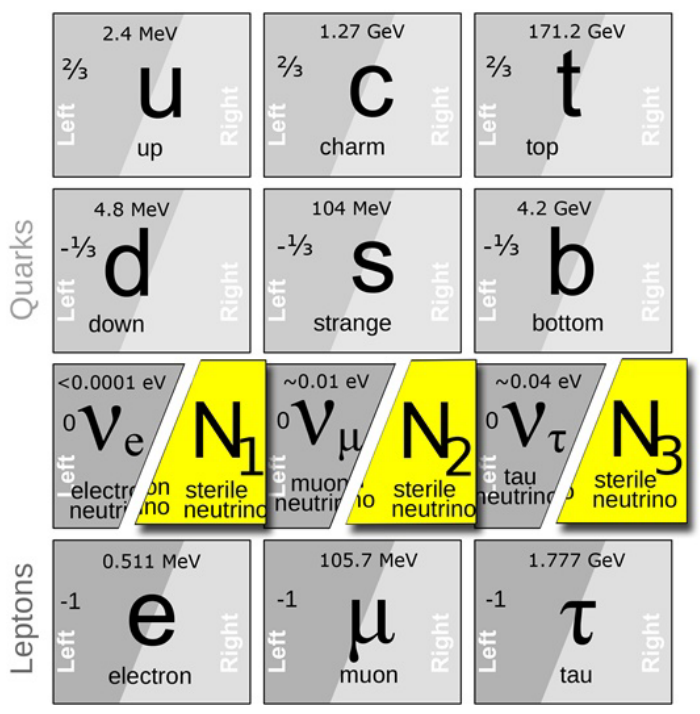

Figure 4.1: The fermions of the Standard Model are replicated in three "generations". There are 3 types of neutrinos: $\nu_{e}, \nu_{\mu}, \nu_{\tau}$. In the Standard Model these neutrinos are massless and always left-chiral. The right-chiral counterparts $N_{1}, N_{2}, N_{3}$ are added. They do not feel the electric, weak or strong forces (thus "sterile neutrinos").

assuming that the mixing elements for other flavours are zero. Although this assumption may not be valid in concrete models, it enables us to derive generic bounds on the mixing parameter without referring to any particular texture or model-building aspects. These bounds have to be translated or scaled appropriately in the context of a given model.

Apart from direct experimental probes, one can also search for HNLs indirectly, e.g. via their contributions to the lepton flavour violating processes (see Sec. 4.5.3).

The right-handed $N_{I}$ can be combined with the left handed $\nu_{\alpha}$ to form the 4-component Dirac spinors. The term (4.1.1) then becomes the Dirac mass term. If the $N_{I}$ transform under global U(1) symmetry, corresponding to the lepton number of the Standard Model, the Dirac mass term preserves this symmetry and does not prevent to build an extension of the SM where the lepton number is conserved.

Alternatively, since $N_{I}$ carry no Standard Model gauge charges, one can write for them Majorana masses:

$$
\text { HNL Majorana mass term }=M_{I} \bar{N}_{I}^{C} N_{I}+\text { h.c. . }
$$

where $N_{I}^{C}$ is a charge-conjugated fermion. For energies below the masses of HNLs, this leads to the appearance of the dimension 5 "Weinberg operator" [314] in the effective Lagrangian of active neutrinos:

$$
\Delta \mathcal{L}_{\mathrm{osc}}=c_{\alpha \beta} \frac{\left(\bar{L}_{\alpha}^{C} \cdot \tilde{\Phi}\right)\left(\tilde{\Phi} \cdot L_{\beta}\right)}{\Lambda}
$$

(where $L_{\alpha}^{C}$ is a charge-conjugation of the left lepton doublet, $L_{\alpha}$, and $\Lambda$ is a parameter having the dimension of mass and defined by $M_{I}$ and $F_{\alpha I}$, and at least some of $c_{\alpha \beta} \sim 1$ ). In case of $\mathcal{N}=1$ HNL the scale is given by $\Lambda \sim M /|F|^{2}$. Therefore, for any value of $\Lambda$, HNLs can have their masses in a very wide range, depending on the magnitude of Yukawa couplings, up to $M_{I} \sim \Lambda$ where Yukawa couplings become $|F| \sim 1$ and the theory is not in the weak coupling regime anymore.

In the Higgs phase the operator (4.1.3) leads to the Majorana masses and changes of flavour of the active neutrinos. Naively this means that for a given HNL mass there is an upper bound 
on Yukawa coupling such the contribution to the neutrino mass matrix via (4.1.3) should not be too large. However, for $\mathcal{N} \geq 2$ contributions of different HNLs can cancel each other, and in fact the Yukawa couplings can be large. To understand this, let us consider the following toy-model. Let two Majorana fermions $N_{1}$ and $N_{2}$ have equal Majorana masses $M_{1}=M_{2}$ and arbitrary Yukawa couplings $F_{\alpha 1}<1$ but $F_{\alpha 2}=0$. One can combine $N_{1}$ and $N_{2}$ into a single Dirac fermion $\Psi=\left(N_{1}, N_{2}^{C}\right)$ with the Dirac mass $M_{1} \bar{\Psi} \Psi$ and carrying lepton number 1 . In such a system the total lepton number is conserved and the operator (4.1.3) is forbidden, making neutrino masses equal to zero, regardless of the values of $F_{\alpha 1}$. See $[315,316]$ and Secs. 4.3.2.2 and 4.3.3.3 where more general cases are analysed. Neutrino masses will only appear if $F_{\alpha 2} \neq 0$ and/or the Majorana mass term $\Delta M \bar{\Psi}^{C} \Psi$ are introduced. Therefore any range of values of $\left|F_{\alpha 1}\right|$ can be made consistent with the experimental data from active neutrino oscillations. However, if an HNL with Yukawa coupling exceeding $\frac{m_{\text {atm }} M_{I}}{v^{2}}$ is found, one can conclude that there must exist other HNLs or some other new physics (see also Fig. 4.5 below).

Experimental data tells us that neutrinos are indeed massive and can change flavours (Secs. 4.2). We do not know the exact masses of the active neutrinos, but we know that the sum of neutrino masses should be $\geq 0.05 \mathrm{eV}$ and that there are at least three mass eigenstates (two mass differences), see Sec. 4.2.2. The Majorana nature of active neutrino masses is tested with neutrinoless double beta decay experiments (Sec. 4.5.1). It is natural to assume that it is the interactions (4.1.1)(4.1.2) that are responsible for this. We can immediately derive several conclusions of this assumption:

(a) The smallness of neutrino masses means that $\Lambda \sim 10^{15} \mathrm{GeV}$.

(b) The number of $N_{I}$, should be $\mathcal{N} \geq 2$ to explain the two observed mass differences for active neutrinos $-\Delta m_{\odot}^{2}$ and $\Delta m_{\text {atm }}^{2}$ (see Table 4.1 on page 67 below). If $\mathcal{N}=2$, the lightest mass eigenstate of active neutrinos is zero. The current experimental bounds on the masses of individual neutrinos (e.g. from those from the kinematics of tritium $\beta$-decay $[156,317,318]$ ) as well as cosmological bounds on the sum of neutrino masses (e.g. [319, 320]) allow for three non-zero mass eigenstates. If all three active neutrinos are massive, we would need $\mathcal{N} \geq 3$.

(c) For any number of HNLs there is a lower bound on the sum of the Yukawa couplings

$$
\sum_{\alpha, I}\left|F_{\alpha I}\right|^{2} \geq \frac{M_{\min } \sum m_{\nu}}{3 v^{2}}=5.5 \times 10^{-16}\left[\frac{\sum m_{\nu}}{0.1 \mathrm{eV}}\right]\left[\frac{M_{\min }}{1 \mathrm{GeV}}\right]
$$

where $\sum m_{\nu}$ is the sum of active neutrino masses, and $M_{\min }$ is a minimal mass among all HNLs that contribute to neutrino oscillations. If all HNLs have similar masses, Eq. (4.1.4) provides the bottom line for searches for the HNLs. If only $\mathcal{N}=2$ HNLs contribute to the neutrino masses, there is a stronger than (4.1.4) lower limit on the sum over flavours $\sum_{\alpha}\left|F_{\alpha 1}\right|^{2}$ for each $N_{I}$ separately (as one HNL is not sufficient to explain two mass differences). If there are $\mathcal{N}=3 \mathrm{HNLs}$, but one of them happens to be the dark matter particle, its Yukawa couplings with the Standard Model should be sufficiently small to satisfy the experimental bounds on stability of dark matter particles (this dark matter candidate, discussed in Section 4.7). In this case the contribution of dark matter HNL to neutrino masses is negligible $[321,322]$ and again there is a lower bound on the sum of $\left|F_{\alpha I}\right|^{2}$ for each $I$ of all the other HNLs (like in the $\mathcal{N}=2$ case). In the more general case with 3 or more HNLs, contributing to neutrino masses, this lower bound can be relaxed [323-325].

Of course, the requirement that the active neutrino masses come exclusively from HNLs is only an assumption, as other mechanisms for active neutrino mass generation are possible (see e.g. Section 4.3.3 below, for review see e.g. [326]). If they operate, the properties of HNLs may be decoupled from neutrino masses, making their parameters less constrained.

It turns out that sterile neutrinos not only can explain the neutrino masses and provide a dark matter candidate, but also provide a mechanism of generation of baryon asymmetry of 

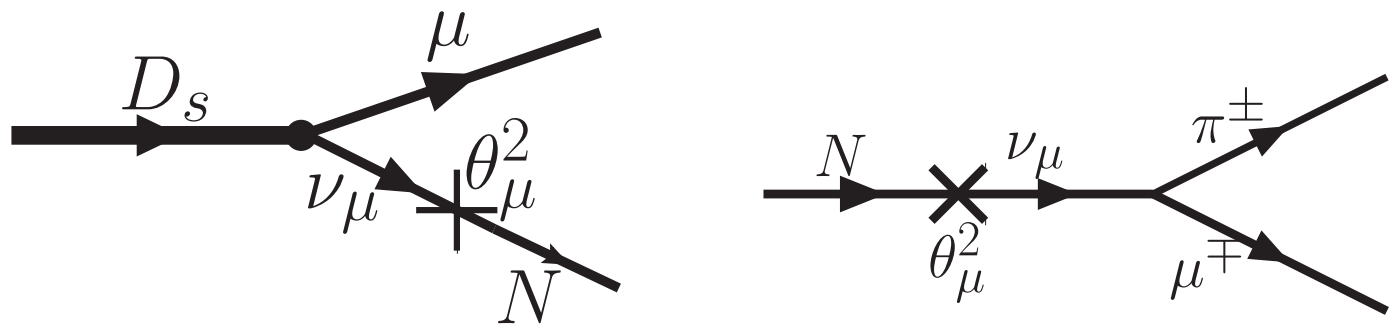

Figure 4.2: Production (left) and subsequent decay (right) of the particle $N_{I}$.

the Universe (see Section 4.6.1 for the formulation of the problem). Moreover, the same particles can be responsible for both neutrino masses and matter-antimatter asymmetry generation. HNLs with the masses ranging from $\mathcal{O}(\mathrm{MeV})$ to $\mathcal{O}\left(10^{12} \mathrm{GeV}\right)$ provide mechanisms of generation of matter-antimatter asymmetry, described in Sections 4.6.2-4.6.4.2 below. In particular, the successful baryogenesis is possible when HNL have experimentally accessible masses (Sections 4.3.2.2, 4.3.2.3). This opens an exciting possibility of direct experimental resolution of these BSM puzzles by finding HNLs experimentally. The phenomenology of neutrino oscillations provides (under certain assumptions, discussed above) the lower bound on Yukawa couplings, while the requirement of successful baryogenesis provides an upper bound on their values.

Right-handed neutrinos can appear as a part of a wider theory, for example as a part of the fermion representation of a gauge group in GUT theories, see Section 4.3.2.1. Interestingly HNLs can be postulated as the only new particles beyond the Standard Model up to a very high energy scale, providing explanations of all major observational BSM phenomena (Section 4.8 below). This brings the questions of the complete UV theory (discussed in Section 4.8.3). The SM supplemented by 3 HNLs, with Majorana mass terms for all of them, and all possible Yukawa couplings with the Higgs boson and left-handed lepton doublets has an intriguing property of charge quantisation. The Majorana mass term (4.1.2) means that the hypercharge of $N_{I}$ is zero and therefore hypercharges of left lepton double and Higgs field are the same. As a result of this, the requirement of cancellation of gauge chiral anomalies has a unique solution in terms of charges [327], quantised exactly as it is observed. In other words, the charge quantisation may be a requirement of the self-consistency of the theory, rather than a consequence of a larger symmetry, as in Grand Unified Theories.

\subsection{Active neutrino phenomenology}

Neutrino physics provides strong motivation for the existence of HNLs. Although properties of HNLs cannot be fully fixed by data from low-energy neutrino experiments, it serves as a source of important constraints. Therefore we review main results of neutrino theory and experiments below.

\subsubsection{Three-flavour neutrino oscillations. A theoretical overview}

A decade of revolutionary neutrino experiments has established that the SM neutrinos are massive and mix like quarks do. The measurement of their tiny masses has been possible thanks to neutrino oscillations, a quantum phenomenon first conjectured by Pontecorvo [328]. Neutrinos are produced and detected via weak processes, therefore by definition they are produced or detected as flavour states (ie. the states that couple to the $e, \mu$ and $\tau$ leptons respectively). However, such states of a definite flavour are superpositions of the vacuum Hamiltonian eigenstates or mass eigenstates 
$\left(\nu_{1}, \nu_{2}, \nu_{3}\right)$ :

$$
\left(\begin{array}{c}
\nu_{e} \\
\nu_{\mu} \\
\nu_{\tau}
\end{array}\right)=U_{\mathrm{PMNS}}\left(\theta_{12}, \theta_{13}, \theta_{23}, \delta, \alpha_{1}, \alpha_{2}\right)\left(\begin{array}{c}
\nu_{1} \\
\nu_{2} \\
\nu_{3}
\end{array}\right)
$$

In complete analogy to the quark sector, the relation between the two bases is a unitary rotation matrix $U_{\mathrm{PMNS}}$, the famous Pontecorvo-Maki-Nakagawa-Sakata [329], which generically depends on three Euler angles, $\left(\theta_{12}, \theta_{13}, \theta_{23}\right)$, a Dirac $\mathrm{CP}$ violating phase, $\delta$, and two more Majorana phases $\left(\alpha_{1}, \alpha_{2}\right)$, in case neutrinos are Majorana particles,

$$
U_{\mathrm{PMNS}}=\left(\begin{array}{ccc}
c_{12} c_{13} & s_{12} c_{13} & s_{13} e^{-i \delta} \\
-c_{23} s_{12}-s_{23} c_{12} s_{13} e^{i \delta} & c_{23} c_{12}-s_{23} s_{12} s_{13} e^{i \delta} & s_{23} c_{13} \\
s_{23} s_{12}-c_{23} c_{12} s_{13} e^{i \delta} & -s_{23} c_{12}-c_{23} s_{12} s_{13} e^{i \delta} & c_{23} c_{13}
\end{array}\right) \times\left(\begin{array}{ccc}
1 & 0 & 0 \\
e^{i \alpha_{1}} & 0 \\
0 & 0 & e^{i \alpha_{2}}
\end{array}\right)
$$

with $s_{i j}=\sin \theta_{i j}, c_{i j}=\cos \theta_{i j}$.

Neutrinos are so weakly interacting that they can keep quantum coherence over macroscopic distances. If a neutrino of flavour $\alpha$ is produced with energy $E$, the probability to detect a different flavour $\beta$ down the beam line at a distance $L$ in vacuum is a sum of oscillatory terms in $L / E$ with wavelengths that depend on the neutrino mass differences as:

$$
\begin{aligned}
P\left(\nu_{\alpha} \rightarrow \nu_{\beta}\right) & =\delta_{\alpha \beta}-4 \sum_{i<j} \operatorname{Re}\left[U_{\alpha i}^{*} U_{\beta i} U_{\alpha j} U_{\beta j}^{*}\right] \sin ^{2}\left(\frac{\left(m_{j}^{2}-m_{i}^{2}\right) L}{4 E}\right) \\
& -2 \sum_{i<j} \operatorname{Im}\left[U_{\alpha i}^{*} U_{\beta i} U_{\alpha j} U_{\beta j}^{*}\right] \sin \left(\frac{\left(m_{j}^{2}-m_{i}^{2}\right) L}{2 E}\right) .
\end{aligned}
$$

The amplitudes of the different terms contain information on different combinations of the mixingmatrix elements. The possibility to measure the mass differences in vacuum relies on performing experiments with $E / L \sim O\left(\left|m_{j}^{2}-m_{i}^{2}\right|\right)$. Much larger values imply that the oscillation is suppressed, while for much smaller values the oscillations cannot be resolved and only an average can be measured. The latter depends on the mixings but quantum coherence is lost, and with it, the sensitivity to the mass differences. The last term in eq. (4.2.3) is CP-odd (changes sign for antineutrinos), and is therefore sensitive to the imaginary entries of the mixing matrix. It can be shown however that only the Dirac phase $\delta$ contributes to it, and CPT symmetry implies that it vanishes for disappearance probabilities, ie. when $\beta=\alpha$.

\subsubsection{Present experimental status of neutrino masses and mixings}

The original indications of flavour transitions in neutrinos came from counting too few solar electron neutrinos [330]. The effect was conclusively settled by the SNO experiment [331] that measured for the first time a flux of muon and/or tau neutrinos coming from the Sun. SuperKamiokande on the other hand measured a distinct oscillatory pattern in the muon neutrino atmospheric flux as a function of the zenith angle [332]. Both oscillations have been confirmed by experiments in the laboratory. The solar oscillation was confirmed by the reactor neutrino experiment KamLAND [333] that observed the oscillatory pattern in the disappearance of reactor neutrinos at $L \sim O(100 \mathrm{~km})$, while the atmospheric one was confirmed by the MINOS [334] and K2K[335] experiments that observed the oscillatory pattern in the disappearance of neutrino muons produced in an accelerator and detected a few hundred kms down the beam line. In 2012, the reactor experiments Daya Bay [336], RENO [337] and Double-Chooz [338], together with the accelerator experiment T2K [339], proved (in two very different energy ranges) that electron neutrinos also oscillate with the 


\begin{tabular}{l|c|c|c|c|c|c} 
& $\Delta m_{\odot}^{2}\left(\mathrm{eV}^{2}\right)$ & $\Delta m_{\text {atm }}^{2}\left(\mathrm{eV}^{2}\right)$ & $\theta_{12}\left(^{\circ}\right)$ & $\theta_{23}\left({ }^{\circ}\right)$ & $\theta_{13}\left({ }^{\circ}\right)$ & $\delta\left(^{\circ}\right)$ \\
\hline \hline $\mathrm{NH}$ & $7.5(2) \times 10^{-5}$ & $2.46(5) \times 10^{-3}$ & $33.5(8)$ & $42.3_{-1.6}^{+3}$ & $8.5(2)$ & $306_{-70}^{+39}$ \\
\hline $\mathrm{IH}$ & idem & $-2.45(5) \times 10^{-3}$ & idem & $49.5_{-2.2}^{+1.5}$ & idem & $254_{-62}^{+63}$ \\
\hline
\end{tabular}

Table 4.1: Results from a global fit to all neutrino data from [340] for normal hierarchy (NH) $m_{3}>m_{1}, m_{2}\left(\Delta m_{\mathrm{atm}}^{2} \equiv m_{3}^{2}-m_{1}^{2}\right)$ and inverted one $(\mathrm{IH}) m_{3}<m_{1}, m_{2}\left(\Delta m_{\mathrm{atm}}^{2} \equiv m_{3}^{2}-m_{2}^{2}\right)$.

atmospheric wavelength even if with a much smaller amplitude than muon neutrinos. This result confirmed the three-fold-mixing way of neutrinos and provided the measurement of the last and smaller mixing angle of the mixing matrix, $U_{\text {PMNS }}$. The present status of the global fits of all neutrino data obtained in [340] are summarised in Table 4.1. There remain two approximate degeneracies that need to be resolved: the octant of the angle $\theta_{23}$ (resulting from an approximate symmetry $\left.\theta_{23} \rightarrow \pi / 4-\theta_{23}\right)$ and the ordering of the mass eigenstates: normal hierarchy (NH) for $m_{3}>m_{1}, m_{2}\left(\Delta m_{\mathrm{atm}}^{2} \equiv m_{3}^{2}-m_{1}^{2}\right)$ or an inverted hierarchy (IH) for $m_{3}<m_{1}, m_{2}\left(\Delta m_{\mathrm{atm}}^{2} \equiv\right.$ $\left.m_{3}^{2}-m_{2}^{2}\right)$. There is at present no evidence for leptonic CP violation, although the global fits are already hinting at a quadrant of the CP phase $\delta$. The new NOVA experiment that has just started taking data might give the first hints of the neutrino ordering thanks to the longer baseline.

The determination of these unknowns, as well as the absolute neutrino mass scale and the Majorana nature of neutrinos is essential to pin down the missing values of the neutrino mass matrix, to establish the existence of a matter-antimatter asymmetry in the lepton sector, and to constrain models of neutrino masses. Fortunately, the prospects to perform these measurements in the future are excellent.

In summary, the standard three-neutrino scenario explains well a wealth of neutrino data from solar, atmospheric, reactor and accelerator neutrino experiments in terms of two distinct mass splittings, the so-called solar, $\Delta m_{\odot}^{2}$, and atmospheric, $\Delta m_{\text {atm }}^{2}$, and three mixing angles.

However, along with the success of the three-neutrino scenario, described in the previous Section, several distinct experiments have revealed anomalies that cannot be accommodated into this picture and can be interpreted as oscillations of three active neutrinos of the Standard Model into additional light (sterile) states [341-346]. They are reviewed in the Section 4.2.3 below.

\subsubsection{Short-Baseline neutrino anomalies}

The LSND anomaly [341], which first manifested itself in the mid 1990's [347], is related to the presence of an excess, at almost the four sigma level, of $\bar{\nu}_{e}$-like events coming from a pion-decay-atrest beam, which is a well-characterized source of $\nu_{\mu}, \nu_{e}$, an $\bar{\nu}_{\mu}$, but not $\bar{\nu}_{e}\left(\pi^{+} \rightarrow \mu^{+} \nu_{\mu}\right.$, followed by $\mu^{+} \rightarrow e^{+} \nu_{e} \bar{\nu}_{\mu}$ ). The LSND anomaly can be interpreted as a nonzero probability (of order $0.1 \%$ ) that a $\bar{\nu}_{\mu}$ will behave as a $\bar{\nu}_{e}$. The associated $L / E$ (baseline over neutrino energy) values are around $0.5 \mathrm{~m} / \mathrm{MeV}$.

The MiniBooNE experiment was designed to explore the region of parameter space preferred by the neutrino oscillation interpretation of the LSND anomaly. It covers $L / E$ values between $0.1 \mathrm{~m} / \mathrm{MeV}$ and $2 \mathrm{~m} / \mathrm{MeV}$. The first results from MiniBooNE [348] analyzed data from positivepion-decay-in-flight, $\pi^{+} \rightarrow \mu^{+} \nu_{\mu}$, and placed bounds on the probability that a $\nu_{\mu}$ would oscillate into a $\nu_{e}$ which were in modest disagreement with LSND data. The analysis of more data [342] revealed an excess of $\nu_{e}$-like events at low energies, $L / E \gtrsim 1 \mathrm{~m} / \mathrm{MeV}$. MiniBooNE also analyzed data from negative-pion-decay-in-flight, $\pi^{-} \rightarrow \mu^{-} \bar{\nu}_{\mu}$ [343]. These data, not as statistically powerful as those from LSND or those from the MiniBooNE neutrino running, are consistent with the neutrino oscillation interpretation of the LSND data and are consistent with the excess observed by the 
MiniBooNE neutrino data. Recent combined analysis of LSND and MiniBooNE data reveal that these are consistent with the hypothesis that there are more than three neutrino mass eigenstates.

Quite independently from the LSND-MiniBooNE developments, recent reevaluations of the neutrino flux from nuclear reactors [344] (later scrutinized and confirmed in [349]) led to a reanalysis of older short-baseline reactor neutrino data [345] which revealed a (more or less) energy independent suppression of the expected flux, which can be interpreted as a survival probability of $\bar{\nu}_{e}$ around $95 \%$. The different experiments span $L / E$ values from $1 \mathrm{~m} / \mathrm{MeV}$ to $20 \mathrm{~m} / \mathrm{MeV}$. Finally, $\nu_{e}$ data from radioactive sources [346], used to calibrate the gallium solar neutrino experiments, also point to a deficit of $\nu_{e}$ for $L / E$ values consistent with those of LSND and the reactor anomaly.

Combined, these can be interpreted as evidence for more than three neutrinos, with a new oscillation length proportional to a new mass-squared difference around $1 \mathrm{eV}^{2}$ (for recent analyses, see [350-353]). Given that the number of active neutrinos is known to be three [354], the extra degrees of freedom must be sterile neutrinos (i.e. should not interact with the $Z^{0}$ boson). The "goodness of fit" of the sterile neutrino hypothesis is not very strong, mostly due to the fact that searches for $\nu_{\mu}$ and $\nu_{e}$ disappearance at similar $L / E$ have not revealed any effects [350-353]. The hypothesis that there are two extra states is modestly preferred over the hypothesis that there is only one new light sterile neutrino, for a couple of reasons. Two extra neutrinos allow for new sources of CP-invariance violation to which the current short-baseline experiments are sensitive, and two extra neutrinos allow for two different mass-squared differences, which can "interfere" with one another and allow one to evade more efficiently the constrains from short-baseline disappearance data. Fig. 4.3 depicts allowed regions of the five-neutrino parameter space, from [352]. We refer to [352] (see also [350,351,353]) for more information and details. It is important to state that new neutrinos with masses around $1 \mathrm{eV}$ and large active components, such as the ones required to explain the short-baseline anomalies, are disfavored by data from cosmic surveys, which are consistent with only three light, neutrino-like degrees of freedom. If the existence of more than three light neutrinos is confirmed by laboratory experiments we will be forced to conclude that our understanding of the thermal history of the university is incomplete, or that there is new physics beyond small neutrino masses and new sterile degrees of freedom.

While our understanding of these so-called short-baseline anomalies remains clouded, there are several experimental proposals aimed at definitively testing the sterile-neutrino interpretation (for an overview see, for example, [355]). It is possible that, in five to ten years, experiments will reveal, beyond reasonable doubt, the existence of new neutrino degrees of freedom.

The eV-Seesaw is severely constrained by data from cosmic surveys and big-bang nucleosynthesis (see [356] for a recent detailed analysis). At face value, cosmological constraints require all but one of the Seesaw right-handed neutrinos to have masses higher than $100 \mathrm{MeV}$, while the remaining one is only allowed to be very light in the limit that the lightest, mostly active neutrino is also very light (mass less than roughly $10^{-3} \mathrm{eV}$ ) [356]. These constraints, however, can be modified, alleviated, or eliminated if our understanding of the thermal history of the universe, or that of fundamental particle physics, is incomplete. For a concrete scenarios see, for example, [357-359].

\subsubsection{Future neutrino experiments}

Future neutrino oscillation experiments can measure the octant, the ordering and discover leptonic $\mathrm{CP}$ violation thanks to the sizeable value of $\theta_{13}$. Three strategies are being pursued in this regard. On the one hand, the huge statistics of atmospheric neutrino events that can be detected in megaton-scale atmospheric neutrino detectors such as the planned Hyper-Kamiokande [360] or future, more finely-instrumented sections of the IceCube or KM3NeT neutrino telescopes, PINGU [361] and ORCA respectively, could provide very valuable information on the octant degeneracy and the neutrino ordering. Next generation LBL reactor neutrino detectors located at $O(50) \mathrm{km}$ from powerful reactors, such as the JUNO project, could significantly improve the precision of $\Delta m_{\odot}^{2}$ 

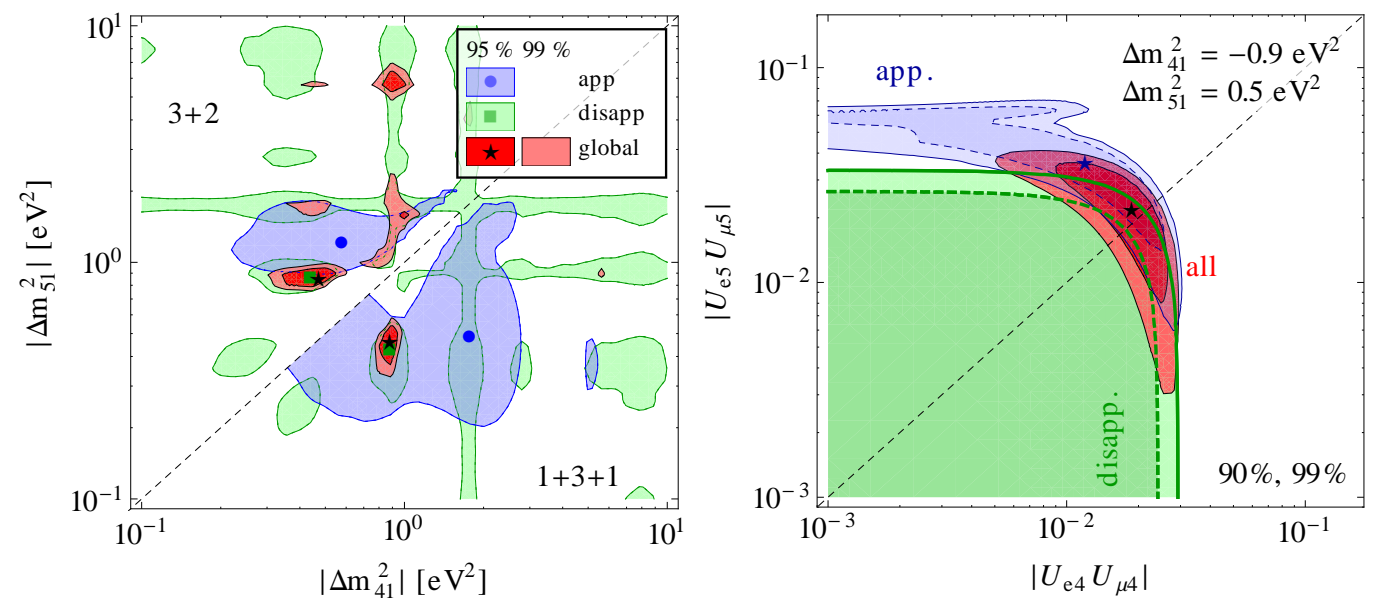

Figure 4.3: Five-neutrino fit to all oscillations data, from [352]. In the left-hand panel are depicted the allowed regions in the plane of $\Delta m_{14}^{2}$ and $\Delta m_{15}^{2}$ in $3+2$ (upper-left part) and $1+3+1$ (lowerright part) mass schemes. Also depicted are the regions for appearance data (light blue) and disappearance data (light green) at 95\% CL (2 dof), and global data (dark and light red) at 95\% and $99 \%$ CL (2 dof). In the right-hand panel are depicted the allowed regions for $3+2$ in the plane of $\left|U_{e 4} U_{\mu 4}\right|$ versus $\left|U_{e 5} U_{\mu 5}\right|$, for fixed values of $\Delta m_{14}^{2}$ and $\Delta m_{15}^{2}$ at $90 \%$ and $99 \%$ CL (2 dof). Also depicted are the regions for appearance data (blue), disappearance data (green), and the global data (red). See [352] for details.

and the angle $\theta_{12}$, as well as provide information on the neutrino ordering through the interference of atmospheric and solar oscillations. Finally, the construction of LBL accelerator neutrino super beams shooting at $\mathcal{O}(10) \mathrm{kton}$-scale detectors located at distances above $1000 \mathrm{~km}$, such as envisioned in the LBNF project, could provide a superb measurement of the neutrino ordering, by exploiting the MSW effect in the propagation of neutrinos through the earth. They will also significantly improve the sensitivity to $\delta$, and can discover leptonic CP violation (at $3 \sigma$ ) for up to $70 \%$ of the possible values of $\delta$ [362].

Concerning the absolute neutrino mass scale, cosmology is already constraining the sum of the three neutrino masses in the sub-ev range. A similar sensitivity is expected in the next generation of $\beta$-decay experiment (KATRIN [363]). The crucial question of whether neutrinos are Majorana particles could be answered in the next-generation of neutrino less double-beta decay experiments that could cover the whole allowed phase space in the case of IH.

\subsection{HNLs and neutrino masses}

\subsubsection{Seesaw formula}

If the neutrinos are Majorana particles, the phenomenology of their oscillations (Section 4.2.2) can be described via Weinberg's operator (4.1.3). This operator of mass dimension 5 can be resolved in many different ways (for a review see e.g. [326]). The simplest way to mediate the interaction (4.1.3) is via the neutrino portal (4.1.1). The Standard Model Lagrangian $\mathcal{L}_{\mathrm{SM}}$ gets extended by $\mathcal{N}$ righthanded neutrinos $N_{I}$ :

$$
\mathcal{L}=\mathcal{L}_{\mathrm{SM}}+i \bar{N}_{I} \not \partial N_{I}-\left(F_{\alpha I} \bar{L}_{\alpha} N_{I} \tilde{\Phi}+\frac{M_{I}}{2} \bar{N}_{I}^{C} N_{I}+\text { h.c. }\right)
$$


(for notations see Eq. (4.1.1)). The particles $N_{I}$ are neutral with respect to all the gauge interactions of the Standard Model (such particles are often called sterile neutrinos or gauge-singlet fermions). In the Higgs phase the combination $\left(\bar{L}_{\alpha} \tilde{\Phi}\right)=\bar{\nu}_{\alpha}$, and therefore the neutrino portal interactions lead to the $\nu_{\alpha}-N_{I}$ mixing. As a result charge eigenstates of the Lagrangian (4.3.1) do not coincide with the mass eigenstates. The latter are obtained by diagonalizing the matrix

$$
\mathcal{M}_{\nu, N}=\left(\begin{array}{c|c}
0 & m_{D} \\
\hline m_{D}^{T} & M_{I}
\end{array}\right)
$$

where $m_{D}$ is $3 \times \mathcal{N}$ Dirac mass matrix, $\left(m_{D}\right)_{\alpha I}=F_{\alpha I} v, v=\sqrt{2}\langle\Phi\rangle$ and $M_{I}$ is $\mathcal{N} \times \mathcal{N}$ matrix of Majorana masses. In the limit $m_{D} \ll M_{I}$, one can easily see that the Lagrangian (4.3.1), indeed, leads to the Weinberg's operator (4.1.3) for neutrinos with

$$
\frac{c_{\alpha \beta} v^{2}}{\Lambda} \equiv\left(\mathcal{M}_{\nu}\right)_{\alpha \beta}=-\sum_{I}\left(m_{D}\right)_{\alpha I} \frac{1}{M_{I}}\left(m_{D}\right)_{\beta I}
$$

The smallness of the Dirac mass term as compared to the Majorana masses $M_{I}$ means that the active neutrino masses (3 eigenvalues of the matrix $\left.\left(\mathcal{M}_{\nu}\right)_{\alpha \beta}\right)$ become much smaller than the scale $M_{I}$ and the electroweak scale. This mechanism is therefore known as the seesaw mechanism [304, $308,364,365$ ], see also [366] and refs. therein. ${ }^{2}$ Other $\mathcal{N}$ eigenstates of the matrix (4.3.2) almost coincide with $N_{I}$ up to a small admixture of $\nu_{\alpha}$. The smallness of this admixture is characterized by the dimensionless ratio of the Dirac and Majorana masses (known as mixing angle or active-sterile mixing):

$$
U_{\alpha I}^{2} \equiv \frac{v^{2}\left|F_{\alpha I}\right|^{2}}{M_{I}^{2}} \ll 1 .
$$

The small weak charge, proportional to $U_{\alpha I}^{2}$, that mass eigenstates $N_{I}$ acquire, allows to probe experimentally for these heavy neutral leptons.

Adding $\mathcal{N}$ new particles $N_{I}$ to the Lagrangian $\mathcal{L}_{\mathrm{SM}}$ adds

$$
\text { Number of HNL parameters }=7 \times \mathcal{N}-3
$$

new parameters to the Lagrangian. These parameters can be chosen as follows: $\mathcal{N}$ real Majorana masses $M_{I}$ plus $3 \times \mathcal{N}$ complex Yukawa couplings $F_{\alpha}$ minus 3 phases absorbed in redefinitions of $\nu_{e}, \nu_{\mu}, \nu_{\tau}$. The PMNS matrix (4.2.2) plus three mass eigenstates $m_{1}, m_{2}, m_{3}$ of the active neutrino sector provide 9 parameters that can be determined experimentally. This shows that one needs $\mathcal{N} \geq 2$ to explain the neutrino oscillations via heavy neutral leptons.

To see how neutrino oscillation data limit the HNL parameters, let us first look at the simplest (unrealistic) toy model with one $H N L, N_{1}$. In this case only one combination of neutrino flavours gets massive and its mass, $m_{\nu}$ allows to limit the sum of the Yukawa couplings, $\left|F_{1}\right|$ :

$$
\left|F_{1}\right|^{2} \equiv \sum_{\alpha}\left|F_{\alpha 1}\right|^{2}=\left(4.1 \times 10^{-8}\right)^{2}\left(\frac{m_{\nu}}{m_{\mathrm{atm}}}\right)\left(\frac{M_{1}}{1 \mathrm{GeV}}\right) \quad \text { One HNL case. }
$$

We see that $\left|F_{1}\right|$ becomes smaller as $M_{1}$ becomes lighter. On the other hand, the mixing element

\footnotetext{
${ }^{2}$ This mechanism is often called Type-I seesaw mechanism because there are other ways to "resolve" the Weinberg's operator (see e.g. [326, 367]). For example, in Type-II seesaw mechanism an extra SU(2) triplet scalar is introduced [309, 368-370], in type-III seesaw mechanism an extra fermion in the adjoint of SU(2) is added to the model [371].
} 
of HNL in the weak interaction is given by

$$
U^{2}=5.0 \times 10^{-11}\left(\frac{m_{\nu}}{m_{\mathrm{atm}}}\right)\left(\frac{1 \mathrm{GeV}}{M_{N}}\right) \quad \text { One HNL case }
$$

which is also very small and the dependence on the HNL mass, $M_{1}$ is opposite to Eq. (4.3.6).

In order to explain two mass differences $\Delta m_{\text {atm }}^{2}$ and $\Delta m_{\odot}^{2}$, one needs $\mathcal{N} \geq 2$. As it turns out, in such cases much larger values of $|F|$ and $U^{2}$ are possible. Even in the simplest case of two HNLs having the same mass $M_{N}$, the mixing angle is expressed via one free parameter $X_{\omega} \geq 1$ (in the notations of [372]) that gives the following equation for $U^{2} \equiv \sum_{\alpha, I} U_{\alpha I}^{2}[315,323,372]$

$$
U^{2}=\frac{\sum_{\nu} m_{\nu}}{2 M_{N}}\left(X_{\omega}^{2}+X_{\omega}^{-2}\right) \simeq 5 \times 10^{-10} \kappa\left(\frac{1 \mathrm{GeV}}{M_{N}}\right)\left(\frac{X_{\omega}}{100}\right)^{2} \quad \text { Two degenerate HNLs }
$$

where $\sum_{\nu} m_{\nu}=\kappa \times m_{\text {atm }}$, i.e. $\kappa \simeq 1$ for $\mathrm{NH}$ and $\kappa \simeq 2$ for IH. One sees that $U^{2}$ can be much larger that the naive estimation (4.3.7).

In case of two HNLs with different masses, the following inequality takes place for each mixing angle $U_{I}^{2}$ (as one can derive from [321]):

$$
U_{I}^{2} \geq \frac{m_{2} m_{3}}{m_{2}+m_{3}} \frac{1}{M_{I}} \simeq \frac{m_{\mathrm{atm}}}{M_{N}}\left\{\begin{aligned}
\frac{m_{\odot}}{m_{\mathrm{atm}}}, & \mathrm{NH} \\
\frac{1}{2}, & \mathrm{IH}
\end{aligned}\right.
$$

where $m_{3}>m_{2}>0$ - two highest mass eigenstates of active neutrinos (c.f. Sec. 4.2.1).

Similar (and more complicated) relation between $U^{2}$ and neutrino matrix parameters exist $\mathcal{N} \geq 3$, see $[324,325]$. In all cases the following inequality holds

$$
U^{2} \geq \frac{\sum_{\nu} m_{\nu}}{3\left(M_{I}\right)_{\max }}
$$

(as one can derive from [321]).

The Lagrangian (4.3.1) remains perturbative with Yukawa coupling $\left|F_{\alpha I}\right| \lesssim 1$ which corresponds to the scale $\Lambda \sim 10^{15} \mathrm{GeV}$ in the Weinberg's operator (4.3.3). Actually, the analysis shows that the theory stays perturbative up to $M_{I} \lesssim 10^{16} \mathrm{GeV}$ [373]. In the opposite limit $M_{I} \rightarrow 0$ the neutrinos become massive Dirac fermions and the smallness of their masses is explained by small Yukawa coupling: $F_{\alpha I} \sim 10^{-11}$. The Lagrangian has in this case an exact, global, non-anomalous $U(1)_{B-L}$ symmetry. ${ }^{3}$ This symmetry is broken when both the $F_{\alpha I}$ and the $M_{I}$ are nonvanishing, a fact that teaches us that any value of $M_{I}$ is technically natural [374], as defined by 'tHooft [375].

If $M_{I}$ is non-zero the neutrino mass is proportional to $\left|F_{\alpha I}\right|$ (rather than $F_{\alpha I}$ ) implying that $F_{\alpha I}$ need not take as small a value as it does in the absence of the Majorana mass. With $N_{I}$ being SM singlet, it can have mass not restricted by physics of the SM (apart from the region $M_{I} \sim 10^{-10}-1 \mathrm{eV}$, that is ruled out by the neutrino oscillation data, see Section 4.3.2.4 for details).

\subsubsection{Seesaw scales}

Heavy neutral leptons are an important window into the nature of physics associated with the origin of neutrino masses. A critical starting point for searches is to know the scale of $M_{I}$. To describe the place of the interesting mass ranges among all possibles values of $M_{I}$, we will review below several

\footnotetext{
${ }^{3}$ This is the only global symmetry, exact at both classical and quantum level, admitted by the Standard Model Lagrangian $\mathcal{L}_{\mathrm{SM}}$.
} 
possible choices and theoretical hints related to that particular values of HNL mass. We discuss this below.

\subsubsection{GUT-scale seesaw}

Two key ingredients of the seesaw formula (4.3.3) are (i) the existence of the singlet $N_{I}$ and (ii) breaking of $B-L$ symmetry implied by the Majorana mass $M_{I}$. One needs to look for plausible models that incorporate these two features. Two models that automatically imply the existence of $N$ 's and also $B-L$ breaking leading to Majorana mass for them are:

- a left-right symmetric extension of the SM

and

- $\mathrm{SO}(10)$ grand unified theory where the basic spinor representation is 16-dimensional and fits all SM fermions of one generation plus a right handed neutrino $N$.

Both models have $B-L$ as a local symmetry whose breaking, needed to recover the standard model at low energies, leads to Majorana mass for the $N_{I}$ 's in the seesaw matrix. These are, therefore, prime candidates as theories of massive neutrinos. In the $\mathrm{SO}(10)$ type theories, fermion unification implies that Yukawa couplings for leptons and quarks are same or of similar order of magnitude, which means that seesaw scale $M_{I} \sim 10^{14} \mathrm{GeV}$. While GUT seesaw models are quite elegant and predictive [376-382] experimentally testing them is not easy as constraints on Yukawa couplings essentially prevent right handed neutrinos from being much lighter than $10^{14} \mathrm{GeV}$ and as a result, their low energy effects are unobservable. In the presence of supersymmetry, they can lead to observable lepton flavor changing processes e.g. $\mu \rightarrow e+\gamma$; however, such rates go as $M_{S S B}^{-4}$ where $M_{S S B}$ is the supersymmetry breaking scale. With supersymmetry breaking being pushed higher by LHC searches, in most supersymmetric models, such rates are unobservable.

In summary: although GUT scale seesaw models predict HNLs that couple to the Standard Model with the Yukawa couplings $|F| \sim \mathcal{O}(1)$, their high masses make direct experimental tests impossible. Their contributions to the lepton flavour violating processes is also strongly suppressed. However, as we will see below (Section 4.3.2.2) HNLs can have significant Yukawa couplings to the $\mathrm{SM}$ neutrinos even for much smaller masses $\mathcal{O}(\mathrm{TeV})$, opening a possibility to probe for these particles at the current and future accelerators and via lepton flavour violating processes. Alternatively, fixed target experiments with intense proton beams [35] can be sensitive for HNL with masses below few GeV. The corresponding HNLs models are discussed in Section 4.3.2.3. Future collider experiments with very large number of produced vector bosons can be sensitive to heavier HNLs with masses up to $M_{W}[383]$.

\subsubsection{TeV-scale seesaw}

From the mass formula for the light neutrinos (4.3.3), it naively follows $|F|^{2} \sim m_{\text {atm }} M_{I} / v^{2} \sim$ $10^{-9} M_{I}$. This translates into a Yukawa coupling $|F|=\mathcal{O}\left(10^{-6}\right)$ for $M_{I} \sim \mathrm{TeV}$, and therefore, although $\mathrm{TeV}$-scale masses are within the range of current accelerators, such low Yukawa couplings would induce too suppressed low energy effects to be observed (unless the right-handed neutrino has additional interactions beyond the Standard Model groups, for instance when it is charged under $\left.U(1)_{B-L}\right)$. However for the physically relevant case $\mathcal{N} \geq 2$ the structure of seesaw mass matrices allows for sizable Yukawa couplings with the right-handed neutrinos having TeV mass (for a simplified discussion see Sec. 4.1). To identify this class of scenarios we first note that there is a continuous family of Dirac neutrino masses which solves the seesaw equation (4.3.2) $\mathcal{M}_{\nu}=-M_{D} M_{N}^{-1} M_{D}^{T}$, given by [384]:

$$
M_{D}=i U^{*} \sqrt{D_{m}} \Omega \sqrt{D_{N}} V^{\dagger}
$$


where $U$ is the unitary matrix which diagonalizes $\mathcal{M}_{\nu}, \mathcal{M}_{\nu}=U^{*} D_{\nu} U^{\dagger}, V$ the matrix which diagonalizes $M_{N}, M_{N}=V^{*} D_{N} V^{\dagger}$, and $\Omega$ is an arbitrary complex orthogonal matrix: $\Omega \Omega^{T}=1$. In the case of two right-handed neutrinos, the matrix $\Omega$ reads [385]:

$$
\Omega_{\mathrm{NH}} \equiv\left(\begin{array}{cc}
0 & 0 \\
\cos \hat{\theta} & \pm \sin \hat{\theta} \\
-\sin \hat{\theta} & \pm \cos \hat{\theta}
\end{array}\right), \quad \Omega_{\mathrm{IH}} \equiv\left(\begin{array}{cc}
\cos \hat{\theta} & \pm \sin \hat{\theta} \\
-\sin \hat{\theta} \pm \cos \hat{\theta} \\
0 & 0
\end{array}\right)
$$

for normal hierarchy (NH) and inverted hierarchy (IH), respectively. It is now possible to exploit the freedom in the choice of $\Omega$ to generate a sizable $m_{D}$ with entries in $U$ of $\mathcal{O}(0.1)$, in $D_{\nu}$ of $\mathcal{O}\left(10^{-10} \mathrm{eV}\right)$ and in $D_{N}$ of $\mathcal{O}\left(10^{3} \mathrm{GeV}\right)$. Decomposing the complex parameter $\hat{\theta}=\omega-i \xi$ and taking $\xi \gg 1$ one finds:

$$
\Omega_{\mathrm{NH}} \simeq \frac{e^{i \omega} e^{\xi}}{2}\left(\begin{array}{cc}
0 & 0 \\
1 & \mp i \\
i & \pm 1
\end{array}\right), \quad \Omega_{\mathrm{IH}} \simeq \frac{e^{i \omega} e^{\xi}}{2}\left(\begin{array}{cc}
1 & \mp i \\
i & \pm 1 \\
0 & 0
\end{array}\right) .
$$

Thus, by adjusting $\xi$ it is possible to construct a viable see-saw scenario with $\mathrm{TeV}$ mass right-handed neutrinos and arbitrarily large Dirac masses [315, 316, 386-388]. It is also important to note that the matrix $\Omega$ introduces in general new sources of flavour structure in $M_{D}$ which are unrelated to the flavour mixing observed in neutrino oscillation experiments. However, in the limit $\xi \gg 1$ the extra flavour structure is fixed, thus rendering a fairly predictive scenario.

The necessary and sufficient condition for the leptonic weak neutral current to be diagonal in mass eigenstates is that all leptons of a given charge and chirality must have the same weak $T$ and $T_{3}$ [389]. Sterile neutrinos generically violate the condition of Ref. [389], so the neutral weak current is, in general nondiagonal. A large Dirac neutrino mass can significantly modify the charged current (CC) and neutral current (NC) leptonic interactions, due to the large mixing between leftand right-handed neutrinos. The $\mathrm{CC}$ and $\mathrm{NC}$ interactions involving the light Majorana neutrinos have the form:

$$
\begin{aligned}
& \mathcal{L}_{C C}^{\nu}=-\frac{g}{\sqrt{2}} \bar{\ell}_{\alpha} \gamma_{\rho}\left[U_{\text {lep }}\right]_{\alpha i} \nu_{i} W^{\rho}+\text { h.c. } \simeq-\frac{g}{\sqrt{2}} \bar{\ell}_{\alpha} \gamma_{\rho}\left[\left(1-\frac{1}{2}(R V)(R V)^{\dagger}\right) U\right]_{\alpha i} \nu_{i} W^{\rho}+\text { h.(44,3.14) } \\
& \mathcal{L}_{N C}^{\nu}=-\frac{g}{2 c_{w}} \overline{\nu_{i}} \gamma_{\rho}\left[U_{\text {lep }}^{\dagger} U_{\text {lep }}\right]_{i j} \nu_{j} Z^{\rho}+\text { h.c. } \simeq-\frac{g}{2 c_{w}} \overline{\nu_{i}} \gamma_{\rho}\left[U^{\dagger}\left(1-(R V)(R V)^{\dagger}\right) U\right]_{i j} \nu_{j} Z^{\rho}+(4.3 .1,5)
\end{aligned}
$$

with $R^{*} \simeq M_{D} M_{M}^{-1}$. Furthermore, the left-right neutrino mixing gives rise to sizable CC and NC couplings of the heavy Majorana neutrinos $N_{j}$ to the $W$ and $Z$ bosons:

$$
\begin{aligned}
& \mathcal{L}_{C C}^{N} \simeq-\frac{g}{2 \sqrt{2}} \bar{\ell}_{\alpha} \gamma_{\rho}(R V)_{\alpha i}\left(1-\gamma_{5}\right) N_{i} W^{\rho}+\text { h.c. }, \\
& \mathcal{L}_{N C}^{N} \simeq-\frac{g}{2 c_{w}} \overline{\nu_{\ell L}} \gamma_{\rho}(R V)_{\alpha i} N_{i} Z^{\rho}+\text { h.c.. }
\end{aligned}
$$

Thus, the combination $R V$ encodes the effects of the heavy neutrinos in low energy phenomenology. Using the parametrization discussed above, this matrix can be cast as $R V \simeq-i U_{\text {lep }} \sqrt{D_{\nu}} \Omega^{*} \sqrt{D_{N}^{-1}}$ [390], which can be explicitly written in terms of the largest neutrino Yukawa eigenvalue, $|F|$, the 
heavy neutrino masses and the low energy neutrino parameters. The result is [388]:

$$
\begin{aligned}
\left|(R V)_{\alpha 1}\right|^{2} & =\frac{1}{2} \frac{y^{2} v^{2}}{M_{1}^{2}} \frac{m_{3}}{m_{2}+m_{3}}\left|U_{\alpha 3}+i \sqrt{m_{2} / m_{3}} U_{\alpha 2}\right|^{2}, \mathrm{NH} \\
\left|(R V)_{\alpha 1}\right|^{2} & =\frac{1}{2} \frac{y^{2} v^{2}}{M_{1}^{2}} \frac{m_{2}}{m_{1}+m_{2}}\left|U_{\alpha 2}+i \sqrt{m_{1} / m_{2}} U_{\alpha 1}\right|^{2} \simeq \frac{1}{4} \frac{y^{2} v^{2}}{M_{1}^{2}}\left|U_{\alpha 2}+i U_{\alpha 1}\right|^{2}, \mathrm{IH} \\
(R V)_{\alpha 2} & = \pm i(R V)_{\alpha 1} \sqrt{\frac{M_{1}}{M_{2}}}
\end{aligned}
$$

The neutral and charged current interactions of the TeV mass right-handed neutrinos with the Standard Model leptons lead to lepton flavor violating processes such as rare leptonic decays or $\mu$-e conversion in nuclei, with a rate that could be at the reach of experiments depending on the value of the Yukawa coupling [388, 391-393] (see Sec. 4.5.3 for details).

Direct searches of HNLs with masses in the range $10 \mathrm{GeV}-1 \mathrm{TeV}$ are discussed in Sec. 4.4.3.

\subsubsection{GeV-scale seesaw}

Let us now discuss the seesaw scenario induced by GeV-scale right-handed neutrinos.

This case is interesting because HNLs with such masses can be efficiently searched for with the existing experimental technologies. In fact, a large number of experiments have been done for searching HNLs in the considered mass range (for reviews see [313, 394, 395]). In addition, in the realistic situation with $\mathcal{N} \geq 2$, the predicted range of $U^{2}$ is widened by the above enhancement factor(s) and can overlap the domain which is probed by the current and near future experiments.

An important motivation for the GeV-scale seesaw scenario is that HNLs for masses of active neutrinos can also generate the baryon asymmetry of the universe by the mechanism via HNL oscillations [396, 397]. Since HNLs at the GeV-scale possess only the Yukawa interaction at the unbroken phase in the early universe and its couplings are very suppressed as mentioned above, they can be out of equilibrium state. Then, the CP violation in the production and evolution of HNLs with oscillation effects generates the asymmetry of left-handed leptons which is partially converted into the baryon asymmetry of the universe. The successful baryogenesis requires an upper bound on the Yukawa couplings (to avoid fast washout of the baryon asymmetry due to the rapid scatterings of HNLs). This upper bound is more strong for $\mathcal{N}=2$ case (Sec. 4.6.4.1) and gets relaxed if more HNLs contribute to the neutrino masses. The details of this baryogenesis scenario will be described in Section 4.6.4.

The $\mathrm{GeV}$-scale seesaw is also motivated by various aspects of particle physics and astrophysics. For instance, HNLs with $0.2 \mathrm{GeV}$ can play an important role in supernova explosions [398]. (See a review [399] for HNLs in astrophysics.) HNLs at GeV scale are also important for lepton number violation (see the discussion in 4.8.2), flavor violation [400], lepton universality violation [310, 311, 401-403] and neutrinoless double beta decay [372, 404-407].

Let us now move on to the current status of the GeV-scale HNLs. Since the weak interaction of HNLs is characterized by the mixing element $U_{\alpha I}$, we shall consider the possible region of the mixings and also the masses.

First of all, as it was already mentioned in Sec. 4.3.1, the successful seesaw mechanism requires that the mixing elements should be in a certain region. For example, in the simplest case with $\mathcal{N}=2, U^{2}$ has the lower bound for the quasi-degenerate HNLs as shown in (4.3.8). The possible value of $U^{2}$ can be different depending on the number $\mathcal{N}$ and mass spectrum of HNLs.

This effective coupling does not spoil the main fundamental electroweak observables in neutrino sector, e.g. $Z$-boson invisible width $\Gamma_{Z \rightarrow \text { invis }}$ (as long as $M_{N} \ll M_{Z}$ ). Indeed, both almost-active (simply active herein after) and almost-sterile (simply sterile herein after) states contribute now, but the coupling of active states gets reduced by the same amount, that determines the coupling of 
sterile states, so the net result remains intact, i.e. the same $\Gamma_{Z \rightarrow \text { invis }}^{\mathrm{SM}}$ as in SM, because, although the width $\Gamma_{Z \rightarrow \nu \bar{\nu}}$ becomes suppressed, additional decay channels $Z \rightarrow N \bar{\nu}, Z \rightarrow \nu N$, and $Z \rightarrow N N$ add up to the same total amount so that $\Gamma_{Z \rightarrow \text { invis }}=\Gamma_{Z \rightarrow \text { invis }}^{\mathrm{SM}}$. Secondly, various search experiments so far have set upper bounds on the interaction strength $U^{2}$ due to the negative results. We should mention again the HNLs can be produced by decays of heavier mesons and weak bosons like the ordinary neutrinos through the neutrino mixing (see Sec. 4.4.2 for details and discussion of various production and decay channels). By using those processes mainly two techniques have been performed in previous search experiments. The first is a peak search (Sec. 4.4.1), which focuses on the two-body decays of charged mesons and searches the peak corresponding to the HNLs in the energy spectrum of the daughter charged leptons. This is based on the fact that the energy of the daughter lepton is determined only by masses of particles in the two-body decay. This puts upper bounds scaling with $U^{2}$. The second is beam-dumps (Sec. 4.4.2), in which HNLs are produced similarly by two-body decays of charged mesons and HNL's decays into charged particles are searched in a detector far from the production point. The results of this type of experiment leads to upper bounds scaling as $U^{4}$. Concrete upper bounds from search experiments are summerized in Refs. [313, 394, 395]. In addition, LHCb [408] and Belle collaborations [409] also reported the bound obtained from B meson decays (Sec. 4.4.3). Moreover, the sensitivity of the search for HNLs originated by the atmosphere [410] or the existing facilities of the accelerator-based neutrino experiments [411] were evaluated.

Thirdly, cosmological observations also restrict HNLs. In the early universe HNLs induce the hadronic decays, the speedup of the expansion of the universe due to the additional energy and the modification of the proton-neutron conversion rate by active neutrino productions. If these phenomena occur around the big bang nucleosynthesis (BBN) era, the abundances of light elements would be altered too much (see Sec. 4.5.4). In order to avoid such a situation BBN puts an upper bound on the lifetime [412-414], which leads to the lower bounds on $\left|U_{\alpha I}\right|$. Moreover, if HNLs are responsible to generate the BAU the interaction strength is restricted further in order to realize the observed amount [415]. When $U^{2}$ is too small, the sufficient asymmetry can't be generated because of the suppression of HNL oscillations and interactions between left-handed leptons and HNLs. On the contrary, the too large $U^{2}$ leads so rapid equilibration that the generated asymmetry vanishes due to the washout effect.

It is interesting to note that we may obtain a limited region of $U^{2}$ by combining the above two constraints, namely the upper bounds from the search experiments as well as the baryogenesis and the lower bounds from BBN and neutrino masses [395]. However, the explicit values of these bounds are strongly dependent on the setup of HNLs and the general expressions are difficult to estimate. As an example, the parameter space allowed from such above constraints have been evaluated in the $\nu$ MSM framework (Sec. 4.8). The results in [416] are summarized in Fig. 4.17. (See also Fig. 4.4 for the recent analysis in [403].)

\subsubsection{4 eV-scale Seesaw}

For non-zero values of $M_{I}$, the Seesaw Lagrangian predicts that the neutrinos are Majorana fermions, and that there exists more neutrinos than the three active ones that have already been accounted for. If $M_{I} \lesssim 1 \mathrm{MeV}$, these extra neutrinos are light sterile neutrinos that can only be directly probed by neutrino-related experiments, including searches for kinematical effects of neutrino masses, searches for neutrinoless double-beta decay, and the observation of the daughters of heavy neutrino decays (for an overview see, for example, $[313,417]$ ). In the case of neutrinoless double-beta decay, the prediction is that, in spite of the fact that there are more neutrinos and that all are Majorana fermions, the expected rate vanishes with very good precision as long as all $M_{I}$ values are smaller than about $50 \mathrm{MeV}[374,418]$. 

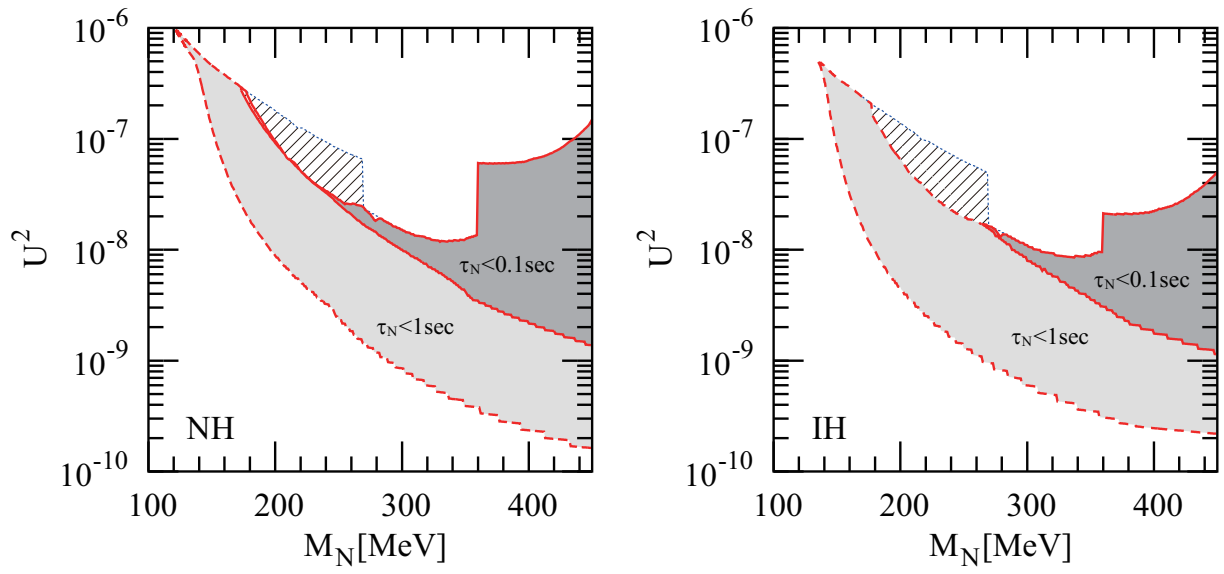

Figure 4.4: Allowed region in the $M_{N}-U^{2}$ plane for the $N H$ case (left panel) and IH case (right panel) from [403]. Allowed regions are shown by the shaded regions with red-solid line or red-dashed line for the case with the cosmological lifetime bound $\tau_{N_{2,3}}<0.1 \mathrm{sec}$ or $\tau_{N_{2,3}}<1 \mathrm{sec}$, respectively. The hatched regions are excluded by the bounds from BNL-E949 experiment.

Consequences of $M_{I}$ values well below $1 \mathrm{MeV}$ can "only" be observed in neutrino oscillation experiments (other options include the search for neutrinoless double-beta decay and precision measurements of $\beta$-decay energy spectra. See, for example, [418-421].) In the extreme case $M_{I} \ll m_{\alpha I}$, the neutrinos are pseudo-Dirac fermions. In this scenario, all neutrino mass states are "doubled", i.e., for every massive neutrino state there is a second state with almost exactly the same mass, and all mass-eigenstates are "50-50" mixtures of active and sterile neutrinos [422]. The new masssquared differences are proportional to $M_{I}$, and solar neutrino data constrain $M_{I} \lesssim 10^{-9} \mathrm{eV}$ $[422,423]$.

For $M_{I} \gg m_{\alpha I}$, it is possible to place an experimental lower bound on $M_{I}$. This is easy to understand. As $M_{I}$ decreases, the active-sterile mixing angles $U_{\alpha I}$ increase for fixed light neutrino masses (remember $\left|U_{\alpha I}\right|^{2} \propto\left|F_{\alpha I}\right|^{2} / M_{I}$ ) while the oscillation lengths proportional to the new neutrino mass states increase (i.e., the new mass-squared differences are of order $M_{I}^{2}$ ). Oscillation experiments rule out new mass-squared differences below $10^{-1} \mathrm{eV}^{2}$, and do not tolerate new mixing angles above $10^{-1}$. Roughly speaking, $M_{I} \lesssim 1 \mathrm{eV}$ [374, 418, 423] are ruled out: hence the generic name "eV-Seesaw" for scenarios with the lowest possible $M_{I}$ that still satisfies $M_{I} \gg m_{\alpha I}$. Fig. 4.5 depicts naive expected value of the active-sterile mixing angles $\left(\sin ^{2} \theta_{a s}\right)$ as a function of $M_{I}$ (in the figure $M_{N}$ ), for different values of the light neutrino masses $m_{\nu}$. The excluded $M_{I}$ values are highlighted in the Fig. 4.5 [422]. The eV-Seesaw is severely constrained by data from cosmic surveys and big-bang nucleosynthesis (see [356] for a recent detailed analysis). At face value, cosmological constraints require all but one of the Seesaw right-handed neutrinos to have masses higher than $100 \mathrm{MeV}$, while the remaining one is only allowed to be very light in the limit that the lightest, mostly active neutrino is also very light (mass less than roughly $10^{-3} \mathrm{eV}$ ) [356]. These constraints, however, can be modified, alleviated, or eliminated if our understanding of the thermal history of the universe, or that of fundamental particle physics, is incomplete. For a concrete scenarios see, for example, [357-359].

The eV-region (i.e., $M_{I} \sim[1,10] \mathrm{eV}^{2}$ ) is particularly interesting, for several of reasons. First and foremost, for those values of $M_{I}$ the predicted values of $U_{\alpha I}$ are within reach of next-generation experiments. Hence this region will be probed experimentally in the next decade. Second, for $M_{I} \sim 1 \mathrm{eV}$, it is possible to explain the so-called short-baseline anomalies, discussed in detail in Sec. 4.2.3, with Seesaw right-handed neutrinos. This means that if the short-baseline anomalies 


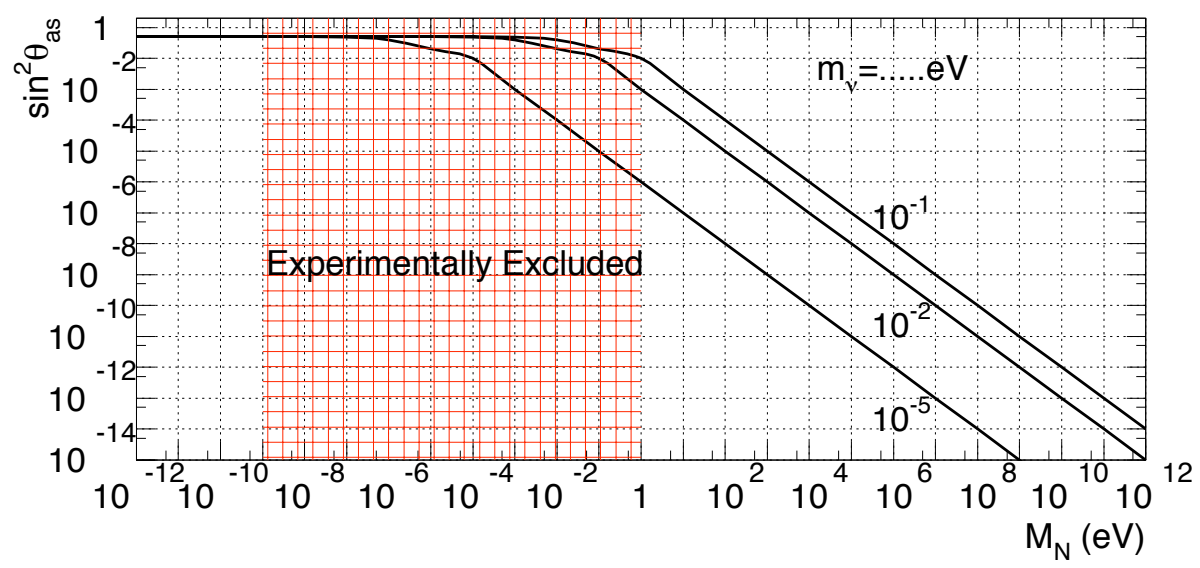

Figure 4.5: Naive expected value of the active-sterile mixing angle $\sin ^{2} \theta_{a s}$ as a function of $M_{I} \equiv$ $M_{N}$, for different values of the light neutrino masses $m_{\nu}$. The $M_{N}$ values highlighted are excluded by the neutrino oscillation data. From [422].

are indeed the consequence of new neutrino states, we may have already detected the existence of right-handed neutrinos. If this is the case (third) the Seesaw Lagrangian allows, in principle, one to test whether sterile neutrinos are, indeed, directly related to the mechanism behind the small neutrino masses. The reason is as follows. The Seesaw Lagrangian does not allow generic values for $U_{\alpha I}$. Instead, these are functions of the neutrino Yukawa couplings, the light neutrino masses $m_{\nu}$, and the heavy neutrino masses $M_{I}$. Detailed analysis have been performed in the literature, both in the context of leptogenesis [384] and in the context of the eV-Seesaw [424]. In a nutshell, once the neutrino masses are known - both the light and the heavy ones - the different $U_{\alpha I}$ are related. For a detailed discussion, we refer readers to, for example, [424], but provide a concrete example here. If $\mathcal{N}=2$ and $m_{5} \gg m_{4}=1 \mathrm{eV}$, and the neutrino mass hierarchy is inverted $\left(m_{3}^{2}<m_{1}^{2}<m_{2}^{2}\right.$, following the usual PDG prescription [156]), the following predictions arise out of the Seesaw Lagrangian, given current neutrino oscillation data:

- $\nu_{e}$ disappearance with an associated effective mixing angle $\sin ^{2} 2 \vartheta_{e e}>0.02$ should be observed. This is with reach of proposed short-baseline $\bar{\nu}_{e}$ disappearance experiments [425, 426].

- $\nu_{\mu}$ disappearance with an associated effective mixing angle $\sin ^{2} 2 \vartheta_{\mu \mu}>0.07$, very close to lower bounds from MINOS [427].

- $\nu_{\mu} \leftrightarrow \nu_{e}$ transitions with an associated effective mixing angle $\sin ^{2} \vartheta_{e \mu}>0.0004$, consistent with the sterile neutrino interpretation of the short-baseline anomalies, see Sec. 4.2.3.

- $\nu_{\mu} \leftrightarrow \nu_{\tau}$ transitions with an associated effective mixing angle $\sin ^{2} \vartheta_{\mu \tau}>0.001$. A $\nu_{\mu} \rightarrow \nu_{\tau}$ appearance search sensitive to probabilities larger than $0.1 \%$ for a mass-squared difference of $1 \mathrm{eV}^{2}$ would definitively rule out $m_{4}=1 \mathrm{eV}$ if the neutrino mass hierarchy is inverted.

\subsubsection{Beyond the minimal seesaw model}

\subsubsection{Left-right symmetric models}

In the Left-Right (LR) symmetric theory [300-303, 428] the left-handedness of the low energy weak interactions is attributed to spontaneous breaking of LR symmetry. This implies the existence of right-handed $(\mathrm{RH})$ neutrino which prophetically predicted a non-vanishing neutrino mass long before experiment. The smallness of neutrino mass is related naturally to the near maximality of 
parity violation at low energies through the seesaw mechanism [304-308]. A curse in the days of the massless neutrino dogma, turned eventually into a blessing.

The theory leads to neutrinoless double beta decay [429-431] through both light left-handed (LH) and heavy right-handed (RH) neutrinos $[308,309]$, denoted $\nu_{i}$ and $N_{I}$, respectively. It also predicts lepton number violation (LNV) at hadronic colliders, which can be used to probe directly the Majorana nature of heavy neutrinos, through the KS process [432]. This process, in a form of same sign charged di-leptons and two jets, without missing energy, is a generic feature of the existence of RH neutrinos. In the minimal version of the theory, the possible observation of this process and the measurements of the $\mathrm{RH}$ charged gauge meson and $\mathrm{RH}$ neutrino masses, allows to make precise predictions [433, 434] (see also [435-441]) for the neutrinoless double beta decay rate and the lepton flavor violating processes. Moreover, the knowledge of $\nu$ and $N_{I}$ masses suffices to predict Dirac Yukawa couplings and the associated decay rates of RH neutrinos [442]. The LR symmetric theory allows to probe the origin of neutrino mass in the same manner that one does for the charged fermions in the SM [443].

The Minimal Model. The Left-Right Symmetric Model, or Left-Right Standard Model (LRSM) is based on the $S U(2)_{L} \times S U(2)_{R} \times U(1)_{B-L}$ gauge group augmented with a LR symmetry. Quarks and leptons come in LR symmetric representations

$$
Q_{L, R}=\left(\begin{array}{l}
u \\
d
\end{array}\right)_{L, R}, \quad \ell_{L, R}=\left(\begin{array}{c}
\nu \\
e
\end{array}\right)_{L, R} .
$$

The formula for the electromagnetic charge becomes

$$
Q_{e m}=I_{3 L}+I_{3 R}+\frac{B-L}{2} .
$$

The unphysical hyper-charge is traded for the gauged $B-L$, the global symmetry of the SM.

The Higgs sector of the LRSM consists of the following multiplets [304, 308]: the bi-doublet $\Phi$ and the $S U(2)_{L, R}$ triplets $\Delta_{L}$ and $\Delta_{R}$

$$
\Phi=\left[\begin{array}{cc}
\phi_{1}^{0} & \phi_{2}^{+} \\
\phi_{1}^{-} & -\phi_{2}^{0 *}
\end{array}\right] \quad \Delta_{L, R}=\left[\begin{array}{cc}
\Delta^{+} / \sqrt{2} & \Delta^{++} \\
\Delta^{0} & -\Delta^{+} / \sqrt{2}
\end{array}\right]_{L, R}
$$

The LR symmetry was chosen originally as a generalised parity $\mathcal{P}$, although it could also be a generalised charge conjugation $\mathcal{C}$. Under these, the fields transform as follows

$$
\mathcal{P}:\left\{\begin{array}{rl}
f_{L} & \leftrightarrow f_{R} \\
\Phi & \leftrightarrow \Phi^{\dagger} \\
\Delta_{L} & \leftrightarrow \Delta_{R}
\end{array} \quad \mathcal{C}:\left\{\begin{array}{c}
f_{L} \leftrightarrow\left(f_{R}\right)^{c} \\
\Phi \leftrightarrow \Phi^{T} \\
\Delta_{L} \leftrightarrow \Delta_{R}^{*}
\end{array}\right.\right.
$$

where $\left(f_{R}\right)^{c}=C \gamma_{0} f_{R}^{*}$ is the usual charge-conjugate spinor. $308]$

The first stage of symmetry breaking down to the SM symmetry takes the following form [303,

$$
\left\langle\Delta_{L}^{0}\right\rangle=0, \quad\left\langle\Delta_{R}^{0}\right\rangle=v_{R}
$$

with $v_{R}$ giving masses to the heavy charged and neutral gauge bosons $W_{R}, Z_{R}$, right-handed neutrinos and all the scalars except for the usual Higgs doublet (the light doublet in the bi-doublet $\Phi$ ). Without assuming LR symmetry one gets for the $W_{R}, Z_{R}$ masses

$$
M_{W_{R}}^{2}=g_{R}^{2} v_{R}^{2}, \quad M_{Z_{R}}^{2}=2\left(g_{R}^{2}+g_{B-L}^{2}\right) v_{R}^{2}
$$


where $g_{R}$ and $g_{B-L}$ gauge couplings correspond to $S U(2)_{R}$ and $(B-L) / 2$, respectively. We get an immediate strict limit $M_{Z_{R}} \gtrsim \sqrt{2} M_{W_{R}}$, which guarantees that $W_{R}$ must be seen before $Z_{R}$. This is true no matter how high the scale of LR symmetry breaking.

In what follows we will use the LR symmetric gauge couplings relevant for the minimal model $g \equiv g_{L}=g_{R}$. One gets then a simplified expression

$$
M_{Z_{R}}=\sqrt{2} \frac{\cos \theta_{W}}{\sqrt{\cos 2 \theta_{W}}} M_{W_{R}} \simeq \sqrt{3} M_{W_{R}} .
$$

The new neutral gauge boson $Z_{R}$ is substantially heavier that its charged counterpart $W_{R}$, which makes it unlikely to be discovered at the LHC (see more below).

Next, the neutral components of $\Phi$ develop vevs and break the SM symmetry down to $U(1)_{e m}$

$$
\langle\Phi\rangle=v \operatorname{diag}\left(\cos \beta,-\sin \beta e^{-i a}\right)
$$

where $v$ is real and positive and $\beta<\pi / 4,0<a<2 \pi$. This gives the mass to the LH charged gauge boson $W_{L}$ (which can be identified with the $W$, since the L-R mixing is rather small), quarks and charged leptons, and to the Higgs boson. This also triggers a small, induced vev for the $\Delta_{L}$ which leads to the so called seesaw II source of neutrino mass [444],[309],[368].

Yukawa sector and the RH quark mixing matrix. The LR symmetry restricts severely the Yukawa couplings. In the case of quarks the Yukawas are either hermitian for generalised parity or symmetric for generalised charge conjugation. In the latter case this guarantees the equality of left and right quark mixing angles, with five extra arbitrary phases in the RH current. The case of $\mathcal{P}$ is more restrictive since the complex vev of (4.3.28) makes quark mass matrices nonhermitian. The search for the RH quark mixing matrix $V_{R}$ has been a great challenge for now forty years, and only recently the following analytic form valid in the entire parameter space was finally obtained $[445,446]$

$$
\left(V_{R}\right)_{i j}=\left(V_{L}\right)_{i j}-i s_{a} t_{2 \beta}\left[t_{\beta}\left(V_{L}\right)_{i j}+\frac{\left(V_{L} m_{d} V_{L}^{\dagger}\right)_{i k}\left(V_{L}\right)_{k j}}{m_{u_{i}}+m_{u_{k}}}+\frac{\left(V_{L}\right)_{i k}\left(V_{L}^{\dagger} m_{u} V_{L}\right)_{k j}}{m_{d_{k}}+m_{d_{j}}}\right]+O\left(s_{a}^{2} t_{2 \beta}^{2}\right)
$$

Remarkably, in spite of near maximal LR symmetry breaking at low energies, the LH and RH mixing angles end up being almost exactly the same, while the new RH phases depend on a single parameter $s_{a} t_{2 \beta}$ which measures the departure from the hermiticity of quark mass matrices.

Limits on the LR scale. Thus, independently of its nature, the LR symmetry guarantees almost the same production strength for $W_{R}$ and $W_{L}$, which in turn implies that the lower limits on the $W^{\prime}$ mass set by CMS and ATLAS (assuming the equal production rates) apply directly to the $W_{R}$. In particular for a large range of $N$ masses $M_{W_{R}} \gtrsim 3 \mathrm{TeV}$ [447].

In recent years there has been a resurgence of interest in the phenomenology of this theory. It has been known for a long time that the $K_{L}-K_{S}$ mass difference implies a lower limit on $M_{W_{R}}$ on the order of a few $\mathrm{TeV}[448,449]$, so that before the advent of the LHC there was little interest in studying in-depth experimental predictions. The limit on the scale was sharpened in recent years [450-452] and it depends on the choice of the LR symmetry. In the case of $\mathcal{C}$ the limit is about $3 \mathrm{TeV}$, while the case of $\mathcal{P}$ is more complicated. From the study of the electric dipole moment of the neutron, the limit was raised to about $7 \mathrm{TeV}$ [453]; however without taking into account the strong CP violation. Meanwhile new studies also improved the chiral perturbation results for this process [454-458] and recently [458] claimed a limit of about $20 \mathrm{TeV}$ by studying carefully the strong CP. This limit depends though on the UV completion of the theory [458], so that strictly one has $M_{W_{R}} \gtrsim 3 \mathrm{TeV}$, independent of what happens at high energy. Similar limit emerges from the LHC direct searches for the RH neutrino masses relevant for the KS process. From (4.3.27) this 
implies $M_{Z_{R}} \gtrsim 5 \mathrm{TeV}$, which makes it hard for $Z_{R}$ to be seen at the LHC.

Moreover, one can obtain a lower limit on the second scalar doublet residing in the bi-doublet $\Phi$, correlated to the mass of $W_{R}$, on the order of $10-15 \mathrm{TeV}$ or so [451,459]. It should be remarked that its heaviness is natural since it gets the mass at the first stage of symmetry breaking, together with $W_{R}, Z_{R}$ and $N_{I}[460]$.

Leptonic sector. In the seesaw picture the Majorana neutrino mass matrix is given by [309]

$$
M_{\nu}=M_{L}-M_{D}^{T} \frac{1}{M_{N}} M_{D},
$$

where $M_{D}$ is the neutrino Dirac mass matrix, while $M_{L} \propto M_{W_{L}}^{2} / M_{W_{R}}$ and $M_{N} \propto M_{W_{R}}$ are the symmetric Majorana mass matrices of left- and right-handed neutrinos, respectively. The above formula connects the smallness of neutrino mass to the scale of parity restoration at high energies.

Since $\mathcal{C}$ can be gauged in four dimensions (in the $\mathrm{SO}(10)$ grand unified theory it is an automatic finite gauge transformation), in what follows we will opt for it unless specified accordingly. This in turn implies symmetric Dirac mass matrices $M_{D}=M_{D}^{T}$. Thus $M_{D}$ can be obtained [442] directly from (4.3.30)

$$
\begin{aligned}
& M_{L}=\frac{v_{L}}{v_{R}} M_{N} \\
& M_{D}=M_{N} \sqrt{\frac{v_{L}}{v_{R}}-\frac{1}{M_{N}} M_{\nu}},
\end{aligned}
$$

and thereby one can determine the mixing between light and heavy neutrinos [442]. Since $M_{\nu}$ and $M_{N}$ can be measured, at least in principle, the only real is unknown is $v_{L}$. The essential point is that the knowledge of $M_{D}$, or equivalently $Y_{D}$ allows for a direct probe of the Higgs-Weinberg origin of the neutrino mass, in analogy with the charged fermions in the SM. Simply, one can predict the associated Higgs (or $W$ ) decays into $N_{I}$, or vice versa if $N_{I}$ are heavy enough [442].

The situation in the case of $\mathcal{P}$ is more subtle, but similar constraints emerge and again Dirac Yukawas get determined [461]. Actually the situation is even maybe more interesting since the system is even more constrained, just as in the quark sector where the $\mathrm{RH}$ mixing matrix is much more constrained that in the case $\mathcal{C}$. However, we have managed to obtain analytic formulas only for the two-generation case, the three generation case is under study [461].

Lepton Number Violation. The Majorana nature of $\nu$ and $N$ implies Lepton Number Violation (LNV). The low energy process is the text-book example of neutrinoless double beta decay. In this theory it is induced by the usual exchange of light Majorana neutrinos and the $W$ boson, and also by its $\mathrm{RH}$ analog exchange of $N_{I}$ and $W_{R}$ as noticed already in [308]. The new contribution can easily dominate the usual neutrino one, and moreover, if neutrino masses turn out to be small, it may be necessary in case this process gets observed in near future. If that were to happen, the $W_{R}$ mass would have to lie tantalisingly close to the LHC reach [433, 434].

The total $0 \nu \beta \beta$ rate can be described by the effective mass parameter [433]

$$
\left|M_{\nu+N}^{e e}\right|=\sqrt{\left|\left(M_{\nu}\right)_{e e}\right|^{2}+\frac{\left|\left(V_{R}\right)_{11}\right|^{4}}{\left|\left(V_{L}\right)_{11}\right|^{4}} \frac{M_{W_{L}}^{8}}{M_{W_{R}}^{8}}\left|\left(\frac{k^{2}}{M_{N}}\right)_{e e}\right|^{2}}
$$

where $k$ is a measure of the neutrino virtuality, $M_{\nu}$ and $M_{N}$ are the mass matrices of light and heavy neutrinos, respectively.

Even more exciting is the KS process, the high energy version of neutrino-less double beta decay: the hadronic collider production of $N$ with the subsequent LNV decay into two same charged leptons [432], as in the Fig. 4.6. The KS process is based on the usual Drell-Yan production 


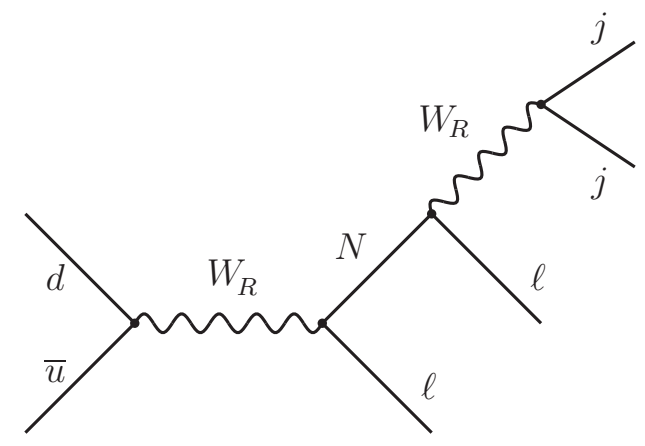

Figure 4.6: Production of lepton number violating same sign di-leptons through the production and subsequent decay of $N$, the KS process. In the LR model the dominant effect is through the on-shell production of $W_{R}$; it can occur also through the $\nu-N$ mixing and the usual $W$ exchange, but that requires huge $M_{D}$.

mechanism for the charged gauge bosons; what is new is the possibility of establishing directly the Majorana nature of $N$ since then both same and opposite sign charged leptons decay products occur with the same probability. It should be stressed that this has become the paradigm for LNV at the hadronic colliders, and it occurs in basically any theory that leads to Majorana light neutrinos.

One can in principle probe both $W_{R}$ and $N$ masses, and also the RH leptonic mixing angles [462, 463], the analog of the PMNS ones. The detailed studies for the LHC were performed in [464, 465] where it was argued that high luminosity of $300 \mathrm{fb}^{-1}$ reach goes all the way to $5-6 \mathrm{TeV}$ (for a roadmap at the LHC, see [466]). Moreover, one can measure the chirality of $N_{I}$ couplings and establish their RH nature [464, 467]. For reviews of this subject, see [468-470]. Since the theory predicts neutrino Dirac Yukawa, this suffices to probe the origin of neutrino masses through the seesaw and the Higgs mechanism.

A question could be asked as what happens if the $N$ masses are small, so small that one ends up effectively with the case of Dirac neutrinos. It requires extremely small Dirac Yukawas for neutrinos, not natural and hard to achieve in the minimal model [471]. For this reason we ignore this possibility; after all the seesaw mechanism emerged as a solution to this unappealing and un-natural scenario.

Probing the Dirac Yukawa couplings at Colliders [442]. As we argued before, the LR symmetric theory not only led to the seesaw mechanism, but it allows for its probe. Through the KS process the RH neutrino mass matrix can be determined in principle, and in order to complete the job all one needs is to measure the neutrino Dirac mass matrix $M_{D}$. Since the LH and RH neutrinos mix through $M_{D}$, it suffices to look for the $N$ decays into SM particles, as in Fig. 4.7.

The crucial thing is that $N$, besides decaying through virtual $W_{R}$ as discussed above, decays also into the left-handed charged lepton through $M_{D} / M_{N}$.

In a physically interesting case when $N$ is heavier than $W_{L}$, which facilitates its search through the KS process, the decay into left-handed leptons proceeds through the on-shell production of $W_{L}$. For the sake of illustration we choose an example of $V_{R}=V_{L}^{*}$ in which case one can estimate the ratio of $N$ decays in the $W_{L}$ and $W_{R}$ channels [442]

$$
\frac{\Gamma_{N \rightarrow \ell_{L} j j}}{\Gamma_{N \rightarrow \ell_{R} j j}} \simeq 10^{3} \frac{M_{W_{R}}^{4}}{M_{W_{L}}^{2} m_{N}^{2}}\left|\frac{v_{L}}{v_{R}}-\frac{m_{\nu}}{m_{N}}\right|,
$$

which is about a permil for naturally small $v_{L}$. The branching ratios for the Higgs-Weinberg and SM gauge bosons are shown in Fig. 4.7 (the SM bosons $W, Z, h$ can decay into a lighter $N$, but the 


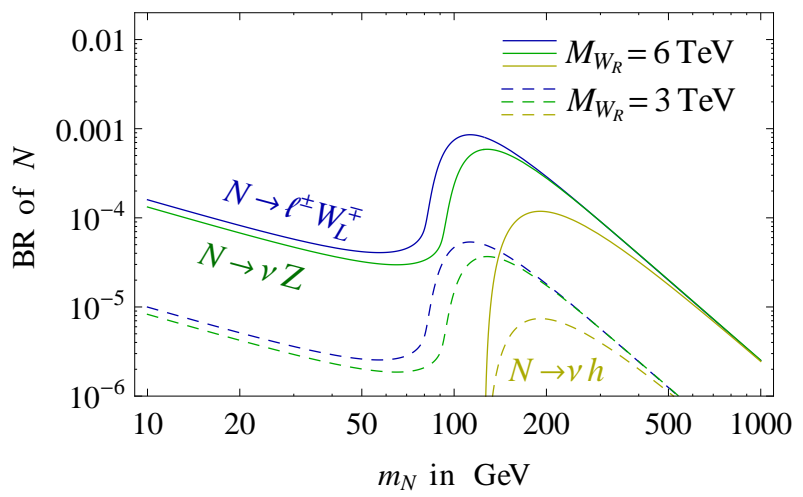

Figure 4.7: Branching ratio for the decay of heavy $N$ to the Higgs-Weinberg and SM gauge bosons, proceeding via Dirac couplings, exemplified $v_{L}=0$ and $V_{R}=V_{L}^{*}$. The solid (dashed) line corresponds to $M_{W_{R}}=6(3) \mathrm{TeV}$.

small couplings make the corresponding branching ratios too tiny to matter at this point).

The issue here is how to observe these rare channels. Ideally, one should measure the chirality of the outgoing charged lepton $[464,467]$ and/or establish the kinematics of the two jets associated with the on-shell production of $W_{L}$. This may be a long shot, but could still be feasible for the LHC with a luminosity in the hundreds of $\mathrm{fb}^{-1}$. The bottom line is that this probes in principle all the matrix elements of $M_{D}$, once the heavy neutrinos are identified through their dominant $W_{R}$ mediated decays. This offers a clear program of bringing the issue of the origin of neutrino mass to the same level of other fermion masses in the SM.

We wish to stress once again that in the LRSM model the neutrino Dirac mass matrix is predicted to take generic seesaw values (4.3.32), except at singular points when the square root is not well defined and can take large values [442]. We shy away from these pathologies, since they are not stable. They can also be achieved by discrete symmetries, but again the symmetries must be exact, which is a mathematical and not a physical possibility (exact global symmetries are not well defined, since there are no zeroes in experiment). This was studied in [438] and argued that production and subsequent decay of $\mathrm{N}$ through the $W$ boson can of course be dominant. However, as we argue here this is by no means necessary in order to measure $M_{D}$; if anything, the $N$ production through $W_{R}$ facilitates the necessary determination of $M_{N}$. In any case, the essential point is the possibility of directly producing $\mathrm{RH}$ neutrinos and probing their Majorana nature independently of the details of production and decays of $N$ in the KS process. The smoking-gun signature is universal: same sign di-leptons and di-jets.

One will wish of course, to probe also the origin of $N$ masses which requires the production of $\Delta_{R}^{0}$ and its decays to two $N$ 's. However, $\Delta_{R}^{0}$ may be too heavy to be produced at the LHC in which case one may look for its effects through the mixing with the SM higgs. This was recently studied in [472].

Dark matter. Just as in the $\nu \mathrm{MSM}$, one can have a warm dark matter in a form of a light sterile neutrino $N_{1}$ with a mass around $\mathrm{keV}[473,474]$. Clearly, there must be a possibility of heavy $W_{R}$ [473], but it is interesting that there is a narrow window for $M_{W_{R}} \simeq 5 \mathrm{TeV}$ [474], accessible to 
the LHC. In any case the masses and mixings of $N_{I}$ are completely predicted [474]

$$
V_{\ell i}^{R} \approx\left(\begin{array}{ccc}
0 & 0 & 1 \\
0 & 1 & 0 \\
1 & 0 & 0
\end{array}\right), \quad \begin{aligned}
& m_{N_{1}} \sim \mathrm{keV}, \\
& m_{N_{2}} \sim m_{\pi}+m_{\mu}, \\
& m_{N_{3}} \sim m_{\pi}+m_{e} .
\end{aligned}
$$

In other words, one ends up with a flavor-diagonal $\mathrm{RH}$ leptonic mixing matrix. The $N_{I}$ are too light for the KS process to be operative, and at the LHC they are seen as the missing energy. The Yukawa Dirac couplings get predicted from (4.3.32). The bottom line is the exciting prediction for the $W_{R}$ to be seen at the LHC.

Baryogenesis. A question often asked: can LRSM account for baryogenesis? First of all, can the usual leptogenesis through the out-of-equilibrium decays of $N$ work here? Well, for heavy enough $W_{R}$ it obviously must work due to the decoupling arguments; however for light $W_{R}$ the $B-L$ violating process involving the $N_{I}$ remain in equilibrium and wash out the $B-L$ number of the universe. A careful analysis shows that the leptogenesis can work for $M_{W_{R}} \gtrsim 20 \mathrm{TeV}$ [475], far above the LHC reach.

Since $B+L$ is washed out by sphalerons, one has a situation where any initial lepton and baryon numbers are washed out, and must be produced during or after the EW phase transition. This is to be contrasted with the SM where the question of genesis cannot be answered, since it depends on the initial $B-L$ number.

What about the EW baryogenesis? The second SM scalar doublet from the bi-doublet $\Phi$, which normally suffices, effectively decouples however at the usual EW phase transition due to its large mass. The only hope are light enough $\Delta_{L, R}$ fields which would allow for the first order phase transition [476]. There are basically no lower limits on their masses, so that this is definitely feasible; the only question whether there is enough CP violation in their couplings to the SM Higgs field (the light component of the bi-doublet). This is now under investigation.

Discrete symmetry breaking: domain wall problem. It is well known that the spontaneous breaking of a discrete symmetry leads to an infamous domain wall problem, which needs to be solved one way or another. A canonical way out is imagined to be inflation, but for a LHC accessible scale of LR symmetry it may not be available. One possibility may be provided by the phenomenon of symmetry non-restoration at high temperature $[477,478]$ which impedes the production of domain walls [479, 480], or at least makes it happen at very high temperature [481], so that the inflation can safely take place. Even if this were not to work [482], the naturally expected tiny breaking of global symmetries by Planck scale effects can be shown to suffice to rid of the domain walls [483].

Large scale of LR symmetry breaking. One can always imagine that $M_{W_{R}}$ is large enough so that $W_{R}$ is decoupled at low energies. If the same is true of all other states of the theory, except for $N_{I}$, we end up with the analog situation of the SM augmented with the seesaw mechanism, but with a residual LR symmetry. One loses though a plethora of physical phenomena that result from the gauge structure of the LRSM. In such a case the $N_{I}$ states would have to be produced through the Dirac Yukawa couplings which allow them to couple to the $W$ boson [313, 484-491]. However, as long as the LR breaking scale is not astronomical, these Yukawas are too small to allow for the observable KS process.

A way out is to give up completely the LR symmetry, although it was originally the raison d'être for the existence of RH neutrinos. In this case the Dirac Yukawas are not determined by the seesaw formula, and depend on the arbitrary complex orthogonal matrix $O$, whose elements can be as large as one wishes [384]. The KS process is then possible, but with large Dirac Yukawas the smallness of neutrino mass becomes an accident, contrary to the idea of the seesaw as a natural explanation behind small neutrino mass. It is also possible that $N_{I}$ induce neutrinoless double beta 
decay [492], if one is willing to pay this big price.

In short, one trades a complete and predictive theory of neutrino mass and associated phenomena for an a posteriori tailor-made model based on pure phenomenological considerations. One can fix the Yukawa Dirac couplings though by asking for dark matter and baryogenesis on top of neutrino mass, as in the $\nu$ MSM. It is often argued that the $\nu \mathrm{MSM}$ is the minimal scheme behind these phenomena; true if you use a number of states and not the structural predictions as a measure of minimality.

\subsubsection{Left-right symmetric models with GeV-scale HNLs}

At low energies the consequences of LR symmetric models are related mainly to right-handed currents mediated by $W_{R}$ bosons. If the right-handed neutrinos have a Majorana mass, they could be observed at the LHC as sterile neutrinos and the Majorana nature can be proved by searching for like-sign leptons plus two jets $[447,493]^{4}$. On the other hand, neutrinoless double beta decay $(0 \nu \beta \beta)$ and the LFV decay $\mu \rightarrow e \gamma$ may also prove parameters associated with LR symmetric models. The experimental results of $0 \nu \beta \beta$ give limits for ${ }^{136} \mathrm{Xe}[497]$ and ${ }^{76} \mathrm{Ge}[498]$ in excess of $10^{25}$ ys, which correspond to a lower limit on the $W_{R}$ mass of roughly $m_{W_{R}} \gtrsim 7 \mathrm{TeV}[451,499,500]$, for masses $m_{N} \sim 1 \mathrm{GeV}$ and $g_{R}=g_{L}$. However, this limit is only sensitive to the mixing of heavy neutrinos $N$ in the electron sector. Moreover, the non-observation of $\mu \rightarrow e \gamma$ imposes also constraints on LR symmetric models, however for masses $m_{N} \sim \mathrm{GeV}$ these bounds are very weak and not comparable to those coming from $0 \nu \beta \beta$ [462].

The SHiP experiment, which is sensitive to heavy neutrinos with masses of the order $m_{N} \sim \mathrm{GeV}$ and large decay lengths, could probe LR symmetric models in a parametric region different than the LHC and $0 \nu \beta \beta$. In particular, the production $D_{s} \rightarrow \mu N$ and the subsequent decay of a heavy neutrino $N \rightarrow \mu \pi$ shown in Fig. 4.2, can be produced in a LR symmetric model through the mediation of a $W_{R}$ boson, as it is shown in Fig. 4.8.

In the "manifest" LR symmetry scenario, i. e. $g_{R}=g_{L}$, the decay length can be written as a function of the masses $m_{N}$ and $m_{W_{R}}[496]$

$$
L=c \bar{\gamma} \tau_{N} \simeq 12 \bar{\gamma}\left(\frac{1 \mathrm{GeV}}{m_{N}}\right)^{5}\left(\frac{m_{W_{R}}}{1 \mathrm{TeV}}\right)^{4}[m]
$$

Therefore, for masses $m_{W_{R}}$ of the order of TeV, the decay length is large enough to be detected by SHiP. Here we have assumed a maximal mixing of the heavy neutrino $N$ in the muon sector.

The number of signal events at SHiP are suppressed by a factor of $\left(m_{W_{L}} / m_{W_{R}}\right)^{8}$. This is because the production of heavy neutrinos $D_{s} \rightarrow \mu N$ is suppressed by $\left(m_{W_{L}} / m_{W_{R}}\right)^{4}$ and since only a small part of heavy neutrinos $N$, proportional to $\tau_{N}^{-1}$, decays inside the detector, an additional suppression $\left(m_{W_{L}} / m_{W_{R}}\right)^{4}$ appears. From the experimental point of view, this process gives the same signal in a LR symmetric model or in a sterile neutrino extension of the SM, where instead, the number of signal events are suppressed by a factor of $\left|U_{\mu I}\right|^{4}$. Therefore, the SHiP limits on the mass $m_{W_{R}}$ (see Fig. 4.9) can be extracted from the SHiP limits on $\left|U_{\mu I}\right|^{2}$, shown in Fig. 4.11, by doing the simple conversion $\left|U_{\mu I}\right|^{2} \rightarrow\left(m_{W_{L}} / m_{W_{R}}\right)^{4}$.

Currently the LHC has imposed limits on the mass $m_{W_{R}} \gtrsim 3.0 \mathrm{TeV}$ [447] in a mass range $m_{N} \sim(0.2-2.0) \mathrm{TeV}$. These limits are expected to be extended in future searches up to $m_{W_{R}} \gtrsim$ $6 \mathrm{TeV}$ [468]. On the other hand, the SHiP experiment could access masses up to $m_{W_{R}} \sim 15 \mathrm{TeV}$ for heavy neutrinos with masses $m_{N} \sim 1 \mathrm{GeV}$ (see Fig. 4.9), making it interesting and complementary to LHC results. ${ }^{5}$

\footnotetext{
${ }^{4}$ Generic processes of this kind have been discussed in [494, 495] as tree-level high-energy completions of lepton number violation operators that generate neutrinoless double beta decay. Moreover, the authors of Ref. [496] studied the possibility of searching for heavy neutrinos at the LHC using displaced vertices.

${ }^{5} \mathrm{~A}$ detailed study of LR symmetric models in meson and tau decays will be given in a future paper.
} 

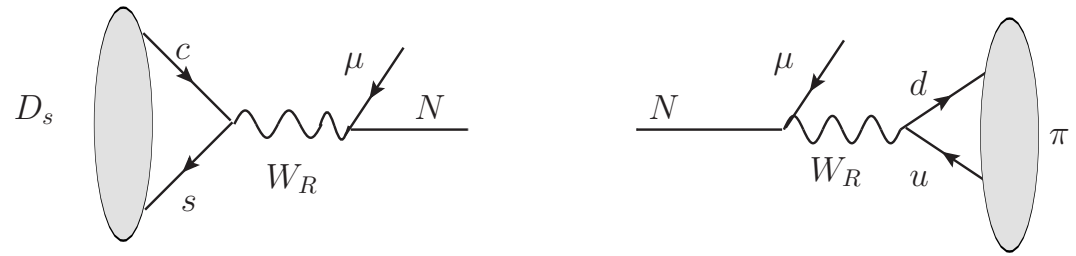

Figure 4.8: Heavy neutrino production (a) and decay (b) at SHiP according to the LR symmetric model.

\subsubsection{Inverse seesaw and GeV scale singlet fermions}

In contrast to type I seesaw, if the SM singlet fermions introduced to understand neutrino masses are Dirac fermions i.e. the singlet fermions come in pairs that form a Dirac mass, then the situation is very different. To see this, we introduce two SM singlet fermions $(N, S)$ per family and write the following Lagrangian:

$$
\mathcal{L}_{\mathrm{ISS}}=F(\bar{L} \cdot \Phi) N+M_{N} \bar{N}^{C} S+\mu \bar{S}^{C} S+\text { h.c. }
$$

This leads to $(\nu, N, S)$ mass matrix of the form:

$$
\mathcal{M}_{\nu, N, S}=\left(\begin{array}{ccc}
0 & F v & 0 \\
F v & 0 & M_{N} \\
0 & M_{N} & \mu
\end{array}\right)
$$

Neutrino mass formula in this case is (again, denoting $m_{D}=F v$ ):

$$
m_{\nu} \simeq m_{D} M_{N}^{-1} \mu M_{N}^{-1} m_{D}
$$

This is known as the inverse seesaw formula for neutrino masses [501, 502]. In this model, the light neutrinos are Majorana fermions but the lepton number violating scale is given by $\mu$ which takes a small value (of order $\mathrm{keV}$ or less). This mechanism is therefore structurally very different from type I seesaw and as such allows lighter $(\sim \mathrm{GeV})$ singlet neutrinos more easily. This lightness enhances the testability of this scenarios in areas such as lepton flavor violation [503-505], leptonic non-unitarity [506]. These models can also be embedded in $\mathrm{SO}(10)$ grand unified theories without any obvious need for fine tuning of coupling parameters to obtain light neutrino masses [507].

It is worth pointing out that inverse seesaw for neutrinos emerges in a natural way in a class of mirror models for dark matter, that were considered recently [508] in a framework where the presence of a heavy right handed neutrino portal to the dark sector explains why the matter and dark matter abundances are of same order. Both the matter and dark matter asymmetry originates from conventional high scale leptogenesis mediated by the heavy right handed neutrino connecting both sectors [509]. A consequence of this set-up is that the neutrinos of the mirror sector end up as light sterile neutrinos with two of them having masses in the $100 \mathrm{MeV}$ to $\mathrm{GeV}$ range that mix with the active neutrinos. Due to the presence of a mass for the heavy right handed neutrinos connecting the mirror sector to the visible sector, the active neutrino masses arise in the model out of radiative one loop effects suggested in [510,511]. These models can be tested via the searches for $\mathrm{MeV}$ to $\mathrm{GeV}$ singlet fermions. 


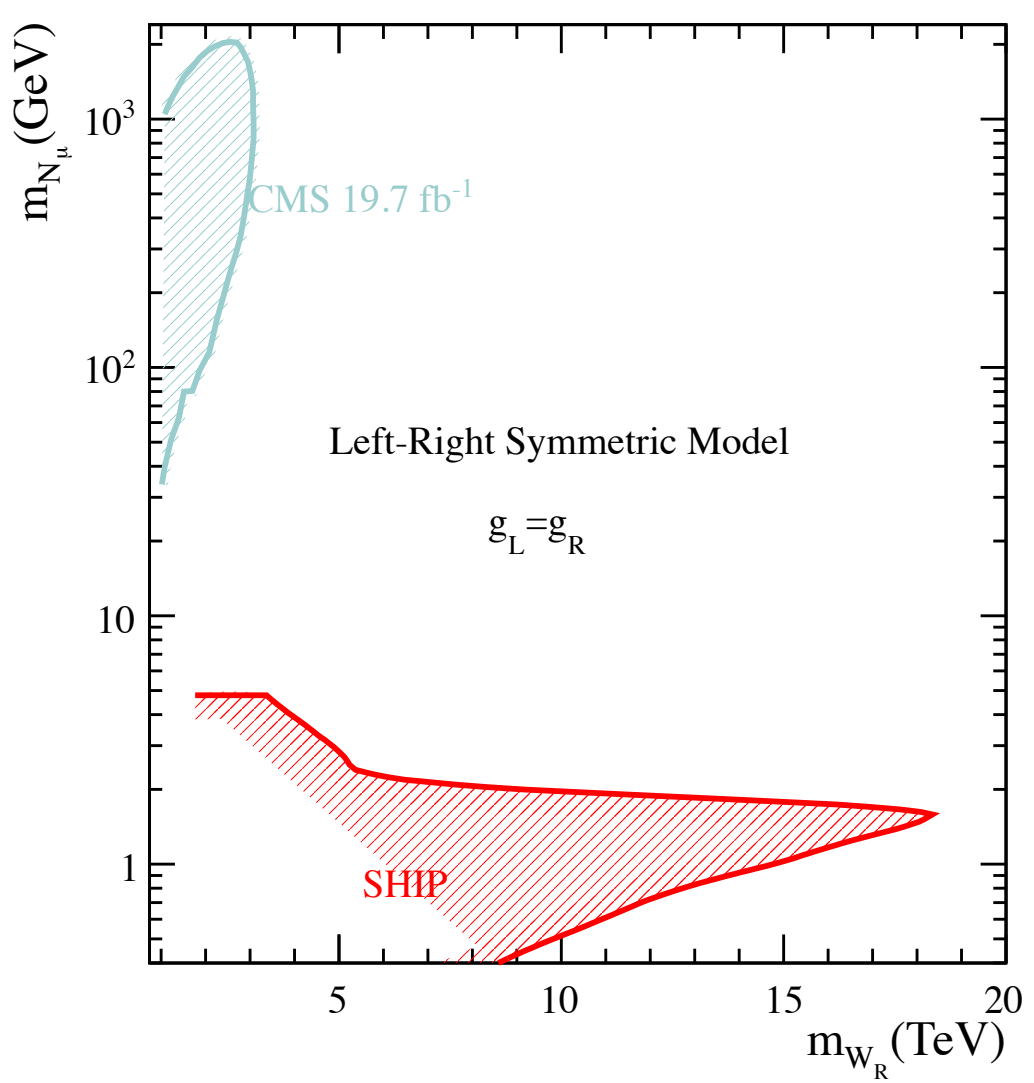

Figure 4.9: Comparison between constraints on LR model from CMS [447] versus HNLs searches at SHiP (from [34]). For details, see text.

\subsubsection{Seesaw I vs inverse seesaw singlet neutrinos at SHiP}

In this section, we look at its possible tests for both type I and inverse seesaw singlet neutrinos at the SHiP experiment, whose goal is to look for light singlet neutrinos with mass of order one $\mathrm{GeV}$ or less in the decay of D-mesons produced at the CERN SPS. In principle in both the cases, the singlet lepton can be in the in the $\mathrm{GeV}$ mass range. However, in the case of type I seesaw, this would require $|F| \sim 10^{-7}$ whereas, the situation is much less constrained in the inverse seesaw (ISS) case. In the later ISS scenario, the smallness of neutrino mass is dictated by the small parameter $\mu$. If we choose $\mu \sim \mathrm{eV}$, then, we could entertain $m_{D} \sim M_{N}$. Even within the grand unified scenario [507], where a plausible choice by quark-lepton unification, one would expect Dirac masses of the order of quark masses, $\left(m_{D}\right)_{1,2,3} \sim m_{u, c, t}$, there would be at least two singlet neutrinos with masses of order GeV. However, if $m_{D} \sim M_{N}$, in the ISS case, it would lead to large corrections to leptonic non-unitarity on which there are strong limits [506]. For instance, the limit on $\left(m_{D}\right)_{11} / M_{N_{1}} \leq 0.05$ which would require that $M_{N_{1}} \geq 20 m_{u}$ or larger than $100 \mathrm{MeV}$.

Coming now to the decay final states, the final states in D-decays can provide a way to distinguish between type I and inverse seesaw scenarios. The goal is to look for $D \rightarrow\left(K^{-}, \pi^{-}\right)+e^{+}+N$ with $N$ subsequently decaying to charged leptons. So in the overall set up, one would look for a dilepton final state. In the case of type I seesaw, the $N_{e}$ will decay with equal branching ratio to both $e^{ \pm}$, so that one would expect both like sign and unlike sign dileptons $\left(e^{-} e^{ \pm}\right)$with equal frequency due to maximal breaking of lepton number. On the other hand, in the case of inverse seesaw, $N$ will decay only to $e^{-}$since the theory mostly conserves lepton number expect for the 
tiny breaking by $\mu$. One would therefore expect only unlike sign dileptons in the D-decay. To estimate the $N$ decay rates, one may look at the current bounds on $U_{e 4}$ in Ref. [313]. Specially important to notice that near $0.5-1 \mathrm{GeV}$, type I singlet lepton will have a mixing severely constrained $\left|U_{e 4}\right| \leq 10^{-4}$ due to neutrinoless double beta decay whereas no such constraint exists on the ISS singlet neutrino. It is only constrained by the peak searches to be $\leq 10^{-3}$ or so.

\subsubsection{Possible origins of the keV-MeV-GeV scale of HNL masses}

Here and below, we use the terms "heavy neutral lepton" and "intermediate-mass neutrino" interchangeably. In the conventional approach to understanding the observed light neutrino masses, one assumes a grand unified theory (GUT). It is also conventional to assume that this is a supersymmetric GUT, since this makes possible gauge coupling unification and can solve the gauge hierarchy problem of the Standard Model (SM). In this approach, Yukawa couplings generate GUT-scale masses for three electroweak-singlet neutrinos, together with a set of Dirac mass terms of order the electroweak (EW) scale and below linking the EW-doublet neutrinos and EW-singlet neutrinos. The diagonalization of the resultant joint Majorana-Dirac mass matrix gives rise to a splitting in the mass eigenvalues of the resultant neutrino mass eigenstates, with one set having GUT-scale masses and the other set having masses given by the eigenvalues of the matrix $M=M_{D} M_{R}^{-1} M_{D}^{T}$, where $M_{D}$ and $M_{R}$ are the Dirac and right-handed Majorana mass matrices. These light neutrino eigenvalues are generically of the form $m_{\nu} \sim m_{D}^{2} / m_{R}$, where $m_{D}$ and $m_{R}$ denote relevant entries in the mass matrices $M_{D}$ and $M_{R}$. For example, with the normal mass hierarchy, for the third generation, with $m_{R} \sim 10^{15} \mathrm{GeV}$ and $m_{D} \sim 2.2 \times 10^{2} \mathrm{GeV}$, one gets $m_{\nu_{3}} \sim 0.05 \mathrm{eV}$, in agreement with the inference of $m_{\nu_{3}} \simeq 0.05 \mathrm{eV}$ from the measured $\left|\Delta m_{32}^{2}\right| \simeq 2.5 \times 10^{-3} \mathrm{eV}^{2}$, where $\Delta m_{i j}=m_{\nu_{i}}^{2}-m_{\nu_{j}}^{2}$.

However, an important property of the seesaw mechanism is that it is invariant under the rescaling

$$
M_{D} \rightarrow z M_{D}, \quad M_{R} \rightarrow z^{2} M_{R}
$$

where $z$ denotes the rescaling factor. Consequently, one can fit the measured value of $\left|\Delta m_{32}^{2}\right|$ mentioned above, and the other measured neutrino mass squared difference, $\Delta m_{21}^{2}=0.8 \times 10^{-4} \mathrm{eV}^{2}$, with values of entries in the matrices $M_{D}$ and $M_{R}$ much lower than the values in the conventional GUT seesaw. Such a theory is commonly called a low-scale seesaw model.

An explicit model with a low-scale seesaw was presented in $[512,513]$ and discussed further in [514-516]. This was a theory with dynamical electroweak symmetry breaking (EWSB). In such models, EWSB occurs as a consequence of the formation of a bilinear condensate of EW-nonsinglet fermions subject to a new gauge interaction, whose coupling gets strong at the TeV scale. To be viable, such models are constructed so that the renormalization group behavior of the new gauge interaction has an approximate infrared fixed point and resultant quasi-scale-invariant behavior. This quasi-dilation symmetry is broken spontaneously by the formation of the bilinear condensate of the new fermions, yielding a light approximate Nambu-Goldstone boson, the dilaton. In these models the $125 \mathrm{GeV}$ Higgs-like scalar boson observed by the ATLAS and CMS experiments at the Large Hadron Collider (LHC) at CERN is a composite particle with a large dilaton component. Although current LHC measurements of the production and decay of the Higgs-like scalar are consistent with it being the fundamental Higgs boson of the Standard Model, they also allow some deviations from SM predictions at the 10-30 percent level, so that a composite structure for this particle remains a possibility. More data from the future runs of the LHC will constrain this compositeness possibility further.

In the model of $[512,513]$, the quark, charged lepton, and neutrino masses are generated dynamically by loop diagrams involving exchanges of a variety of different massive gauge bosons resulting from the breaking of a gauge symmetry in which the generational quantum number is 
gauged. This generational symmetry breaks in three stages, corresponding by inverse powers to the three families of SM quarks and leptons. Thus, diagrams involving virtual exchanges of vector bosons whose masses result from the highest generational gauge symmetry breaking scale, $\Lambda_{1} \sim 10^{3}$ $\mathrm{TeV}$, are responsible for the masses of the $u$ and $d$ quarks and the electron, while diagrams involving virtual exchanges of vector bosons whose masses result from the lowest breaking scale, $\Lambda_{3} \sim$ few $\mathrm{TeV}$, are responsible for the masses of $t$ and $b$ quarks and the $\tau$ lepton, and similarly with the second generation and a breaking scale $\Lambda_{2} \sim 10^{2} \mathrm{GeV}$. The Majorana masses of the largely EW-singlet neutrinos also arise via loop diagrams, and at least one of these could be of order the GeV scale. The low-scale seesaw of $[512,513]$ results because there is similar suppression of Dirac neutrino masses for the neutrinos, so that, in accordance with the general rescaling property (4.3.40), the model can fit the observed neutrino masses and mixing. Although the model of $[512,513]$ is not fully realistic, its low-scale neutrino mass seesaw is of considerable interest and is testable in the SHiP experiment. The intermediate-mass neutrinos, when expressed as linear combinations of weak eigenstates, are mainly EW-singlets ("sterile"). That is, the associated couplings $\left|U_{e I}\right|,\left|U_{\mu I}\right|$, and $\left|U_{\tau I}\right|$ are predicted to be quite small.

The models with keV-GeV sterile neutrino emerge in a straightforward manner if the righthanded neutrinos are charged under a new symmetry broken at the PeV scale [517]. Sterile neutrinos with the $\mathrm{MeV}-\mathrm{GeV}$ masses generically appear in the low scale gauged $U(1)_{B L}$ extension of the standard model [518] or other gauge extensions of the Standard Model [473]. The origin of HNL with the mass $>100 \mathrm{MeV}$ are discussed in the context of the extended double seesaw model for neutrino mass spectrum and low scale leptogenesis [519] Sub-GeV sterile neutrinos that arise in the 5-dimensional Randall-Sundrum-like models [520,521]). Ref. [522] discusses leptogenesis and neutrino oscillations in the classically conformal Standard Model with the Higgs portal. GeVscale Majorana masses are induced by the Coleman-Weinberg field and lead to the generation of active neutrino masses through the standard see-saw mechanism (mass range $200 \mathrm{MeV}-500 \mathrm{GeV}$ is possible). A number of theoretical explanations based on the underlying symmetries was put forward to predict the spectrum with HNLs in both $\mathrm{keV}$ and $\mathrm{GeV}$ masses in radiative seesaw models [523], or by imposing additional global symmetries [315, 524-527]. Intermediate-mass neutrinos also have important astrophysical and cosmological consequences (see e.g. [399, 528] or the books $[529,530]$ for reviews).

\subsection{Direct HNL searches}

Sterile neutrinos mix with active ones, so that mass and gauge (flavour) states in the neutral fermion sector are misaligned. At small mixing the almost-active-neutrino mass states contain tiny amount of sterile neutrinos. Therefore, the mass eigenstates in sterile neutrinos effectively couple to SM particles through the weak interactions provided by the small but nonzero mixing $U_{\alpha I}$, Eq. (4.3.4). Actually because of this mixing HNLs participate in any weak processes the active neutrino do, but the strength is accordingly suppressed by the sterile-active mixing angles, and kinematics reflects the fact that sterile neutrinos are appreciably massive particles. For a particular weak process one can select a region in the phase space where an associated to sterile neutrino kinematics feature gets mostly pronounced. These features are exploited in direct searches for sterile neutrinos.

\subsubsection{Direct signatures of HNL: kink searches; peak searches}

The models with sifficiently light sterile neutrinos may be probed in nuclei $\beta$-decays, e.g.

$$
\mathrm{T} \rightarrow{ }^{3} \mathrm{He}+e+(\bar{\nu}, N)
$$


The natural observable here is the spectrum of outgoing electrons, which is proportional to the following kinematical factor [531, 532]

$$
\left|U_{e I}\right|^{2} \sqrt{\left(E_{0}-E_{e}\right)^{2}-M_{I}^{2}}+\left(1-\left|U_{e I}\right|^{2}\right)\left(E_{0}-E_{e}\right)
$$

with electron energy $E_{e}$ and the $\beta$-spectrum endpoint $E_{0}$. At $E_{e}<E_{0}+M_{I}$ only the second term in (4.4.1) contributes, which comes from decays into active neutrino, while at higher energies $E_{e}$ sterile neutrinos contribute as well. Thus at critical energy $E_{e}=E_{0}+M_{I}$ one expects a kink in the electron spectrum. Its absence places an upper limit on sterile-electron neutrino mixing $\left|U_{e I}\right|^{2}$ for a given mass of sterile neutrino $M_{I}$. The relevant mass range here is below $\mathrm{MeV}$, that is beyond the scope of SHiP. The present limits and references may be found in [156], see also Fig. 2 in Ref. [313].

Sterile neutrinos of masses above $\mathrm{MeV}$ range are searched for in weak decays of charged heavy leptons $(\mu, \tau)$ and hadrons (mesons and heavy flavor baryons). The most promising here are twobody decays of electrically charged mesons into charged leptons and neutrino (dubbed leptonic decays), $X \rightarrow l N$, suggested for testing sterile neutrino models in 1980s [310,531]. There for a fixed meson momentum one expects two peaks in the charged lepton spectrum: one is for active neutrino in the final state, while another one is for sterile neutrino in the final state ${ }^{6}$. In the meson rest frame the charged lepton spectrum is monotonic with two lines at 3-momenta

$$
p_{e}=\frac{M_{X}^{2}+m_{l}^{2}}{2 M_{X}} \quad \text { and } \quad p_{e}=\frac{\sqrt{\left(\left(M_{X}-M_{I}\right)^{2}+m_{l}^{2}\right)\left(\left(M_{X}+M_{I}\right)^{2}+m_{l}^{2}\right)}}{2 M_{X}} .
$$

Note that for sterile neutrino heavier than the charged lepton the lepton mass in the chirality suppression factor inherent in leptonic decay rate is replaced with sterile neutrino mass [531], which makes electron and muon final states equally interesting. Searches for the second peaks in charge lepton spectrum have been performed at KEK, SIN/PSI, TRIUMF and BNL in the channels $K \rightarrow \mu N$ [533-535]; $K \rightarrow e N$ [536]; $\pi \rightarrow \mu N$ [537-539]; $\pi \rightarrow e N$ [540-543]; see also [156] for more references. The present limits on active-sterile mixing in the mass range relevant for SHiP are outlined in Figs. 4.10, 4.11. At small neutrino masses precise measurement of pion partial decay width into electron and neutrino (called $\mu$-e universality) done in TRIUMF $[542,544]$ by observing the electrons of "standard energy" $E_{e} \approx M_{\pi} / 2$ also places limits on mixing of HNLs with electron neutrinos.

There are also limits on the model parameters from 3-body decays of muons where sterile neutrino contribution may distort the spectrum of Michel electrons, see Ref. [311, 545] for discussion. For $\tau$-lepton more promising are two-body decays into mesons, like $\tau \rightarrow N \pi, N K$, which may be probed at $B$-factories. It was argued [546] that multihadronic states can be utilized in the peak searches with $\tau$-leptons as well, see Fig. 4.12.

\subsubsection{Direct signatures of HNL: fixed target experiments}

Sterile neutrinos can be produced in 3-body meson decays into light (pseudoscalar or vector) meson, neutrino and charged lepton (dubbed semileptonic decay). Formulas for the decay rates can be found in [395]. However, in practice, it is rather difficult to recognize the contribution of sterile neutrino into the continuos spectra of SM particles in the final state, since only part of the phase space density is usually available for study, and the theoretical SM predictions suffer from QCD uncertainties. Similar problems persist for heavy baryon weak decays into sterile neutrino [547].

Another method to probe sterile neutrino models is searches for sterile neutrino decays. Indeed, sterile neutrinos are unstable because of the very same mixing with active neutrinos. The decay

\footnotetext{
${ }^{6}$ Obviously there could be several peaks for several sterile neutrinos of different masses.
} 
rate is that typical for all the weak decays multiplied by the squared mixing angle,

$$
\Gamma_{N \rightarrow \text { weak }} \propto\left|U_{\alpha I}\right|^{2} G_{F}^{2} M_{I}^{5},
$$

where we neglected the masses of final state particles. Even for $\mathcal{O}(1 \mathrm{eV})$ sterile neutrino the decay into 3 active neutrinos is kinematically allowed. Heavier neutrino may decay into the final state with two charged leptons,

$$
N_{I} \rightarrow e^{+} e^{-} \nu, \mu^{+} \mu^{-} \nu, \mu^{ \pm} e^{\mp} \nu, \tau^{+} \tau^{-} \nu, \text { etc },
$$

and final states with hadrons,

$$
N_{I} \rightarrow \pi^{ \pm} e^{\mp}, \pi^{ \pm} \mu^{\mp}, K^{ \pm} e^{\mp}, K^{ \pm} \pi^{\mp} \nu, \text { etc . }
$$

For the list of decay rates see e.g. [395, 548].

Then two different types of experimental installations are capable of searching for sterile neutrino decays. The first type is an experiment, where both sterile neutrino production and decay happen inside a single detector volume. A sterile neutrino is electrically neutral, so its propagation in the detetctor media is unobservable. What is observable is the mixture of two states: particles accompanying sterile neutrino production (e.g. muon for $K \rightarrow \mu N$ ) and products of sterile neutrino decays (e.g. pion and muon for $N \rightarrow \pi \mu$ ), mimicking the many-body decay of the initial hadron (e.g. $K \rightarrow \pi \mu \mu$ ). Obviously, the invariant mass of decay product (e.g. $\pi \mu$-pair) equals $M_{I}$. This signal must be recognized and two features may help. First, sterile neutrino can decay at some finite distance from the production point, which gives a signature of displaced secondary vertex (e.g. for the example above among the triples $\pi \mu \mu$ the signal ones are those with $\pi \mu$ coming from a single point at some distance from $K$-meson decay). Second, since sterile neutrino, being Majorana fermion and hence selfconjugated state, violates C-symmetry and L-symmetry, the accompanying particles and decay products may form final states which are rare or forbidden within the SM physics given the type of initial particle (in our example those are states with the same sign leptons, $\left.K^{ \pm} \rightarrow \pi^{\mp} \mu^{ \pm} \mu^{ \pm}\right)$.

The typical example of this type of experiments is a collider, and all the searches performed there are of this type, see Sec. 4.4.3. For sterile neutrino of $\mathcal{O}(1) \mathrm{GeV}$ mass the limit from Belle experiment is an example [409]: sterile neutrinos were supposed to appear in leptonic and semileptonic decay of one of two B-mesons coming from $\Upsilon(4 s)$ resonance, and the decay channels $\pi^{ \pm} \mu^{\mp}$ and $\pi^{\mp} e^{ \pm}$have been accounted for. For an analysis of similar limits valid for sterile neutrinos of sub-GeV range, see e.g. [394].

If the sterile neutrino decay length is shorter than the detector size the number of signal events is suppressed as

$$
N_{S} \propto\left|U_{\alpha I}\right|^{2} .
$$

In the opposite case, when the decay length $l_{N}$ exceeds the detector size $L_{d}$, so that sterile neutrinos decay mostly outside the detector, the number of events is more strongly suppressed,

$$
N_{S} \propto\left|U_{\alpha I}\right|^{2} \times \frac{l_{N}}{L_{d}} \propto\left|U_{\alpha I}\right|^{4}
$$

With decreasing mixing the sterile neutrino lifetime grows, so for a finite-size detector one naturally arrives at the second case. Obviously, this significantly diminishes the sensitivity of the direct search experiments to the mixing angles.

One may overcome $\left|U_{\alpha I}\right|^{4}$ by increasing the statistics of initial hadrons (i.e. increase the production) which is achieved at a fixed-target experiment exploiting a high current proton beam. 
Each scattering produces hadrons, which subsequently decay, sometimes into sterile neutrinos. Unfortunately, the multiparticle production inherent in hadron scatterings yields enormous amount of secondary hadrons and leptons, which forms heavy background mimicking the signal events. The natural way out is adopting the appropriate beam-dump absorbing strongly and electromagnetically interacting particles. Then at some distance downstream the beam-line one deploys a detector where freely propagating through the beam dump sterile neutrinos can decay. These experiments form the second type of installations capable of testing the models with sterile neutrinos. The signal events are very clear: two charged particles appearing from nowhere. The signature is two tracks coming from a single point. The invariant mass of the two particles equals sterile neutrino mass, if they come from a two-body decay, see (4.4.3) for examples.

Several beam-dump experiments have been installed at Fermilab (E613, FMMF, NuTeV), IHEP, and many more at CERN (PS191, CDHS, CHARM, CHARMII, BEBC, NOMAD, WA66, NA3), see [156] for complete set of references. The present limits are outlined in Figs. 4.10, 4.11, 4.12.

\subsubsection{Direct Signatures of HNL: Collider Searches}

HNLs in the $\mathrm{GeV}-\mathrm{TeV}$ mass range can be directly probed at collider experiments; for a review, see e.g. [549]. For $M_{I}$ below the $Z$-boson mass, $95 \%$ C.L. direct limits on the mixing parameter $\left|U_{\ell I}\right|^{2}$ were set by L3 [550] and DELPHI [551] collaborations, searching for $Z$ decay to HNLs through NC interactions in the LEP1 data. These limits are shown by the contour labeled 'L3' (pink, dashed) in Figures 4.10 and 4.11, and by the contours labeled 'DELPHI' (dark green, dashed) in Figures 4.104.12. For higher masses, a direct search was performed in $e^{+} e^{-}$annihilation at LEP $[552,553]$, where a single HNL can be produced via its mixing with active neutrinos: $e^{+} e^{-} \rightarrow N_{I} \nu_{\ell}$, and can subsequently decay via $\mathrm{NC}$ or CC interaction: $N_{I} \rightarrow \ell W, \nu_{\ell} Z, \nu_{\ell} H$, if kinematically allowed. Concentrating on the decay channel $N_{I} \rightarrow e W$ with $W \rightarrow$ jets, which would lead to a single isolated electron plus hadronic jets, the L3 collaboration put a 95\% C.L. upper limit on the mixing parameter $\left|U_{e I}\right|^{2}$ in a HNL mass range between 80 and $205 \mathrm{GeV}$ [553], as shown by the (red, solid) contour labeled 'LEP2' in Figure 4.10. Similar limits can be obtained [554] by using the $e^{+} e^{-} \rightarrow W^{-} W^{+} \rightarrow \bar{\nu} \ell^{-} \ell^{+} \nu$ data at LEP-II [555]. The LEP searches were mainly limited by the maximum available center-of-mass energy $\sqrt{s}=208 \mathrm{GeV}$. Future lepton colliders can significantly improve the sensitivity and extend it to higher mass regions, as illustrated in Figure 4.10 by the projected limit labeled 'ILC', which was obtained assuming a $\sqrt{s}=500 \mathrm{GeV}$ ILC with luminosity of $500 \mathrm{fb}^{-1}[549,556]$.

After the inauguration of the LHC era, the CMS and ATLAS collaborations have performed direct searches for the on-shell production of HNLs above the $Z$-threshold. Assuming the HNLs to be Majorana particles, they have looked for the smoking gun lepton-number violating signature of same-sign dilepton plus jets with no missing transverse energy: $p p \rightarrow W^{*} \rightarrow N \ell^{ \pm} \rightarrow \ell^{ \pm} \ell^{ \pm} j j[432$, 485-487, 557-559]. The previous searches with $4.7 \mathrm{fb}^{-1}$ data at $\sqrt{s}=7 \mathrm{TeV}$ LHC set limits on $\left|U_{\ell I}\right|^{2}$ (with $\ell=e, \mu$ ) for HNL masses up to $300 \mathrm{GeV}[560,561]$. More recently, these limits were extended for masses up to $500 \mathrm{GeV}$ with $20 \mathrm{fb}^{-1}$ data at $\sqrt{s}=8 \mathrm{TeV}[562,563]$, as shown by the contours labeled 'CMS' (red, solid) and 'ATLAS' (blue, solid) in Figure 4.11 (and for electron channel, by the contour labeled 'ATLAS' in Figure 4.10). Similar limits have been derived for the case of Dirac HNLs [564], using the trilepton channel: $p p \rightarrow W^{*} \rightarrow N \ell^{ \pm} \rightarrow \ell^{ \pm} \ell^{\mp} \ell^{ \pm}+\mathbb{E}_{T}$ [488, 565-569] and analyzing the recent trilepton data from the $\sqrt{s}=8 \mathrm{TeV}$ LHC [570].

For $M_{I} \sim 100 \mathrm{GeV}$, the direct limits in the muon sector are comparable to the indirect limits on $\left|U_{\mu I}\right|^{2} \lesssim 10^{-3}$ imposed by the electroweak precision data (EWPD) [554, 571-575] and LHC Higgs data [576]. The 90\% C.L. indirect constraints from a recent global fit to electroweak precision observables and leptonic non-unitarity parameters [554] are shown in Figures 4.10-4.12 (dark brown, dotted contours labeled 'EWPD'). 
With the run-II phase of the LHC starting later this year with more energy and higher luminosity, the direct search limits could be extended for HNL masses up to a TeV or so. It is worth noting here that all the direct searches at the LHC so far have only considered the simplest production process for HNLs through an $s$-channel $W$-exchange [313, 485-487, 557-559]. However, there exists another, infra-red enhanced, production mode involving $t$-channel exchange of $W$ bosons and photons [577], which gives a dominant contribution to the HNL production cross section for higher $M_{I}$ and at higher $\sqrt{s}$. This is mainly because of the fact that with increasing HNL mass, the production cross section for the $t$-channel process drops at a rate slower than that of the $s$-channel process, though the exact cross-over point depends crucially on the selection cut for the $p_{T}$ of the additional jet associated with the virtuality of the $t$-channel photon [491, 577]. In any case, including the infra-red enhancement effect could further enhance the HNL signal sensitivity at the next run of the LHC [549, 577]. As an illustration, we have shown in Figures 4.10 and 4.11 projected conservative limits with $300 \mathrm{fb}^{-1}$ data at $\sqrt{s}=14 \mathrm{TeV}$ (blue, dashed contours labeled 'LHC 14'), assuming that the cross-section limits are at least as good as the existing ones at $\sqrt{s}=8 \mathrm{TeV}$, as reported in Ref. [563]. The direct collider limits for $M_{I}<100 \mathrm{GeV}$ are not likely to improve significantly with higher collision energy, due to the increased pile-up effects, thus obfuscating the low- $p_{T}$ leptons produced by the decay of a low-mass HNL. Instead, a displaced vertex search might be useful to probe the low-mass range between 3-80 GeV for mixing values $10^{-7} \lesssim\left|U_{\ell I}\right|^{2} \lesssim 10^{-5}$ [496].

$B$-physics experiments offer a complementary probe of the low-mass region. In particular, the $\mathrm{LHCb}$ experiment has searched for a heavy Majorana neutrino produced in the $B^{-} \rightarrow \pi^{+} \mu^{-} \mu^{-}$ decay mode and set $95 \%$ C.L. upper limits on $\left|U_{\mu I}\right|^{2}=10^{-2}-10^{-3}$ in the HNL mass range 250 $\mathrm{MeV}$ to $5 \mathrm{GeV}$ using $3 \mathrm{fb}^{-1}$ of integrated luminosity collected at $\sqrt{s}=7$ and $8 \mathrm{TeV}$ [408], as shown by the (yellow, solid) contour labeled 'LHCb' in Figure 4.11. Similarly, the Belle experiment has set $90 \%$ C.L. upper limits on both $\left|U_{e I}\right|^{2}$ and $\left|U_{\mu I}\right|^{2}$ in the HNL mass range $500 \mathrm{MeV}$ to $5 \mathrm{GeV}$, as shown by the (green, solid) contours labeled 'Belle' in Figures 4.10 and 4.11, obtained using the decay mode $B \rightarrow X \ell N_{I}$ followed by $N_{I} \rightarrow \ell \pi$ (with $\ell=e, \mu$ ) in a data sample of 772 million $B \bar{B}$ pairs [409]. These limits are expected to improve significantly, as more data is accumulated in future $B$-factories.

Finally, we note that there exist no direct collider searches for HNLs involving tau lepton final states. This is mainly due to the experimental challenges of $\tau$ reconstruction at a hadron collider. The only existing constraints on $\left|U_{\tau I}\right|^{2}$ in the $\mathrm{GeV}$-range are from the $Z$-decay experiments at DELPHI [551], as shown in Figure 4.12. Future $B$-factories with a large dataset of $\sim 10$ million $\tau^{-}$ decays could offer a powerful probe of the HNLs in the tau sector. This is illustrated in Figure 4.12 by the projected $95 \%$ C.L. limits, as shown by the red solid (conservative) and dashed (optimistic) contours, obtained from a recent Monte-Carlo simulation [546].

The complementary searches in the proposed SHiP experiment at the intensity frontier will dramatically improve the HNL sensitivity in the low-mass regime for all flavors, as can be seen from its projected 90\% C.L. limits [35] in Figures 4.10-4.12. For a direct comparison with the existing limits, these projection limits have been derived in a model-independent manner, assuming a single-flavor dominance. For comparison, we also show the corresponding projected sensitivity from another proposed intensity-frontier experiment, namely, the FCC-ee [383]. Here we have assumed $10^{12} Z$-boson decays occurring between 10-100 $\mathrm{cm}$ from the interaction point. Increasing the number of $Z$-bosons and/or the range of decay length could further enhance these sensitivity limits. 


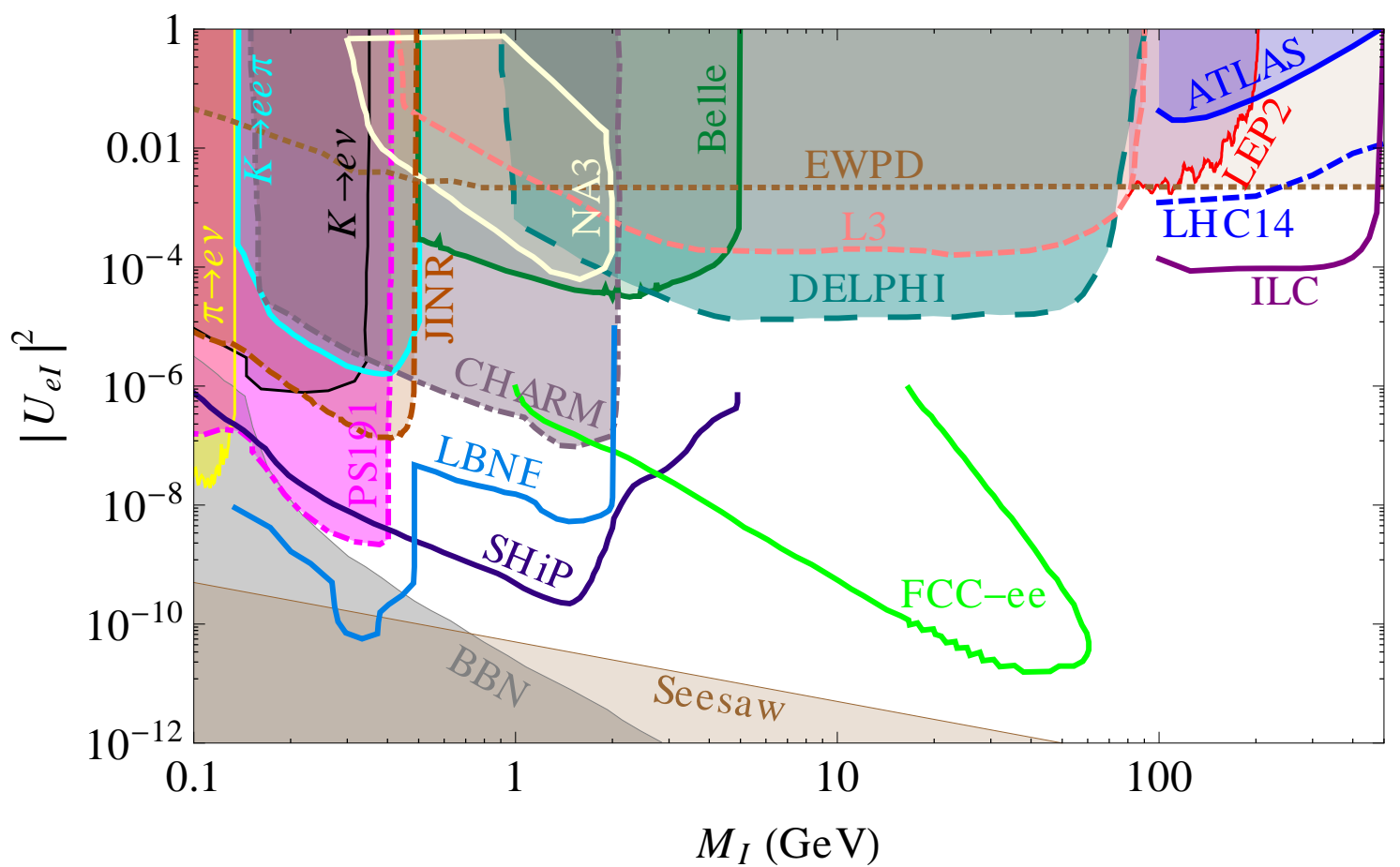

Figure 4.10: Limits on the mixing between the electron neutrino and a single HNL in the mass range $100 \mathrm{MeV}-500 \mathrm{GeV}$. The (gray, dotted) contour labeled 'BBN' corresponds to an HNL lifetime $>1 \mathrm{sec}$, which is disfavored by BBN [395, 414, 528]. The (brown, dashed) line labeled 'Seesaw' shows the scale of mixing naively expected in the canonical seesaw (see Section 4.3.2.3). The (dotted, dark brown) contour labeled 'EWPD' is the 90\% C.L. exclusion limit from electroweak precision data [554]. The contours labeled ' $\pi \rightarrow e \nu$ ' (yellow, solid) $[542,544]$ and ' $K \rightarrow e \nu$ ' (black, solid) [536] are excluded at $90 \%$ C.L. by peak searches (see Section 4.4.1). Those labeled 'PS191' (magenta, dot-dashed) [578], 'CHARM' (dark blue, dot-dashed) [579], 'NA3' (light yellow, solid) [580] and 'JINR' [581] are excluded at 90\% C.L. from beam-dump experiments (see Section 4.4.2). The (cyan, solid) contour labeled ' $K \rightarrow e e \pi$ ' is the exclusion region at 90\% C.L. from $K$-meson decay search with a detector size of $10 \mathrm{~m}$ [313]. The (green, solid) contour labeled 'Belle' is the exclusion region at $90 \%$ C.L from HNL searches in B-meson decays at Belle [409]. The contours labeled 'L3' (pink, dashed) [550] and 'DELPHI' (dark green, dashed) [551] are excluded at 95\% C.L. by analyzing the LEP data for $Z$-boson decay to HNLs. The (red, solid) contour labeled 'LEP2' is excluded at 95\% C.L. by direct searches for HNL at LEP [553]. The (blue, solid) contour labeled 'ATLAS' is excluded at $95 \%$ C.L. from direct searches by ATLAS at $\sqrt{s}=8$ TeV LHC [563]. The (blue, dashed) curve labeled 'LHC 14' is a projected exclusion limit from the $\sqrt{s}=14$ TeV LHC with $300 \mathrm{fb}^{-1}$ data [549]. The (purple, solid) contour labeled 'ILC' is a projected sensitivity at $\sqrt{s}=500 \mathrm{GeV}$ ILC with $500 \mathrm{fb}^{-1}$ data $[549,556]$. The (light blue, solid) contour labeled 'LBNE' is the expected 5 -year sensitivity of the LBNE near detector with an exposure of $5 \times 10^{21}$ protons on target for a detector length of $30 \mathrm{~m}$ and assuming a normal hierarchy of neutrinos [582]. The (dark green, solid) contour labeled 'FCC-ee' is the projected reach of FCC-ee for $10^{12} Z$ decays occurring between 10-100 $\mathrm{cm}$ from the interaction vertex [383]. The (violet, solid) contour labeled ' $\mathrm{SHiP}$ ' is the projected reach of SHiP at $90 \%$ C.L. [35, 583]. 


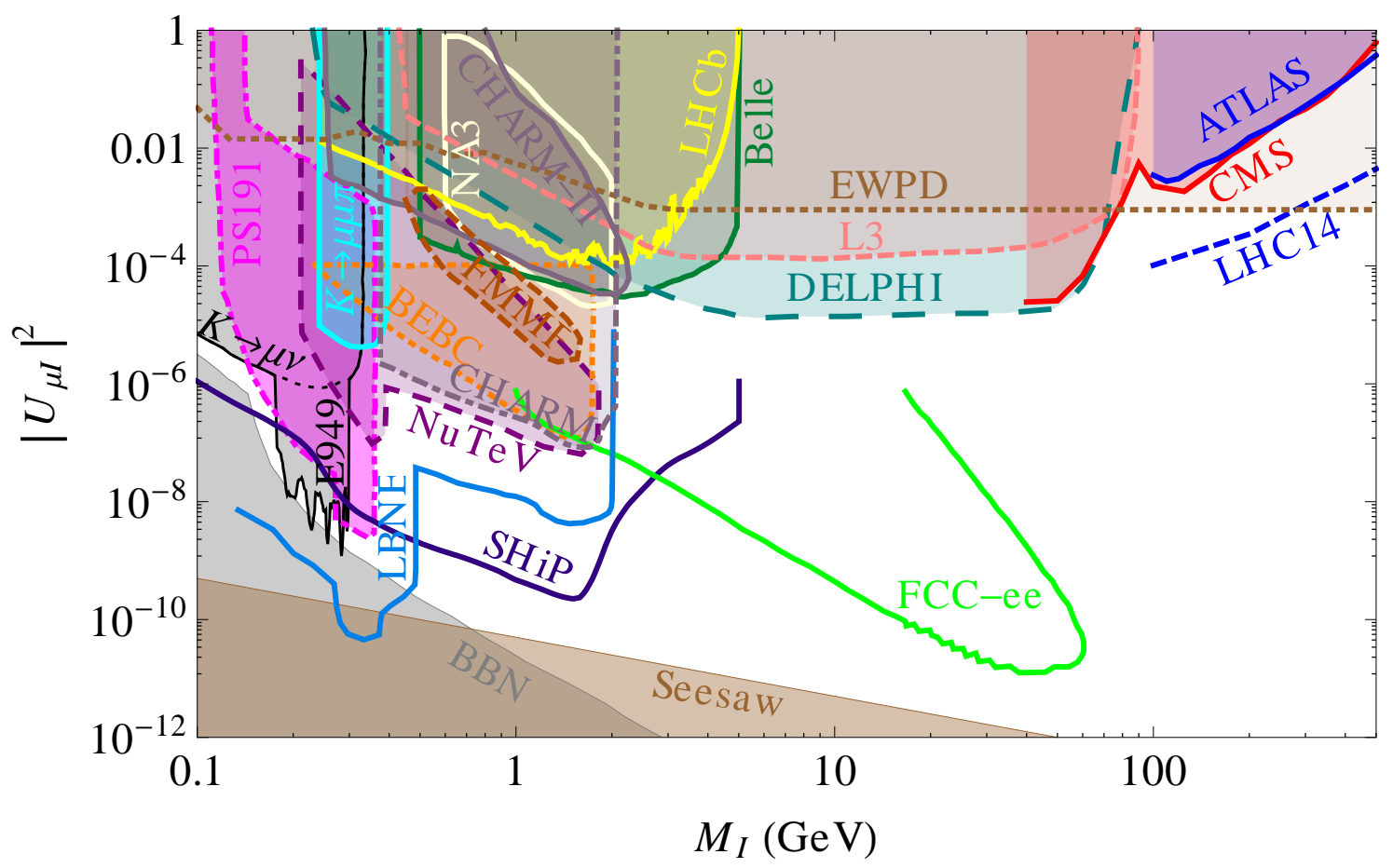

Figure 4.11: Limits on the mixing between the muon neutrino and a single HNL in the mass range $100 \mathrm{MeV}-500 \mathrm{GeV}$. The (gray, dotted) contour labeled BBN corresponds to an HNL lifetime $>1$ sec, which is disfavored by BBN [395, 414, 528]. The (brown, dashed) line labeled 'Seesaw' shows the scale of mixing naively expected in the canonical seesaw (see Section 4.3.2.3). The (dotted, dark brown) contour labeled 'EWPD' is the 90\% C.L. exclusion limit from electroweak precision data [554]. The contour labeled ' $K \rightarrow \mu \nu$ ' (black, solid) is excluded at 90\% C.L. by peak searches [535, 536]. Those labeled 'PS191' (magenta, dot-dashed) [578], 'NA3' (light yellow, solid) [580], 'BEBC' (orange, dotted) [584], 'FMMF' (light cyan, dashed) [585], 'NuTeV' (purple, dashed) [586] and 'CHARM' (dark blue, dot-dashed) [587] are excluded at 90\% C.L. from beamdump experiments. The (cyan, solid) contour labeled ' $K \rightarrow \mu \mu \pi$ ' is the exclusion region at $90 \%$ C.L. from $K$-meson decay search with a detector size of $10 \mathrm{~m}$ [313]. The (green, solid) contour labeled 'Belle' is the exclusion region at 90\% C.L from HNL searches in B-meson decays at Belle [409]. The (yellow, solid) contour labele1d ' $\mathrm{LHCb}$ ' is the exclusion region at 95\% C.L from HNL searches in B-meson decays at $\mathrm{LHCb}$ [408]. The (dark blue, dot-dashed) contour labeled 'CHARM-II' [588] is excluded at 90\% C.L. from the search for direct HNL production with a wide-band neutrino beam at CERN. The (pink, dashed) contour labeled 'L3' [550] and (dark green, dashed) labeled 'DELPHI' [551] are excluded at 95\% C.L. by analyzing the LEP data for Z-boson decay to HNL. The (blue, solid) contour labeled 'ATLAS' [563] and (red, solid) labeled 'CMS' [589] are excluded at $95 \%$ C.L. from direct searches at $\sqrt{s}=8 \mathrm{TeV}$ LHC. The (blue, dashed) curve labeled 'LHC 14' is a projected exclusion limit from the $\sqrt{s}=14 \mathrm{TeV} \mathrm{LHC}$ with $300 \mathrm{fb}^{-1}$ data [549]. The (light blue, solid) contour labeled 'LBNE' is the expected 5-year sensitivity of the LBNE near detector with an exposure of $5 \times 10^{21}$ protons on target for a detector length of $30 \mathrm{~m}$ and assuming a normal hierarchy of neutrinos [582]. The (dark green, solid) contour labeled 'FCC-ee' is the projected reach of FCC-ee for $10^{12} Z$ decays and $10-100 \mathrm{~cm}$ decay length [383]. The (violet, solid) contour labeled ' $\mathrm{SHiP}$ ' is the projected reach of $\mathrm{SHiP}$ at $90 \%$ C.L. [35]. 


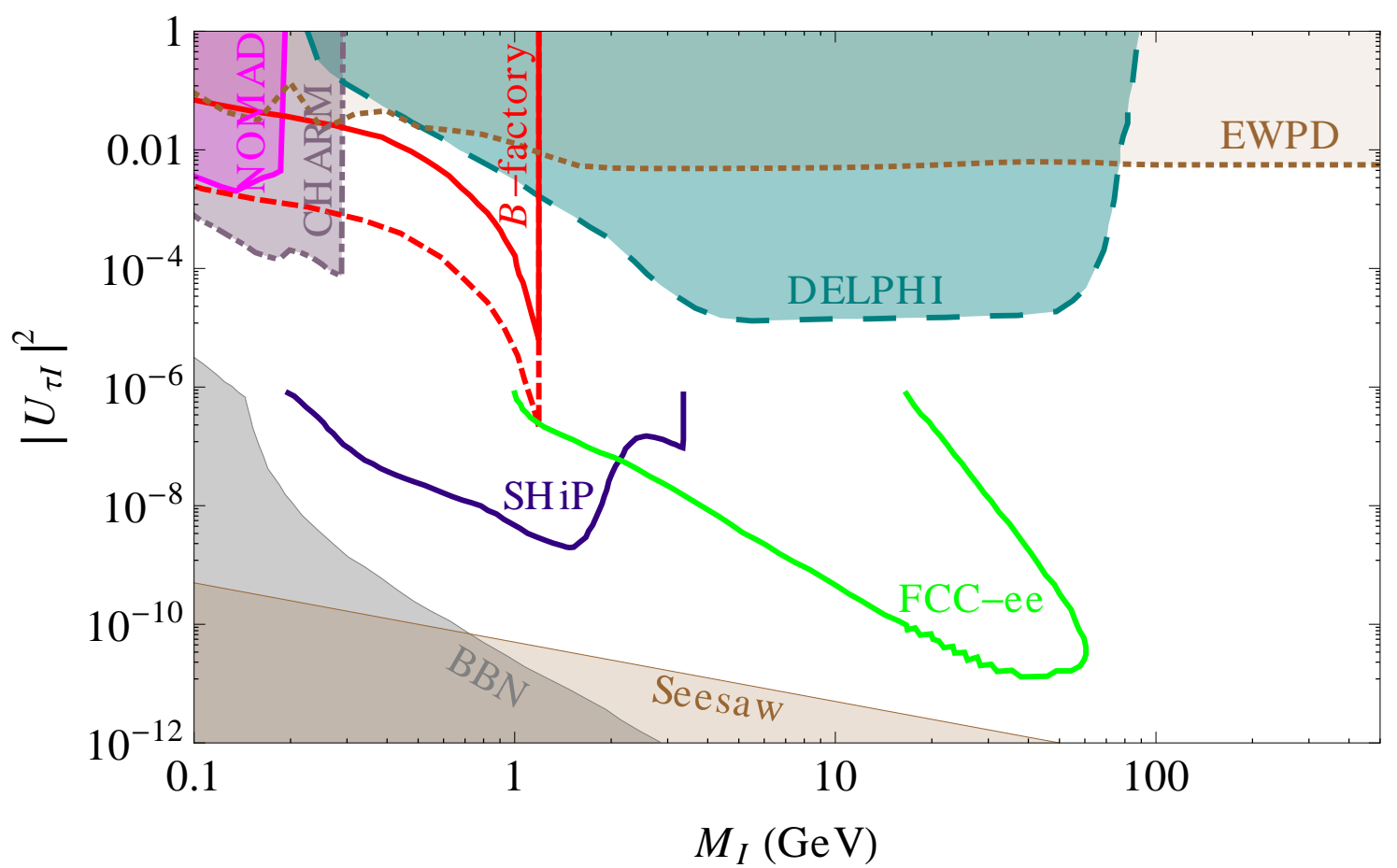

Figure 4.12: Limits on the mixing between the tau neutrino and a single HNL in the mass range $100 \mathrm{MeV}-500 \mathrm{GeV}$. The (gray, dotted) contour labeled BBN corresponds to an HNL lifetime $>1$ sec, which is disfavored by BBN [395, 414, 528]. The (brown, dashed) line labeled 'Seesaw' shows the scale of mixing naively expected in the canonical seesaw (see Section 4.3.2.3). The (dotted, dark brown) contour labeled 'EWPD' is the $90 \%$ C.L. exclusion limit from electroweak precision data [554]. The (dark blue, dot-dashed) contour labeled 'CHARM' [590] and (purple, solid) contour labeled 'NOMAD' [591] are excluded at 90\% C.L. from the search for direct HNL production. The (dark green, dashed) contour labeled 'DELPHI' [551] is excluded at 95\% C.L. by analyzing the LEP data for $Z$-boson decay to HNL. The (red, solid and dashed) contours labeled ' $B$-factory' are the conservative and optimistic projected limits at $90 \%$ C.L. from $\sim 10$ million $\tau$-decays at a future $B$-factory [546]. The (dark green, solid) contour labeled 'FCC-ee' is the projected reach of FCC-ee for $10^{12} Z$ and $10-100 \mathrm{~cm}$ decay length [383]. The (violet, solid) contour labeled 'SHiP' is the projected reach of SHiP at $90 \%$ C.L. [35]. 


\subsection{Indirect HNL probes}

\subsubsection{Neutrinoless double beta decay for non-seesaw HNL}

The experimental search of baryon/lepton number violations is a major entrance door to extensions of the Standard Model and offers us a chance of addressing the question of the origin of matter. In particular, the evidences of neutrino oscillations motivate further testing of the lepton number via the search for neutrinoless double beta decay $(0 \nu \beta \beta)$.

It is plausible to assume the $0 \nu \beta \beta$ rate to be dominated by the exchange of the 3 ordinary neutrinos with Majorana mass. Such a mechanism is usually the leading one in theories where the scale of new physics is much larger than the electroweak scale.

Within this scenario, next generation experiments will be able to probe Majorana effective mass values greater than $(70-100) \mathrm{meV}$ in optimistic assumptions [592], but the bound on the cosmological mass of $140 \mathrm{meV}$ [320] already implies $m_{\beta \beta}<39 \mathrm{meV}$ (55 meV) for normal (inverted) mass hierarchy.

Therefore, the observation of a $0 \nu \beta \beta$ signal in the next generation of experiments would indicate that some other mechanisms compatible with a faster decay rate are at work. This possibility has been noted a long time ago [593, 594], and it is currently regarded with renewed interest, see e. g. [313, 433, 492].

In fact, a fast decay rate could be attributed to the same particles that are added to the standard model spectrum to explain oscillations, e. g. heavy neutrinos. This hypothesis, along with the radiative stability of the tree-level neutrino masses, implies that the heavy neutrino masses cannot exceed $10 \mathrm{GeV}$ or so [492]. Indeed, within the conventional seesaw model, the amplitudes of light and heavy neutrino exchange are linked. Naively, the former is proportional to $m_{\nu} /(100 \mathrm{MeV})^{2}$ where $m_{\nu}=m_{D}^{2} / M_{N}$, while the latter to $m_{D}^{2} / M_{N}^{3}$, thus suggesting that heavy neutrinos give a small contribution when $M_{N} \gg 100 \mathrm{MeV}$. This is not true if the flavor structure implies that the seesaw contribution to neutrino mass is suppressed. In this context one can assume that the exchange of $M_{N}>100 \mathrm{MeV}$ saturates the $0 \nu \beta \beta$ decay rate, also reproducing the ordinary neutrino masses. But one cannot exceed $M_{N} \sim 10 \mathrm{GeV}$ without spoiling the seesaw model, since this would mean that the one-loop contribution dominates the neutrino mass. Heavy neutrinos masses and mixing angles, compatible with the rate of $0 \nu \beta \beta$, depend on the nuclear matrix elements of the transition (compare e.g. [313] and [492]). Thus, nuclear physics has an impact on the limits that are relevant for a direct search for heavy neutrinos with accelerators.

Recent studies outlined the following conclusions about nuclear matrix elements calculations:

1. Each scheme of calculation can estimate its intrinsic uncertainty. This is usually found to be small in modern computations (about $16 \%$ for light neutrino exchange and about $28 \%$ for heavy neutrino exchange, [595]).

2. The free nucleon couplings are likely to be lowered in the nuclear medium (quenching, [596]) diminishing $0 \nu \beta \beta$ matrix elements by $\left(A^{-0.18}\right)^{2}$ where $A$ is the mass number, [597].

In a conservative treatment, these two factors of uncertainty should be taken into account. It has to be noticed that if the $0 \nu \beta \beta$ is due to a pointlike (dimension-9) operator, as for heavy neutrino exchange, 2 nucleons are in the same point. Therefore, the effect of a hard core repulsion, estimated modeling the short range correlations, plays an important role in the determination of the uncertainties. A significant step forward has been recently made, pushing down this source of theoretical error of about an order of magnitude, [595]. The error quoted in point 1. already takes it into account. 
Using the nuclear matrix elements evaluated with the IBM-2 model [595] and focussing on the case of heavy neutrino exchange, one gets for ${ }^{76} \mathrm{Ge}$ :

$$
\mathscr{M}(\mathrm{Ge})= \begin{cases}104 \pm 29 & (\text { point } 1 .) \\ 22 \pm 6 & (\text { point } 1 .+ \text { point 2. })\end{cases}
$$

Therefore, it can be seen that if we consider the different reasons of increase and of decrease of the value of the matrix element, we get a very wide range.

From the experimental point of view, the limits on $0 \nu \beta \beta$ indicate that the mixings of heavy neutrinos $\left|U_{\mathrm{eI}}\right|^{2}$ are small. The present lower limit on the decay half-life for ${ }^{76} \mathrm{Ge}$ is $3.0 \cdot 10^{25} \mathrm{yr}$ [498]. Using the phase space factor from [598], one gets:

$$
\left|\sum_{I} \frac{U_{\mathrm{e}}^{2}}{M_{I}}\right|<\frac{7.8 \cdot 10^{-8}}{m_{\mathrm{p}}} \cdot\left[\frac{104}{\mathscr{M}(\mathrm{Ge})}\right] \cdot\left[\frac{3 \cdot 10^{25} \mathrm{yr}}{T_{1 / 2}}\right]^{\frac{1}{2}}
$$

where $m_{\mathrm{p}}$ is the proton mass and the heavy neutrino masses $M_{I}$ are assumed to be $\gtrsim \mathrm{GeV}$.

Note that Fig. 4.13 illustrates the case of a single heavy neutrino. However, in the full case, e. g. in Eq. (4.5.2), the various contributions may lead to to cancellations among them, just as it can happen in the case of light neutrino exchange. A specific example when this happens can be found in Sec. 4.5.2.

However, the bound is still uncertain. It weakens by one order of magnitude if the axial vector coupling constant is strongly quenched in the nuclear medium, as it can be seen from Fig. 4.13. This plot shows the case of the mixing for ${ }^{76} \mathrm{Ge}$ assuming that a single heavy neutrino dominates the amplitude. The two regimes of heavy and light neutrino exchange are matched as suggested by [599]. The colored bands reflect the different sources of theoretical uncertainty.

Therefore, for some regions of the parameter space, the limits obtained more than 15 years ago with accelerators are more restrictive than the current limits coming from $0 \nu \beta \beta$ search. Further direct exploration of the mass region around the $\mathrm{GeV}$ can thus provide a competitive approach to search for heavy neutrinos.

\subsubsection{Neutrinoless double beta decay for two seesaw HNLs}

The generic case of neutrinoless double decay may be rather complicated and require analysis of all possible lepton number violating physics. For example, if the model has additional heavy sterile neutrinos which do not participate in the see-saw generation of the active neutrino masses (i.e. there are more than three sterile neutrinos or some type II or III see-saw generates active neutrino masses) non-observation of fast $0 \nu \beta \beta$ decay can bound their masses, as described in Section 4.5.1. Here we constrain the model to the pure type I seesaw.

The neutrinoless double beta decay width is usually given by the formula

$$
\Gamma_{0 \nu \beta \beta}=G \cdot\left|\mathcal{M}^{2}\right| \cdot\left|m_{\beta \beta}^{\nu}\right|^{2},
$$

where $G$ is an exactly calculable phase space factor, and $\mathcal{M}$ is the nuclear matrix element is, schematically

$$
\left|\mathcal{M}^{2}\right|=|\langle f|O \cdot F(r)| i\rangle|^{2} .
$$

In this expression $i$ and $f$ are initial and final states of the nucleus, $O$ is some hadronic operator, and $F(r)$ represents the neutrino potential. Neglecting the structure of energy levels (which is of the order of $10 \mathrm{MeV}$ ) and neutrino mass in comparison to the characteristic neutrino virtual 


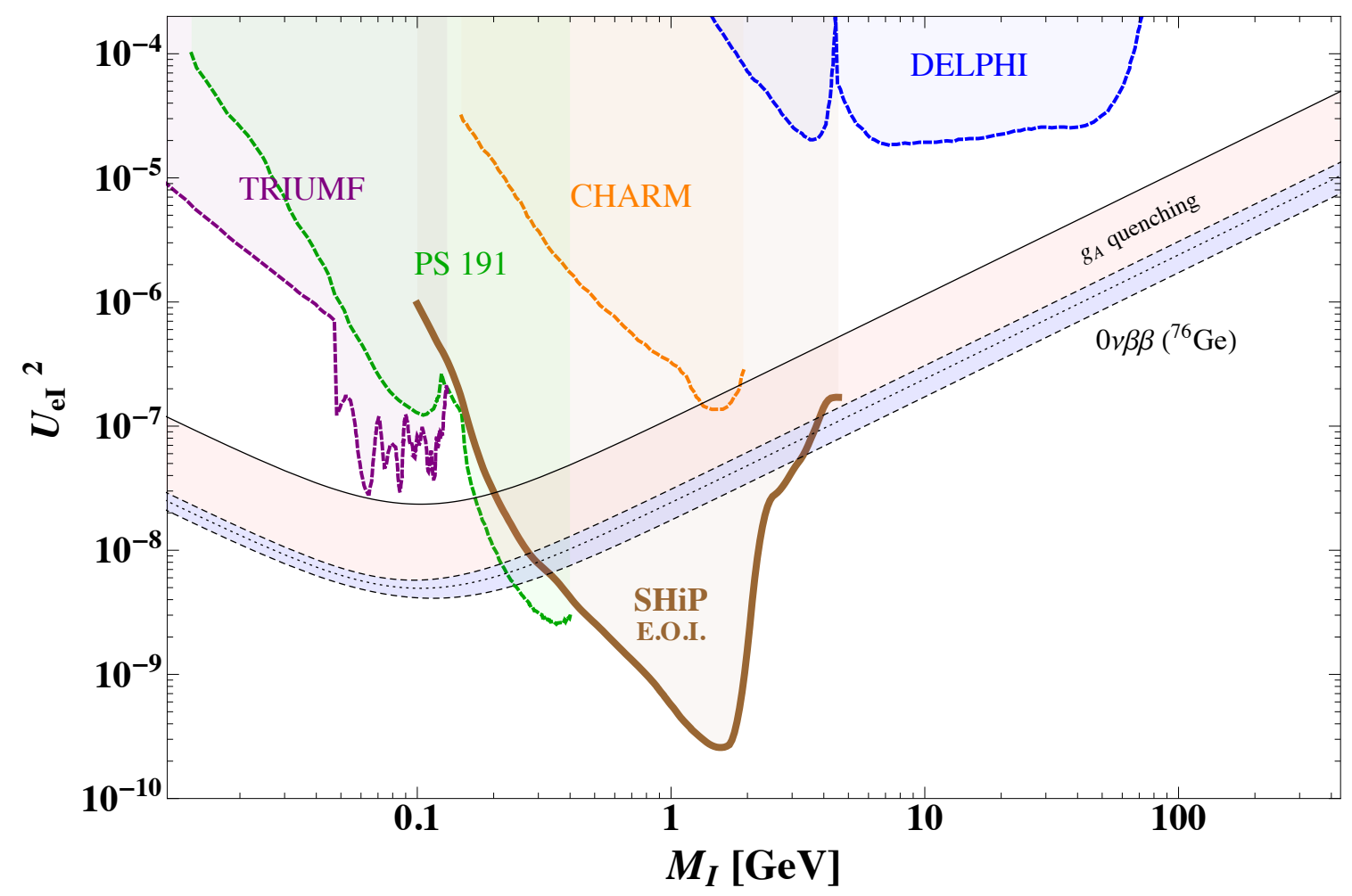

Figure 4.13: Bounds on the mixing between the electron neutrino and a single heavy neutrino from the combination of bounds obtained with $\mathrm{Ge} 0 \nu \beta \beta$ experiments [498] using the representation introduced in [599]. This limit is not valid for seesaw HNLs because of the different cancellations discussed in Sec. 4.5.2. The bands correspond to the uncertainties discussed in the text. The dashed contours indicate the mass regions excluded by some of the accelerator experiments considered in [313]: CHARM (90\% C. L., [587]), DELPHI (95\% C. L., [551]), PS 191 (90\% C. L., [578]), TRIUMF (90\% C. L., [542, 544]).

momentum (about $100 \mathrm{MeV}$ ) the neutrino contribution is given by

$$
F(r) m_{\beta \beta}^{\nu}=m_{\beta \beta}^{\nu} \frac{1}{r}
$$

This corresponds to the usual Majorana type neutrino contribution.

In the pure see-saw case, the contribution to the neutrinoless double beta decay goes by exchange of the heavy states $M_{I}$. Thus instead of simple Majorana propogator $m / k^{2}$ for light neutrino this gives two massless electron neutrino propagators, two Yukawas, one massive propagator

$$
\sum_{I=1}^{3} \frac{F_{e I} v M_{I} F_{e I} v}{k^{2}\left(k^{2}-M_{I}^{2}\right)}=\sum_{I=1}^{3}\left(-\frac{F_{e I}^{2} v^{2} / M_{I}}{k^{2}}+\left(\frac{F_{e I} v}{M_{I}}\right)^{2} \frac{M_{I}}{k^{2}-M_{I}^{2}}\right)
$$

Alternatively, in the mass diagonal basis, this can be understood as the sum of contributions of light 'active' neutrinos (first term) and heavy 'sterile' neutrinos (second term). Note, that these terms have opposite signs (because of opposite CP parity), leading to cancellation of the terms in the formula if the typical nuclear momentum can be neglected compared to $M_{I}$.

These means that instead of the Majorana mass $m_{\beta \beta}^{\nu}$ in the (4.5.3) we should write effective 
Majorana mass

$$
\left|m_{\beta \beta}^{\mathrm{eff}}\right|=\left|\sum_{I=1}^{3} \frac{F_{e I}^{2} v^{2}}{M_{I}}\left\langle\frac{1}{r}-\frac{e^{-M_{I} r}}{r}\right\rangle /\left\langle\frac{1}{r}\right\rangle\right|,
$$

where $\langle\cdot\rangle$ means average over nuclear distribution function.

Let us analyze this formula in more detail. First, in case of heavy Majorana masses, $M_{I} \gg \Lambda \sim$ $1 \mathrm{GeV}$, one can neglect the second term. Thus the mass dependence factorizes in this expression, and one arrives to the usual result for light Majorana active neutrinos,

$$
m_{\beta \beta}=\left|\sum_{I} F_{e I}^{*} \frac{v^{2}}{M_{I}} F_{I e}^{\dagger}\right|=\left|\sum_{i} m_{i} U_{\mathrm{PMNS}, e i}^{2}\right|,
$$

where the last expression sums over the active neutrino mass states.

In the opposite limit of small Majorana masses $M_{I}$ one can neglect the mass in the exponent of the denominator of the second term, and both terms cancel. Note that because of the see-saw mechanism the phases of contributions to the effective double beta decay mass from active and sterile neutrinos (first and second terms in (4.5.6)) are automatically opposite.

Slightly simplifying the discussion (neglecting the effects of the nuclear matrix elements for the neutrino masses close to the typical momentum transfer $100 \mathrm{MeV}$ ) the discussion is summarized by the observation that there is the cancellation

$$
\sum_{\text {active }} m_{i} U_{e i}^{2}+\sum_{\text {light }} M_{I} U_{e I}^{2}+\sum_{\text {heavy }} M_{I} U_{e I}^{2}=0
$$

while the observed rate of the $0 \nu \beta \beta$ decay is controlled by

$$
m_{\beta \beta}=\left|\sum_{\text {active }} m_{i} U_{e i}^{2}+\sum_{\text {light }} M_{I} U_{e I}^{2}\right| .
$$

Here "light" and "heavy" correspond to the sterile neutrinos with $M_{I}$ smaller or larger than $100 \mathrm{MeV}$. First term in (4.5.10) corresponds to the contribution of the active states, and can be guessed from the knowledge of the neutrino mixing angles, mass differences and active neutrino mass scale. The second term gives the contribution of the light right handed neutrinos, and can be significant only if some of the masses $M_{I}$ are above and some are below the typical nuclear momentum transfer [405, 600, 601].

Let us consider the specific case of $\nu \mathrm{MSM}$ (see Section 4.8) the situation is simplified by the following two observations:

- One sterile neutrino is DM with mass $M_{1}=\mathcal{O}(10) \mathrm{keV}$ and mixing angle bound by (4.7.1)

- Apart from the DM neutrino there are only two sterile neutrinos with $M_{2,3}=\mathcal{O}(10) \mathrm{GeV}$, with some level of degeneracy $\Delta M / M \ll 1$ required by the leptogenesys, c.f. Section 4.6.4.

One can easily see, that though $M_{1}$ is a "light" sterile neutrino, its contribution to (4.5.10) is negligible [404, 602]. As far as the other two sterile neutrinos are degenerate in mass, they either both do not contribute significantly to the $0 \nu \beta \beta$ if they are heavy. They can cancel (partially) the contribution of the active neutrinos, if they are light [372] (c.f. (4.5.9)), but this situation is bound in the pure $\nu \mathrm{MSM}$ by the $\mathrm{BBN}$, which requires the heavy neutrino to decay before $\mathrm{BBN}$ (c.f. Section 4.5.4)

Thus, in $\nu$ MSM the $0 \nu \beta \beta$ decay can be calculated only from the contributions of the active neutrinos, assuming strongly hierarchical neutrino spectrum (which is again guaranteed by the DM 
requirement [321]). From the most recent oscillation measurements the bounds for the mass in the $0 \nu \beta \beta$ in the $\nu \mathrm{MSM}$ are [603]

$$
\begin{gathered}
1.5 \mathrm{meV}<m_{\beta \beta}^{\mathrm{NH}}<3.9 \mathrm{meV}, \\
17 \mathrm{meV}<m_{\beta \beta}^{\mathrm{IH}}<49 \mathrm{meV},
\end{gathered}
$$

for the normal and inverse mass hierarchies of the active neutrinos. There is no mechanism within $\nu \mathrm{MSM}$ to make the decay faster, but it may be suppressed for $M_{2,3} \sim 0.1 \mathrm{GeV},[372,603]$.

\subsubsection{Charged lepton flavour violating processes}

The search for charged lepton flavor violation (CLFV) is expected to know in the next few years a real step forward. This concerns a long series of processes with a $\mu-e, \tau-\mu$ or $\tau-e$ transition. For $\mu-e$ processes an improvement of up to 4 to 6 orders of magnitude could be expected, in particular for the $\mu \rightarrow$ eee [604] decay and $\mu$ to $e$ conversion in atomic nuclei [605]-[606]. Important improvements are also expected for the $\mu \rightarrow e \gamma$ decay [607]. On the theory side such transitions could be induced by a large variety of beyond the SM physics models. This new physics could lead to observable rates even if the associated energy scale is in some cases as large as few thousands of $\mathrm{TeV}$. In this section we briefly review the possibility to get observable rates from processes mediated by heavy neutral leptons, and more generally from the physics associated to the neutrino masses. It is well known that neutrino oscillations guarantee non-vanishing CLFV but at a very suppressed level. Beyond this experimentally unreachable contribution we will review the possibilities that the seesaw states, that are generally expected to be at the origin of the neutrino masses, could induce rates that are not suppressed by the smallness of these masses. This offers an opportunity to probe better the yet unknown neutrino mass origin. It is known that the 3 possible seesaw neutrino mass origins (with fermion singlets, scalar triplet(s) of fermion triplets, see Section 2.2), cannot be distinguished only from the knowledge of the neutrino mass matrix, as they all lead to the same dim-5 neutrino mass effective interactions (and all can lead to any flavor structure for it). Instead they lead to totally different dimension 6 interactions, i.e. to a different CLFV phenomenology. The relevant dimension 6 operator induced by the exchange of heavy seesaw state(s) are for the 3 seesaw options

$$
\begin{aligned}
\mathcal{L}_{\text {eff-I }}^{d=6} & =c_{\alpha \beta}^{d=6} \bar{L}_{\alpha} \tilde{\phi} i \not \partial\left(\tilde{\phi}^{\dagger} L_{\beta}\right) \\
\mathcal{L}_{\text {eff-II }}^{d=6} & =c_{\alpha \beta \delta \gamma}^{d=6}\left(\bar{L}_{\beta} \gamma_{\mu} L_{\delta}\right)\left(\bar{L}_{\alpha} \gamma^{\mu} L_{\gamma}\right) \\
\mathcal{L}_{\text {eff-III }}^{d=6} & =c_{\alpha \beta}^{d=6} \bar{L}_{\alpha} \tau^{a} \tilde{\phi} i \not D\left(\tilde{\phi}^{\dagger} \tau^{a} L_{\beta}\right)
\end{aligned}
$$

with $c_{\alpha \beta}^{d=6}=\left(Y_{N}^{\dagger} \frac{1}{M_{N}^{2}} Y_{N}\right)_{\alpha \beta}, c_{\alpha \beta \delta \gamma}^{d=6}=\left(1 / 2 m_{\Delta}^{2}\right) Y_{\Delta_{\alpha \beta}}^{\dagger} Y_{\Delta_{\delta \gamma}}$ and $c_{\alpha \beta}^{d=6}=\left(Y_{\Sigma}^{\dagger} \frac{1}{M_{\Sigma}^{2}} Y_{\Sigma}\right)_{\alpha \beta}$ respectively (where the various notations have been defined in Section 2.2).

These dimension 6 effects are in general expected to be far too faint to be observed soon. For example using the naive type-I seesaw expectation $Y_{N}^{2} \sim m_{\nu} m_{N} / v^{2}$, for $m_{N} \sim 10^{14} \mathrm{GeV}$ $\left(m_{N} \sim 100 \mathrm{GeV}\right)$ one gets typically $\operatorname{Br}(\mu \rightarrow e \gamma) \sim 10^{-52}\left(10^{-26}\right)$. However this naive seesaw expectation used here to relate Yukawa couplings to neutrino masses has no reason to be necessarily valid. The $c_{\alpha \beta}^{d=6}$ coefficients involve the Yukawa couplings in a $Y_{N} Y_{N}^{\dagger} / m_{N} L$ conserving combination, whereas the $c^{d=5}$ coefficients involve them in a $Y_{N}^{T} Y_{N} / m_{N}^{2} L$ violating one. Since both combinations differ on the basis of a symmetry they have no reason to be related in such a simple way. It turns out that the fermion seesaw states can have masses and Yukawa interactions without breaking lepton number, see e.g. Refs. [608, 609]. This means that, without adding any new particles or interactions, the seesaw framework could have an approximately lepton number conserving structure with large Yukawa couplings which do lead to large CLFV rates (if the seesaw scale is not too high) without 

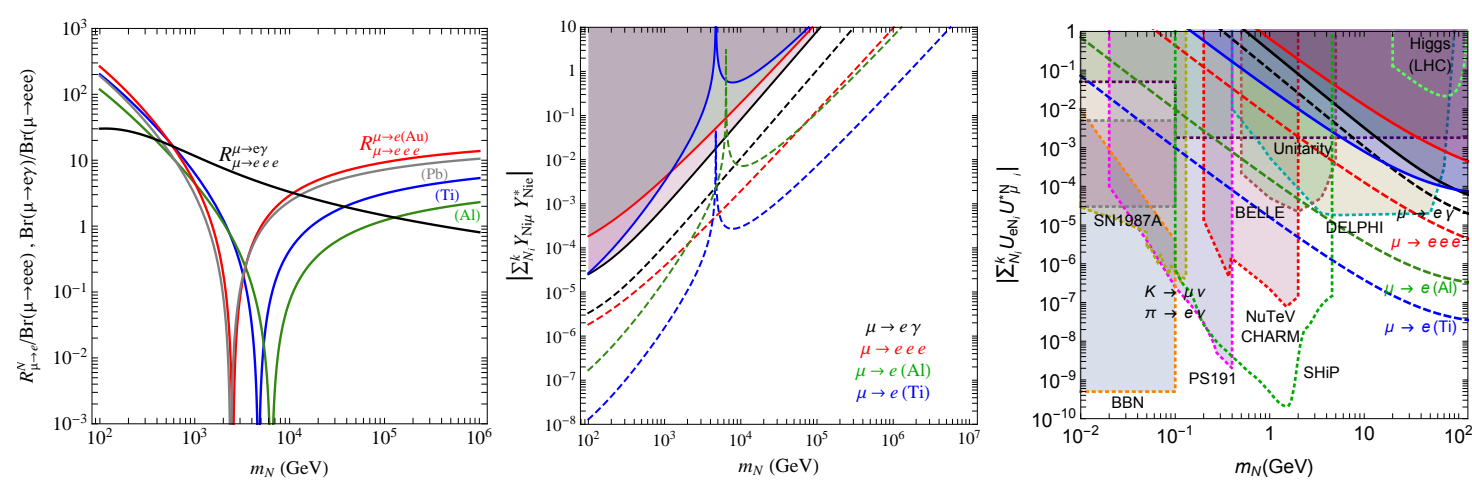

Figure 4.14: Left pannel: $\operatorname{Br}(\mu \rightarrow e \gamma) / \operatorname{Br}(\mu \rightarrow$ eee $)$ and $R_{\mu \rightarrow e e e}^{\mu \rightarrow e}=R_{\mu \rightarrow e}^{N} / \operatorname{Br}(\mu \rightarrow$ eee $)$ as a function of the right-handed neutrino mass scale $m_{N}$, for $\mu \rightarrow e$ conversion in various nuclei, from Ref. [392, 612]. Middle pannel: from Ref. [392], present bounds and future sensitivity on $\left|\sum_{i}^{k} Y_{\Sigma_{i \mu}} Y_{\Sigma_{i e}^{*}}\right|$ for scenarios characterized by one right-handed neutrino mass scale. The solid lines are obtained from present experimental upper bounds: from $R_{\mu \rightarrow e}^{T i}<4.3 \cdot 10^{-12}$ [613] and $\operatorname{Br}(\mu \rightarrow e \gamma)<5.7 \cdot 10^{-13}[614], \operatorname{Br}(\mu \rightarrow e e e)<10^{-12}$ [615]. The dashed lines are obtained from the expected experimental sensitivities: from $R_{\mu \rightarrow e}^{T i} \lesssim 10^{-18}[605,616], R_{\mu \rightarrow e}^{A l} \lesssim 10^{-16}$ [605]-[606] and $\operatorname{Br}(\mu \rightarrow e \gamma)<10^{-14}$ [607], $\operatorname{Br}(\mu \rightarrow e e e)<10^{-16}$ [604]. Right panel: from [392], $\left|\sum_{i} U_{e N_{i}} U_{\mu N_{i}}^{*}\right|$ (with $U_{j N_{i}} \equiv Y_{N_{j i}}^{\dagger} v / M_{N_{i}}$ the mixing angles with $v=174 \mathrm{GeV}$ ) versus mass sensitivity regions for present (continuous curves) and future (dashed curves) $e-\mu$ flavour experiments. Black, red, green and blue curves result from $\operatorname{Br}(\mu \rightarrow e \gamma), \operatorname{Br}(\mu \rightarrow e e e), R_{\mu \rightarrow e}^{A l}$ and $R_{\mu \rightarrow e}^{T i}$, respectively. The regions already excluded by non-unitarity limits, $\pi$ and $K$ peak searches, $\pi, K, D, Z$ decay searches, BBN, SN1987A and LHC collider searches (dotted lines) are also indicated. Shaded areas signal the regions already excluded experimentally. The right panel shows the nice future complementarity between CLFV experiments and SHiP.

inducing too large neutrino masses. A particularity of these setups is that they predict that the heavy neutral lepton which do induce large CLFV rate are quasi-degenerate in mass. This will play an important role for the testability of these setups below. Same structures can also be considered for the type-III case. For the type-II case the decoupling of the dimension 6 and 5 coefficient is manifest from the start, as the coefficient are proportional to $Y_{\Delta}^{4}$ and $Y_{\Delta} \mu_{\Delta} / m_{\Delta}$ respectively. Large $Y_{\Delta}$ couplings with small $\mu_{\Delta}$ coupling and not too large $m_{\Delta}$ gives large CLFV rates and small neutrino masses.

Let's start with the singlet heavy neutral lepton CLFV predictions. In this setup all CLFV processes are necessarily induced at the loop level because flavor violation occurs only at the level of the neutral leptons, requiring at least one internal $W$ boson inside a loop diagram. For analytical calculation of the $\mu \rightarrow e \gamma, \mu \rightarrow e e e$ and $\mu \rightarrow e$ rates see Refs. [304]-[610], Ref. [611].and Ref. [392] respectively (and Refs. therein). To establish the existence of heavy neutral lepton the use of a single CLFV rate is by no means enough, as neither the dimension- 6 coefficients, nor the seesaw scale can be known from neutrino mass matrix constraints alone. (This is possible tough for particular minimal flavor violation patterns [387, 612]). As pointed out in Refs. [612] and [392], the most promising possibility of tests is to consider ratios of two CLFV processes involving a same $l \rightarrow l^{\prime}$ transition. As said above in the seesaw configurations that do lead to observable CLFV rates the heavy neutral leptons at the origin of these large rates are quasi-degenerate in mass. As a result it is easy to see that the dimension 6 coefficients factorize out from the ratio, leaving a dependence only in the single $m_{N}$ scale. This allows for a long series of possible tests.

In Fig. 4.14 (left panel) are plotted the various ratios one gets for the various CLFV processes, for $m_{N}>100 \mathrm{GeV}$. First of all, for the $\operatorname{Br}(\mu \rightarrow e \gamma)$ to $\operatorname{Br}(\mu \rightarrow e e e)$ ratio, one observes [392,612] a 
monotonous function that is always larger than $\sim 2.5$ (for values of $m_{N}$ that do give an observable rate), which means that the measurement of this ratio would basically allow for a determination of $m_{N}$, or for an exclusion of the scenario if a ratio smaller than $\sim 2.5$ is found. In fact the observation of a single rate could already be sufficient to exclude the model, if together with the experimental upper bound on the other one, it leads to a ratio incompatible with the expectations of Fig. 4.14. A same discussion holds for the other ratios [392] given in Fig. 4.14, except for the fact that the $R_{\mu \rightarrow e}^{N}$ conversion rates (which depend crucially on the nuclei considered) turn out to vanish for a particular value of $m_{N}$, see Refs [391, 392]. Consequently the ratios are not monotonous functions of $m_{N}$. The value of $m_{N}$ which gives a vanishing $R_{\mu \rightarrow e}^{N}$ depends on the nuclei considered. This illustrates how important it would be to search for $\mu \rightarrow e$ conversion, not only with one nuclei, but with several of them.

Fig. 4.14 (middle panel) shows the lower bounds resulting for the Yukawa couplings, if the various rates are required to be large enough to be observed in planned experiments. It also shows the upper bounds which hold today on these quantities from the non-observation of these processes. This figure illustrates well the impact of future $\mu \rightarrow e$ conversion measurements/bounds, as they will become increasingly dominant in exploring flavor physics in the $\mu-e$ charged lepton sector. Values of the Yukawa couplings as low as $10^{-2}, 10^{-3}$ and $10^{-4}$ could be probed, for $m_{N}=100 \mathrm{TeV}, 1 \mathrm{TeV}$ and $100 \mathrm{GeV}$, respectively, with Titanium experiments being the most sensitive. These bounds can be rephrased as upper bounds on the $m_{N}$ scale for fixed values of the Yukawa couplings. For example if the later are of order unity, we get $m_{N} \lesssim 2000 \mathrm{TeV}, m_{N} \lesssim 300 \mathrm{TeV}, m_{N} \lesssim 100 \mathrm{TeV}$, $m_{N} \lesssim 300 \mathrm{TeV}$ from $R_{\mu \rightarrow e}^{T i}, R_{\mu \rightarrow e}^{A l}, \operatorname{Br}(\mu \rightarrow e \gamma), \operatorname{Br}(\mu \rightarrow e e e)$ respectively. Overall, this exercise shows that future experiments may in principle probe heavy neutral leptons beyond the $\sim 1000 \mathrm{TeV}$ scale, and that $\mu \rightarrow e$ conversion future experiments could become with time the most sensitive ones. For a similar discussion on CLFV processes involving a $\tau$, including the experimentally very promising $\tau \rightarrow \mu \mu \mu$ process, see Ref. [612,617].

The sensitivity of CLFV processes to heavy neutral lepton singlets lighter than $100 \mathrm{GeV}$ is also outstanding. On the right panel of Fig. 4.14 we give the sensitivity of the various CLFV processes down to the $10 \mathrm{MeV}$ scale. It shows that above the GeV scale future CLFV processes, in particular $\mu \rightarrow e$ conversion experiments will go below the sensitivity of any present constraint. Possibilities to observe a ratio of 2 same CLFV processes involving a same flavor transition do exist down to $\sim 50 \mathrm{TeV}$ if this ratio involves the $\mu \rightarrow e \gamma$ or $\mu \rightarrow e e e$ process. Below $100 \mathrm{GeV}$ the $R_{\mu \rightarrow e}^{T i} / R_{\mu \rightarrow e}^{A l}$ is predicted to be equal to 2 , which can be checked down to $\sim 5 \mathrm{GeV}$.

There is a crucial difference between the type-I and type-III seesaw models for what concerns CLFV processes. While in the type-I case there is flavor mixing only at the level of the neutral leptons, for the type-III case there is flavor mixing directly at the level of the charged leptons. For instance a $\mu^{-}-e^{-}$transition can proceed directly through charged lepton/charged $\Sigma$ mixing, i.e. through the $\mu^{-} \stackrel{Y}{\rightarrow} \Sigma^{-} \stackrel{Y}{\rightarrow} e^{-}$chain, with the $Y$ index referring to a vertex involving a Yukawa coupling (i.e. the insertion of a SM scalar boson or its vev). As a result, if for the type-I case all processes necessarily occurs at the loop level, for the type-III case the $l \rightarrow 3 l$ and $\mu \rightarrow e$ conversion process in atomic nuclei proceed at tree level. Only $\mu \rightarrow e \gamma$ still has to proceed at loop level because the QED coupling remains flavor diagonal in the charged fermion mass eigenstate basis (unlike Z couplings, see Refs. $[608,618])$. The tree level processes obviously do not induce logarithmic terms. Moreover it turns out that for $\mu \rightarrow e \gamma$ there is no logarithmic term either [608,618]. As a result the ratio of 2 same flavor transition processes are predicted to a fixed value! The measurement of such a ratio could then easily rule-out or strongly support the type-III scenario as possible explanation of these processes. We find $\operatorname{Br}(\mu \rightarrow e \gamma)=1.3 \cdot 10^{-3} \cdot \operatorname{Br}(\mu \rightarrow e e e)=3.1 \cdot 10^{-4} \cdot R_{\mu \rightarrow e}^{T i}$. Larger $\mu \rightarrow$ eee and $\mu \rightarrow e$ conversion rates, as compared to the $\mu \rightarrow e \gamma$ rate, are characteristic of this model unlike for the majority of other beyond the standard model scenarios that can lead to observable CLFV 
rates. See Ref. $[608,618]$ for the ratios involving a $\tau$ lepton.

Finally for what concerns the type-II seesaw model it presents a situation intermediate to the type I and III cases, as $\mu \rightarrow$ eee proceeds at tree level whereas $\mu \rightarrow e \gamma$ and $\mu \rightarrow e$ conversion proceeds at one loop. For predictions of ratios of 2 CLFV rates in this case see Ref. [617].

In conclusion clear possibilities of observing CLFV processes associated to heavy neutral leptons (or other seesaw states which display quite different patterns) do exist. These processes are competitive probes of heavy neutral leptons all the way from the $\mathrm{GeV}$ scale up to beyond $1000 \mathrm{TeV}$. The crucial way to distinguish the seesaw models, and which also allows for a determination of the seesaw mass scale, is to measure the ratio of $2 \mathrm{CLFV}$ processes invilving a same $l \rightarrow l^{\prime}$ transition.

\subsubsection{HNL and primordial nucleosynthesis}

The primordial nucleosynthesis (BBN) describes the formation of all the light elements, such as deuterium, helium, lithium etc, at a time when the universe was between 1 second and few minutes old, and went from a temperature of $3 \mathrm{MeV}$ to $50 \mathrm{keV}$. When one changes the properties of the universe around 1 second one affects the predicted abundance of helium and deuterium, which can then be compared to present astronomical observations [156]

The existence of HNL may affect the BBN in various way. The most important is throught the increased energy density of the Universe. A higher energy density gives a faster expansion rate, and this implies that the weak reaction rates between neutrons and protons $\left(n, p, \nu_{e}, e\right)$ will become slow in comparison to the expansion rate. Thus the neutron-to-proton ratio, $r_{p} \equiv n_{n} / n_{p}$, will freeze out at a higher value. Since $r_{p}$ is directly linked to the observable deuterium and helium abundances, this gives us the possibility of testing the existence of HNL using observations of the light elements [619].

Very generally, two properties are required of the HNL in order to affect the BBN: $i$ ) it must be produced in sufficient abundance before the time of BBN, and ii) it must not have decayed much before the time of BBN. The first implies that very small mixing angles will not affect the BBN (typically $U^{2}>10^{-12}$ for $M=100 \mathrm{MeV}$ ). The second implies that large mixing angles also do not affect BBN (typically $U^{2}<10^{-4}$ for $M=100 \mathrm{MeV}$ ). This renders BBN constraints complementary to direct experimental searches [323].

The calculation of the effect of HNL on BBN is divided into 4 mass regions separated at $2 \mathrm{MeV}$, $140 \mathrm{MeV}$, and $500 \mathrm{MeV}$.

Below $2 \mathrm{MeV}$. BBN effectively excludes these small masses. For such masses the lifetime is around $\tau=100 \mathrm{sec} /\left((M / 2 \mathrm{MeV})^{5} U^{2}\right)$, which implies that the lifetime is longer than the BBN time of a few minutes, even for mixing $U^{2} \sim 1$. The relativistic HNL would increase the expansion rate, leading to a higher helium abundance than observed.

Between $2 \mathrm{MeV}$ and $140 \mathrm{MeV}$. In this mass range detailed calculations have shown, that BBN constrain a wide band in $U^{2}$. These calculations include both the effect of the increased energy density (which increases $r_{p}$ ), the decay produced, non-thermal spectrum of the electron neutrinos which preserves equilibrium between neutrons and protons for longer (decreasing $r_{p}$ ), and finally decays into the electromagnetic part of the primeval plasma will reduce the relative contribution of the energy density of the light neutrions and hence diminish $r_{p}$ [412-414]. The result is that mixing angles are allowed above $U^{2}>0.4$ @ NHL mass of $10 \mathrm{MeV}$ and above $U^{2}>4 \times 10^{-5}$ @ NHL mass of $140 \mathrm{MeV}$. The main reason is that when the HNL decays sufficiently early, the thermal equilibrium between the neutrinos and the electromagnetic sector erases all signs of the NHL. When translated into decaytime, the excluded region goes between 2 and $1 / 20$ second for masses between 10 and 140 $\mathrm{MeV}$ respectively.

Also very small mixing angles are allowed, since they would lead to very small energy density of the HNL at the time of BBN. An estimate of this effect gives the constraint $U^{2}(M / 50 \mathrm{MeV})^{2}=$ 
$10^{-10}$.

Between $140 \mathrm{MeV}$ and $500 \mathrm{MeV}$. At these masses one must include the decay channels including the charged and neutral pions [413,620,621]. An estimate on the effect hereof on BBN was made in [413]. The pion from, $\nu_{s} \rightarrow \pi_{0}+\nu_{\alpha}$ decays into 2 photons, which heats the electromagnetic component of the plasma, which diminishes the role of the active neutrinos. This leads to an underproduction of helium. The result is that lifetimes larger than approximately $\tau=0.1$ sec are excluded, which can be translated into an excluded region for large masses and mixing angles.

Above $500 \mathrm{MeV}$. At these high masses new decay channels open up (including kaons, eta, etc), and the calculations become significantly more complicated [620]. No reliable estimate on the effect on BBN appears in the literature.

\subsection{HNL and baryon asymmetry of the Universe}

One of the most important arguments for the existence of physics beyond the Standard Model is baryon asymmetry of the Universe (BAU). A number of attempts to explain BAU within the Standard Model were made some 20 years ago, when no experimental evidence for physics beyond the SM existed [622-625]. They failed because of two reasons. First, the Kobayashi-Maskawa CPviolation happened to be too small to account for baryogenesis [622, 626, 627]. Second, it has been shown that the non-equilibrium first order electroweak phase transition, necessary for baryogenesis in the SM, can only occur if the Higgs mass is smaller than $M_{H} \simeq 72 \mathrm{GeV}$ [628, 629] (see also $[630])$.

A convincing experimental proof of incompleteness of the Standard Model came at the end of nineties and is associated with observation of neutrino oscillations (see Section 4.2 for an overview). It calls for extension of the SM in the neutrino sector, and thus to existence of new leptonic states, new sources of CP-violation and new possibilities for baryogenesis. Guided by the principle of "minimality" (explain the maximum number of physical effects with the smallest number of new particles introduced) we will discuss below the prospects for baryogenesis motivated by the discoveries in neutrino physics and associated with HNLs. Many other mechanisms, suggested in the literature, will not be discussed here (for reviews see, e.g. [631-637].).

We will start with a short overview of Sakharov conditions and then describe the physics of baryogenesis for different choices of HNL masses: the GUT scale, the TeV scale, and finally the $\mathrm{GeV}$ scale, relevant for the SHiP experiment .

\subsubsection{Sakharov conditions. Leptogenesis}

The night sky is decorated with stars, and a wide range of observations, from $\gamma$-ray spectra [638] to the Cosmic Microwave Background(CMB) [272] passing via primordial nuclear abundances [156], agree that all stars are made of matter. This universal excess of matter over anti-matter is refered to as the "Baryon Asymmetry of the Universe" (BAU), because the lightest baryons are the constituents of matter, the neutron and the proton. This means that matter and baryon asymmetries are proportional, whereas a large and different lepton asymmetry could be present in the Cosmic Neutrino Background. The best current determination of the baryon minus anti-baryon number density $n_{B}$, normalised to the entropy density $s$, is from the CMB (PLANCK [272]):

$$
\frac{n_{B}}{s}=(8.59 \pm 0.13) \times 10^{-11} \quad(1 \sigma)
$$

Today, $s \simeq 7 n_{\gamma}$, so for every 1600 million photons, there is a baryon.

The origin of the BAU is a puzzle for modern physics. It is improbable that the Universe was born with an asymmetry, because the coherence of large-scale CMB fluctuations strongly suggests the Universe underwent a period of inflation, during which any original asymmetry would have been 
diluted to irrelevance. To separate baryons from antibaryons on scales of order our Universe today, in a baryon-symmetric Universe, is at least as difficult as generating an asymmetry. So one is left with the option of generating the BAU after inflation. However, there is no known way to do so in the standard models of particle physics and cosmology. So the BAU shares the rare distinction of indicating Beyond the Standard Model physics, and a multitude of models ( see,eg [632, 639-643]) have been proposed to explain the number given in equation (4.6.1).

Fortunately, generating the BAU is like making a cake: there may be many recipes, but a few key ingredients ensure success. For the BAU, these were succinctly given by Sakharov [644] long ago, and are refered to as "Sakharov conditions":

1. Baryon number $(B)$ violation

To evolve from a state with $B=0$, to a state with $B \neq 0$, requires $B$ violation.

$\mathrm{B}$ violation can be obtained by adding to the Lagrangian interactions that change $\mathrm{B}$. In the case of interactions which change B by one unit, it can be challenging to obtain a sufficient BAU in the first few seconds of the Universe, while respecting the lower limit on the proton lifetime [645]: $\tau_{p}>8.2 \times 10^{33}$ years for the process $p \rightarrow \pi_{0} e^{+}$. Alternatively, the Standard Model has non-perturbative $\mathrm{B}+\mathrm{L}$ violation [646] as a consequence of the chiral anomaly. These interactions change $\mathrm{B}$ and $\mathrm{L}$ by one unit in each generation (so $\Delta B=\Delta L=3$ ), are exponentially suppressed in the Universe today, but relatively fast before [647] and during the Electroweak Phase Transition [648].

2. $\mathrm{C}$ and $\mathrm{CP}$ violation

Particles and anti-particles must behave differently — otherwise particles and antiparticles could simultaneously use the $B$ violation to make cancelling $B$ and anti- $B$ asymetries. Since some Standard Model particles are transformed to their antiparticle by $\mathrm{C}$, and some by $\mathrm{CP}$, violation of both is required.

This can be obtained from the phases of fields or couplings.

3. departure from thermal equilibrium

In thermal equilibrium, there are no asymmetries in unconserved quantum numbers, and $B$ is not conserved by condition 1 . So generating the BAU is a dynamical process; phase transitions and the expansion of the Universe are sources of non-equilibrium.

The Standard Models of particle physics and cosmology verify the Sakharov conditions: there is non-perturbative $\mathrm{B}+\mathrm{L}$ violation, and $\mathrm{CP}$ violation in the $\mathrm{CKM}$ matrix, however the non-equilibrium (from the Universe expansion or the electroweak phase transition) appears insufficient. In particular, all perturbative SM interactions are in equilibrium at the electroweak scale, the electroweak phase transition is a cross-over (so there are no bubble walls to provide dynamics), and the $\mathrm{B}+\mathrm{L}$ violation turns off after the phase transition [648].

Using the SM of particle physics to generate the BAU in alternate cosmologies could be possible $[649,650]$. The more popular approach is to extend the Standard Model of particle physics, in standard cosmology. Such models usually contain a large number of new parameters, but the BAU is only one number. So it is judicious to study the BAU in models that are motivated for other reasons. For instance, supersymmetric models contain many scalars to address the hierarchy problem, and the Affleck-Dine mechanism [651] uses the dynamics of these $B$ - or $L$-carrying scalar fields to generate the BAU. Or leptogenesis [652] is a class of mechanisms, which use the $L$ violation present in Majorana neutrino mass models.

Leptogenesis is defined here to include all scenarios which produce a lepton (anti-)asymmetry via $\mathrm{CP}$-violating out-of-equilibrium processes, and rely on the $\mathrm{SM}$ non-perturbative $\mathrm{B}+\mathrm{L}$ violation, in equilibrium, to partially transform the lepton deficit into a baryon excess. Leptogenesis therefore 


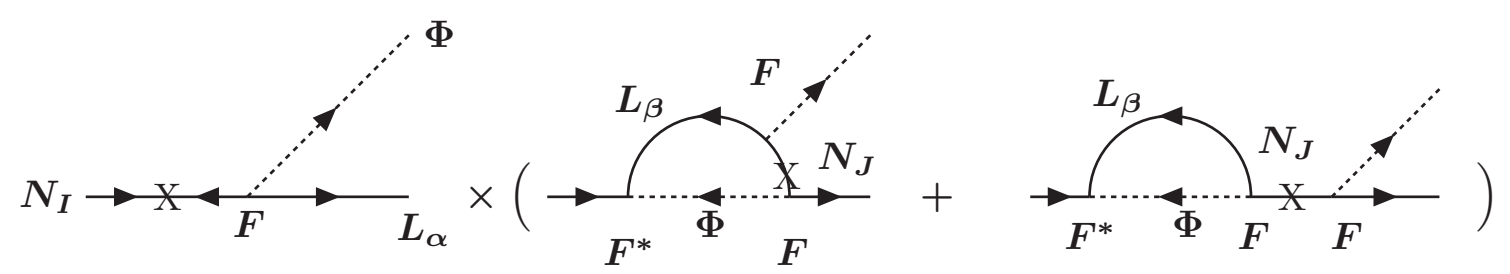

Figure 4.15: The diagrams contributing to the $\mathrm{CP}$ asymmetry $\epsilon_{1}^{\alpha}$. The flavour of the internal lepton $L_{\beta}$ is summed. The internal $L_{\beta}$ and Higgs $\Phi$ are on-shell. The X represents a Majorana mass insertion. Line direction is "left-handedness", assigning to scalars the handedness of their SUSY partners.

occurs before/at the electroweak phase transition. A minor attraction is that there are no $\Delta B=1$ interactions, so no concerns with proton decay. More importantly, a natural way to understand why neutrinos are much lighter than other SM fermions, is to suppose that their masses are Majorana, that is, $L$-violating. So in such extensions of the SM, the first Sakharov condition comes for free.

Leptogenesis was proposed by Fukugita and Yanagida [652] in the type I seesaw, and has been extensively studied in type I (for reviews, see e.g. [643, 653]), type II [370, 654, 655], and type III [656, 657] seesaws, mostly using Boltzmann Equations as pioneered by [658]. Recent interest has focussed on improving the credibility and accuracy by calculating interaction rates at finite temperature [659-665], and by obtaining quantum equation of motion [666-668]. The next sections summarise the scenario in the type I seesaw with hierarchical heavy singlets, and degenerate singlets at various mass scales.

\subsubsection{Thermal leptogenesis}

Consider the type I seesaw model in three generations, containing heavy gauge singlet neutrinos with a hierarchical spectrum $\left(10^{9} \mathrm{GeV} \lesssim M_{1} \ll M_{2} \ll M_{3} \lesssim 10^{15} \mathrm{GeV}\right)$, and whose only interactions are the Yukawa $F_{\alpha I} \bar{L}_{\alpha} \Phi N_{I}$. The Yukawa matrix $F$ is taken complex; recall also that the combination of Majorana masses $M_{I}$ and the Yukawa couplings $F$ is $L$ violating. This area of parameter space is GUT-motivated, because $F$ is comparable to other SM Yukawa matrices. However, there are loop contributions to the Higgs mass, $\delta m_{\Phi}^{2} \sim m_{\nu} M_{I}^{3} /\left(4 \pi v^{2}\right)$ which greatly exceed the measured Higgs mass, and which somehow must be cancelled.

After inflation, suppose that the Universe reheats to a bath of particles with gauge interactions (no heavy singlets $N_{I}$ ), of temperature $T_{\text {reheat }}$, such that $10^{9} \mathrm{GeV} \lesssim M_{1} \lesssim T_{\text {reheat }} \lesssim M_{2}$. This ensures that the dynamics of leptogenesis are simple, allowing credible analytic estimates.

A population of $N_{1} \mathrm{~s}$ will develop due to their Yukawa interactions. This is a non-equilibrium process, so assuming there are phases in $F$, asymmetries will simultaneously be generated in the distinguishable flavours of SM lepton doublets. The finite temperature production rate requires resummation even at leading order, and has been calculated in [663-665].

If the interaction rate of the $N_{1} \mathrm{~s}$ is $\gtrsim H$ ( $H \equiv$ the Hubble expansion rate), as is possible, then an equilibrium distribution of $N_{1}$ s will be produced, and the asymmetries in lepton doublets will be washed out. This "strong washout" area of parameter space is predictive, because pre-existing asymmetries vanish, so the BAU will be generated in the decay of $N_{1}$ (see e.g. [669]).

When the temperature of the expanding Universe drops below $M_{1}$, the equilibrium $N_{1}$ abundance crashes exponentially, so the $N_{1}$ population goes away. Individual $N_{1}$ s decay to a Higgs and lepton, or anti-Higgs and anti-lepton. The CP asymmetry in doublet flavour $\alpha$ arising from the 
tree $\times$ loop diagrams for the decay of $N_{1}$ (see figure 4.15 ) can be written, at $T=0$, as

$$
\epsilon_{I}^{\alpha}=\frac{\Gamma\left(N_{I} \rightarrow \Phi L_{\alpha}\right)-\Gamma\left(N_{I} \rightarrow \bar{\Phi} \bar{L}_{\alpha}\right)}{\Gamma\left(N_{I} \rightarrow \Phi L\right)+\Gamma\left(N_{I} \rightarrow \bar{\Phi} \bar{L}\right)}=\frac{1}{(8 \pi)} \frac{1}{\left[F^{\dagger} F\right]_{I I}} \sum_{j} \operatorname{Im}\left\{\left(F_{\alpha 1 I}^{*}\right)\left(F^{\dagger} F\right)_{I J} F_{\alpha J}\right\} g\left(x_{J}\right)
$$

(see [660] for a finite temperature calculation), where $x_{J} \equiv M_{J}^{2} / M_{I}^{2}$, and, within the SM [670],

$$
g(x)=\sqrt{x}\left[\frac{1}{1-x}+1-(1+x) \ln \left(\frac{1+x}{x}\right)\right] \stackrel{x \gg 1}{\longrightarrow}-\frac{3}{2 \sqrt{x}}-\frac{5}{6 x^{3 / 2}}+\ldots
$$

The asymmetries in distinguishable lepton flavours should be evolved individually in Boltzmann equations. A flavour is "distinguishable", if its charged lepton Yukawa is in equilibrium, which is true for the $\tau$ Yukawa at the temperatures $10^{9} \lesssim T \lesssim 10^{12} \mathrm{GeV}$ considered here (see [643] for flavoured Boltzmann equations). So the flavour index $\alpha$ runs over $\tau, o$, where $o$ is a linear combination of $\mu$ and $e$.

These CP asymmetries are in general uncorrelated to the phases of the lepton mixing matrix at low energy [671]. However, there is an upper bound [672, 673]

$$
\left|\epsilon_{1}\right|=\left|\sum_{\alpha} \epsilon_{1}^{\alpha}\right| \leq 3 m_{\mathrm{atm}} M_{1} /\left(8 \pi v^{2}\right)
$$

which applies for $M_{1} \ll M_{2,3}$, because in this case the $N_{2}, N_{3}$ propagators in the loop can be approximated as contact interactions (so effectively, the dimension-five light neutrino mass operator sits in the loop).

In the strong washout scenario, a deficit of lepton number $L_{\alpha}$ from the decay of $N_{1}$, will no longer be washed out by the inverse decays, once these go out of equilibrium, which occurs at a temperature $T_{f \alpha}$, defined such that $\Gamma\left(\Phi L_{\alpha} \rightarrow N_{1}\right) \lesssim H\left(T_{f \alpha}\right)$. This is the third Sakharov condition. So the final asymmetry in flavour $\alpha$ can be estimated as

$$
\frac{n_{L_{\alpha}}-n_{\overline{L_{\alpha}}}}{s} \simeq \frac{n_{N}\left(T_{f \alpha}\right)}{s} \epsilon_{1}^{\alpha} \sim \frac{\Gamma\left(N_{1} \rightarrow \Phi L_{\alpha}\right)}{H\left(T=M_{1}\right)} \frac{n_{\nu}}{s} \epsilon_{1}^{\alpha}
$$

Using $\Gamma\left(\Phi L_{\alpha} \rightarrow N_{1}\right) \simeq e^{-M_{1} / T_{f \alpha}} \Gamma\left(N_{1} \rightarrow \Phi L_{\alpha}\right)$, and $n_{N}\left(T_{f(\alpha)}\right) \simeq e^{-M_{1} / T_{f \alpha}} n_{\nu}$ gives the second approximation. More accurate estimates can be obtained by solving the Boltzmann equations, or, for some parameter ranges, the equations for two-point functions obtained in closed-time-path quantum field theory.

The non-perturbative Standard Model $\mathrm{B}+\mathrm{L}$ violating processes are expected to be in equilibrium, and fast, at the temperatures considered here. So the lepton asymmetries generated in $N_{1}$ interactions are immediately redistributed among other SM particles, resulting in a baryon asymmetry today

$$
\frac{n_{B}}{s} \simeq \frac{12}{37} \frac{135 \zeta(3)}{4 \pi^{4} g_{*}} \sum_{\alpha} \eta_{\alpha} \epsilon_{1}^{\alpha}
$$

where $\eta_{\alpha}=\Gamma\left(N_{1} \rightarrow \Phi L_{\alpha}\right) / H$ is the "efficiency factor", and $g_{*}=106$ is the number of degrees of freedom in the SM plasma. The coefficient of the sum is $\simeq 1.4 \times 10^{-3}$, so since $\eta_{\alpha} \lesssim 1$, one needs $\epsilon_{1}^{\alpha} \gtrsim 10^{-6}$ to reproduce the observed asymmetry. Combined with eqn (4.6.4), this implies that $M_{1} \gtrsim 10^{9} \mathrm{GeV}$, for thermal leptogenesis with hierarchical singlet masses.

That the same Yukawa interactions are responsable for the production and decay of the $N_{I}$ s, distinguishes the minimal seesaw leptogenesis from other out-of-equilibrum-decay scenarios, such as the decay of GUT bosons. If the initial heavy particle distribution is generated by gauge interactions, then the asymmetry is generated only in decay, and if the decay is out-of-equilibrium, there is no 
washout. However, if washout is neglected in leptogenesis, then the doublet asymmetry generated with the heavy singlets, will exactly cancel the anti-asymmetry generated in their decays, so the final asymmetry is zero.

This scenario works naturally for $T_{\text {reheat }}>10^{9} \mathrm{GeV}$, but is challenging to confirm, because the high-scale Lagrangian has 18 parameters beyond the SM, whereas at low energy, we can at best measure three neutrino masses, three mixing angles and three phases. However, this scenario could be ruled out, if cosmological observations determined that $T_{\text {reheat }}<10^{9} \mathrm{GeV}[674,675]$. A lower scale for $M$ would be more testable.

\subsubsection{Resonant Leptogenesis}

The GUT-scale leptogenesis scenario (Sec. 4.6.2) runs into certain difficulties, if one attempts to explain the flatness of the Universe and other cosmological data [676] within supergravity models of inflation. To avoid overproduction of gravitinos $\widetilde{G}$ whose late decays may ruin the successful predictions of BBN, the reheat temperature $T_{\text {reh }}$ of the Universe should be lower than $10^{9}-10^{6} \mathrm{GeV}$, for $m_{\widetilde{G}}=8-0.2 \mathrm{TeV}$ [677-680]. This implies that the heavy Majorana neutrinos should accordingly have masses as low as $T_{\text {reh }} \lesssim 10^{9} \mathrm{GeV}$, thereby rendering the relation of these particles with GUT-scale physics less natural. On the other hand, it proves very difficult to directly probe the heavy-neutrino sector of such a model at high-energy colliders, e.g. at the LHC or in any other foreseeable experiment.

A potentially interesting and phenomenological testable scenario that circumvents the above problems may be obtained within the framework of Resonant Leptogenesis (RL) [681], where the isosinglet Majorana mass scale could be lowered well below TeV scale. The key aspect of RL is that self-energy effects dominate the leptonic asymmetries [670,682, 683], when two heavy Majorana neutrinos happen to have a small mass difference with respect to their actual masses (for earlier works on resonant baryogenesis see [684]). If this mass difference becomes comparable to the heavy neutrino widths, a resonant enhancement of the leptonic asymmetries takes place that may reach values $\mathcal{O}(1)[681,685,686]$. An indispensable feature of $\mathrm{RL}$ models is that flavour effects due to the light-to-heavy neutrino Yukawa couplings [687-689] play a dramatic role and can modify the predictions for the BAU by many orders of magnitude [687, 688]. Most importantly, these flavour effects enable the modelling [688] of minimal RL scenarios with electroweak-scale heavy Majorana neutrinos that could be tested at the LHC $[313,316,485,577,690]$ and in other non-accelerator experiments, while maintaining agreement with the low-energy neutrino data.

Many variants of RL have been proposed in the literature, which include:

- Soft RL [691, 692]. In this scenario, leptogenesis results from sneutrino decays in the MSSM. CP-violating soft SUSY-breaking parameters may split the degeneracy within a single generation of right-handed sneutrino states, which leads to a resonant enhancement of the leptonic asymmetries.

- Radiative RL [693-695]. In this case, the heavy Majorana neutrinos are exactly degenerate at the GUT scale. Then, small mass differences among the heavy Majorana neutrino states are generated via renormalization group (RG) running from the GUT scale down to the leptogenesis scale. These small mass differences give rise to RL.

- Leptogenesis via sterile neutrino oscillations [396, 397, 696]. This scenario relies on the possibility that sterile neutrinos with masses well below the Fermi scale, typically in the range of 1 to $10 \mathrm{GeV}$, maintain the coherence of their $\mathrm{CP}$ asymmetric oscillations and produce an enhanced leptonic asymmetry which is of $\mathcal{O}\left(F_{\alpha I}^{6}\right)$ but with the proper amount needed to create the observed BAU. This scenario can be tested by the SHIP experiment and is discussed in more detail in the subsequent sections. 
Making use of the corresponding terminology of the dynamics of the $K^{0} \bar{K}^{0}$ system in the medium, there are three distinct mechanisms that contribute to the CP-violating leptonic decays of the heavy Majorana neutrinos:

- $\varepsilon^{\prime}$-type $C P$ violation. In this case, the required $\mathrm{CP}$ violation arises from the interference of the tree-level graph $N_{I} \rightarrow L_{\alpha} \Phi$ with the absorptive part of the vertex correction. The $\varepsilon^{\prime}$-type leptonic asymmetry is given by

$$
\varepsilon_{N_{I}}^{\prime}=\frac{\operatorname{Im}\left(F^{\dagger} F\right)_{I J}^{2}}{\left(F^{\dagger} F\right)_{I I}\left(F^{\dagger} F\right)_{J J}}\left(\frac{\Gamma_{J}}{M_{I}}\right) f\left(\frac{M_{J}^{2}}{M_{I}^{2}}\right),
$$

where

$$
\Gamma_{J}=\frac{\left(F^{\dagger} F\right)_{J J}}{8 \pi} M_{J}
$$

is the tree-level decay width of $N_{J}$, and $f(x)=-\sqrt{(x)}(1+x) \ln [(1+x) / x]$ is the FukugitaYanagida loop function [697], which is part of the function $\mathrm{g}(\mathrm{x})$ that appeared in Eq. (4.6.3) of the previous section.

- $\varepsilon$-type CP violation. This is due to off-diagonal absorptive transitions from one heavy neutrino to another which lead to CP-mixed sterile neutrino states. Based on an effective LSZ-type formalism [681, 685], the $\varepsilon$-type leptonic asymmetry is found to be

$$
\varepsilon_{N_{I}}^{\operatorname{mix}}=\frac{\operatorname{Im}\left(F^{\dagger} F\right)_{I J}^{2}}{\left(F^{\dagger} F\right)_{I I}\left(F^{\dagger} F\right)_{J J}}\left(\frac{\Gamma_{J}}{M_{J}}\right) \frac{\left(M_{I}^{2}-M_{J}^{2}\right) M_{I} M_{J}}{\left(M_{I}^{2}-M_{J}^{2}\right)^{2}+M_{I}^{2} \Gamma_{J}^{2}}
$$

Note that $\varepsilon_{N_{I}}^{\text {mix }}$ dominate over the $\varepsilon_{N_{I}}^{\prime}$ when $\left|M_{I}-M_{J}\right| \ll M_{I, J}$. In the limit $M_{I} \rightarrow M_{J}$, the would-be singularity is regularized by the decay width of the heavy neutrino $N_{J}$.

- Regenerative CP violation. This third source of CP violation occurs via the regeneration of the heavy sterile neutrinos from the thermal plasma. In analogy to the phenomenon of regeneration of $K_{S}$ states in the medium [698], the inverse decays create different coherences of heavy netrino states from the ones involved in the direct decays, leading to oscillations [699, 700]. The leptonic asymmetry due to these sterile-neutrino oscillations is $\mathcal{O}\left(F_{\alpha I}^{4}\right)$ and is approximately given by

$$
\varepsilon_{N_{I}}^{\mathrm{osc}} \approx \frac{\operatorname{Im}\left(F^{\dagger} F\right)_{I J}^{2}}{\left(F^{\dagger} F\right)_{I I}\left(F^{\dagger} F\right)_{J J}}\left(\frac{\Gamma_{J}}{M_{J}}\right) \frac{\left(M_{I}^{2}-M_{J}^{2}\right) M_{I} M_{J}}{\left(M_{I}^{2}-M_{J}^{2}\right)^{2}+M_{N}^{2}\left(\Gamma_{N_{1}}+\Gamma_{N_{2}}\right)^{2}},
$$

where $M_{N}=\left(M_{N_{1}}+M_{N_{2}}\right) / 2$. Note that the CP-violating source due to $\varepsilon_{N_{I}}^{\text {osc }}$ has the same sign with the one arising from $\varepsilon_{N_{I}}^{\text {mix }}$, giving rise to an enhancement of a factor of $\sim 2$ in scenarios of resonant leptogenesis. Recently, these results have independently been confirmed in [700] by an approach based on the CTP KadanoffBaym formalism. Still, the situation remains somewhat debatable, as in Refs. [667, 701], where $\varepsilon$-type CP-violation and CP-violation from mixing are identified, no enhancement by a factor $\sim 2$ is reported.

The inclusion of phenomena due to charged-lepton decoherence and heavy-neutrino flavour oscillations may increase the predictions for the BAU by up to one order of magnitude in RL models, according to a recent study based on a fully flavour-covariant formalism [699].

\subsubsection{Leptogenesis via HNL oscillations}

In this subsection we are going to describe how the baryon asymmetry is generated via oscillation of relatively light right-handed neutrinos, described in Sec. 4.3.2.3, following [396, 397]. In the considering mass range of right-handed neutrinos, say $M_{N} \lesssim \mathcal{O}(10) \mathrm{GeV}$, the lepton-number violation 
due to Majorana masses is negligible at the baryogenesis temperatures $T>T_{\mathrm{sph}} \sim 100 \mathrm{GeV}\left(T_{\mathrm{sph}}\right.$ is the temperature when the rapid baryon number violation due to the sphaleron process is switched off.). It is, therefore, crucial that the lepton asymmetry is distributed into left-handed leptons $L_{\alpha}$ and right-handed neutrinos $N_{I}$ rather than the generation of the total asymmetry as in the case of the conventional leptogenesis scenario [697]. In this case, the asymmetry stored in the left-handed sector is partially transferred into the baryon asymmetry due to the sphaleron transitions which are rapid for $T>T_{\mathrm{sph}}[647]$.

The generation of these asymmetries are described by the kinetic equations for matrices of densities $\rho_{N}$ of right-handed neutrinos and chemical potentials $\mu_{\nu}$ of left-handed neutrinos [397] (see also $[415,702,703]$ )

$$
\begin{aligned}
\frac{d \rho_{N}}{d t}= & -i\left[H_{N}^{0}+V_{N}, \rho_{N}\right]-\frac{3}{2} \gamma\left\{F^{\dagger} F, \rho_{N}-\rho_{N}^{e q}\right\}+3 \gamma F^{\dagger} \mu_{\nu} F \\
\frac{d \mu_{\nu_{\alpha}}}{d t}= & -\gamma\left(F F^{\dagger}\right)_{\alpha \alpha} \mu_{\nu_{\alpha}}-\frac{\gamma}{4}\left[F \rho_{N} F^{\dagger}-F^{*} \rho_{\bar{N}} F^{T}\right]_{\alpha \alpha} \mu_{\nu_{\alpha}} \\
& +\frac{3 \gamma}{4}\left[F \rho_{N} F^{\dagger}-F^{*} \rho_{\bar{N}} F^{T}\right]_{\alpha \alpha},
\end{aligned}
$$

where $H_{N}^{0}$ denote the free Hamiltonian, $V_{N}=(T / 16) F^{\dagger} F$, and $\gamma=3 h_{t}^{2} T /\left(64 \pi^{3}\right)$ [396]. The kinetic equation for $\rho_{\bar{N}}$ is obtained by replacing $F \rightarrow F^{*}$ and $\mu_{\nu} \rightarrow-\mu_{\nu}$ in (4.6.8).

These equations incorporate the medium effects of surrounding hot plasma, i.e., the thermal potential $V_{N}$ which induces the coherent oscillations of right-handed neutrinos and the decoherent terms which describe the production and destruction of $N_{I}$ [396]. Furthermore, they contain the terms which express the exchange of asymmetries between left and right-handed sectors [397].

The inclusion of such terms by [397] is crucial in the following respects. The one important point is that such terms make the baryogenesis effective even if the number of right-handed neutrinos is $\mathcal{N}=2$. The other one is that the yield of baryon asymmetry does depend on the parameters of active neutrinos. This is because such terms depend on the neutrino Yukawa couplings in a very complicated way (rather than the simple $F^{\dagger} F$ ) and hence they depend on, for example, the values of the PMNS mixing matrix. Therefore, the $\mathrm{CP}$ violations of active neutrino physics can be directly related with the cosmic baryon asymmetry in this framework. This is a distinct feature of the mechanism compared with the simple leptogenesis scenario [697].

The coupled equations (4.6.8) and (4.6.9) can be solved not only numerically, but also analytically by using the perturbative expansion of the Yukawa coupling constants $F$ [397]. At the order $\mathcal{O}\left(F^{2}\right)$ right-handed neutrinos are created due to the production term and start to oscillate due to the mixing induced by the effective potential. The temperature of such an oscillation is determined from the mass-squared difference of right-handed neutrinos. Then, at the order $\mathcal{O}\left(F^{4}\right)$, the third term in the right-hand side of (4.6.9) generate the asymmetries of left-handed leptons due to the $\mathrm{CP}$ violation in the leptonic sector. the total asymemtry in the left-handed leptons is zero $\sum_{\alpha} \mu_{\alpha}=0$ at this order, but becomes non-vanishing at the order $\mathcal{O}\left(F^{6}\right)$ because of the exchange of asymmetries between left- and right-handed leptons. Therefore, the baryon asymmetry in this mechanism is generated at $\mathcal{O}\left(F^{6}\right)$.

The yield of baryon asymmetry depends on the Yukawa couplings and the masses of righthanded neutrinos. Especially, the mass differences are important to determine the time of their flavor oscillations which is essential in this mechanism. As shown below, when two right-handed neutrinos participate the baryogenesis (as in the $\nu \mathrm{MSM}$ ), enough baryon asymmetry can be generated if the mass difference is sufficiently small.

We should comment that in the description above the momentum dependence of the matrices of densities is approximately taken into account. This issue has been discussed in Ref. [703] and it is found that the final value of the yield of baryon asymmetry does not change much by the correct 
treatment of the momentum dependence.

\subsubsection{Leptogenesis via oscillations with two HNLs}

Now we are at the position to discuss the baryogenesis in the $\nu \mathrm{MSM}$ with three right-handed neutrinos (HNLs) $\mathcal{N}=3$. In this case the lightest HNL $N_{1}$ is a candidate of dark matter. This particle is allowed to possess very suppressed Yukawa couplings (see the discussion in Section 2.2.5), and then $N_{1}$ gives only a minor contribution to the seesaw mass matrix of active neutrinos [321, 322] and also the generation of baryon asymmetry [397] via the mechanism [396, 397]. Therefore, $N_{2}$ and $N_{3}$ are responsible to these origins and the masses and mixings of active neutrinos and the baryon asymmetry of the universe correlate to each other through physics of $N_{2}$ and $N_{3}$.

To ensure the successful seesaw mechanism, the Yukawa couplings of $N_{2}$ and $N_{3}$ can be parametrized as in Eq. (4.3.11). We can choose to work in the basis where the right-handed neutrino mass matrix is diagonal, then $V=1$ and $D_{N}=\operatorname{diag}\left(M_{2}, M_{3}\right)$, which we take $M_{2}=M_{N}-\Delta M / 2$ and $M_{3}=M_{N}+\Delta M / 2$. Moreover, we assume that the couplings of $N_{1}$ are zero $F_{\alpha 1}=0$ for simplicity, and hence the masses of active neutrinos are $m_{3}>m_{2}>m_{1}=0$ for the NH case and $m_{2}>m_{1}>m_{3}=0$ for the IH case.

The CP violation in leptonic sector is essential for the considering baryogenesis. There are three violating parameters in the Yukawa couplings of $N_{2,3}$, the Dirac and Majorana phases, $\delta$ and $\eta$ and the complex parameter $\hat{\theta}$. When $F_{\alpha 1}=0$, the number of Majorana phase in $U_{\mathrm{PMNS}}$ is equal to unity (rather than two in the case with three massive active neutrinos), and we can take $\alpha_{1}=\eta$ and $\alpha_{2}=0$ without loss of generality. It should be emphasized that all these parameters can contribute to the yield of the baryon asymmetry. This is because the communication terms between left-handed and right-handed leptons do depend on the PMNS matrix. Therefore, in the $\nu$ MSM the $\mathrm{CP}$ violating phases of active neutrinos which may probed in the future neutrino experiments can directly connect with the baryon asymmetry of the universe. For instance, when the CP violation in the HNL sector is absent (i.e., $\operatorname{Im} \hat{\theta}$ ), the dependence of $\delta$ and $\eta$ in the baryon asymmetry is explicitly presented in Ref. [702].

The complex parameter $\hat{\theta}$, which is denoted by $-\omega$ in Ref. [372], is a very important parameter in the considering baryogenesis. As demonstrated in Ref. [372], its imaginary part determines the typical magnitudes of the Yukawa couplings since $\left|F_{\alpha I}\right| \propto X_{\omega} \equiv \exp (\operatorname{Im} \omega)=\exp (-\operatorname{Im} \hat{\theta})$ for $X_{\omega} \gg 1$. Note that the Yukawa couplings can be enhanced by larger $X_{\omega}$ even keeping the masses of active neutrinos as the observational values (which can be seen in the parameterization in Eq. (4.3.11)). Although the production of asymmetry is boosted due to such enhanced couplings, the baryon asymmetry eventually receives the wash-out effects since $N_{2}$ and $N_{3}$ get in equilibrium for larger $X_{\omega}$. Thus, the successful baryogenesis requires the certain range of $X_{\omega}$ and it can be translated into the range of the mixing through

$$
U^{2}=\frac{\sum_{i} m_{i}}{2 M_{N}}\left(X_{\omega}^{2}+X_{\omega}^{-2}\right)
$$

See Fig. 4.17. Similar discussion can be done for the masses of $N_{2}$ and $N_{3}$, since the seesaw mechanism shows the proportionality $\left|F_{\alpha I}\right| \propto M_{N}$.

The flavor oscillation between $N_{2}$ and $N_{3}$ is essential for baryogenesis in the $\nu \mathrm{MSM}$, which starts at the cosmic temperature $T_{\text {osc }}$ as

$$
T_{\mathrm{osc}} \simeq 2.3 \times 10^{4} \mathrm{GeV}\left(\frac{M_{N}}{1 \mathrm{GeV}}\right)^{1 / 3}\left(\frac{\Delta M / M_{N}}{10^{-4}}\right)^{1 / 3} .
$$

The yield of asymmetry depends significantly on $T_{\text {osc }}$ and so $\Delta M$. When the wash-out effect is negligible, its dependence is found to be $\eta_{B} \propto T_{\mathrm{osc}}^{-2}$ for $T_{\mathrm{osc}}>T_{\mathrm{sph}}$ and $\eta_{B} \propto T_{\mathrm{osc}}^{3}$ for $T_{\mathrm{osc}}<T_{\mathrm{sph}}$, 


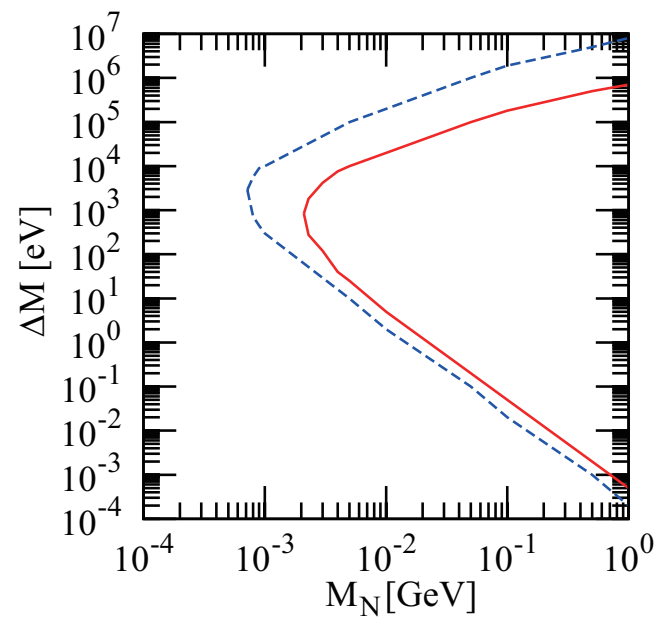

Figure 4.16: The region of $M_{N}$ and $\Delta M$ accounting for the cosmic baryon asymmetry from Ref. [406]. The observational data can be explained in the regions inside the (red) solid and (blue) dashed lines for the $\mathrm{NH}$ and $\mathrm{IH}$ cases, respectively.

and hence the most effective baryogenesis occurs when $T_{\text {osc }} \sim T_{\text {sph }}$ which requires $\Delta M \ll M_{N}$ as we will show below.

Let us then discuss the domain of the parameter space of the $\nu$ MSM in which the observed value of $\eta_{B}$ (as well as the results of the neutrino oscillations) can be explained. The lower bound on the masses of $N_{2}$ and $N_{3}$ are found in Refs. [406, 415] The recent analysis [406] shows that $M_{N} \geq 2.1 \mathrm{MeV}(0.7 \mathrm{MeV})$ for the $\mathrm{NH}(\mathrm{IH})$ case, respectively. (Cf. Fig. 4.16.) It is very interesting that baryogenesis in the $\nu \mathrm{MSM}$ can work even when the masses of HNLs are small as MeV scale if the mass difference is sufficiently small. On the other hand, the upper bound on $M_{N} \lesssim 17 \mathrm{GeV}$ can be obtained to avoid the thermalization of $N_{2}$ and $N_{3}$ for $T>T_{\mathrm{sph}}[396,397]$. Therefore, enough baryon asymmetry can be generated in the $\nu \mathrm{MSM}$ via the mechanism of oscillation of the quasi-degenerate NHLs, $N_{2}$ and $N_{3}$, when $M_{N}=\mathcal{O}(1) \mathrm{MeV}-\mathcal{O}(10) \mathrm{GeV}$.

We should note that a part of the above mass range is excluded by various experimental constraints from direct search of $N_{2}$ and $N_{3}$ and also by the cosmological constraint on the late decays of $N_{2}$ and $N_{3}$. See the analysis in Refs. [395, 403, 406]. The former (latter) constraints put the upper (lower) bound on the mixing $U^{2}$, respectively, and hence we can obtain a certain range of the allowed region in the $M_{N}-U^{2}$ plane. (Cf. Fig. 4.17.) The recent analysis in Ref. [403] shows the lower bound on $M_{N}$ becomes severer as $M_{N}>173 \mathrm{MeV}(264 \mathrm{MeV})$ for the NH (IH) case, respectively. (Cf. Fig. 4.4.)

\subsubsection{Leptogenesis via oscillations with three HNLs}

The number $\mathcal{N}$ of HNLs that can be added to the SM is a free parameter: In contrast to the light neutrinos, the heavy right handed neutrinos are SM gauge singlets, and there are no restrictions from any anomaly cancellation. If the seesaw mechanism is the sole origin of neutrino masses, $\mathcal{N} \geq 2$ is necessary because two non-zero mass differences between the light SM neutrinos have been observed, and the seesaw mechanism requires one $\mathrm{RH}$ neutrino per observed non-zero light neutrino mass. Leptogenesis from neutrino oscillations [396] as described in section 4.6.4 also requires $\mathcal{N} \geq 2$ for any oscillations to occur. Most studies have been focused on the case $\mathcal{N}=2$ (or even $\mathcal{N}=1$ ), either for simplicity or because only two out of three HNLs contribute to neutrino mass generations and leptogenesis, see e.g. Refs. [323, 372, 403, 406, 408-411, 414, 416, 704] and Refs. $[313,528,549,554,705,706]$ for a summary. The latter situation is e.g. realised in the 

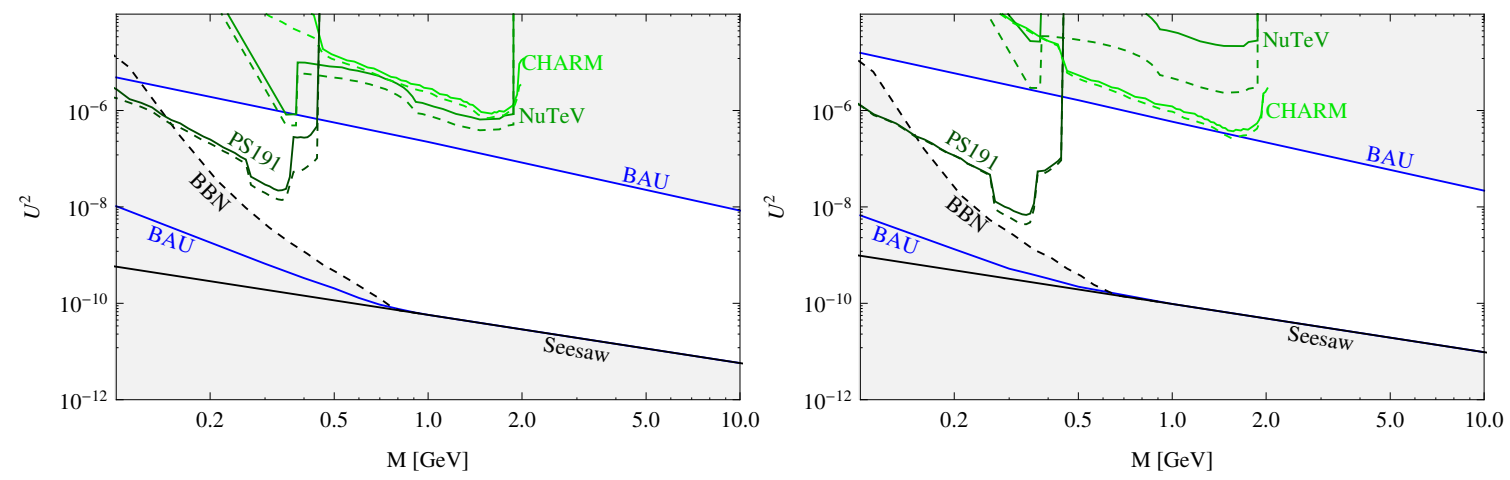

Figure 4.17: Constraints on the $N_{2,3}$ masses $M_{2,3} \simeq M$ and mixing $U^{2}=\operatorname{tr}\left(\theta^{\dagger} \theta\right)$ from baryogenesis; upper panel - normal hierarchy, lower panel - inverted hierarchy. In the region between the solid blue "BAU" lines, the observed BAU can be generated. The regions below the solid black "seesaw" line and dashed black "BBN" line are excluded by neutrino oscillation experiments and BBN, respectively. The areas above the green lines of different shade are excluded by direct search experiments, as indicated in the plot. The solid lines are exclusion plots for all choices of $\nu$ MSM parameters, for the dashed lines the phases were chosen to maximize the BAU, consistent with the blue lines. The uncertainties in theoretical computations may move the lines up or down by a factor of few $\sim 2-3$. From [416].

$\nu$ MSM [397] described in section 4.8 because the lightest HNL $N_{1}$ is assumed to be a Dark Matter candidate; bounds on the DM lifetime require its mixings $U_{\alpha 1}^{2}$ to be so feeble that it effectively decouples, and the $\nu \mathrm{MSM}$ is equivalent to $\mathcal{N}=2$ as far as baryogenesis and neutrino masses are concerned. However, in the seesaw framework $\mathcal{N} \geq 3$ is required if the lightest neutrino turns out to be massive.

The interpretation of experimental constraints and cosmological considerations both strongly depend on $\mathcal{N}$ and the mass of the lightest neutrino [325, 706]. For instance, for $\mathcal{N}=3$ there is no lower bound on $\sum_{\alpha} U_{\alpha I}^{2}$ from neutrino oscillation data if the lightest neutrino is massless. On the other hand, if its mass is near the upper limit of $0.23 \mathrm{eV}$ from cosmology [319], then there is a lower bound $U_{I}^{2}>10^{-10}[325]$, see Fig. 4.18, which is near the anticipated SHiP sensitivity. Moreover, the range of $N_{I}$ parameters for which leptogenesis can be realised with $M_{I}$ below the electroweak scale turns out to be very different for $\mathcal{N}=2$ and $\mathcal{N}=3$. For $\mathcal{N}=2$ the two masses masses $M_{1}$ and $M_{2}$ have to be quasi-degenerate in order to explain the observed BAU [707], see [681, 686] (leptogenesis during $N_{I}$ decay) and $[324,397,415,416,703,704,708]$ (leptogenesis during $N_{I}$ production). Moreover, leptogenesis with $\mathcal{N}=2$ requires that the Yukawa coupling constants $F_{\alpha I}$ are very tiny, making it very challenging to find the $N_{I}$ in existing experiments [316, 388, 416, 704]. This provides strong motivation for SHiP. With $\mathcal{N}=3$ both of these restrictions can be overcome [709-711]; in this case leptogenesis does not require a mass degeneracy [709], and the parameter region where the BAU can be explained is within reach of the existing experiments LHCb and BELLE [710]. This is illustrated in figure 4.19 for the observable $U_{\mu 2}^{2}$. However, B-factories can only improve existing bounds if the $N_{I}$ have masses between the $D$-meson and $B$-meson mass. Even in this mass range, they can probably only find the $N_{I}$ if their mixings $U_{\alpha I}^{2}$ are less than two orders of magnitude below the current bounds [710]. SHiP could probe much smaller mixings and exclude most of the viable parameter space below the $D$-meson mass shown in Fig. 4.18 if the lightest neutrino is massive. At larger masses, ATLAS and CMS [712] or future high energy colliders [383, 549, 554, 713] could complement SHiP.

At first sight it seems surprising that leptogenesis in the case $\mathcal{N}=3$ is phenomenologically so different from $\mathcal{N}=2$. The reason lies in the fact that leptogenesis with $M_{I} \ll T_{\text {sph }}$ is primarily driven by lepton flavour violating processes, while total lepton number violation is suppressed by 

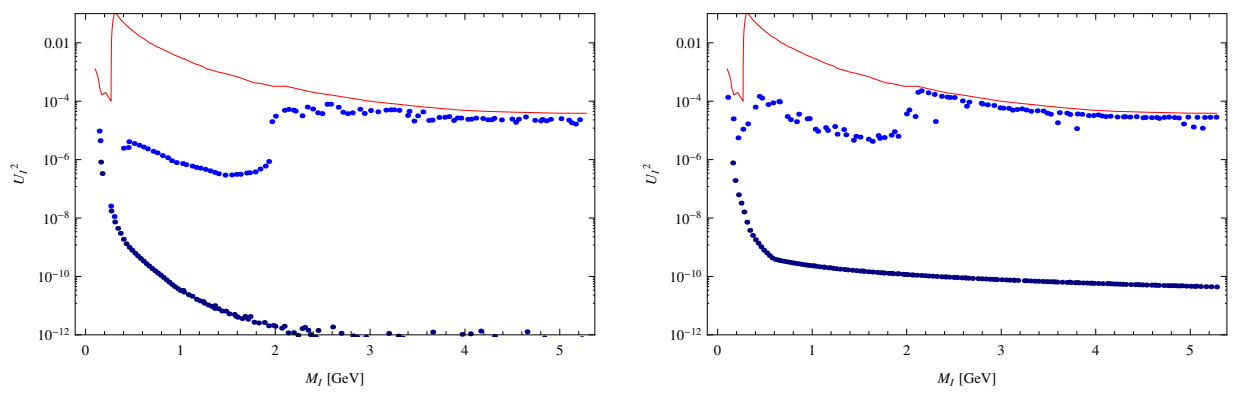

Figure 4.18: For $\mathcal{N}=3$, there is no lower bound on the mixings $U_{\alpha I}^{2}$ of a given HNL $N_{I}$ with the individual active flavours [324, 325]. However, their sum $U_{I}^{2} \equiv \sum_{\alpha} U_{\alpha I}^{2}$ is bound from below by $\mathrm{BBN}$. If the lightest neutrino is relatively heavy, then there is also a lower bound from neutrino oscillation data. The dark blue dots in the left and right panel show the smallest $U_{I}^{2}$ for given $M_{I}$ found in a numerical scan performed in Ref. [325] that are consistent with neutrino oscillation data, $\mathrm{BBN}$, neutrinoless double $\beta$-decay, $\mu \rightarrow e \gamma$ and past collider searches in the $\mathcal{N}=3$ scenario. In the left panel the lightest neutrino is massless, and the only lower bound comes from BBN. In the right panel the lightest neutrino has a mass at the cosmological upper limit $0.23 \mathrm{eV}$. The light blue dots show the largest mixing found to be consistent with the above requirements. The red line shows the collider bound from direct searches alone.

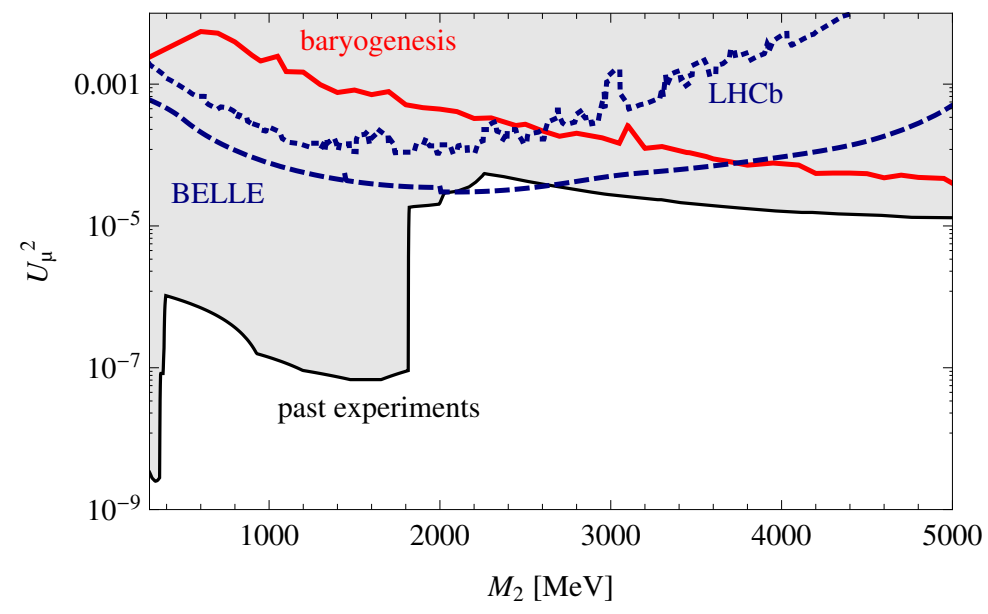

Figure 4.19: The red line shows the maximal mixing $U_{\mu 2}^{2}$ consistent with baryogenesis found in [710], i.e. below the line there exist parameter choices for which the observed BAU can be generated. Here the masses $M_{1}=1 \mathrm{GeV}$ and $M_{3}=3 \mathrm{GeV}$ are fixed while $M_{2}$ is varied. The resonant cases $M_{2} \simeq M_{1}$ and $M_{2} \simeq M_{3}$ were excluded, which allows to use standard methods to describe the propagation of the $N_{I}$ even in the early universe [709, 714-716]. The small mass splitting in the degenerate case introduces a new oscillation time scale, which can give rise to resonant phenomena that require special treatment $[667,700,701,717]$ and possibly in the laboratory $[718,719]$. The scatter is a result of the Monte Carlo method and not physical. It indicates that we have not found the global maxima, but the density of valid points decreases rapidly for larger $\left|U_{\mu 2}\right|^{2}$. The gray area represents bounds from the past experiments PS191 [578], NuTeV [586] (both re-analysed in [323]), NA3 [580], CHARMII [588] and DELPHI [551] (as given in [313]). They are stronger than those from violation of lepton universality [401, 402, 549, 554, 573, 720], see [313, 705] for a discussion of other experimental constraints. The blue lines indicate the current bounds from LHCb [408] (dotted) and BELLE [409] (dashed), which will improve in the future. 
$M_{I} / T$. A sizable lepton number violation can only be realised with a resonant enhancement caused by a mass degeneracy $\frac{M_{I} M_{J}}{\left|M_{I}^{2}-M_{J}^{2}\right|} \gg 1$. The source of lepton flavour violation, on the other hand, scales as $\frac{T^{2}}{\left|M_{I}^{2}-M_{J}^{2}\right|}$, which for $M_{I}<M_{W}$ is large at temperatures $T \gg T_{\mathrm{sph}}$, even if $\frac{M_{I} M_{J}}{\left|M_{I}^{2}-M_{J}^{2}\right|} \sim 1$. This argument applies in principle to both cases, $\mathcal{N}=2$ and $\mathcal{N}=3$. However, the generation of the asymmetries at $T \gg T_{\mathrm{sph}}$ and their washout at $T \gtrsim T_{\mathrm{sph}}$ are driven by the same Yukawa interactions $F_{\alpha I}$. Hence, the Yukawa interactions $F_{\alpha I}$ have to be large enough to generate significant lepton asymmetries $L_{\alpha}$ at $T \gg T_{\mathrm{sph}}$, but small enough to prevent the complete washout of all $L_{\alpha}$ before $T=T_{\mathrm{sph}}$. This is most easily achieved if individual elements $F_{\alpha I}$ are sufficiently different in size that one active flavour $\alpha$ couples much more weakly to the $N_{I}$ than the other two, leading to a flavour asymmetric washout that allows the asymmetry in that flavour to survive until $T=T_{\text {sph }}$. For $\mathcal{N}=2$ this is difficult to achieve because the strengths of the active-sterile couplings $F_{\alpha I}$ in all flavours are tied together, as they are essentially governed by just one parameter [324, 372, 395, 696, 708]. In the popular Casas-Ibarra parametrisation (4.3.11) [384] this is the imaginary part of the one complex "Euler angle" $\omega$ in the "rotation matrix" $\Omega$. This generally leads to very small baryon asymmetries because a large asymmetry generation at $T \gg T_{\text {sph }}$ is necessarily antagonised by a large washout at $T \gtrsim T_{\mathrm{sph}}$ in all flavours, and the observed BAU can only be explained if it is resonantly enhanced by a degeneracy in the masses at the level $<10^{-3}[416,708]$. The situation changes drastically in the $\mathcal{N}=3$ scenario. The reason is that in this case there are three complex angles $\omega_{i j}$ in $\Omega$. This enlarged parameter space contains considerable regions in which the individual $\left|F_{\alpha I}\right|$ are vastly different. If the $N_{I}$-mixings with two active flavours are relatively large (allowing for an efficient asymmetry production without mass degeneracy) while mixing with the third one is small (and prevents a complete washout of the asymmetry prior to sphaleron freezeout), then the $N_{I}$ that can generate the BAU are within reach of existing experiments [710]. However, for the parameters shown in figure 4.19, "large" $\left|F_{\alpha I}\right|>10^{-7}$ can only be made consistent with the small observed neutrino masses if there are cancellations amongst the different elements of (4.3.3) that keep the physical mass eigenvalues small. From a theoretical viewpoint this may be considered as "tuning", and it has been argued that the region with small mixings is more "natural" [708]. As far as baryogenesis is concerned, the required amount of "tuning" can be reduced if the HNLs couple to other new degrees of freedom in addition to the SM particles, such as an additional Higgs doublet [708, 709]. This leads to larger thermal production rates in the early universe while still keeping neutrino masses small.

The scenario outlined above works particularly well if the $M_{I}$ are in the GeV range; smaller masses are excluded by the combination of bounds from big bang nucleosynthesis and direct searches of past experiments [325, 415, 416, 704], larger masses are strongly disfavoured because the seesaw relation (4.3.3) naturally leads to larger $F_{\alpha I}$ and a more efficient washout. Theoretically this choice can be motivated by e.g. classical scale invariance [522], in the framework of the "inverse seesaw" [501, 502], "no new scale"-considerations [721] or by applying Ockham's razor to the number of new particles required to explain the known phenomena beyond the SM [397]. In this mass range, the $N_{I}$ can be produced and studied in meson decays [395]. Though B-factories can probe a part of the leptogenesis parameter space with $U_{I}^{2}>10^{-6}$ [710], SHiP would be by far the most powerful instrument to probe the scenario of baryogenesis from neutrino oscillations with $\mathcal{N}=3$. In particular, with its expected sensitivity SHiP could enter deeply into the "natural" parameter region of smaller $U_{I}^{2}$ where no significant tuning is required to keep the neutrino masses small within the minimal type-I seesaw framework. Besides, we emphasise that baryogenesis via HNLs can be easily accommodated within some of the most plausible and simple extensions of the SM [522, 708, 709]. 

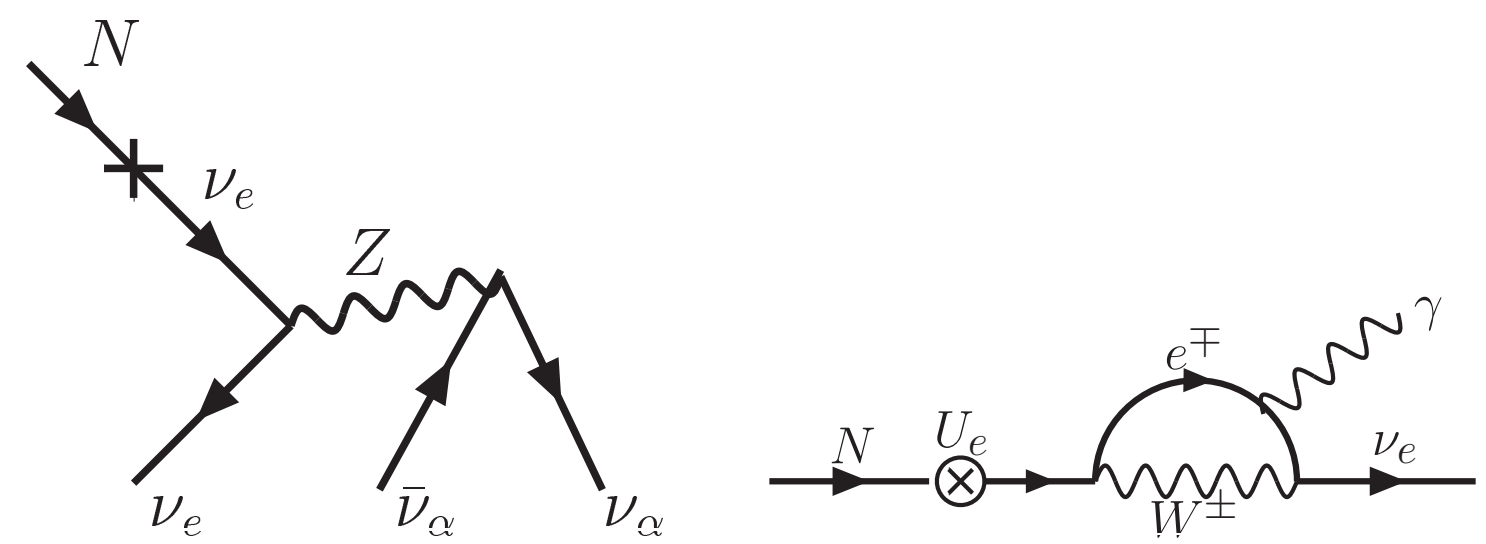

Figure 4.20: Decay channels of the sterile neutrino with the mass below twice the electron mass. Right panel shows radiative decay channel that allows to look for the signal of sterile neutrino dark matter in the spectra of dark matter dominated objects.

\subsection{HNL and dark matter}

The nature of dark matter remains one of the most intriguing questions of modern physics. There is a body of strong and convincing evidence which points at the existence of a new substance, universally distributed in objects of all scales and providing a contribution to the total energy density of the Universe at the level of about $25 \%$. Various attempts to explain this phenomenon by the presence of macroscopic compact objects (such as, for example, old stars) or by modifications of the laws of gravity (or of dynamics) failed to provide a consistent description of all the observed phenomena. Therefore, a microscopic origin of dark matter phenomenon (i.e. a new particle or particles) remains the most plausible hypothesis.

The only electrically neutral and long-lived particle in the Standard Model are neutrinos. As the experiments show that neutrinos have mass, they could play the role of dark matter particles. Neutrinos are involved in weak interactions that keep these particles in the early Universe in thermal equilibrium down to the temperatures of few $\mathrm{MeV}$. At smaller temperatures, the interaction rate of weak reactions drops below the expansion rate of the Universe and neutrinos "freeze out" from the equilibrium. Therefore, a background of relic neutrinos was created just before primordial nucleosynthesis. As interaction strength and, therefore, decoupling temperature and concentration of these particles are known, their present day density is fully defined by the sum of the masses for all neutrino flavours. To constitute the whole DM this mass should be about $11.5 \mathrm{eV}$ (see e.g. [722]). This mass is in conflict with current experimental data (see Section 4.2.2). However even in the past when such a sum of neutrino masses was not excluded by particle physics data, another problem signified that neutrinos could constitute only a fraction of DM. Indeed, if DM particle is a fermion, the phase-space number density of the faintest galaxies should not exceed the density of degenerate Fermi gas. Provided that DM mass density is bounded from below observationally, this puts a lower bound on the mass of fermionic DM particles to be a few hundreds of eV (the so-called "Tremaine-Gunn bound" [723]). ${ }^{7}$ Therefore, cosmological and astrophysical requirements to neutrino dark matter contradict to each other. Therefore, the DM particle hypothesis necessarily implies an extension of the Standard Model.

\footnotetext{
${ }^{7}$ There are appreciable astronomical uncertainties in deducing phase-space density from astronomical observations and under reasonable assumptions they can be changed by a factor $\sim 2$, see e.g. [724]. Depending on how the uncertainties are treated, this bound ranges from $0.4 \mathrm{keV}$ [724] to $1 \mathrm{keV}$ [725].
} 
The sterile neutrino allows to resolve the contradiction that have ruled out active neutrinos as dark matter candidate. Its interaction with the Standard Model particles is similar to that of active neutrino, but suppressed by the mixing angle $U^{2}$. Therefore number density of the relic sterile neutrino background can be much lower and account for the correct DM abundance in the much larger range of masses of the particle, easily satisfying Tremaine-Gunn bound. The production through mixing with active neutrinos always contributes to the relic sterile neutrino abundance $[116,118,726,727]$. If a large lepton asymmetry or new particles and fields are present in the model then additional production mechanism are possible [117, 119, 120, 728, 729] (see $[399,528,730]$ for review).

\subsubsection{Bounds on HNL as dark matter. No assumptions on production mechanism}

The sterile neutrino has finite lifetime. It can decay to 3 active (anti)neutrinos (Figure 4.20). To be a dark matter candidate its lifetime should be greater than the lifetime of the Universe, which implies [726]

$$
U^{2}<4 \times 10^{-8}\left(\frac{50 \mathrm{keV}}{M_{N}}\right)^{5}
$$

In fact, due to the existence of the subdominant radiative decay channel $N \rightarrow \gamma+\nu$ (Figure 4.20, right panel) it should be even longer. The decay width is [731, 732]

$$
\left.\Gamma_{N \rightarrow \gamma \nu}=\frac{9 \alpha G_{F}^{2} U^{2} M_{N}^{5}}{256 \pi^{4}}=\begin{array}{c}
5.55 \times 10^{-30} \mathrm{sec}^{-1} \\
\text { or } \\
3.66 \times 10^{-48} \mathrm{keV}
\end{array}\right\} \times\left[\frac{U^{2}}{10^{-8}}\right]\left[\frac{M_{N}}{1 \mathrm{keV}}\right]^{5}
$$

and the energy of the emitted photon is fixed to be $E_{\gamma}=\frac{1}{2} M_{N}$ which means that one expects a monochromatic line in the spectra of dark matter-dominated objects (galaxies and galaxy clusters) [726, 733, 734]. Even if Eq. (4.7.1) is satisfied, the huge amount of dark matter particles in galaxies will give rise to a strong signal that would be immediately observed. Even the first look at the diffuse extragalactic X-ray background tells us that this limit is stronger than Eq. (4.7.1) roughly by a factor of $3 \times 10^{6}[322,735]$.

The bound on dark matter decay signal can be made even stronger if one looks at high dark matter overdensities i.e. nearby galaxies and galaxy clusters. In the $\mathrm{keV}-\mathrm{MeV}$ mass range the search of the DM decay signal was conducted using space telescopes: XMM-Newton [735, 737-743], Chandra [744-750], Suzaku [751, 752], Swift [753], INTEGRAL [754, 755] and HEAO-1 [735] cosmic missions, as well as rocket-borne X-ray microcalorimeter [756] (see also [757] for extension for higher energies). The resulting bounds are shown in Fig. 4.21, left panel.

This means that the contribution of the sterile neutrino dark matter particle to the neutrino masses and oscillations is negligible and if the neutrino oscillations are to be explained by sterile neutrinos, at least two more heavy neutrinos, interacting much stronger that the dark matter one, should be present in the model [321, 322].

\subsubsection{Bounds on DM HNL if produced via mixing with active neutrinos only}

The X-ray bounds provide a upper limit on the mixing angle $U^{2}$ for each value of the mass $M_{N}$ under the only assumption that all dark matter is made of sterile neutrinos. In addition to that in the minimal model where sterile neutrino dark matter is produced solely via its mixing with active neutrinos, the abundance of DM is a unique function of DM mass, mixing angle $U^{2}$ and the lepton asymmetry present at the plasma at the time of production. Due to thermal effects the effective coupling between active and sterile neutrinos becomes temperature-dependent and reaches its maximum at temperatures of few hundreds $\mathrm{MeV}$. The value of the lepton asymmetry at these 


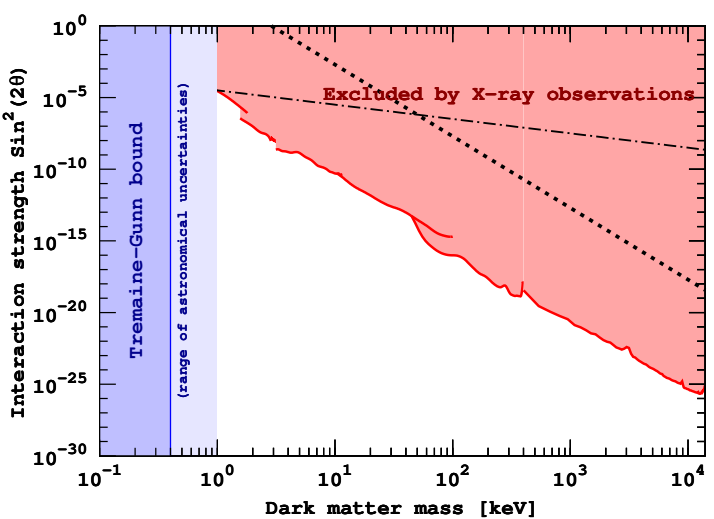

(a) The most conservative constraints on the mixing angle and mass of Dark Matter HNL. Left vertical line is the Tremaine-Gunn bound [723] (for lighter particles the phase space density of DM particles in the small galaxies would exceed the Tremaine \& Gunn bound $[724,725])$. Along the dotted line the HNL has the lifetime equal the age of the Universe. Along the dashed-dotted line the contribution of the HNL to the neutrino masses is of the order of $U^{2} M_{1}=m_{\odot} \sim$ $8 \times 10^{-3} \mathrm{eV}$.

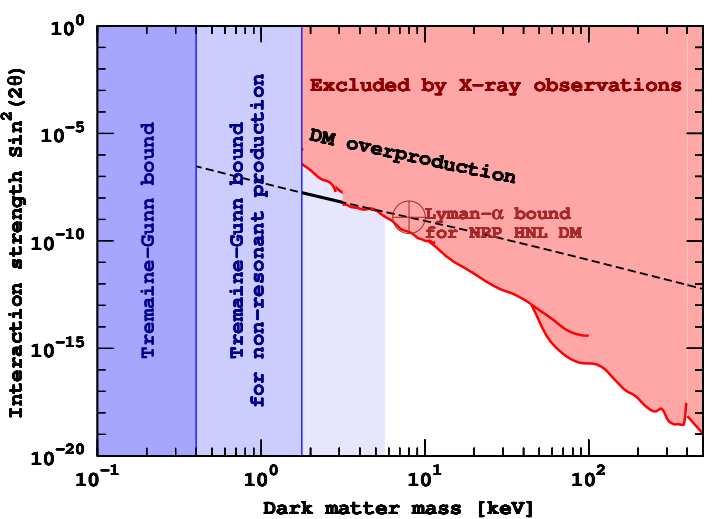

(b) Constraints on HNL produced via (non-resonant) mixing with neutrinos [116, 727]. The Tremaine-Gunn bound becomes stronger [724] (light blue shadow extended to $5.7 \mathrm{keV}$ shows the bound from [725]). Along the black curve the correct DM abundance is produced. The confrontation of production and (nonobservation of) decay leaves a small window of masses between $\sim 1.7 \mathrm{keV}$ and $\sim 4 \mathrm{keV}$ allowed for this scenario. In addition the Lyman- $\alpha$ bound $(M \gtrsim$ $8 \mathrm{keV}$ [736], marked by the cross) is in tension with the other bounds.

Figure 4.21: Constraints of DM particles made of HNLs under the assumption that HNLs constitute $100 \%$ of dark matter.

temperatures depends on the initial conditions. if lepton asymmetry is assumed to be zero at the moment of DM production, the requirement of correct DM abundance imposes a unique between DM mass and its lifetime. Combined with X-ray constraints on the lifetime, this give an upper bound on the mass $M_{N} \lesssim \mathcal{O}(2-4) \mathrm{keV}$. Therefore the remaining window for such a DM becomes narrow as the universal (Tremaine-Gunn) lower bound is $\sim \mathcal{O}(0.5-1) \mathrm{keV}$.

If the mixing angle is above the line of non-resonant production (black solid line in Figs. 4.21, right panel) the dark matter mixes with the ordinary neutrinos too strongly and as a result is overproduced.

Confronting this relation between $M_{1}$ and $U^{2}$ with X-ray bounds puts an upper bound on the mass:

- $M_{1} \lesssim 4 \mathrm{keV}$, non-resonant production (Fig. 4.21, right panel)

- $M_{1} \lesssim 50 \mathrm{keV}$, resonant production (Fig. 4.22).

The production via mixing predicts a specific shape of primordial phase-space distribution function and the Tremaine-Gunn bound becomes stronger:

- its lower bound for $M_{1}$ for non-resonant production is between $1.7 \mathrm{keV}$ [724] and $5.7 \mathrm{keV}$ [725].

- $M_{1} \gtrsim 1 \mathrm{keV}$, resonant production [724]

Moreover, as the primordial momentum distribution of DM particles is quasi-thermal in this case, structure formation data favors heavier masses (although the systematic uncertainty there is significant and hard to estimate).

HNLs produced via mixing with ordinary neutrinos are born relativistic and as they cool down they erase small-scale density perturbations. The non-resonantly produced HNL DM behaves as warm dark matter [727, 758], while the resonantly produced one is sufficiently colder and can be approximated as cold-plus-warm dark matter [736, 759]. 
The measurement of the structures at small scales is done via the Lyman- $\alpha$ forest method [736, 759-761]. This method is very complicated, involves cosmological simulations of both clustering of DM particles and complicated astrophysical properties in inter-galactic medium. The systematic errors can be therefore quite significant and hard to estimate (see the discussion in [736]). The current Lyman- $\alpha$ bounds can be summarized as follows:

- The conservative lower bound for non-resonantly produced particles is $M_{1} \gtrsim 8 \mathrm{keV}(99.7 \%$ C.L.) [736]. This bound is based on a statistically large sample of SDSS quasars. ${ }^{8}$

- $M_{1} \gtrsim 2 \mathrm{keV}$ for resonantly produced [759].

As a result, for non-resonant production the Lyman- $\alpha$ constraints comes at tension with the X-ray constraints (even if systematic uncertainties are taken into account). Moreover even without taking into account structure formation, X-ray bound is at tension with Tremaine-Gunn bound for the non-resonant production mechanism $[724,725]$. Non-zero values of initial lepton asymmetry remove the tension and open up the whole parameter space up to the masses $M_{N} \sim \mathcal{O}(100) \mathrm{keV}$.

In summary: assumption of production of HNL DM via mixing with ordinary neutrino (and no other new particles in the primordial plasma at temperatures below $\sim 1 \mathrm{GeV}$ ) leads to the conclusion that large lepton asymmetry should be present in the plasma at these temperatures. Alternatively, one needs to assume other production mechanisms of HNL, for example HNLs can be produced via decay of scalars [315, 729].

There can be different origins of large lepton asymmetry at temperature of interest (e.g. [696, 763]). Interestingly the required lepton asymmetry may be produced in the minimal model that contains only 3 new particles: one sterile neutrino, $N_{1}$, as a dark matter particle, and two others, $N_{2}, N_{3}$, responsible for neutrino masses (see Section 4.8 below). The value of lepton asymmetry at the dark matter production epoch is defined by the properties of $N_{2}$ and $N_{3}$. Therefore X-ray constraints on the properties of $N_{1}$, translated into possible range of value of lepton asymmetry, select a certain part of the parameter space of $N_{2}, N_{3}$. In particular, if the dark matter origin of the $3.5 \mathrm{keV}$ line is confirmed, it will imply definite range of values of the parameters of $N_{2}, N_{3}$. However, the value of the lepton asymmetry at the temperatures of interest may depend also on the complicated dynamics of this asymmetry in primordial plasma. Therefore the explicit relation between properties of $N_{1}$ and $N_{2}, N_{3}$ within the minimal model are still under investigation. When fully established, this relation will provide a promising way to make cosmological predictions for particle physics searches of HNLs.

\subsection{3 $\quad 3.5 \mathrm{keV}$ line}

Recently two groups have reported an unidentified spectral line at the energy $E \sim 3.5 \mathrm{keV}$ in the stacked X-ray spectra of Andromeda galaxy, Perseus galaxy clusters, stacked galaxy clusters and the Galactic Center of the Milky Way [297, 298, 764]. This result is being widely discussed in the literature. Existing data do not allow to completely exclude the possibility that in some of the objects this line may originate from an atomic transition [765-772]. However the distribution of this signal over the sky and the ratios of its strengths observed in different objects are consistent with predictions for decaying dark matter with the mass $M_{N} \approx 7.1 \pm 0.1 \mathrm{keV}$.

\footnotetext{
${ }^{8}$ To emphasize the degree of uncertainty of the method, we mention that a stronger bound of $M_{1} \gtrsim 15 \mathrm{keV}(99.7 \%$ C.L.) has been claimed in [762] for a sample of 25 high resolution quasars. The same ref. [762] showed, however, that by relaxing astrophysical assumptions about the thermal history of the intergalactic medium, this bound can be relaxed down to about $9 \mathrm{keV}$, making it largely consistent with [736].
} 


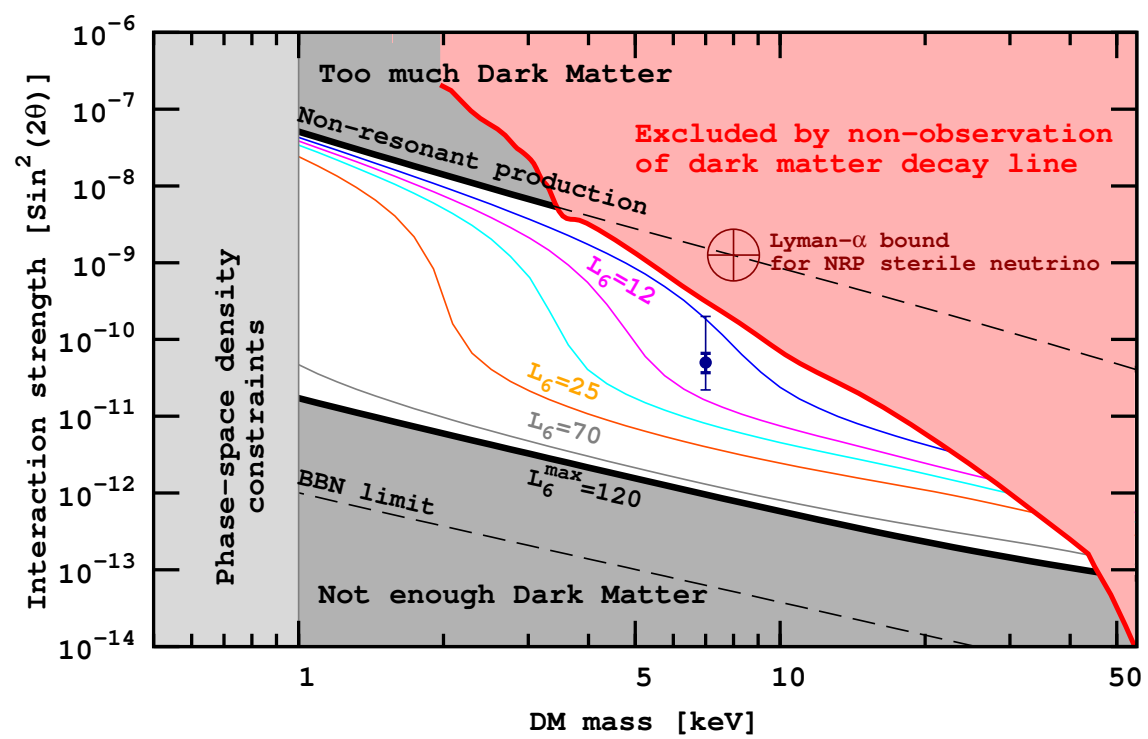

Figure 4.22: The parameter space of sterile neutrino dark matter produced via mixing with the active neutrinos (unshaded region). The two thick black lines bounding this region are production curves for nonresonant production [727] (upper line, "NRP") and for resonant production (RP) with the maximal lepton asymmetry, attainable in the $\nu$ MSM (lower line, marked " $L_{6}^{\max }=120$ ") $[416,696,728]$ ( $L_{6}$ is defined as the ratio of the lepton density to the entropy density times $10^{6}$ ). The thin coloured curves between these lines represent production curves for different values of lepton asymmetry. The red shaded upper right corner represents X-ray constraints [738, 740, 741, 751, 755] (rescaled by a factor of two to account for possible systematic uncertainties in the determination of DM content). The black dashed-dotted line approximately shows the RP models with the largest cold component. The region below $1 \mathrm{keV}$ is ruled out according to the phase-space density arguments [725] (see text for details). The point at $\sim 7.1 \mathrm{keV}$ corresponds to the unidentified spectral detected in stacked X-ray spectra of galaxies and galaxy clusters [297, 298]. Thick errorbars are $\pm 1 \sigma$ limits on the flux as determine from data. Thin errorbars correspond to the uncertainty in the DM distribution.

\section{$4.8 \nu \mathrm{MSM}$}

Let us explain here the framework of the so-called $\nu \mathrm{MSM}$ (neutrino Minimal Standard Model). This is a simple extension of the SM by introducing $\mathcal{N}$ right-handed neutrinos $N_{I}(I=1,2, \cdots, \mathcal{N})$ in order to explain the three observational phenomenon which cannot be explained by the SM, i.e., the non-zero masses of active neutrinos, the cosmic dark matter, and the baryon asymmetry of the universe. These right-handed neutrinos are introduced with Majorana masses $M_{I}$

$$
\left|m_{D}\right|_{\alpha I} \ll M_{I}<\mathcal{O}\left(10^{2}\right) \mathrm{GeV},
$$

where the Dirac mass is given by $\left|m_{D}\right|_{\alpha I}=\left|F_{\alpha I}\right|\langle\Phi\rangle$. The first inequality between Dirac and Majorana masses is imposed for the seesaw mechanism. The scale of Majorana mass for the seesaw mechanism cannot be determined from active neutrino masses and can vary in a wide range (see Sections 4.3.2.1-4.3.2.4). The possibility, discussed here, is to choose Majorana masses that are comparable to or smaller than the electroweak scale $\mathcal{O}\left(10^{2}\right) \mathrm{GeV}$, so that masses of HNLs are comparable to or smaller than masses of quarks and charged leptons. Interestingly, even when HNLs are lighter than the electroweak scale, enough baryon asymmetry can be generate via oscillations, as discussed in Section 4.6.4.

The two mass scales, $\Delta m_{\mathrm{atm}}^{2}$ and $\Delta m_{\odot}^{2}$, confirmed by various oscillation experiments require that there must be at least two massive states of active neutrinos which mass eigenvalues are 
different, and then the number of right-handed neutrino must be equal to or larger than two $(\mathcal{N} \geq 2)$. Notice that the lightest active neutrino becomes exactly massless for the minimal choice $\mathcal{N}=2$.

We then discuss the possible candidate for dark matter in this framework. The candidate is HNL with $\mathcal{O}(10) \mathrm{keV}$ mass (see the discussions in Sec. 4.7) We might expect that HNLs needed to explain $\Delta m_{\text {atm }}^{2}$ and $\Delta m_{\odot}^{2}$ also may play the role of dark matter. However it is impossible because of the following reasons. First, it is shown [321] that HNLs for masses of active neutrinos must has sizable Yukawa couplings which produce too much dark matter particles by the Dodelson-Widrow mechanism [116], and then the present abundance exceeds the observed value. In addition, such HNLs cannot be dark matter since they give too much $X$-rays from their radiative decays and conflict with observations [322]. Therefore, we must introduce right-handed neutrino(s) for dark matter in addition to at least two right-handed neutrinos for active neutrino masses. In this case the number of right-handed neutrino must be $\mathcal{N} \geq 3$.

Finally, let us consider the baryogenesis in the $\nu$ MSM. As explained in Section 4.6.4, HNLs with $\mathrm{GeV}$-scale masses can create the baryon asymmetry through the mechanism via oscillations [396, 397]. It is interesting that even in the minimal option required for $\Delta m_{\mathrm{atm}}^{2}$ and $\Delta m_{\odot}^{2}$, say the two HNLs case, the enough baryon asymmetry can be generate as demonstrated in Ref. [397].

As a result, the minimal number of right-handed neutrinos explaining the neutrino masses, dark matter, and the baryon asymmetry at the same time is $\mathcal{N}=3$. In this case the HNL $N_{1}$ plays a role of dark matter (see Section 4.7) and the heavier HNLs $N_{2}$ and $N_{3}$ are then responsible to the seesaw mechanism and baryogenesis. The model with $\mathcal{N}=3$ introduces 18 new parameters in addition to the parameters of the SM, which are three Majorana masses $M_{I}$ and 15 (physical) parameters in the neutrino Yukawa couplings $F_{\alpha I}$. The parameters associated with the heavier HNLs $N_{2}$ and $N_{3}$ are 11. Among them 7 are parameters of active neutrinos (two mass-squared-differences and three mixing angles of active neutrinos and one Dirac-type phase and one Majorana-type phase in the PMNS matrix), and 4 are parameters of HNLs (their masses $M_{2,3}=M_{N} \pm \Delta M / 2$ and one complex parameter). The residual 7 parameters are for dark matter $N_{1}$ (mass of $N_{1}$, three its mixing elements $\left|U_{\alpha 1}\right|$ and three $\mathrm{CP}$ violating phases).

One important consequence of this model is that the lightest active neutrino is lighter than $\mathcal{O}\left(10^{-5}\right) \mathrm{eV}[321,322]$. This comes from the fact that the dark matter HNL is allowed to give a tiny contribution to the seesaw. Therefore, the mass eigenvalues of heavier active neutrinos can be identified from $\Delta m_{\mathrm{atm}}^{2}$ and $\Delta m_{\odot}^{2}$ (the ordering of masses are still unknown). This point is crucial for the neutrinoless double beta decays in this framework (see the discussion 4.5.2).

As described in Section 4.6.4, the successful baryogenesis requires that $N_{2}$ and $N_{3}$ are quasidegenerate $(\Delta M / M \ll 1)$ and $M_{N}$ is bounded from below. The constraints on $N_{2}$ and $N_{3}$ from direct searches and cosmology make such bound on $M_{N}$ more stringent. The most recent analysis [403] shows that $M_{N}>173 \mathrm{MeV}(264 \mathrm{MeV})$ for the $\mathrm{NH}(\mathrm{IH})$ case, respectively. Further, the allowed range of mixing of $N_{2}$ and $N_{3}$ is summarized in Figure 4.17. The region for $M_{N}$ and $U^{2}$ of $N_{2}$ and $N_{3}$ indicates that experimental searches of $N_{2}$ and $N_{3}$ are very important [395].

\subsubsection{Lepton asymmetry and dark matter production in the model with 3 HNL}

It was shown in the Section 4.7 that bounds on dark matter HNL, produced solely from mixing with active neutrinos require presence of large lepton asymmetry as initial conditions in the plasma.

In the presence of 3 HNLs ( $\nu$ MSM) the existence of lepton assymetry can be naturally generated as a consequence of baryogenesis or via out-of-equilibrium processes involving HNL at lower temperatures.

Let us introduce an extra assumption: no production of Dark Matter HNL above tempera- 
tures of few TeV. ${ }^{9}$ The interactions contained in Lagrangian (4.3.1) allow to generate large lepton asymmetry at temperatures below $T \sim 140 \mathrm{GeV}$. This may happen in distinct 3 regimes

I. at temperatures $T \sim 50-100 \mathrm{GeV}$, when $N_{2,3}$ come to thermal equilibrium

II. at temperatures $T \sim$ few $\mathrm{GeV}$, when $N_{2,3}$ go out of thermal equilibrium

III. at temperatures $T<M_{2,3} \mathrm{GeV}$, when $N_{2,3}$ start to decay

Let us assume that the Big Bang theory is valid below the temperatures of few $\mathrm{TeV}$ and that no baryon asymmetry existed beforehand. Then lepton number is generated by $N_{2}, N_{3}$. Baryon number non-conservation due to sphalerons [321, 397, 415] results in conversion of this lepton number in the baryon number (baryogenesis). To produce baryon asymmetry, the parameters of $N_{2,3}$ should be in the region shown in Fig. 4.17.

While sphalerons are active at temperatures above $T>140 \mathrm{GeV}$, the lepton asymmetry continues to be produced below this temperature and can reach quite high values (unrelated to the baryon asymmetry [696]) — case I above. The question of the subsequent evolution of this lepton asymmetry and what abundance it has at temperatures of HNL DM generation is under active investigation [774]. If significant $\eta_{L}$ survives, this would allow to relate the properties of $N_{2,3}$ with (potentially observable) properties of Dark Matter.
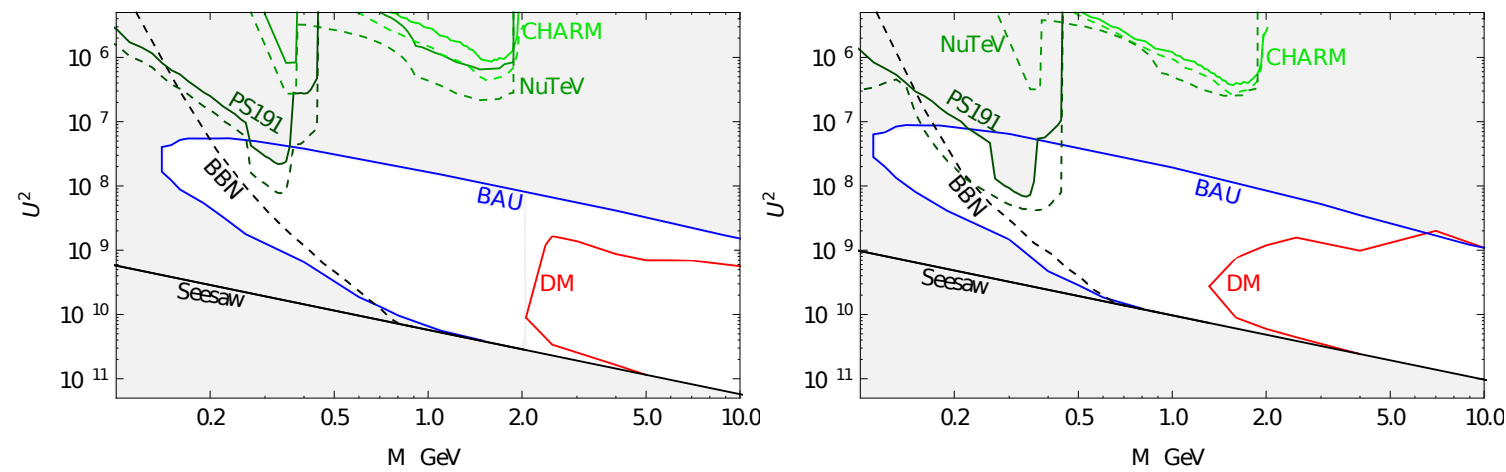

Figure 4.23: Constraints on the $N_{2,3}$ masses $M_{2,3} \simeq M$ and mixing $U^{2}=\operatorname{tr}\left(\theta^{\dagger} \theta\right)$ in the $\nu$ MSM leading to sufficient production of lepton asymmetry in the regimes [ii], [iii], to enhance the DM production; left panel - normal hierarchy, right panel - inverted hierarchy. In the region between the solid blue "BAU" lines, the observed BAU can be generated. Inside the solid red "DM" line the lepton asymmetry at $T=100 \mathrm{MeV}$ can be large enough that the resonant enhancement of $N_{1}$ production is sufficient to explain the observed $\Omega_{D M}$ - The CP-violating phases were chosen to maximize the asymmetry at $T=100 \mathrm{MeV}$. The regions below the solid black "seesaw" line and dashed black "BBN" line are excluded by neutrino oscillation experiments and BBN, respectively. The areas above the green lines of different shade are excluded by direct search experiments, as indicated in the plot. The solid lines are exclusion plots for all choices of $\nu$ MSM parameters, for the dashed lines the phases were chosen to maximize the late time asymmetry, consistent with the red line. From [416].

The regimes [II] and [III] have been investigated in [416]. The results are shown in Figs. 4.22, 4.23. In Fig. 4.22, the new element in comparison with Fig. 4.21 appears - the bottom line below which HNL DM cannot be produced without additional assumptions. In this regime a large degeneracy (fine-tuning) between masses $M_{2}$ and $M_{3}$ is required $\left|M_{2}-M_{3}\right| \ll m_{\text {atm }}$ and in addition

\footnotetext{
${ }^{9}$ This is quite a strong hypothesis even in the framework of the conjecture that there is no any new physics beyond the $\nu$ MSM up to the very high scale such as the Planck scale or inflationary scale. In particular, it is shown in [773] that the DM HNL can be produced in sufficient amounts at the reheating of the Universe after Higgs inflation without addidion of any new particles.
} 
$M_{2,3}$ are limited from below by $1-2 \mathrm{GeV}$. There are several theoretical uncertainties involved in computation of the bounds 4.23. These uncertainties may lead to a factor $\sim 2-3$ change in the positions of red and blue curves. The comparison between Fig. 4.17 and Fig. 4.23 shows, that the constraints on $N_{2,3}$ are more tight, as it is more challenging to get large lepton asymmetry in addition to the small baryon asymmetry.

In a non-minimal model, large lepton asymmetry required for resonant production of DM $N_{1}$ can be present in the plasma regardless the properties of $N_{2,3}$. $N_{1}$ can also play the role of DM, but be produced not only from its interactions with the SM neutrinos but, for example, from inflaton decay or other non-minimal interactions [120, 221, 222, 315, 729, 773], see [299] for a particular discussion of $7 \mathrm{keV}$ sterile neutrino dark matter.

\subsubsection{Lepton number violation in the $\nu \mathrm{MSM}$}

Heavy neutral leptons with Majorana type mass naturally give rise to processes with lepton number violation and charged lepton flavor violating processes as discussed in Sec. 4.5.3. In $\nu$ MSM, apart of the standard for sterile neutrino models lower limit on sterile-active mixing following from the seesaw mechanism, [395, 704]

$$
U^{2}>5 \times \kappa \times 10^{-11}\left(\frac{\mathrm{GeV}}{M_{N}}\right)
$$

there is also upper limit on the mixing, [603, 704]

$$
U^{2}<2.5 \times \kappa \times 10^{-7} \times\left(\frac{\mathrm{GeV}}{M_{N}}\right)^{3 / 2}
$$

associated with succesful generation of the baryon asymmetry of the Universe (BAU) via oscillations between sterile and active neutrinos in primordial plasma as discussed in Sec. 4.6.4.1; these limits are presented in Figs. 4.17 for two possible mass hierarchies in the active neutrino sector: normal $(\kappa=1)$ and inverted $(\kappa=2)$. For light HNL, $M_{N} \lesssim 700-800 \mathrm{MeV}$ the lower limit (4.8.2) is superseded by the limits form Big Bang Nucleosynthesis: with smaller mixing neutrino decay after onset of the light nuclei production in the early Universe, which may destort the process. This BBN limit meets the limit from BAU (4.8.3), that places lower bound on sterile neutrino mass, $M_{I} \gtrsim 150 \mathrm{MeV}$. The upper bound on the sterile neutrino mass is not fixed, but the mechanism of the leptogenesis via osciallations implies sterile neutrino mass below electroweak scale. Thus, the $\nu$ MSM parameter space is finite and hence can be fully explored.

Given the value of $M_{I}$, sterile neutrinos can participate in lepton flavor and lepton number violating processes as real or virtual particle. We discuss them in turn below.

Real HNL contributions. If kinematically allowed, sterile neutrino may replace active neutrino in all the weak processes paying the price of small mixing obeying the limits (4.8.3) and (4.8.2). In particular, sterile neutrino can appear in weak decays of SM elementary and composite (mesons, baryons) particles. Since the two heavy HNL are almost degenerate in $\nu$ MSM, both contribute to the processes, and corresponding branching ratios are suppressed by squared mixing angles summed over the sterile neutrinos,

$$
\operatorname{Br} \propto \sum_{I}\left|U_{\alpha I}\right|^{2} .
$$

Upper and lower limits on branching ratios of various decay rates have been calculated in Ref. [395] (see also [547] for general formulas for semileptonic baryon decays). The most interesting numbers are within $10^{-7}-10^{-11}$ for $M_{I} \simeq 500 \mathrm{MeV}$ and decrease as $\propto 1 / M_{I}^{2}$ and $\propto 1 / M_{I}$ in accordance with (4.8.3) and (4.8.2), respectively. Note that two-body meson leptonic decays going through the chirallity-flipping are somewhat enhanced here, since the suppression factor is proportional to the 
heaviest of the final lepton mass, that can be the HNL mass. Generally the branching ratios of heavy mesons are smaller than similar ones of the light mesons, since the former have more final states to decay into.

HNL decay into SM particles (if kinematically allowed) via weak interactions induced by mixing with active neutrinos. HNL lifetime is $\tau_{N}=0.1-10^{-5} \mathrm{~s}$ for $M_{I} \simeq 1 \mathrm{GeV}$ and the interval borders decrease as $\propto 1 / M_{I}^{2}$ and $\propto 1 / M_{I}$ in accordance with (4.8.3) and (4.8.2), respectively. Sterile neutrino branching ratios are gouverned by flavor structure of sterile-active mixing. Sterile neutrino decays look as production of SM particles out of nowhere and becomes a realistic process for fixed target and collider experiments, where sterile neutrinos can be produced in decays of heavy unstable particles participating in weak interactions. $\nu \mathrm{MSM}$ phenomenology at fixed target experiment has been discussed in [395, 775]. For a detector of realistic size $L$ the number of signal events from sterile neutrino decays are suppressed by $U^{2} \times U^{2}$, since production is suppressed by $U^{2}$, and only small part of sterile neutrinos, $\sim \tau_{N} L$ decays inside detector, which gives additional $U^{2}$. Prospects of future $e^{+} e^{-}$colliders in searches for $\nu \mathrm{MSM}$ sterile neutrino inspired lepton number violating processes have been highlighted in [383]. The most promising is decays of weak bosons into sterile neutrinos with their subsequent decays into charged SM particles within detector yet at some distance from the colliding point, so that the background-free signature is argued to be the displaced vertex. The number of such events are suppressed only by $U^{2}$, and even for decreasing with mass mixing (4.8.3) is reasonably high given the expected high statistics of $Z$-bosons at future $e^{+} e^{-}$-machines.

Virtual HNL contributions. Virtual HNL contribute to the rare and forbidden in the SM processes with amplitude suppressed by squared mixing, so that their contribution to the rates are very strongly suppressed by $|U|^{4}$ which starts with $10^{-12}$ at best, provided eq. (4.8.3). The general conclusion about HNL impact on such processes obtained in [603] is that it is extremely small, usually much smaller than that of the active neutrinos, if any. This situation is illustratted in the previous section devoted to neutrinoless double beta decay: at masses in exceed of $500 \mathrm{MeV}$ the chances to distinguish HNL contribution are negligibly small given the uncertainties of the hadronic matrix elements entering the process amplitude.

Among the lepton flavor violating processes with virtual sterile neutrinos, the most promising is $\mu$-e conversion in atoms with conversion rate starting at $10^{-22}[400,603]$ that is far below the expected sensitivity of realistic experiments. Then $\mu \rightarrow$ eee is expected at the level below $10^{-24}$. Branching ratios for rare decays of $\tau$-leptons are naturally even smaller.

One of the most prominent processes associated with virtual sterile neutrino exchange is production of the same sign charged leptons, e.g. $K^{ \pm} \rightarrow \pi^{\mp} e^{ \pm} e^{ \pm}$. A well-known example of such a process is neutrinoless double beta decay. The process is very attractive because of very clear signature easily recognized even with heavy hadronic background. Unfortunately, HNL contribution in $\nu$ MSM is expected to be miniscule. SImilar situation with same sign hadrons iin final state. Say, for $M_{I}=5 \mathrm{GeV}$ the decay branching rate of $\tau^{ \pm} \rightarrow e^{\mp} \pi^{ \pm} \pi^{ \pm}$is expected at the level below $10^{-30}$, which shows that indirect searches for HNL are hopeless within $\nu$ MSM framework.

\subsubsection{Inflation, vacuum stability, dark energy and naturalness in the $\nu \mathrm{MSM}$}

In addition to explanation of neutrino masses and oscillations, of providing the dark matter candidate, and of ensuring the generation of the baryon asymmetry of the Universe, any particle theory pretending to describe Nature up to the scale of quantum gravity should be able to provide a mechanism of cosmological inflation and of the present accelerating expansion of the Universe. In addition, it should address in same way the hierarchy problem and the naturalness issues.

We will provide here a short overview of the minimal models, based on the $\nu$ MSM, dealing with these extra problems. 
Inflation. The most economical way to incorporate inflation to $\nu \mathrm{MSM}$ (and to the Standard Model) is through the non-minimal coupling of the SM Higgs field to gravitational Ricci scalar $R$ [292], $\frac{\xi h^{2}}{2} R$. The presence of the non-minimal coupling modifies the $\lambda h^{4}$ behaviour of the Higgs potential in the Einstein frame, making it flat at large values of the Higgs field. The standard inflationary computations allow to fix the unknown parameter $\xi$ in terms of the scalar self-coupling as $\xi=49000 \sqrt{\lambda}$ whereas the scalar spectral index and for the tensor-to-scalar ratio are [292] $n_{s}=0.97, \quad r=0.003$. Moreover, as is the case for most single field inflationary models, the perturbations are Gaussian. All these predictions are consistent with Planck results. After the slow-roll the Higgs field starts to oscillate and produce particles of the SM, heating the Universe up to the temperatures $\sim 10^{14} \mathrm{GeV}[776,777]$. Different aspects of the Higgs inflation (radiative corrections, higher-dimensional operators, self-consistency, etc) were discussed in a large number of papers, for an overview see [778] and references therein.

Dark energy in the $\nu$ MSM. The most minimal way to describe the accelerated expansion of the Universe at the present epoch in any theory, including the $\nu \mathrm{MSM}$, is simply to add the cosmological constant $\Lambda$. The extremely small value of $\Lambda$ remains without explanation (this is exactly the cosmological constant problem), but this "solution" fits all the cosmological data.

Yet another proposal is based on quantum scale-invariant extension of the $\nu$ MSM with gravity $[779,780]$. For this end all dimensionful parameters are replaced by the dynamical dilaton field, $M_{P}^{2} \rightarrow \xi_{\chi} \chi^{2}, \Lambda \rightarrow \beta \chi^{4}, M_{H}^{2} \rightarrow \alpha \chi^{2}$, and $M_{N} \rightarrow f_{N} \chi^{2}$, where $\xi_{\chi} \sim 1$, and $\beta, \alpha, f_{N}$ are all very small. The spontaneous breakdown of scale symmetry leads to a non-zero vacuum expectation value of the dilaton field and generates the Planck constant, mass of the Higgs boson, and HNL masses. The dilaton interacts with the matter-fields only through derivatives and thus evades all constraints coming from the search of the "fifth force". The smallness of the cosmological constant comes from the smallness of the dilaton self-coupling $\beta \lll 1$, what, as previously, has no explanation. In the case $\beta=0$ (absence of the cosmological constant) the dynamical dark energy may come from the dilaton field, if the theory is based on the unimodular gravity [779].

Hierarchy problem, naturalness, and the $\nu$ MSM. The $\nu$ MSM (as well as the SM) coupled to gravity suffers from the lack of explanation why the Fermi scale is so small in comparison with the Planck scale (the celebrated Higgs hierarchy problem). In its scale-invariant version discussed above this is converted to the absence of explanation why the dilaton-Higgs coupling $\alpha$ is so small. So, the $\nu$ MSM does not provide a solution to the hierarchy problem. Nevertheless, it does address the naturalness issues. As has been shown in [780], if $\alpha$ is chosen to be small, it remains small in all orders of scale-invariant perturbation theory, and, therefore, the value of $\alpha$ (the Higgs mass) is not fine tuned. The key point is the masslessness of the graviton in any dilaton background and the absence of particles with masses much higher than the Fermi scale. It remains to be seen if this argument survives beyond perturbation theory.

It is also interesting to note that the quantisation of electric charges in the $\nu$ MSM is a requirement of self-consistency and comes from the condition of cancellation of gauge and gravitational anomalies [781]. Contrary to Grand Unification, also insuring the electric charge quantisation, this does not imply the existence of any particles with masses between the Fermi and Planck scales, which appear in the loops and may lead to the problem with the naturalness.

Vacuum stability and the $\nu$ MSM. The experimentally measured values of the Higgs mass and of the top Yukawa coupling lead to quite a peculiar behaviour of the scalar self-coupling $\lambda$ in the SM (and also in the $\nu$ MSM, since HNLs couplings are small and can be safely neglected), see Fig. 4.24. This constant decreases with energy, reaches its minimum at energies close to the Planck scale, and then increases [782]. Depending on the values of the Higgs mass and top Yukawa coupling allowed by experiments, $\lambda$ can cross zero at energies as small as $10^{10} \mathrm{GeV}$ and remain negative around the Planck scale, or be positive at any energy, or just touch zero at an energy 


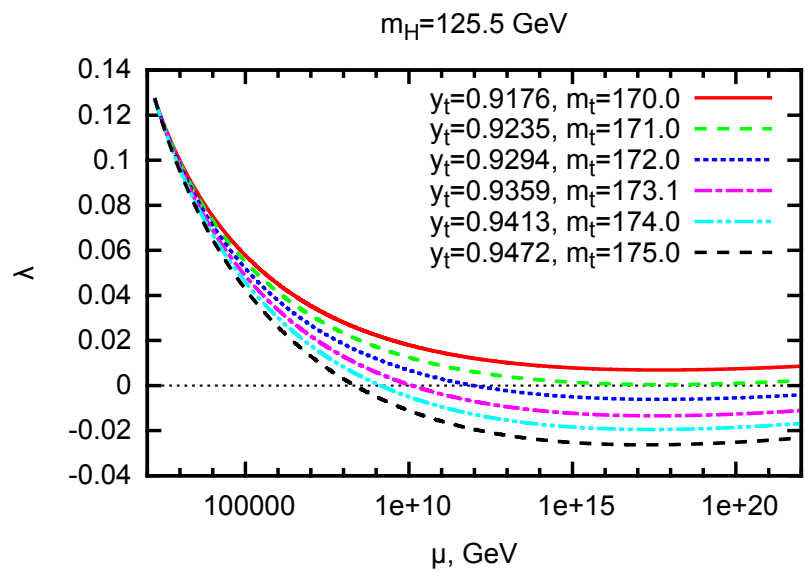

Figure 4.24: Renormalization group running of the Higgs coupling constant $\lambda$ for the Higgs mass $M_{h}=125.7 \mathrm{GeV}$ and several values of the top quark Yukawa $y_{t}(\mu=173.2 \mathrm{GeV})$.

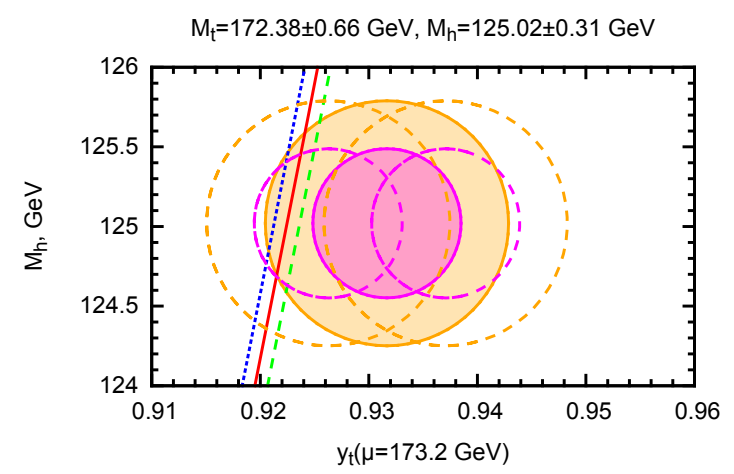

Figure 4.25: The figure shows the borderline between the regions of absolute stability and metastability of the SM vacuum on the plane of the Higgs boson mass and top quark Yukawa coupling in the $\overline{\mathrm{MS}}$ scheme taken at $\mu=173.2 \mathrm{GeV}$. The diagonal line stands for the critical value of the top Yukawa coupling $y_{t}^{\text {crit }}$ as a function of the Higgs mass and the dashed lines account for the uncertainty associated to the error in the strong coupling constant $\alpha_{s}$. The SM vacuum is absolutely stable to the left of these lines and metastable to the right. The filled ellipses correspond to experimental values of $y_{t}$ extracted from the latest CMS determination [786] of the Monte-Carlo top quark mass $M_{t}=172.38 \pm 0.10$ (stat) \pm 0.65 (syst) $\mathrm{GeV}$, if this is identified with the pole mass. The Higgs mass $M_{h}=125.02 \pm 0.27$ (stat) \pm 0.15 (syst) GeV is taken from CMS measurements [787]. Dashed ellipses encode the shifts associated to the ambiguous relation between pole and Monte Carlo masses. See [788] and references therein for more discussion.

scale close to the Planck one [30-32, 783-785]. The behaviour of the Higgs self-coupling is closely related to the problem of stability of the SM vacuum: if $\lambda$ is negative in some domain of energies, the effective potential of the scalar field without gravity develops a second, deeper minimum at the scalar field values of the order of Planck scale. In this case the SM vacuum becomes metastable. The situation is uncertain: the SM vacuum can be absolutely stable or metastable within experimental and theoretical error-bars, see Fig. 4.25.

If the SM vacuum is indeed metastable, there is a danger of transition from our vacuum to another, unwanted one, with Planck scale physics. Though the life-time of the SM vacuum exceeds the age of the Universe by many orders of magnitude [789], it may happen that the Universe 
evolution during or after inflation could drive the system out of our vacuum. To prevent this, some kind of new physics should intervene to save the Universe from the collapse in the Planck vacuum. In [778] has been demonstrated that the specific threshold effects in the SM (and in the $\nu$ MSM) with non-minimal coupling to gravity at the energy scale $M_{P} / \xi$ may lead to relaxation of the system in the SM metastable vacuum. No new heavy particles are needed for this to happen, keeping the perturbative naturalness, discussed above, intact.

\subsection{Conclusions}

The motivation for existence of neutrino portal steams from both experiment and theory and points out to existence of heavy neutral leptons. These particles may play essential role in cosmology, providing a dark matter candidate and producing baryon asymmetry of the Universe. In neutrino physics they provide the source of neutrino masses and mixings. If the masses of HNLs are smaller than $\sim 2 \mathrm{GeV}$, their direct searches are possible in decays of heavy mesons carrying strangeness, charm or beauty, created in high intensity fixed target experiments such as SHiP. Heavier HNL's can be searched for in collider experiments at the LHC and in future experimental facilities like FCC-ee. Virtual HNLs lead to lepton flavour number violation that can potentially be seen in the processes like $\mu \rightarrow 3 e, \mu \rightarrow e \gamma$ or $\tau \rightarrow 3 \mu$. They also generically imply the lepton number violation that can manifest itself in neutrino-less double beta decays. It goes without saying that the discovery of such particles or indirect indication of their existence would revolutionise our understanding of particle physics and cosmology, whereas constraining their properties would help to elucidate different ideas on physics beyond of the Standard Model. 


\section{Chapter 5}

\section{ALPs and other PNGBs at SHiP}

\subsection{ALPs and why they are interesting}

The discovery of the Higgs boson provides strong evidence that fundamental scalar bosons exist in nature. It is thus a timely and well-motivated task to search for further light scalar or pseudoscalar particles. The presence of such additional states are predicted by many extensions of the Higgs sector (e.g. Two-Higgs Doublet Models [250] but also models with extra non-doublet scalars such as the NMSSM see, e.g. [790]). Alternatively, they can arise as pseudo-Nambu-Goldstone bosons (PNGB) of a spontaneously broken $U(1)$ symmetry. In both cases, the new state can be light and have highly suppressed couplings to Standard Model (SM) particles.

To be concrete let us motivate the properties of the particles we are interested in from the example of PNGB of spontaneously broken global symmetries. These symmetries can be $U(1)$ s or other global symmetry groups which are often encountered in field theories, but it could also be shift symmetries typically present in string theory models. For our purposes such PNGBs have two crucial properties,

- Small couplings: Their interactions are suppressed by the scale of spontaneous symmetry breaking $f_{A}$ of the symmetry in question. If the latter is high, the interactions are very small.

- Small mass: If the symmetry is exact, the particles would be proper massless Goldstone bosons. But even if there is a small explicit breaking of the symmetry (as there often is) associated with a scale $\Lambda$ their mass is typically suppressed by the scale of spontaneous symmetry breaking, $m_{A} \sim \Lambda^{2} / f_{A}$, and the PNGB can be very light, even if the underlying physics scales $\Lambda$ and $f_{A}$ are high.

The prime example of such a very light pseudo-Goldstone boson is the axion [248, 791-793] introduced to solve the strong CP problem in QCD. It is a PNGB arising from a $U(1)$ symmetry, the so-called Peccei-Quinn symmetry, that is anomalously (and therefore explicitly) broken by the axial anomaly of QCD. Depending on the model it features

- couplings to two gauge bosons $\sim A F \tilde{F} / f_{A}$

- derivative couplings to SM fermions $\sim\left(1 / f_{A} \partial_{\mu} A\right) \bar{f} \gamma^{\mu} \gamma^{5} f$.

These are the couplings we will focus on below. The mass of the axion is essentially fixed by the QCD anomaly and the scale of spontaneous symmetry breaking with only a relatively small model dependency.

Beyond the axion there exists a wide range of interesting (pseudo-)scalar particles which typically feature very similar interactions as the axion, but which may have different masses. We will call those particles axion-like particles or ALPs. 


\subsubsection{ALP origins}

ALPs - being pseudo Nambu-Goldstone bosons - are, like the axion, associated with the breaking of approximate global symmetries; the differences being that the symmetry does not necessarily have a QCD anomaly and the explicit breaking of the symmetry then gives the ALP its mass. Indeed, an ALP can only have an appreciable coupling to QCD if its mass is significantly greater than that of the axion, otherwise it would instead usurp the role of the QCD axion.

\section{ALPs from string theory}

String theory naturally provides significant motivation for ALPs, since string compactifications contain many different candidates; see e.g. [794-796]. In particular it has been proposed that string theory predicts an axiverse [797-799], a host of axion-like particles with masses potentially taking values distributed across every scale of energy. For example in the LARGE volume scenario we have two classes of axions, called local and non-local. The non-local ones have couplings to the visible sector suppressed by the Planck mass (so are essentially unobservable) and are effectively massless, while local ones have couplings suppressed by the string scale and masses exponentially suppressed from either the Planck scale or the gravitino mass (depending on the origin of the mass term); they can therefore have essentially any mass, but the couplings to the SM gauge groups are fixed, giving $f_{A} \sim M_{\text {string }}, g_{A i} \sim \frac{\alpha_{i}}{2 \pi M_{\text {string }}}$, where the index $i$ indicates the gauge group in question. On the other hand, the couplings of local axions to matter fields are more model dependent, given by either $g_{A f} \sim \frac{v g^{2}}{M_{\text {string }}}$ (where $g$ is a gauge coupling) or zero depending on the setup [799]. Due to this model dependency one should aim to test both types of couplings (to gauge bosons and SM fermions).

\section{Pseudoscalars in extended Higgs sectors}

A class of models which contain a pseudoscalar that can be in the correct mass range and with sufficiently strong couplings to be searched for by SHiP is that of extended Higgs sectors [232, 252, 800]. We also refer the reader to Section 3.4 where an explicit model of this based on the NMSSM is considered.

If we add a (complex) singlet scalar (under the SM gauge groups) to the theory, then the associated pseudoscalar has no direct couplings to the SM fields, only acquiring them through mixing with the would-be Goldstone boson and any other pseudoscalars in the theory. It would then couple at tree-level to quarks via their Yukawa couplings (and only with loop-suppressed couplings to photons). This results in couplings to the SM fermions which are proportional to the SM particle masses. This feature also occurs quite naturally for scalars mixing with the Higgs. Indeed in experiments such as SHiP the scalar and pseudoscalar phenomenologies are quite similar, as we can see by comparing Figs. 3.9 and 5.2.

\section{Other motivations for light pseudoscalars}

Besides string theory, ALPs may also appear in the context of quantum field theory on torsionful manifolds [801-803]. In 4 dimensions the axion coupling is typically suppressed by the Planck scale and therefore beyond the scope of current experiments. Nevertheless, in extra-dimensional models $[804,805]$, the fundamental Planck scale might be significantly lower, leading to a sizeable axion coupling. For instance, considering a fundamental Planck scale $M_{*} \sim$ few $\mathrm{TeV}$ one gets an axionic decay constant $f_{A} \sim$ few $\mathrm{TeV}$ which lies in the potentially observable regime.

An additional interesting motivation for PNGBs are spontaneously broken global flavour symmetries, resulting in so-called familons [806] (which could also be interesting dark matter candidates $[807,808])$. Familons naturally couple to SM fermions, typically with couplings of non-trivial flavour structure. 


\subsubsection{Connection to Dark Matter}

ALPs also provide an interesting connection to the puzzle of dark matter (DM), because they can mediate the interactions between the DM particle and SM states and allow for additional annihilation channels relevant for the thermal freeze-out of DM. Indeed, it is a critical challenge for any DM model to explain the observed DM relic abundance in terms of the interactions of the DM particle. Direct detection experiments [275, 809-811] as well as bounds on the invisible width of the SM Higgs $[812,813]$ challenge the idea that DM couples directly to a SM mediator.

An attractive possibility to relax these constraints is to assume the presence of an additional new particle that mediates the interactions of DM while coupling only weakly to the visible sector. In particular, if the new particle is a pseudoscalar event rates in direct detection experiments are strongly suppressed so that some of the most stringent bounds on DM interactions can be avoided $[814,815]$. This is in stark contrast to the scalar case where direct detection (depending on the putative dark matter mass) can lead to severe restrictions on the parameter space as shown in Fig. 3.9. For the pseudoscalar this is not the case and a much wider parameter range is viable (cf. Fig. 5.2).

As pointed out in the context of asymmetric DM [816], if the pseudoscalar mass is sub-GeV it can furthermore evade detection at the Large Hadron Collider (LHC), for example from LHC monojet searches. Another advantage of a very light pseudoscalar $A$ is that it allows for the possibility that DM can obtain the appropriate relic density from thermal freeze-out even if it couples very weakly to SM particles. The reason is that, provided the pseudoscalar mass is less than the mass of the DM particle $\chi$, the annihilation channel $\chi \bar{\chi} \rightarrow A A$, followed by decays of the pseudoscalars into SM particles, potentially allows for the highly efficient annihilation of DM particles. The only significant constraint is that the pseudoscalars produced in DM annihilations must decay before BBN, which intriguingly provides a lower bound on the couplings between the pseudoscalar and SM states.

Finally, light mediators for DM have also received great interest in the context of DM selfinteractions. Such self-interactions can potentially explain the discrepancies between $N$-body simulations of collisionless cold DM and the observations of small-scale structures and help solve the cusp-core problem as well as the missing satellite problem [122]. Light mediators are particularly interesting in this context, because they naturally lead to a velocity dependence in the momentum transfer cross section which helps to evade constraints from high-velocity systems such as the Bullet Cluster [150]. Moreover, for a light mediator there can be additional enhancements at low velocities due to non-perturbative effects, which have been studied in a lot of detail recently [149, 154].

\subsection{Interactions, phenomenological features and existing limits}

The interactions between an ALP $A$ and SM states are usually written in the form

$$
\begin{aligned}
\mathcal{L}_{\mathrm{ALPSM}} & =\sum_{f} \frac{C_{A f}}{2 f_{A}} \bar{f} \gamma^{\mu} \gamma^{5} f \partial_{\mu} A-\sum_{i} \frac{\alpha_{i}}{8 \pi} \frac{C_{A i}}{f_{A}} F_{\mu \nu}^{b(i)} \tilde{F}_{b}^{(i) \mu \nu} A \\
& =\sum_{f} \frac{C_{A f}}{2 f_{A}} \bar{f} \gamma^{\mu} \gamma^{5} f \partial_{\mu} A-\frac{\alpha}{8 \pi} \frac{C_{A \gamma}}{f_{A}} F_{\mu \nu} \tilde{F}^{\mu \nu} A-\frac{\alpha_{3}}{8 \pi} \frac{C_{A 3}}{f_{A}} G_{\mu \nu}^{b} \tilde{G}^{b \nu} A+\ldots,
\end{aligned}
$$

where $f=\{q, \ell, \nu\}, q=\{u, d, s, c, b, t\}, \ell=\{e, \mu, \tau\}$, and $i=\{Y, 2,3\}$. In the second line we focus on the gauge groups relevant below the electroweak scale and $F_{\mu \nu}$ is the electromagnetic field strength tensor, $G_{\mu \nu}^{b}$ the field strength for the strong force. We have furthermore defined the dual field strength tensors $\tilde{F}^{\mu \nu}=\frac{1}{2} \epsilon^{\mu \nu \rho \sigma} F_{\rho \sigma}$. We also have the definitions of ALP couplings to the gauge 

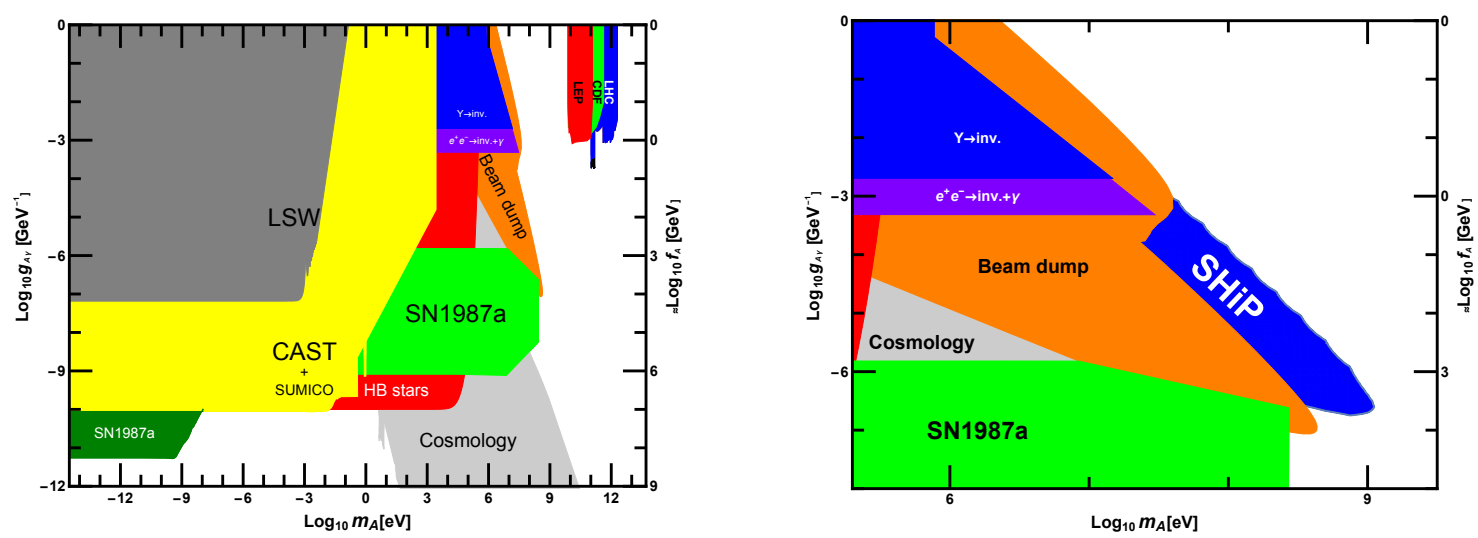

Figure 5.1: Excluded parameter regions for a pseudoscalar $A$ with couplings to two photons/gauge bosons. Compilation adapted and combined from [817-824]. The right panel focusses on the (sub) GeV region of masses and shows the potential improvement that can be achieved with the future modification of the SHiP detector, sensitive to all neutral final states (see footnote 1).

field strengths given by

$$
g_{A i} \equiv \frac{\alpha_{i}}{2 \pi} \frac{C_{A i}}{f_{A}} .
$$

An ALP, in a crucial difference to the QCD axion, has in addition a (significant) mass term in the lagrangian. If the ALP has a coupling to QCD, then it will acquire a contribution to its mass from QCD effects and mix with the QCD axion, but for the ALPs of interest to SHiP their mass will be sufficiently large that this is not significant.

For on-shell fermions the ALP coupling to fermions can be rewritten using the equation of motion:

$$
\mathcal{L} \supset i \sum_{f} g_{A f} \frac{m_{f}}{v} A \bar{f} \gamma_{5} f, \quad g_{A f} \equiv-C_{A f} \frac{v}{f_{A}}
$$

\subsection{ALPs coupled to two gauge bosons}

Let us first consider an axion-like particle which couples to two gauge bosons, e.g. these could be two photons or two gluons. Let us write the ALP coupling to gauge bosons $i$ as

$$
\mathcal{L} \supset-\frac{g_{A i}}{4} A F_{\mu \nu}^{(i) b} \tilde{F}_{b}^{(i) \mu \nu} .
$$

Then the partial width is

$$
\Gamma(A \rightarrow i i)=\frac{d(G) g_{A i}^{2} m_{A}^{3}}{64 \pi}
$$

where $d(G)$ is the number of generators in the group.

Here we will focus on the exemplary case of a coupling purely to two photons. But more general couplings to SM gauge bosons are of interest and offer opportunities for SHiP.

The current constraints on the two photon coupling are shown in Fig. 5.1. A zoom on the region of interest for SHiP is shown in the right panel. We note that this is also the mass region of interest in models where ALPs serve as mediators to a DM sector. 


\subsubsection{Prospects for SHiP}

To obtain an estimate of the sensitivity of SHiP, we have implemented the ALP-photon vertex in CalcHEP 3.4 [825]. We find the dominant process to be Drell-Yan production of a photon followed by Primakoff production of the ALP. To estimate the geometric acceptance for the detector, we require the ALP to travel in a direction within 0.5 degrees from the direction of the initial beam. If the total production cross section is $\sigma_{A}$, we can obtain the number of ALPs produced at SHiP by comparing this cross section to the total proton nucleon cross section $\sigma_{p N} \sim 10 \mathrm{mb}$, $N_{A}=N_{\mathrm{PoT}}\left(\sigma_{A} / \sigma_{p N}\right)$. For our estimate we use for the number of protons on target, $N_{\mathrm{PoT}} \sim 2 \cdot 10^{20}$.

The number of decays in the detector region can then be calculated by multiplying the number $N_{A}$ of ALPs produced with the probability that the ALP decays inside the detector. If the detector is placed at a distance $l$ and has a length $\Delta l$, the number of events in the detector region is

$$
N_{\mathrm{det}} \sim N_{A}\left[\exp \left(-\frac{l}{\gamma \beta c \tau_{A}}\right)-\exp \left(-\frac{l+\Delta l}{\gamma \beta c \tau_{A}}\right)\right] .
$$

where $\tau_{A}=\Gamma_{A}^{-1}$ is the ALP lifetime and $\gamma=E_{A} / m_{A}$ is its relativistic gamma factor.

In practice, the energy $E_{A}$ of the ALPs produced at SHiP is not fixed. We therefore need to determine the differential cross section $\mathrm{d} \sigma_{A} / \mathrm{d} E_{A}$ and integrate over the ALP energy to obtain

$$
N_{\text {det }} \sim \int \frac{N_{\pi}}{\sigma_{\pi^{0}}} \frac{\mathrm{d} \sigma_{A}}{\mathrm{~d} E_{A}}\left[\exp \left(-\frac{l m_{A}}{E_{A} \beta c \tau_{A}}\right)-\exp \left(-\frac{(l+\Delta l) m_{A}}{E_{A} \beta c \tau_{A}}\right)\right] \mathrm{d} E_{A} .
$$

We take $l=70 \mathrm{~m}, \Delta l=55 \mathrm{~m}$. To indicate the interesting parameter region, we show in the right panel of Fig. 5.1 the sensitivity for SHiP to observe 3 or more events. ${ }^{1}$

\subsection{ALPs coupled to SM fermions}

Similar to the case of scalars discussed in Section 3.2 flavor observables also provide a path to probe pseudoscalars. While similar constraints have been studied for light vector mediators [46, 47], many other cases remain relatively unexplored. Recently, some of us have presented ${ }^{2}$ an exhaustive study of the constraints on pseudoscalar ALPs from rare meson decays and fixed target experiments for different coupling structures between the ALP and SM fermions [232]. Here, we will closely follow [232] and use these findings to set the stage for our study of the prospects of SHiP for ALP physics.

\subsubsection{Interactions, phenomenological features and existing limits}

The interactions between ALPs and SM matter states are usually written in the form

$$
\mathcal{L}=\sum_{f} \frac{C_{A f}}{2 f_{A}} \bar{f} \gamma^{\mu} \gamma^{5} f \partial_{\mu} A
$$

In the following we will concentrate on flavour-diagonal couplings. But in principle also flavour non-diagonal couplings are possible and of interest as, e.g. in the case of familons [806].

For a pseudoscalar arising from an extended Higgs sector, one would conventionally write

$$
\mathcal{L}=-i g_{Y} \sum_{f} \frac{m_{f}}{v} A \bar{f} \gamma^{5} f
$$

\footnotetext{
${ }^{1}$ The SHiP detector in its current configuration, as described in [34] cannot detect all neutral final states. The modifications of electromagnetic calorimeter's design is required for the effective detection at the SHiP SPS facility (currently under investigation).

${ }^{2}$ F. Kahlhoefer and K. Schmidt-Hoberg together with M. Dolan and C. McCabe
} 
where $m_{f}$ is the fermion mass, $v \simeq 246 \mathrm{GeV}$ is the vacuum expectation value (vev) of the SM Higgs field and we introduce a factor of $i$ so that the coupling $g_{Y}$ is real. This coupling structure is expected because the couplings of the pseudoscalar to SM fermions arise from mixing with the SM Higgs boson and are therefore automatically proportional to the SM Yukawa couplings. For the kinds of processes that we are interested in, both ways of writing the ALP-fermion interactions are equivalent and one can be obtained from the other by integrating by parts, using the equations of motion and setting $g_{Y}=C_{A f} v / f_{A}$. Following the conventions of [232], we will adopt the notation from Eq. (5.4.2) in the following.

Since the effective coupling of ALPs to SM fermions is proportional to the mass of the SM fermions an ALP is expected to couple only very weakly to electrons. Consequently, the production rate from bremsstrahlung in electron beam dump experiments is very small. Proton beam dump experiments, on the other hand, offer a unique opportunity to search for such particles. The reason is that ALPs can be produced in rare decays of heavy mesons produced in the target. We will therefore now briefly discuss the effective flavour-changing interactions of ALPs and the resulting branching ratios for various rare decays.

\subsubsection{ALP-pion mixing}

Replacing ${ }^{3} \partial^{\mu}\left(\bar{q} \gamma_{\mu} \gamma^{5} \frac{\tau^{3}}{2} q\right)$ by $m_{\pi}^{2} f_{\pi} \pi^{0}$ it becomes clear that the interaction between ALPs and quarks introduced above leads to a non-diagonal mass term of the form

$$
\frac{C_{A f} f_{\pi}}{2 f_{A}} m_{\pi}^{2} \pi^{0} A
$$

Diagonalisation of the mass matrix then leads to ALP-pion mixing proportional to

$$
\kappa=\frac{C_{A f} f_{\pi}}{2 f_{A}} \frac{m_{\pi}^{2}}{m_{\pi}^{2}-m_{A}^{2}}
$$

assuming that $\kappa \ll 1 .^{4}$ As a result, any physical process producing pions will have a non-zero chance of producing ALPs as well. The respective production cross sections are simply related by

$$
\sigma_{A}=\kappa^{2} \sigma_{\pi^{0}}
$$

\subsubsection{Effective flavour-changing interactions}

Flavour changing interactions allow efficient production of ALPs from heavy meson decays. This is most obvious for ALPs which have flavour changing interactions at tree-level. But even if the initial ALP couplings appear flavour diagonal, flavour-changing neutral currents (FCNCs) arise at the one-loop level from diagrams with heavy quarks and $W$-bosons (similar to those shown in Fig. 3.7 but with $A$ replacing $S$ ). We will be interested in the transitions $b \rightarrow s A$ and $s \rightarrow d A$. The relevant flavour-changing terms can be parameterised in the form

$$
\mathcal{L}_{\mathrm{FCNC}} \supset h_{d s}^{R} A \bar{d}_{L} s_{R}+h_{d s}^{L} A \bar{d}_{R} s_{L}+h_{s b}^{R} A \bar{s}_{L} b_{R}+h_{s b}^{L} A \bar{s}_{R} b_{L}+\text { h.c. },
$$

where $q_{R, L}=\left(1 \pm \gamma^{5}\right) / 2 q$.

In the approximation that the top-quark mass $m_{t}$ and the $W$-boson mass $M_{W}$ are large com-

\footnotetext{
${ }^{3}$ As a caveat we note that this replacement is justified only for $m_{A} \lesssim m_{\pi}$. Above this mass results obtained via ALP-pion mixing should be interpreted with caution.

${ }^{4}$ For $m_{\pi} \sim m_{A}$ mixing will be large even if the off-diagonal terms are small.
} 
pared to all other masses one obtains

$$
\begin{aligned}
h_{s b}^{R} & =i \frac{\alpha m_{b}}{4 \sqrt{2} \pi \sin \left(\theta_{W}\right)^{2} v} f\left(x_{t}\right) V_{t b} V_{t s}^{*}, \\
h_{s b}^{L} & =-i \frac{\alpha m_{s}}{4 \sqrt{2} \pi \sin \left(\theta_{W}\right)^{2} v} f\left(x_{t}\right) V_{t b} V_{t s}^{*},
\end{aligned}
$$

where $\alpha \equiv e^{2} /(4 \pi), \theta_{W}$ is the Weinberg angle, $V$ is the CKM matrix and

$$
f\left(x_{t}\right)=x_{t}\left(x_{t}-2\right)\left[-\frac{1}{x_{t}-1}+\frac{\log x_{t}}{\left(x_{t}-1\right)^{2}}\right]
$$

with $x_{t} \equiv m_{t}^{2} / M_{W}^{2}$.

\subsubsection{ALP-mediated rare decays}

Using these results, we can now calculate the partial decay widths for flavour changing meson decays. The most interesting decay in the present context is $B \rightarrow K A[233,826]$ :

$$
\Gamma(B \rightarrow K A)=\frac{1}{16 \pi m_{B}^{3}} \lambda^{1 / 2}\left(m_{B}^{2}, m_{K}^{2}, m_{A}^{2}\right)\left|f_{0}^{B^{0}}\left(m_{A}^{2}\right)\right|^{2}\left(\frac{m_{B}^{2}-m_{K}^{2}}{m_{b}-m_{s}}\right)^{2}\left|h_{s b}^{S}\right|^{2},
$$

where

$$
h_{s b}^{S}=\frac{h_{s b}^{L}+h_{s b}^{R}}{2} .
$$

For the form factor we use the parameterisation from [827]:

$$
f_{0}^{B^{0}}\left(q^{2}\right)=\frac{0.33}{1-q^{2} /\left(38 \mathrm{GeV}^{2}\right)},
$$

which should be accurate within $10 \%$. To avoid these theoretical uncertainties, it is possible to study the more inclusive decays $B \rightarrow X_{s} A$, where $X_{s}$ can be any strange meson. One then finds [233]

$$
\Gamma\left(B \rightarrow X_{s} A\right)=\frac{1}{8 \pi} \frac{\left(m_{b}^{2}-m_{A}^{2}\right)^{2}}{m_{b}^{3}}\left|h_{s b}^{S}\right|^{2} .
$$

To obtain the branching ratios (BR) for rare meson decays into SM final states, we need to divide these partial widths by the total meson decay width and multiply with the branching ratio of the ALP into the appropriate final state. For example,

$$
\begin{aligned}
\operatorname{BR}\left(B \rightarrow K \mu^{+} \mu^{-}\right) & =\frac{\Gamma\left(B \rightarrow K \mu^{+} \mu^{-}\right)}{\Gamma_{B}} \\
& =\frac{\Gamma(B \rightarrow K A) \times \operatorname{BR}\left(A \rightarrow \mu^{+} \mu^{-}\right)}{\Gamma_{B}}+\mathrm{BR}\left(B \rightarrow K \mu^{+} \mu^{-}\right)_{\mathrm{SM}},
\end{aligned}
$$

where we have made use of the narrow width approximation in the second line. Since decays into two pseudoscalar pions violate $C P^{5}$, the ALP will decay almost exclusively into charged leptons and photons, so that a clean experimental signal can be expected across the entire mass range $1 \mathrm{MeV} \lesssim m_{A} \lesssim 1 \mathrm{GeV}$ [232]. Decays into electrons dominate for ALP masses of about (1-100) MeV. As the ALP mass approaches the $\mu^{+} \mu^{-}$threshold, the branching ratio for $A \rightarrow \gamma \gamma$ becomes sizeable, while above the threshold the $\mu^{+} \mu^{-}$decay channel is the most important one.

\footnotetext{
${ }^{5}$ Scalars on the other hand can decay into two pions.
} 
Many searches for rare decays, for example $B \rightarrow K \mu^{+} \mu^{-}$at LHCb [828] rely on the reconstruction of the primary vertex and hence require the ALP to decay more or less instantaneously. For ALPs with smaller couplings and longer lifetimes these searches therefore cease to be constraining even though ALPs may still be produced in abundance. Beam-dump experiments in contrast are particularly sensitive to long-lived light new states, which can travel through the hadron absorber before decaying. Many beam-dump experiments operate using electron beams which, as already mentioned, are not very sensitive to new light scalars or pseudoscalars with Yukawa-like couplings or couplings only to quarks. This is where the proton beam of SHiP proves advantageous. Nevertheless there are already a number of proton beam-dump results in the literature including CHARM [234], NuCal [829] and E613 [830] as well as the recent DAE $\delta$ ALUS [174] proposal. As we will see below, SHiP can significantly extend the sensitivity beyond those.

\subsubsection{Prospects at SHiP}

There are two main ways to produce ALPs with quark couplings at SHiP: Direct ALP production from ALP-pion mixing and indirect ALP production from $B$-meson decays. If a total number of $N_{\pi}$ pions is produced in the direction of the detector, the corresponding number of ALPs produced via ALP-pion mixing is simply given by $N_{A}=\kappa^{2} N_{\pi}$. We estimate that there are about 4 pions produced per proton nucleon interaction giving a total of $N_{\pi} \sim 8 \cdot 10^{20}$.

For the production of ALPs via $B$-mesons we follow the same strategy as in Section 3.2 by comparing the total cross sections for proton nucleon collisions $\sigma_{p N} \sim 10 \mathrm{mb}$ with that of $B$-mesons $\sigma_{B} \sim 3.6 \mathrm{nb}$. From this we obtain $N_{B}=N_{\mathrm{PoT}} \sigma_{B} / \sigma_{p N}=7 \cdot 10^{13}$. For the number of ALPs produced we then use $N_{A}=N_{B} \times \operatorname{BR}(B \rightarrow A+X)[221,230]^{6}$. These ALPs then need to decay inside the detector volume. Note that, in agreement with the discussion of the decays of a light pseudoscalar in [232], we take the branching ratio of the ALP into electrons, muons and photons to be $100 \%$ for $m_{A}<2 m_{\tau}$. We note again the (small) difference to the scalar case where one also has decays to pions.

As in [234] we use $E_{A} \sim 25 \mathrm{GeV}$ for the typical energy of the produced ALPs and assume that SHiP will observe no background events, so that it can make a discovery with $N_{\text {det }} \geq 3$ events. The projected sensitivity region (together with current bounds on the ALP parameters) is depicted in Fig. 5.2. For clarity we show separately the exclusion region resulting from ALP-pion mixing (purple, dotted) and from $B$-meson decays (cyan, dot-dashed), as well as the combined reach (blue, dashed). The probed region is determined by the requirement that the ALPs decay inside the detector volume. Therefore their lifetimes should neither be too large (small coupling and mass) nor too small (large coupling and mass). Along the lines of tis argument the feature at $m_{A} \sim 210 \mathrm{MeV}$ results from the rapidly decreasing lifetime of the ALP as decays into muons become allowed.

Finally, we want to emphasise that, crucially, there is a lower bound on the coupling $g_{Y}$, so that the interesting parameter range is finite (this is indicated by the red dotted line in Fig. 5.2). The key observation is that the ALPs produced in the early Universe (e.g. from dark matter annihilation) should decay before primordial nucleosynthesis in order to avoid changes to the expansion rate and the entropy density during BBN or the destruction of certain elements. For $m_{A}<2 m_{\mu}$ current bounds (e.g. from CHARM) are still about two orders of magnitude away from this limit, but SHiP would be able to shift this bound significantly.

Furthermore, SHiP is also sensitive to the case $m_{A}>m_{K}-m_{\pi}$, where ALPs can only be produced in B-meson decays. The resulting exclusion from SHiP is highly complementary to the ones from conventional searches for rare decays, such as $B \rightarrow \pi^{0} \ell^{+} \ell^{-}$, which require the ALPs

\footnotetext{
${ }^{6}$ As in Section 3.2 we assume that the $B$-mesons still have a sufficiently large boost that the majority of the ALPs produced in the decays will travel in the direction of the detector.
} 


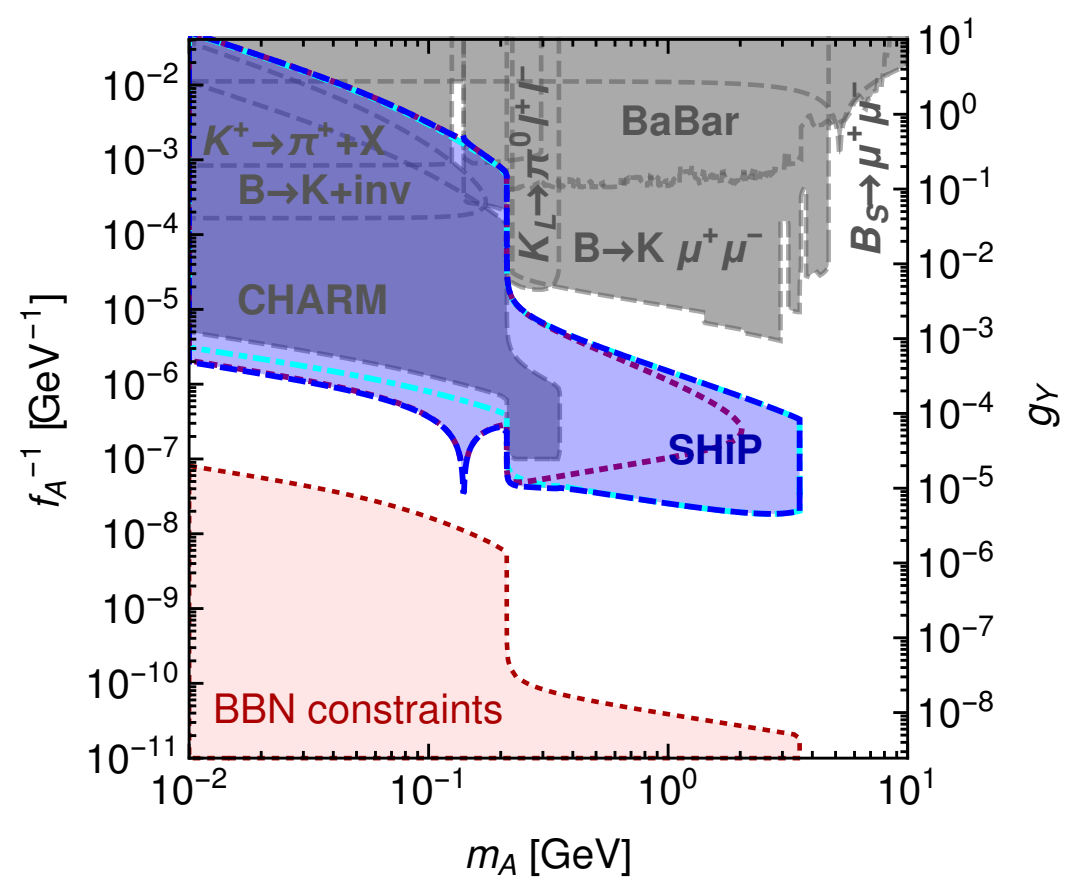

Figure 5.2: Projected sensitivity of SHiP for an ALP $A$ with Yukawa-like couplings to all fermions, in comparison to the existing bounds. The purple dotted line indicates the sensitivity resulting from ALP-pion mixing, while the cyan dot-dashed line indicates the sensitivity from $B$-meson decays. The combined sensitivity for both production mechanisms is shown in blue. The red dotted line indicates the minimal coupling required to avoid constraints from BBN.

to decay instantaneously, while SHiP is most sensitive to long-lived states. Combining results from SHiP and $\mathrm{LHCb}$ will be able to probe the entire parameter region $2 m_{\mu}<m_{A}<2 m_{\tau}$ and $g_{Y}>10^{-5}$. As discussed in [232], DM direct and indirect detection experiments can only typically probe $g_{Y} \gtrsim 10^{-1}$ (assuming that the ALP-DM coupling is fixed by the relic density requirement). SHiP therefore offers a unique possibility to probe a range of couplings that is inaccessible for both low-background and collider searches.

\subsection{Concluding remark}

SHiP can probe significant untested regions in parameter space both for ALPs coupled to two gauge bosons and for those coupled to SM fermions. Particularly interesting search channels include,

$$
p p \rightarrow A+X, A \stackrel{\text { long lived }}{\longrightarrow} \gamma \gamma
$$

to probe couplings to gauge bosons, and

$$
p p \rightarrow B+X \rightarrow A+K+X, A \stackrel{\text { long lived }}{\longrightarrow} \mu^{+} \mu^{-}
$$

for the couplings to SM fermions. While the muon channel is a relatively simple search channel for the SHiP, the all-neutral photon channel is more challenging. Encouraged by the prospects for having significant reach for ALPs and other particles (see, e.g. next chapter) this is currently being investigated (c.f. footnote 1). 
The new area probed is especially relevant for the case of ALPs coupled to SM fermions because it probes a significant part of the parameter space suggested by models where an ALP can act as a mediator for DM. 


\section{Chapter 6}

\section{SUSY}

\subsection{Introduction}

Supersymmetry is one of the most popular, but also one of the best motivated extensions of the Standard Model. Connecting bosons with fermions, by giving each boson a fermionic superpartner and vice versa, its structure is theoretically appealing but it also helps to address or at least alleviate some of the most significant challenges to the Standard Model, e.g. the hierarchy problem or grand unification.

Of course, observation (e.g. the absence of a selectron with mass of $511 \mathrm{keV}$ ) tells us that supersymmetry must be broken. Indeed supersymmetry softly broken at or slightly above the scale of electroweak symmetry breaking would provide a natural way to address the hierarchy problem of the Standard Model. In this situation one expects superpartners with masses in the $100 \mathrm{GeV}$ to few $\mathrm{TeV}$ range. This directly leads one to search for them at high energy colliders such as the LHC [26, 27, 831-833]. Alternatively, heavy particles can also be detected via their effects in loop processes and searches, in particular for flavor violating effects, have been performed using for example $B$-mesons (see, e.g., [834]). While these searches are ongoing and hopes are high, in particular for Run II of the LHC, one has to face the fact that existing searches pose significant constraints on this scenario. Especially the idea of a very high degree of naturalness seems under pressure.

In this situation it seems prudent to rethink our "natural" expectations of supersymmetry, go beyond the simplest models assumptions (such as, e.g. the CMSSM, or R-parity conservation), and explore suitable complementary search strategies. For example in the Standard Model the electron is quite light compared to the electroweak scale. Therefore it may be sensible to also search for SUSY particles a couple of orders of magnitude lighter than the mass of the $\mathrm{W}$ and $\mathrm{Z}$ bosons. Indeed such lightness could be "accidental" or it could be due to some mechanism. In this chapter we explore and survey some possibilities for light superpartners. Interestingly we find that there are quite a variety of opportunities for light superpartners that to date have avoided detection.

The next step is, of course detection of these particles. As this is a physics case study for the SHiP experiment we focus on this proposal and illuminate the potential of SHiP to search for SUSY particles.

We note that actually there is a history of searching for SUSY at fixed target facilities [835]. While the required light gluinos for those searches are excluded by now, we show in the following that there are still interesting opportunities for finding SUSY at fixed target facilities such as SHiP.

In this chapter we discuss a variety of SUSY particles and models, review their individual motivations and discuss the discovery potential at SHiP. For convenience we highlight at the end of each section the relevant search channel at SHiP. 


\subsection{A Very Light Supersymmetric Neutralino and R-Parity Violation}

\subsubsection{Motivation for a very light neutralino}

Within the CMSSM the mass of the lightest neutralino, $\widetilde{N}_{1}^{0}$, is constrained to be [156]

$$
m_{\widetilde{N}_{1}^{0}}>46 \mathrm{GeV} .
$$

However this assumes a supersymmetric GUT relation among the electroweak gaugino masses

$$
M_{1}=\frac{5}{3} \tan ^{2} \theta_{\mathrm{W}} M_{2} \approx \frac{1}{2} M_{2},
$$

and then uses the LEP II chargino search. If the relation Eq. (6.2.2) is dropped, and $M_{1}$ and $M_{2}$ are left as free parameters, there is no laboratory bound on the neutralino mass and even a massless neutralino is allowed $[836,837]$. This can be understood as follows. Setting the determinant of the MSSM neutralino mass matrix to zero and solving for $M_{1}$ we obtain [837]

$$
M_{1}=\frac{M_{2} M_{Z}^{2} \sin (2 \beta) \sin ^{2} \theta_{\mathrm{W}}}{\mu M_{2}-M_{Z}^{2} \sin (2 \beta) \cos ^{2} \theta_{\mathrm{W}}} .
$$

Thus for every choice of the supersymmetric parameters $M_{2}, \mu, \tan \beta$, there is always an $M_{1}$ resulting in at least one massless neutralino. ${ }^{1}$ The resulting $M_{1}$ is typically a factor 50 or so smaller than $\mu, M_{2}$, and the lightest neutralino is almost pure bino. It therefore does not couple directly to the $Z^{0}$, evading LEP I bounds [836]. The pair production of neutralinos at a collider [838] then necessarily involves virtual squarks or sleptons. If these are heavier than about $300 \mathrm{GeV}$ the rate is sufficiently small to avoid bounds.

The main bounds on the neutralino are then astrophysical from supernova [839] and white dwarf cooling [840], or cosmological from the resulting dark matter energy density [837, 841, 842]. The supernova bounds [839] are evaded for neutralino masses above about $250 \mathrm{MeV}$. A massless neutralino is allowed for selectron masses above $1275 \mathrm{GeV}$ or below $320 \mathrm{GeV}$. For $m_{\tilde{e}}>1275 \mathrm{GeV}$ too few neutralinos are produced. For $m_{\tilde{e}}<320 \mathrm{GeV}$ the neutralinos are trapped in the supernova, similar to neutrinos and must be included in the full supernova simulation. Since this has not been done to-date the supernova does not give a reliable bound in this region.

Cosmologically the Cowsik-McClelland bound [843, 844] on a very light neutrino translates into the upper neutralino mass bound [837]

$$
m_{\widetilde{N}_{1}^{0}}<0.7 \mathrm{eV}
$$

The neutralino in this case provides hot dark matter, but not enough to negatively affect structure formation. The observed dark matter energy density must then originate elsewhere.

If one requires the lightest neutralino to provide the correct dark matter energy density one obtains a lower mass bound, corresponding to the Lee-Weinberg bound [98, 845, 846]. The proper bound is obtained by scanning over the supersymmetric parameter space, while dropping the relation in Eq. (6.2.2) and taking into account the latest collider constraints on the supersymmetric parameters. $^{2}$ This is thus an on-going process [847-851]. The most recent bound including the Higgs-discovery data and also constraints from stau searches gives [841, 842, 852]

$$
m_{\widetilde{N}_{1}^{0}}>24 \mathrm{GeV}
$$

\footnotetext{
${ }^{1}$ Note that we assumed here that $M_{1}, M_{2}, \mu \in \mathbb{R}$; for complex parameters a solution need not exist [837].

${ }^{2}$ For a semi-analytical treatment see [837].
} 

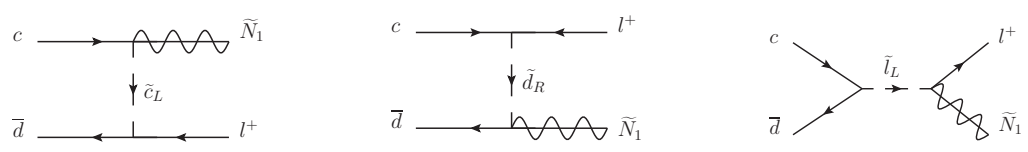

Figure 6.1: Relevant Feynman Diagrams for $D^{+} \rightarrow \widetilde{N}_{1}^{0}+\ell^{+}$.

Therefore the mass range

$$
0.7 \mathrm{eV}<m_{\widetilde{N}_{1}^{0}}<24 \mathrm{GeV}
$$

is excluded for a stable neutralino LSP as it gives too much dark matter. A neutralino LSP in this mass range is only allowed if it decays, i.e. R-parity is violated.

Note that there is no particular motivation to expect $M_{1}$ to approximately satisfy Eq. (6.2.3), resulting in a light neutralino. However, there is also no reason the neutralino must be heavy. Since we have no convincing theory of supersymmetry breaking, which would predict $M_{1}, M_{2}, \mu$, and $\tan \beta$, experimental searches are our best bet.

\subsubsection{R-parity Violation}

When supersymmetrizing the Standard Model (SM), an extra Higgs doublet must be introduced and then the particle content must be doubled. With this minimal particle content the most general renormalizable superpotential, which governs the non-gauge interactions, is fixed by the $\mathrm{SU}(3) \mathrm{xSU}(2) \mathrm{xU}(1)$ gauge symmetry to contain two sets of terms $W_{M S S M}=W_{R P C}+W_{R P V}$ with

$$
\begin{aligned}
& W_{R P C}=\left(Y_{e}\right)_{i j} L_{i} H_{1} \bar{E}_{j}+\left(Y_{d}\right)_{i j} Q_{i} H_{1} \bar{D}_{j}+\left(Y_{u}\right)_{i j} Q_{i} H_{2} \bar{U}_{j}+\mu H_{1} H_{2}, \\
& W_{R P V}=\lambda_{i j k} L_{i} L_{j} \bar{E}_{k}+\lambda_{i j k}^{\prime} L_{i} Q_{j} \bar{D}_{k}+\lambda_{i j k}^{\prime \prime} \bar{U}_{i} \bar{D}_{j} \bar{D}_{k}+\kappa_{i} L_{i} H_{2}
\end{aligned}
$$

in the notation of [853]. The terms in Eq. (6.2.7) correspond to the known Yukawa couplings in the SM. The terms in Eq. (6.2.8) have no correspondence and violate lepton- or baryon number. If all the latter terms are present and have significant couplings there is rapid proton decay which is experimentally excluded. A further symmetry must be added to forbid at least a subset of the terms $W_{R P V}$. One standard approach is to use a discrete multiplicative symmetry R-parity, which forbids all the terms in $W_{R P V}$, this is the widely considered MSSM. An alternative symmetry, e.g. baryontriality, see for example [854], just forbids the terms proportional to $\lambda_{i j k}^{\prime \prime}$. Baryon-number is then conserved and the proton is also stable. Both discrete symmetries are discrete gauge anomaly-free $[855,856]$, and are thus theoretically equally well motivated.

The main features of an additional R-parity violating (RPV) term [857] are that the lightest supersymmetric particle is no longer stable. It is thus not a dark matter candidate. This could instead be the axino, or the axion [858] (cf. also Chapter 5). At colliders we would also not expect to have significant amount of missing transverse momentum as a result of supersymmetric production. Furthermore at colliders one can have resonant single production of supersymmetric particles. As long as the nature of the dark matter particle is not known, and supersymmetry has not been discovered at the LHC, R-parity conservation and RPV are equally well motivated.

\subsubsection{Finding Neutralinos at SHiP via R-Parity violation}

In the following, we assume that there exists an $\mathrm{R}$-parity violating interaction $\Delta W=\lambda_{i 21}^{\prime}\left(L_{i}\right.$. $\left.Q_{2}\right) \bar{D}_{1}$. in the superpotential. Moreover we focus on production via the more numerous $D$ mesons. Production via $B$ mesons is also possible and allows to extend the mass reach. We leave this option for future more detailed studies.

For the case at hand, a $D^{ \pm}$meson produced at SHiP could decay into a neutralino and a charged lepton via the diagrams in Fig. 6.1, if the neutralino is light enough. The respective partial 
width for this decay reads

$$
\Gamma\left(D^{ \pm} \rightarrow \widetilde{N}_{1}^{0}+\ell^{ \pm, i}\right)=\frac{9 g_{2}^{2}}{256 \pi}\left(Z_{12}+\tan \theta_{W} Z_{11}\right)^{2} \frac{\left|\lambda_{i 21}^{\prime}\right|^{2}}{m_{\widetilde{f}}^{4}} f_{D}^{2} \frac{m_{D^{ \pm}}^{2}\left(m_{D^{ \pm}}^{2}-m_{\widetilde{N}_{1}^{0}}^{2}-m_{\ell^{i}}^{2}\right)}{\left(m_{c}+m_{d}\right)^{2}}\left|\vec{p}_{\widetilde{N}_{1}^{0}}\right|,
$$

with $\vec{p}_{\widetilde{N}_{1}^{0}}=\lambda^{\frac{1}{2}}\left(m_{D^{ \pm}}^{2}, m_{\widetilde{N}_{1}^{0}}^{2}, m_{\ell^{i}}^{2}\right) /\left(2 m_{D^{ \pm}}\right)$being the 3-momentum of the neutralino in the $D^{ \pm}$ meson's rest frame ${ }^{3}$. For a pure bino-like lightest neutralino, the neutralino mixing matrix elements $Z_{11}=1, Z_{12}=0$, and vice versa for a pure wino lightest neutralino. We have not considered higgsino admixtures, as the corresponding couplings are very small for the first two quark/lepton generations we consider here. $f_{D}$ is the decay constant of the $D$ meson which we set to $207 \mathrm{MeV}$ according to [156]. For the sake of simplicity we here assume all sfermion masses to have a common value $m_{\tilde{f}}$. Comparing this partial width to the total width of the charged D-meson, $\Gamma_{\text {tot }}^{D^{ \pm}}=6.33 \times 10^{-13} \mathrm{GeV}$ [156], one can find the branching ratio $\operatorname{Br}\left(D^{ \pm} \rightarrow \widetilde{N}_{1}^{0} \ell^{ \pm, i}\right)$ for the above decay of interest, which can range between $10^{-10}$ and $10^{-2}$ for the considered range of parameters shown in Fig. 6.2.

The same R-parity violating operator yields a coupling which could induce subsequent decays $\widetilde{N}_{1}^{0} \rightarrow \overline{K_{S}^{0}} \nu^{i}, \overline{K_{L}^{0}} \nu^{i}, K_{S}^{0} \overline{\nu^{i}}, K_{L}^{0} \overline{\nu^{i}}$, which all have equal decay probability in the limit of negligible CPviolating effects. Decays into charged Kaons exist in addition if the operator $\Delta W^{\prime}=\lambda_{i 12}^{\prime}\left(L_{i} \cdot Q_{1}\right) \bar{D}_{2}$ (note the different order of the indices) is non-vanishing. The diagrams are similar to those shown in Fig. 6.1. The corresponding decay widths for the lightest neutralino are then as follows

$$
\begin{gathered}
\Gamma\left(\widetilde{N}_{1}^{0} \rightarrow K_{S / L}^{0}+\nu^{i}\right)=\frac{g_{2}^{2}}{512 \pi}\left(3 Z_{12}-\tan \theta_{W} Z_{11}\right)^{2} \frac{\left|\lambda_{i 21}^{\prime}\right|^{2}}{m_{\widetilde{f}}^{4}} f_{K}^{2} \frac{m_{K_{0}}^{4}\left(m_{\widetilde{N}_{1}^{0}}^{2}-m_{K^{0}}^{2}\right)}{m_{\widetilde{N}_{1}^{0}}^{2}\left(m_{s}+m_{d}\right)^{2}}\left|\vec{p}_{\nu^{i}}\right|, \\
\Gamma\left(\widetilde{N}_{1}^{0} \rightarrow K^{\mp}+\ell^{ \pm, i}\right)=\frac{9 g_{2}^{2}}{256 \pi}\left(Z_{12}+\tan \theta_{W} Z_{11}\right)^{2} \frac{\left|\lambda_{i 12}^{\prime}\right|^{2}}{m_{\widetilde{f}}^{4}} f_{K}^{2} \frac{m_{K^{+}}^{4}\left(m_{\widetilde{N}_{1}^{0}}^{2}-m_{K^{+}}^{2}-m_{\ell^{i}}^{2}\right)}{m_{\widetilde{N}_{1}^{0}}^{2}\left(m_{s}+m_{u}\right)^{2}}\left|\vec{p}_{\ell^{i}}\right|,
\end{gathered}
$$

with $\vec{p}_{\nu^{i}}=\left(m_{\widetilde{N}_{1}^{0}}^{2}-m_{K_{0}}^{2}\right) /\left(2 m_{\widetilde{N}_{1}^{0}}\right), \vec{p}_{\ell^{i}}=\lambda^{\frac{1}{2}}\left(m_{K^{+}}^{2}, m_{\widetilde{N}_{1}^{0}}^{2}, m_{\ell^{i}}^{2}\right) /\left(2 m_{\widetilde{N}_{1}^{0}}\right)$ and the decay constant of the Kaon $f_{K}$ with value $156 \mathrm{MeV}$ [156]. Depending on the size of the supersymmetric parameters, the resulting proper decay length of the neutralino can range from $\mathcal{O}(\mathrm{mm})$ for $\lambda_{i 21}^{\prime} / m_{\widetilde{f}}^{2}=10^{-5} \mathrm{GeV}^{-2}$ up to $\mathcal{O}(1000 \mathrm{~km})$, for $\lambda_{i 21}^{\prime} / m_{\widetilde{f}}^{2}=10^{-9} \mathrm{GeV}^{-2}$.

To estimate the kinematic properties of the neutralino, we use Pythia 8.1.7.5 [286]. We generate proton-proton-collisions in the lab frame by setting $E\left(p_{1}\right)=400 \mathrm{GeV}$ and $E\left(p_{2}\right)=m_{p}$, the proton mass. Since we are here only interested in the properties of $D^{ \pm}$mesons, we exclusively generate HardQCD : hardccbar matrix elements, which include the partonic interactions $q \bar{q}, g g \rightarrow c \bar{c}$. We find that $1 c \bar{c}$ event on average creates $0.53 D^{ \pm}$mesons. We demand exclusive decays $D^{ \pm} \rightarrow$ $\widetilde{N}_{1}^{0} \ell^{ \pm, i}, \widetilde{N}_{1}^{0} \rightarrow K^{\mp} \ell^{ \pm, i}$ to increase the Monte Carlo statistics. The lifetime of the $D^{ \pm}$is kept fixed at the experimental value, whereas we set the lifetime of the neutralino to the numerical value calculated from Eqs. (6.2.10) and (6.2.11).

We are interested in the probability that a given neutralino candidate decays within the detector at SHiP. We assume the distance target-detector to be $L_{t \rightarrow d}=68.8 \mathrm{~m}$, the total length of the detector to be $L_{d}=55 \mathrm{~m}$ and an elliptic face with semiaxes $5 \mathrm{~m}$ and $2.5 \mathrm{~m}$. For each neutralino candidate, we determine the velocity $\beta_{i}^{z}$ of the neutralino in the beam direction, the boost factor $\gamma_{i}$,

\footnotetext{
${ }^{3} \lambda^{\frac{1}{2}}(a, b, c) \equiv \sqrt{a^{2}+b^{2}+c^{2}-2 a b-2 a c-2 b c}$

${ }^{4}$ Since the kinematics of the neutralino decay products are not important, we use the same single neutralino decay mode for all scenarios and just change the total width formula according to the scenario.
} 
which relates the neutralino-rest frame to the lab frame, and the angle $\theta_{i}$ between the neutralino's momentum and the beam axis. The average values of $\bar{\gamma} \approx 30$ and $\bar{\theta} \approx 60 \mathrm{mrad}$ show that their consideration is important; the boost significantly increases the observed decay length in the lab frame and the angles are such that the neutralinos can easily leave the detector in radial direction before they decay, as it only covers polar angles in the range $25 \mathrm{mrad}$ to $100 \mathrm{mrad}$. Using this information, we can find the probability for each such candidate to have a decay vertex with coordinates $z_{i} \in\left[L_{t \rightarrow d}, L_{t \rightarrow d}+L_{d}\right]$ and $\rho_{i} \in\left[0, R_{d\left(\phi_{\widetilde{N}_{1}}\right.}\right]$. From averaging the probabilities of 5,000 individual Monte Carlo Events one finds an estimate for the overall fraction of neutralinos that decay within the detector, $r_{\widetilde{N}}^{\text {detector }}$, with statistical uncertainty of less than $5 \%$. From [35] we expect $\approx 9 \times 10^{16}$ produced $c \bar{c}$ pairs, which according to our Pythia study corresponds to roughly $N_{D^{ \pm}}^{\text {produced }}=5 \times 10^{16}$ produced $D^{ \pm}$mesons in 5 years of operation. Using the results from above, we find the expected number of observable neutralino decays to be

$$
N_{\widetilde{N}}^{\text {observed }}=N_{D^{ \pm}}^{\text {produced }} \times \operatorname{Br}\left(D^{ \pm} \rightarrow \widetilde{N}_{1}^{0} \ell^{ \pm, i}\right) \times r_{\widetilde{N}}^{\text {detector }} .
$$

The resulting event yields for different combinations of $m_{\widetilde{N}_{1}^{0}}$ and $\lambda^{\prime} / m_{\widetilde{f}}^{2}$ can be found in Fig. 6.2, assuming the neutralino to be a pure bino.

\subsubsection{Comparison with Previous Bounds}

The best bounds we are currently aware of are from [859]. These bounds are at the $2 \sigma$ level

$$
\begin{aligned}
\lambda_{121}^{\prime}<0.043 \frac{m_{\tilde{d}_{R}}}{100 \mathrm{GeV}}, & \lambda_{221}^{\prime}<0.18 \frac{m_{\tilde{s}_{R}}}{100 \mathrm{GeV}}, \\
\lambda_{112}^{\prime}<0.021 \frac{m_{\tilde{s}_{R}}}{100 \mathrm{GeV}}, & \lambda_{212}^{\prime}<0.059 \frac{m_{\tilde{s}_{R}}}{100 \mathrm{GeV}} .
\end{aligned}
$$

As can be seen, these bounds scale differently, than our event rates at SHiP. We have thus inserted into Fig. 6.2 the bounds for fixed sfermion mass. To the left of the line is the new regime which can be explored by SHiP.

\subsubsection{Concluding remarks}

Using the channels ${ }^{5}$

$$
\begin{aligned}
& p p \rightarrow D+X \rightarrow \widetilde{N}_{1}^{0}+X, \widetilde{N}_{1}^{0} \stackrel{\text { long lived }}{\longrightarrow} K_{S / L}^{0}+\stackrel{(-)}{\nu}^{\circ} \\
& p p \rightarrow D+X \rightarrow \widetilde{N}_{1}^{0}+X, \widetilde{N}_{1}^{0} \stackrel{\text { long lived }}{\longrightarrow} K^{ \pm}+\ell^{\mp}
\end{aligned}
$$

and similar channels for $B$ meson decays, allows SHIP to significantly extend the discovery reach for light neutralinos in a scenario with R-parity violation. This direct probe is complementary to SUSY searches at the LHC and allows to (indirectly) probe sfermions in the region of a few 10s of $\mathrm{TeV}$.

\footnotetext{
${ }^{5}$ Similar to the photon channels discussed in Chapter 5 the first, neutral channel is quite challenging experimentally. We note however, that it probes different couplings and that the experimental options are being investigated.
} 

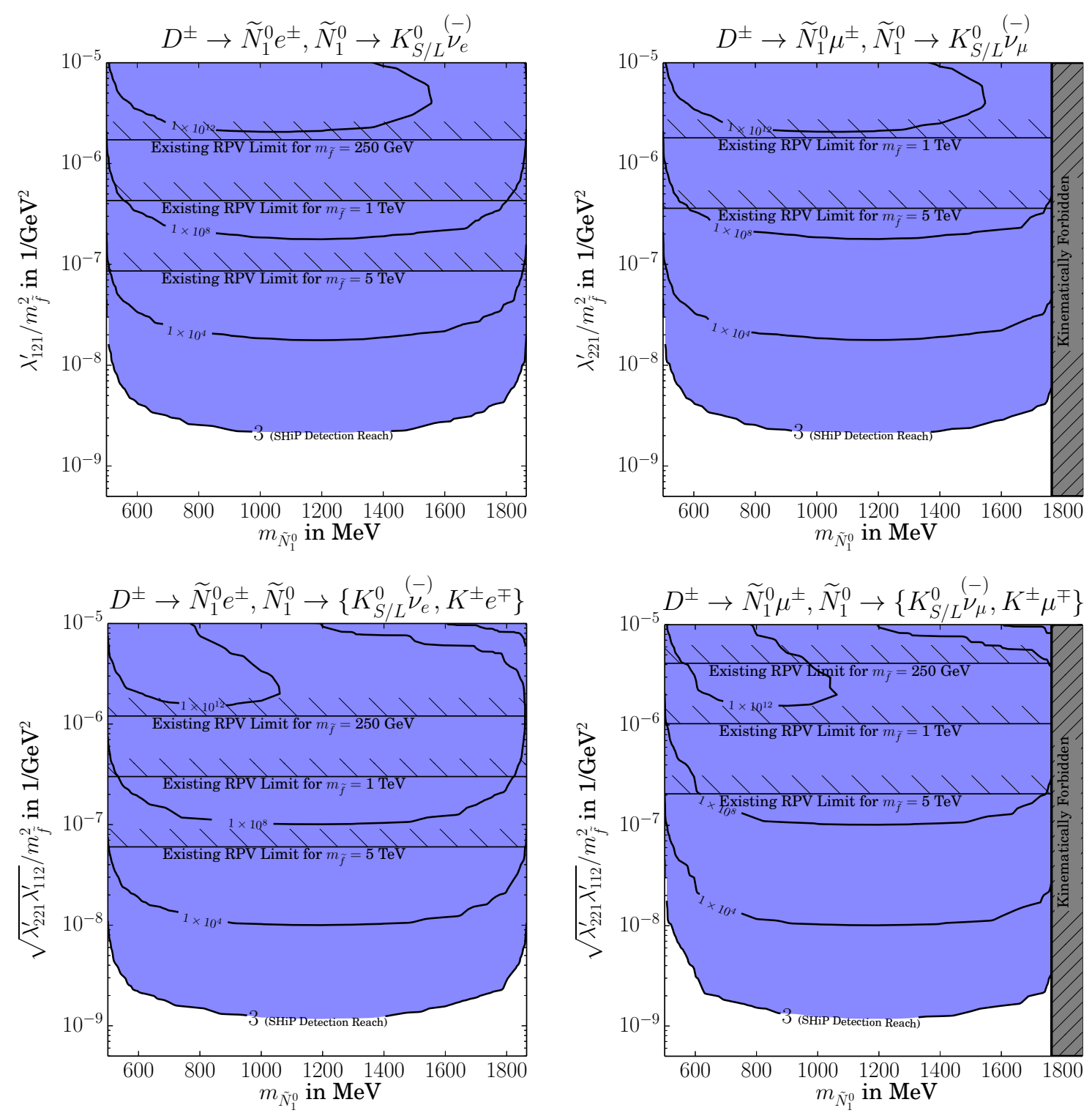

Figure 6.2: Expected event yields after a runtime of 5 years for RPV-produced neutralinos decaying into kaons and leptons with the decay vertex being inside the detector. Shown contour lines are determined by interpolating results of $28 \times 29$ points. The horizontal lines correspond to the existing bounds in Eq. (4.19) for fixed sfermion mass, as labelled. Previously excluded is always above the horizontal line, below is the potentially new region tested by SHiP.

\subsection{Light particles from the SUSY breaking sector}

\subsubsection{Origin of light sgoldstinos}

Supersymmetry, if it exists, must be spontaneously broken. This happens when an auxiliary component of some chiral superfield gains nonzero vacuum expectation value (vev). In a particular model the situation may be more complicated, involving several chiral and/or vector superfields, but for the purposes here it is sufficient to consider the simplest setup. The chiral superfield $\Phi$ is 
called the Goldstone supermultiplet. It includes the auxiliary component $F_{\Phi}$, with non-zero vev

$$
\left\langle F_{\phi}\right\rangle \equiv F
$$

responsible for the SUSY breaking, the Majorana fermion-massless Goldstone particle - called goldstino $\psi$, and the spin-0 component $\phi$ forming scalar $S$ and pseudoscalar $P$ sgoldstinos [860]. Introducing the superspace with Grassmanian coordinates $\theta$ and $\bar{\theta}$, one can write the Goldstone superfield ${ }^{6}$ as

$$
\Phi=\phi+\sqrt{2} \theta \psi+F_{\phi} \theta \theta .
$$

If supersymmetry is promoted to a local symmetry, supergravity, the gravitino $\tilde{G}_{\mu}$ (spin-3/2 superpartner of graviton) acquires a mass $m_{\tilde{G}}$ as a result of the super Higgs mechanism [862] and the goldstino disappears from the spectrum becoming the longitudinal component of the gravitino,

$$
\tilde{G}_{\mu} \rightarrow \tilde{G}_{\mu}+\frac{i}{m_{\tilde{G}}} \sqrt{\frac{2}{3}} \partial_{\mu} \psi
$$

The gravitino mass is then proportional to the supersymmetry breaking parameter,

$$
m_{\tilde{G}}=\sqrt{\frac{8 \pi}{3}} \frac{F}{M_{\mathrm{Pl}}} .
$$

In many simple cases, like O'Raifeartaigh and Fayet-Iliopolous models, scalar and pseudoscalar sgoldstinos

$$
S \equiv \frac{1}{\sqrt{2}}\left(\phi+\phi^{\dagger}\right), \quad P \equiv \frac{1}{i \sqrt{2}}\left(\phi-\phi^{\dagger}\right)
$$

remain massless at tree level. Higher order corrections to the scalar effective potential contribute to their masses. Their values are largely model dependent, and we keep them as free parameters $m_{S}$ and $m_{P}$ in what follows. For example they can be of the order of the gravitino mass [863] or much heavier. For the SHiP experiment the interesting mass range is $m_{S(P)} \sim 1 \mathrm{MeV}-10 \mathrm{GeV}$.

\subsubsection{Sgoldstino couplings and phenomenology}

To the leading order in $1 / F$, the goldstino $\psi$ couples to all the fields through the GoldbergerTreiman formula

$$
\mathcal{L}_{\psi} \propto \frac{1}{F} J_{\text {SUSY }}^{\mu} \partial_{\mu} \psi
$$

where $J_{\text {SUSY }}^{\mu}$ is the supercurrent. Using the transformation (6.3.3) induces interactions between the gravitino and other fields suppressed not by the inversed Planck mass, but by the inverse SUSYviolating parameter $1 / F$. Therefore, for low scale of SUSY breaking $\Lambda_{S U S Y} \sim \sqrt{F}$, the gravitino couples to other fields mostly through its longitudinal component, i.e. the goldstino.

The effective interactions of the sgoldstino can be obtained by making use of a supersymmetric transformation of (6.3.4). Alternatively, following Ref. [864], one can use the spurion technique for the Goldstone superfield $\Phi$ to derive its coupling to the SM particles and their superpartners. ${ }^{7}$

\footnotetext{
${ }^{6}$ In any case a chiral superfield must participate in the supersymmetry breaking [861], and if many, $\Phi$ is replaced by a linear combination of the chiral superfields, which plays the same role that we attribute to the Goldstone supermultiplet. In particular, the fermionic component of this linear combination is massless, that is the goldstino, and the scalar components are sgoldstinos, if no strong mixing exists in the spin-0 sector.

${ }^{7}$ This procedure is transparent and provides the sgoldstino interaction terms to the leading order in $1 / F$ valid in many classes of supersymmetric models. In other cases, e.g. [865], the relation between sgoldstino couplings and the soft supersymmetry breaking MSSM parameters may be more complicated as compared to what we obtain following this procedure.
} 
Namely, the effective interaction

$$
\frac{M_{\lambda}}{F} \int d \theta^{2} \Phi W^{\alpha} W_{\alpha}+\text { h.c. }
$$

yields SUSY violating gaugino mass terms $M_{\lambda} \lambda \lambda+$ h.c., when the auxiliary component of $\Phi(6.3 .2)$ is substituted by a nonzero vev (6.3.1). For sgoldstinos it provides couplings to the SM gauge bosons. In particular, the interaction terms with photons and gluons read ${ }^{8}$

$$
\begin{aligned}
\mathcal{L}_{e f f} & =-\frac{1}{2 \sqrt{2}} \frac{M_{\gamma \gamma}}{F} S F^{\mu \nu} F_{\mu \nu}+\frac{1}{4 \sqrt{2}} \frac{M_{\gamma \gamma}}{F} P \epsilon^{\mu \nu \rho \sigma} F_{\mu \nu} F_{\rho \sigma} \\
& -\frac{1}{2 \sqrt{2}} \frac{M_{3}}{F} S G^{\mu \nu a} G_{\mu \nu}^{a}+\frac{1}{4 \sqrt{2}} \frac{M_{3}}{F} P \epsilon^{\mu \nu \rho \sigma} G_{\mu \nu}^{\alpha} G_{\rho \sigma}^{\alpha}
\end{aligned}
$$

where $F_{\mu \nu}$ is the electromagnetic field strength, $G_{\mu \nu}^{a}, a=1, \ldots, 8$ refers to gluons, and $M_{\gamma \gamma}=$ $M_{1} \sin ^{2} \theta_{W}+M_{2} \cos ^{2} \theta_{W}$ with $M_{1}$ and $M_{2}$ denoting $U(1)_{Y}$ and $S U(2)_{W}$ gaugino SUSY breaking mass terms, and $M_{3}$ stands for the gluino mass.

Similarly, the effective interaction

$$
\int d \theta^{2}\left(\frac{A_{i j}^{u}}{F} \int \Phi H_{u} Q_{i} U_{j}^{C}+\frac{A_{i j}^{d}}{F} \int \Phi H_{d} Q_{i} D_{j}^{C}+\frac{A_{i j}^{l}}{F} \int \Phi H_{d} L_{i} E_{j}^{C}\right)+\text { h.c. }
$$

leads for (6.3.2), (6.3.1) to the soft supersymmetry breaking trilinear terms. After electroweak symmetry breaking it provides with sgoldstino Yukawa-like couplings to the SM fermions,

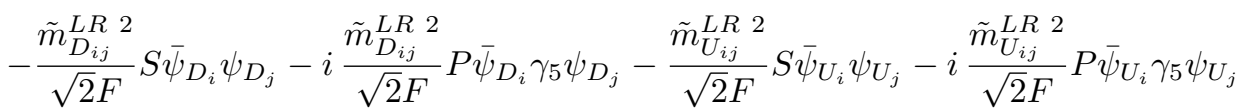

$$
\begin{aligned}
& -\frac{\tilde{m}_{L_{i j}}^{L R} 2}{\sqrt{2} F} S \bar{\psi}_{L_{i}} \psi_{L_{j}}-i \frac{\tilde{m}_{L_{i j}}^{L R} 2}{\sqrt{2} F} P \bar{\psi}_{L_{i}} \gamma_{5} \psi_{L_{j}}
\end{aligned}
$$

where $\psi_{L_{i}}$ are leptons, $\psi_{U_{i}}$ and $\psi_{D_{i}}$, are up- and down-type quarks and $i$ runs over generations; the SUSY violating entries in the slepton, up-squark and down-squark left-right squared mass matrices are introduced as follows

$$
\tilde{m}_{L_{i j}}^{L R} 2 \equiv A_{i j}^{l} v_{d}, \quad \tilde{m}_{D_{i j}}^{L R 2} \equiv A_{i j}^{d} v_{d}, \quad \tilde{m}_{U_{i j}}^{L R 2} \equiv A_{i j}^{u} v_{u}
$$

where $v_{d}$ and $v_{u}$ are vevs of the Brout-Englert-Higgs fields $H_{d}$ and $H_{u}$, respectively.

While the gravitino (goldstino) is $R$-odd, sgoldstinos are $R$-even, and hence (if kinematically allowed) may be singly produced in collisions and decays of the SM particles and may decay into pairs of the SM particles. Interaction Lagrangians (6.3.5)-(6.3.9) ensure that the rates of all these processes are proportional to $1 / F^{2}$. This defines the sgoldstino phenomenology, whose main attraction is the unique possibility to determine the scale of supersymmetry breaking $\Lambda_{\mathrm{SUSY}} \sim \sqrt{F}$ in the whole theory. The sgoldstino couplings are proportional to the SUSY breaking MSSM soft terms and inversely proportional to $F$. Thus the higher the SM superpartner scale and the lower the scale of SUSY breaking in the whole theory, the larger the sgoldstino couplings to the SM particles. Among the models with relatively low scale of SUSY breaking are models with no-scale supergravity [863, 868] and models with gauge mediation of supersymmetry breaking [869, 870].

The list of processes where light sgoldstinos may show up is long, since both flavor conserving and flavor violating couplings are present in the sgoldstino interaction sector. The latter

\footnotetext{
${ }^{8}$ See Refs. [866, 867] for complete set of interaction terms including all the MSSM soft parameters, and goldstinosgoldstino leading order couplings.
} 
(6.3.8),(6.3.9) are proportional to the off-diagonal SUSY-violating entries in the slepton and squark mass matrices, which are fixed by the pattern of the trilinear soft terms (6.3.10). The off-diagonal mass terms and soft trilinear couplings give rise to the FCNC processes through the squark and slepton virtual contribution to the SM rare processes. Their absence places limits on these couplings, and hence the numerators in the sgoldstino coupling constants (6.3.8),(6.3.9) (which are the ratios of the MSSM soft parameters and SUSY breaking parameter $F$ ) are strongly constrained. As to the flavor conserving sgoldstino couplings to vector bosons, e.g. (6.3.5),(6.3.6), the largest are those to the particles whose superpartners are the heaviest. Flavor conserving couplings to fermions are proportional to the diagonal trilinear soft parameters (6.3.10). For the universal (or close to universal) conditions for the soft SUSY breaking parameters, the trilinear terms are proportional to the SM fermion Yukawas $Y_{i}^{l(d, u)}$, and hence

$$
\tilde{m}_{L_{i i}}^{L R} 2=A^{l} Y_{i}^{l} v_{d}=A^{l} m_{i}^{l}, \quad \tilde{m}_{D_{i i}}^{L R} 2=A^{d} Y_{i}^{d} v_{d}=A^{d} m_{i}^{d}, \quad \tilde{m}_{U_{i i}}^{L R} 2=A^{u} Y_{i}^{u} v_{u}=A^{u} m_{i}^{u},
$$

where $m_{i}^{l(d, u)}$ denotes the corresponding fermion mass. If the trilinear parameters are of the same order,

$$
A^{l} \sim A^{d} \sim A^{u},
$$

the strongest are couplings to the heaviest fermions.

The sgoldstino phenomenology depends on the sgoldstino mass scale and is governed by the pattern of the MSSM soft terms. It has been thoroughly studied in the literature. Very light sgoldstinos can play important roles in astrophysics and cosmology, and the absence of a visible imprint left by the sgoldstino places strong limits on its couplings to the SM fields [866, 871]. At the same time, relic sgoldstinos of $7 \mathrm{keV}$ mass have been suggested [872] to explain the excess of $3.5 \mathrm{keV} X$-rays [297, 298] observed by the orbital telescopes.

Heavier sgoldstinos can contribute to rare processes including flavor violation [866, 873]. They can appear in lepton, meson and baryon decays [866], as well as $Z$-boson decays [874]. Sgoldstino pair production in $B$-meson decays [875] has been recently searched for at the LHCb experiment [876]. Note that the HyperCP anomalous events [877] in $\Sigma^{+} \rightarrow p \mu^{+} \mu^{-}$with dimuon invariant mass of $214.3 \pm 0.5 \mathrm{MeV}$ can be explained as pseudoscalar sgoldstino production in the $\Sigma$-hyperon decay through the flavor violating coupling, with subsequent sgoldstino decay into $\mu^{+} \mu^{-}$. The suggested tests of the model in kaon [878] and $B$-mesons [879] physics have been performed with negative results [880, 881], closing a part of the model parameter space.

Sgoldstinos can be directly produced in high energy collision processes. Sgoldstino phenomenology at $e^{+} e^{-}$-colliders has been addressed in $[860,874,882]$. The DELPHI Collaboration has performed searches for a sgoldstino signal in the collected data revealing no hints [883]. Signatures of massive sgoldstinos at the TeVatron have been considered in [884-886]. Heavy sgoldstinos are produced mostly through gluon fusion [886]. Prospects of sgoldstino searches at LHC have been discussed in [887, 888], including tests of possible sgoldstino mixing with the Higgs boson [889] and of flavor violatings interaction with top-quark [890].

\subsubsection{Sgoldstinos at SHiP}

If kinematically allowed, sgoldstinos may be directly produced by $400 \mathrm{GeV}$ protons scattering on a target and indirectly through the decays of mesons and baryons emerging from proton scattering and the subsequent hadron shower in the dump. The direct sgoldstino production here is naturally dominated by the gluon fusion $g g \rightarrow S, g g \rightarrow P$. The coupling to gluons is expected to be the strongest among the flavor conserving, since in many particular supersymmetric extensions of the $\mathrm{SM}$, the gluino is naturally the heaviest gaugino (as, e.g. in case of universal boundary conditions for the soft terms, in models with gauge mediation of SUSY breaking, etc). The production cross 


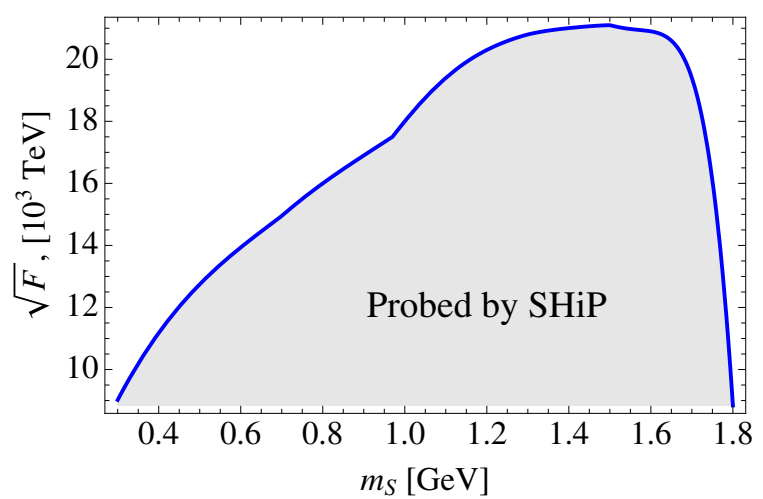

Figure 6.3: SUSY breaking scale $\sqrt{F}$ probed by SHiP as a function of the sgoldstino mass $m_{S}<$ $m_{D}$ in the lepton flavour violating case, Eq. (6.3.8). The down-squark left-right mass matrix element was chosen $\tilde{m}_{D_{12}}^{L R 2}=(100 \mathrm{GeV})^{2}$ and the gaugino mass $M_{3}=3 \mathrm{TeV}$. The result scales with $\tilde{m}_{D_{12}}^{L R 2}$ and $M_{3}$ as shown in Eq. (6.3.13).

section can be obtained by simple rescaling of the known results for the fusion of the SM Higgs boson of the same mass, sgoldstino coupling to gluons is proportional to $M_{3} / F(6.3 .6)$. The results for scalar and pseudoscalar sgoldstinos of the same mass coincide.

Indirect sgoldstino production may go through the flavor conserving and flavor violating couplings, constrained by the absence of rare processes and superpartners at the LHC. For the flavor conserving case the dominant channel for $\mathcal{O}(1) \mathrm{GeV}$ mass sgoldstino is $B \rightarrow K S(P)$, the same one-loop process as would dominate the SM Higgs boson production of the same mass. For lighter sgoldstinos $K \rightarrow \pi S(P)$ dominates. In the flavor violating case the result depends on the chosen pattern of the flavor violating model parameters. In particular, sgoldstinos of (sub) GeV mass may be produced in the two body $D$-meson decays like $D_{s} \rightarrow K+S(P), D^{0} \rightarrow \pi^{0}+S(P)$, and $D^{ \pm} \rightarrow \pi^{ \pm}+S(P)$.

Sgoldstinos decay into the SM particles mostly through the flavor conserving couplings. Here the final states are

$$
S(P) \rightarrow \gamma \gamma, \quad \mu^{+} \mu^{-}, \quad \pi^{+} \pi^{-}, \quad \pi^{0} \pi^{0}, \quad e^{+} e^{-}, \ldots
$$

with hadronic channels (if open) presumably dominant (provided by heavy gluino mass and large number of gluons). Indeed, for the ratio of sgoldstino decay rates into photons and gluons one obtains from eqs. (6.3.5),(6.3.6),

$$
\frac{\Gamma(S(P) \rightarrow g g)}{\Gamma(S(P) \rightarrow \gamma \gamma)}=\left(N_{c}^{2}-1\right) \frac{M_{3}^{2}}{M_{\gamma \gamma}^{2}} .
$$

For the leptonic channels one finds with the universal diagonal trilinear terms (6.3.11)

$$
\frac{\Gamma\left(S(P) \rightarrow e^{+} e^{-}\right)}{\Gamma\left(S(P) \rightarrow \mu^{+} \mu^{-}\right)}=\frac{m_{e}^{2}}{m_{\mu}^{2}} \ll 1,
$$

therefore the heavy fermions dominate. For the rates to fermions and gluons one obtains $[860,866]$

$$
\frac{\Gamma\left(S(P) \rightarrow \mu^{+} \mu^{-}\right)}{\Gamma(S(P) \rightarrow g g)}=\frac{A^{l 2}}{4 M_{3}^{2}} \frac{m_{\mu}^{2}}{m_{S(P)}^{2}} .
$$

If the sgoldstino decay rates are saturated by hadronic channels, sgoldstino lifetimes may be esti- 
mated as (for sgoldstino masses $2 m_{\pi}<m_{S}<2 m_{K}$ the decay $S \rightarrow \pi^{+} \pi^{-}$dominates)

$$
\tau_{S(P)}=2 \times 10^{-4} \mathrm{~s} \times\left(\frac{\sqrt{F}}{10000 \mathrm{TeV}}\right)^{4}\left(\frac{3 \mathrm{TeV}}{M_{3}}\right)^{2}\left(\frac{1 \mathrm{GeV}}{m_{S(P)}}\right)^{3} \quad 2 m_{\pi}<m_{S(P)}<2 m_{K}
$$

(for $m_{S}>2 m_{K}$ the lifetime becomes shorter by $40 \%$ due to additional channels $S(P) \rightarrow K^{0} \bar{K}^{0}$ and $\left.S(P) \rightarrow K^{+} K^{-}\right)$. Here the difference between scalar and pseudoscalar sgoldstino is only due to their masses, which are usually different. The same conclusion is true for all the processes, if parity is violated by sgoldstino couplings, so both scalar and pseudoscalar sgoldstinos couple through the parity odd and parity even interaction terms. If parity is conserved by sgoldstino couplings [891], the phenomenology of scalar and pseudoscalar sgoldstinos, especially those involving meson decays, differ.

For the reference values of the model parameters adopted in Eq. (6.3.12) sgoldstinos produced by $400 \mathrm{GeV}$ protons on target cover a distance of several kilometers before they decay. Hence the corresponding signature - a couple of charged particles emerging at a single point with invariant mass equal to that of the sgoldstino - may be searched for in the SHiP detector. For a $60 \mathrm{~m}$ length detector with $2 \times 10^{20}$ PoTs one expects the following number of charged pion pairs appearing from the light sgoldstino decays,

$$
N_{\pi^{+} \pi^{-}} \simeq 4 \times 10^{4}\left(\frac{\tilde{m}_{D_{i i}}^{L R} 2}{(100 \mathrm{GeV})^{2}}\right)^{2}\left(\frac{10000 \mathrm{TeV}}{\sqrt{F}}\right)^{8}\left(\frac{M_{\lambda_{g}}}{3 \mathrm{TeV}}\right)^{2}\left(\frac{m_{S, P}}{1 \mathrm{GeV}}\right)^{4}, \quad 2 m_{\pi}<m_{S(P)}<2 m_{K}
$$

Here we take into account the Lorentz $\gamma$-factor for sgoldstino, produced in decays of $6.8 \times 10^{17}$ $D$-mesons (see Appendix A) via lepton flavour violating coupling $\tilde{m}_{D_{12}}^{L R 2}$. The results of the detailed MC simulations, taking into account the acceptance of the detector, are presented in Fig. 6.3. The scaling of the sensitivity plot with $\tilde{m}_{D_{12}}^{L R 2}$ and $M_{3}$ remains the same as in Eq. (6.3.13) also for $m_{S}>2 m_{K}$. We note that the reference numbers in (6.3.13) are all in agreement with present experimental limits on MSSM parameters.

\subsubsection{Concluding remarks}

Sgoldstinos are a natural consequence of F-term SUSY breaking and their typical interaction strength are directly linked to the scale at which SUSY is broken. Phenomenologically they share features with scalars from Higgs portal models and pseudoscalar axion-like particles (ALPs) and similar search strategies can be applied (cf. Chapters 3 and 5). Promising search channels at SHiP include,

$$
\begin{aligned}
& p p \rightarrow S \text { (gluon fusion), } \mathrm{S} \stackrel{\text { long lived }}{\longrightarrow} \ell^{+} \ell^{-}, \pi^{+} \pi^{-}, \pi^{0} \pi^{0} \\
& p p \rightarrow D+X \rightarrow S+X^{\prime}, S \stackrel{\text { long lived }}{\longrightarrow} \ell^{+} \ell^{-}, \pi^{+} \pi^{-}, \pi^{0} \pi^{0},
\end{aligned}
$$

and similar for the pseudoscalar $P$.

\subsection{Light Dirac gauginos}

\subsubsection{Origins of Pseudo-Dirac fermions}

A pseudo-Dirac fermion, where an originally Dirac fermion is split into two Majorana components, is an interesting candidate for dark matter, since it allows co-annihilation between the eigenstates but would appear as a Majorana particle for the purposes of direct and indirect detection experiments 
- provided that the mass splitting is larger than the typical kinetic energy of the particles, of order $100 \mathrm{keV}$, but less than the energy of the thermal bath during freeze-out, typically a few GeV.

Pseudo-Dirac fermions can occur quite naturally in supersymmetric theories. For example in the MSSM we could have a Higgsino which is pseudo-Dirac. However this is excluded for consideration at the masses $\lesssim 20 \mathrm{GeV}$ which are of interest to SHiP as it would be excluded by the Z-width measurement.

A better candidate is a Dirac gaugino [892-896] which is an extension of the MSSM which is well motivated by the existence of an (approximate) R-symmetry.

If the Dirac particle is predominantly bino-like then it will have weak enough interactions to generate sufficient dark matter, and will also evade the Z-width constraint for models accessible by SHiP. There are typically two classes of Dirac gaugino models: those which preserve an R-symmetry [897-905] and those which allow a small breaking [906-913]. In the latter case, a minimal extension of the Higgs sector is allowed, and the neutralino is split into (at least) six majorana fermions. The splitting between the bino eigenstates induced by electroweak symmetry breaking is [894]

$$
\Delta m \simeq-\sin 2 \beta \frac{M_{Z}^{2} s_{W}^{2}}{\mu}\left(\frac{2 \lambda_{S}^{2}-g_{Y}^{2}}{g_{Y}^{2}}\right) .
$$

Hence if $\mu \sim \mathrm{TeV}$ we have a mass splitting of the order of $2 \mathrm{GeV}$.

Typically even in models that do not have an explicit R-symmetry breaking on the field theoretical level such a breaking is naturally induced by gravitational effects. For example a splitting $\Delta m \gtrsim 100 \mathrm{keV}$, required to have a genuine pseudo-Dirac candidate as regards direct-detection experiments, is induced through anomaly mediation if the gravitino mass $m_{\tilde{G}}$ is given by

$$
m_{\tilde{G}} \simeq \frac{16 \pi^{2}}{g_{Y}^{2}} \Delta m \simeq 0.1 \mathrm{GeV} \times\left(\frac{\Delta m}{100 \mathrm{keV}}\right) .
$$

There are several possibilities how pseudo-Dirac particles could arise in supersymmetric models with Dirac gauginos. Due to the variety of model-building possibilities we shall adopt in the following an effective action approach along the lines of [895] and leave detailed investigation of specific examples to future work.

\subsubsection{Effective model, phenomenological features}

For models of interest to SHiP, the pseudo-Dirac particle must be much lighter than other supersymmetric particles - and also the electroweak gauge bosons. We can therefore integrate them out and parametrise the leading couplings of the intially Dirac fermion $\Psi$ to standard model fermions $f$ as $[895]$

$$
\mathcal{L}_{\text {int }}=\frac{1}{\Lambda^{2}}\left[\bar{\Psi} \gamma^{\mu}\left(c_{L} P_{L}+c_{R} P_{R}\right) \Psi\right]\left[\bar{f} \gamma_{\mu}\left(c_{L}^{f} P_{L}+c_{R}^{f} P_{R}\right) f\right] .
$$

The LHC has already put limits on the strength of these interactions; from the point of view of the LHC the splitting of the Dirac fermion into two components is irrelevant. From [914, 915] we find that for light dark matter,

$$
\frac{c_{L, R}^{u, d}}{\Lambda} \lesssim 900 \mathrm{GeV} .
$$

These are also the important bounds for the production of the particles in SHiP. On the other hand, there is no reason that the couplings to up and down quarks must be the important couplings for the dark matter relic abundance. In [895] an approximate formula for the relic density was given 


$$
\frac{\Lambda}{C_{D M}} \simeq 0.8 \mathrm{TeV}\left(\frac{\Omega h^{2}}{0.11}\right)^{1 / 4}\left(\frac{m_{1}}{100 \mathrm{GeV}}\right)^{1 / 2} \exp \left[-6 \frac{\Delta m}{m}\right]
$$

Here

$$
C_{D M}^{4} \equiv \frac{1}{4}\left|c_{L}+c_{R}\right|^{2} \sum_{f}\left(\left|c_{L}^{f}\right|^{2}+\left|c_{R}^{f}\right|^{2}\right)
$$

where the sum is over all fermions relativistic at freezeout. Clearly for a light dark matter particle, given the above collider bounds, either we should expect to have an overabundance of dark matter or the annihilations should proceed to leptons rather than quarks. Both cases are possible. In the first case a notional excess of dark matter is acceptable because a non-standard thermal history of the universe - such as with a late-decaying particle that injects additional entropy and dilutes the density to the observed value, e.g. in the "non-thermal WIMP miracle" [916] - would then render such a scenario viable.

\subsubsection{Origin of the effective model}

Let us now consider the origin of the couplings present in the effective model in the context of TeVscale SUSY Dirac gaugino models (as opposed to Split-SUSY variants, such as [917-920], where the dark matter properties are very different), where they can come through the Z, sfermion and Higgs portals. We shall imagine that our dark matter candidate consists of neutral components from $S U(2)$ singlets $b$ (which include the bino), triplets $w$ (including the wino) and doublets $r$ (such as the higgsinos or R-higgsinos) so that we can write schematically

$$
P_{L} b=L_{s} P_{L} \Psi+\ldots, \quad P_{L} w=L_{t} P_{L} \Psi+\ldots, \quad P_{L} r=L_{r_{i}} P_{L} \Psi+\ldots .
$$

We note that for the purposes of SHiP it is perfectly acceptable to integrate out particles with masses of the order of the $Z$ or the Higgs mass and use the effective description (for LHC this may not always be appropriate).

\subsubsection{Z portal}

Given the above interaction is vector-like and our Dirac particle is neutral, it is natural to consider first the Z-portal for interactions between the dark matter and the visible sector. However, this is strongly constrained by the Z-width; it is not possible to have a light pseudo-Dirac particle coming from an $S U(2)$ doublet such as a Higgsino. On the other hand, if there is some mixing between a bino and Higgsino, then we can write the effective coupling between the Dirac fermion (before splitting the eigenvalues) as

$$
\mathcal{L}_{Z} D M=\frac{e}{2 s_{W} c_{W}} c_{Z} Z_{\mu} \bar{\Psi} \gamma^{\mu} P_{L} \Psi
$$

In the decomposition of our Dirac particle in (6.4.7) we have $c_{Z}=2 \sum_{i} Y_{i} L_{r_{i}}^{2}$. The Z-width then constrains

$$
\left|c_{Z}\right| \lesssim 0.1
$$

For pure Z-portal dark matter with interactions of the above type, [921] found that dark matter would always be overproduced for $m_{\Psi} \lesssim 22 \mathrm{GeV}$ when we satisfy the Z-width constraint. As mentioned above, however, from the perspective of SHiP this merely means that the thermal history of the universe in this case would be non-thermal. 


\subsubsection{Higgs portal}

If the Pseudo-Dirac fermion couples to the Higgs then it annihilate via Higgs exchange, which will determine the pattern of couplings to Standard Model fermions to be proportional to their masses. If the Higgs sector preserves R-symmetry, then we can write the coupling of the Dirac fermion prior to the mass splitting to be

$$
\mathcal{L}_{H i g g s} D M=H \bar{\Psi}\left(c_{H} P_{L}+c_{H}^{*} P_{R}\right) \Psi
$$

Here the couplings can have gauge current and superpotential origin.

The Higgs portal is constrained by the invisible Higgs width, from monojet searches [922] and $t \bar{t} H$ production [923]; the latter gives a bound

$$
\operatorname{Br}(H \rightarrow \text { invisible })<0.4,
$$

which, since the width of the Standard Model Higgs with a mass of $125 \mathrm{GeV}$ is $4.04 \times 10^{-3} \mathrm{GeV}$, translates into a bound (in the limit of the Dirac particle much lighter than the Higgs mass) of

$$
\begin{aligned}
\left|c_{H}\right|^{2} & <8 \pi \times 0.4 / 0.6 \times \frac{\Gamma_{H}}{m_{H}} \\
& <5 \times 10^{-4} .
\end{aligned}
$$

This leads to a massive overproduction of dark matter; for $m_{\Psi}=1 \mathrm{GeV}$ we find

$$
\Omega h^{2} \simeq 2.5 \times 10^{6} \times\left(\frac{10^{-4}}{\left|c_{H}\right|^{2}}\right)
$$

As in the Z-portal case, this could still be interesting if the thermal history of the universe is non-standard. However, the amount of entropy injection required here is much greater.

\subsubsection{Sfermion portal}

The natural possibility to consider is the scalar portal, which corresponds in SUSY theories to squark or slepton exchange. In the Dirac basis, there are direct couplings of the sfermions $\tilde{f}$ to the gauginos of the form

$$
\mathcal{L}_{\text {Scalar DM }}=\tilde{f}_{i}^{*}\left(a_{L, i} \overline{f^{c}} P_{L} \Psi+a_{R, i} \bar{\Psi} P_{R} f\right)+\text { h.c. }
$$

Let us consider an approximately R-symmetric theory. Then there is no mixing between left- and right-handed sfermions, and in the decomposition of our Dirac particle in (6.4.7) we have either $a_{L, i}=0$ or $a_{R, i}=0$. We then have $a_{(L, R), i}=\sqrt{2} g_{Y} Y L_{s}+\sqrt{2} g_{2} T L_{t}$.

The sfermion portal is the most natural way of obtaining a reasonable relic density while evading other constraints: the sleptons can be light (with masses of $\mathcal{O}(100) \mathrm{GeV}$ ), in contrast to the squarks - as claimed in [896], the dark matter could be as light as $10 \mathrm{GeV}$ without being overproduced for a pure bino.

\subsubsection{Detection at SHiP}

\subsubsection{Decays}

One of the essential requirements for an experiment like SHiP is a particle with a macroscopic decay length. If we consider the decays of our pseudo-Dirac particle, we shall assume that the dark matter density is dominated by lepton interactions and therefore pairs of leptons will be produced. The 
decay length is [895]

$$
L\left(\chi_{2} \rightarrow \ell^{+} \ell^{-} \chi_{1}\right) \simeq 250 \mathrm{~m} \times \frac{E_{2}}{m_{2}} \times\left(\frac{\Lambda / C_{D M}}{100 \mathrm{GeV}}\right)^{4}\left(\frac{50 \mathrm{MeV}}{\Delta m}\right)^{5} .
$$

where $\chi_{1}$ and $\chi_{2}$ denote the heavier and lighter components of the pseudo-Dirac fermion, respectively.

Since the decay length is very sensitive to the mass splitting of the states - which then does not affect any of our other observables - it is not possible to place definitive exclusions; but we may make a detection!

\subsubsection{Production}

The production of light fermions coupled to the standard model by the effective operator (6.4.3) is suppressed by the fraction of anti-up and anti-down quarks in the proton at low momenta. Taking for sake of argument $c_{L}=c_{R} \equiv c, c_{u}^{L}=c_{u}^{R}=c_{d}^{L}=c_{d}^{R} \equiv c_{u, d}, \frac{\Lambda^{2}}{c c_{u, d}}=(2 \mathrm{TeV})^{2}$ with equal couplings to up and down quarks, and $m_{\Psi}=1 \mathrm{GeV}$; we find

$$
\sigma(p+p \rightarrow \Psi+\bar{\Psi}) \simeq 2 \times 10^{-6} \mathrm{pb} \times\left(\frac{(2 \mathrm{TeV})^{2}}{\Lambda^{2} /\left(c \times c_{u, d}\right)}\right)^{2} .
$$

This should be compared to the proton cross-section, which at the centre-of-mass energy of $\sqrt{s}=27$ $\mathrm{GeV}$ is $40 \mathrm{mb}$ (cf. [924]). Hence for $2 \times 10^{20}$ protons on target we expect roughly $10^{4}$ raw events. We must then take the geometric factors and decays into account; we do this via a rough estimate with a geometric factor of $8 \times 10^{-4}$, and taking the decay length in the lab frame to be $90 \mathrm{~m}$ for reference, we have about 2 expected events - just enough to make a detection, and with much weaker couplings than the direct LHC bounds. The prospects improve as we decrease the particle mass; we plot in figure 6.4 the estimated optimistic SHiP reach in this approach over a range of masses and couplings, and find good prospects for detection.

\subsubsection{Concluding remarks}

For pseudo-Dirac particles suitable for dark matter models the channel,

$$
p p \rightarrow \chi_{2}+\chi_{1,2}, \chi_{2} \stackrel{\text { long lived }}{\longrightarrow} \chi_{1}+\ell^{+} \ell^{-}
$$

offers significant discovery potential beyond current LHC limits. 


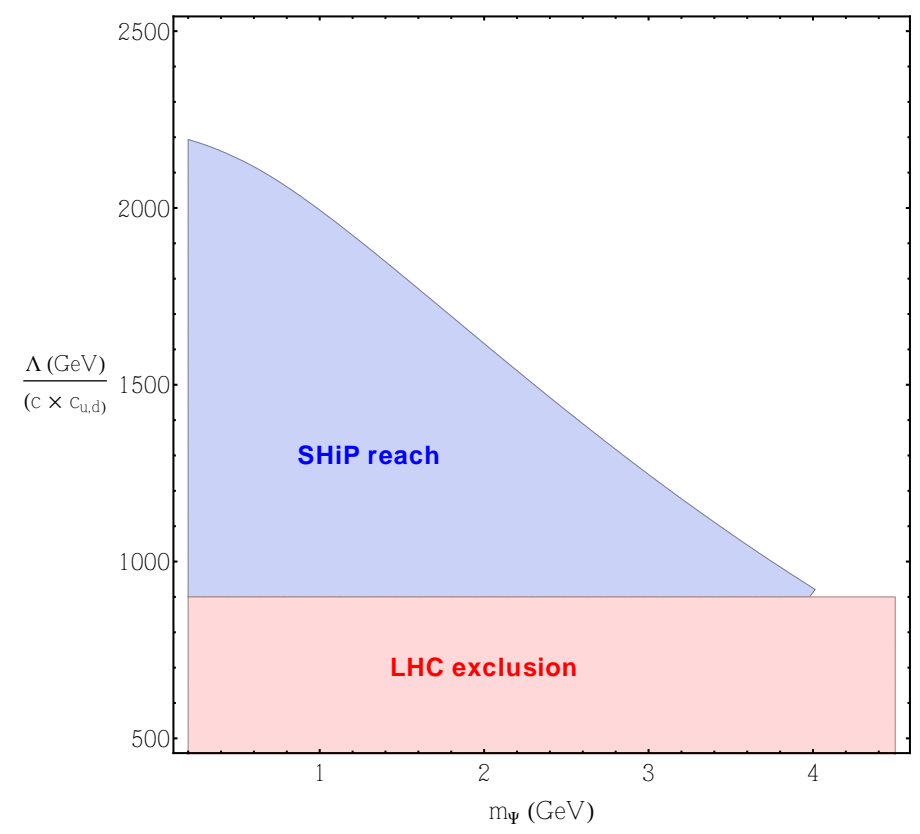

Figure 6.4: Reach of SHiP for a pseudo-Dirac fermion $\Psi$ and compared to existing constraints from LHC.

\subsection{SUSY vector portal I: Hidden Photinos}

In this and the following section we will consider extensions of the MSSM by extra U(1) gauge groups. Here, we will consider the case where the extra U(1) gauge boson is massless (and not accompanied by any hidden matter). In the next section we will then turn to the case where the gauge boson is massive an accompanied by Higgses. As we will discuss below, in the first case the observable signal arises from the fermionic superpartner of the massless gauge boson, the hidden photino, whereas in the second case interesting signals arise from the decay of the U(1) gauge boson into the extra Higgses or extra neutralinos.

\subsubsection{Motivation}

A novel possibility for new light fermionic states coupled to the Standard Model arises in supersymmetric extensions of the Standard Model with additional massless $U(1)$ gauge sectors (cf. also the Chapter 2 on dark vector particles). The gauge bosons of these additional $U(1)$ factors may kinetically mix with hypercharge, while the gauge fermions (hidden photinos) may mix with the bino through both kinetic mixing and mass mixing. If there is light matter charged under these $U(1)$ factors, then the light matter inherits fractional electromagnetic charge and provides the primary means for probing the new sector. However, if there is no light matter charged under the additional $U(1)$ sectors, then the kinetic mixing between abelian gauge bosons is unphysical and the only portal is the kinetic and mass mixing of the photinos [925, 926]. Hidden photinos may be comparable in mass to, or significantly lighter than, MSSM neutralinos and couple through renormalizable couplings with small mixing angles, making them ideal candidates for exploration at a fixed target experiment.

Beyond their phenomenological novelty, hidden photinos have a natural realization in string theory. Realistic string constructions typically involve the compactification of extra dimensions on manifolds with non-trivial topology, which is then reflected by the spectrum of light states 
after compactification. In particular [927, 928], dimensional reduction of Ramond-Ramond (RR) $n$-forms gives rise to massless $U(1)$ fields labeled by the independent cycles of dimension $(n-1)$. For example, dimensional reduction of the RR four-form in Type IIB string theory gives rise to a $U(1)$ gauge group for every 3-cycle of the compactification manifold; the multitude of such cycles in realistic compactifications leads to a potential plethora of RR $U(1)$ 's. There are generically no light states charged under these RR $U(1)$ s, making them ideal sources of hidden photinos.

\subsubsection{Features}

Hidden photinos couple to the MSSM through kinetic and mass mixing with the bino. In a theory with $n$ additional $U(1) \mathrm{s}$, the mass spectrum consists of four mostly-MSSM-like neutralinos $\tilde{N}_{i}$ $(i=1, \ldots 4)$ that are principally admixtures of the bino, wino, and neutral higgsinos and $n$ mostlyphotino neutralinos $\tilde{\gamma}_{a}(a=1, \ldots n)$. These photinos couple to the MSSM through small mixings $\kappa$ that are parametrically of the order of the kinetic mixing $\epsilon$ times the ratio of photino mass to bino mass,

$$
\kappa \sim \epsilon \frac{m_{i}}{M_{1}} .
$$

Photino pairs couple to the Standard Model through the $s$-channel exchange of $Z$ or Higgs bosons and through the $t$-channel exchange of squarks and sleptons. These couplings are suppressed by powers of $\kappa$ as well as possibly small mixings $\eta \sim M_{Z} / \mu, M_{Z} / M_{1,2}$ corresponding to further suppression of photino-higgsino and photino-wino mixing.

If $R$-parity is conserved the lightest photino is stable. Heavier photinos decay into the lightest photino via three-body decays primarily through an off-shell $Z$ boson with lifetime

$$
\tau\left(\tilde{\gamma}_{a} \rightarrow \tilde{\gamma}_{1}+\ell^{+} \ell^{-}\right) \simeq 30 \mathrm{~cm} \times\left(\frac{10^{-2}}{\kappa}\right)^{4}\left(\frac{1}{\eta}\right)^{4}\left(\frac{10 \mathrm{GeV}}{\delta m}\right)^{5}
$$

where $\delta m$ is the inter-photino mass splitting. If $R$-parity is violated then even the lightest photino can decay directly to the Standard Model. The lifetime depends on the details of the $R$-parityviolating interaction, but in general the lifetime is enhanced by a factor of $\kappa^{-2}$ relative to the equivalent three-body decay of a bino.

\subsubsection{Existing bounds}

\subsubsection{Direct bounds}

Direct bounds on hidden photinos come from precision measurements of the $Z$ width and potentially from direct production. LEP direct search limits on processes such as $e^{+} e^{-} \rightarrow \tilde{N}_{1} \tilde{N}_{2}$ [929] place a negligible constraint on photino masses and mixings. There are no meaningful bounds from LHC Run 1, though photinos appearing in sparticle cascade decays may weaken the sensitivity of existing searches for supersymmetry [930]. The invisible $Z$ width, in contrast, provides a sensitive probe of light photinos. In the presence of $N$ photinos lighter than $M_{Z} / 2$, the contribution to the decay width is

$$
\delta \Gamma_{Z} \sim \frac{G_{F}}{6 \sqrt{2} \pi} N^{2} \kappa^{4} \eta^{4} M_{Z}^{3} \sim 0.03 \mathrm{MeV} \times N^{2}\left(\frac{\kappa}{0.1}\right)^{4}\left(\frac{\eta}{1}\right)^{4}
$$

Given LEP bounds on the invisible $Z$ width [354] this corresponds to a constraint on the mixing parameters $\kappa \eta \lesssim 0.3 / \sqrt{N}$ and represents the strongest constraint on photinos from precision electroweak observables. Similarly, the contribution to the invisible Higgs width is

$$
\delta \Gamma_{h} \sim \frac{G_{F}}{2 \sqrt{2} \pi} N^{2} \kappa^{4} \eta^{2} M_{W}^{2} m_{h} \sim 0.1 \mathrm{MeV} \times N^{2}\left(\frac{\kappa}{0.1}\right)^{4}\left(\frac{\eta}{1}\right)^{2} .
$$


Bounds on the Higgs invisible width on the order of $20 \%$ from Higgs coupling fits (e.g. [931]) provide the somewhat stronger constraint $\kappa \sqrt{\eta} \lesssim 0.17 / \sqrt{N}$.

\subsubsection{Cosmological bounds}

If a hidden photino is stable and constitutes the LSP, then there are relatively strong constraints coming from the observed dark matter relic abundance [925, 926]. Photinos are thermalized by MSSM interactions provided $\kappa \gtrsim 10^{-6}$, in which case the thermal relic abundance of such a photino dark matter candidate is typically too large by a factor of $\kappa^{-4}$. Even in the case that photinos do not reach thermal equilibrium with the MSSM for $\kappa \lesssim 10^{-6}$, out-of-equilibrium production may overclose the universe unless $\kappa \lesssim 10^{-11}$. Of course, these constraints may be ameliorated by a low reheating temperature, a phase of thermal inflation at low energies, or decay of photinos either into new hidden degrees of freedom or back into the Standard Model via $R$-parity-violating couplings, and place no irreducible bound on hidden photinos. Another potential cosmological bound on exceptionally light photinos with masses $\lesssim 30 \mathrm{MeV}$ comes from supernova cooling via photino-strahlung, but this does not place a significant constraint on the parameter space for hidden photinos.

\subsubsection{What SHiP can do}

The production of light photinos at low-energy experiments is much akin to the production of light neutralinos, albeit with extra $\kappa^{4}$ suppression due to mixing. Light photinos could be produced in pairs at SHiP either through the two-body decay of mesons or through three-body decays such as $B \rightarrow K \tilde{\gamma} \tilde{\gamma}$; the precise meson production modes depend on the flavor structure of the MSSM sfermion sector, which determines the flavor structure of $q q \tilde{\gamma} \tilde{\gamma}$ couplings via squark exchange. The rates for production are akin to those for light neutralinos [932] with an additional suppression factor $\sim \kappa^{4}$ for pair production (this can be reduced to $\sim \kappa^{2}$ if there is also a light ordinary neutralino and one produces $\tilde{N}_{1}^{0} \widetilde{\gamma}$ ). The signatures of photino pair production then depend on whether or not $R$-parity is conserved.

\subsubsection{R-parity conserving photinos}

If $R$-parity is conserved then the lightest photino $\tilde{\gamma}_{1}$ is generically stable and we can have only pair production of $\tilde{\gamma}_{1}$ in meson decays. However, it is also possible for mesons to decay first to heavier photinos which in turn undergo three-body decays into lighter photinos, as in pair production of $\tilde{\gamma}_{1} \tilde{\gamma}_{2}$ in meson decays followed by (possibly displaced) decay of the $\tilde{\gamma}_{2}$ via $\tilde{\gamma}_{2} \rightarrow q \bar{q} \tilde{\gamma}_{1}$ or $\tilde{\gamma}_{2} \rightarrow \ell \bar{\ell} \tilde{\gamma}_{1}$.

To obtain a (very rough) estimate for the potential sensitivity at SHiP, let us consider the case with several light photinos produced from the decay of charged $B$ mesons (one could also consider the even more numerous $D$ mesons at a slight expense of a reduced mass reach). The production of light neutralinos can be quite high if there exist flavor changing terms from SUSY breaking (even in absence of these production is possible via loop diagrams) [932]. Indeed, high gluino masses as required by the LHC measurements, weaken constraints on flavor changing terms. Optimistically those could then be such that they maximize the production of photinos. As a guide let us take the maximal allowed branching ratio for one of the $B$ decay channels [933, 934]

$$
B R\left(B^{-} \rightarrow K^{-}+\nu \bar{\nu}\right)=1.410^{-5} \sim 10^{-5}
$$

which is currently within the allowed regime. Analog to Section 5.4 .2 we estimate that $\sim 7 \times 10^{13}$ $B$ mesons being produced at SHiP. Using a geometric factor of $\sim 10^{-3}$ for the produced hidden photinos to be in the suitable detector region we would have about $\sim 10^{5}-10^{6}$ hidden photinos inside the detector region. With $\kappa$ in the $10^{-2.5}$ region a significant number of those would decay inside the detector volume, giving an intriguing possibility for a discovery. 
It should be said that this is, of course, a very optimistic estimate. The true branching fraction and production rate depends on the detailed model parameters (superpartner masses and flavor changing terms) and can and will in general be suppressed by $\kappa^{4}\left(\sim \kappa^{2}\right.$ in the case of a light neutralino). Yet, at the same time before suppression by $\kappa^{4}$ there are no hard constraints that the branching fraction into hidden photinos could not even be bigger and only the $\kappa^{4}$ suppression brings it into the experimentally allowed region.

\subsubsection{R-parity-violating photinos}

$R$-parity violation is a compelling setting for hidden photinos, as cosmological bounds on photinos are naturally avoided and LHC bounds on the scale of MSSM states may be lowered. In this case, the phenomenology is much like that of R-parity violating neutralino decays. Production of the lightest photino proceeds either in pair production or single production through meson decays followed by three-body decay to Standard Model final states. In particular the latter proceeds along very similar lines as the RPV scenario discussed in Section 6.2 and from there we are optimistic that it yields a significant step into untested parameter regions.

\subsubsection{Concluding remarks}

Searches for the gauginos of extra "hidden" U(1) gauge symmetries are a well motivated setup offering interesting possibilities at SHiP. Of particular interest are channels such as,

$$
\begin{aligned}
& p p \rightarrow B^{-} \rightarrow K^{-}+\widetilde{\gamma} \widetilde{\gamma}, \widetilde{\gamma}_{i} \stackrel{\text { long lived }}{\longrightarrow} \widetilde{\gamma}_{j}+\ell^{+} \ell^{-} \\
& p p \rightarrow B+X \rightarrow \widetilde{\gamma}+X^{\prime}, \widetilde{\gamma} \stackrel{\text { long lived }}{\longrightarrow} D^{ \pm}+\ell^{\mp}
\end{aligned}
$$

and similar channels with $D$ mesons instead of the $B$ mesons. While further more detailed studies are clearly needed, estimates indicate that SHiP will have interesting sensitivity.

\subsection{SUSY vector portal II: Novel Hidden Photon decays}

In the previous section we have considered the case of an extra "hidden" U(1) gauge group with a massless gauge boson. In this case the signals originate directly from the additional hidden photinos. We will now turn to the alternative option where the extra $\mathrm{U}(1)$ gauge symmetry is broken and the corresponding gauge boson is massive and therefore directly observable. In this case SUSY offers interesting new channels and signatures compared to the case of a simple dark vector scenario as diskussed in Chapter 2.

\subsubsection{Setup}

In much the same way that supersymmetry can stabilize the electroweak scale against large quantum corrections, it can also stabilize the mass of a light Abelian vector boson [202]. To do so, there must exist additional light particles beyond just the dark vector. These new particles can produce new signals in lower-energy experiments at the intensity frontier [166].

Extending the vector portal to be supersymmetric implies that the dark vector $X^{\mu}$ of the exotic $U(1)_{x}$ gauge invariance must be accompanied by a dark gaugino fermion $\widetilde{\gamma}^{\alpha}$. Supersymmetry also implies that the kinetic mixing interaction must be extended to include new interactions involving the dark gaugino and any scalars charged under the dark gauge group or SM hypercharge:

$$
\mathscr{L} \supset-\frac{\epsilon}{2} B_{\mu \nu} X^{\mu \nu}+\epsilon\left[\widetilde{B}^{\dagger} i \bar{\sigma} \cdot \partial \widetilde{\gamma}+(\text { h.c. })\right]+\epsilon D_{x} D_{Y},
$$

where $D_{i}=g_{i} \sum_{a} q_{a}^{i}\left|\phi_{a}\right|^{2}$ sums over all the scalar fields in the theory weighted by their charge $q_{a}^{i}$ under the corresponding $U(1)_{i}$ factor, $i=x, Y$. The first term in Eq. (6.6.1) is the familiar vector 
portal interaction, the second is a kinetic mixing between the dark gaugino and the hypercharge gaugino $\widetilde{B}$, and the third term is an extension of the Higgs portal interaction to the new scalar fields required by supersymmetry.

In this section we will focus on a scenario where the dark photon gets a mass $m_{x}$ from a dark Higgs mechanism [91]. This occurs when a scalar dark Higgs field charged under $U(1)_{x}$ develops a vacuum expectation value (VEV). A minimal supersymmetric realization of this consists of two dark Higgs supermultiplets $H$ and $H^{\prime}$ with equal and opposite $U(1)_{x}$ charges $\pm x_{H}$. Each supermultiplet contains a complex scalar and a Weyl fermion. Scalar VEVs can be induced by the $D$-term interaction of Eq. (6.6.1) or by soft supersymmetry breaking terms in the dark sector.

When both dark Higgs scalars develop VEVs, the Weyl fermion Higgsinos mix with the dark gaugino, one of the dark Higgs scalars is eaten by dark vector, and the remaining Higgs scalars mix amongst themselves. The physical states in this minimal Higgsed dark vector realization consist of a single massive dark vector $Z^{x}$, two real dark scalars $h_{1,2}^{x}$, one dark pseudoscalar $A^{x}$, and three Majorana dark neutralinos $\widetilde{N}_{1,2,3}^{x}$. If $R$-parity is conserved, the lightest dark neutralino will be stable and contribute to the dark matter density.

The natural size of the dark vector mass is of the same order as the soft supersymmetry breaking terms in the dark sector. These can be much smaller than the corresponding terms in the visible sector (which must be larger than about a $\mathrm{TeV}$ ) if supersymmetry breaking is communicated less efficiently to the dark sector [202]. However, the soft terms in the dark sector should be larger than at least $m_{\text {soft }}^{x} \gtrsim \min \left\{\sqrt{\epsilon} M_{Z}, \epsilon m_{\text {soft }}^{\text {vis }}\right\}$ due to the kinetic mixing interaction [91, 935].

\subsubsection{SHiP Sensitivity}

The signals of this minimal supersymmetric dark vector theory in fixed-target experiments depend on how the dark vector decays. There are four main cases [166]:

A. $Z^{x} \rightarrow S M+S M$ : signals are similar to the non-supersymmetric dark vector (cf. Chapter 2).

B. $Z^{x} \rightarrow \widetilde{N}_{1}^{x} \widetilde{N}_{1}^{x}$ : vector decays are invisible.

C. $Z^{x} \rightarrow h_{1}^{x} A^{x}$ : scalars decay slowly to the SM and can give rise to displaced signals.

D. $Z^{x} \rightarrow \widetilde{N}_{1}^{x} \widetilde{N}_{2}^{x}$ : can give displaced decays when the 3-body mode $\widetilde{N}_{2}^{x} \rightarrow \widetilde{N}_{1}^{x} Z^{x *}$ is slow.

Cases $\mathrm{A}$ and $\mathrm{B}$ are similar to minimal dark vector and minimal dark matter theories respectively, so we will concentrate on cases C and D. For this, we will set $\alpha_{x}=g_{x}^{2} / 4 \pi=\alpha$ and scale all the dimensionful parameters of the theory as fixed ratios of the dark vector mass $m_{x}$ as in Ref. [166].

Adapting the same strategy and techniques as in [166] to the geometry of SHiP in the left and right panels of Fig. 6.5 we show the reach of the SHiP experiment to cases $\mathrm{C}$ and D of this minimal supersymmetric dark photon theory relative to other searches. A significant improvement is achievable.

\subsubsection{Concluding remarks}

For SUSY models with an extra, spontaneously broken U(1) gauge symmetry SHiP offers significant improvement over existing limits based on channels such as, e.g.

$$
\begin{aligned}
& p p \rightarrow Z^{x}+X \rightarrow h_{1}^{x} A_{1}^{x}+X, A_{1}^{x} \stackrel{\text { long lived }}{\longrightarrow} h_{1}^{x}+\ell^{+} \ell^{-} \\
& p p \rightarrow Z^{x}+X \rightarrow \widetilde{N}_{2}^{x} \widetilde{N}_{1}^{x}+X, \widetilde{N}_{2}^{x} \stackrel{\text { long lived }}{\longrightarrow} \widetilde{N}_{2}^{x}+\ell^{+} \ell^{-} .
\end{aligned}
$$



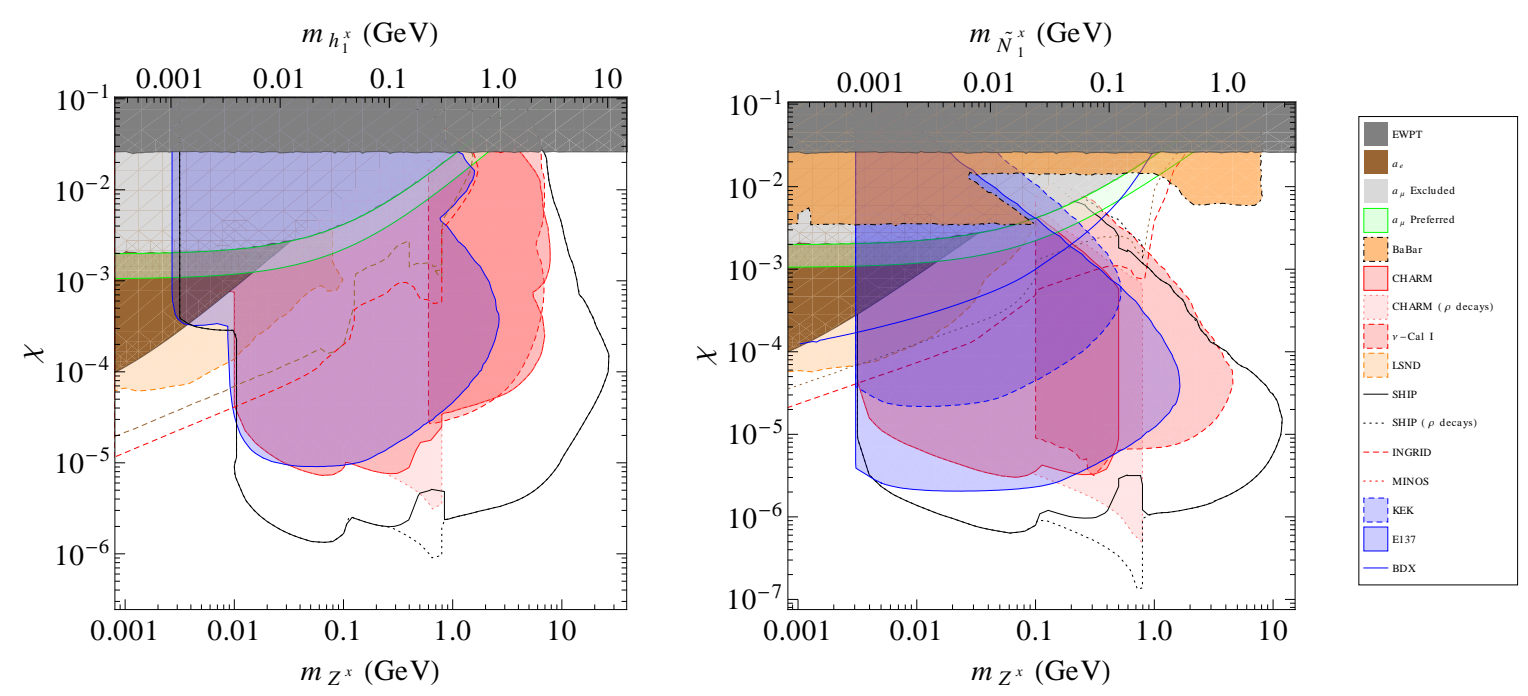

Figure 6.5: Sensitivity estimates for Cases C (left) and D (right) of the minimal supersymmetric dark vector theory discussed in the text. Benchmark slopes are as in [166].

\subsection{Axinos and saxions, ALPinos and sALPs}

\subsubsection{Motivation}

As discussed in Chapter 5 axions and axion-like particles (ALP) are well motivated in string theory but also in field theoretic settings with spontaneously broken global approximate symmetries. In a supersymmetric version of axion models ${ }^{9}$ (cf. Chapter 5), the axion $A$ forms a supermultiplet with a fermonic partner, axino $\tilde{A}$, and a scalar partner, saxion $s$.

The interactions for these partner particles are typically very weak (often suppressed by the large scale of spontaneous symmetry breaking $f_{A}$ ) and therefore testing them requires a high precision/intensity experiment such as SHiP.

\subsubsection{Phenomenology of saxions and axinos and possibilities at SHiP}

The most direct constraints typically arise from searching for the axion itself (cf. Chapter 5 for those constraints). However, if the axino and/or the saxion are relatively light it is interesting to search for these particles directly.

Masses of axino and saxion are naively considered to be of the order of the gravitino mass, however those can be lower in specific cases and take values between $\mathrm{MeV}$ and $\mathrm{GeV}$ [936-938].

\subsubsection{Saxions}

Since saxions are just the scalar partners of the corresponding pseudo-scalar axions they feature interactions very similar to that of the axion itself. The interactions typically include couplings to two axions as well as to two gauge bosons, e.g. photons and gluons,

$$
\mathcal{L}=\frac{s}{\sqrt{2} f_{A}}\left(\partial_{\mu} A\right)^{2}+C \frac{\alpha}{8 \pi f_{A}} s F^{a \mu \nu} F_{\mu \nu}^{a}+C_{3} \frac{\alpha_{3}}{8 \pi f_{A}} s G^{a \mu \nu} G_{\mu \nu}^{a}
$$

\footnotetext{
${ }^{9}$ For notational simplicity we will from now on talk about axions, axinos and saxions but unless specified otherwise this includes ALPs, ALPinos and sALPs.
} 


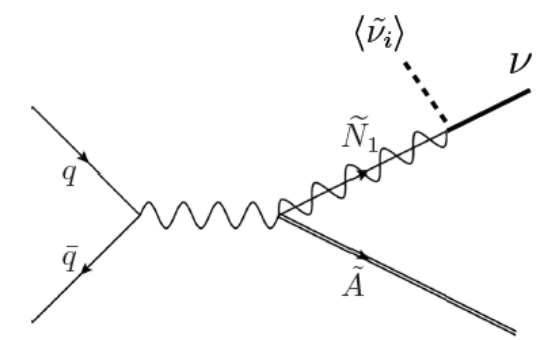

Figure 6.6: Feynman diagram for the R-parity violating production of an axino.

with some constants $C$ and $C_{3}$. This is essentially the scalar equivalent to the pseudo-scalar interactions of ALPs discussed in Chapter 5 plus an additional interaction with two axions. In addition we can also have (strongly model dependent) interactions with Standard Model fermions.

Let us briefly discuss the two main differences: the parity and the interaction with two axions. For most direct experimental tests such as SHiP the difference in parity only leads to minor modifications (see, e.g., Figs. 3.9 and 5.2 where we consider scalars and pseudoscalars interacting with SM fermions; we expect this level of similarity also for other couplings such as one to two gauge bosons). The interaction with two axions is more important. If the axion mass is less than half the saxion mass, the saxion can dominantly decay via $s \rightarrow A A$. Depending on the value of $f_{A}$ and the mass of the axion this can be an invisible decay, or the axion $A$ itself can decay into something visible. If the decay is invisible this can lead to a significant reduction in the observed number of events and a corresponding reduction in sensitivity. One can always consider this as the worst case scenario for the sensitivity. Decays of the produced axions can only lead to additional events. Aside from this modification we expect for saxions at SHiP very similar signatures and quantitatively also similar improvements to those for ALPs, and we refer the reader to Chapter 5 for details.

\subsubsection{Axino LSP with R-parity breaking}

If R-parity is broken, an axino LSP can decay and produce signatures in the SHiP experiment. In bilinear R-parity violation models, the light axinos dominantly decay to photon and neutrino with a lifetime ${ }^{10}[939]$

$$
\tau_{\tilde{A}} \sim 1 \mathrm{~m}\left(\frac{\left|U_{\tilde{\gamma} \tilde{Z}}\right| \xi_{i}}{10^{-1}}\right)^{-2}\left(\frac{m_{\tilde{A}}}{10 \mathrm{GeV}}\right)^{-3}\left(\frac{f_{A}}{10^{5} \mathrm{GeV}}\right)^{2},
$$

with $\xi_{i}=\left\langle\tilde{\nu}_{i}\right\rangle / v$ and $\left\langle\tilde{\nu}_{i}\right\rangle$ being the sneutrino vev which parametrizes the size of R-parity violation. In models with DSFZ type interactions axinos can additionally decay into $e^{-} e^{+} \nu$ with similar decay rate. The QCD axino with $f_{A} \gtrsim 10^{9} \mathrm{GeV}$ is found to be hard to decay inside the SHiP experiment. However, for general ALPinos, the corresponding Peccei-Quinn breaking $f_{A}$ scale might be smaller.

With R-parity violation, a single axino can be produced together with a neutrino, e.g. from two quarks via an s-channel $Z$ boson as shown in Fig. 6.6.

\subsubsection{Axinos with R-parity conservation}

If $\mathrm{R}$ parity is conserved, supersymmetric particles must be produced in pairs and this, of course also holds for the axinos. Typical R-parity conserving production channels are shown in Fig. 6.7. Since the diagram on the left hand side is suppressed by two factors of $f_{A}$, the corresponding production is likely to be too small to be observed, and a light neutralino is required for direct

\footnotetext{
${ }^{10}$ Here we consider parameter values that are more appropriate for ALP models and not for the QCD axion. Nevertheless we model the interactions (also for the superpartners) on those of the KSVZ axion. However, one should take this with a grain of salt, as strong model dependencies are possible.
} 

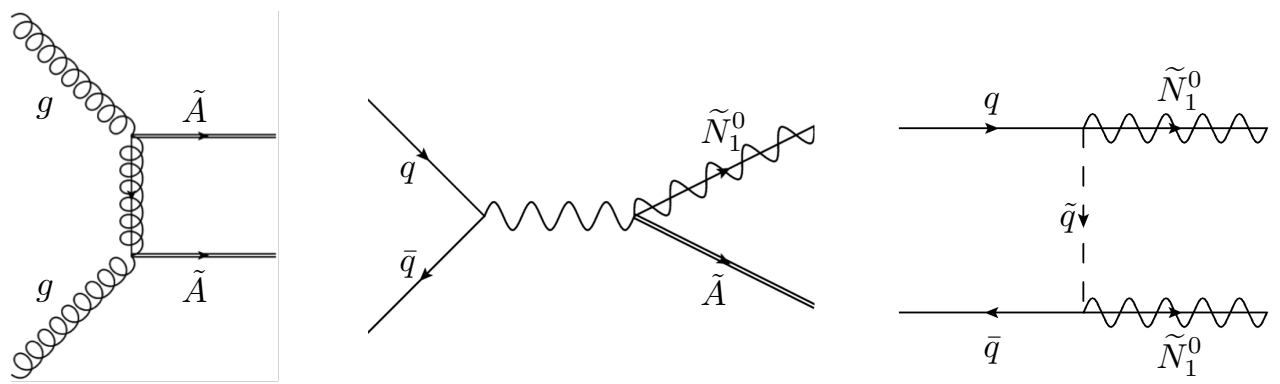

Figure 6.7: Typical Feynman diagrams for axino/neutralino pair production from gluons (left) or in association with a light neutralino (middle) or production of two light neutralinos. All of these production channels also work if R-parity is unbroken. According to the number of axinos produced the production amplitude is suppressed by $1 / f_{A}^{2}, 1 / f_{A}$ and 1 .
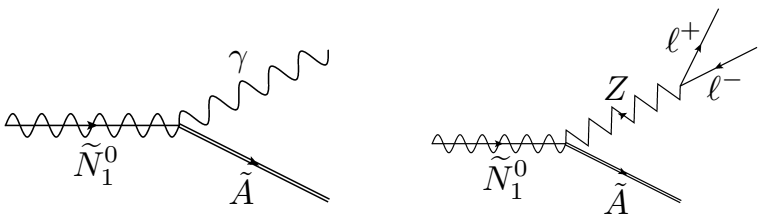

Figure 6.8: Neutralino to axino decay channels.

R-parity conserving production. In this case we can have axino/neutralino production (middle) or neutralino pair production (right).

An interesting option then also opens up for the decay. The heavier of the two (either the axino or the neutralino) can decay into the lighter one, as shown in Fig. 6.8, for the case when the neutralino is heavier. The corresponding lifetime can be quite large and therefore suitable to the SHiP setup since the relevant interaction is suppressed by $f_{A}$. The decay shown on the right hand side then leads to an interesting but also challenging mono-photon signature in SHiP. In addition we can also have (somewhat suppressed) decays with two charged tracks as shown on the right hand side of Fig. 6.8. If the axino is heavier, we can just reverse the roles of axino and neutralino in the decay diagrams.

\subsubsection{Concluding remarks}

If axion/ALP models are embedded in a supersymmetric context two additional particles appear that lend themselves to searches at SHiP. The first option is the saxion/sALP whose phenomenological features are qualitatively relatively close to that of the axion/ALP and can be searched at SHiP with the strategies discussed in Chapter 5. However, if the decay of the saxion into two axions is kinematically allowed, it is typically dominant, which can lead to reductions in the observed number of events with SM particles.

The fermionic axino/ALPino leads to different signatures and strategies. If R-parity is broken 
possible channels include,

$$
\begin{aligned}
& p p \rightarrow \tilde{A} \nu, \tilde{A} \stackrel{\text { long lived }}{\longrightarrow} \nu+\ell^{+} \ell^{-}, \\
& p p \rightarrow \tilde{A} \nu, \tilde{A} \stackrel{\text { long lived }}{\longrightarrow} \nu+\gamma,
\end{aligned}
$$

If R-parity is conserved interesting signatures exist if there is an additional light neutralino. In this case one could have mono-photon signatures such as

$$
p p \rightarrow \widetilde{N}_{1}^{0} \widetilde{N}_{1}^{0}, \widetilde{N}_{1}^{0} \stackrel{\text { long lived }}{\longrightarrow} \tilde{A}+\gamma
$$

but also signals with charged track such as

$$
p p \rightarrow \widetilde{N}_{1}^{0} \widetilde{N}_{1}^{0}, \tilde{N}_{1}^{0} \stackrel{\text { long lived }}{\longrightarrow} \tilde{A}+\ell^{+} \ell^{-},
$$

More studies are needed for quantitative statements on the sensitivity.

\subsection{Additional Possibilities}

In this section we briefly mention several other options that could lead to observable signatures at SHiP, but which are in very early stages of discussion and need further investigation to determine the sensitivity at SHiP and for comparison with existing limits.

\subsubsection{Pair production of light neutralinos and decay to gravitino}

In Section 6.2 we already discussed the possibility of light neutralinos. The focus was on R-parity violating production and decays.

Even if R-parity is conserved, the neutralino is not necessarily the lightest superpartner. In theories with gauge mediated supersymmetry breaking this is typically the gravitino. For example the neutralino can decay into a photon and a gravitino (cf. [869]),

$$
\tau\left(\widetilde{N}_{1}^{0} \rightarrow \tilde{G} \gamma\right)=\frac{16 \pi F^{2}}{k^{2} \kappa_{\gamma} m_{\widetilde{N}_{1}^{0}}^{5}}=10 \mathrm{~m} \frac{1}{k^{2} \kappa_{\gamma}}\left(\frac{10 \mathrm{GeV}}{m_{\widetilde{N}_{1}^{0}}}\right)^{5}\left(\frac{\sqrt{F}}{100 \mathrm{TeV}}\right)^{4}
$$

Here $F$ is the scale of supersymmetry breaking, $k$ is a coupling strength to the SUSY breaking sector and $\kappa_{\gamma}$ encodes the relevant neutralino mixing angles for the neutralino-photon-gravitino coupling.

Pair production of such light neutralinos could occur via squark exchange in the t-channel. (On the parton level the diagrams are similar to what we will have in the case of flavor violating production discussed in the next subsection and shown in Fig. 6.9, but due to the absence of flavor violation, formation of intermediate mesons is not necessarily preferred.)

In this setup the channel of interest would be,

$$
p p \rightarrow \widetilde{N}_{1}^{0} \widetilde{N}_{1}^{0}, \widetilde{N}_{1}^{0} \stackrel{\text { long lived }}{\longrightarrow} \tilde{G}+\gamma
$$

somewhat similar to the axino case discussed above.

\subsubsection{Flavor violating production of light neutralinos}

Flavor violating squark terms could lead to a significantly enhanced pair production of light neutralinos. A possible diagram is shown in Fig. 6.9, where we have production from the decay of 


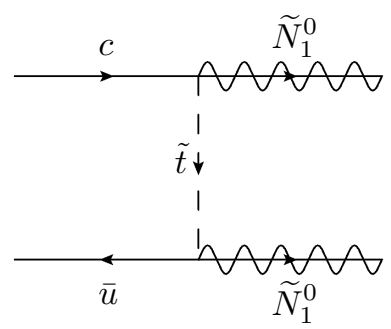

Figure 6.9: Neutralino pair production from neutral $D$ mesons via flavor violating processes with t-channel stop exchange.

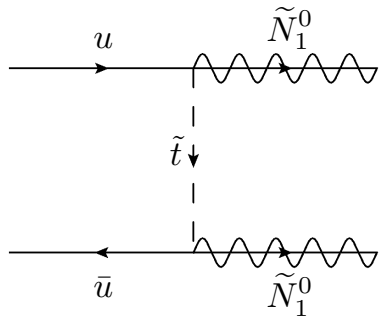

Figure 6.10: Direct neutralino pair production in a process with t-channel stop exchange. Compared with production via mesons shown in Fig. 6.9 this can benefit from larger parton distribution functions and from somewhat less constrained mixings between up- and top-squarks [940, 941].

$D$-mesons. Alternatively one could also have direct production of neutralino pairs as shown in Fig. 6.10. The case of a stop exchange is particularly interesting since this can still be fairly light.

For R-parity conserving setups decay can proceed to a gravitino and a photon as discussed above. If R-parity is violated we could have decays similar to those discussed in Section 6.2.

All in all we have potential channels,

$$
\begin{aligned}
& p p \rightarrow D^{0}+X \rightarrow \widetilde{N}_{1}^{0} \widetilde{N}_{1}^{0}+X, \widetilde{N}_{1}^{0} \stackrel{\text { long lived }}{\longrightarrow} \tilde{G}+\gamma \quad(\mathrm{R}-\text { parity conserved }) \\
& p p \rightarrow D^{0}+X \rightarrow \widetilde{N}_{1}^{0} \widetilde{N}_{1}^{0}+X, \widetilde{N}_{1}^{0} \stackrel{\text { long lived }}{\longrightarrow} K^{ \pm}+\ell^{\mp} \quad(\mathrm{R}-\text { parity violated })
\end{aligned}
$$

and similar direct processes where the intermediate meson production and decay step is absent. It will be interesting to see if SHiP can compete with or exceed constraints from B-physics for those couplings.

\subsection{SUSY at SHiP: Final remarks}

Many supersymmetric models still allow for new light neutral particles in the MeV to few GeV range which is accessible to SHiP. In these models SHiP often allows for significant discovery potential beyond existing constraints.

Importantly supersymmetry not only provides additional theoretical motivation but also encourages to consider additional interesting but also challenging signatures in the SHiP detector. In 
particular we can have the following decays of a long lived neutral particle,

$$
\begin{aligned}
& X \rightarrow \ell^{+} \ell^{-} \\
& X \rightarrow \pi^{+} \pi^{-} \\
& X \rightarrow K^{ \pm}+\ell^{\mp} \\
& X \rightarrow K^{0}+\not \notin \\
& X \rightarrow \pi^{0}+\pi^{0} \\
& X \rightarrow \gamma+\gamma \\
& X \rightarrow \gamma+\not \mathbb{E}
\end{aligned}
$$

where especially the lower ones may be challenging. The above also shows that there are significant opportunities in these neutral channels which strongly motivates the study of their experimental sensitivity, which is currently under way. 


\section{Chapter 7}

\section{Tau neutrino physics and other precision measurements in SHiP}

The high intensity proton beam dump experiment under consideration produces an high intensity neutrino flux with all three flavors: electron, muon and tau neutrinos and antineutrinos. In this section we will present a list of observables that could be measured exploiting the variety of such a neutrino flux. The basic assumptions are that $2 \times 10^{20}$ protons with $400 \mathrm{GeV}$ energy from the SPS at CERN will be stopped in a target of Molybdenum and Tungsten, and that on the path of the emerging flux of neutrinos there will be a neutrino detector, consisting for the most part of lead, that allows to identify the flavor of all the three kinds of charged leptons produced in charged current neutrino interactions and also the sign of the charge for tau and muon charged leptons, as well as the other relevant kinematical variables of the Deep Inelastic Scattering (DIS) processes for both the charged and the neutral current ( $\mathrm{CC}$ and $\mathrm{NC}$ ) interactions. We will further assume that the neutrino detector will allow to identify the production and decay of charmed resonances. As we will discuss later in this section, under these assumptions there will be the possibility to measure tau neutrino charged current cross sections. This is currently the least known charged current interaction vertex of the Standard Model (SM) with 7.5 events detected by the DONUT collaboration [942] and 4 by the OPERA collaboration [943]. As we will see in section 7.1, with the SHiP experiment the statistics of such events can be increased by three orders of magnitude allowing to perform new studies of the cross sections both at the inclusive and differential level. To the extent of performing detailed measurements of tau neutrino cross sections it is crucial to asses the precision in the determination of the tau neutrino flux. This is discussed in section 7.1.1. The SHiP experiment covers the DIS kinematics in a typical range of the Bjorken scaling variable $x$ down to $x \sim 5 \times 10^{-4}$ for tau neutrinos with energy $E_{\nu}=50 \mathrm{GeV}$ and $x \sim 5 \times 10^{-3}$ for muon neutrinos with energy $E_{\nu}=20 \mathrm{GeV}$. Given the high rate of muon neutrinos and anti-neutrinos DIS events expected, in section 7.2 we review the current status of deep-inelastic scattering with emphasis on charged current interactions off unpolarized fixed targets. We address the most relevant open questions in our fundamental understanding of the proton and discuss the physics potential of neutrino-nucleon deep-inelastic scattering. Finally, we consider other limits that can be improved exploiting the neutrino flux in SHiP; among them there are the limit on the tau neutrino magnetic moment (section 7.3) and the limit on the production cross section of a weakly decaying charmed baryon with five valence quarks (section 7.4). We will draw our conclusion in section 7.5. 


\subsection{Tau neutrino physics}

Direct measurements of tau neutrino charged-current (CC) interactions are a fairly recent phenomenon. The DONUT experiment reported 9 tau neutrino events with a background of 1.5 events from their neutrino beam produced with the $800 \mathrm{GeV}$ Tevatron beam at Fermilab [942]. Their cross section measurement agrees well with the related muon neutrino CC cross section, however, their statistical and systematic errors are each $33 \%$ of the best fit measurement respectively [942]. More specifically, in the DONUT experiment it was not possible to identify the charge of the outgoing tau lepton, for this reason the collaboration quoted the average of $\nu_{\tau}$ and $\bar{\nu}_{\tau}$ charged current cross sections as:

$$
\begin{aligned}
\sigma_{\nu_{l}} & =\sigma_{\nu_{l}}^{\text {const }} E K(E), \quad l=e, \mu, \tau \\
\sigma_{\nu_{l}}^{\text {const }} & =0.39 \pm 0.13 \pm 0.13 \times 10^{-38} \mathrm{~cm}^{2} \mathrm{GeV}^{-1}
\end{aligned}
$$

where $K(E)$ describes the kinematical suppression due to the tau mass and $\sigma_{\nu_{l}}^{\text {const }}$ has to be compared with the average of $\nu_{\mu}$ and $\bar{\nu}_{\mu}$ charged current cross sections, that is $0.51 \times 10^{-38} \mathrm{~cm}^{2} \mathrm{GeV}^{-1}$. As a final remark concerning the averaged tau neutrino cross section measured by the DONUT collaboration, we note that at the time of the analysis of Ref. [942] the charm quark fragmentation in $D_{s}$ and the $D_{s} \rightarrow \tau \nu_{\tau}$ decay branching ratio were known with less precision. Together with the charm production cross section in proton-nucleon collisions, these are the three basic ingredients to estimate the tau neutrino flux in a beam dump experiment as they enter directly as overal factors in the normalization of the flux. The $D_{s} \rightarrow \tau \nu_{\tau}$ branching ratio, for example, in Ref. [942] was assumed to be $(6.4 \pm 1.5) \%$, as suggested in the PDG of 2006, while recently it has been measured with increased precision to be $(5.54 \pm 0.24) \%$, as reported by the PDG of 2014 [156].

The OPERA experiment reported 4 tau neutrino events with practically no background [943]. In OPERA these events were found while searching for $\nu_{\mu} \rightarrow \nu_{\tau}$ oscillation starting from an almost pure muon neutrino flux. For this reason in OPERA only $\tau^{-}$leptons have been detected and not $\tau^{+}$. Even if the OPERA collaboration could now publish a measurement of the $\nu_{\tau}$ cross section for the first time, within SHiP there will be the possibility to measure separately $\nu_{\tau}$ and $\bar{\nu}_{\tau}$ cross sections in the same experiment and with higher precision, identifying for the first time also $\bar{\nu}_{\tau}$ interactions through the detection of the positively charged tau leptons produced. According to our preliminary estimations, the number of detectable tau neutrino and anti-neutrino charged current events in the SHiP neutrino detector will be several thousands. Indeed, such a large sample of events will bring opportunities to make new measurements of the structure functions in tau neutrino and antineutrino charged current events. With the usual DIS variables: $x \equiv Q^{2} / 2 p \cdot q$ and $y \equiv p \cdot q / p \cdot k$, where the momentum assignments are:

$$
\begin{gathered}
\nu_{\tau} / \bar{\nu}_{\tau}(k)+N \rightarrow \tau^{-} / \tau^{+}\left(k^{\prime}\right)+X \\
q^{2} \equiv\left(k-k^{\prime}\right)^{2}=-Q^{2},
\end{gathered}
$$

the tau neutrino and anti-neutrino charged current cross sections in terms of the structure functions $F_{1}, \ldots, F_{5}$ are [944]:

$$
\begin{aligned}
\frac{d^{2} \sigma^{\nu(\bar{\nu})}}{d x d y} & =\frac{G_{F}^{2} M E_{\nu}}{\pi\left(1+Q^{2} / M_{W}^{2}\right)^{2}}\left(\left(y^{2} x+\frac{m_{\tau}^{2} y}{2 E_{\nu} M}\right) F_{1}+\left[\left(1-\frac{m_{\tau}^{2}}{4 E_{\nu}^{2}}\right)-\left(1+\frac{M x}{2 E_{\nu}}\right) y\right] F_{2}\right. \\
& \left. \pm\left[x y\left(1-\frac{y}{2}\right)-\frac{m_{\tau}^{2} y}{4 E_{\nu} M}\right] F_{3}+\frac{m_{\tau}^{2}\left(m_{\tau}^{2}+Q^{2}\right)}{4 E_{\nu}^{2} M^{2} x} F_{4}-\frac{m_{\tau}^{2}}{E_{\nu} M} F_{5}\right),
\end{aligned}
$$

where $+F_{3}$ applies to neutrino scattering and $-F_{3}$ to antineutrinos, $M$ and $m_{\tau}$ are the nucleon and 
$\tau$ lepton masses respectively, $E_{\nu}$ is the initial neutrino energy and $G_{F}$ is the Fermi constant. As we will see, at the lower $\nu_{\tau}$ energies the SHiP experiment offers the first opportunity to measure the structure functions $F_{4}$ and $F_{5}$. At the Born level, neglecting target mass corrections, the Albright-Jarlskog relations apply [944]

$$
\begin{aligned}
& F_{4}=0, \\
& F_{5}=\frac{F_{2}}{2 x} .
\end{aligned}
$$

The QCD predictions for the DIS structure functions $F_{4}$ and $F_{5}$ are known up to NLO accuracy [945], including full dependence on heavy-quark masses, though. The detailed relationships between the five structure functions, including NLO QCD together with target mass and charm quark mass corrections, are discussed, for example, in Refs. [945-948].

To evaluate neutrino-lead charged current scattering, we find the neutrino cross section per nucleon via

$$
\sigma_{C C}=\frac{Z \sigma_{\nu p}+(A-Z) \sigma_{\nu n}}{A}
$$

and similarly for antineutrinos.

In evaluating the cross section, we have extrapolated the low $Q$ structure functions according to Ref. [949] below $Q^{2}=2 \mathrm{GeV}^{2}$. To avoid double counting with quasi-elastic and few particle production, a minimum hadronic final state invariant mass $W$ is set. A frequent choice consist of taking $W_{\min }=1.4 \mathrm{GeV}$. A second choice, $W_{\text {min }}=m_{p}+m_{\pi}$, approximates single and few pion production with the DIS cross section. We show both choices for $W_{\min }$ in the next few figures. Fig. 7.1 (a) shows the $\nu_{\tau} \mathrm{CC}$ cross section per nucleon per GeV as a function of energy for two
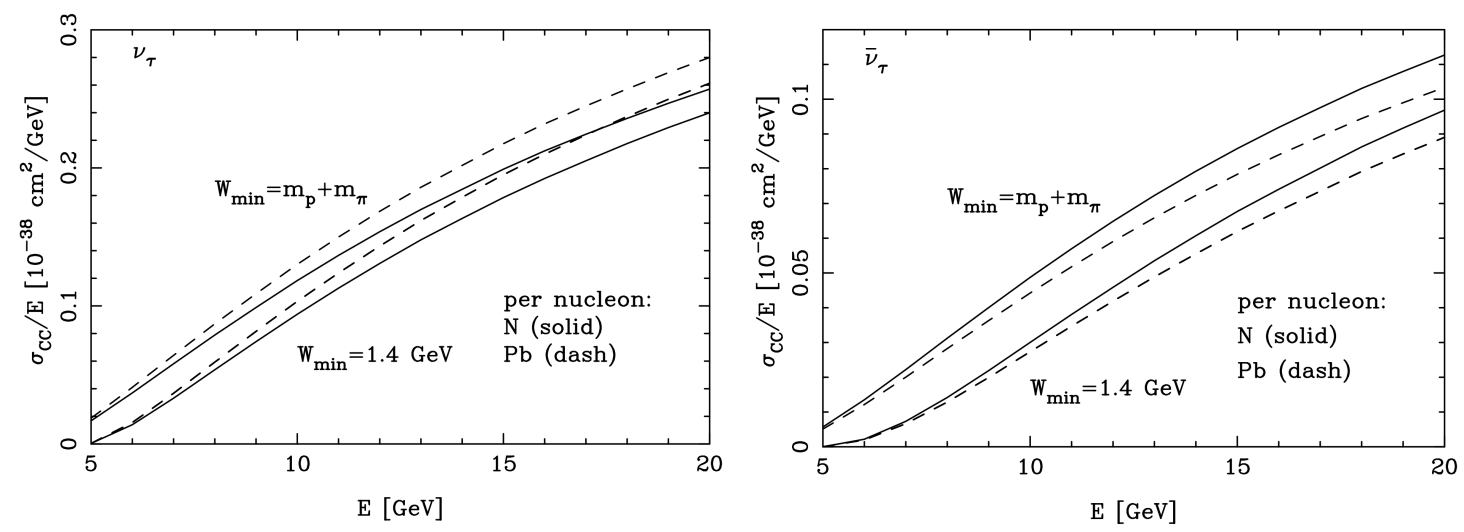

Figure 7.1: The charged current cross section per nucleon, scaled by incident energy for (a) $\nu_{\tau}$ and (b) $\bar{\nu}_{\tau}$ scattering with isoscalar nucleons (solid) and lead (dashed) targets. The upper solid and dashed curves are for $W_{\min }=m_{p}+m_{\pi}$, while the lower solid and dashed curves are for $W_{\min }=1.4$ $\mathrm{GeV}$.

targets: isoscalar nucleons ( $\mathrm{N}$, solid curves) and lead ( $\mathrm{Pb}$, dashed curves) for two choices of $W_{\min }$. At $E=20 \mathrm{GeV}$, the cross section per nucleon on lead is almost $10 \%$ larger than on an isoscalar target. The neutrino-neutron cross section is larger than the neutrino proton cross section since the valence contribution $\nu_{\tau} d \rightarrow \tau u$ is large. Fig. 7.1(b) shows that the cross section per nucleon for $\bar{\nu}_{\tau}$ scattering with lead is smaller than with isoscalar nucleon targets since $\bar{\nu}_{\tau} u \rightarrow \bar{\tau} d$ is the dominant valence process. At low energies, the CC cross sections for $\nu_{\tau} p$ and $\bar{\nu}_{\tau} p$ are comparable. The additional structure functions $F_{4}$ and $F_{5}$ have an impact on the charged current cross section, as shown in Fig. 7.2(a) and (b). This is primarily due to $F_{5} \simeq F_{2} /(2 x)$, reducing the cross section. 

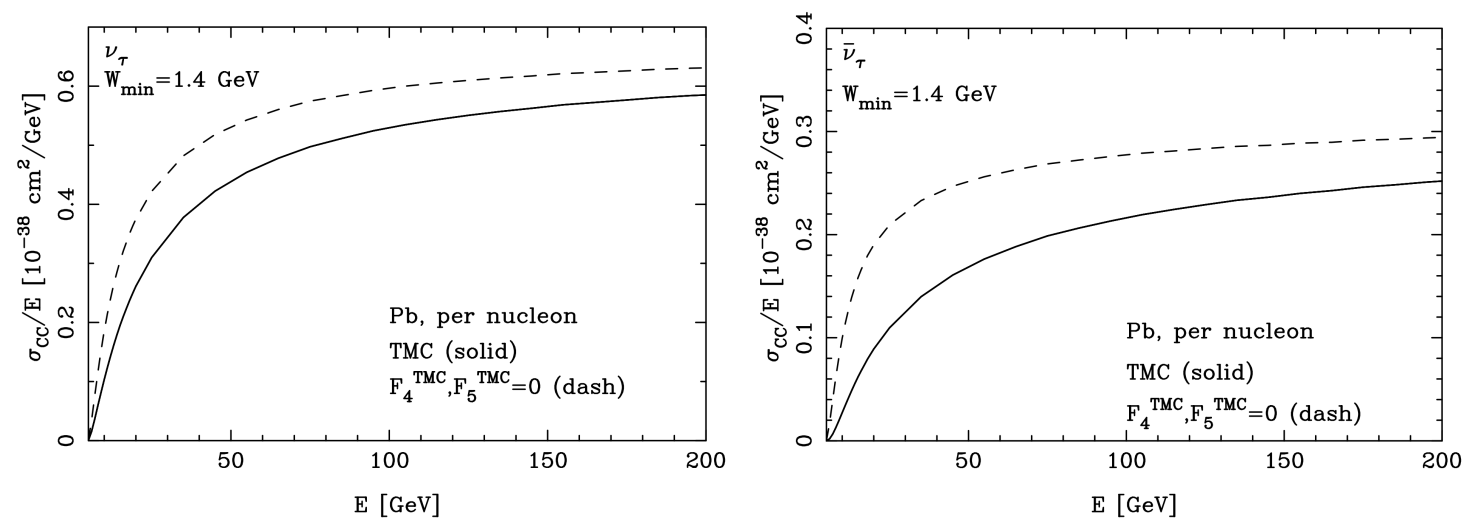

Figure 7.2: The charged current cross section per nucleon, scaled by incident energy for (a) $\nu_{\tau}$ and (b) $\bar{\nu}_{\tau}$ scattering with a lead target with $W_{\min }=1.4 \mathrm{GeV}$. The dashed curve has $F_{4}$ and $F_{5}$ set to zero, while the solid curve has the full expression for the target mass corrected (TMC) cross section.

At $E=20 \mathrm{GeV}$, for tau neutrino $\mathrm{CC}$ scattering, the full expression for the cross section yields a result about $30 \%$ lower than when these terms are neglected. At $E=200 \mathrm{GeV}$, the contribution of these terms reduces the cross section by about $7 \%$. For $\bar{\nu}_{\tau} \mathrm{CC}$ scattering, the solid line is about $53 \%$ lower than the dashed line for $E=20 \mathrm{GeV}$ and about $14 \%$ lower for $200 \mathrm{GeV}$. Target mass corrections are included here. They amount to about a $7 \%$ correction for $\nu_{\tau} \mathrm{CC}$ scattering at $E=10 \mathrm{GeV}$.

\subsubsection{Flux of tau neutrinos}

The precision on the tau neutrino cross section measurement is linked to the precision in the estimation of the flux of neutrinos. As sketched in Fig. 7.3, the prompt flux of $\nu_{\tau}+\bar{\nu}_{\tau}$ comes

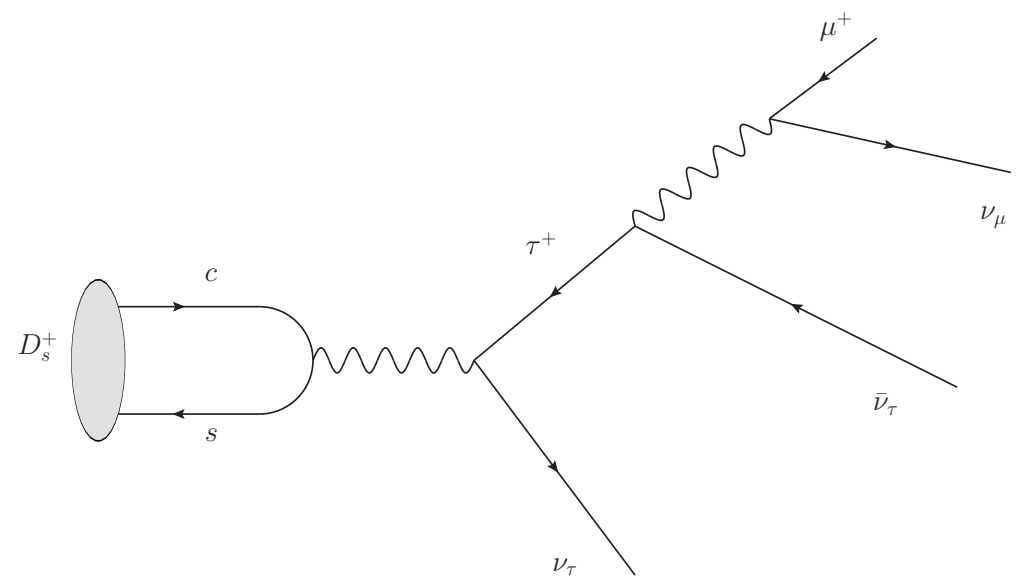

Figure 7.3: Example of $D_{s}$ decay chain producing a $\nu_{\tau}$ and a $\bar{\nu}_{\tau} .18 \%$ of the tau leptons decay to a muon (see also Table 7.2), in all tau decay modes another tau neutrino is produced.

from the leptonic decay $D_{s} \rightarrow \tau \nu_{\tau}$, with a decay length of $c \tau_{D_{s}}=149.9 \mu \mathrm{m}$ and branching ratio $B\left(D_{s} \rightarrow \tau \nu_{\tau}\right)=(5.54 \pm 0.24) \%$ [156]. In this decay channel, two tau neutrinos are produced: one directly from the decay and the other from the "chain decay" $D_{s} \rightarrow \tau \rightarrow \nu_{\tau}$. For a $D_{s}$ with energy 
$E_{D}$, the lepton energy distributions are constant, constrained by

$$
\begin{aligned}
0 & \leq E_{\nu} \leq\left(1-R_{\tau}\right) E_{D} \\
R_{\tau} E_{D} & \leq E_{\tau} \leq E_{D}
\end{aligned}
$$

for $R_{\tau}=m_{\tau}^{2} / m_{D}^{2}$. With $m_{\tau} \sim m_{D_{s}}$, the neutrino energy range is much lower than the tau energy range: $\left\langle E_{\nu}\right\rangle \simeq 0.09 E_{D}$ while $\left\langle E_{\tau}\right\rangle \simeq 0.91 E_{D}$. The tau itself decays promptly, $c \tau_{\tau}=87.03 \mu \mathrm{m}$, to $\nu_{\tau}$ with approximately $1 / 3$ of the tau energy going to the neutrino. Except for the lowest energies, it is the chain decay $D_{s} \rightarrow \tau \rightarrow \nu_{\tau}$ that dominates the $\nu_{\tau}+\bar{\nu}_{\tau}$ flux. Here, we estimate the prompt tau neutrino plus antineutrino fluxes from $D_{s}$ decays. We show, for comparison, the prompt muon neutrino plus antineutrino fluxes from charmed hadrons using the same approximations. In the illustrative results below, we use two approximations for particles produced, starting with a $400 \mathrm{GeV}$ proton beam incident on a series of two $58 \mathrm{~cm}$ targets, first molybdenum, then tungsten (combined column depth $1,712 \mathrm{~g} / \mathrm{cm}^{2}$ ). First we use the collinear approximation to get the energy distribution of all of the charmed particles produced in the hadronic interaction. In a second approximation, we account for the detector geometry of a $2 \mathrm{~m} \times 0.75 \mathrm{~m}$ detector downstream $51.5 \mathrm{~m}$ from the front of the target. The fragmentation and decays then proceed in the collinear approximation. For definiteness, we discuss $D_{s}$ production, but this applies more generally to charmed hadrons $h_{c}$. Given the differential cross section for target $A, d \sigma\left(p A \rightarrow D_{s} X\right) / d E_{D}$, the number of neutrinos produced per unit energy is approximately

$$
\phi_{\nu_{\tau}+\bar{\nu}_{\tau}} \simeq 2 \int_{E_{\nu}}^{E_{b}} d E_{D_{s}} \frac{N_{p}}{\sigma_{p A}} \frac{d \sigma_{p A \rightarrow D_{s} X}}{d E_{D_{s}}}\left(E_{b}, E_{D_{s}}\right) \times \sum_{i} \frac{d n_{i}}{d E_{\nu}}\left(E_{D_{s}}, E_{\nu}\right) .
$$

This approximate expression depends on $N_{p}$, the number of protons on target, the total $p A$ cross section, and the differential energy distribution of the $D_{s}$ (and its charge conjugate). The column depth of either sample target is much larger than the interaction lengths of the incident proton and the $D_{s}$, both of which are larger than the decay length of the $D_{s}$. Regenerated protons, emerging with lower energies from the first interaction, have been neglected in Eq. (7.1.9). As discussed below, the factor of two accounts for the sum of neutrinos and antineutrinos, equal for prompt decays since the energy distributions of $c$ and $\bar{c}$ are equal, as are the subsequent decay distributions. The summation of $d n_{i} / d E_{\nu}$ is over $i=$ direct or $i=$ chain decay energy distributions.

Over the full energy range of $E_{\nu}$ that come from $D_{s}$ decays, Eq. (7.1.9) gives

$$
N_{\nu_{\tau}+\bar{\nu}_{\tau}}\left(E_{\nu}^{m i n}=0\right)=\int_{0}^{E_{b}} d E_{\nu} \phi_{\nu_{\tau}+\bar{\nu}_{\tau}}=4 \times N_{p} \frac{\sigma_{p A \rightarrow c \bar{c} X}}{\sigma_{p A}} f_{D_{s}} B\left(D_{s} \rightarrow \tau \nu_{\tau}\right) .
$$

Here $f_{D_{s}}$ is the fragmentation fraction for $c \rightarrow D_{s}$. The factor of four accounts for $c+\bar{c}$ and the two neutrinos associated with each $D_{s}$ produced.

The interaction length of protons on a molybdenum target is $\lambda_{p M o}=A_{M o} /\left(N_{A} \sigma_{p M o}\right)=156$ $\mathrm{g} / \mathrm{cm}^{2}$ [156], giving a cross section per nucleon of $\sigma_{p N}=\sigma_{p M o} / A_{M o}=10.7 \mathrm{mb}$, corresponding to an approximate $A$ dependence of $A^{0.71}$. The $A$ dependence of the charm production cross section is approximated by $\sigma_{p A \rightarrow h_{c} X} \simeq A \sigma_{p N \rightarrow h_{c} X}$ for $A_{M o}=95.95$ [950, 951]. For $D_{s}$ production, we use NLO QCD [952-954] as implemented in the numerical program of Cacciari et al. [955, 956], to calculate $d \sigma_{p N \rightarrow h_{c} X} / d E_{c}$ and the Kniehl and Kramer fragmentation functions (LO at the initial scale $\mu_{0}$ ) [957] to convert to the hadronic energy distribution. The normalizations of the fragmentation functions are rescaled to match the fragmentation fractions of Ref. [958]. In particular, the fragmentation fraction for $c \rightarrow D_{s}$ is set to $f_{D_{s}}=0.077$.

Inputs to the differential cross section are the charm mass, the factorization scale $M_{F}$, the 
renormalization scale $\mu_{R}$ and the parton distribution functions. We take $m_{c}=1.27 \mathrm{GeV}$ [156] and $\left(M_{F}, \mu_{R}\right)=\left(n_{F}, n_{R}\right) m_{c}$ with values guided by Ref. [959]. In Ref. [959], Nelson, Vogt and Frawley vary the factorization and renormalization scales to determine the range of scales consistent with fixed target and RHIC data on charm production. We use the scale combinations $\left(n_{F}, n_{R}\right)=(1.25,1.48)$ (dashed), $(2.1,1.6)$ (solid) and $(4.65,1.71)$ (dot-dashed) that bracket the data, as shown in Fig. 7.4(a), for protons incident on an isoscalar target. The data are shown from the compilation in Ref. [960] and from HERA-B in Ref. [950]. With these choices for the scale factors, the charm production cross section, evaluated with the CT10 NLO parton distribution functions [961] range between 15.1-20.1 $\mu \mathrm{b}$ for $\sqrt{s}=27.4 \mathrm{GeV}$. For $E_{b}=400 \mathrm{GeV}$, using the central (solid line) factorization scale, the charm production cross section per nucleon is $\sigma_{p N \rightarrow c \bar{c} X}=18.1 \mu \mathrm{b}$. We stress that the procedure outlined above cannot be considered as a genuine QCD prediction in the usual sense. This is because the overall normalization of the cross section is an input taken from data that provide it with an uncertainty lower then the current theoretical uncertainty on the $c \bar{c}$ cross section for high energy hadron collisions. Nevertheless, we consider NLO QCD corrections reliable for correlating measurements at different energies first and, second, to correlate the overall normalization of the charm production cross section with the tail of the energy distribution of the charmed particles produced. For the incident proton beam energy of $E_{b}=400 \mathrm{GeV}(\sqrt{s} \simeq 27.4$
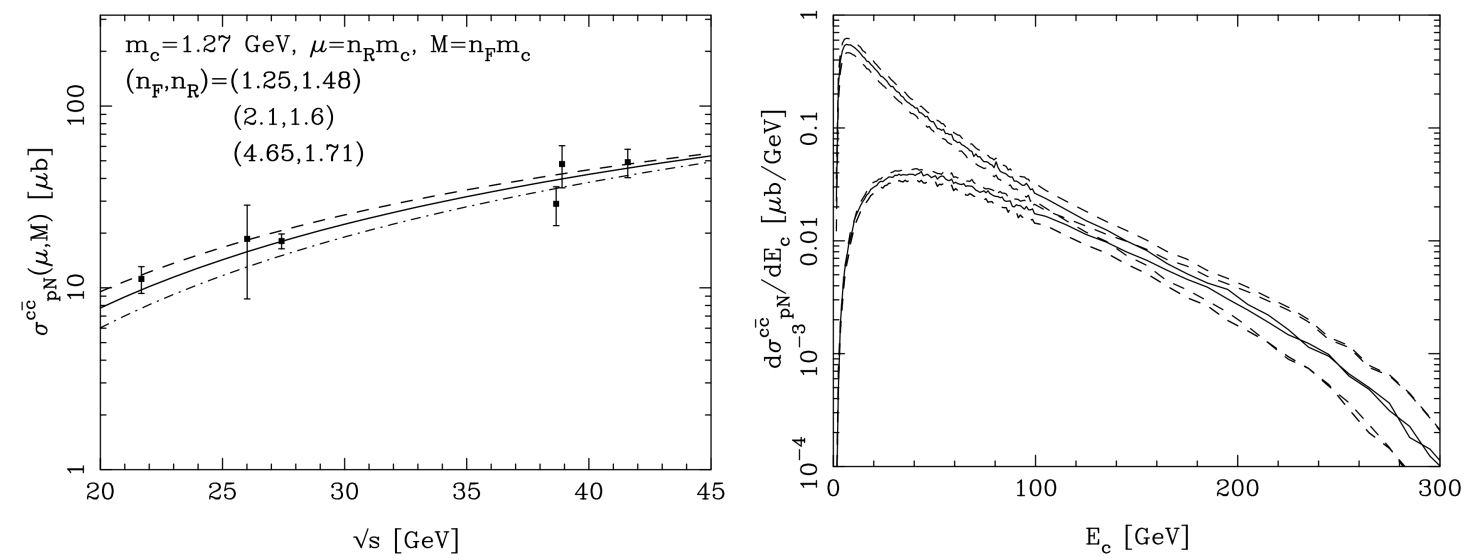

Figure 7.4: (a) The charm production cross section $\sigma_{p N \rightarrow c \bar{c}+X}$ at NLO with $m_{c}=1.27 \mathrm{GeV}$ using the CT10 parton distributions, as a function of $\sqrt{s}$. The central curve has factorization and renormalization scales $M_{F}=2.10 m_{c}$ and $\mu_{R}=1.6 m_{c}$, respectively. Data points are shown from the compilation of fixed target results collected in Ref. [960] and from HERA-B [950]. (b) The differential cross section $d \sigma(p A \rightarrow c \bar{c} X) / d E_{c}$ for the charmed quark, as a function of $E_{c}$ for $E_{b}=400 \mathrm{GeV}$. The upper solid line uses the central scale choices $\left(M_{F}, \mu_{R}\right)=(2.1,1.6) m_{c}$ and the collinear approximation, while the lower solid line uses the central scale choices with the detector direction requirement for the charmed quark momentum vector. The dashed lines are for $\left(n_{F}, n_{R}\right)=(1.25,1.48)$ and $(4.65,1.71)$ to represent a range of uncertainty in the NLO perturbative evaluation with $m_{c}=1.27 \mathrm{GeV}$.

$\mathrm{GeV}$ ), the differential energy distributions of the outgoing charmed quark are shown in Fig. 7.4(b). The upper curves show the energy distribution in the collinear approximation. The lower solid line is the same distribution with the requirement that the charmed quark momentum vector intersects the detector $51.5 \mathrm{~m}$ downstream from the front of the target. For $E_{c} \simeq 10 \mathrm{GeV}$, the lowest dashed curve is about $\sim 20 \%$ lower than the solid curve. At $100 \mathrm{GeV}$, there is a wider range, from a few percent higher than the solid curve, to $\sim 30 \%$ lower. The scale dependences of the differential charm production cross section contribute to the tau neutrino flux uncertainties. The $D_{s}$ and leptonic tau 
decay distributions have simple forms [962]. For example, $D_{s}$ decay to $\nu_{\tau}$ has

$$
\frac{d n_{\text {direct }}}{d E_{\nu}}\left(E_{D_{s}}, E_{\nu}\right)=\frac{1}{\Gamma\left(E_{D_{s}}\right)} \frac{d \Gamma\left(E_{D_{s}}, E_{\nu}\right)}{d E_{\nu}}=\frac{1}{E_{D_{s}}} F_{D_{s}}^{\nu}\left(\frac{E_{\nu}}{E_{D_{s}}}\right)
$$

where

$$
F_{D_{s}}^{\nu}(x)=B\left(D_{s} \rightarrow \nu_{\tau} \tau\right) /\left(1-R_{\tau}\right) \times \theta\left(1-R_{\tau}-x\right)
$$

for $R_{\tau}=m_{\tau}^{2} / m_{D_{s}}^{2}$. The energy distribution $F_{D_{s}}^{\nu}$ is the same for neutrinos and antineutrinos. For $\tau$ decays to neutrinos, the decay distribution of $\tau^{-}$at rest differs from the decay distribution of $\tau^{+}$. Because of the opposite helicities of $\nu_{\tau}$ and $\bar{\nu}_{\tau}$ in the $D_{s}$ decay, the polarizations of $\tau^{-}$ and $\tau^{+}$are also opposite. The net effect is that in the laboratory frame, the neutrino energy distribution in the chain decay $D_{s}^{+} \rightarrow \tau^{+} \rightarrow \bar{\nu}_{\tau}$ is the same as for $D_{s}^{-} \rightarrow \tau^{-} \rightarrow \nu_{\tau}$ in the absence of depolarization [962, 963]. For the tau decay, in the energies of interest here, electromagnetic energy loss is negligible (see, for example, Ref. $[964,965]$ ), so the polarization of the produced tau is retained in the evaluation of the tau decays. Therefore, the $\nu_{\tau}$ and the $\bar{\nu}_{\tau}$ fluxes from both the direct $D_{s} \rightarrow \nu_{\tau}$ and the chain decays are identical. A summary of the function forms for $D_{s} \rightarrow \tau \rightarrow \nu_{\tau}$ can be found in Ref. [966]. We include the leptonic decays of the tau and the two body decays of $\left.\tau \rightarrow\left(\pi, \rho, a_{1}\right) \nu\right) \tau$, overall accounting for $\sim 87 \%$ of the tau decays. The parameters for the charm fragmentation and the PDG central values for the relevant branching ratios of the tau lepton are reported in Appendix A.

Taking $N_{p}=2 \times 10^{20}$ protons, our results for the number of neutrinos per unit energy are shown in Fig. 7.5. We use Eq. (7.1.9) to find $\phi_{\nu}$ for $\nu=\nu_{\tau}+\bar{\nu}_{\tau}$ and $\nu=\nu_{\mu}+\bar{\nu}_{\mu}$ from $h_{c}=$ $D^{0}, \bar{D}^{0}, D^{ \pm}, D_{s}^{ \pm}, \Lambda_{c}^{ \pm}$, were we take the relativistic limit. Following Ref. [967], we approximate the decays of the charmed mesons to $\nu_{\mu}$ as approximate three-body decays. We take the $\nu_{\mu}$ energy distribution equal to the muon energy distribution parameterized in Ref. [967]. For the $\Lambda_{c}$, we also approximate the decay by a three-body distribution. As indicated above, we use the collinear approximation, and with Eq. (7.1.9), we account only for the first $p A$ interaction to produce $c \bar{c}$.

The $D_{s} \rightarrow \tau \rightarrow \nu_{\tau}$ decay chain dominates the direct $D_{s} \rightarrow \nu_{\tau}$ contribution above a few $\mathrm{GeV}$. With $D_{s}$ decays, the tau neutrino flux equals the tau antineutrino flux. The charmed hadron contributions to the muon neutrino flux are similar in shape. The $D^{ \pm}$and the $D^{0}+\bar{D}^{0}$ contributions to $\nu_{\mu}+\bar{\nu}_{\mu}$ are nearly equal.

Fig. 7.6 shows the integral of $\phi_{\nu}$ from $E_{\nu}=E_{\nu}^{\min } \rightarrow 400 \mathrm{GeV}, N_{\nu}\left(E_{\nu}^{\min }\right)$, for $\nu=\nu_{\tau}+\bar{\nu}_{\tau}$ (lower curves) and $\nu=\nu_{\mu}+\bar{\nu}_{\mu}$ (upper curves) for $\left(n_{F}, n_{R}\right)=(2.1,1.6)$. Overall, in the collinear approximation, these parameters give $5.7 \times 10^{15} \nu_{\tau}+\bar{\nu}_{\tau}$ for $N_{p}=2 \times 10^{20}$ protons for all neutrino energies, and $1.8 \times 10^{15}$ for $E_{\nu} \geq 4 \mathrm{GeV}$.

The relative number of neutrinos of the two flavor shifts as a function of energy, and there is a fairly strong dependence on factorization and renormalization scales. As an estimate of the theoretical error in the flux predictions, the quantity $\phi_{\nu}$ as a function of $E_{\nu}$ for $\nu=\nu_{\tau}+\bar{\nu}_{\tau}$, for the central scale choices $M_{F}=2.10 m_{c}$ and the scale variation given above are shown in Fig. 7.7. One expects additional uncertainties associated with the choice of charmed quark mass and fragmentation functions, the latter especially at high neutrino energies where the flux is low. The additional uncertainties from the fragmentation fraction $(<10 \%)$ and branching fraction for $D_{s} \rightarrow \tau \nu_{\tau}(<5 \%)$ are however small compared to the scale dependence. The ratio of $\nu_{\tau} / \nu_{\mu}$ is much less sensitive to the scale dependence than the absolute flux. For the integrated flux, the ratio differs by just a few percent for $E_{\nu}^{\min }=10 \mathrm{GeV}$ and by about $20 \%$ for $E_{\nu}^{\min }=50 \mathrm{GeV}$. Therefore, the relative contributions by neutrino flavor of the prompt fluxes should help pin down the tau neutrino flux. 


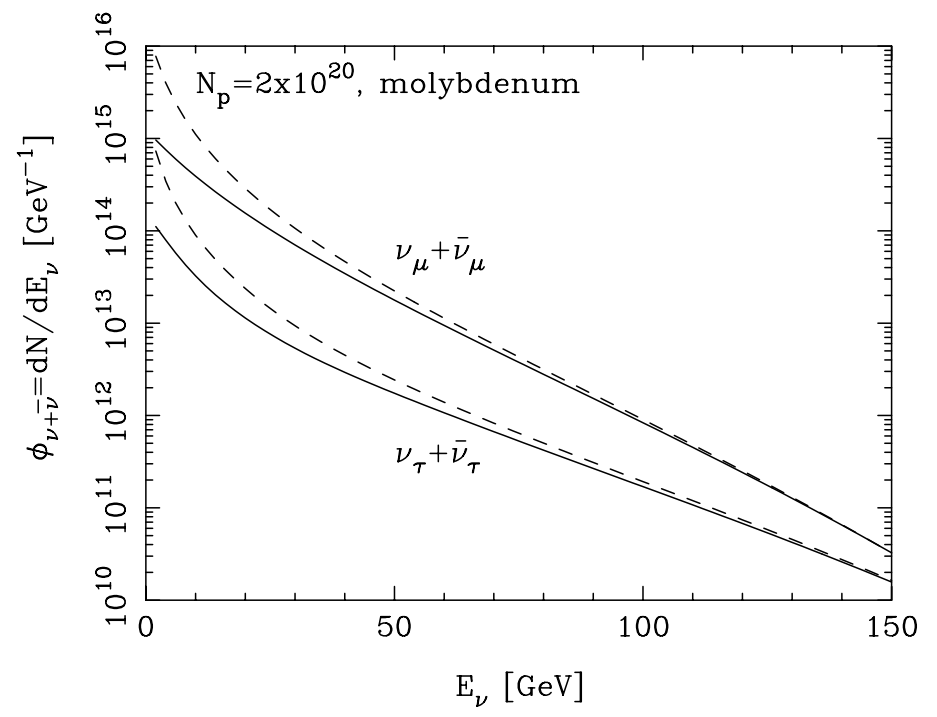

Figure 7.5: The number of neutrinos plus antineutrinos per unit energy, $\phi_{\nu+\bar{\nu}}$, as a function of energy for $\nu+\bar{\nu}=\nu_{\tau}+\bar{\nu}_{\tau}$ (lower curves) and $\nu+\bar{\nu}=\nu_{\mu}+\bar{\nu}_{\mu}$ (upper curves) for $N_{p}=2 \times 10^{20}$ from charmed hadron decays, with $M_{F}=2.10 m_{c}$ and $\mu_{R}=1.6 m_{c}$ for a molybdenum target. The contribution from $D_{s} \rightarrow \tau \rightarrow \nu_{\tau}$ dominates the tau neutrino flux for most of the energy range. The dashed lines use the collinear approximation, while the solid lines account for the detector direction requirement for the charmed quark momentum vector.

\subsubsection{Expected sensitivity}

The theoretical uncertainty associated to the cross sections plotted in Fig. 7.2 are mainly related to the scale choices, to the Parton Distribution Functions (PDF's) uncertainty and the modeling of the target mass corrections. Furthermore, the disentanglement of the nuclear effects will also be a source of uncertainty in the extraction of the cross section from the measurements; we estimate that these sources will give all together an uncertainty of $\sim 10 \%$ or less for a large interval of relevant neutrino energies. The largest source of uncertainty will be then given by the one on the knowledge of the fluxes $\sim 20 \%$. To estimate the inclusive number of events that might be collected with the neutrino detector, we take the convolution of the flux entering the surface of the detector with the cross sections above.

Using the tau neutrino plus antineutrino fluxes of Fig. 7.7 including the detector geometry restriction, the number of events is

$$
N_{\text {evts }}=\int_{E_{\min }}^{E_{\max }} d E_{\nu}\left(\phi_{\nu} \frac{L_{P b}}{\lambda_{\nu P b}}+\phi_{\bar{\nu}} \frac{L_{P b}}{\lambda_{\bar{\nu} P b}}\right)
$$

where $\phi_{\nu}=\phi_{\bar{\nu}}=\phi_{\nu+\bar{\nu}} / 2$ for tau neutrinos. For $M=1$ ton $=10^{3} \mathrm{~kg}$, and $\rho_{P b}=11.35 \mathrm{~g} / \mathrm{cm}^{3}$, the depth of the detector is $d=5.87 \mathrm{~cm}$ for a cross sectional detector area of $2.0 \mathrm{~m}$ by $0.75 \mathrm{~m}$. The column depth of the lead is $L_{P b}=d \rho_{P b}=66.7 \mathrm{~g} / \mathrm{cm}^{2}$. The interaction length of neutrinos in lead is $\lambda_{\nu P b}=A /\left(N_{A} \sigma_{\nu A}\right)$ for $A=207$ and $Z=82$, with $\sigma_{\nu A} / A$ given in Eq. (7.1.6).

Fig. 7.8 and 7.9 show the separate neutrino and antineutrino contributions to the integrand in Eq. (7.1.13), and Fig. 7.10 shows the sum of the two terms. Fig. 7.11 shows the number of events for 


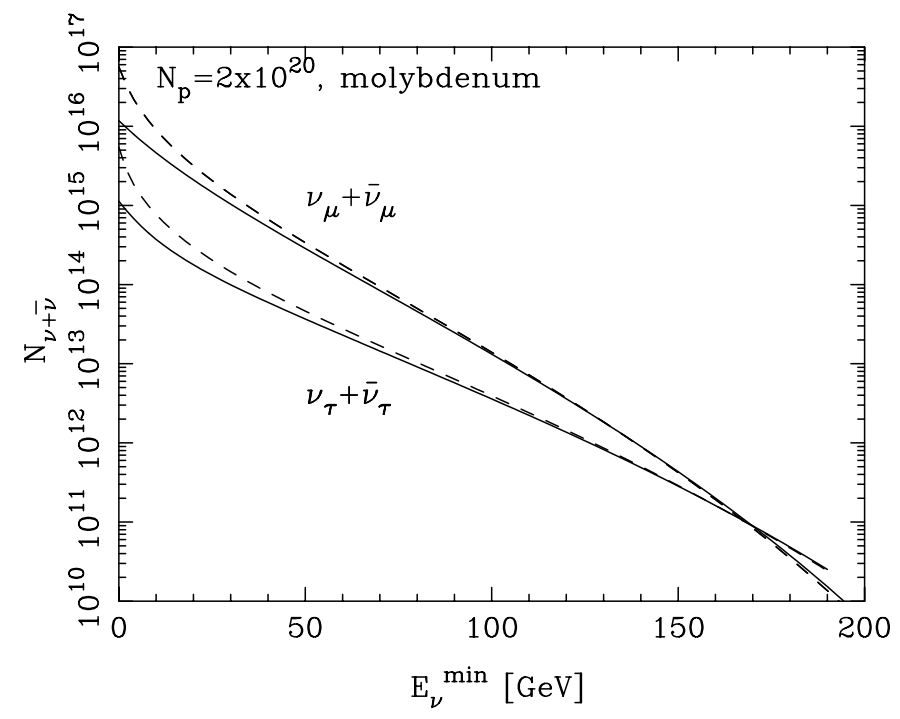

Figure 7.6: The integral of $\phi_{\nu+\bar{\nu}}$ as a function of $E_{\nu}^{\text {min }}$ of the prompt tau and muon neutrino plus anti-neutrino fluxes for $N_{p}=2 \times 10^{20}$ on a molybdenum target. The upper curves are $\nu+\bar{\nu}=\nu_{\mu}+\bar{\nu}_{\mu}$ and lower curves are $\nu+\bar{\nu}=\nu_{\tau}+\bar{\nu}_{\tau}$, as in Fig. 7.5.

the central scale choice (solid lines in Figs. 7.8 and 7.9) integrated in $5 \mathrm{GeV}$ bins from $E=5 \mathrm{GeV}$ to $E=200 \mathrm{GeV}$. The sum of the number of events is 936 events for the central scale choice (685 tau neutrino interactions and 252 tau antineutrino interactions). For $\left(n_{F}, n_{R}\right)=(4.65,1.71)$, there are 723 events (529 tau neutrino and 194 tau antineutrino), while for $\left(n_{F}, n_{R}\right)=(1.25,1.48)$, we find 1151 events (840 tau neutrino and 311 tau antineutrino). The number of neutrino and antineutrino interactions corresponding to Figs. 7.8 and 7.9, integrated over energy in $5 \mathrm{GeV}$ intervals, is shown in Fig. 7.11. The sum of the entries in each bin, from $5 \mathrm{GeV}$ through the $195-200 \mathrm{GeV}$ bin, gives the total number of events quoted above.

We note that for a good range of neutrino energies among 10 and $50 \mathrm{GeV}$, where a significant fraction of CC tau neutrino DIS interactions is expected, the theoretical uncertainty on the flux, that is the largest one, can be estimated to be uniform of the order of $20 \%$. We assume that the current knowledge of the structure functions $F_{1}, F_{2}$ and $F_{3}$ from previous precise experimental measurements in $\nu_{\mu}$ and $\bar{\nu}_{\mu}$ CC has a much smaller error, parametrized in terms of scale and PDF uncertainties, to be lower then $10 \%$. By subtracting the contribution of $F_{1}, F_{2}$ and $F_{3}$ from the expected number of events observed, that is about twice the expected contribution of $F_{4}$ and $F_{5}$, we deduce that in SHiP there will be the opportunity to asses the cumulative impact of the structure functions $F_{4}$ and $F_{5}$ differentially in the interval of energies among 10 and $50 \mathrm{GeV}$. In the left panel of Fig. 7.12 we show the ratio of the averaged $\nu_{\tau}$ and $\bar{\nu}_{\tau}$ cross sections on lead as a function of the incident neutrino energy, while in the right panel the same ratio is plotted for $\bar{\nu}_{\tau}$ cross section only. Systematic uncertainties on these ratios are expected to cancel and, given the relatively large number of events expected, we assume that the theoretical error is the dominant source of uncertainty. Under these assumptions in the two plots we also included the lines for one and two standard deviations from the hypothesis of no contribution from $F_{4}$ and $F_{5}$ structure functions. However, we point out that, even including radiative corrections, $F_{4}$ is not expected to give an important contribution for the cross section in the Standard Model, indeed it contributes 


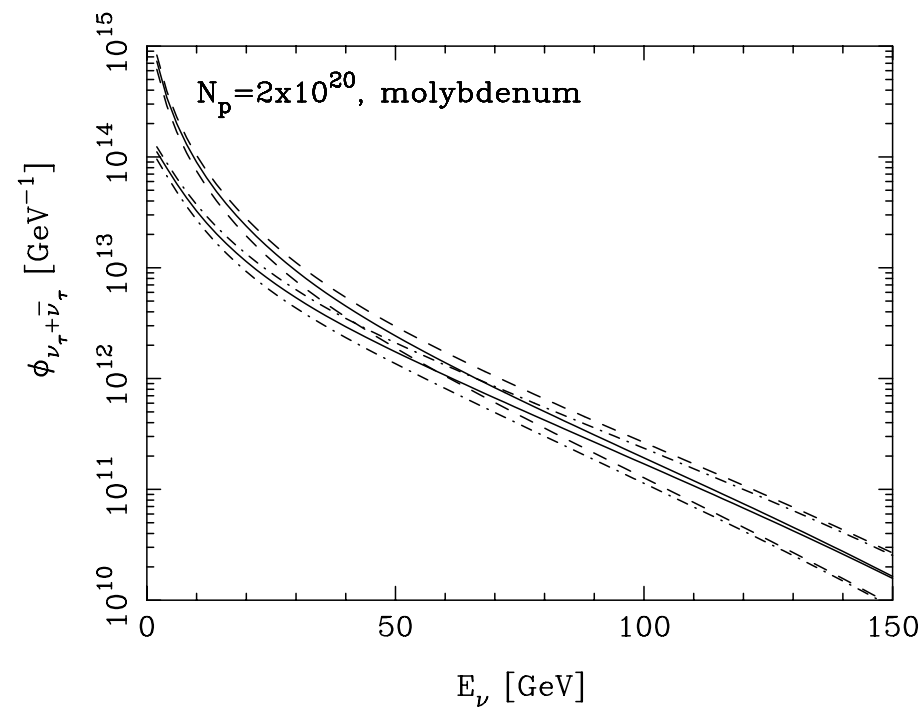

Figure 7.7: The flux $\phi_{\nu+\bar{\nu}}$ as a function of $E_{\nu}$ for $\nu+\bar{\nu}=\nu_{\tau}+\bar{\nu}_{\tau}$, for the central scale choices, $M_{F}=2.10 m_{c}$ and $\mu_{R}=1.6 m_{c}$ (solid line) for a molybdenum target and $N_{p}=2 \times 10^{20}$ protons. The upper curves used the collinear approximation with other scale choices shown with the dashed lines, while the lower curves account for the detector direction requirement for the charmed quark momentum vector and other scale choices (dot-dashed lines).

$-5 \%$ at $5 \mathrm{GeV}$, but only $1 \%$ at $10 \mathrm{GeV}$ and further decreasing with energy. As a final remark we stress here that the total number of events quoted above is affected by the geometrical acceptance and the collinear approximation used. Including a more detailed description of the $D_{s} \rightarrow \tau \rightarrow \nu_{\tau}$ chain would lower the expected number of events estimated here. On the other hand the collinear approximation that we have adopted here does not spoil the relative theoretical uncertainties quoted. 


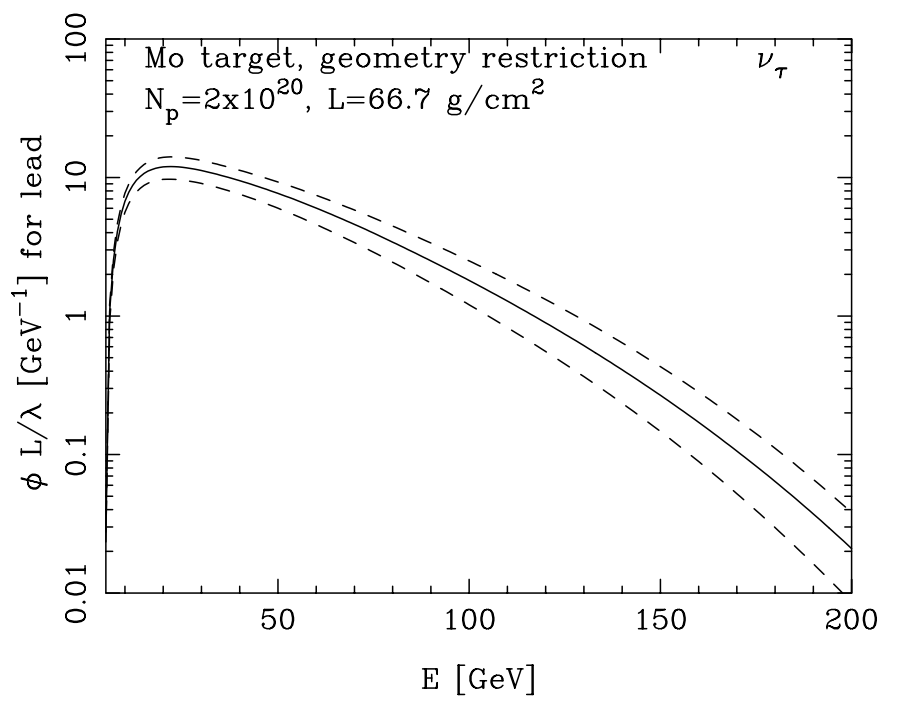

Figure 7.8: The quantity $\phi_{\nu} \times L / \lambda$ as a function of $E_{\nu}$ for for the central scale choices, $M_{F}=2.10 m_{c}$ and $\mu_{R}=1.6 m_{c}$ (solid line). The scale choices $\left(n_{F}, n_{R}\right)=(1.25,1.48)$ and $(4.65,1.71)$ are shown with the dashed lines. The flux is evaluated for a molybdenum target and $N_{p}=2 \times 10^{20}$ protons with the detector geometry restriction on the charmed quark momentum. The lead detector column depth is taken to be $L=66.7 \mathrm{~g} / \mathrm{cm}^{2}$.

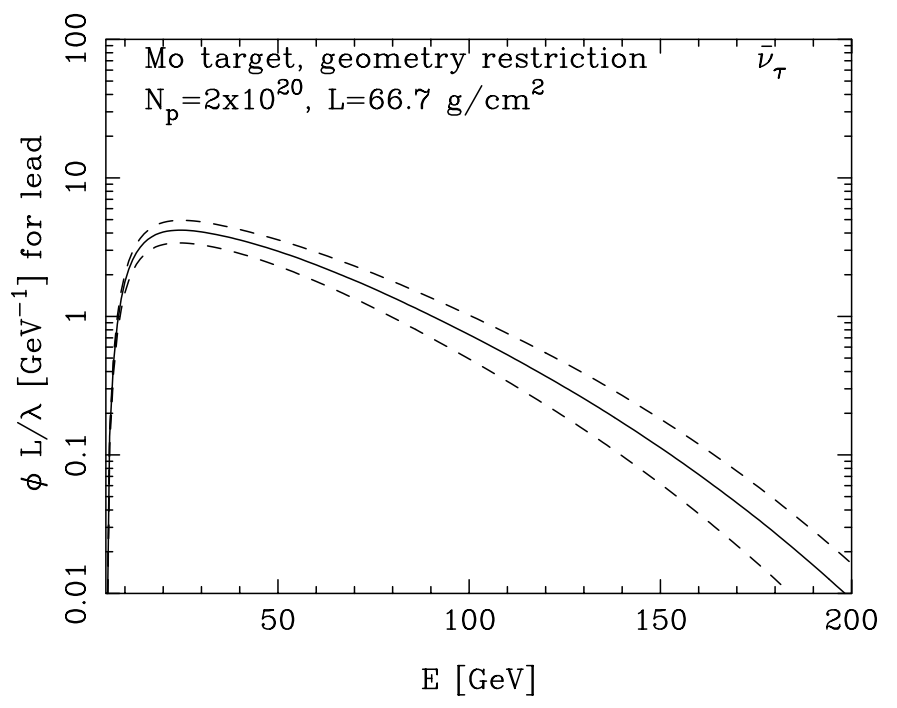

Figure 7.9: As in Fig. 7.8, for anti-neutrinos. 


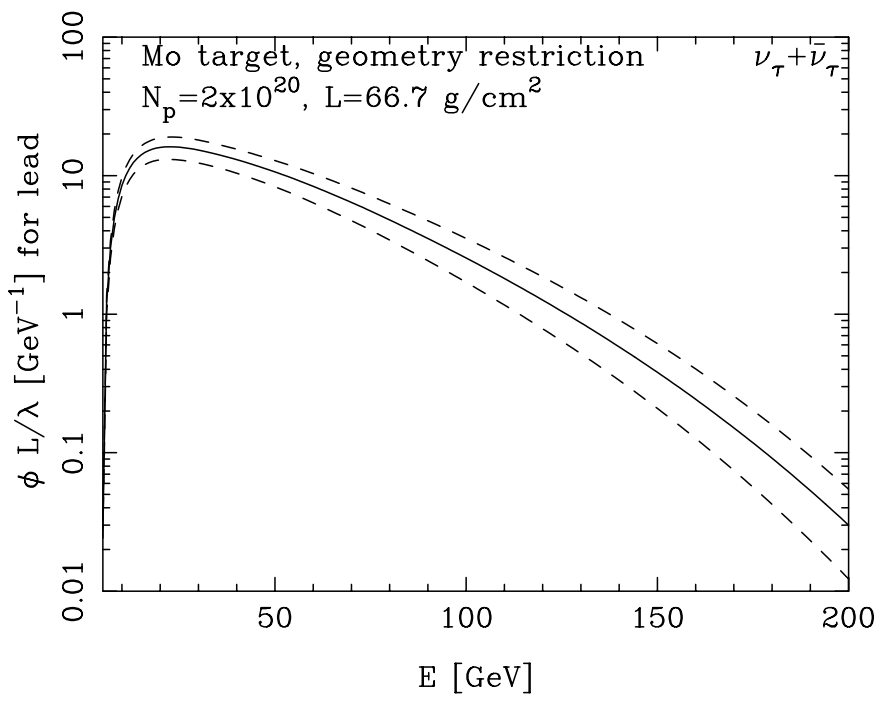

Figure 7.10: The quantity $\phi_{\nu} \times L / \lambda_{\nu}+\phi_{\bar{\nu}} \times L / \lambda_{\bar{\nu}}$ as a function of $E_{\nu}$ for for the central scale choices, $M_{F}=2.10 m_{c}$ and $\mu_{R}=1.6 m_{c}$ (solid line). The scale choices $\left(n_{F}, n_{R}\right)=(1.25,1.48)$ and $(4.65,1.71)$ are shown with the dashed lines. The flux is evaluated for a molybdenum target and $N_{p}=2 \times 10^{20}$ protons with the detector geometry restriction on the charmed quark momentum. The lead detector column depth is taken to be $L=66.7 \mathrm{~g} / \mathrm{cm}^{2}$. 


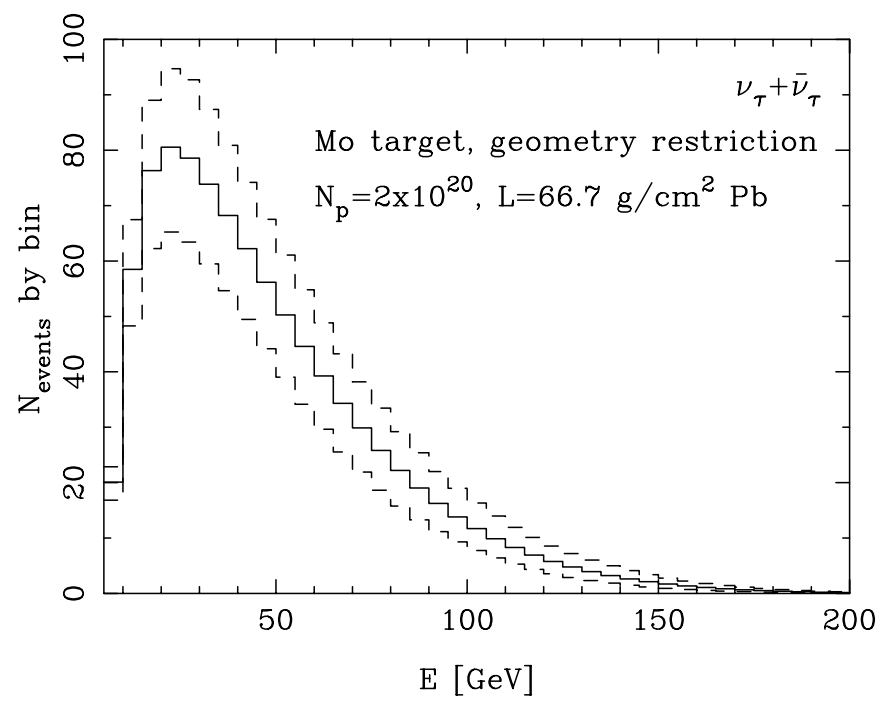

Figure 7.11: The number of events in each $5 \mathrm{GeV}$ energy bin for $E_{\nu}=5-200 \mathrm{GeV}$ for for the central scale choices, $M_{F}=2.10 m_{c}$ and $\mu_{R}=1.6 m_{c}$. The scale choices $\left(n_{F}, n_{R}\right)=(1.25,1.48)$ and $(4.65,1.71)$ are shown with the dashed lines. The flux is evaluated for a molybdenum target and $N_{p}=2 \times 10^{20}$ protons with the detector geometry restriction on the charmed quark momentum. The lead detector column depth is taken to be $L=66.7 \mathrm{~g} / \mathrm{cm}^{2}$.
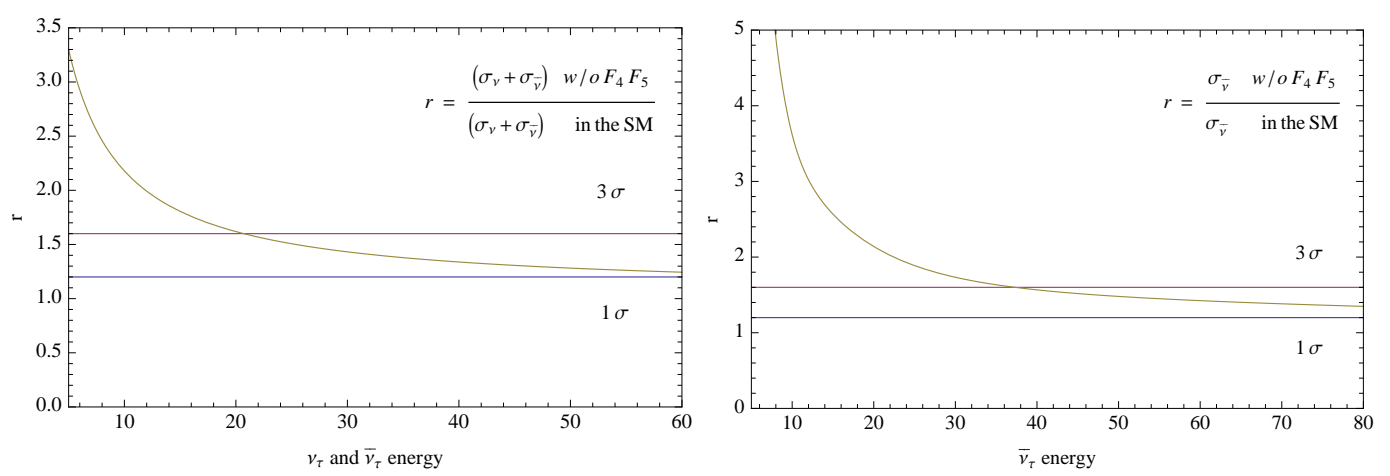

Figure 7.12: Left panel: ratio of the averaged tau neutrino cross sections off lead as a function of the incident neutrino energy. Right panel: the same ratio for the anti-neutrino cross section. 


\subsection{Deep inelastic muon and electron neutrino scattering}

Historically, the formulation of QCD as the gauge theory of the strong interactions [968, 969] has been closely related to the early deep-inelastic scattering (DIS) experiments and the observed scaling violations in inclusive structure functions, which parametrize the hadronic interaction. Over time experiments with lepton- and neutrino scattering off fixed targets at CERN, FNAL and SLAC as well as collisions of electrons and positrons on protons at DESY's HERA collider have provided unique information on the nucleon structure. The available experimental data is of very high precision. At the same time, it spans a large range in the kinematics of the Bjorken- $x$ variable and of the measured values of momentum transfer $Q^{2}$ between the scattered lepton or neutrino and the nucleon.

The current picture of the proton structure from DIS data is complemented by measurements at the Large Hadron Collider (LHC) for a number of hadro-production processes. These include, for instance, cross sections for the hadro-production of jets or differential distributions for the production of $W^{ \pm}$- and $Z$-gauge bosons, possibly in association with charm quarks. These data can contribute to our understanding of the flavor composition of the nucleon, it can constrain the gluon distribution at large values of Bjorken- $x$ and it is sensitive to the value of the strong coupling constant $\alpha_{s}\left(M_{Z}\right)$.

Dramatic further improvements in different ranges of kinematics can be expected from a number of planned future colliders. The proposal for a Large Hadron Electron Collider (LHeC) to collide an electron beam of $60 \mathrm{GeV}$, up to possibly $140 \mathrm{GeV}$, with the intense hadron beams of the LHC, would allow for coverage of a kinematic range extended by a factor of twenty in $Q^{2}$, and in the inverse Bjorken- $x$, if compared to HERA, and with great physics potential [970] regarding high precision DIS measurements.

The design of the planned electron-ion collider EIC aims instead at center-of-mass energies in the range from 50 to $140 \mathrm{GeV}$, but allows to collide electrons and positrons with polarized protons and with light to heavy nuclei at high luminosity. Major research themes of the EIC are the spin and flavor structure of the proton as well as three-dimensional images of the nucleon structure [971].

The planned SHiP experiment [35] is distinct from these activities to the extend that it realizes intense neutrino beams. Beyond establishing for first time the direct observation of tau antineutrinos, the resulting neutrino-nucleon DIS interactions from intense electron and muon neutrino beams will allow to study the strangeness of the nucleon. Besides the tau neutrino events discussed in the previous subsection, the $\mathrm{SHiP}$ experiment is expected to collect $\mathcal{O}(2 \mathrm{M})$ charged current interactions from muon neutrinos and $\mathcal{O}(1 \mathrm{M})$ from electron neutrinos, the latter with an average energy of the interacting neutrinos around $30 \mathrm{GeV}$. Previous studies of the physics potential with neutrino beams have focused on experiments at the front-end of a muon storage ring [972] and date back to the pre-LHC era, so that an update is mandatory. In the following we will review the status of perturbative QCD computations and the most important DIS measurements from muon and electron neutrinos that can be performed in the SHiP experiment.

\subsubsection{Status of perturbative QCD calculations}

The key observables for tests of perturbative QCD are either inclusive structure functions or, in the semi-inclusive case, differential cross sections, which describe the hard hadronic interaction in the QCD improved parton model. The particle data group (PDG) [156] provides a summary of DIS, including the definitions of kinematic variables.

Precision predictions in perturbative QCD rest on factorization. Sensitivity to the dynamics at scales of the proton mass is separated from hard high-energy scattering at a large scale of the momentum transfer $Q^{2}$. In Fig. 7.13 this is illustrated for lepton-nucleon DIS in the one-boson exchange approximation. With the help of factorization at a scale $\mu$ the structure functions $F_{k}$ 


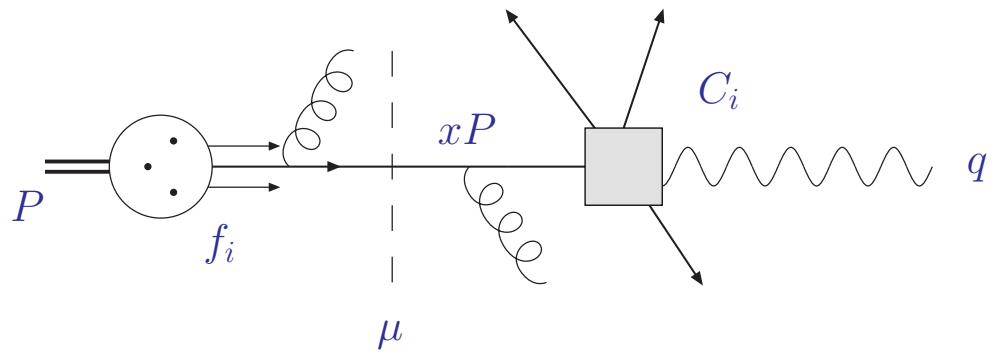

Figure 7.13: QCD factorization of the cross section for the scattering of a deeply virtual gaugeboson with (space-like) momentum $q\left(-q^{2}=Q^{2}>0\right)$ off a proton with momentum $P$ in their center-of-mass frame, see Eq. (7.2.1).

$(k=2,3, L)$ can be expressed as convolutions of parton distributions (PDFs) $f_{i}(i=q, \bar{q}, g)$ in the nucleon and short-distance Wilson coefficient functions $C_{k, i}$,

$$
F_{k}\left(x, Q^{2}\right)=\sum_{i=q, \bar{q}, g} \int_{x}^{1} d z f_{i}\left(\frac{x}{z}, \mu^{2}\right) C_{k, i}\left(z, Q^{2}, \alpha_{s}(\mu), \mu^{2}\right)
$$

in so-called leading twist approximation up to power corrections of $\mathcal{O}\left(1 / Q^{2}\right)$. Such higher twist contributions are relevant in the analysis of DIS data at low $Q^{2}$, see, e.g., [973].

QCD factorization has also been established for differential distributions in DIS, e.g., for semiinclusive DIS where the cross section $d^{2} \sigma / d x d Q^{2}$ is subject to a decomposition similar to Eq. (7.2.1). In the differential case, the hard parton scattering cross sections are augmented by an additional prescription for any exclusive final state parton, e.g., a jet algorithm or fragmentation functions, in order to ensure infrared finiteness.

The coefficient functions $C_{k, i}$ in Eq. (7.2.1) can be calculated perturbatively in powers of the strong coupling constant $\alpha_{s}$,

$$
C=C^{(0)}+\alpha_{s} C^{(1)}+\alpha_{s}^{2} C^{(2)}+\alpha_{s}^{3} C^{(3)}+\ldots
$$

where the expansion coefficients $C^{(0)}$ are denoted as the leading order (LO), $C^{(1)}$ as the next-toleading order (NLO) and so on. The PDFs $f_{i}$ are non-perturbative objects describing the nucleon momentum fraction $x=Q^{2} /(2 P \cdot q)$ (see Fig. 7.13) carried by the quark or gluon and have to be determined in global fits to experimental data. Within perturbation theory, PDFs at different scales are related, though, by the well-known evolution equations,

$$
\frac{d}{d \ln \mu^{2}}\left(\begin{array}{c}
f_{q_{i}}\left(x, \mu^{2}\right) \\
f_{g}\left(x, \mu^{2}\right)
\end{array}\right)=\sum_{j} \int_{x}^{1} \frac{d z}{z}\left(\begin{array}{cc}
P_{q_{i} q_{j}}(z) & P_{q_{i} g}(z) \\
P_{g q_{j}}(z) & P_{g g}(z)
\end{array}\right)\left(\begin{array}{c}
f_{q_{j}}\left(x / z, \mu^{2}\right) \\
f_{g}\left(x / z, \mu^{2}\right)
\end{array}\right)
$$

where the so-called splitting functions $P_{i j}$ enter to account for the different possible parton splittings in the collinear limit. The $P_{i j}$ are universal quantities in QCD and, like the $C_{k, i}$, can be computed in a power series in $\alpha_{s}$,

$$
P=\alpha_{s} P^{(0)}+\alpha_{s}^{2} P^{(1)}+\alpha_{s}^{3} P^{(2)}+\ldots
$$

The QCD predictions for DIS observables have reached an unprecedented level of precision. The Wilson coefficients $C_{k, i}$ and the splitting functions $P_{i j}$ are known to high enough orders in perturbation theory, so that the effect of radiative corrections on structure functions is well under 
control. The splitting functions $P_{i j}$ in Eq. (7.2.4) are known to next-to-next-to-leading order (NNLO) [974, 975] and, likewise, the coefficient functions $C_{k, i}[976-979]$ in Eq. (7.2.2). Moreover, for photon and charged current $W^{ \pm}$-boson exchange, the hard corrections at order next-to-next-tonext-to-leading order $\left(\mathrm{N}^{3} \mathrm{LO}\right)$ are also available [980-982].

For the production of heavy quarks in DIS, like charm, mass effects need to included. The theoretical description of DIS heavy-quark production in the kinematical range of SHiP can be based on the fixed-flavor-number (FFN) factorization scheme with three light quarks in the initial state and the heavy-quarks appearing in the final state only. The respective Wilson coefficients for the heavy-quark electro-production are known to NLO completely [983, 984] and approximately to [985], the latter based on the massive operator matrix elements at three loop order [986, 987]. This ensures the exact description of all logarithmically enhanced terms in the heavy-quark mass $m$ up to $\alpha_{s}^{3} \ln ^{k}\left(Q^{2} / m^{2}\right)$ with $k=1, \ldots, 3$. Similarly, the heavy-quark coefficient functions for charm quark production in charged current DIS are known exactly at NLO [988-990] and at asymptotic values of $Q^{2} \gg m^{2}$ at NNLO [991-993]. The exact quark mass dependence for charm quark production in charged current DIS to NNLO is not available at present, though, it could, in principle, be extracted from Ref. [994]. Available QCD theory predictions and experimental data for inclusive

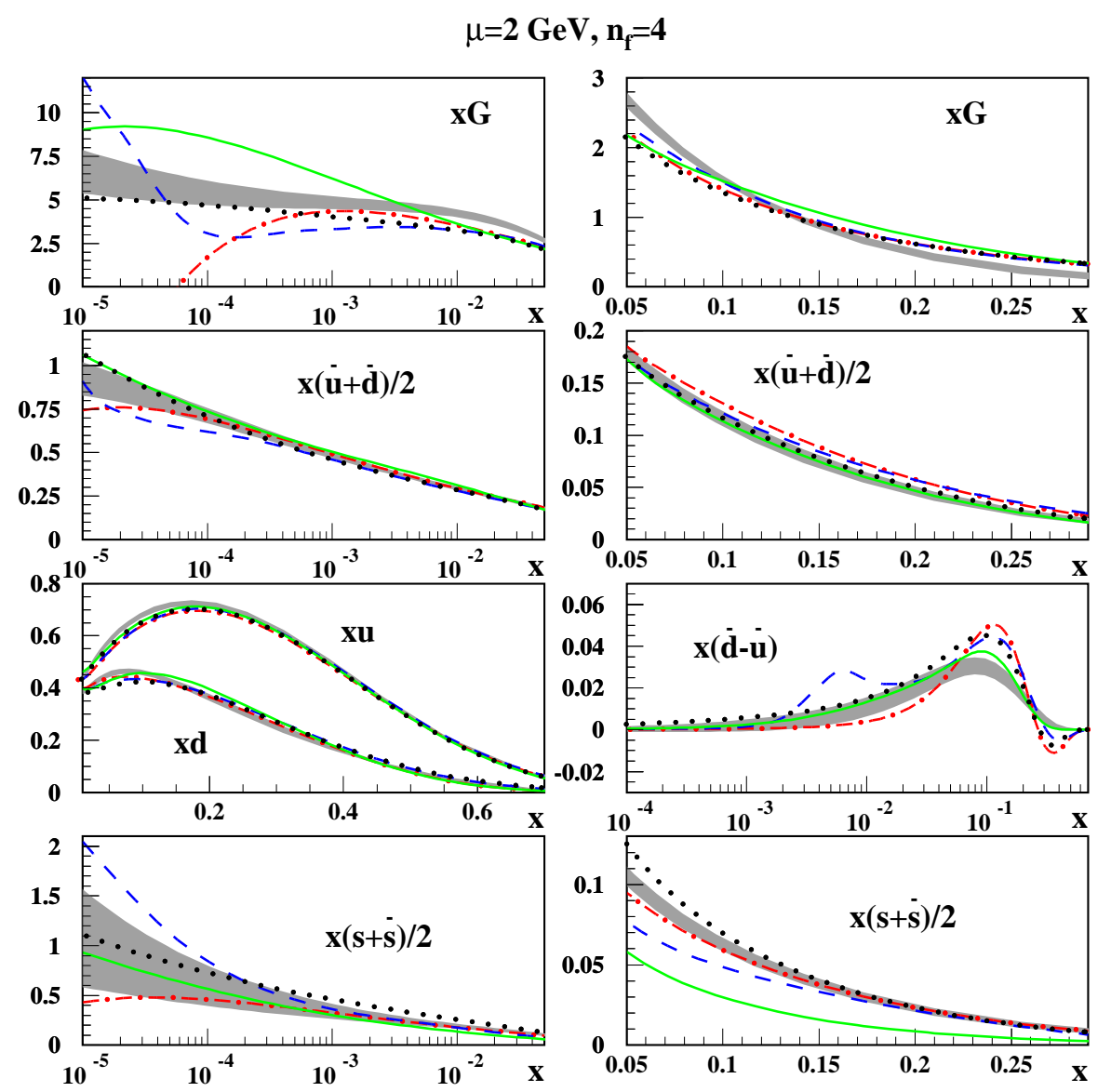

Figure 7.14: The $1 \sigma$ band obtained for the 4-flavor NNLO ABM12 PDFs [995] at the scale of $\mu=2 \mathrm{GeV}$ versus $x$ (shaded area) compared with the central fits obtained by JR09 [996] (solid lines), MSTW [997] (dashed dots), NNPDF (v2.3) [998] (dashes), and CT10 [999] (dots). Plot taken from [995].

DIS allow to deduce important information about PDFs or the value of the strong coupling constant $\alpha_{s}\left(M_{Z}\right)$ as the non-perturbative input to QCD precision predictions. This is, of course, a situation 
that, generally, needs to be addressed beyond DIS, since experimental data from the (anti-)protonproton colliders Tevatron at FNAL and the LHC provide invaluable further constraints, e..g., on the flavor composition of the proton. Analyses of the proton structure are, therefore, carried out as global fits, which are typically accurate to NNLO in QCD. Results for such NNLO PDF sets have been obtained by ABM11 [973], ABM12 [995], CT10 [999], HERAPDF (v1.5) [1000, 1001], JR09 [996], MSTW [997], NNPDF (v2.3) [998] and the recent updates MMHT 2014[1002] and NNPDF (v3.0)[1003]. Some of the results are displayed in Fig. 7.14. Given the current status of

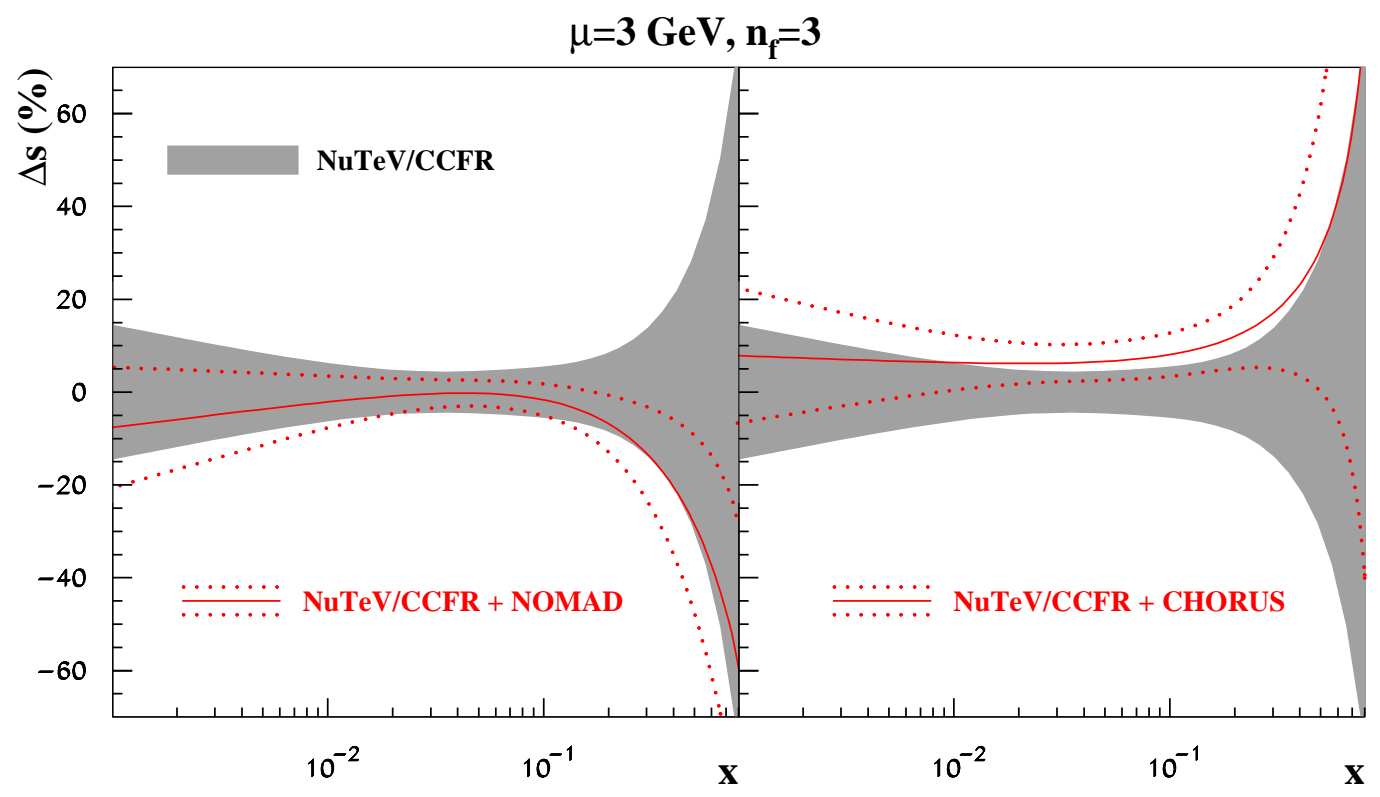

Figure 7.15: The relative strange sea uncertainty obtained from variants of the ABM12 PDF analysis [995] with only NuTeV and CCFR data [1004] employed to constrain the strange sea (grey area) and with the solid lines displaying the relative change in the strange sea due to the NOMAD [1005] (left panel) and CHORUS [1006] (right panel) data sets. The dots correspond to the strange sea uncertainty after inclusion of the new data sets. Plot taken from [993].

perturbative QCD and the precision of the experimental data a future physics program of neutrino nucleon DIS as in the SHiP experiment can help to address a number of very relevant questions. For PDFs improvements can be made with respect to the flavor asymmetry of sea quarks, in particular the strangeness. The information on the strange sea quark content of the nucleon shown in Fig. 7.14 relies almost entirely on the data by the $\mathrm{NuTeV}$ and CCFR experiments [1004] from charm di-muon production in (anti)neutrino-iron DIS interactions. The recent publication of new data samples for charm di-muon production in neutrino-iron DIS interactions by the NOMAD experiment [1005] and for inclusive charm production in nuclear emulsions by the CHORUS experiment [1006] shown a clear improvement of the situation as illustrated in Fig. 7.15. Note that, however, even if combining $\mathrm{NuTeV} / \mathrm{CCFR}$ data with the NOMAD or CHORUS data the bands shrink, the two new best fit represented in Fig. 7.15 appear to go in opposite directions. For this reason we believe that only with a new and large data sample we might have a definitive leap in our knowledge of the nucleon strangeness.

\subsubsection{Strangeness from heavy-quark DIS in CC interactions}

Anti-charm production in charged current anti-neutrino interactions selects anti-strange quark in the nucleon. This is because while $\bar{d}$ quarks are not privileged with respect to the $\bar{s}$ quarks in the proton sea, the latter are favoured to produce charm by the Cabibbo angle. The process is 
sketched in Fig. 7.16. Precise knowledge of the strangeness is an important information for many

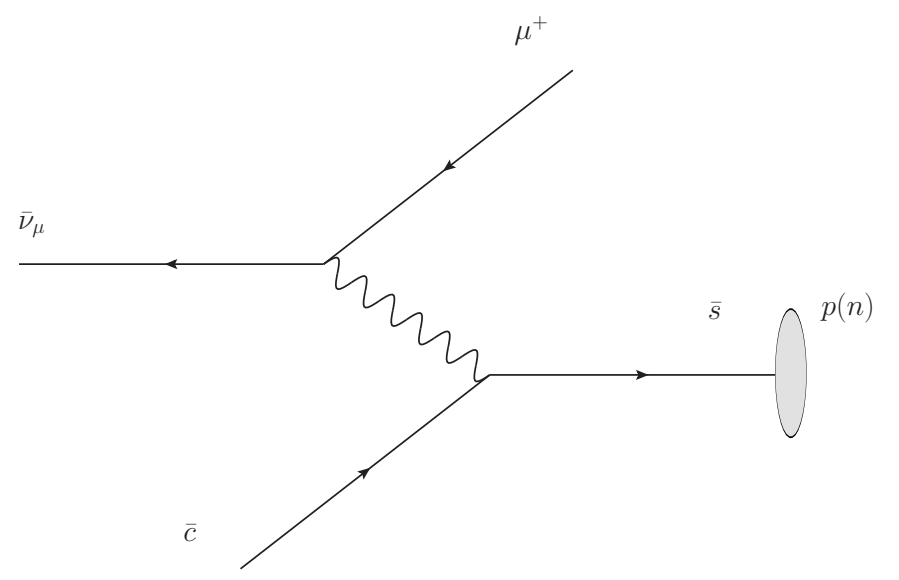

Figure 7.16: Diagram for anti-charm production in anti-neutrino charged current interactions.

precision tests of the SM as well as for BSM searches at the LHC. The $W$ mass is a fundamental parameter of the model and at the LHC $W$ boson production proceeds for the $80 \%$ through the $u \bar{d}$ channel and $20 \%$ through the $c \bar{s}$ channel. For a detailed discussion about the strong impact of the uncertainty of the strange quark content of the proton on $W$ mass measurements see for example Ref. [1007]. In Fig. 7.17 the current situation for the proton strangeness $(s+\bar{s})$ is summarized including uncertainty bands. Although the four PDF collaborations: NNPDF3.0, MMHT, CT10 and ABM12, use approximately the same data points, the final results are quite different in the $x$ range $(0.001,0.1)$. The strange see quark determination by all the PDF groups relies mainly on the

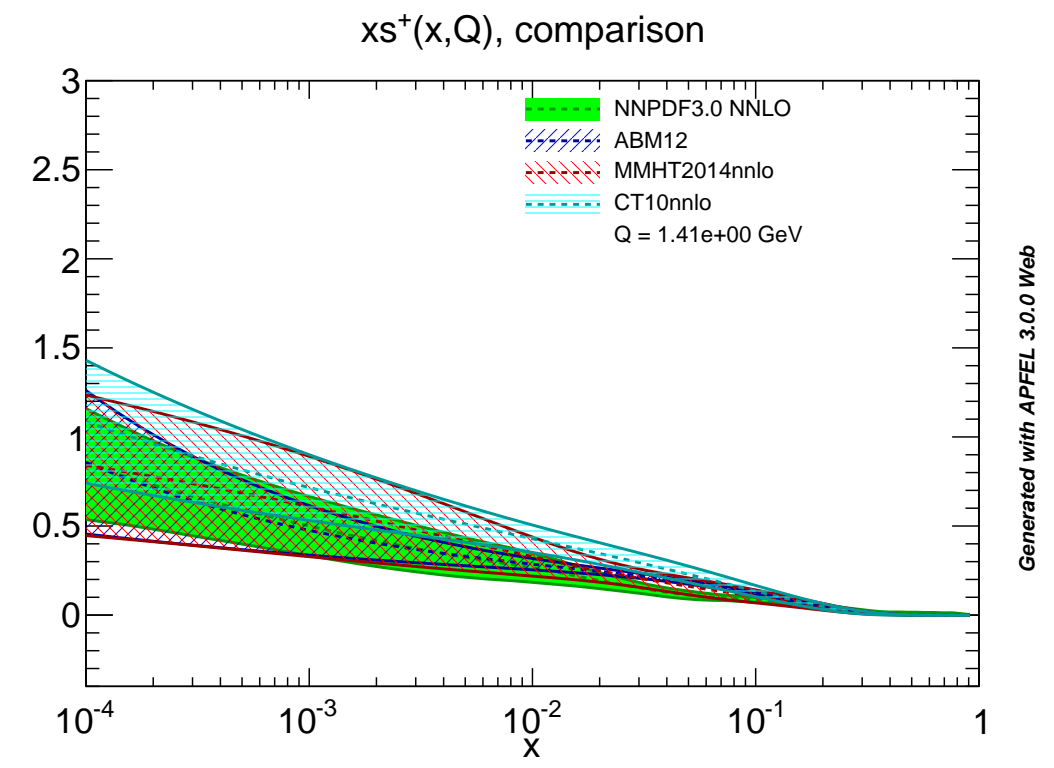

Figure 7.17: $s+\bar{s}$ distribution in the proton.

dimuon data collected by the $\mathrm{NuTeV} / \mathrm{CCFR}$ collaboration. Despite the high number of charged current $\nu_{\mu}$ and $\bar{\nu}_{\mu}$ events, 1.280 .000 and 270.000 respectively, the detection of charm production was limited by the selection based on the muon decay channel for the charmed resonances produced. 
Furthermore a cut of $5 \mathrm{GeV}$ was imposed for the muon identification. For these reasons only 5.102 $\nu_{\mu}$ and $1.458 \bar{\nu}_{\mu}$ events were identified as charm production in charged current interactions.

The neutrino detector under consideration in SHiP would have a lower cut for the muon identification and, more important, would allow the detection of any decay of the produced charmed hadrons. For these reasons, even if the number of expected CC muon neutrino events is comparable with the ones detected by $\mathrm{NuTeV} / \mathrm{CCFR}$ the charmed data collection in SHiP will be much larger. Indeed, in the whole data taking of the SHiP experiment 1.8 million of $\nu_{\mu}$ and 0.6 million of $\bar{\nu}_{\mu}$ events are expected [1008]. This would translate into the order of 70 thousands charm and 27 thousands anti-charm produced. Furthermore, the electron neutrino charged current are also expected to contribute about 20 thousands induced charm production. Under these assumptions we expect a considerable improvement in the knowledge of the nucleon strangeness. In the following we consider only the contribution of the CC muon neutrino interactions.

To be more specific, we used a simulated data sample normalized to $2 \times 10^{20} \mathrm{POT}$ to estimate number and the distribution of muon neutrino interactions with charm production. We followed closely the NuTeV analysis [1004] where the collected data were divided in five bins for the $x$ variable, three for $y$ and three energies per event sample. In SHiP the energies will be lower than $\mathrm{NuTeV}$, nevertheless distributing the events in bins with an equal content we find similar values for the other two variables $x$ and $y$. As a result we estimate that the statistical uncertainty in each bin will reduce from $\sim 10-20 \%$ in [1004] for both $\nu_{\mu}$ and $\bar{\nu}_{\mu}$ interactions, to $\sim 2-3 \%$ for charm production and $\sim 4-5 \%$ for anti-charm production. Note that in the $\mathrm{NuTeV}$ analysis the systematic error was dominant. To make a realistic estimation of the level of improvement in our analysis we included also a systematic uncertainty. We assessed the potential impact of the simulated charm data by adding them to the NNPDF3.0 NNLO fit [1003] using the Bayesian reweighting technique introduced and described in $[1009,1010]$. Pseudodata are generated fluctuating the central value around the central NNPDF3.0 NNLO predictions. Their distribution is assumed to be a gaussian with central value 0 and standard deviation equal to the sum in quadrature of the statistical uncertainty inferred from simulation, and the total systematic uncertainty, assumed to be uniform $5 \%$ (and uncorrelated) for all pseudodata points. In the method of Refs. $[1009,1010]$ one first generate an ensamble of unweighted replicas representing the probability distribution function under consideration. It is then possible to attribute a weight expressing the relative probability of the new data for different PDF's. Finally one can use the weights to sample the new probability density using the original replica reweighted. The constraing power of the SHiP pseudodata is measured by the Shannon entropy of the reweighted set, which measures the number of effective $\left(N_{\text {eff }}\right)$ replicas after reweighting. In this analysis, starting from a prior set with 1000 replicas, we obtain $N_{\text {eff }}=62$. The reweighted $s^{+}$and $s^{-}$distributions are showed in Fig. 7.18. The reduction of the associated uncertainty is significant in the ranges $0.03-0.3$ for $s^{+}$and $0.08-0.3$ for $s^{-}$respectively.

\subsubsection{Nuclear effects in $\nu N$ DIS and global analyses of nuclear PDFs}

High statistics neutrino deep-inelastic scattering experiments have generated significant interest in the literature as they provide crucial information for global fits of parton distribution functions as has been discussed in the preceding section. The neutrino DIS data provide the most stringent constraints on the strange quark distribution in the proton [1011], and allow for a flavor decomposition of the PDFs which is essential for precise predictions of the benchmark gauge boson production processes at the LHC. Moreover, the neutrino experiments have been used to make precision tests of the Standard Model (SM) in the neutrino sector. A prominent example is the extraction of the weak mixing angle $\theta_{W}$ in a Paschos-Wolfenstein type analysis. A good knowledge of the neutrino DIS cross sections is also very important for long baseline experiments of the next generation which aim at measuring small parameters of the NMS mixing matrix such as the mixing angle $\theta_{13}$ with more precision and eventually the $\mathrm{CP}$ violating phase $\delta$. 

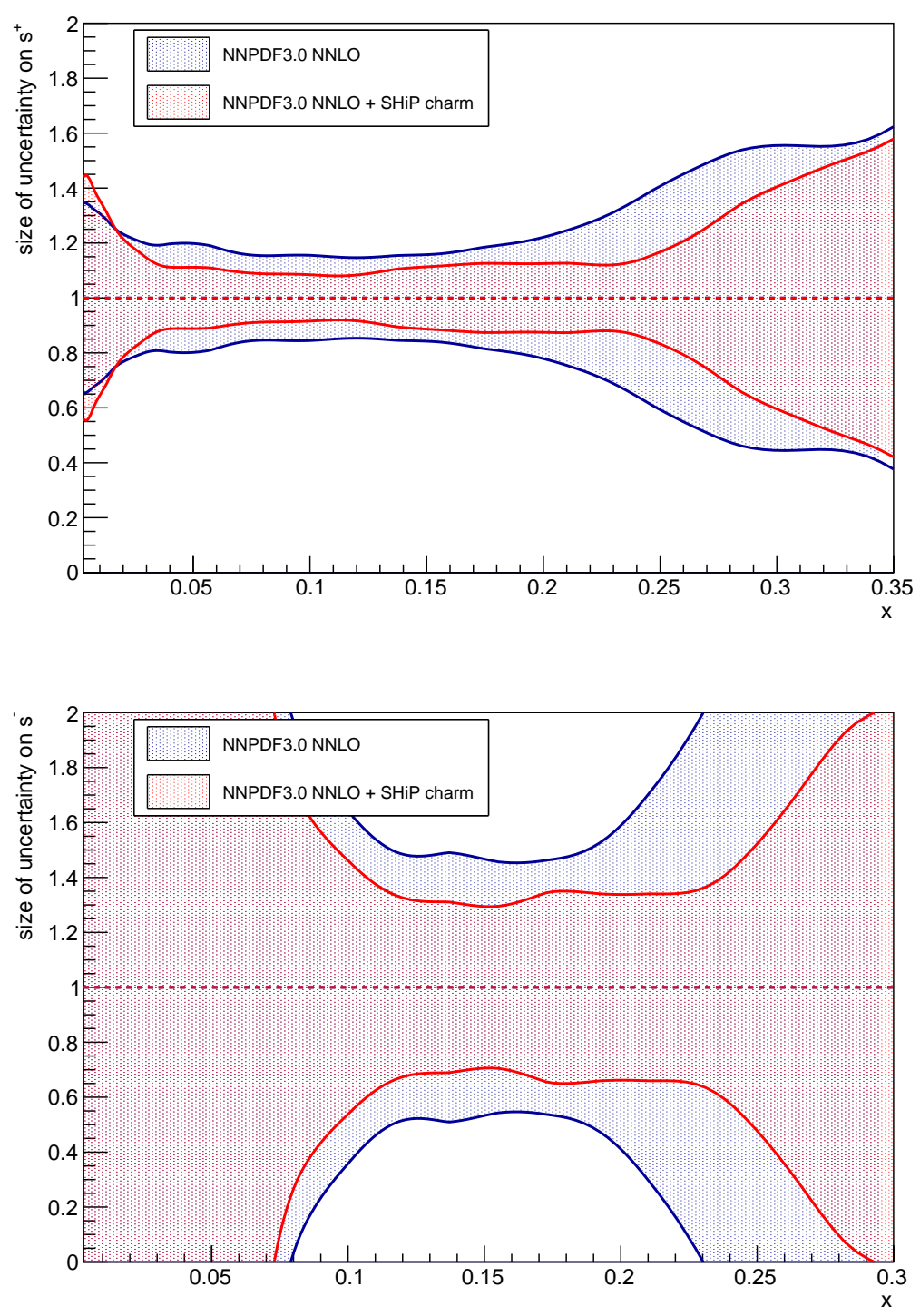

Figure 7.18: $s+\bar{s}$ (top) and $s-\bar{s}$ (bottom) uncertainty bands with and without adding simulated charm data from the SHiP experiment to the NNPDF3.0 NNLO fit [1003].

Due to the weak nature of neutrino interactions the use of heavy nuclear targets is unavoidable, and this complicates the analysis of the precision physics discussed above since model-dependent nuclear corrections must be appplied to the data. Our understanding of the nuclear corrections relies largely on charged-lepton-nucleus $(\ell A)$ DIS data. In the early 80s, the European Muon Collaboration (EMC) [1012] found that the nucleon structure functions $F_{2}$ for iron and deuterium differ as a function of $x$. This discovery triggered a vast experimental program to investigate the nuclear modifications of the ratio $R\left[F_{2}^{\ell A}\right]=F_{2}^{\ell A} /\left(A F_{2}^{\ell N}\right)$ for a wide range of nuclear targets with atomic number $A$, see, e.g., Table 1 in [1013]. By now, such modifications have been established in a kinematic range from relatively small Bjorken $x\left(x \sim 10^{-2}\right)$ to large $x(x \sim 0.8)$ in the deepinelastic region with squared momentum transfer $Q^{2}>1 \mathrm{GeV}^{2}$. The behavior of the ratio $R\left[F_{2}^{\ell A}\right]$ can be divided into four regions: (i) $R>1$ for $x \gtrsim 0.8$ (Fermi motion region), (ii) $R<1$ for 

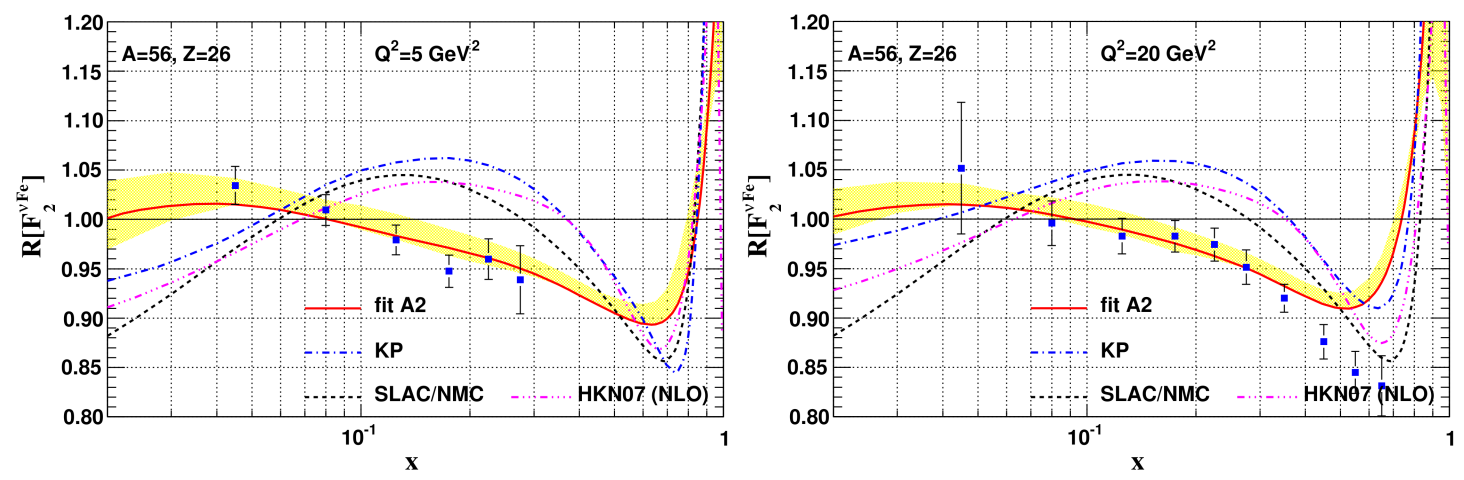

Figure 7.19: The nuclear correction factor $R\left[F_{2}^{\nu F e}\right] \simeq F_{2}^{\nu F e} / F_{2}^{\nu D}$ as a function of $x$ for $Q^{2}=$ $5 \mathrm{GeV}^{2}$ (left) and $Q^{2}=20 \mathrm{GeV}^{2}$ (right). The solid (red) line is the prediction of a fit to $\nu F e$ DIS data [1021]. This fit is compared with predictions based on $\ell^{ \pm} A$ DIS data. In particular, with the SLAC/NMC parametrization, the model from Kulagin and Petti (KP) [1022], and the predictions using the HKN'07 NPDFs [1020].

$0.25 \lesssim x \lesssim 0.8$ (EMC region), (iii) $R>1$ for $0.1 \lesssim x \lesssim 0.25$ (antishadowing region), and (iv) $R<1$ for $x \lesssim 0.1$ (shadowing region). Various models and physics mechanisms have been proposed to explain the nuclear modifications in the different kinematic regions. The shadowing suppression at small $x$ is generally assumed to occur due to coherent multiple scattering inside the nucleus of a $q \bar{q}$ pair coming from the virtual photon [1014] with destructive interference of the amplitudes [1015]. The antishadowing region is theoretically less well understood but might be explained by the same mechanism with constructive interference of the multiple scattering amplitudes [1015] or by the application of momentum, charge, and/or baryon number sum rules. Conversely, the modifications at medium and large $x$ are usually explained by nuclear binding and medium effects and the Fermi motion of the nucleons [1016], however, a complete understanding is still lacking and subject of ongoing investigations.

The charged-lepton-nucleus $(\ell A)$ DIS data form the backbone of global analyses of nuclear parton distribution functions (NPDFs) [1017-1020]. The NPDFs differ from the PDFs inside free nucleons such that the measured ratios of structure functions is reproduced. The NPDFs can be used to compute nuclear correction factors for different observables in a flexible way. Furthermore, as in the free nucleon case, neutrino-nucleus DIS data are very useful for a better flavor separation of the NPDFs. In particular, the opposite charge di-muon data from NuTEV and CCFR allow to constrain the nuclear strange quark distribution in an iron target.

However, in a recent analysis [1021] of $\nu F e$ DIS data from the NuTeV collaboration it was found that the nuclear correction factors are surprisingly different from the predictions based on the $\ell^{ \pm} F e$ DIS data with important implications for global analyses of proton PDFs. This can be seen in Fig. 7.19 where the nuclear correction factor $R\left[F_{2}^{\nu F e}\right] \simeq F_{2}^{\nu F e} / F_{2}^{\nu D}$ is shown as a function of $x$ for $Q^{2}=5 \mathrm{GeV}^{2}$ (left) and $Q^{2}=20 \mathrm{GeV}^{2}$ (right). Here, the solid line is the prediction of a fit to $\nu F e$ DIS data from the NuTeV collaboration [1021]. The other three curves are based on $\ell^{ \pm} A$ data and have quite a different $x$-shape. At the same time, the nCTEQ fits to $\ell A$ DIS and DY data [1018] are well in agreement with the charged-lepton data as can be seen in Fig. 7.20.

The study in [1021] left open the question, whether the neutrino DIS data could be reconciled with the charged-lepton DIS data by a better flavor separation of the NPDFs. This question was addressed in Ref. [1013] by performing a global $\chi^{2}$-analysis of the combined data from $\nu A$ DIS, $\ell A$ DIS and the DY process. This study confirmed that the neutrino DIS cross-section data are indeed slightly incompatible with the charged lepton DIS+DY data if the precision correlated errors of the neutrino data are taken into account. In case that the less precise uncorrelated errors are used the 

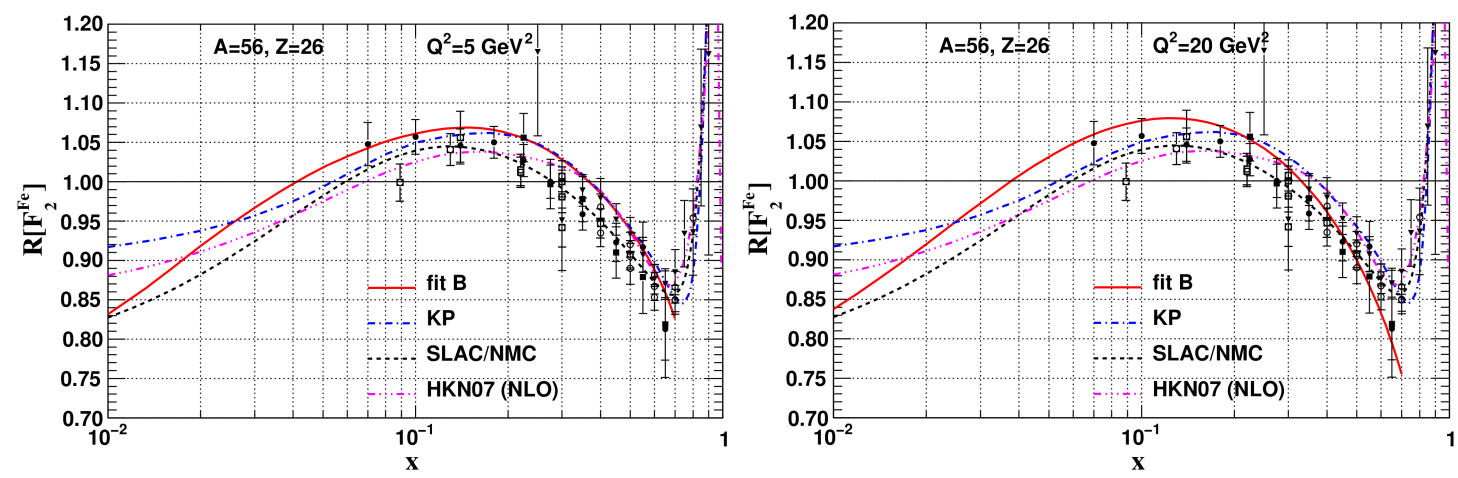

Figure 7.20: The nuclear correction factor $R\left[F_{2}^{\ell F e}\right] \simeq F_{2}^{\ell F e} / F_{2}^{\ell D}$ as a function of $x$ for $Q^{2}=5 \mathrm{GeV}^{2}$ (left) and $Q^{2}=20 \mathrm{GeV}^{2}$ (right). The solid (red) line is the prediction of a fit (fit B =decut3) to $\ell F e$ DIS data and Drell-Yan data in the $A$-dependent framework of [1018]. This fit is compared with predictions based on $\ell^{ \pm} A$ DIS data. In particular, with the SLAC/NMC parametrization, the model from Kulagin and Petti (KP) [1022], and the predictions using the HKN'07 NPDFs [1020].

tension between the NuTeV/CCFR and the charged-lepton DIS data sets disappears [1017, 1023].

In conclusion, precise measurements of neutrino-lead DIS cross sections with the SHiP experiment have a great potential to (i) improve the knowledge of NPDFs and (ii) help resolve the tension between the $\mathrm{NuTeV} / \mathrm{CCFR}$ data and charged-lepton DIS data. This would improve our knowledge of the nuclear correction factors needed for the proton global analyses. Most importantly, for the determination of the strange PDF in the nucleon using di-muon data a solid understanding of the nuclear modifications of the individual parton flavors is necessary. An improved understanding of these questions will then allow to test theoretical models predicting these nuclear modifications. An intriguing point is also that a large DIS data sample for lead together with proton-lead data from the LHC would allow dedicated determinations of lead PDFs without the need to model the nuclear $A$-dependence.

\subsection{4 $\alpha_{S}$ measurement via Gross-Llewellin Smith sum rule}

Inclusive quantities in DIS such as several sum rules are known to high orders in QCD. Of particular interest for neutrino-induced DIS is the Gross-Llewellyn Smith (GLS) sum rule, which is well studied in the literature. The GLS integral is given by

$$
S_{\mathrm{GLS}}\left(Q^{2}\right)=\frac{1}{2} \int_{0}^{1} d x\left(F_{3}^{\nu p}+F_{3}^{\nu n}\right),
$$

and is known to $\mathrm{N}^{3} \mathrm{LO}$ in QCD, see [1024], up to higher twist corrections.

New high statistics data from a DIS experiment can also be used for precision determinations of the strong coupling constant $\alpha_{s}$ exploiting Eq. (7.2.5). The current precision is reviewed, e.g., in [1025].

Measurements of $\alpha_{s}$ with the GLS sum rule need to account for the correlation of the value of $\alpha_{s}$ with higher twist contributions. A phenomenological parametrization of the higher twist contributions to structure functions considers them as independent from the leading twist ones

$$
F_{i}^{\mathrm{ht}}\left(x, Q^{2}\right)=F_{i}^{\mathrm{TMC}}\left(x, Q^{2}\right)+\frac{H_{i}^{\tau=4}(x)}{Q^{2}}+\frac{H_{i}^{\tau=6}(x)}{Q^{4}}+\ldots,
$$

where $F_{i}^{\mathrm{TMC}}$ are the structure functions at leading twist supplemented by kinematic corrections 
due to hadron mass effects i.e., the so-called target mass correction (TMC). The coefficients $H_{i}$ are some polynomial in $x$ and any $Q^{2}$-dependence of the higher twist operators due to the QCD evolution is neglected.

Various studies have quantified the effect of higher twist contributions on the value of $\alpha_{s}$ in global analysis, which generally depend on the order of perturbation theory considered. Twist six $H_{i}^{\tau=6}$ contributions are generally found to be consistent with zero in global fits, while the twist four terms $H_{i}^{\tau=4}$ can be significant, see e.g., [973, 1026]. For $\alpha_{s}$ measurements via the GLS sum rule additional uncertainties arise from extrapolation into the unmeasured kinematics region at small and large $x$.

Previous studies (cf. Sec.5 of [972]) for physics at the front-end of a neutrino factory have estimated the error on $\alpha_{s}$ measurements from Eq. (7.2.5) in a kinematical range similar to SHiP, i.e., $1.5 \leq Q^{2} \leq 80 \mathrm{GeV}$ to be

$$
\Delta \alpha_{s}=0.0035
$$

assuming the simultaneous determination of higher twist terms and $\mathcal{O}\left(\alpha_{s}^{2}\right)$ QCD corrections. If higher twist terms are fixed, the uncertainty reduces by roughly one third to $\Delta \alpha_{s}=0.0026$. Currently, even the $\mathrm{N}^{3} \mathrm{LO}$ coefficient functions are known, higher twist contributions are better understood and constrained, so that a significant further reduction of the uncertainty on $\alpha_{s}$ in Eq.(7.2.7) seems to be feasible. For these reasons the SHiP experiment could make an important contribution here.

\subsubsection{Precise Ratios for Neutrino Nucleon Interactions}

The SHiP experiment offers also the possibility to perform precise measurements of Electroweak parameters. An open question in particle physics is still the significance of the "NuTeV anomaly" which reported a $3 \sigma$ deviation in the measument of $\sin ^{2} \theta_{W}$. A theoretical resolution of the anomaly or new experimental measurements will be important. The SHiP experiment will be using high intensity $\nu$ and $\bar{\nu}$ beams over a large range of energies and will have the oppportunity to check and improve previous results.

The NuTeV analysis followed a simultaneous fitting of $R^{\nu(\bar{\nu})}=\sigma_{N C}^{\nu(\bar{\nu})} / \sigma_{C C}^{\nu(\bar{\nu})}$ which is related but not identical to the PW-relation [1027]

$$
R^{-}=\frac{\sigma_{N C}^{\nu}-\sigma_{N C}^{\bar{\nu}}}{\sigma_{C C}^{\nu}-\sigma_{C C}^{\bar{\nu}}}=\frac{1}{2}-\sin ^{2} \theta_{W}+\delta R_{A}^{-}+\delta R_{Q C D}^{-}+\delta R_{E W}^{-} .
$$

The original relation was derived for isoscalar targets but in the $\mathrm{NuTeV}$ experiment the target was Iron which brings the corrections included on the rhs of equation 7.2.8. The corrections have been studied in several articles and we will not repeat them here. We simply refer to the articles in Refs. [1028-1030]. Other ratios of neutral to charged current reactions are also determined accurately because the largest contribution is determined by isospin symmetry and the smaller terms are estimated by using available data and/or theoretical relations. These ratios can be used, with sufficient care, either to determine the Weinberg angle or to search for new effects manifested in the change of the ratios as function of energy or as function of the distance traveled by the neutrinos. An attractive ratio is [1031]

$$
P=\frac{\sigma_{N C}^{\nu}+\sigma_{N C}^{\bar{\nu}}}{\sigma_{C C}^{\nu}+\sigma_{C C}^{\bar{\nu}}}
$$

Before comparing the ratio above with the number of events detected in SHiP we have to take into account the differences in the $\nu$ and $\bar{\nu}$ fluxes. Indeed, first estimates based on full Monte Carlo 
simulations of the events in the proton target show that above $40 \mathrm{GeV}$ the fluxes of $\nu$ and $\bar{\nu}$ are not far from each other in the absolute values and have the same shape. We estimate that with the help of a detailed Monte Carlo simulation tuned to reproduce the number of charged leptons and anti-leptons detected respectively in the experiment, and the total number of neutral current events, one can correct for the relative difference in the fluxes and keep the uncertainty on $\sin ^{2} \theta_{W}$ associated to this procedure at the level of $1 \%$ or lower. Note that $\sin ^{2} \theta_{W}$ has been measured with $1 \%$ accuracy in neutrino nucleon scattering by CDHS [1032] and CHARM [1033] at CERN. We note that Monte Carlo uncertainty in signal modeling has also been used by other experiments to determine the level of purity of a muon neutrino beam with respect to muon antineutrinos and the electron neutrino contamination in a muon neutrino beam. Here, Monte Carlo simulations would address the difference in the energy spectra of the sum of muon and electron neutrinos and the antineutrinos. In the assumption that the electrons are identified, they do not represent a limitation. In general one has to correct the $P$ ratio above for quark mixing, quark sea effects, $c$-quark threshold effects, non-isoscalarity, $W-Z$ propagator differences, the finite muon mass, QED and EW radiative corrections. Indeed, quark sea effects and $c$-quark threshold effects could be measured within the same experiment with high level of accuracy and we are lead to the conclusion that after the flux asymmetry mentioned above the non-isoscalarity is the next higher source of uncertainty. As we will see below, the correction for non isoscalarity on the $P$ ratio is less then $1 \%$ so that the correction for the asymmetry in the fluxes sets the sensitivity on a possible measurement of $\sin ^{2} \theta_{W}$. An important property of this ratio is the disappearance of the interference terms between vector and axial-vector currents in both numerator and denominator. The weak hadronic current is given by

$$
J_{\mu}=x V_{\mu}^{3}+y A_{\mu}^{3}+\gamma V_{\mu}^{0}+\delta A_{\mu}^{0} .
$$

The currents $V_{\mu}^{3}$ and $A_{\mu}^{3}$ are the isospin partners of the charged currents and $V_{\mu}^{0}, A_{\mu}^{0}$ are iscalar components. The quark content of the currents is

$$
V_{\mu}^{3}=\frac{1}{2}\left(\bar{u} \gamma_{\mu} u-\bar{d} \gamma_{\mu} d\right) \quad A_{\mu}^{3}=\frac{1}{2}\left(\bar{u} \gamma_{\mu} \gamma_{5} u-\bar{d} \gamma_{\mu} \gamma_{5} d\right)
$$

and

$$
V_{\mu}^{0}=\frac{1}{2}\left(\bar{u} \gamma_{\mu} u+\bar{d} \gamma_{\mu} d\right) \quad A_{\mu}^{0}=\frac{1}{2}\left(\bar{u} \gamma_{\mu} \gamma_{5} u+\bar{d} \gamma_{\mu} \gamma_{5} d\right)
$$

In the Standard Model

$$
\begin{array}{ll}
x=1-2 \sin ^{2} \theta_{W} & y=-1 \\
\gamma=-\frac{2}{3} \sin ^{2} \theta_{W} & \delta=0 .
\end{array}
$$

As already mentioned the $A_{\mu}^{3} \otimes V_{\mu}^{0}$ term in Eq. (7.2.9) is eliminated for any target (even if the target is not isoscalar).

For the denominator in $P$ we write

$$
\sigma\left(\nu_{l} N \rightarrow l^{-} X\right)=A+I+V
$$

where $V$ comes from the vector current alone, $A$ from the axial current alone and the interference term $I$ is eliminated by the averaging over neutrinos and antineutrinos. The expression is written as

$$
P=\frac{1}{2} \frac{A+x^{2} V+\gamma^{2} S+2 x \gamma\left(V^{3} \otimes V^{0}\right)}{A+V}
$$

where $S$ comes from the current $V_{\mu}^{0}$ alone and the last term is the interference between $V_{\mu}^{3}$ and $V_{\mu}^{0}$. 
The last two currents also appear in electromagnetism and their contributions can be extracted from experimental data. Since the SHiP experiment will cover high energies we will estimate these terms using deep inelastic distribution functions. We can mention already that coefficients in front of the terms are smaller and thus reduce the uncertainties entering the final answer.

We can simplify the right hand side of Eq. (7.2.16) by appealing to propreties that we already known for the reactions.

(i) At high energies deep inelastic scattering dominates, so that the scattering off Nuclei is the incoherent sum over protons and neutrons (the EMC effect can be included separately). It is the incoherent sum of parton distribution functions on the quarks. The quark content of the currents $V_{\mu}^{0}$ and $V_{\mu}^{3}$ is the same except for a minus sign which for incoherent scattering on quarks is irrelevant. Thus

$$
V=S
$$

This means that the first three terms in the numerator of Eq. (7.2.16) see the isoscalar content of the target. Their contribution over neutrons and protons is proportional to the atomic number $A$.

(ii) The intereference in the last term of the numerator involves electromagnetic currents and is related to the difference of electron reactions on protons and neutrons. In fact in the average over equal numbers of protons and neutrons this term vanishes, thus only the excess of neutrons in the target nucleus contributes to the ratio making the correction small.

(iii) Defining

$$
\frac{d V^{e m}}{d x d y}=\frac{G^{2}}{\pi} \frac{Q^{4}}{4 \pi \alpha} \frac{\sigma^{e m}}{d x d y}
$$

we find

$$
\frac{d V_{e p}^{e m}}{d x}=\frac{2 G^{2} M E}{\pi}\left[\frac{4}{9}(u+\bar{u})+\frac{1}{9}(d+\bar{d})+\frac{1}{9}(s+\bar{s})\right] .
$$

Then normalizing the denominator in Eq. (7.2.16) to an isoscalar target with atomic number $A$ we obtain

$$
P=\frac{1}{2}\left\{1-2 \sin ^{2} \theta_{W}+\frac{20}{9} \sin ^{4} \theta_{W}-\lambda\left(1-2 \sin ^{2} \theta_{W}\right) \sin ^{2} \theta_{W}\right\},
$$

with $\lambda$ originating from the unequal numbers of protons $Z$ and neutrons $(A-Z)$ in the target. The functional form is

$$
\lambda=\frac{A-2 Z}{A} \frac{V_{p}^{e m}-V_{n}^{e m}}{\frac{1}{2}\left[\left(\sigma_{c c}^{\nu p}+\sigma_{c c}^{\bar{\nu} p}\right)+\left(\sigma_{c c}^{\nu n}+\sigma_{c c}^{\bar{\nu} n}\right)\right]} .
$$

All quantities are known and we can proceed to numerical estimates. For an isoscalar target $Z=A / 2$ and $\lambda=0$, then

$$
P=R_{1}=\frac{1}{2}\left\{1-2 \sin ^{2} \theta_{W}+\frac{20}{9} \sin ^{4} \theta_{W}\right\}
$$

If the target is made of lead we estimate $\lambda=0.042$ with an error at the level of a few $\%$, showing that the ratio is rather stable and non-isoscalarity correction introduces a per mille level uncertainty.

\subsection{Limit on Tau neutrino magnetic moment}

Neutrinos are electrically neutral fundamental particles that couple to the other particles only through weak interaction in the Standard Model (SM). Nevertheless, in the minimal extension of the SM where neutrinos are proposed to be Dirac particles, they can acquire a magnetic moment $\left(\mu_{\nu}\right)$ and give rise to electromagnetic interactions. This is possible because of loop diagrams in which a neutrino radiates a virtual $W$-boson transforming into a lepton and these combine again 
to give back the original neutrino [1034]. The intermediate particles can then couple to an external electromagnetic field. However, the magnetic moment produced in this manner is extremely small and is proportional to the mass of the neutrino [1034]:

$$
\mu_{\nu}=\frac{3 e G_{F} m_{\nu}}{8 \pi^{2} \sqrt{2}} \simeq\left(3.2 \times 10^{-19}\right)\left(\frac{m_{\nu}}{1 \mathrm{eV}}\right) \mu_{B}
$$

The magnetic moment of neutrinos can be enhanced in other new physics models (see, e.g., [1035]). An increase in the cross section $\sigma_{\nu e}$ for the elastic scattering of neutrino on electron, which involves only the neutral current in the SM, would imply a larger magnetic moment and would be a clear signal of new physics. The SM contribution to the energy $(T)$ distribution of the scattered electron in the laboratory frame is given by $[1036,1037]$

$$
\left.\frac{\sigma_{(\nu e, \bar{\nu} e)}}{d T}\right|_{S M}=\frac{G_{F}^{2} m_{e}}{2 \pi}\left[\left(g_{V} \pm g_{A}\right)^{2}+\left(g_{V} \mp g_{A}\right)^{2} \times\left(1-\frac{T}{E_{\nu}}\right)^{2}-\left(g_{V}^{2}-g_{A}^{2}\right) \frac{m_{e} T}{E_{\nu}^{2}}\right]
$$

where the upper (lower) sign refers to the interaction with $\nu(\bar{\nu})$. The contribution arising from magnetic moment of neutrino is given by the additional term [1038]:

$$
\left.\frac{\sigma_{(\nu e, \bar{\nu} e)}}{d T}\right|_{\mu_{\nu}}=\frac{\pi \alpha_{e m}^{2} \mu_{\nu}^{2}}{m_{e}^{2}}\left(\frac{1}{T}-\frac{1}{E_{\nu}}\right) .
$$

There is no interference between the SM and magnetic moment amplitudes as the latter involves a flip in the spin of the neutrino.

In the laboratory frame the kinematical constraint on the angle between the incoming neutrino and the scattered electron is [1039]

$$
\theta_{\nu-e}^{2}<2 m_{e} / E_{e}
$$

and for electron energies of about $1 \mathrm{GeV}, \theta_{\nu-e}<30 \mathrm{mrad}$. This constraint can be used as a criterion to suppress the background events arising from $\nu$-nucleon scattering. The actual limits on $\nu_{e}$ and $\nu_{\mu}$ magnetic moment are $2.9 \times 10^{-11} \mu_{B}$ [1040] and $6.8 \times 10^{-10} \mu_{B}$ [1041] respectively, while for the tau neutrino the limit of $3.9 \times 10^{-7} \mu_{B}$ has been set by the DONUT experiment [1042]. In DONUT one event was found, within the acceptance cuts, while 2.3 were expected from the background processes. For this reason the new limit was deduced by comparing the expected rate for a given value of the magnetic moment with the level of background events. To get an estimate of the number of events to be found in SHiP for a given value of the tau neutrino magnetic moment we can integrate the differential cross section in Eq. (7.3.3) above assuming a minimum energy for the produced electron of $10 \mathrm{GeV}$. Convoluting with the spectrum of tau neutrino and anti-neutrino given in Fig. 7.5, rescaling to $2 \times 10^{20}$ POT and considering a target of 10 tons, the number of expected events is

$$
N_{e v}=4.3 \times 10^{15} \frac{\mu_{\nu}^{2}}{\mu_{B}^{2}}
$$

Backgrounds events consist of charged current $\nu_{e}$ scattering off nuclei for which only the high energy electron is detected, and neutral current Standard Model neutrino electron scattering of Eq. (7.3.2). The number of background events and the relative uncertainly can only be evaluated with a detailed simulation of the full neutrino flux and of the performance of the detector [1008]. In Fig. 7.21 we show the number of events with an outgoing electron with energy greather then $10 \mathrm{GeV}$, that would be produced in the SHiP experiment assuming a value for the magnetic moment of tau neutrino 
equal to the upper limit set by the DONUT experiment of $3.9 \times 10^{-7} \mu_{B}$ [1042].

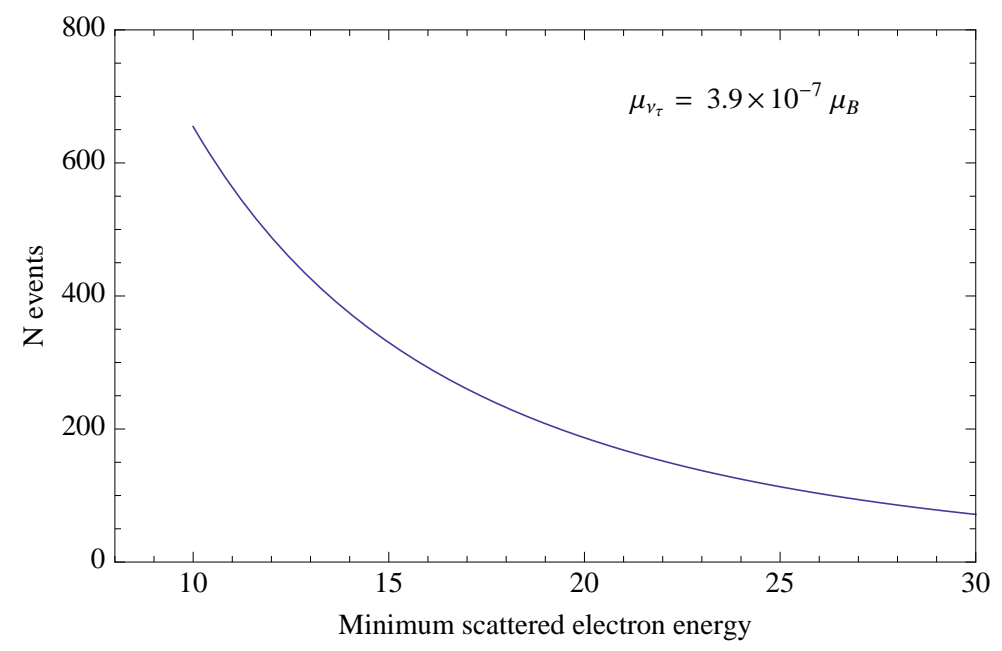

Figure 7.21: Number of expected events in SHiP producing an electron with energy greather then $10 \mathrm{GeV}$ assuming for $\mu_{\nu_{\tau}}$ the upper limit set by the DONUT experiment [1042].

\subsection{Charmed pentaquark searches}

The recent discovery of charged charmonia and bottomonia states is a proof of the existence of particles with four valence quarks. The nature of the new states found is still debated, they could be systems bound by QCD forces between colored objects (tetraquarks) or by residual forces between color singlet objects like hadron molecules. The correlation of the mass of the new states to meson-meson thresholds favors the hypothesis of these states to be molecules of mesons. On the other hand the sizeable production of these states at hadron colliders disfavors extended structures with respect to compact structures. For a recent status report of the situation see, for example, Ref. [1043]. Once we might consider a violation of the paradigm of hadronic particles being made of $q \bar{q}$ or $q q q$ states only, we can also consider the existence of five quark states. Indeed, the neutrino detector of the SHiP experiment provides a unique opportunity to improve the limits on the production cross section of a weakly decaying baryon state $(B=1)$ with $C=-1$, called $\Theta_{c}^{0}$. Negative searches of such a state have been reported in three experiments. The Babar collaboration searched for a $\Theta_{c}^{0}$ resonance in $e^{+} e^{-}$scattering at the energy of $10.58 \mathrm{GeV}$ [1044], while the Delphi collaboration used $Z$ boson decays [1045]. However, such experimental situations could be not optimal for pentaquark generation. The lack of valence quarks available in these reactions could represent a detractor for the creation of a five valence quark state. Finally, in the analysis reported by the CHORUS collaboration, a weakly decaying charmed pentaquark state was searched among the charged current anti-neutrino DIS events in [1046]. The situation is sketched in Fig. 7.22. The analysis of Ref. [1046] produced as a result an upper limit for the production cross section of a $\Theta_{c}^{0}$

$$
\frac{\sigma_{\Theta_{c}^{0}}}{\sigma_{\bar{\nu}}}<0.039 \quad \text { at } 90 \% \text { C.L. for } \tau_{\Theta_{\mathrm{c}}^{0}} \sim 0.5 \tau_{\mathrm{D}_{0}},
$$

and below 0.085 at $90 \%$ C.L. for longer lifetime of the $\Theta_{c}^{0}$. These limits were obtained with a relatively low statistic. Indeed, 2262 charged current anti-neutrino events were collected by the CHORUS experiment, with only 32 events with anti-charm production. This is because the CHORUS detector was using an almost pure $\nu_{\mu}$ beam. On the other hand, in the SHiP neutrino detector, one expects about 0.6 million of anti neutrino interactions and, of them, about 50.000 


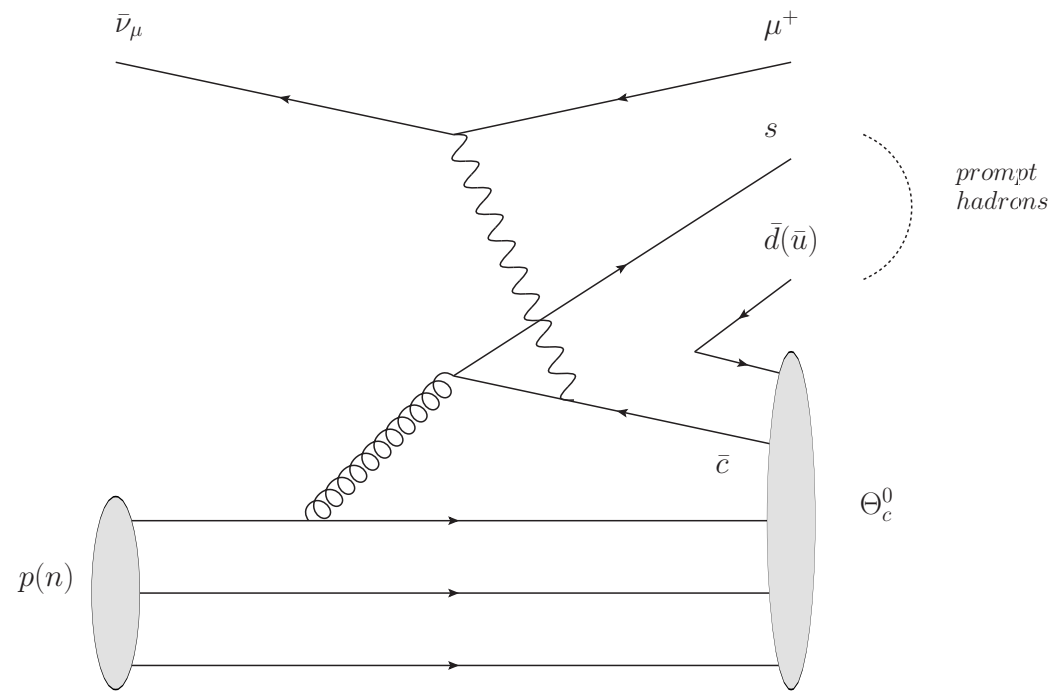

Figure 7.22: $\Theta_{c}^{0}$ production in $\bar{\nu}_{\mu}$ interactions.

events with anti-charm production. Assuming that the neutrino detector in SHiP would have a detection efficiency similar to the one of the CHORUS experiment, the higher statistic would give an improvement of the limit in Eq. (7.4.1) of about three orders of magnitude.

\subsection{Summary}

In this section we have discussed a collection of physics observables that could be measured exploiting the features of an high intense neutrino flux from a $400 \mathrm{GeV}$ proton beam dump experiment. We presented results for the tau neutrino physics and the opportunities to identify tau anti-neutrino interactions and measure the impact of the $F_{5}$ nucleon structure function on DIS for the first time. Furthermore, we have briefly summarized the current status of DIS experiments. The theoretical description of DIS data can build on a very mature understanding, which could be confronted with future experimental data from a new neutrino DIS fixed target experiments in order to improve our knowledge about the fundamental structure of matter and the important dynamics of quarks and gluons in nucleons. In the last part we have considered the actual limits on the neutrino magnetic moment and the production cross section of five quark states as examples of observables that might be studied with an high level of accuracy. 


\begin{tabular}{|l|c|c|c|c|}
\hline Particle & $N_{K K}$ & $B_{c}\left(2 m_{c}\right)$ & $N$ & $\epsilon$ \\
\hline$D^{0}$ & 0.694 & 0.728 & $0.694 \times 54.5 / 72.8$ & 0.101 \\
\hline$D^{+}$ & 0.282 & 0.289 & $0.282 \times 22.2 / 28.9$ & 0.104 \\
\hline$D_{s}^{+}$ & 0.0498 & 0.123 & $0.050 \times 7.7 / 12.3$ & 0.0322 \\
\hline$\Lambda_{c}^{+}$ & 0.00677 & 0.0617 & 0.00677 & 0.00418 \\
\hline
\end{tabular}

Table 7.1: Parameters for the charm quark fragmentation used in the simulation. The numbers $N_{K K}$ and $\epsilon$ come from Kniehl and Kramer's [957] Table I. The fragmentation fraction $B_{c}$ is from their Table III.

\begin{tabular}{|l|c|}
\hline Process & $B$ \\
\hline$D^{0} \rightarrow \nu_{\mu}$ & 0.067 \\
\hline$D^{+} \rightarrow \nu_{\mu}$ & 0.176 \\
\hline$D_{s}^{+} \rightarrow \nu_{\mu}$ & 0.065 \\
\hline$\Lambda_{c}^{+} \rightarrow \nu_{\mu}$ & 0.045 \\
\hline$D_{s}^{+} \rightarrow \nu_{\tau} \tau$ & 0.0554 \\
\hline$\tau \rightarrow e$ & 0.178 \\
\hline$\tau \rightarrow \mu$ & 0.178 \\
\hline$\tau \rightarrow \pi$ & 0.12 \\
\hline$\tau \rightarrow \rho$ & 0.26 \\
\hline$\tau \rightarrow a_{1}$ & 0.13 \\
\hline
\end{tabular}

Table 7.2: Parameters for the branching fractions used in this section. For the tau decays, the formulae are listed in [966].

\section{Appendix A: Parameters used to describe chram quark fragmentation and tau lepton decays}

The parameters for the fragmentation functions used are shown in Table 7.1, where the fragmentation function has the form

$$
D_{c}\left(x, \mu_{0}^{2}\right)=\frac{N x(1-x)^{2}}{\left[(1-x)^{2}+\epsilon x\right]^{2}} .
$$

In Ref. [957] the authors take $\mu_{0}=1.5 \mathrm{GeV}$. They also report the fragmentation fraction in their Table III, listed here in the column labeled $B_{c}\left(2 m_{c}\right)$. To account for different fragmentation fractions, rather than using the $N=N_{K K}$ of Kniehl-Kramer in the fragmentation function, we have used $N$ rescaled by fragmentation fractions from Ref. [958]. Note, we have used the fragmentation function parameterized at $\mathrm{LO}$ with our NLO differential distribution, a fragmentation parameterization where $m_{c}=1.5 \mathrm{GeV}$. Table 7.2 shows the branching fractions used. Finally, also note the approximations in $H_{c} \rightarrow \nu_{\mu} X$ using three body decays, further approximated by the muon energy distribution parameterized in [965] as discussed above. 


\section{Chapter 8}

\section{Searches of lepton flavour violating processes $\tau \rightarrow 3 \mu$}

\subsection{Motivation as a null-test of the standard model}

The observation of neutrino oscillations is the first evidence of the lepton flavour violation (LFV) in the neutral sector. This observation leads to non-zero neutrino masses and implies that lepton flavour should not be conserved in charged sector either. The effect corresponds to the branching fractions of the order of $10^{-40}$ and lower, but a number of models beyond the standard model predict the enhancement of the LFV in the charged sector (CLFV).

In this Section several representative models are reviewed alongside with the example predictions for the branching ratio of the $\tau \rightarrow 3 \mu$ decay. From the experimental point of view $\mu \rightarrow \mathrm{e} \gamma$ or $\mu \rightarrow 3$ e decays are contained more stringently than $\tau \rightarrow 3 \mu$ one, but in case LFV occurs in the scalar sector, the decay of $\tau \rightarrow 3 \mu$ can be significantly enhanced compared to the $\mu$ decays.

\section{$8.2 \tau \rightarrow 3 \mu$ in seesaw scenarios}

The neutrino masses could be added to the SM via various seesaw mechanisms which were discussed in detail in Section 4.3. Here we concentrate on the phenomenological outcome for the $\tau \rightarrow 3 \mu$ observable which were summarized in Ref. [608] and Ref. [617].

- Type I seesaw. In this scenario the CLFV processes occur only at the loop level since flavour violations happens only in neutral leptons. The prevalence os $\tau$ over $\mu$ flavour violating decays depends on the mass hierarchy. The current experimental data do not provide a constraint on this conclusion, and neutrino data allows for LFV $\tau$ decays to have higher rate than LFV $\mu$ decays. Examples scenarios are present in Refs. [387, 612].

- Type III seesaw. Here the CFLV can happen at the tree-level through a mixing between a charged lepton and charged components of the fermion triplets $\Sigma$, therefore processes of $\ell \rightarrow 3 \ell^{\prime}$ and $\mu \rightarrow$ e conversion occur at the tree level while $\ell \rightarrow \ell^{\prime} \gamma$ still proceed only through a loop. The calculations in Ref. [618] give an amplitude as

$$
T_{\ell \rightarrow \ell^{\prime}}=\sum_{\Sigma_{i}} \frac{\left|\Upsilon_{\Sigma_{i \ell^{\prime}}} \Upsilon_{\Sigma_{i \ell}}^{\dagger}\right|}{m_{\Sigma_{i}^{4}}} \cdot c
$$

where c depends on $m_{\ell}$ and $m_{\mathrm{W}, \mathrm{Z}}$. Therefore, measurement of the ratio of a same flavour transitions could help to identify if type III seesaw is in effect. In general, in this scenario three- 
lepton CLFV transitions have larger rates than radiative CLFV decays. Current experimental observations lead to $\epsilon_{\mu \tau}<4.9 \times 10^{-4}$ with $\mathcal{B}(\tau \rightarrow 3 \mu)<3.2 \times 10^{-8}$ [618].

- Type II seesaw. In this case the intermediate situation is in place. The CFLV decay to three leptons can occur at the tree level while the radiative decays proceeds only through loops. Therefore the trilepton decay is dominating.

\subsection{Supersymmetric models}

In the context of the R-parity conserving supersymmetric models lepton number is not violated and neutrino masses are zero. The solution which preserves R-parity and at the same time explains neutrino oscillations can be the same as in the SM and lead to extension of the fields of the MSSM in the scope of one of the seesaw mechanisms. A comprehensive review of an extension of the MSSM with three singlet heavy neutrinos in a scope of a type I seesaw can be found in Ref. [504].

While in the SM extension with type I seesaw mechanism the heavy neutrinos are usually decoupled from low energy sector leading to very low CLFV rates, in the MSSM they interfere with a low energy sector through a renormalisation group and lead to large CLFV in slepton sector at mass scale of a $\mathrm{TeV}$ order.

Computations in Ref. [504] lead to rates of the CLFV in the context of low-scale seesaw model in the MSSM which are larger than the corresponding effect in the SM, except for radiative CLFV decays which are suppressed in the supersymmetric limit.

Interesting phenomenological consequence arise in the scope of the inverse seesaw model [505] which can realise both in non-SUSY and SUSY scenarios. It is a low-scale model which has large Yukawa couplings and $\mathrm{TeV}$ mass seesaw mediators. The neutrino masses are suppressed with a slight breaking of lepton number in the singlet sector.

The rate of $\tau \rightarrow 3 \mu$ decay is found to be $10^{-11}$ and smaller therefore are beyond the sensitivity of the nearest experiments. But at the same time this decay is sensitive to the relative size of non-SUSY and SUSY contributions. There are also set of parameters which make LFV $\tau$-decays enhanced to $10^{-9}$ and therefore observable in the next experiments.

\subsection{Relation to two-body LVF decays of Z boson, neutral pseudoscalar and vector mesons}

Another example of CLFV processes are $\mu-e$ or $\tau-\mu$ conversions in nuclei $\mu^{-}(\tau)+(A, Z) \longrightarrow$ $e^{-}\left(\mu^{-}\right)+(A, Z)^{*}$. They are well-known to be one of the most sensitive probes of CLFV and underlying physics BSM (for reviews, see [1047-1050]). Up to now there have been undertaken significant efforts aimed at searching for CLFV via $\mu-e$ conversion in various nuclei with negative results [1047], thus setting upper limits on the $\mu-e$ conversion rate $R_{\mu e}^{A}=\Gamma\left(\mu^{-}+(A, Z) \rightarrow\right.$ $\left.e^{-}+(A, Z)\right) / \Gamma\left(\mu^{-}+(A, Z) \rightarrow \nu_{\mu}+(A, Z-1)\right)$. The SINDRUM II experiment at PSI has set stringent upper bounds on $\mu-e$ conversion rate $R_{\mu e} \leq 4.3 \times 10^{-12}, 7.0 \times 10^{-13}, 4.6 \times 10^{-11}$ in ${ }^{48} \mathrm{Ti}$ [613], ${ }^{197} \mathrm{Au}$ [1051] and ${ }^{208} \mathrm{~Pb}$ [1052] as stopping targets, respectively. Several new proposals for $\mu-e$ experiments are aimed at a significant improvement of the SINDRUM II sensitivity. Among them we mention the planned nearest future DeeMe experiment at J-PARC [1053], the next generation muon-to-electron conversion experiment by Mu2e Collaboration at Fermilab [1054] and COMET at J-PARC [1055] with planned sensitivities around $10^{-14}, 7 \times 10^{-17}$ and $10^{-16}$ respectively, as well as the more distant future proposal PRISM/PRIME [1056] at J-PARC, with estimated sensitivity $10^{-18}$.

The theoretical studies of $\mu-e$ conversion, presented in the literature, cover various aspects of this CLFV process: the adequate treatment of structure effects $[1050,1057,1058]$ of the nucleus 
participating in the reaction and the underlying mechanisms of CLFV at the quark level within different scenarios of physics beyond the SM (see [1049] and references therein). As is known there are two categories of $\mu-e$ conversion mechanisms: photonic and non-photonic. In the photonic case photon connects the CLFV leptonic and the electromagnetic nuclear vertices. The non-photonic mechanisms are induced by the four-fermion lepton-quark LFV contact interactions. These mechanisms significantly differ from each other, receiving different contributions from new physics and requiring different description of the nucleon and the nuclear structure.

In Refs. [1059-1061] the non-photonic scenario in CLFV process of $\mu-e$ conversion in nuclei has been used to estimate microscopic $\mu e q q$ contact interactions and their contribution to the effective CLFV lepton-hadronic coupling govern the two mechanisms of the $\mu-e$ conversion in nuclei on the hadronic level direct nucleon and vector and scalar meson exchange ones. In the former case the quarks are embedded directly into the nucleons while in the latter in mesons which then interact with nucleons in a nucleus. Using general framework of effective Lagrangians without referring to any particular CLFV model beyond the SM and experimental upper bounds on the $\mu-e$ conversion rate lower limits on the mass scales $\Lambda_{\mu e}$ of the CLFV lepton-quark contact vector and scalar terms qque involved in this process for all quark flavors have been extracted. It was shown that these limits are more stringent than the similar ones existing in the literature, including the limits from the present experimental data on meson decays and the limits expected from the future experiments at the LHC.

The abundant production of vector mesons and $Z^{0}$-bosons in current experiments naturally suggests to search for their two-body decays in the $\mu^{ \pm} e^{\mp}$ final state, which is rather convenient for event identification. Recently the SND Collaboration at the BINP (Novosibirk) [1062] reported on the search for the CLFV process $e^{+} e^{-} \rightarrow e \mu$ in the energy region $\sqrt{s}=984-1060 \mathrm{MeV}$ at the VEPP$2 \mathrm{M} e^{+} e^{-}$collider. They give a model independent upper limit on the $\phi \rightarrow e \mu$ branching fraction of $\operatorname{Br}(\phi \rightarrow e \mu)<2 \times 10^{-6}$. There are also experimental limits for the $e \mu$ decay mode of $J / \psi$ and of the $Z^{0}$ boson [156]: $\operatorname{Br}(J / \psi \rightarrow e \mu)<1.6 \times 10^{-7}, \operatorname{Br}\left(Z^{0} \rightarrow e \mu\right)<1.7 \times 10^{-6}$ and $\mu \tau$ decay mode of $\Upsilon$ [156]: $\operatorname{Br}(\Upsilon \rightarrow \mu \tau)<6.0 \times 10^{-6}$. In the near future this list may be extended by the results of other experimental collaborations. However, a natural question, which arises in this context, touches upon the prospects of this category of searches in view of possible theoretical limitations on the rates of these CLFV decays. In Refs. [1063] and [1060] there already exist stringent limits of this sort. For example, unitarity relations between the vector boson LFV decays $M \rightarrow \mu^{ \pm} e^{\mp}$ with $M=\rho^{0}, \omega, \phi, J / \psi, \Upsilon, Z^{0}$ and the pure leptonic LFV decay $\mu \rightarrow 3 e$ have been exploited in Ref. [1063]. From the existing experimental bounds on the latter process the following stringent bounds were deduced [1063] $\operatorname{Br}(\phi \rightarrow e \mu) \leq 4 \times 10^{-17}, \operatorname{Br}(J / \psi \rightarrow e \mu) \leq 4 \times 10^{-13}, \operatorname{Br}(\Upsilon \rightarrow e \mu)<2 \times 10^{-9}$, $\operatorname{Br}\left(Z^{0} \rightarrow e \mu\right)<5 \times 10^{-13}$. In Ref. [1060] these decay widths have been constrained from $\mu^{-}-e^{-}$ conversion $\operatorname{Br}\left(\rho^{0} \rightarrow e \mu\right) \leq 3.5 \times 10^{-24}, \operatorname{Br}(\omega \rightarrow e \mu) \leq 6.2 \times 10^{-27}, \operatorname{Br}(\phi \rightarrow e \mu) \leq 1.3 \times 10^{-21}$, $\operatorname{Br}(J / \psi \rightarrow e \mu) \leq 3.5 \times 10^{-13}, \operatorname{Br}(\Upsilon \rightarrow e \mu) \leq 3.9 \times 10^{-6}, \operatorname{Br}\left(Z^{0} \rightarrow e \mu\right) \leq 8.0 \times 10^{-15}$. The limit for the $J / \psi \rightarrow e \mu$ mode is compatible with the corresponding number extracted from $\mu \rightarrow 3 e$ as done in Ref. [1063]. The upper limits on the rates of $\phi \rightarrow e \mu$ and $Z^{0} \rightarrow e \mu$ decay modes derived in Ref. [1060] are significantly more stringent than in Ref. [1063] while for the limit on $\Upsilon \rightarrow e \mu$ the situation is opposite. The bounds for the decays $\rho^{0}, \omega \rightarrow e \mu$ were derived in Ref. [1060] for the first time in literature.

SHiP experiment will search for the CLFV decay $\tau \rightarrow 3 \mu$, which is analogous to the CLFV decay $\mu \rightarrow 3 e$. In this view experimental study of the $\tau \rightarrow 3 \mu$ decays and unitarity inspired relatations [1063] between two- and three-body LFV decays would constraint the couplings and rates of $Z^{0}$ boson, neutral pseudoscalar and vector mesons $\left(\eta_{c}, \eta_{b}, J / \psi, \Upsilon, \ldots\right)$ decays into $\tau^{ \pm} \mu^{\mp}$ pairs. Also there is a clear link of $\tau \rightarrow 3 \mu$ decay to $\tau-\mu$ conversion in nuclei. This is because the latter is realized by the exchange of $Z^{0}$ boson or neutral pseudoscalar and vector mesons between nucleon and lepton currents. 


\subsection{Current and future experimental sensitivities}

The most stringent limits on the $\tau \rightarrow 3 \mu$ rate are set by the BaBar and Belle [1064] collaborations, which bounds $\mathcal{B}(\tau \rightarrow 3 \mu)<2.1 \times 10^{-8}$ at $90 \% \mathrm{CL}$. Also the first result from LHCb is published $\left(\mathcal{B}(\tau \rightarrow 3 \mu)<8.0 \times 10^{-8}\right)$ and it is to be improved in the near future [1065]. In the next years, the Belle II experiment could bring the sensitivity up by one order of magnitude and reach the $10^{-9}$ range [1066]. Another contribution could come from the Super Tau-Charm factory [1067] which provides a possibility to search for a $\tau \rightarrow \mu \gamma$ decay.

\subsection{Proposal for a fixed-target facility}

The SHiP facility proposes a new opportunity for the investigating intensity frontier in the flavour physics.This is a fixed-target experiment to be based at the CERN SPS proton beam (400 GeV), which will collect $2 \times 10^{20}$ proton-on-target in 5 years. This amount of data will contain $3 \times 10^{15} \tau$ in the forward region, which provides a window to target rare $\tau$ decays at the unprecedented level.

The search for a $\tau \rightarrow 3 \mu$ decay at the fixed target experiment has been proposed before [1068], but at the time it aimed at the sensitivity at the level of $10^{-6}-10^{-8}$. Several orders of magnitude gain in senstitivity pose new constraints on the parameters of the detector performance which require some concept modification. Compared with the collider searches, a fixed target experiment has several orders of magnitude higher charged particles multiplicity in an event, therefore it requires careful estimation of combinatorial backgrounds in addition to the the ones considered in the collider experiments. The initial concept of the detector [1068] relies on a long target followed by a hadron absorber and a muon system after it. Such target ensures nearly full efficiency of each spill interaction providing efficient use of the beam, and the absorber removes hadrons allowing only muons to reach a muons system. At the same time, a multiple scattering of the muons in a long target combined with an absorber deteriorates trimuon invariant mass resolution to values of about $200 \mathrm{MeV}$, and also does not allow for an accurate $\tau$ decay vertex reconstruction, which significantly undermines the targeted sensitivity of $10^{-10}$.

In order to overcome these drawbacks, a new layout of the experiment is proposed. Two main features are introduced. First, the tungsten target thickness is decreased to a value of $1 \mathrm{~mm}$. This reduces the total number of produced $\tau$ by a factor of 100 , but in this case over $90 \%$ of produced $\tau$ are decaying outside the target (see Fig. 8.1). Second, a precise tracking system, as for instance silicon pixel, is installed between the target and the hadron absorber, which allows to retain the muon momentum information before the multiple scattering in the hadron absorber. In this configuration, the main contributors to the trimuon mass resolution are the track momentum resolution and the matching inefficiency of muons before and after the absorber. We can assume to have a pencil-like beam and a secondary vertex locator similar to LHCb VELO [1069]. The tracking needs to be in magnetic field to measure momentum. The silicon detector should be as close as possible to the beam, similar to LHCb VELO ( $8 \mathrm{~mm}$ from the beam). The tracking system is followed by a hadron absorber, which is followed by a less precise spectrometer (trigger spectrometer). One of the possibilities for the hadron absorber is magnetized iron. The trigger spectrometer is needed for particle identification and for triggering. The trigger will select three muons with invariant mass close to the $\tau$ mass. One important point to keep under control is the penetration depth and the multiple scattering of the muons in the absorber. In particular it is necessary to measure the momentum of all three muons from the $\tau$. The dimensions and material of the absorber need a careful optimization with the simulation comparing the efficiency and resolution with respect to the misidentification probability.

Due to specific demands on the target configuration, this experiment cannot be carried out with the SHiP target, but it can utillize the same beam and be positioned upstream to the main 
SHiP experiment. In such way, the $\tau \rightarrow 3 \mu$ experiment becomes the part of the SHiP facility, and benefits from the same data-taking timeline.
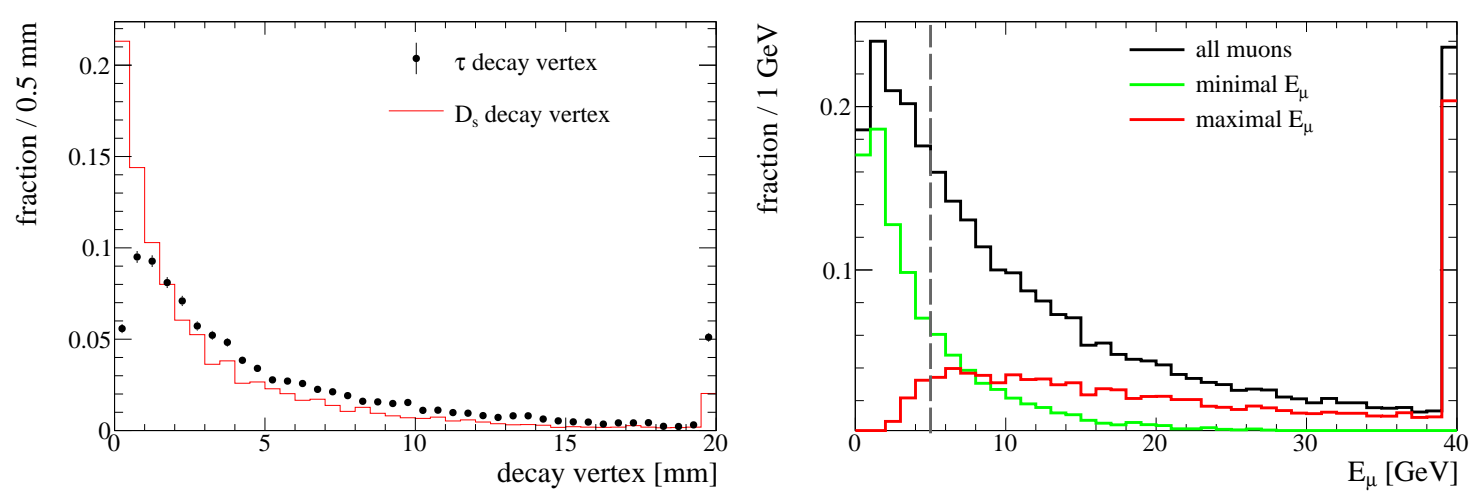

Figure 8.1: (left) The position of the decay vertex along the beam axis. (right) The energy spectrum of the muons in $\tau \rightarrow 3 \mu$ decay. Dashed line shows the requirement of $E_{\mu}>5 \mathrm{GeV}$.

For the estimates of main background sources to this search, the following parameters of the experimental setup are assumed. An event is considered to be in acceptance, if the energy of all three muons is greater than $5 \mathrm{GeV}$ in order for muons to be detectable after passing the hadron absorber. Figure 8.1 (right) shows the spectrum of the muons originating from a $\tau \rightarrow 3 \mu$ decay. The geometrical acceptance of the detector is defined by outer muon chamber size of $50 \mathrm{~cm}$ at maximum distance of $3 \mathrm{~m}\left(\theta_{\max }=0.17\right)$, and by inner muon chamber radius of $5 \mathrm{~mm}$ at $1.5 \mathrm{~m}$ $\left(\theta_{\min }=0.003\right)$. These requirements lead to $33 \%$ signal acceptance of the $\tau \rightarrow 3 \mu$ process.

Standard model backgrounds can be grouped in the following categories:

- Combinatorial background consisting on muons coming form different sources (mainly $\eta, \eta^{\prime}$ and $\omega$ decays ). Since these are accidental combinations, the level of this background strongly depends on the beam parameters (intensity and spread in space and time) and the detector resolution. Accidental overlap of three independent muons and two resonance muons and one independent muon is estimated with PYTHIA8 [286, 1070] simulation. For the numerical estimation the following beam parameters are assumed: $6 \mathrm{~mm}$ in diameter uniform beam profile, vertex resolution $\sigma_{\mathrm{xy}}=10 \mu \mathrm{m}$, spill duration $1 \mathrm{~s}$, detector timing resolution $\sigma_{\mathrm{t}}=10 \mathrm{~ns}$. The single muon production cross section within the detector acceptance is estimated with PYTHIA to be $\sigma\left(\mathrm{pp} \rightarrow \mu+X, E_{\mu}>5 \mathrm{GeV}\right)=3.8 \mu \mathrm{b}$ which for a spill with $4.5 \times 10^{13}$ protons yields $4 \times 10^{7} \mu /$ spill. This leads to a number of accidental $3 \mu$ combinations in the $\pm 5 \mathrm{MeV}$ mass window around nominal $\tau$ mass of 10 events in total after collecting $1 \%$ of $2 \times 10^{20}$ pot.

The production cross section for two resonant muons in the acceptance is determined as $3.0 \mu \mathrm{b}$. Combinatorial background from $2 \mu+1 \mu$ processes is estimated to be $2.6 \times 10^{5}$ background events in $\pm 5 \mathrm{MeV}$ mass window. This estimate does not take into account the additional handle provided by requiring a reconstructed $\tau$ decay vertex to be displaced from the target. Given that two muons form a vertex not detached from the target, a further significant suppression of this background can be achieved.

- Misidentified $\mathrm{D} \rightarrow 3 \mathrm{~h}$ decays. This background category includes misidentification of hadrons, punch-through and decay in flight. The study of this background requires assumption on particle identification. In pp collision, number of produced pions is 2 orders of magnitude higher than the number of muons. In case misidentification rate is at the level of $10^{-3}$, the contribution from this background is negligible compared to the backgrounds due to muons. 
- Specific muon backgrounds are listed in Table 8.1 and consist of two subcategories: semileptonic $\mathrm{D}_{(\mathrm{s})}$ decays with three muons in the final state and irreducible $\tau \rightarrow 3 \mu 2 \nu$ background. The total background is estimated as $0.3 \times 10^{6}$ events, the main contribution being from the $D_{\mathrm{s}}^{+} \rightarrow \eta \mu^{+} \nu_{\mu}, \eta \rightarrow \mu^{+} \mu^{-} \gamma$ process. Additional suppression for this process comes from a requirement of dimuon invariant mass to be greater than $450 \mathrm{MeV}$, which significantly reduces $\eta \rightarrow \mu^{+} \mu^{-}$contribution, and leads to a total background at $1.5 \times 10^{5}$ events. At the same time the signal efficiency of the additional requirement is $84 \%$.

With total number of $\tau$ collected at SHiP facility of $0.8 \times 10^{13}$ and the above number of background events, the exclusion can be made at the level of $10^{-10}$. Moreover, an important conclusion is drawn that the operation mode of the fixed-target experiment has the same challenges in terms of the standard model background as a collider experiment, given that the contributions from the combinatorial backgrounds and hadron misidentification are subdominant to the processes yielding three muons in the final state.

Further improvements could include several thin targets with a spectrometer posed one after another. Each additional target scales the sensitivity with $\sqrt{N_{\text {targets }}}$.

Table 8.1: Summary of the processes yielding three muons in the final state.

\begin{tabular}{|c|c|}
\hline Decay & $\mathcal{B}$ \\
\hline$\overline{D^{+}} \rightarrow \eta \mu^{+} \nu_{\mu}$ & $1.14 \cdot 10^{-3}$ \\
\hline$\eta \rightarrow \mu^{+} \mu^{-}$ & $5.8 \cdot 10^{-6}$ \\
\hline \multicolumn{2}{|l|}{ or } \\
\hline$\eta \rightarrow \mu^{+} \mu^{-} \gamma$ & $3.1 \cdot 10^{-4}$ \\
\hline$D^{+} \rightarrow \rho \mu^{+} \nu_{\mu}$ & $2.4 \cdot 10^{-3}$ \\
\hline$\rho \rightarrow \mu^{+} \mu^{-}$ & $4.55 \cdot 10^{-5}$ \\
\hline$D^{+} \rightarrow \eta^{\prime} \mu^{+} \nu_{\mu}$ & $2.2 \cdot 10^{-4}$ \\
\hline$\eta \prime \rightarrow \mu^{+} \mu^{-} \gamma$ & $1.8 \cdot 10^{-4}$ \\
\hline$D^{+} \rightarrow \omega \mu^{+} \nu_{\mu}$ & $1.6 \cdot 10^{-3}$ \\
\hline$\omega \rightarrow \mu^{+} \mu^{-}$ & $9.0 \cdot 10^{-5}$ \\
\hline$D_{s}^{+} \rightarrow \eta \mu^{+} \nu_{\mu}$ & $2.7 \cdot 10^{-2}$ \\
\hline$\eta \rightarrow \mu^{+} \mu^{-}$ & $5.8 \cdot 10^{-6}$ \\
\hline \multicolumn{2}{|l|}{ or } \\
\hline$\eta \rightarrow \mu^{+} \mu^{-} \gamma$ & $3.1 \cdot 10^{-4}$ \\
\hline$D_{s}^{+} \rightarrow \eta^{\prime} \mu^{+} \nu_{\mu}$ & $9.9 \cdot 10^{-3}$ \\
\hline$\eta \prime \rightarrow \mu^{+} \mu^{-} \gamma$ & $1.8 \cdot 10^{-4}$ \\
\hline
\end{tabular}




\section{Chapter 9}

\section{Conclusion}

In this document we demonstrated a potential of proton fixed target experiments and in particular of a proposed facility to search for hidden particles at the SPS in CERN (SHiP) in probing wide class of physically motivated models and discovering new particles with masses in the range from hundreds of $\mathrm{MeV}$ to few $\mathrm{GeV}$. In particular, the experiments like SHiP provide an opportunity for direct experimental resolution of several major observational puzzles of modern particle physics and cosmology that cannot be explained within the Standard Model of elementary particles.

The SHiP experiment is capable:

- to find a dark matter candidate directly or shed new light on the properties of dark matter particles (see Chapter 4, Sections 4.7, 4.8; Chapter 2, Sections 2.4.2, 2.3.2, 2.6.3, Chapter 33.5 for discussion of different candidates)

- to find particles that lead to neutrino masses and oscillations (Chapter 4)

- to find particles generating the baryon asymmetry of the Universe (Chapter 4 , Sections 4.6 and in particular 4.6.4; Chapter 3, Section 3.1.2)

- find particles that could inflate the Universe (Section 3.7)

There are no clear experimental indications where one should expect to find new physics. Given the current experimental status of BSM searches it is possible that the experiments like SHiP would be the only way to discover these particles.

Additionally, the SHiP experiment is capable to probe models where the BSM phenomena are explained by the particles heavier than the electroweak scale (SUSY, extra dimensions, new strong dynamics, etc). To detect these particles directly the LHC Run-II or some post-LHC energy frontier experiments are required. The SHiP experiment would not be able to find particle, directly responsible for the BSM puzzles. However, these models often predict light, super-weakly interacting particles in the spectrum, that can be probed with SHiP. Generically, such particles are pseudo-Goldstone bosons (or fermions) of symmetries, broken at high scales. Axions and their supersymmetric counterparts (Chapter 5 or Section 6.7) and sgoldstinos (Section 6.3) are examples of such situations. The discoveries at SHiP can serve as a motivation and a guiding principle for the future energy frontier experiments to search for new physics above the electroweak scale. In particular, the SHiP experiment has a potential of probing a wide class of light supersymmetric particles (Chapter 6, Chapter 2, Section 2.2.2; Chapter 3, Section 3.1.3).

It is possible that the new particles, responsible for the BSM phenomena, are very heavy (much above $\mathrm{TeV}$ ) and the only interaction of new sectors with the Standard Model is via light particles coupled to the gauge singlet combinations of the SM fields or portals (Chapters 4, 2, 3, 5). These 
light mediator particles (scalars, pseudoscalars, fermions, vector particles) can then be the only ones that can be directly found to the accelerator experiments in the foreseeable future. In addition, currently inaccessible high energy physics can manifest itself via rare processes like $\tau \rightarrow 3 \mu$ that can be probed at the same fixed target facility at the SPS beyond the SHiP experiment (Chapter 8).

Along with the searches for new physics, the SHiP experiment will check Standard Model predictions that has not been verified by the previous experiments. In particular it will detect for the first time the $\bar{\nu}_{\tau}$ and measure separately the cross-sections of $\nu_{\tau}$ and $\bar{\nu}_{\tau}$ (Chapter 7 ). 


\section{Appendix A}

\section{The SHiP experiment}

In this chapter we provide a brief description of the SHiP experiment. Although not intended to be exhaustive it allows to do the estimates of the sensitivity towards the detection of new physics. The details may be found at $[34,35]$ and at http://ship.web.cern.ch.

\section{Experimental setup}

A dedicated beam line extracted from the SPS will convey a $400 \mathrm{GeV} / c$ proton beam at the SHiP facility $[34,35]$. The beam will be stopped in a Molybdenum and Tungsten target, at a center-ofmass energy $E_{C M}=\sqrt{2 E_{b} m_{p}} \simeq 27 \mathrm{GeV}$. Approximately $2 \times 10^{20}$ proton-target collisions $($ PoT $)$ are foreseen in 5 years of operation.

The target will be followed by a hadron stopper, intended to stop all $\pi^{ \pm}$and $K$ mesons before they decay, and by a system of shielding magnets to sweep muons away from the fiducial decay volume.

A neutrino detector consisting of OPERA-like bricks of laminated lead and emulsions, placed in a magnetic field downstream of the muon shield, will allow to measure and identify charged particles produced in charged current neutrino interactions. It is followed by a tracking system and muon magnetic spectrometer.

An upstream tagger, together with the muon spectrometer of the neutrino detector, will allow to detect and veto charged particles produced outside the main decay volume. The fiducial decay volume begins approximately $63.8 \mathrm{~m}$ downstream of the primary target, and is contained in a $60 \mathrm{~m}$ long cylindrical vacuum tank with elliptical section of $x$ and $y$ semiaxes $2.5 \mathrm{~m}$ and $5 \mathrm{~m}$ long, respectively.

An straw tagger is placed in vacuum $5 \mathrm{~m}$ downstream of the entrance lid of the vacuum tank. Its purpose is to help reducing background arising from interactions in the material upstream of the decay volume.

An additional background tagger surrounds the fiducial decay volume, which walls enclose $30 \mathrm{~cm}$ of liquid scintillator.

The tracking system aimed at measuring the decay products of hidden particles is located at the end of the decay volume. It will consist of $5 \mathrm{~m}$ long straw tubes organized in 4 stations, with a magnetic field of $1 \mathrm{Tm}$ between the first and the second pair of stations. The high-accuracy timing information provided by a dedicated detector following the straw tracker will be used to discriminate combinatorial background.

The particle identification system is placed outside the vacuum tank, and it features an electromagnetic and an hadronic calorimeter, followed by a muon system made of four active layers interlaced with iron. 


\section{Number of mesons and adopted cross-sections}

The number of charm and beauty mesons produced at the SHiP target can be estimated as

$$
N_{\text {mesons }}=2 \times X_{q \bar{q}} \times N_{P o T}
$$

where $X_{q \bar{q}}$ represents the $q \bar{q}$ production rate. The following cross sections have been used for the estimates:

- the proton-nucleon cross section is $\sigma(p N) \simeq 10.7$ mbarn.

- $\sigma(c c)=18 \mu$ barn $[950]$ and the fraction $X_{c c}=1.7 \times 10^{-3}$

- $\sigma(b b)=1.7$ nbarn [960] and the fraction $X_{b b}=1.6 \times 10^{-7}$

The relative abundances of charmed mesons are approximately as follows (PYTHIA8 simulations):

$$
\begin{aligned}
& D^{ \pm} 30 \% \\
& D^{0}, \bar{D}^{0} 62 \% \\
& D_{s}^{ \pm} 8 \% \\
& J / \psi 1 \%
\end{aligned}
$$

The expected number of $\tau$ leptons for $N_{P o T}=2 \times 10^{20}$ is $N_{\tau}=3 \times 10^{15}$.

\section{Number of events in the detector}

In order to estimate the number long-lived particles produced in decays of heavy mesons (c-and $b$-mesons) we used the following formula:

$$
n_{\text {prod }} \simeq N_{P o T} \times \chi(p p \rightarrow X) \times \epsilon_{\text {geom }} \times \frac{60 \mathrm{~m}}{\gamma c \tau}
$$

where

$$
\chi(p p \rightarrow X)=\left(N_{\text {mesons }} / N_{P o T}\right) \times B R(\text { meson } \rightarrow X) .
$$

In Eqn. A.0.2, $\epsilon_{\text {geom }}$ is the geometric acceptance, computed as ratio between the solid angle covered by the detector and the average divergence of new particles $X$, and the factor $\gamma=\langle E\rangle / m_{X}$ is the average energy of $X$ divided by its mass $m_{X}$. The last factor of Eqn. A.0.2 approximates the longitudinal acceptance if the average energy and lifetime are such that $\gamma c \tau \gg 60 \mathrm{~m}$ (the length of the decay volume). This formula must be further corrected for the acceptance of the final state in the detector, in addition to reconstruction and selection efficiency.

Finally, we consider as "detectable" the final states with two or more charged particles. 


\section{Appendix B}

\section{Notations}

Throughout the document we use the following conventions:

- Index $\alpha=\{e, \mu, \tau\}$ is the flavour index

- Charged leptons $\left(e^{ \pm}, \mu^{ \pm}, \tau^{ \pm}\right)$collectively are $\ell_{\alpha}$

- Neutrinos $\nu_{\alpha}$

- Left lepton doublet $L_{\alpha}=\left(\begin{array}{c}\nu_{\alpha} \\ \ell_{\alpha}\end{array}\right)$

- $\Phi$ denotes Higss field (two-component SU(2) doublet); $\tilde{\Phi}_{i} \equiv \epsilon_{i j} \Phi_{j}$

- VEV of the Higgs field $\langle\Phi\rangle=\frac{v}{\sqrt{2}}$, where $v=246 \mathrm{GeV}$

Chapter 4 uses the following additional notations:

- $N_{I}$ denotes right-handed (two component) gauge singlet neutrinos

- Index $I=1,2, \ldots \mathcal{N}$ runs over HNL species $(\mathcal{N} \geq 2)$

- Active-sterile $F_{\alpha I}$ - matrix $3 \times \mathcal{N}$

- Majorana mass of HNL: $M_{I}$

- Dirac mass $\left(m_{D}\right)_{\alpha I}$

- Sterile neutrino mixing angle

$$
U_{\alpha I}^{2}=\frac{v^{2}\left|F_{\alpha I}\right|^{2}}{M_{I}^{2}}
$$

- Experimental constraints are put either on $U_{\alpha}^{2}$, defined as

$$
U_{\alpha}^{2}=\sum_{I} U_{\alpha I}^{2}
$$

the total mixing angle $U^{2}$ is defined as $^{1}$

$$
U^{2}=\sum_{\alpha} U_{\alpha}^{2}
$$

Cosmological notations

- $n_{B}$ - baryonic number density

${ }^{1}$ The notation $\sin ^{2}(2 \theta)$ is sometimes used instead of $U^{2}$. The correspondence is $\sin ^{2}(2 \theta)=U^{2}$ with no extra factors. 
- Baryon asymmetry of the Universe:

$$
\eta_{B} \equiv \frac{n_{B}}{s}
$$

- $s$ - entropy density

\section{B.1 Abbreviations:}

Axion-like particle

Baryon asymmetry of the Universe

BroutEnglertHiggs (field, particle,...)

Beyond the Standard Model

Big Bang Nucleosynthesis

Charged (sector) lepton flavour violation

Closed time path (formalism)

Confidence Level

Dark Matter

Deep-inelastic scattering

Electroweak

Electroweak phase transition

Electroweak symmetry breaking

Future Circular Collider

Grand Unified Theory

Gross-Llewellyn Smith (sum rule)

Neutrinoless double beta decay

Heavy neutral lepton

Left-right symmetric model

lepton flavour violation

lepton number violation

Right-handed (neutrino/current)

Super Proton Synchrotron

Pseudo-Nambu-Goldstone boson

Pontecorvo-Maki-Nakagawa-Sakata

protons-on-target

R-parity violating/violation

Standard Model
ALP

BAU

$\mathrm{BEH}$

BSM

BBN

CLFV

CTP

C.L. or CL

DM

DIS

EW

EWPT

EWSB

FCC

GUT

GLS

$0 \nu \beta \beta$

HNL

LRSM

LFV

LNV

RH

SPS

PNGB

PMNS

PoT or p.o.t.

RPV

SM

\section{Financial support acknowledgements}

The work of T. Asaka was supported in part by JSPS KAKENHI Grant Numbers 25400249 and 26105508 .

The research by K. Bondarenko was supported by the Netherlands Science Foundation (NWO/OCW).

The work of C. Corral was supported by CONICYT (Chile) under Grant No.21130179.

The work of P. S. B. Dev and A. Pilaftsis is supported by the Lancaster-Manchester-Sheffield Consortium for Fundamental Physics under STFC grant ST/L000520/1.

The work of Christophe Grojean was supported in part by the Marie Curie Career Integration Grant 631962, by the Spanish Ministry MICINN under contract FPA2010-17747 and by the Generalitat de Catalunya grant 2014-SGR-1450. 
The work of S. Eijima, O. Ruchayskiy and M. Shaposhnikov was supported in part by the Swiss National Science Foundation.

A. Ibarra acknowledges the support of DFG cluster of excellence "Origin and Structure of the Universe".

The Dark Cosmology Centre is funded by the Danish National Research Foundation.

The work of J. C. Helo was supported by Fondecyt (Chile) under Grant No. 11121557.

The work of A. Ivashko was supported in part by funding from the European Research Council under the European Union's Seventh Framework Programme (FP/2007-2013) / ERC Grant Agreement nr. 226043 .

The work of C. S. Kim and Y. S. Jeong was supported by the National Research Foundation of Korea (NRF) grant funded by the Korea government of the Ministry of Education, Science and Technology (MEST) (No. 2011-0017430), (No. 2011-0020333) and Institute of Physics and Applied Physics (IPAP).

The work of S. Kovalenko was supported by FONDECYT (Chile) under Grant No.1150792.

The work of V. E. Lyubovitskij was supported by Tomsk State University Competitiveness Improvement Program.

M. McCullough acknowledges support from a CERN COFUND Fellowship.

The work of S. Moch has been supported by Bundesministerium für Bildung und Forschung through contract (05H12GU8).

The work of R. N. Mohapatra was supported by the National Science Foundation grant No. PHY1315155 .

The work of M. H. Reno was supported in part by the US Department of Energy contract DESC0010114.

This work of Osamu Seto was supported in part by the Grant-in-Aid for Scientific Research on Innovative Areas No. 26105514 from the Ministry of Education, Culture, Sports, Science and Technology in Japan.

The work of R. Shrock is partially supported by the NSF grant NSF-PHY-13-16617.

The work of Martin W. Winkler was supported in part by SFB-Transregio TR33 "The Dark Universe" (Deutsche Forschungsgemeinschaft). 


\section{Bibliography}

[1] ATLAS Collaboration, G. Aad et al., Combined search for the Standard Model Higgs boson using up to $4.9 \mathrm{fb}^{-1}$ of pp collision data at $\sqrt{\mathrm{s}}=7 \mathrm{TeV}$ with the ATLAS detector at the LHC, Phys.Lett. B710 (2012) 49-66, [arXiv:1202.1408].

[2] ATLAS Collaboration, G. Aad et al., Observation of a new particle in the search for the Standard Model Higgs boson with the ATLAS detector at the LHC, Phys.Lett. B716 (2012) 1-29, [arXiv: 1207.7214].

[3] CMS Collaboration, S. Chatrchyan et al., Combined results of searches for the standard model Higgs boson in pp collisions at $\sqrt{s}=7$ TeV, Phys.Lett. B710 (2012) 26-48, [arXiv:1202.1488].

[4] CMS Collaboration, S. Chatrchyan et al., Observation of a new boson at a mass of 125 GeV with the CMS experiment at the LHC, Phys.Lett. B716 (2012) 30-61, [arXiv:1207.7235].

[5] ATLAS, CMS Collaboration, G. Aad et al., Combined Measurement of the Higgs Boson Mass in pp Collisions at $\sqrt{s}=7$ and $8 \mathrm{TeV}$ with the ATLAS and CMS Experiments, arXiv:1503.07589.

[6] ATLAS Collaboration, X. Zhuang, Early Search for Supersymmetry at ATLAS, PoS KRUGER2010 (2011) 051, [arXiv:1104.2907].

[7] ATLAS Collaboration, G. Aad et al., Search for massive supersymmetric particles decaying to many jets using the ATLAS detector in pp collisions at $\sqrt{s}=8 \mathrm{TeV}$, arXiv:1502.05686.

[8] ATLAS Collaboration, G. Aad et al., Search for new phenomena in final states with an energetic jet and large missing transverse momentum in pp collisions at $\sqrt{s}=8$ TeV with the ATLAS detector, arXiv: 1502.01518.

[9] ATLAS Collaboration, G. Aad et al., Search for direct pair production of a chargino and a neutralino decaying to the 125 GeV Higgs boson in $\sqrt{s}=8$ TeV pp collisions with the ATLAS detector, arXiv:1501.07110.

[10] ATLAS Collaboration, G. Aad et al., Search for squarks and gluinos in events with isolated leptons, jets and missing transverse momentum at $\sqrt{s}=8 \mathrm{TeV}$ with the ATLAS detector, arXiv: 1501.03555.

[11] ATLAS Collaboration, G. Aad et al., Search for Scalar-Charm Pair Production in pp Collisions at $\sqrt{s}=8 \mathrm{TeV}$ with the ATLAS Detector, arXiv:1501.01325.

[12] CMS Collaboration, V. Khachatryan et al., Searches for supersymmetry using the MT2 variable in hadronic events produced in pp collisions at $8 \mathrm{TeV}$, arXiv:1502.04358.

[13] CMS Collaboration, V. Khachatryan et al., Search for physics beyond the standard model in events with two leptons, jets, and missing transverse momentum in pp collisions at sqrt(s) $=8$ TeV, arXiv: 1502.06031.

[14] CMS Collaboration, V. Khachatryan et al., Search for supersymmetry using razor variables in events with b-tagged jets in pp collisions at $\sqrt{s}=8 \mathrm{TeV}$, arXiv:1502.00300.

[15] CMS Collaboration, V. Khachatryan et al., Searches for supersymmetry based on events with $b$ jets and four $W$ bosons in pp collisions at $8 \mathrm{TeV}$, arXiv:1412.4109. 
[16] ATLAS Collaboration, G. Aad et al., Search for dark matter in events with heavy quarks and missing transverse momentum in pp collisions with the ATLAS detector, Eur.Phys.J. C75 (2015), no. 2 92, [arXiv:1410.4031].

[17] ATLAS Collaboration, G. Aad et al., Search for production of $W W / W Z$ resonances decaying to a lepton, neutrino and jets in pp collisions at $\sqrt{s}=8 \mathrm{TeV}$ with the ATLAS detector, arXiv: 1503.04677.

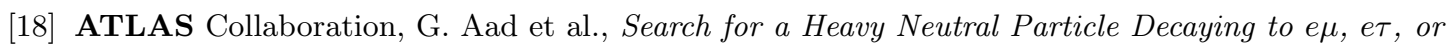
$\mu \tau$ in pp Collisions at $\sqrt{s}=8 \mathrm{TeV}$ with the ATLAS Detector, arXiv:1503.04430.

[19] ATLAS Collaboration, N. Bousson, ATLAS Exotic Searches, EPJ Web Conf. 28 (2012) 09012, [arXiv:1201.5256].

[20] J. Tam, Summary of results on exotic searches from ATLAS, Tech. Rep. ATL-PHYS-PROC-2014-212, CERN, Geneva, Oct, 2014.

[21] ATLAS, CDF, CMS, Do Collaboration, S. Rahatlou, Exotic Searches at LHC and Tevatron, arXiv: 1201.4810.

[22] P. Bargassa, Beyond the Standard Model physics: Strong Susy production searches at ATLAS ES $C M S$, in Augé et al. [1071], pp. 359-368.

[23] ATLAS, CMS Collaboration, T. R. T. Fernandez, Exotic searches in the large hadron collider, in Augé et al. [1071], pp. 431-438.

[24] ATLAS, CMS Collaboration, M. Flowerdew, Electroweak SUSY production searches at ATLAS and $C M S$, in Augé et al. [1071], pp. 375-382.

[25] ATLAS, CMS Collaboration, P. Thompson, Beyond the Standard Model Higgs Searches at the $L H C$, in Augé et al. [1071], pp. 347-352.

[26] CMS Collaboration, "CMS Supersymmetry Physics Results." https://twiki.cern.ch/twiki/bin/view/CMSPublic/PhysicsResultsSUSY, 2015.

[27] ATLAS Collaboration, "ATLAS exotics public results." https://twiki.cern.ch/twiki/bin/view/AtlasPublic/SupersymmetryPublicResults, 2015.

[28] ATLAS Collaboration, "ATLAS Supersymmetry Searches." https://twiki.cern.ch/twiki/bin/view/AtlasPublic/ExoticsPublicResults, 2015.

[29] CMS Collaboration, "CMS Exotica Public Physics Results." https://twiki.cern.ch/twiki/bin/view/AtlasPublic/ExoticsPublicResults, 2015.

[30] D. Buttazzo, G. Degrassi, P. P. Giardino, G. F. Giudice, F. Sala, et al., Investigating the near-criticality of the Higgs boson, JHEP 1312 (2013) 089, [arXiv:1307.3536].

[31] G. Degrassi, S. Di Vita, J. Elias-Miro, J. R. Espinosa, G. F. Giudice, et al., Higgs mass and vacuum stability in the Standard Model at NNLO, JHEP 1208 (2012) 098, [arXiv:1205.6497].

[32] F. Bezrukov, M. Y. Kalmykov, B. A. Kniehl, and M. Shaposhnikov, Higgs Boson Mass and New Physics, JHEP 1210 (2012) 140, [arXiv:1205.2893].

[33] I. Antoniadis, A. Boyarsky, S. Espahbodi, O. Ruchayskiy, and J. D. Wells, Anomaly driven signatures of new invisible physics at the Large Hadron Collider, Nucl.Phys. B824 (2010) 296-313, [arXiv:0901.0639].

[34] SHiP Collaboration, Technical proposal: A facility to search for hidden particles (SHiP) at the SPS, tech. rep., 2015. CERN-SPSC-2015-016 (SPSC-P-350).

[35] W. Bonivento, A. Boyarsky, H. Dijkstra, U. Egede, M. Ferro-Luzzi, et al., Proposal to Search for Heavy Neutral Leptons at the SPS, arXiv:1310.1762.

[36] P. Langacker, The Physics of Heavy Z' Gauge Bosons, Rev.Mod.Phys. 81 (2009) 1199-1228, [arXiv:0801.1345]. 
[37] ATLAS Collaboration, G. Aad et al., Search for high-mass dilepton resonances in pp collisions at $\sqrt{s}=8 \mathrm{TeV}$ with the ATLAS detector, Phys.Rev. D90 (2014), no. 5 052005, [arXiv:1405.4123].

[38] CMS Collaboration, V. Khachatryan et al., Search for physics beyond the standard model in dilepton mass spectra in proton-proton collisions at sqrt(s) $=8 \mathrm{TeV}$, arXiv:1412.6302.

[39] R. Essig, J. A. Jaros, W. Wester, P. H. Adrian, S. Andreas, et al., Dark Sectors and New, Light, Weakly-Coupled Particles, arXiv:1311.0029.

[40] L. Okun, LIMITS OF ELECTRODYNAMICS: PARAPHOTONS?, Sov.Phys.JETP 56 (1982) 502.

[41] B. Holdom, Two U(1)'s and Epsilon Charge Shifts, Phys.Lett. B166 (1986) 196.

[42] H. An, M. Pospelov, and J. Pradler, New stellar constraints on dark photons, Phys.Lett. B725 (2013) 190-195, [arXiv:1302.3884].

[43] P. Galison and A. Manohar, TWO Z's OR NOT TWO Z's?, Phys.Lett. B136 (1984) 279.

[44] R. Foot and X.-G. He, Comment on Z Z-prime mixing in extended gauge theories, Phys.Lett. B267 (1991) 509-512.

[45] N. Borodatchenkova, D. Choudhury, and M. Drees, Probing MeV dark matter at low-energy e+ecolliders, Phys.Rev.Lett. 96 (2006) 141802, [hep-ph/0510147].

[46] P. Fayet, Constraints on Light Dark Matter and U bosons, from psi, Upsilon, K+, piO, eta and eta-prime decays, Phys.Rev. D74 (2006) 054034, [hep-ph/0607318].

[47] P. Fayet, U-boson production in e+ e- annihilations, psi and Upsilon decays, and Light Dark Matter, Phys.Rev. D75 (2007) 115017, [hep-ph/0702176].

[48] J. D. Bjorken, R. Essig, P. Schuster, and N. Toro, New Fixed-Target Experiments to Search for Dark Gauge Forces, Phys.Rev. D80 (2009) 075018, [arXiv:0906.0580].

[49] S. Andreas, C. Niebuhr, and A. Ringwald, New Limits on Hidden Photons from Past Electron Beam Dumps, Phys.Rev. D86 (2012) 095019, [arXiv:1209.6083].

[50] S. Gninenko, Constraints on dark photons from $\pi^{0}$ decays, Phys.Rev. D87 (2013), no. 3 035030, [arXiv: 1301.7555].

[51] PHENIX Collaboration, A. Adare et al., Search for dark photons from neutral meson decays in $p+p$ and $d+A u$ collisions at $\sqrt{s_{N N}}=200 \mathrm{GeV}$, arXiv:1409.0851.

[52] BaBar Collaboration, J. Lees et al., Search for a Dark Photon in $e^{+} e^{-}$Collisions at BaBar, Phys.Rev.Lett. 113 (2014), no. 20 201801, [arXiv:1406.2980].

[53] KLOE-2 Collaboration, D. Babusci et al., Search for light vector boson production in $e^{+} e^{-} \rightarrow \mu^{+} \mu^{-} \gamma$ interactions with the KLOE experiment, Phys.Lett. B736 (2014) 459-464, [arXiv: 1404.7772].

[54] NA48/2 Collaboration, E. Goudzovski, Search for the dark photon in $\pi^{0}$ decays by the NA48/2 experiment at CERN, arXiv:1412.8053.

[55] A1 Collaboration, H. Merkel et al., Search for Light Gauge Bosons of the Dark Sector at the Mainz Microtron, Phys.Rev.Lett. 106 (2011) 251802, [arXiv:1101.4091].

[56] APEX Collaboration, S. Abrahamyan et al., Search for a New Gauge Boson in Electron-Nucleus Fixed-Target Scattering by the APEX Experiment, Phys.Rev.Lett. 107 (2011) 191804, [arXiv: 1108.2750].

[57] M. Battaglieri, S. Boyarinov, S. Bueltmann, V. Burkert, A. Celentano, et al., The Heavy Photon Search Test Detector, Nucl.Instrum.Meth. A777 (2014) 91-101, [arXiv:1406.6115].

[58] J. Balewski, J. Bernauer, J. Bessuille, R. Corliss, R. Cowan, et al., The DarkLight Experiment: A Precision Search for New Physics at Low Energies, arXiv:1412.4717. 
[59] X. He, G. C. Joshi, H. Lew, and R. Volkas, NEW Z-prime PHENOMENOLOGY, Phys.Rev. D43 (1991) 22-24.

[60] X.-G. He, G. C. Joshi, H. Lew, and R. Volkas, Simplest Z-prime model, Phys.Rev. D44 (1991) $2118-2132$.

[61] S. Baek, N. Deshpande, X. He, and P. Ko, Muon anomalous g-2 and gauged L(muon) - L(tau) models, Phys.Rev. D64 (2001) 055006, [hep-ph/0104141].

[62] E. Ma, D. Roy, and S. Roy, Gauged L(mu) - L(tau) with large muon anomalous magnetic moment and the bimaximal mixing of neutrinos, Phys.Lett. B525 (2002) 101-106, [hep-ph/0110146].

[63] E. Salvioni, A. Strumia, G. Villadoro, and F. Zwirner, Non-universal minimal Z' models: present bounds and early LHC reach, JHEP 1003 (2010) 010, [arXiv:0911.1450].

[64] J. Heeck and W. Rodejohann, Gauged $L_{\mu}-L_{\tau}$ Symmetry at the Electroweak Scale, Phys.Rev. D84 (2011) 075007, [arXiv:1107.5238].

[65] M. Pospelov, Neutrino Physics with Dark Matter Experiments and the Signature of New Baryonic Neutral Currents, Phys.Rev. D84 (2011) 085008, [arXiv:1103.3261].

[66] B. A. Dobrescu and C. Frugiuele, Hidden GeV-scale interactions of quarks, Phys.Rev.Lett. 113 (2014) 061801, [arXiv: 1404.3947].

[67] S. Tulin, New weakly-coupled forces hidden in low-energy QCD, Phys.Rev. D89 (2014), no. 11 114008, [arXiv: 1404.4370].

[68] B. Batell, P. deNiverville, D. McKeen, M. Pospelov, and A. Ritz, Leptophobic Dark Matter at Neutrino Factories, Phys.Rev. D90 (2014), no. 11 115014, [arXiv:1405.7049].

[69] J. Preskill, Gauge anomalies in an effective field theory, Annals Phys. 210 (1991) 323-379.

[70] J. F. Kamenik and C. Smith, Could a light Higgs boson illuminate the dark sector?, Phys.Rev. D85 (2012) 093017, [arXiv:1201.4814].

[71] B. A. Dobrescu, Massless gauge bosons other than the photon, Phys.Rev.Lett. 94 (2005) 151802, [hep-ph/0411004].

[72] I. Antoniadis, E. Kiritsis, and T. Tomaras, A D-brane alternative to unification, Phys.Lett. B486 (2000) 186-193, [hep-ph/0004214].

[73] C. Coriano, N. Irges, and E. Kiritsis, On the effective theory of low scale orientifold string vacua, Nucl.Phys. B746 (2006) 77-135, [hep-ph/0510332].

[74] N. Irges, C. Coriano, and S. Morelli, Stuckelberg Axions and the Effective Action of Anomalous Abelian Models 2. A SU(3)C $x S U(2) W x U(1) Y x U(1) B$ model and its signature at the LHC, Nucl.Phys. B789 (2008) 133-174, [hep-ph/0703127].

[75] I. Antoniadis, A. Boyarsky, and O. Ruchayskiy, Axion alternatives, hep-ph/0606306.

[76] I. Antoniadis, A. Boyarsky, and O. Ruchayskiy, Anomaly induced effects in a magnetic field, Nucl.Phys. B793 (2008) 246-259, [arXiv:0708.3001].

[77] P. Anastasopoulos, M. Bianchi, E. Dudas, and E. Kiritsis, Anomalies, anomalous U(1)'s and generalized Chern-Simons terms, JHEP 0611 (2006) 057, [hep-th/0605225].

[78] P. Anastasopoulos, F. Fucito, A. Lionetto, G. Pradisi, A. Racioppi, et al., Minimal Anomalous U(1)-prime Extension of the MSSM, Phys.Rev. D78 (2008) 085014, [arXiv:0804.1156].

[79] J. A. Harvey, C. T. Hill, and R. J. Hill, Standard Model Gauging of the Wess-Zumino-Witten Term: Anomalies, Global Currents and pseudo-Chern-Simons Interactions, Phys.Rev. D77 (2008) 085017, [arXiv:0712.1230].

[80] E. Dudas, Y. Mambrini, S. Pokorski, and A. Romagnoni, (In)visible Z-prime and dark matter, JHEP 0908 (2009) 014, [arXiv:0904.1745]. 
[81] J. Kumar, A. Rajaraman, and J. D. Wells, Probing the Green-Schwarz Mechanism at the Large Hadron Collider, Phys.Rev. D77 (2008) 066011, [arXiv:0707.3488].

[82] E. D'Hoker and E. Farhi, Decoupling a Fermion in the Standard Electroweak Theory, Nucl. Phys. B248 (1984) 77.

[83] E. D'Hoker and E. Farhi, Decoupling a Fermion Whose Mass Is Generated by a Yukawa Coupling: The General Case, Nucl. Phys. B248 (1984) 59.

[84] L3 Collaboration, M. Acciarri et al., Search for new physics in energetic single photon production in $e^{+} e^{-}$annihilation at the $Z$ resonance, Phys.Lett. B412 (1997) 201-209.

[85] B. Batell, M. Pospelov, and A. Ritz, Probing a Secluded U(1) at B-factories, Phys.Rev. D79 (2009) 115008, [arXiv:0903.0363].

[86] M. Pospelov, Secluded U(1) below the weak scale, Phys.Rev. D80 (2009) 095002, [arXiv:0811.1030].

[87] H. Davoudiasl, H.-S. Lee, and W. J. Marciano, 'Dark' Z implications for Parity Violation, Rare Meson Decays, and Higgs Physics, Phys.Rev. D85 (2012) 115019, [arXiv:1203.2947].

[88] K. R. Dienes, C. F. Kolda, and J. March-Russell, Kinetic mixing and the supersymmetric gauge hierarchy, Nucl.Phys. B492 (1997) 104-118, [hep-ph/9610479].

[89] D. Hooper and K. M. Zurek, A Natural Supersymmetric Model with MeV Dark Matter, Phys.Rev. D77 (2008) 087302, [arXiv:0801.3686].

[90] M. Baumgart, C. Cheung, J. T. Ruderman, L.-T. Wang, and I. Yavin, Non-Abelian Dark Sectors and Their Collider Signatures, JHEP 0904 (2009) 014, [arXiv:0901.0283].

[91] D. E. Morrissey, D. Poland, and K. M. Zurek, Abelian Hidden Sectors at a GeV, JHEP 0907 (2009) 050, [arXiv:0904.2567].

[92] Muon G-2 Collaboration, G. Bennett et al., Final Report of the Muon E821 Anomalous Magnetic Moment Measurement at BNL, Phys.Rev. D73 (2006) 072003, [hep-ex/0602035].

[93] A. Czarnecki and W. J. Marciano, The Muon anomalous magnetic moment: A Harbinger for 'new physics', Phys.Rev. D64 (2001) 013014, [hep-ph/0102122].

[94] S. Gninenko and N. Krasnikov, The Muon anomalous magnetic moment and a new light gauge boson, Phys.Lett. B513 (2001) 119, [hep-ph/0102222].

[95] R. Essig, J. Mardon, M. Papucci, T. Volansky, and Y.-M. Zhong, Constraining Light Dark Matter with Low-Energy $e^{+} e^{-}$Colliders, JHEP 1311 (2013) 167, [arXiv:1309.5084].

[96] W. Altmannshofer, S. Gori, M. Pospelov, and I. Yavin, Quark flavor transitions in $L_{\mu}-L_{\tau}$ models, Phys.Rev. D89 (2014), no. 9 095033, [arXiv:1403.1269].

[97] W. Altmannshofer, S. Gori, M. Pospelov, and I. Yavin, Neutrino Trident Production: A Powerful Probe of New Physics with Neutrino Beams, Phys.Rev.Lett. 113 (2014) 091801, [arXiv:1406.2332].

[98] B. W. Lee and S. Weinberg, Cosmological Lower Bound on Heavy Neutrino Masses, Phys.Rev.Lett. 39 (1977) 165-168.

[99] M. Vysotsky, A. Dolgov, and Y. Zeldovich, Cosmological Restriction on Neutral Lepton Masses, JETP Lett. 26 (1977) 188-190.

[100] C. Boehm and P. Fayet, Scalar dark matter candidates, Nucl.Phys. B683 (2004) 219-263, [hep-ph/0305261].

[101] R. Essig, J. Mardon, and T. Volansky, Direct Detection of Sub-GeV Dark Matter, Phys.Rev. D85 (2012) 076007, [arXiv:1108.5383].

[102] P. Jean, J. Knoedlseder, V. Lonjou, M. Allain, J.-P. Roques, et al., Early SPI / INTEGRAL measurements of $511 \mathrm{keV}$ line emission from the 4 th quadrant of the Galaxy, Astron.Astrophys. 407 (2003) L55, [astro-ph/0309484]. 
[103] N. Prantzos, C. Boehm, A. Bykov, R. Diehl, K. Ferriere, et al., The 511 keV emission from positron annihilation in the Galaxy, Rev.Mod.Phys. 83 (2011) 1001, [arXiv:1009.4620].

[104] C. Boehm, D. Hooper, J. Silk, M. Casse, and J. Paul, MeV dark matter: Has it been detected?, Phys.Rev.Lett. 92 (2004) 101301, [astro-ph/0309686].

[105] PAMELA Collaboration, O. Adriani et al., An anomalous positron abundance in cosmic rays with energies 1.5-100 GeV, Nature 458 (2009) 607-609, [arXiv:0810.4995].

[106] PAMELA Collaboration, O. Adriani et al., PAMELA results on the cosmic-ray antiproton flux from $60 \mathrm{MeV}$ to $180 \mathrm{GeV}$ in kinetic energy, Phys.Rev.Lett. 105 (2010) 121101, [arXiv:1007.0821].

[107] Fermi LAT Collaboration, M. Ackermann et al., Measurement of separate cosmic-ray electron and positron spectra with the Fermi Large Area Telescope, Phys.Rev.Lett. 108 (2012) 011103, [arXiv: 1109.0521].

[108] AMS Collaboration, M. Aguilar et al., First Result from the Alpha Magnetic Spectrometer on the International Space Station: Precision Measurement of the Positron Fraction in Primary Cosmic Rays of 0.5-350 GeV, Phys.Rev.Lett. 110 (2013) 141102.

[109] N. Arkani-Hamed, D. P. Finkbeiner, T. R. Slatyer, and N. Weiner, A Theory of Dark Matter, Phys.Rev. D79 (2009) 015014, [arXiv:0810.0713].

[110] M. Pospelov and A. Ritz, Astrophysical Signatures of Secluded Dark Matter, Phys.Lett. B671 (2009) 391-397, [arXiv:0810.1502].

[111] M. Pospelov, A. Ritz, and M. B. Voloshin, Secluded WIMP Dark Matter, Phys.Lett. B662 (2008) 53-61, [arXiv: 0711.4866].

[112] S. Profumo, Dissecting cosmic-ray electron-positron data with Occam's Razor: the role of known Pulsars, Central Eur.J.Phys. 10 (2011) 1-31, [arXiv:0812.4457].

[113] D. Hooper, P. Blasi, and P. D. Serpico, Pulsars as the Sources of High Energy Cosmic Ray Positrons, JCAP 0901 (2009) 025, [arXiv:0810.1527].

[114] LAT Collaboration, M. Ackermann et al., Fermi LAT Search for Dark Matter in Gamma-ray Lines and the Inclusive Photon Spectrum, Phys.Rev. D86 (2012) 022002, [arXiv:1205.2739].

[115] B. Shuve and I. Yavin, Dark matter progenitor: Light vector boson decay into sterile neutrinos, Phys.Rev. D89 (2014), no. 11 113004, [arXiv:1403.2727].

[116] S. Dodelson and L. M. Widrow, Sterile-neutrinos as dark matter, Phys.Rev.Lett. 72 (1994) 17-20, [hep-ph/9303287].

[117] X.-D. Shi and G. M. Fuller, A New dark matter candidate: Nonthermal sterile neutrinos, Phys.Rev.Lett. 82 (1999) 2832-2835, [astro-ph/9810076].

[118] K. Abazajian, G. M. Fuller, and M. Patel, Sterile neutrino hot, warm, and cold dark matter, Phys. Rev. D64 (2001) 023501, [astro-ph/0101524].

[119] M. Shaposhnikov and I. Tkachev, The nuMSM, inflation, and dark matter, Phys.Lett. B639 (2006) 414-417, [hep-ph/0604236].

[120] A. Kusenko, Sterile neutrinos, dark matter, and the pulsar velocities in models with a Higgs singlet, Phys.Rev.Lett. 97 (2006) 241301, [hep-ph/0609081].

[121] A. Merle, V. Niro, and D. Schmidt, New Production Mechanism for keV Sterile Neutrino Dark Matter by Decays of Frozen-In Scalars, JCAP 1403 (2014) 028, [arXiv:1306.3996].

[122] D. N. Spergel and P. J. Steinhardt, Observational evidence for selfinteracting cold dark matter, Phys.Rev.Lett. 84 (2000) 3760-3763, [astro-ph/9909386].

[123] R. Dave, D. N. Spergel, P. J. Steinhardt, and B. D. Wandelt, Halo properties in cosmological simulations of selfinteracting cold dark matter, Astrophys.J. 547 (2001) 574-589, [astro-ph/0006218]. 
[124] B. Moore, Evidence against dissipationless dark matter from observations of galaxy haloes, Nature 370 (1994) 629.

[125] R. A. Flores and J. R. Primack, Observational and theoretical constraints on singular dark matter halos, Astrophys.J. 427 (1994) L1-4, [astro-ph/9402004].

[126] J. D. Simon, A. D. Bolatto, A. Leroy, L. Blitz, and E. L. Gates, High-resolution measurements of the halos of four dark matter-dominated galaxies: Deviations from a universal density profile, Astrophys.J. 621 (2005) 757-776, [astro-ph/0412035].

[127] S.-H. Oh, W. de Blok, E. Brinks, F. Walter, and J. Kennicutt, Robert C., Dark and luminous matter in THINGS dwarf galaxies, Astron.J. 141 (2011) 193, [arXiv:1011.0899].

[128] M. G. Walker and J. Penarrubia, A Method for Measuring (Slopes of) the Mass Profiles of Dwarf Spheroidal Galaxies, Astrophys.J. 742 (2011) 20, [arXiv:1108.2404].

[129] J. F. Navarro, C. S. Frenk, and S. D. White, A Universal density profile from hierarchical clustering, Astrophys.J. 490 (1997) 493-508, [astro-ph/9611107].

[130] W. de Blok, S. S. McGaugh, A. Bosma, and V. C. Rubin, Mass density profiles of LSB galaxies, Astrophys.J. 552 (2001) L23-L26, [astro-ph/0103102].

[131] R. Kuzio de Naray, S. S. McGaugh, and W. de Blok, Mass Models for Low Surface Brightness Galaxies with High Resolution Optical Velocity Fields, Astrophys.J. 676 (2008) 920-943, [arXiv:0712.0860].

[132] R. K. de Naray and K. Spekkens, Do Baryons Alter the Halos of Low Surface Brightness Galaxies?, Astrophys.J. 741 (2011) L29, [arXiv:1109.1288].

[133] W. de Blok and A. Bosma, High-resolution rotation curves of low surface brightness galaxies, Astron.Astrophys. 385 (2002) 816, [astro-ph/0201276].

[134] A. B. Newman, T. Treu, R. S. Ellis, and D. J. Sand, The Density Profiles of Massive, Relaxed Galaxy Clusters: II. Separating Luminous and Dark Matter in Cluster Cores, Astrophys.J. $\mathbf{7 6 5}$ (2013), no. issue 25, [arXiv: 1209.1392].

[135] M. Boylan-Kolchin, J. S. Bullock, and M. Kaplinghat, Too big to fail? The puzzling darkness of massive Milky Way subhaloes, Mon.Not.Roy.Astron.Soc. 415 (2011) L40, [arXiv:1103.0007].

[136] M. Boylan-Kolchin, J. S. Bullock, and M. Kaplinghat, The Milky Way's bright satellites as an apparent failure of LCDM, Mon.Not.Roy.Astron.Soc. 422 (2012) 1203-1218, [arXiv:1111.2048].

[137] E. J. Tollerud, M. Boylan-Kolchin, and J. S. Bullock, M31 Satellite Masses Compared to LCDM Subhaloes, Mon.Not.Roy.Astron.Soc. 440 (2014) 3511, [arXiv:1403.6469].

[138] S. Garrison-Kimmel, M. Boylan-Kolchin, J. S. Bullock, and E. N. Kirby, Too Big to Fail in the Local Group, Mon.Not.Roy.Astron.Soc. 444 (2014) 222, [arXiv:1404.5313].

[139] M. Rocha, A. H. Peter, J. S. Bullock, M. Kaplinghat, S. Garrison-Kimmel, et al., Cosmological Simulations with Self-Interacting Dark Matter I: Constant Density Cores and Substructure, Mon.Not.Roy.Astron.Soc. 430 (2013) 81-104, [arXiv:1208.3025].

[140] A. H. Peter, M. Rocha, J. S. Bullock, and M. Kaplinghat, Cosmological Simulations with Self-Interacting Dark Matter II: Halo Shapes vs. Observations, Mon.Not.Roy.Astron.Soc. 430 (2013) 105, [arXiv: 1208.3026].

[141] M. Vogelsberger, J. Zavala, and A. Loeb, Subhaloes in Self-Interacting Galactic Dark Matter Haloes, Mon.Not.Roy.Astron.Soc. 423 (2012) 3740, [arXiv:1201.5892].

[142] J. Zavala, M. Vogelsberger, and M. G. Walker, Constraining Self-Interacting Dark Matter with the Milky Way's dwarf spheroidals, Monthly Notices of the Royal Astronomical Society: Letters 431 (2013) L20-L24, [arXiv:1211.6426]. 
[143] A. Pontzen and F. Governato, How supernova feedback turns dark matter cusps into cores, Mon.Not.Roy.Astron.Soc. 421 (2012) 3464, [arXiv:1106.0499].

[144] F. Governato, A. Zolotov, A. Pontzen, C. Christensen, S. Oh, et al., Cuspy No More: How Outflows Affect the Central Dark Matter and Baryon Distribution in Lambda CDM Galaxies, Mon.Not.Roy.Astron.Soc. 422 (2012) 1231-1240, [arXiv:1202.0554].

[145] A. Zolotov, A. M. Brooks, B. Willman, F. Governato, A. Pontzen, et al., Baryons Matter: Why Luminous Satellite Galaxies Have Reduced Central Masses, Astrophys.J. 761 (2012) 71, [arXiv:1207.0007].

[146] R. Teyssier, B. Moore, D. Martizzi, Y. Dubois, and L. Mayer, Mass Distribution in Galaxy Clusters: the Role of AGN Feedback, Mon.Not.Roy.Astron.Soc. 414 (2011) 195, [arXiv: 1003.4744].

[147] L. Ackerman, M. R. Buckley, S. M. Carroll, and M. Kamionkowski, Dark Matter and Dark Radiation, Phys.Rev. D79 (2009) 023519, [arXiv:0810.5126].

[148] J. L. Feng, M. Kaplinghat, and H.-B. Yu, Halo Shape and Relic Density Exclusions of Sommerfeld-Enhanced Dark Matter Explanations of Cosmic Ray Excesses, Phys.Rev.Lett. 104 (2010) 151301, [arXiv:0911.0422].

[149] M. R. Buckley and P. J. Fox, Dark Matter Self-Interactions and Light Force Carriers, Phys.Rev. D81 (2010) 083522, [arXiv:0911.3898].

[150] A. Loeb and N. Weiner, Cores in Dwarf Galaxies from Dark Matter with a Yukawa Potential, Phys.Rev.Lett. 106 (2011) 171302, [arXiv:1011.6374].

[151] T. Lin, H.-B. Yu, and K. M. Zurek, On Symmetric and Asymmetric Light Dark Matter, Phys.Rev. D85 (2012) 063503, [arXiv:1111.0293].

[152] L. G. van den Aarssen, T. Bringmann, and C. Pfrommer, Is dark matter with long-range interactions a solution to all small-scale problems of $\Lambda$ CDM cosmology?, Phys.Rev.Lett. 109 (2012) 231301, [arXiv:1205.5809].

[153] S. Tulin, H.-B. Yu, and K. M. Zurek, Resonant Dark Forces and Small Scale Structure, Phys.Rev.Lett. 110 (2013), no. 11 111301, [arXiv:1210.0900].

[154] S. Tulin, H.-B. Yu, and K. M. Zurek, Beyond Collisionless Dark Matter: Particle Physics Dynamics for Dark Matter Halo Structure, Phys.Rev. D87 (2013), no. 11 115007, [arXiv: 1302.3898].

[155] M. Kaplinghat, S. Tulin, and H.-B. Yu, Direct Detection Portals for Self-interacting Dark Matter, Phys.Rev. D89 (2014), no. 3 035009, [arXiv:1310.7945].

[156] PDG Collaboration, K. Olive et al., Review of Particle Physics, Chin.Phys. C38 (2014) 090001.

[157] B. Batell, M. Pospelov, and A. Ritz, Exploring Portals to a Hidden Sector Through Fixed Targets, Phys.Rev. D80 (2009) 095024, [arXiv:0906.5614].

[158] J. Blümlein and J. Brunner, New Exclusion Limits on Dark Gauge Forces from Proton Bremsstrahlung in Beam-Dump Data, Phys.Lett. B731 (2014) 320-326, [arXiv:1311.3870].

[159] D. Gorbunov, A. Makarov, and I. Timiryasov, Decaying light particles on board the SHiP (I): Signal rate estimates for hidden photons, Phys.Rev. D91 (2015) 035027, [arXiv:1411.4007].

[160] P. deNiverville, D. McKeen, and A. Ritz, Signatures of sub-GeV dark matter beams at neutrino experiments, Phys.Rev. D86 (2012) 035022, [arXiv:1205.3499].

[161] P. deNiverville, M. Pospelov, and A. Ritz, Observing a light dark matter beam with neutrino experiments, Phys.Rev. D84 (2011) 075020, [arXiv:1107.4580].

[162] E. Izaguirre, G. Krnjaic, P. Schuster, and N. Toro, New Electron Beam-Dump Experiments to Search for MeV to few-GeV Dark Matter, Phys.Rev. D88 (2013) 114015, [arXiv:1307.6554]. 
[163] Y. S. Jeong, C. Kim, and M. H. Reno, Majorana Dark Matter Cross Sections with Nucleons at High Energies, Phys.Rev. D86 (2012) 094025, [arXiv:1207.1526].

[164] D. E. Soper, M. Spannowsky, C. J. Wallace, and T. M. P. Tait, Scattering of Dark Particles with Light Mediators, Phys.Rev. D90 (2014), no. 11 115005, [arXiv:1407.2623].

[165] B. A. Dobrescu and C. Frugiuele, GeV-scale dark matter: production at the Main Injector, JHEP 1502 (2015) 019, [arXiv:1410.1566].

[166] D. E. Morrissey and A. P. Spray, New Limits on Light Hidden Sectors from Fixed-Target Experiments, JHEP 1406 (2014) 083, [arXiv:1402.4817].

[167] P. Schuster, N. Toro, and I. Yavin, Terrestrial and Solar Limits on Long-Lived Particles in a Dark Sector, Phys.Rev. D81 (2010) 016002, [arXiv:0910.1602].

[168] J. Heeck, Unbroken B-L Symmetry, Phys.Lett. B739 (2014) 256-262, [arXiv:1408.6845].

[169] BaBar Collaboration, J. Lees et al., Search for Low-Mass Dark-Sector Higgs Bosons, Phys.Rev.Lett. 108 (2012) 211801, [arXiv:1202.1313].

[170] KLOE-2 Collaboration, D. Babusci et al., Search for dark Higgsstrahlung in e+ e- - $\dot{z} \mathrm{mu}+\mathrm{mu-}$ and missing energy events with the KLOE experiment, arXiv:1501.06795.

[171] S. Andreas, S. Donskov, P. Crivelli, A. Gardikiotis, S. Gninenko, et al., Proposal for an Experiment to Search for Light Dark Matter at the SPS, arXiv:1312.3309.

[172] E. Izaguirre, G. Krnjaic, P. Schuster, and N. Toro, Testing GeV-Scale Dark Matter with Fixed-Target Missing Momentum Experiments, arXiv:1411.1404.

[173] B. Batell, R. Essig, and Z. Surujon, Strong Constraints on Sub-GeV Dark Sectors from SLAC Beam Dump E137, Phys.Rev.Lett. 113 (2014), no. 17 171802, [arXiv:1406.2698].

[174] Y. Kahn, G. Krnjaic, J. Thaler, and M. Toups, DAEdALUS and Dark Matter, Phys.Rev. D91 (2015) 055006, [arXiv:1411.1055].

[175] MiniBooNE Collaboration, R. Dharmapalan et al., Low Mass WIMP Searches with a Neutrino Experiment: A Proposal for Further MiniBooNE Running, arXiv:1211.2258.

[176] P. D. Serpico and G. G. Raffelt, MeV-mass dark matter and primordial nucleosynthesis, Phys. Rev. D70 (2004) 043526, [astro-ph/0403417].

[177] K. M. Nollett and G. Steigman, BBN And The CMB Constrain Light, Electromagnetically Coupled WIMPs, Phys.Rev. D89 (2014), no. 8 083508, [arXiv:1312.5725].

[178] G. Raffelt and D. Seckel, Bounds on Exotic Particle Interactions from SN 1987a, Phys.Rev.Lett. 60 (1988) 1793.

[179] P. Fayet, D. Hooper, and G. Sigl, Constraints on light dark matter from core-collapse supernovae, Phys.Rev.Lett. 96 (2006) 211302, [hep-ph/0602169].

[180] J. Alwall, R. Frederix, S. Frixione, V. Hirschi, F. Maltoni, et al., The automated computation of tree-level and next-to-leading order differential cross sections, and their matching to parton shower simulations, JHEP 1407 (2014) 079, [arXiv: 1405.0301].

[181] F. Englert and R. Brout, Broken Symmetry and the Mass of Gauge Vector Mesons, Phys.Rev.Lett. 13 (1964) 321-323.

[182] P. W. Higgs, Broken symmetries, massless particles and gauge fields, Phys.Lett. 12 (1964) 132-133.

[183] P. W. Higgs, Broken Symmetries and the Masses of Gauge Bosons, Phys.Rev.Lett. 13 (1964) 508-509.

[184] G. Guralnik, C. Hagen, and T. Kibble, Global Conservation Laws and Massless Particles, Phys.Rev.Lett. 13 (1964) 585-587. 
[185] D. E. Morrissey and M. J. Ramsey-Musolf, Electroweak baryogenesis, New J.Phys. 14 (2012) 125003, [arXiv: 1206.2942].

[186] V. Silveira and A. Zee, SCALAR PHANTOMS, Phys.Lett. B161 (1985) 136.

[187] D. Feldman, B. Kors, and P. Nath, Extra-weakly Interacting Dark Matter, Phys.Rev. D75 (2007) 023503, [hep-ph/0610133].

[188] Z. Chacko, H.-S. Goh, and R. Harnik, The Twin Higgs: Natural electroweak breaking from mirror symmetry, Phys.Rev.Lett. 96 (2006) 231802, [hep-ph/0506256].

[189] G. Burdman, Z. Chacko, H.-S. Goh, and R. Harnik, Folded supersymmetry and the LEP paradox, JHEP 0702 (2007) 009, [hep-ph/0609152].

[190] N. Craig and K. Howe, Doubling down on naturalness with a supersymmetric twin Higgs, JHEP 1403 (2014) 140, [arXiv:1312.1341].

[191] N. Craig, S. Knapen, and P. Longhi, Neutral Naturalness from Orbifold Higgs Models, Phys.Rev.Lett. 114 (2015), no. 6 061803, [arXiv:1410.6808].

[192] G. Burdman, Z. Chacko, R. Harnik, L. de Lima, and C. B. Verhaaren, Colorless Top Partners, a 125 GeV Higgs, and the Limits on Naturalness, Phys.Rev. D91 (2015), no. 5 055007, [arXiv:1411.3310].

[193] M. J. Strassler and K. M. Zurek, Echoes of a hidden valley at hadron colliders, Phys.Lett. B651 (2007) 374-379, [hep-ph/0604261].

[194] T. Binoth and J. van der Bij, Influence of strongly coupled, hidden scalars on Higgs signals, Z.Phys. C75 (1997) 17-25, [hep-ph/9608245].

[195] R. Schabinger and J. D. Wells, A Minimal spontaneously broken hidden sector and its impact on Higgs boson physics at the large hadron collider, Phys.Rev. D72 (2005) 093007, [hep-ph/0509209].

[196] B. Patt and F. Wilczek, Higgs-field portal into hidden sectors, hep-ph/0605188.

[197] M. J. Strassler, Possible effects of a hidden valley on supersymmetric phenomenology, hep-ph/0607160.

[198] J. L. Feng and J. Kumar, The WIMPless Miracle: Dark-Matter Particles without Weak-Scale Masses or Weak Interactions, Phys.Rev.Lett. 101 (2008) 231301, [arXiv:0803.4196].

[199] D. E. Kaplan, M. A. Luty, and K. M. Zurek, Asymmetric Dark Matter, Phys.Rev. D79 (2009) 115016, [arXiv:0901.4117].

[200] N. Craig, A. Katz, M. Strassler, and R. Sundrum, Naturalness in the Dark at the LHC, arXiv: 1501.05310

[201] I.-W. Kim and K. M. Zurek, Flavor and Collider Signatures of Asymmetric Dark Matter, Phys.Rev. D89 (2014), no. 3 035008, [arXiv:1310.2617].

[202] N. Arkani-Hamed and N. Weiner, LHC Signals for a SuperUnified Theory of Dark Matter, JHEP 0812 (2008) 104, [arXiv:0810.0714].

[203] J. Shelton and K. M. Zurek, Darkogenesis: A baryon asymmetry from the dark matter sector, Phys.Rev. D82 (2010) 123512, [arXiv:1008.1997].

[204] G. Burdman, Z. Chacko, H.-S. Goh, R. Harnik, and C. A. Krenke, The Quirky Collider Signals of Folded Supersymmetry, Phys.Rev. D78 (2008) 075028, [arXiv:0805.4667].

[205] N. Craig, S. Knapen, and P. Longhi, The Orbifold Higgs, arXiv:1411.7393.

[206] N. Craig, H. K. Lou, M. McCullough, and A. Thalapillil, The Higgs Portal Above Threshold, arXiv: 1412.0258.

[207] J. E. Juknevich, Pure-glue hidden valleys through the Higgs portal, JHEP 1008 (2010) 121, [arXiv:0911.5616]. 
[208] H. M. Lee, S. Raby, M. Ratz, G. G. Ross, R. Schieren, et al., A unique $Z_{4}^{R}$ symmetry for the MSSM, Phys.Lett. B694 (2011) 491-495, [arXiv: 1009.0905].

[209] H. M. Lee, S. Raby, M. Ratz, G. G. Ross, R. Schieren, et al., Discrete R symmetries for the MSSM and its singlet extensions, Nucl.Phys. B850 (2011) 1-30, [arXiv:1102.3595].

[210] G. G. Ross and K. Schmidt-Hoberg, The Fine-Tuning of the Generalised NMSSM, Nucl.Phys. B862 (2012) 710-719, [arXiv:1108.1284].

[211] G. G. Ross, K. Schmidt-Hoberg, and F. Staub, The Generalised NMSSM at One Loop: Fine Tuning and Phenomenology, JHEP 1208 (2012) 074, [arXiv: 1205.1509].

[212] R. Kappl, M. Ratz, and M. W. Winkler, Light dark matter in the singlet-extended MSSM, Phys.Lett. B695 (2011) 169-173, [arXiv:1010.0553].

[213] J. Erler, P. Langacker, S. Munir, and E. Rojas, Z' Bosons at Colliders: a Bayesian Viewpoint, JHEP 1111 (2011) 076, [arXiv:1103.2659].

[214] S. Khalil and A. Masiero, Radiative B-L symmetry breaking in supersymmetric models, Phys.Lett. B665 (2008) 374-377, [arXiv:0710.3525].

[215] P. Fileviez Perez and S. Spinner, The Fate of R-Parity, Phys.Rev. D83 (2011) 035004, [arXiv: 1005.4930].

[216] B. O'Leary, W. Porod, and F. Staub, Mass spectrum of the minimal SUSY B-L model, JHEP 1205 (2012) 042, [arXiv:1112.4600].

[217] L. Basso, B. O'Leary, W. Porod, and F. Staub, Dark matter scenarios in the minimal SUSY B-L model, JHEP 1209 (2012) 054, [arXiv:1207.0507].

[218] D. Lopez-Fogliani and C. Munoz, Proposal for a Supersymmetric Standard Model, Phys.Rev.Lett. 97 (2006) 041801, [hep-ph/0508297].

[219] A. Bartl, M. Hirsch, A. Vicente, S. Liebler, and W. Porod, LHC phenomenology of the mu nu SSM, JHEP 0905 (2009) 120, [arXiv:0903.3596].

[220] P. Ghosh, D. E. Lopez-Fogliani, V. A. Mitsou, C. Munoz, and R. R. de Austri, Probing the $\mu \nu S S M$ with light scalars, pseudoscalars and neutralinos from the decay of a SM-like Higgs boson at the LHC, JHEP 1411 (2014) 102, [arXiv:1410.2070].

[221] F. Bezrukov and D. Gorbunov, Light inflaton Hunter's Guide, JHEP 1005 (2010) 010, [arXiv: 0912.0390].

[222] F. Bezrukov and D. Gorbunov, Light inflaton after LHC8 and WMAP9 results, JHEP 1307 (2013) 140, [arXiv:1303.4395].

[223] M. Voloshin, Once Again About the Role of Gluonic Mechanism in Interaction of Light Higgs Boson with Hadrons, Sov.J.Nucl.Phys. 44 (1986) 478.

[224] S. Raby and G. B. West, The Branching Ratio for a Light Higgs to Decay Into $\mu^{+} \mu^{-}$Pairs, Phys.Rev. D38 (1988) 3488.

[225] T. N. Truong and R. Willey, Branching Ratios for Decays of Light Higgs Bosons, Phys.Rev. D40 (1989) 3635 .

[226] J. F. Donoghue, J. Gasser, and H. Leutwyler, The Decay of a Light Higgs Boson, Nucl.Phys. B343 (1990) 341-368.

[227] D. McKeen, Constraining Light Bosons with Radiative Upsilon(1S) Decays, Phys.Rev. D79 (2009) 015007, [arXiv:0809.4787].

[228] T. Robens and T. Stefaniak, Status of the Higgs Singlet Extension of the Standard Model after LHC Run 1, Eur.Phys.J. C75 (2015), no. 3 104, [arXiv:1501.02234].

[229] K. Schmidt-Hoberg, F. Staub, and M. W. Winkler, Constraints on light mediators: confronting dark matter searches with B physics, Phys.Lett. B727 (2013) 506-510, [arXiv:1310.6752]. 
[230] J. D. Clarke, R. Foot, and R. R. Volkas, Phenomenology of a very light scalar (100 MeV $<m_{h}<$ $10 \mathrm{GeV}$ ) mixing with the SM Higgs, JHEP 1402 (2014) 123, [arXiv:1310.8042].

[231] B. Batell, M. Pospelov, and A. Ritz, Multi-lepton Signatures of a Hidden Sector in Rare B Decays, Phys.Rev. D83 (2011) 054005, [arXiv:0911.4938].

[232] M. J. Dolan, C. McCabe, F. Kahlhoefer, and K. Schmidt-Hoberg, A taste of dark matter: Flavour constraints on pseudoscalar mediators, arXiv:1412.5174.

[233] G. Hiller, B physics signals of the lightest CP odd Higgs in the NMSSM at large tan beta, Phys.Rev. D70 (2004) 034018, [hep-ph/0404220].

[234] CHARM Collaboration, F. Bergsma et al., Search for Axion Like Particle Production in 400-GeV Proton - Copper Interactions, Phys.Lett. B157 (1985) 458.

[235] D. Curtin, R. Essig, S. Gori, P. Jaiswal, A. Katz, et al., Exotic decays of the 125 GeV Higgs boson, Phys.Rev. D90 (2014), no. 7 075004, [arXiv:1312.4992].

[236] M. J. Strassler and K. M. Zurek, Discovering the Higgs through highly-displaced vertices, Phys.Lett. B661 (2008) 263-267, [hep-ph/0605193].

[237] M. J. Strassler, Why Unparticle Models with Mass Gaps are Examples of Hidden Valleys, arXiv:0801.0629.

[238] J. E. Juknevich, D. Melnikov, and M. J. Strassler, A Pure-Glue Hidden Valley I. States and Decays, JHEP 0907 (2009) 055, [arXiv:0903.0883].

[239] D. Curtin, R. Essig, S. Gori, and J. Shelton, Illuminating Dark Photons with High-Energy Colliders, arXiv:1412.0018.

[240] C. Bird, P. Jackson, R. V. Kowalewski, and M. Pospelov, Search for dark matter in $b \rightarrow s$ transitions with missing energy, Phys.Rev.Lett. 93 (2004) 201803, [hep-ph/0401195].

[241] C. Bird, R. V. Kowalewski, and M. Pospelov, Dark matter pair-production in $b \rightarrow s$ transitions, Mod.Phys.Lett. A21 (2006) 457-478, [hep-ph/0601090].

[242] A. Ali, P. Ball, L. Handoko, and G. Hiller, A Comparative study of the decays $B \rightarrow\left(K, K^{*}\right) \ell^{+} \ell^{-}$ in standard model and supersymmetric theories, Phys.Rev. D61 (2000) 074024, [hep-ph/9910221].

[243] J. Alwall, M. Herquet, F. Maltoni, O. Mattelaer, and T. Stelzer, MadGraph 5 : Going Beyond, JHEP 1106 (2011) 128, [arXiv:1106.0522].

[244] L. H. C. S. W. Group. https://twiki.cern.ch/twiki/bin/view/LHCPhysics/HiggsEuropeanStrategy, 2014.

[245] S. Dawson, A. Gritsan, H. Logan, J. Qian, C. Tully, et al., Working Group Report: Higgs Boson, arXiv: 1310.8361.

[246] M. E. Peskin, Estimation of LHC and ILC Capabilities for Precision Higgs Boson Coupling Measurements, arXiv:1312.4974.

[247] H. E. Haber and G. L. Kane, The Search for Supersymmetry: Probing Physics Beyond the Standard Model, Phys.Rept. 117 (1985) 75-263.

[248] R. Peccei and H. R. Quinn, CP Conservation in the Presence of Instantons, Phys.Rev.Lett. 38 (1977) 1440-1443.

[249] J. E. Kim, Light Pseudoscalars, Particle Physics and Cosmology, Phys.Rept. 150 (1987) 1-177.

[250] J. F. Gunion, H. E. Haber, G. L. Kane, and S. Dawson, The Higgs Hunter's Guide, Front.Phys. 80 (2000) 1-448.

[251] J. F. Gunion and H. E. Haber, The CP conserving two Higgs doublet model: The Approach to the decoupling limit, Phys.Rev. D67 (2003) 075019, [hep-ph/0207010]. 
[252] G. Branco, P. Ferreira, L. Lavoura, M. Rebelo, M. Sher, et al., Theory and phenomenology of two-Higgs-doublet models, Phys.Rept. 516 (2012) 1-102, [arXiv:1106.0034].

[253] U. Ellwanger, C. Hugonie, and A. M. Teixeira, The Next-to-Minimal Supersymmetric Standard Model, Phys.Rept. 496 (2010) 1-77, [arXiv:0910.1785].

[254] R. Barbieri, L. J. Hall, Y. Nomura, and V. S. Rychkov, Supersymmetry without a Light Higgs Boson, Phys.Rev. D75 (2007) 035007, [hep-ph/0607332].

[255] L. J. Hall, D. Pinner, and J. T. Ruderman, A Natural SUSY Higgs Near 126 GeV, JHEP 1204 (2012) 131, [arXiv:1112.2703].

[256] M. Farina, M. Perelstein, and B. Shakya, Higgs Couplings and Naturalness in $\lambda$-SUSY, JHEP 1404 (2014) 108, [arXiv: 1310.0459].

[257] Y. Nomura and J. Thaler, Dark Matter through the Axion Portal, Phys.Rev. D79 (2009) 075008, [arXiv:0810.5397].

[258] B. A. Dobrescu and K. T. Matchev, Light axion within the next-to-minimal supersymmetric standard model, JHEP 0009 (2000) 031, [hep-ph/0008192].

[259] R. Dermisek and J. F. Gunion, The NMSSM Close to the R-symmetry Limit and Naturalness in $h \rightarrow$ aa Decays for $m_{a}<2 m_{b}$, Phys.Rev. D75 (2007) 075019, [hep-ph/0611142].

[260] D. E. Morrissey and A. Pierce, Modified Higgs Boson Phenomenology from Gauge or Gaugino Mediation in the NMSSM, Phys.Rev. D78 (2008) 075029, [arXiv:0807.2259].

[261] E. Chun, Natural $\mu$-term with Peccei-Quinn symmetry, Phys.Lett. B348 (1995) 111-114, [hep-ph/9411290].

[262] P. Ciafaloni and A. Pomarol, Dynamical determination of the supersymmetric Higgs mass, Phys.Lett. B404 (1997) 83-88, [hep-ph/9702410].

[263] L. J. Hall and T. Watari, Electroweak supersymmetry with an approximate U(1)(PQ), Phys.Rev. D70 (2004) 115001, [hep-ph/0405109].

[264] D. Miller and R. Nevzorov, The Peccei-Quinn axion in the next-to-minimal supersymmetric standard model, hep-ph/0309143.

[265] P. Draper, T. Liu, C. E. Wagner, L.-T. Wang, and H. Zhang, Dark Light Higgs, Phys.Rev.Lett. 106 (2011) 121805, [arXiv: 1009.3963].

[266] D. Miller, S. Moretti, and R. Nevzorov, Higgs bosons in the NMSSM with exact and slightly broken $P Q$-symmetry, hep-ph/0501139.

[267] L. J. Hall and M. B. Wise, FLAVOR CHANGING HIGGS - BOSON COUPLINGS, Nucl.Phys. B187 (1981) 397.

[268] J. Frere, J. Vermaseren, and M. Gavela, The Elusive Axion, Phys.Lett. B103 (1981) 129-133.

[269] M. Freytsis, Z. Ligeti, and J. Thaler, Constraining the Axion Portal with $B \rightarrow K \ell^{+} \ell^{-}$, Phys.Rev. D81 (2010) 034001, [arXiv:0911.5355].

[270] BaBar Collaboration, J. Lees et al., Search for Long-Lived Particles in $e^{+} e^{-}$Collisions, arXiv: 1502.02580.

[271] CMS Collaboration, V. Khachatryan et al., Search for long-lived particles that decay into final states containing two electrons or two muons in proton-proton collisions at $\sqrt{s}=8 \mathrm{TeV}$, arXiv: 1411.6977.

[272] Planck Collaboration, P. Ade et al., Planck 2013 results. XVI. Cosmological parameters, Astron.Astrophys. (2014) [arXiv: 1303.5076].

[273] M. W. Winkler, "Light Dark Matter in Theory and Experiment." PhD thesis, TUM, 2012. 
[274] J. M. Cline, K. Kainulainen, P. Scott, and C. Weniger, Update on scalar singlet dark matter, Phys.Rev. D88 (2013) 055025, [arXiv: 1306.4710].

[275] LUX Collaboration, D. Akerib et al., First results from the LUX dark matter experiment at the Sanford Underground Research Facility, Phys.Rev.Lett. 112 (2014), no. 9 091303, [arXiv: 1310.8214].

[276] SuperCDMS Collaboration, A. Anderson, Constraints on Light WIMPs from SuperCDMS, arXiv: 1405.4210.

[277] P. Junnarkar and A. Walker-Loud, Scalar strange content of the nucleon from lattice QCD, Phys.Rev. D87 (2013), no. 11 114510, [arXiv:1301.1114].

[278] G. Belanger, B. Dumont, U. Ellwanger, J. Gunion, and S. Kraml, Global fit to Higgs signal strengths and couplings and implications for extended Higgs sectors, Phys.Rev. D88 (2013) 075008, [arXiv:1306.2941].

[279] L. J. Hall, K. Jedamzik, J. March-Russell, and S. M. West, Freeze-In Production of FIMP Dark Matter, JHEP 1003 (2010) 080, [arXiv:0911.1120].

[280] T. Cohen, D. J. Phalen, A. Pierce, and K. M. Zurek, Asymmetric Dark Matter from a GeV Hidden Sector, Phys.Rev. D82 (2010) 056001, [arXiv:1005.1655].

[281] M. T. Frandsen, S. Sarkar, and K. Schmidt-Hoberg, Light asymmetric dark matter from new strong dynamics, Phys.Rev. D84 (2011) 051703, [arXiv:1103.4350].

[282] Y. Bai and P. Schwaller, Scale of dark QCD, Phys.Rev. D89 (2014), no. 6063522 , [arXiv: 1306.4676].

[283] K. Petraki and R. R. Volkas, Review of asymmetric dark matter, Int.J.Mod.Phys. A28 (2013) 1330028, [arXiv: 1305.4939].

[284] K. M. Zurek, Asymmetric Dark Matter: Theories, Signatures, and Constraints, Phys.Rept. 537 (2014) 91-121, [arXiv: 1308.0338].

[285] P. Schwaller, D. Stolarski, and A. Weiler, Emerging Jets, arXiv:1502.05409.

[286] T. Sjostrand, S. Mrenna, and P. Z. Skands, A Brief Introduction to PYTHIA 8.1, Comput.Phys.Commun. 178 (2008) 852-867, [arXiv:0710.3820].

[287] D. S. Gorbunov and V. A. Rubakov, Introduction to the theory of the early universe: cosmological perturbations and inflationary theory. Hackensack, USA: World Scientific, 2011.

[288] Planck Collaboration, P. Ade et al., Planck 2013 results. XXII. Constraints on inflation, Astron.Astrophys. 571 (2014) A22, [arXiv:1303.5082].

[289] A. Anisimov, Y. Bartocci, and F. L. Bezrukov, Inflaton mass in the $\nu$ MSM inflation, Phys. Lett. B671 (2009) 211-215, [arXiv:0809.1097].

[290] F. Vissani, Do experiments suggest a hierarchy problem?, Phys.Rev. D57 (1998) 7027-7030, [hep-ph/9709409].

[291] G. F. Giudice, Naturalness after LHC8, PoS EPS-HEP2013 (2013) 163, [arXiv:1307.7879].

[292] F. L. Bezrukov and M. Shaposhnikov, The Standard Model Higgs boson as the inflaton, Phys.Lett. B659 (2008) 703-706, [arXiv:0710.3755].

[293] D. I. Kaiser and A. T. Todhunter, Primordial Perturbations from Multifield Inflation with Nonminimal Couplings, Phys.Rev. D81 (2010) 124037, [arXiv:1004.3805].

[294] R. N. Greenwood, D. I. Kaiser, and E. I. Sfakianakis, Multifield Dynamics of Higgs Inflation, Phys.Rev. D87 (2013) 064021, [arXiv:1210.8190].

[295] D. I. Kaiser and E. I. Sfakianakis, Multifield Inflation after Planck: The Case for Nonminimal Couplings, Phys.Rev.Lett. 112 (2014), no. 1 011302, [arXiv:1304.0363]. 
[296] WMAP Collaboration, G. Hinshaw et al., Nine-Year Wilkinson Microwave Anisotropy Probe (WMAP) Observations: Cosmological Parameter Results, Astrophys.J.Suppl. 208 (2013) 19, [arXiv: 1212.5226].

[297] E. Bulbul, M. Markevitch, A. Foster, R. K. Smith, M. Loewenstein, et al., Detection of An Unidentified Emission Line in the Stacked X-ray spectrum of Galaxy Clusters, Astrophys.J. $\mathbf{7 8 9}$ (2014) 13, [arXiv:1402.2301].

[298] A. Boyarsky, O. Ruchayskiy, D. Iakubovskyi, and J. Franse, Unidentified Line in X-Ray Spectra of the Andromeda Galaxy and Perseus Galaxy Cluster, Phys.Rev.Lett. 113 (2014) 251301, [arXiv: 1402.4119].

[299] F. Bezrukov and D. Gorbunov, Relic Gravity Waves and 7 keV Dark Matter from a GeV scale inflaton, Phys.Lett. B736 (2014) 494-498, [arXiv: 1403.4638].

[300] J. C. Pati and A. Salam, Lepton Number as the Fourth Color, Phys. Rev. D 10 (1974) 275.

[301] R. N. Mohapatra and J. C. Pati, A Natural Left-Right Symmetry, Phys. Rev. D 11 (1975) 2558.

[302] R. N. Mohapatra and J. C. Pati, Left-Right Gauge Symmetry and an Isoconjugate Model of CP Violation, Phys.Rev. D11 (1975) 566-571.

[303] G. Senjanovic and R. N. Mohapatra, Exact Left-Right Symmetry and Spontaneous Violation of Parity, Phys.Rev. D12 (1975) 1502.

[304] P. Minkowski, mu $\rightarrow$ e gamma at a Rate of One Out of 1-Billion Muon Decays?, Phys. Lett. B67 (1977) 421.

[305] T. Yanagida, HORIZONTAL SYMMETRY AND MASSES OF NEUTRINOS, Conf.Proc. C7902131 (1979) 95-99.

[306] S. Glashow, The Future of Elementary Particle Physics, NATO Sci.Ser.B 59 (1980) 687.

[307] M. Gell-Mann, P. Ramond, and R. Slansky, Complex Spinors and Unified Theories, Conf.Proc. C790927 (1979) 315-321, [arXiv:1306.4669].

[308] R. N. Mohapatra and G. Senjanovic, Neutrino mass and spontaneous parity nonconservation, Phys. Rev. Lett. 44 (1980) 912.

[309] R. N. Mohapatra and G. Senjanovic, Neutrino Masses and Mixings in Gauge Models with Spontaneous Parity Violation, Phys. Rev. D 23 (1981) 165.

[310] R. E. Shrock, General Theory of Weak Leptonic and Semileptonic Decays. 1. Leptonic Pseudoscalar Meson Decays, with Associated Tests For, and Bounds on, Neutrino Masses and Lepton Mixing, Phys.Rev. D24 (1981) 1232.

[311] R. E. Shrock, General Theory of Weak Processes Involving Neutrinos. 2. Pure Leptonic Decays, Phys.Rev. D24 (1981) 1275.

[312] M. Gronau, C. N. Leung, and J. L. Rosner, Extending Limits on Neutral Heavy Leptons, Phys.Rev. D29 (1984) 2539.

[313] A. Atre, T. Han, S. Pascoli, and B. Zhang, The Search for Heavy Majorana Neutrinos, JHEP 0905 (2009) 030, [arXiv:0901.3589].

[314] S. Weinberg, Varieties of Baryon and Lepton Nonconservation, Phys.Rev. D22 (1980) 1694.

[315] M. Shaposhnikov, A Possible symmetry of the nuMSM, Nucl.Phys. B763 (2007) 49-59, [hep-ph/0605047].

[316] J. Kersten and A. Y. Smirnov, Right-Handed Neutrinos at LHC and the Mechanism of Neutrino Mass Generation, Phys. Rev. D76 (2007) 073005, [arXiv:0705.3221].

[317] C. Kraus, B. Bornschein, L. Bornschein, J. Bonn, B. Flatt, et al., Final results from phase II of the Mainz neutrino mass search in tritium beta decay, Eur.Phys.J. C40 (2005) 447-468, [hep-ex/0412056]. 
[318] Troitsk Collaboration, V. Aseev et al., An upper limit on electron antineutrino mass from Troitsk experiment, Phys.Rev. D84 (2011) 112003, [arXiv:1108.5034].

[319] Planck Collaboration, P. Ade et al., Planck 2015 results. XIII. Cosmological parameters, arXiv: 1502.01589.

[320] N. Palanque-Delabrouille, C. Yche, J. Lesgourgues, G. Rossi, A. Borde, et al., Constraint on

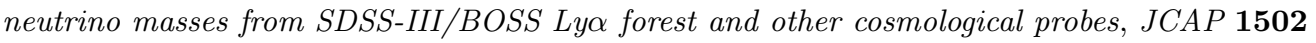
(2015) 045, [arXiv: 1410.7244].

[321] T. Asaka, S. Blanchet, and M. Shaposhnikov, The nuMSM, dark matter and neutrino masses, Phys. Lett. B631 (2005) 151-156, [hep-ph/0503065].

[322] A. Boyarsky, A. Neronov, O. Ruchayskiy, and M. Shaposhnikov, The Masses of active neutrinos in the nuMSM from X-ray astronomy, JETP Lett. 83 (2006) 133-135, [hep-ph/0601098].

[323] O. Ruchayskiy and A. Ivashko, Experimental bounds on sterile neutrino mixing angles, JHEP 1206 (2012) 100, [arXiv:1112.3319].

[324] D. Gorbunov and A. Panin, On the minimal active-sterile neutrino mixing in seesaw type I mechanism with sterile neutrinos at GeV scale, Phys.Rev. D89 (2014) 017302, [arXiv:1312.2887].

[325] M. Drewes and B. Garbrecht, Experimental and cosmological constraints on heavy neutrinos, arXiv: 1502.00477

[326] A. Strumia and F. Vissani, Neutrino masses and mixings and..., hep-ph/0606054.

[327] K. Babu and R. N. Mohapatra, Quantization of Electric Charge From Anomaly Constraints and a Majorana Neutrino, Phys.Rev. D41 (1990) 271.

[328] B. Pontecorvo, Mesonium and anti-mesonium, Sov.Phys.JETP 6 (1957) 429.

[329] Z. Maki, M. Nakagawa, and S. Sakata, Remarks on the unified model of elementary particles, Prog.Theor.Phys. 28 (1962) 870-880.

[330] J. Davis, Raymond, D. S. Harmer, and K. C. Hoffman, Search for neutrinos from the sun, Phys.Rev.Lett. 20 (1968) 1205-1209.

[331] SNO Collaboration, Q. Ahmad et al., Measurement of the rate of $\nu_{e}+d \rightarrow p+p+e^{-}$interactions produced by ${ }^{8} B$ solar neutrinos at the Sudbury Neutrino Observatory, Phys.Rev.Lett. 87 (2001) 071301, [nucl-ex/0106015].

[332] Super-Kamiokande Collaboration, Y. Fukuda et al., Evidence for oscillation of atmospheric neutrinos, Phys.Rev.Lett. 81 (1998) 1562-1567, [hep-ex/9807003].

[333] KamLAND Collaboration, K. Eguchi et al., First results from KamLAND: Evidence for reactor anti-neutrino disappearance, Phys.Rev.Lett. 90 (2003) 021802, [hep-ex/0212021].

[334] MINOS Collaboration, D. Michael et al., Observation of muon neutrino disappearance with the MINOS detectors and the NuMI neutrino beam, Phys.Rev.Lett. 97 (2006) 191801, [hep-ex/0607088].

[335] K2K Collaboration, E. Aliu et al., Evidence for muon neutrino oscillation in an accelerator-based experiment, Phys.Rev.Lett. 94 (2005) 081802, [hep-ex/0411038].

[336] DAYA-BAY Collaboration, F. An et al., Observation of electron-antineutrino disappearance at Daya Bay, Phys.Rev.Lett. 108 (2012) 171803, [arXiv:1203.1669].

[337] RENO Collaboration, J. Ahn et al., Observation of Reactor Electron Antineutrino Disappearance in the RENO Experiment, Phys.Rev.Lett. 108 (2012) 191802, [arXiv:1204.0626].

[338] DOUBLE-CHOOZ Collaboration, Y. Abe et al., Indication for the disappearance of reactor electron antineutrinos in the Double Chooz experiment, Phys.Rev.Lett. 108 (2012) 131801, [arXiv: 1112.6353]. 
[339] T2K Collaboration, K. Abe et al., Observation of Electron Neutrino Appearance in a Muon Neutrino Beam, Phys.Rev.Lett. 112 (2014) 061802, [arXiv:1311.4750].

[340] M. Gonzalez-Garcia, M. Maltoni, and T. Schwetz, Updated fit to three neutrino mixing: status of leptonic CP violation, JHEP 1411 (2014) 052, [arXiv: 1409.5439].

[341] LSND Collaboration, A. Aguilar-Arevalo et al., Evidence for neutrino oscillations from the observation of anti-neutrino(electron) appearance in a anti-neutrino(muon) beam, Phys.Rev. D64 (2001) 112007, [hep-ex/0104049].

[342] MiniBooNE Collaboration, A. Aguilar-Arevalo et al., Unexplained Excess of Electron-Like Events From a 1-GeV Neutrino Beam, Phys. Rev. Lett. 102 (2009) 101802, [arXiv:0812.2243].

[343] MiniBooNE Collaboration, A. Aguilar-Arevalo et al., Improved Search for $\bar{\nu}_{\mu} \rightarrow \bar{\nu}_{e}$ Oscillations in the MiniBooNE Experiment, Phys. Rev. Lett. 110 (2013) 161801, [arXiv:1207.4809].

[344] G. Mention, M. Fechner, T. Lasserre, T. Mueller, D. Lhuillier, et al., The Reactor Antineutrino Anomaly, Phys. Rev. D83 (2011) 073006, [arXiv:1101.2755].

[345] T. Mueller, D. Lhuillier, M. Fallot, A. Letourneau, S. Cormon, et al., Improved Predictions of Reactor Antineutrino Spectra, Phys. Rev. C83 (2011) 054615, [arXiv:1101.2663].

[346] D. Frekers, H. Ejiri, H. Akimune, T. Adachi, B. Bilgier, et al., The Ga-71(He-3, t) reaction and the low-energy neutrino response, Phys. Lett. B706 (2011) 134-138.

[347] LSND Collaboration, C. Athanassopoulos et al., Candidate events in a search for anti-muon-neutrino to anti-electron-neutrino oscillations, Phys.Rev.Lett. 75 (1995) 2650-2653, [nucl-ex/9504002].

[348] MiniBooNE Collaboration, A. Aguilar-Arevalo et al., A Search for electron neutrino appearance at the $\Delta m^{2} \sim 1 e V^{2}$ scale, Phys.Rev.Lett. 98 (2007) 231801, [arXiv:0704.1500].

[349] P. Huber, On the determination of anti-neutrino spectra from nuclear reactors, Phys.Rev. C84 (2011) 024617, [arXiv:1106.0687].

[350] C. Giunti, M. Laveder, Y. Li, Q. Liu, and H. Long, Update of Short-Baseline Electron Neutrino and Antineutrino Disappearance, Phys. Rev. D86 (2012) 113014, [arXiv:1210.5715].

[351] J. Conrad, C. Ignarra, G. Karagiorgi, M. Shaevitz, and J. Spitz, Sterile Neutrino Fits to Short Baseline Neutrino Oscillation Measurements, Adv. High Energy Phys. 2013 (2013) 163897, [arXiv: 1207.4765].

[352] J. Kopp, P. A. N. Machado, M. Maltoni, and T. Schwetz, Sterile Neutrino Oscillations: The Global Picture, JHEP 1305 (2013) 050, [arXiv: 1303.3011].

[353] C. Giunti, M. Laveder, Y. Li, and H. Long, Pragmatic View of Short-Baseline Neutrino Oscillations, Phys.Rev. D88 (2013) 073008, [arXiv:1308.5288].

[354] ALEPH Collaboration, DELPHI Collaboration, L3 Collaboration, OPAL Collaboration, SLD Collaboration, LEP Electroweak Working Group, SLD Electroweak Group, SLD Heavy Flavour Group Collaboration, S. Schael et al., Precision electroweak measurements on the $Z$ resonance, Phys.Rept. 427 (2006) 257-454, [hep-ex/0509008].

[355] Intensity Frontier Neutrino Working Group Collaboration, A. de Gouvêa et al., Working Group Report: Neutrinos, arXiv:1310.4340.

[356] P. Hernandez, M. Kekic, and J. Lopez-Pavon, $n_{\mathrm{eff}}$ in low-scale seesaw models versus the lightest neutrino mass, Phys.Rev. D90 (2014), no. 6 065033, [arXiv:1406.2961].

[357] G. Gelmini, S. Palomares-Ruiz, and S. Pascoli, Low reheating temperature and the visible sterile neutrino, Phys.Rev.Lett. 93 (2004) 081302, [astro-ph/0403323].

[358] B. Dasgupta and J. Kopp, Cosmologically Safe eV-Scale Sterile Neutrinos and Improved Dark Matter Structure, Phys.Rev.Lett. 112 (2014), no. 3 031803, [arXiv:1310.6337]. 
[359] S. Hannestad, R. S. Hansen, and T. Tram, How Self-Interactions can Reconcile Sterile Neutrinos with Cosmology, Phys.Rev.Lett. 112 (2014), no. 3 031802, [arXiv:1310.5926].

[360] K. Abe, T. Abe, H. Aihara, Y. Fukuda, Y. Hayato, et al., Letter of Intent: The Hyper-Kamiokande Experiment - Detector Design and Physics Potential —, arXiv:1109.3262.

[361] IceCube-PINGU Collaboration, M. Aartsen et al., Letter of Intent: The Precision IceCube Next Generation Upgrade (PINGU), arXiv:1401.2046.

[362] LBNE Collaboration, M. Bass et al., Baseline optimization for the measurement of CP violation and mass hierarchy in a long-baseline neutrino oscillation experiment, Phys.Rev. D89 (2014) 123506, [arXiv: 1311.0212].

[363] KATRIN Collaboration, A. Osipowicz et al., KATRIN: A Next generation tritium beta decay experiment with sub-eV sensitivity for the electron neutrino mass. Letter of intent, hep-ex/0109033.

[364] P. Ramond, The Family Group in Grand Unified Theories, hep-ph/9809459.

[365] T. Yanagida, Horizontal gauge symmetry and masses of neutrinos, Prog. Theor. Phys. 64 (1980) 1103.

[366] Seesaw mechanism. Proceedings, International Conference, SEESAW25, Paris, France, June 10-11, 2004, (Singapore), World Scientific, 2005.

[367] E. Ma, Pathways to naturally small neutrino masses, Phys.Rev.Lett. 81 (1998) 1171-1174, [hep-ph/9805219].

[368] G. Lazarides, Q. Shafi, and C. Wetterich, Proton Lifetime and Fermion Masses in an SO(10) Model, Nucl.Phys. B181 (1981) 287-300.

[369] J. Schechter and J. Valle, Neutrino Masses in SU(2) x U(1) Theories, Phys.Rev. D22 (1980) 2227.

[370] E. Ma and U. Sarkar, Neutrino masses and leptogenesis with heavy Higgs triplets, Phys.Rev.Lett. 80 (1998) 5716-5719, [hep-ph/9802445].

[371] R. Foot, H. Lew, X. He, and G. C. Joshi, Seesaw Neutrino Masses Induced by a Triplet of Leptons, Z.Phys. C44 (1989) 441.

[372] T. Asaka, S. Eijima, and H. Ishida, Mixing of Active and Sterile Neutrinos, JHEP 1104 (2011) 011, [arXiv: 1101.1382].

[373] F. Maltoni, J. Niczyporuk, and S. Willenbrock, Upper bound on the scale of Majorana neutrino mass generation, Phys.Rev.Lett. 86 (2001) 212-215, [hep-ph/0006358].

[374] A. de Gouvea, See-saw energy scale and the LSND anomaly, Phys.Rev. D72 (2005) 033005, [hep-ph/0501039].

[375] G. 't Hooft, Naturalness, chiral symmetry, and spontaneous chiral symmetry breaking, NATO Sci.Ser.B 59 (1980) 135.

[376] K. Babu and R. Mohapatra, Predictive neutrino spectrum in minimal SO(10) grand unification, Phys.Rev.Lett. 70 (1993) 2845-2848, [hep-ph/9209215].

[377] T. Fukuyama and N. Okada, Neutrino oscillation data versus minimal supersymmetric SO(10) model, JHEP 0211 (2002) 011, [hep-ph/0205066].

[378] B. Bajc, G. Senjanovic, and F. Vissani, $b$ - tau unification and large atmospheric mixing: A Case for noncanonical seesaw, Phys.Rev.Lett. 90 (2003) 051802, [hep-ph/0210207].

[379] H. Goh, R. Mohapatra, and S.-P. Ng, Minimal SUSY SO(10), b tau unification and large neutrino mixings, Phys.Lett. B570 (2003) 215-221, [hep-ph/0303055].

[380] K. Babu and C. Macesanu, Neutrino masses and mixings in a minimal SO(10) model, Phys.Rev. D72 (2005) 115003, [hep-ph/0505200]. 
[381] A. S. Joshipura and K. M. Patel, Viability of the exact tri-bimaximal mixing at $M_{G U T}$ in $S O(10)$, JHEP 1109 (2011) 137, [arXiv: 1105.5943].

[382] G. Altarelli and D. Meloni, A non supersymmetric SO(10) grand unified model for all the physics below $M_{G U T}$, JHEP 1308 (2013) 021, [arXiv: 1305.1001].

[383] A. Blondel, E. Graverini, N. Serra, and M. Shaposhnikov, for the FCC-ee study team, Search for Heavy Right Handed Neutrinos at the FCC-ee, arXiv:1411.5230.

[384] J. Casas and A. Ibarra, Oscillating neutrinos and muon to e gamma, Nucl.Phys. B618 (2001) 171-204, [hep-ph/0103065].

[385] A. Ibarra and G. G. Ross, Neutrino phenomenology: The Case of two right-handed neutrinos, Phys.Lett. B591 (2004) 285-296, [hep-ph/0312138].

[386] M. Raidal, A. Strumia, and K. Turzynski, Low-scale standard supersymmetric leptogenesis, Phys.Lett. B609 (2005) 351-359, [hep-ph/0408015].

[387] M. Gavela, T. Hambye, D. Hernandez, and P. Hernandez, Minimal Flavour Seesaw Models, JHEP 0909 (2009) 038, [arXiv:0906.1461].

[388] A. Ibarra, E. Molinaro, and S. Petcov, Low Energy Signatures of the TeV Scale See-Saw Mechanism, Phys.Rev. D84 (2011) 013005, [arXiv:1103.6217].

[389] B. W. Lee and R. E. Shrock, Natural Suppression of Symmetry Violation in Gauge Theories: Muon - Lepton and Electron Lepton Number Nonconservation, Phys.Rev. D16 (1977) 1444.

[390] A. Ibarra, E. Molinaro, and S. Petcov, TeV Scale See-Saw Mechanisms of Neutrino Mass Generation, the Majorana Nature of the Heavy Singlet Neutrinos and $(\beta \beta)_{0 \nu}$-Decay, JHEP 1009 (2010) 108, [arXiv: 1007.2378].

[391] D. Dinh, A. Ibarra, E. Molinaro, and S. Petcov, The $\mu-e$ Conversion in Nuclei, $\mu \rightarrow e \gamma, \mu \rightarrow 3 e$ Decays and TeV Scale See-Saw Scenarios of Neutrino Mass Generation, JHEP 1208 (2012) 125, [arXiv: 1205.4671].

[392] R. Alonso, M. Dhen, M. Gavela, and T. Hambye, Muon conversion to electron in nuclei in type-I seesaw models, JHEP 1301 (2013) 118, [arXiv:1209.2679].

[393] D. Aristizabal Sierra, A. Degee, and J. Kamenik, Minimal Lepton Flavor Violating Realizations of Minimal Seesaw Models, JHEP 1207 (2012) 135, [arXiv:1205.5547].

[394] A. Kusenko, S. Pascoli, and D. Semikoz, New bounds on MeV sterile neutrinos based on the accelerator and Super-Kamiokande results, JHEP 0511 (2005) 028, [hep-ph/0405198].

[395] D. Gorbunov and M. Shaposhnikov, How to find neutral leptons of the $\nu$ MSM?, JHEP 0710 (2007) 015, [arXiv:0705.1729].

[396] E. K. Akhmedov, V. A. Rubakov, and A. Y. Smirnov, Baryogenesis via neutrino oscillations, Phys. Rev. Lett. 81 (1998) 1359-1362, [hep-ph/9803255].

[397] T. Asaka and M. Shaposhnikov, The nuMSM, dark matter and baryon asymmetry of the universe, Phys. Lett. B620 (2005) 17-26, [hep-ph/0505013].

[398] G. M. Fuller, A. Kusenko, and K. Petraki, Heavy sterile neutrinos and supernova explosions, Phys.Lett. B670 (2009) 281-284, [arXiv:0806.4273].

[399] A. Kusenko, Sterile neutrinos: The Dark side of the light fermions, Phys.Rept. 481 (2009) 1-28, [arXiv: 0906.2968].

[400] L. Canetti and M. Shaposhnikov, The $\nu$ MSM and muon to electron conversion experiments, Hyperfine Interact. 214 (2013), no. 1-3 5-11.

[401] A. Abada, D. Das, A. Teixeira, A. Vicente, and C. Weiland, Tree-level lepton universality violation in the presence of sterile neutrinos: impact for $R_{K}$ and $R_{\pi}$, JHEP 1302 (2013) 048, [arXiv:1211.3052]. 
[402] A. Abada, A. Teixeira, A. Vicente, and C. Weiland, Sterile neutrinos in leptonic and semileptonic decays, JHEP 1402 (2014) 091, [arXiv:1311.2830].

[403] T. Asaka, S. Eijima, and K. Takeda, Lepton Universality in the $\nu$ MSM, Phys.Lett. B742 (2015) 303-309, [arXiv: 1410.0432].

[404] F. Bezrukov, nu MSM-predictions for neutrinoless double beta decay, Phys.Rev. D72 (2005) 071303, [hep-ph/0505247].

[405] M. Blennow, E. Fernandez-Martinez, J. Lopez-Pavon, and J. Menendez, Neutrinoless double beta decay in seesaw models, JHEP 1007 (2010) 096, [arXiv: 1005.3240].

[406] T. Asaka and S. Eijima, Direct Search for Right-handed Neutrinos and Neutrinoless Double Beta Decay, PTEP 2013 (2013), no. 11 113B02, [arXiv:1308.3550].

[407] A. Faessler, M. Gonzalez, S. Kovalenko, and F. Simkovic, Arbitrary mass Majorana neutrinos in neutrinoless double beta decay, Phys.Rev. D90 (2014) 096010, [arXiv:1408.6077].

[408] LHCb Collaboration, R. Aaij et al., Search for Majorana neutrinos in $B^{-} \rightarrow \pi^{+} \mu^{-} \mu^{-}$decays, Phys.Rev.Lett. 112 (2014), no. 13 131802, [arXiv:1401.5361].

[409] Belle Collaboration, D. Liventsev et al., Search for heavy neutrinos at Belle, Phys.Rev. D87 (2013), no. 7 071102, [arXiv:1301.1105].

[410] T. Asaka and A. Watanabe, Atmospheric Sterile Neutrinos, JHEP 1207 (2012) 112, [arXiv: 1202.0725].

[411] T. Asaka, S. Eijima, and A. Watanabe, Heavy neutrino search in accelerator-based experiments, JHEP 1303 (2013) 125, [arXiv:1212.1062].

[412] A. D. Dolgov, S. H. Hansen, G. Raffelt, and D. V. Semikoz, Cosmological and astrophysical bounds on a heavy sterile neutrino and the KARMEN anomaly, Nucl. Phys. B580 (2000) 331-351, [hep-ph/0002223].

[413] A. Dolgov, S. Hansen, G. Raffelt, and D. Semikoz, Heavy sterile neutrinos: Bounds from big bang nucleosynthesis and SN1987A, Nucl.Phys. B590 (2000) 562-574, [hep-ph/0008138].

[414] O. Ruchayskiy and A. Ivashko, Restrictions on the lifetime of sterile neutrinos from primordial nucleosynthesis, JCAP 1210 (2012) 014, [arXiv:1202.2841].

[415] L. Canetti and M. Shaposhnikov, Baryon Asymmetry of the Universe in the NuMSM, JCAP 1009 (2010) 001, [arXiv: 1006.0133].

[416] L. Canetti, M. Drewes, T. Frossard, and M. Shaposhnikov, Dark Matter, Baryogenesis and Neutrino Oscillations from Right Handed Neutrinos, Phys.Rev. D87 (2013), no. 9093006, [arXiv: 1208.4607].

[417] A. Y. Smirnov and R. Zukanovich Funchal, Sterile neutrinos: Direct mixing effects versus induced mass matrix of active neutrinos, Phys.Rev. D74 (2006) 013001, [hep-ph/0603009].

[418] A. de Gouvêa, J. Jenkins, and N. Vasudevan, Neutrino Phenomenology of Very Low-Energy Seesaws, Phys. Rev. D75 (2007) 013003, [hep-ph/0608147].

[419] J. Formaggio and J. Barrett, Resolving the Reactor Neutrino Anomaly with the KATRIN Neutrino Experiment, Phys. Lett. B706 (2011) 68-71, [arXiv:1105.1326].

[420] W. Rodejohann, Neutrino-less Double Beta Decay and Particle Physics, Int. J. Mod. Phys. E20 (2011) 1833-1930, [arXiv:1106.1334].

[421] A. Esmaili and O. L. Peres, KATRIN Sensitivity to Sterile Neutrino Mass in the Shadow of Lightest Neutrino Mass, Phys. Rev. D85 (2012) 117301, [arXiv:1203.2632].

[422] A. de Gouvea, W.-C. Huang, and J. Jenkins, Pseudo-Dirac Neutrinos in the New Standard Model, Phys.Rev. D80 (2009) 073007, [arXiv:0906.1611]. 
[423] A. Donini, P. Hernandez, J. Lopez-Pavon, M. Maltoni, and T. Schwetz, The minimal 3+2 neutrino model versus oscillation anomalies, JHEP 1207 (2012) 161, [arXiv:1205.5230].

[424] A. de Gouvêa and W.-C. Huang, Constraining the (Low-Energy) Type-I Seesaw, Phys.Rev. D85 (2012) 053006, [arXiv:1110.6122].

[425] A. Bungau, A. Adelmann, J. Alonso, W. Barletta, R. Barlow, et al., Proposal for an Electron Antineutrino Disappearance Search Using High-Rate ${ }^{8}$ Li Production and Decay, Phys. Rev. Lett. 109 (2012) 141802, [arXiv:1205.4419].

[426] PROSPECT Collaboration, J. Ashenfelter et al., PROSPECT - A Precision Reactor Oscillation and Spectrum Experiment at Very Short Baselines, arXiv:1309.7647.

[427] MINOS Collaboration, P. Adamson et al., Search for sterile neutrino mixing in the MINOS long baseline experiment, Phys.Rev. D81 (2010) 052004, [arXiv:1001.0336].

[428] G. Senjanovic, Spontaneous Breakdown of Parity in a Class of Gauge Theories, Nucl.Phys. B153 (1979) 334-364.

[429] G. Racah, On the symmetry of particle and antiparticle, Nuovo Cim. 14 (1937) 322-328.

[430] W. Furry, Note on the Theory of the Neutral Particle, Phys.Rev. 54 (1938) 56-67.

[431] W. Furry, On transition probabilities in double beta-disintegration, Phys.Rev. 56 (1939) 1184-1193.

[432] W.-Y. Keung and G. Senjanovic, Majorana Neutrinos and the Production of the Right-handed Charged Gauge Boson, Phys.Rev.Lett. 50 (1983) 1427.

[433] V. Tello, M. Nemevsek, F. Nesti, G. Senjanovic, and F. Vissani, Left-Right Symmetry: from LHC to Neutrinoless Double Beta Decay, Phys.Rev.Lett. 106 (2011) 151801, [arXiv:1011.3522].

[434] M. Nemevsek, F. Nesti, G. Senjanovic, and V. Tello, Neutrinoless Double Beta Decay: Low Left-Right Symmetry Scale?, arXiv:1112.3061.

[435] J. Chakrabortty, H. Z. Devi, S. Goswami, and S. Patra, Neutrinoless double- $\beta$ decay in TeV scale Left-Right symmetric models, JHEP 1208 (2012) 008, [arXiv: 1204.2527].

[436] F. F. Deppisch, M. Hirsch, and H. Pas, Neutrinoless Double Beta Decay and Physics Beyond the Standard Model, J.Phys. G39 (2012) 124007, [arXiv:1208.0727].

[437] J. Barry and W. Rodejohann, Lepton number and flavour violation in TeV-scale left-right symmetric theories with large left-right mixing, JHEP 1309 (2013) 153, [arXiv:1303.6324].

[438] C.-Y. Chen, P. S. B. Dev, and R. Mohapatra, Probing Heavy-Light Neutrino Mixing in Left-Right Seesaw Models at the LHC, Phys.Rev. D88 (2013) 033014, [arXiv:1306.2342].

[439] C.-H. Lee, P. Bhupal Dev, and R. Mohapatra, Natural TeV-scale left-right seesaw mechanism for neutrinos and experimental tests, Phys.Rev. D88 (2013), no. 9 093010, [arXiv:1309.0774].

[440] W.-C. Huang and J. Lopez-Pavon, On neutrinoless double beta decay in the minimal left-right symmetric model, Eur.Phys.J. C74 (2014) 2853, [arXiv:1310.0265].

[441] P. S. B. Dev, S. Goswami, and M. Mitra, TeV Scale Left-Right Symmetry and Large Mixing Effects in Neutrinoless Double Beta Decay, arXiv:1405.1399.

[442] M. Nemevsek, G. Senjanovic, and V. Tello, Connecting Dirac and Majorana Neutrino Mass Matrices in the Minimal Left-Right Symmetric Model, Phys.Rev.Lett. 110 (2013), no. 15151802 , [arXiv: 1211.2837].

[443] S. Weinberg, A Model of Leptons, Phys. Rev. Lett. 19 (1967) 1264-1266.

[444] M. Magg and C. Wetterich, Neutrino Mass Problem and Gauge Hierarchy, Phys.Lett. B94 (1980) 61.

[445] G. Senjanovi and V. Tello, Right Handed Quark Mixing in Left-Right Symmetric Theory, Phys.Rev.Lett. 114 (2015) 071801, [arXiv:1408.3835]. 
[446] G. Senjanovi and V. Tello, Restoration of Parity and the Right-Handed Analog of the CKM Matrix, arXiv:1502.05704.

[447] CMS Collaboration, V. Khachatryan et al., Search for heavy neutrinos and W bosons with right-handed couplings in proton-proton collisions at $\sqrt{s}=8$ TeV, Eur.Phys.J. C74 (2014), no. 11 3149, [arXiv: 1407.3683].

[448] G. Beall, M. Bander, and A. Soni, Constraint on the Mass Scale of a Left-Right Symmetric Electroweak Theory from the K(L) K(S) Mass Difference, Phys.Rev.Lett. 48 (1982) 848.

[449] R. N. Mohapatra, G. Senjanovic, and M. D. Tran, Strangeness Changing Processes and the Limit on the Right-handed Gauge Boson Mass, Phys.Rev. D28 (1983) 546.

[450] Y. Zhang, H. An, X. Ji, and R. N. Mohapatra, General CP Violation in Minimal Left-Right Symmetric Model and Constraints on the Right-Handed Scale, Nucl.Phys. B802 (2008) 247-279, [arXiv: 0712.4218].

[451] A. Maiezza, M. Nemevsek, F. Nesti, and G. Senjanovic, Left-Right Symmetry at LHC, Phys.Rev. D82 (2010) 055022, [arXiv: 1005.5160].

[452] S. Bertolini, A. Maiezza, and F. Nesti, Present and Future $K$ and B Meson Mixing Constraints on TeV Scale Left-Right Symmetry, Phys.Rev. D89 (2014) 095028, [arXiv:1403.7112].

[453] F. Xu, H. An, and X. Ji, Neutron Electric Dipole Moment Constraint on Scale of Minimal Left-Right Symmetric Model, JHEP 1003 (2010) 088, [arXiv:0910.2265].

[454] J. Engel, M. J. Ramsey-Musolf, and U. van Kolck, Electric Dipole Moments of Nucleons, Nuclei, and Atoms: The Standard Model and Beyond, Prog.Part.Nucl.Phys. 71 (2013) 21-74, [arXiv: 1303.2371].

[455] C.-Y. Seng, J. de Vries, E. Mereghetti, H. H. Patel, and M. Ramsey-Musolf, Nucleon electric dipole moments and the isovector parity- and time-reversal-odd pionnucleon coupling, Phys.Lett. B736 (2014) 147-153, [arXiv:1401.5366].

[456] W. Dekens, J. de Vries, J. Bsaisou, W. Bernreuther, C. Hanhart, et al., Unraveling models of CP violation through electric dipole moments of light nuclei, JHEP 1407 (2014) 069, [arXiv: 1404.6082].

[457] J. Bsaisou, J. de Vries, C. Hanhart, S. Liebig, U.-G. Meiner, et al., Nuclear Electric Dipole Moments in Chiral Effective Field Theory, JHEP 1503 (2015) 104, [arXiv:1411.5804].

[458] A. Maiezza and M. Nemevek, Strong P invariance, neutron electric dipole moment, and minimal left-right parity at LHC, Phys.Rev. D90 (2014), no. 9 095002, [arXiv:1407.3678].

[459] M. Blanke, A. J. Buras, K. Gemmler, and T. Heidsieck, Delta $F=2$ observables and $B \rightarrow X_{q} \gamma$ decays in the Left-Right Model: Higgs particles striking back, JHEP 1203 (2012) 024, [arXiv:1111.5014].

[460] G. Senjanovic and P. Senjanovic, Suppression of Higgs Strangeness Changing Neutral Currents in a Class of Gauge Theories, Phys.Rev. D21 (1980) 3253.

[461] M. Nemevšek, G. Senjanović, and V. Tello to appear, 2015.

[462] S. Das, F. Deppisch, O. Kittel, and J. Valle, Heavy Neutrinos and Lepton Flavour Violation in Left-Right Symmetric Models at the LHC, Phys.Rev. D86 (2012) 055006, [arXiv:1206.0256].

[463] J. C. Vasquez, Right-handed lepton mixings at the LHC, arXiv:1411.5824.

[464] A. Ferrari, J. Collot, M.-L. Andrieux, B. Belhorma, P. de Saintignon, et al., Sensitivity study for new gauge bosons and right-handed Majorana neutrinos in pp collisions at $s=14$-TeV, Phys.Rev. D62 (2000) 013001.

[465] S. Gninenko, M. Kirsanov, N. Krasnikov, and V. Matveev, Detection of heavy Majorana neutrinos and right-handed bosons, Phys.Atom.Nucl. 70 (2007) 441-449. 
[466] M. Nemevsek, F. Nesti, G. Senjanovic, and Y. Zhang, First Limits on Left-Right Symmetry Scale from LHC Data, Phys.Rev. D83 (2011) 115014, [arXiv:1103.1627].

[467] T. Han, I. Lewis, R. Ruiz, and Z.-g. Si, Lepton Number Violation and $W^{\prime}$ Chiral Couplings at the LHC, Phys.Rev. D87 (2013), no. 3 035011, [arXiv:1211.6447].

[468] G. Senjanovic, Seesaw at LHC through Left - Right Symmetry, Int.J.Mod.Phys. A26 (2011) 1469-1491, [arXiv: 1012.4104].

[469] G. Senjanovic, Neutrino mass: From LHC to grand unification, Riv. Nuovo Cim. 034 (2011) 1-68.

[470] V. Tello, Connections between the high and low energy violation of Lepton and Flavor numbers in the minimal left-right symmetric model, .

[471] G. Branco and G. Senjanovic, The Question of Neutrino Mass, Phys.Rev. D18 (1978) 1621.

[472] A. Maiezza, M. Nemevsek, and F. Nesti, Lepton Number Violation in Higgs Decay, arXiv: 1503.06834

[473] F. Bezrukov, H. Hettmansperger, and M. Lindner, keV sterile neutrino Dark Matter in gauge extensions of the Standard Model, Phys.Rev. D81 (2010) 085032, [arXiv:0912.4415].

[474] M. Nemevsek, G. Senjanovic, and Y. Zhang, Warm Dark Matter in Low Scale Left-Right Theory, JCAP 1207 (2012) 006, [arXiv: 1205.0844].

[475] J.-M. Frere, T. Hambye, and G. Vertongen, Is leptogenesis falsifiable at LHC?, JHEP 0901 (2009) 051, [arXiv:0806.0841].

[476] M. Nemevšek, G. Senjanović, and Y. Zhang unpublished, 2012.

[477] S. Weinberg, Gauge and Global Symmetries at High Temperature, Phys.Rev. D9 (1974) 3357-3378.

[478] R. N. Mohapatra and G. Senjanovic, Broken Symmetries at High Temperature, Phys.Rev. D20 (1979) 3390-3398.

[479] G. Dvali and G. Senjanovic, Is there a domain wall problem?, Phys.Rev.Lett. 74 (1995) 5178-5181, [hep-ph/9501387].

[480] G. Dvali, A. Melfo, and G. Senjanovic, Nonrestoration of spontaneously broken $P$ and $C P$ at high temperature, Phys.Rev. D54 (1996) 7857-7866, [hep-ph/9601376].

[481] R. N. Mohapatra and G. Senjanovic, High Temperature Behavior of Gauge Theories, Phys.Lett. B89 (1979) 57.

[482] G. Bimonte and G. Lozano, On Symmetry nonrestoration at high temperature, Phys.Lett. B366 (1996) 248-252, [hep-th/9507079].

[483] B. Rai and G. Senjanovic, Gravity and domain wall problem, Phys.Rev. D49 (1994) 2729-2733, [hep-ph/9301240].

[484] D. A. Dicus, D. D. Karatas, and P. Roy, Lepton nonconservation at supercollider energies, Phys.Rev. D44 (1991) 2033-2037.

[485] A. Datta, M. Guchait, and A. Pilaftsis, Probing lepton number violation via majorana neutrinos at hadron supercolliders, Phys.Rev. D50 (1994) 3195-3203, [hep-ph/9311257].

[486] T. Han and B. Zhang, Signatures for Majorana neutrinos at hadron colliders, Phys.Rev.Lett. 97 (2006) 171804, [hep-ph/0604064].

[487] F. del Aguila, J. Aguilar-Saavedra, and R. Pittau, Heavy neutrino signals at large hadron colliders, JHEP 0710 (2007) 047, [hep-ph/0703261].

[488] F. del Aguila and J. Aguilar-Saavedra, Electroweak scale seesaw and heavy Dirac neutrino signals at LHC, Phys.Lett. B672 (2009) 158-165, [arXiv:0809.2096].

[489] J. Aguilar-Saavedra, F. Deppisch, O. Kittel, and J. Valle, Flavour in heavy neutrino searches at the LHC, Phys.Rev. D85 (2012) 091301, [arXiv:1203.5998]. 
[490] J. Aguilar-Saavedra and F. Joaquim, Measuring heavy neutrino couplings at the LHC, Phys.Rev. D86 (2012) 073005, [arXiv: 1207.4193].

[491] D. Alva, T. Han, and R. Ruiz, Heavy Majorana Neutrinos from $W \gamma$ Fusion at Hadron Colliders, JHEP 1502 (2015) 072, [arXiv:1411.7305].

[492] M. Mitra, G. Senjanovic, and F. Vissani, Neutrinoless Double Beta Decay and Heavy Sterile Neutrinos, Nucl. Phys. B 856 (2012) 26-73.

[493] ATLAS Collaboration, G. Aad et al., Search for heavy neutrinos and right-handed $W$ bosons in events with two leptons and jets in pp collisions at $\sqrt{s}=7$ TeV with the ATLAS detector, Eur.Phys.J. C72 (2012) 2056, [arXiv:1203.5420].

[494] J. Helo, M. Hirsch, H. Ps, and S. Kovalenko, Short-range mechanisms of neutrinoless double beta decay at the LHC, Phys.Rev. D88 (2013) 073011, [arXiv:1307.4849].

[495] J. Helo, M. Hirsch, S. Kovalenko, and H. Pas, Neutrinoless double beta decay and lepton number violation at the LHC, Phys.Rev. D88 (2013), no. 1 011901, [arXiv:1303.0899].

[496] J. Helo, M. Hirsch, and S. Kovalenko, Heavy neutrino searches at the LHC with displaced vertices, Phys. Rev. D 89 (2014) 073005, [arXiv:1312.2900].

[497] EXO-200 Collaboration, J. Albert et al., Search for Majorana neutrinos with the first two years of EXO-200 data, Nature 510 (2014) 229234, [arXiv:1402.6956].

[498] GERDA Collaboration, M. Agostini et al., Results on neutrinoless double beta decay of ${ }^{76}$ Ge from GERDA Phase I, Phys. Rev. Lett. 111 (2013) 122503.

[499] D. Guadagnoli and R. N. Mohapatra, TeV Scale Left Right Symmetry and Flavor Changing Neutral Higgs Effects, Phys.Lett. B694 (2011) 386-392, [arXiv:1008.1074].

[500] M. Hirsch, H. Klapdor-Kleingrothaus, and O. Panella, Double beta decay in left-right symmetric models, Phys.Lett. B374 (1996) 7-12, [hep-ph/9602306].

[501] R. Mohapatra, Mechanism for Understanding Small Neutrino Mass in Superstring Theories, Phys.Rev.Lett. 56 (1986) 561-563.

[502] R. Mohapatra and J. Valle, Neutrino Mass and Baryon Number Nonconservation in Superstring Models, Phys.Rev. D34 (1986) 1642.

[503] A. Abada, D. Das, A. Vicente, and C. Weiland, Enhancing lepton flavour violation in the supersymmetric inverse seesaw beyond the dipole contribution, JHEP 1209 (2012) 015, [arXiv: 1206.6497].

[504] A. Ilakovac, A. Pilaftsis, and L. Popov, Charged lepton flavor violation in supersymmetric low-scale seesaw models, Phys.Rev. D87 (2013), no. 5 053014, [arXiv:1212.5939].

[505] A. Abada, M. E. Krauss, W. Porod, F. Staub, A. Vicente, et al., Lepton flavor violation in low-scale seesaw models: SUSY and non-SUSY contributions, JHEP 1411 (2014) 048, [arXiv: 1408.0138].

[506] S. Antusch, C. Biggio, E. Fernandez-Martinez, M. Gavela, and J. Lopez-Pavon, Unitarity of the Leptonic Mixing Matrix, JHEP 0610 (2006) 084, [hep-ph/0607020].

[507] P. B. Dev and R. Mohapatra, TeV Scale Inverse Seesaw in SO(10) and Leptonic Non-Unitarity Effects, Phys.Rev. D81 (2010) 013001, [arXiv:0910.3924].

[508] Y. Zhang, X. Ji, and R. N. Mohapatra, A Naturally Light Sterile neutrino in an Asymmetric Dark Matter Model, JHEP 1310 (2013) 104, [arXiv:1307.6178].

[509] H. An, S.-L. Chen, R. N. Mohapatra, and Y. Zhang, Leptogenesis as a Common Origin for Matter and Dark Matter, JHEP 1003 (2010) 124, [arXiv:0911.4463].

[510] P. B. Dev and A. Pilaftsis, Minimal Radiative Neutrino Mass Mechanism for Inverse Seesaw Models, Phys.Rev. D86 (2012) 113001, [arXiv:1209.4051]. 
[511] P. Bhupal Dev and A. Pilaftsis, Light and Superlight Sterile Neutrinos in the Minimal Radiative Inverse Seesaw Model, Phys.Rev. D87 (2013), no. 5 053007, [arXiv:1212.3808].

[512] T. Appelquist and R. Shrock, Neutrino masses in theories with dynamical electroweak symmetry breaking, Phys.Lett. B548 (2002) 204-214, [hep-ph/0204141].

[513] T. Appelquist and R. Shrock, Dynamical symmetry breaking of extended gauge symmetries, Phys.Rev.Lett. 90 (2003) 201801, [hep-ph/0301108].

[514] T. Appelquist, M. Piai, and R. Shrock, Fermion masses and mixing in extended technicolor models, Phys.Rev. D69 (2004) 015002, [hep-ph/0308061].

[515] T. Appelquist, N. D. Christensen, M. Piai, and R. Shrock, Flavor-changing processes in extended technicolor, Phys.Rev. D70 (2004) 093010, [hep-ph/0409035].

[516] N. D. Christensen and R. Shrock, Implications of dynamical generation of standard-model fermion masses, Phys.Rev.Lett. 94 (2005) 241801, [hep-ph/0501294].

[517] S. B. Roland, B. Shakya, and J. D. Wells, Neutrino Masses and Sterile Neutrino Dark Matter from the PeV Scale, arXiv:1412.4791.

[518] S. Khalil and O. Seto, Sterile neutrino dark matter in B - L extension of the standard model and galactic 511-keV line, JCAP 0810 (2008) 024, [arXiv:0804.0336].

[519] S. K. Kang and C. Kim, Extended double seesaw model for neutrino mass spectrum and low scale leptogenesis, Phys.Lett. B646 (2007) 248-252, [hep-ph/0607072].

[520] K. L. McDonald, Light Neutrinos from a Mini-Seesaw Mechanism in Warped Space, Phys.Lett. B696 (2011) 266-272, [arXiv:1010.2659].

[521] M. Duerr, D. P. George, and K. L. McDonald, Neutrino Mass and $\mu \rightarrow e+\gamma$ from a Mini-Seesaw, JHEP 1107 (2011) 103, [arXiv: 1105.0593].

[522] V. V. Khoze and G. Ro, Leptogenesis and Neutrino Oscillations in the Classically Conformal Standard Model with the Higgs Portal, JHEP 1310 (2013) 075, [arXiv:1307.3764].

[523] D. Aristizabal Sierra, J. Kubo, D. Restrepo, D. Suematsu, and O. Zapata, Radiative seesaw: Warm dark matter, collider and lepton flavour violating signals, Phys.Rev. D79 (2009) 013011, [arXiv: 0808.3340].

[524] T. Araki and Y. Li, Q6 flavor symmetry model for the extension of the minimal standard model by three right-handed sterile neutrinos, Phys.Rev. D85 (2012) 065016, [arXiv:1112.5819].

[525] A. Merle and V. Niro, Deriving Models for keV sterile Neutrino Dark Matter with the Froggatt-Nielsen mechanism, JCAP 1107 (2011) 023, [arXiv:1105.5136].

[526] K. Allison, Dark matter, singlet extensions of the nuMSM, and symmetries, JHEP 1305 (2013) 009, [arXiv: 1210.6852].

[527] M. Lindner, A. Merle, and V. Niro, Soft $L_{e}-L_{\mu}-L_{\tau}$ flavour symmetry breaking and sterile neutrino keV Dark Matter, JCAP 1101 (2011) 034, [arXiv: 1011.4950].

[528] A. Boyarsky, O. Ruchayskiy, and M. Shaposhnikov, The Role of sterile neutrinos in cosmology and astrophysics, Ann.Rev.Nucl.Part.Sci. 59 (2009) 191-214, [arXiv:0901.0011].

[529] G. Raffelt, Stars as laboratories for fundamental physics: The astrophysics of neutrinos, axions, and other weakly interacting particles. University of Chicago Press, 1996. ISBN 0-226-70272-3.

[530] M. Fukugita and T. Yanagida, Physics of neutrinos and applications to astrophysics. Springer, 2003.

[531] R. E. Shrock, New Tests For, and Bounds On, Neutrino Masses and Lepton Mixing, Phys. Lett. B96 (1980) 159. 
[532] A. Belesev, A. Berlev, E. Geraskin, A. Golubev, N. Likhovid, et al., An upper limit on additional neutrino mass eigenstate in 2 to $100 \mathrm{eV}$ region from 'Troitsk nu-mass' data, JETP Lett. 97 (2013) 67-69, [arXiv: 1211.7193].

[533] Y. Asano, R. Hayano, E. Kikutani, S. Kurokawa, T. Miyachi, et al., Search for a Heavy Neutrino Emitted in $K^{+} \rightarrow \mu^{+}$Neutrino Decay, Phys.Lett. B104 (1981) 84.

[534] R. Hayano, T. Taniguchi, T. Yamanaka, T. Tanimori, R. Enomoto, et al., HEAVY NEUTRINO SEARCH USING K(mu2) DECAY, Phys.Rev.Lett. 49 (1982) 1305.

[535] E949 Collaboration, A. Artamonov et al., Search for heavy neutrinos in $K^{+} \rightarrow \mu^{+} \nu_{H}$ decays, Phys.Rev. D91 (2015) 052001, [arXiv:1411.3963].

[536] T. Yamazaki, T. Ishikawa, Y. Akiba, M. Iwasaki, K. Tanaka, et al., Search for Heavy Neutrinos in Kaon Decay, Conf.Proc. C840719 (1984) I.262.

[537] R. Abela, M. Daum, G. Eaton, R. Frosch, B. Jost, et al., Search for an admixture of heavy neutrino in pion decay, Phys.Lett. B105 (1981) 263-266.

[538] R. Minehart, K. Ziock, R. Marshall, W. Stephens, M. Daum, et al., A Search for Admixture of Massive Neutrinos in the Decay $\pi \rightarrow \mu_{\nu}$, Phys.Rev.Lett. 52 (1984) 804-807.

[539] M. Daum, B. Jost, R. Marshall, R. Minehart, W. Stephens, et al., Search for admixtures of massive neutrinos in the decay pit $\rightarrow$ mu+ neutrino, Phys.Rev. D36 (1987) 2624.

[540] G. Azuelos, D. Britton, D. Bryman, E. Clifford, P. Kitching, et al., Constraints on Massive Neutrinos in $\pi \rightarrow \nu_{e}$ Decay, Phys.Rev.Lett. 56 (1986) 2241.

[541] N. De Leener-Rosier, J. Deutsch, M. Lebrun, O. Naviliat-Cuncic, R. Prieels, et al., Search for massive neutrinos in pi $\rightarrow$ e neutrino decay, Phys.Rev. D43 (1991) 3611-3618.

[542] D. Britton, S. Ahmad, D. Bryman, R. Burnham, E. Clifford, et al., Improved search for massive neutrinos in $\pi^{+} \rightarrow e^{+} \nu$ decay, Phys.Rev. D46 (1992) 885-887.

[543] PIENU Collaboration, M. Aoki et al., Search for Massive Neutrinos in the Decay $\pi \rightarrow e \nu$, Phys.Rev. D84 (2011) 052002, [arXiv:1106.4055].

[544] D. Britton, S. Ahmad, D. Bryman, R. Burnbam, E. Clifford, et al., Measurement of the $\pi^{+} \rightarrow e^{+} \nu$ branching ratio, Phys.Rev.Lett. 68 (1992) 3000-3003.

[545] R. E. Shrock, PURE LEPTONIC DECAYS WITH MASSIVE NEUTRINOS AND ARBITRARY LORENTZ STRUCTURE, Phys.Lett. B112 (1982) 382.

[546] A. Kobach and S. Dobbs, Heavy Neutrinos and the Kinematics of Tau Decays, Phys.Rev. D91 (2015) 053006, [arXiv:1412.4785].

[547] S. Ramazanov, Semileptonic decays of charmed and beauty baryons with sterile neutrinos in the final state, Phys.Rev. D79 (2009) 077701, [arXiv:0810.0660].

[548] L. M. Johnson, D. W. McKay, and T. Bolton, Extending sensitivity for low mass neutral heavy lepton searches, Phys.Rev. D56 (1997) 2970-2981, [hep-ph/9703333].

[549] F. F. Deppisch, P. S. B. Dev, and A. Pilaftsis, Neutrinos and Collider Physics, arXiv:1502.06541.

[550] L3 Collaboration, O. Adriani et al., Search for isosinglet neutral heavy leptons in Z0 decays, Phys.Lett. B295 (1992) 371-382.

[551] DELPHI Collaboration, P. Abreu et al., Search for neutral heavy leptons produced in Z decays, Z.Phys. C74 (1997) 57-71.

[552] L3 Collaboration, M. Acciarri et al., Search for heavy isosinglet neutrinos in $e^{+} e^{-}$annihilation at 130-GeV less than $S^{(1 / 2)}$ less than 189-GeV, Phys.Lett. B461 (1999) 397-404, [hep-ex/9909006].

[553] L3 Collaboration, P. Achard et al., Search for heavy isosinglet neutrino in $e^{+} e^{-}$annihilation at LEP, Phys.Lett. B517 (2001) 67-74, [hep-ex/0107014]. 
[554] S. Antusch and O. Fischer, Testing sterile neutrino extensions of the Standard Model at future lepton colliders, arXiv: 1502.05915.

[555] ALEPH Collaboration, A. Heister et al., Measurement of W-pair production in e+ e-collisions at centre-of-mass energies from 183-GeV to 209-GeV, Eur.Phys.J. C38 (2004) 147-160.

[556] S. Banerjee, P. S. B. Dev, A. Ibarra, T. Mandal, and M. Mitra, Prospects of Heavy Neutrino Searches at Future Lepton Colliders, arXiv:1503.05491.

[557] J. Almeida, F.M.L., Y. D. A. Coutinho, J. A. Martins Simoes, and M. do Vale, On a signature for heavy Majorana neutrinos in hadronic collisions, Phys.Rev. D62 (2000) 075004, [hep-ph/0002024].

[558] O. Panella, M. Cannoni, C. Carimalo, and Y. Srivastava, Signals of heavy Majorana neutrinos at hadron colliders, Phys.Rev. D65 (2002) 035005, [hep-ph/0107308].

[559] S. Bray, J. S. Lee, and A. Pilaftsis, Resonant CP violation due to heavy neutrinos at the LHC, Nucl.Phys. B786 (2007) 95-118, [hep-ph/0702294].

[560] CMS Collaboration, S. Chatrchyan et al., Search for heavy Majorana neutrinos in $\mu^{+} \mu^{+}\left[\mu^{-} \mu^{-}\right]$ and $e^{+} e^{+}\left[e^{-} e^{-}\right]$events in pp collisions at $\sqrt{s}=7$ TeV, Phys.Lett. B717 (2012) 109-128, [arXiv:1207.6079].

[561] ATLAS Collaboration, Search for Majorana neutrino production in pp collisions at sqrt(s)=7 TeV in dimuon final states with the ATLAS detector, 2012. ATLAS-CONF-2012-139, ATLAS-COM-CONF-2012-165.

[562] CMS Collaboration, J. W. Wetzel, A search for a heavy Majorana neutrino and a radiation damage simulation for the HF detector, PhD Thesis, University of Iowa (2014).

[563] ATLAS Collaboration, J. Klinger, Search for heavy Majorana neutrinos in pp collisions at $\sqrt{s}=8$ TeV with the ATLAS detector, PhD Thesis, University of Manchester (2014).

[564] A. Das, P. S. B. Dev, and N. Okada, Direct bounds on electroweak scale pseudo-Dirac neutrinos from $\sqrt{s}=8 \mathrm{TeV}$ LHC data, Phys.Lett. B735 (2014) 364-370, [arXiv:1405.0177].

[565] F. del Aguila and J. Aguilar-Saavedra, Distinguishing seesaw models at LHC with multi-lepton signals, Nucl.Phys. B813 (2009) 22-90, [arXiv:0808.2468].

[566] F. del Aguila, J. Aguilar-Saavedra, and J. de Blas, Trilepton signals: the golden channel for seesaw searches at LHC, Acta Phys.Polon. B40 (2009) 2901-2911, [arXiv:0910.2720].

[567] C.-Y. Chen and P. S. B. Dev, Multi-Lepton Collider Signatures of Heavy Dirac and Majorana Neutrinos, Phys.Rev. D85 (2012) 093018, [arXiv:1112.6419].

[568] A. Das and N. Okada, Inverse seesaw neutrino signatures at the LHC and ILC, Phys.Rev. D88 (2013), no. 11 113001, [arXiv:1207.3734].

[569] G. Bambhaniya, S. Goswami, S. Khan, P. Konar, and T. Mondal, Looking for hints of a reconstructible seesaw model at the Large Hadron Collider, arXiv:1410.5687.

[570] CMS Collaboration, S. Chatrchyan et al., Search for anomalous production of events with three or more leptons in pp collisions at $\sqrt{(}(s)=8$ TeV, Phys.Rev. D90 (2014) 032006, [arXiv:1404.5801].

[571] F. del Aguila, J. de Blas, and M. Perez-Victoria, Effects of new leptons in Electroweak Precision Data, Phys.Rev. D78 (2008) 013010, [arXiv:0803.4008].

[572] E. Akhmedov, A. Kartavtsev, M. Lindner, L. Michaels, and J. Smirnov, Improving Electro-Weak Fits with TeV-scale Sterile Neutrinos, JHEP 1305 (2013) 081, [arXiv:1302.1872].

[573] L. Basso, O. Fischer, and J. J. van der Bij, Precision tests of unitarity in leptonic mixing, Europhys.Lett. 105 (2014), no. 1 11001, [arXiv: 1310.2057].

[574] J. de Blas, Electroweak limits on physics beyond the Standard Model, EPJ Web Conf. 60 (2013) 19008, [arXiv: 1307.6173]. 
[575] S. Antusch and O. Fischer, Non-unitarity of the leptonic mixing matrix: Present bounds and future sensitivities, JHEP 1410 (2014) 94, [arXiv: 1407.6607].

[576] P. S. B. Dev, R. Franceschini, and R. Mohapatra, Bounds on TeV Seesaw Models from LHC Higgs Data, Phys.Rev. D86 (2012) 093010, [arXiv:1207.2756].

[577] P. B. Dev, A. Pilaftsis, and U.-k. Yang, New Production Mechanism for Heavy Neutrinos at the LHC, Phys.Rev.Lett. 112 (2014), no. 8 081801, [arXiv:1308.2209].

[578] G. Bernardi, G. Carugno, J. Chauveau, F. Dicarlo, M. Dris, et al., FURTHER LIMITS ON HEAVY NEUTRINO COUPLINGS, Phys.Lett. B203 (1988) 332.

[579] CHARM Collaboration, F. Bergsma et al., A SEARCH FOR DECAYS OF HEAVY NEUTRINOS IN THE MASS RANGE 0.5-GeV TO 2.8-GeV, Phys.Lett. B166 (1986) 473.

[580] NA3 Collaboration, J. Badier et al., Direct Photon Production From Pions and Protons at 200-GeV/c, Z.Phys. C31 (1986) 341.

[581] S. A. Baranov et al., Search for heavy neutrinos at the IHEP-JINR neutrino detector, Phys.Lett. B302 (1993) 336-340.

[582] LBNE Collaboration, C. Adams et al., The Long-Baseline Neutrino Experiment: Exploring Fundamental Symmetries of the Universe, arXiv:1307.7335.

[583] E. Graverini, Heavy neutral leptons in ship,, . Talk given at 2nd SHiP workshop at CERN, September 24, 2014.

[584] WA66 Collaboration, A. M. Cooper-Sarkar et al., Search for Heavy Neutrino Decays in the BEBC Beam Dump Experiment, Phys.Lett. B160 (1985) 207.

[585] FMMF Collaboration, E. Gallas et al., Search for neutral weakly interacting massive particles in the Fermilab Tevatron wide band neutrino beam, Phys.Rev. D52 (1995) 6-14.

[586] NuTeV Collaboration, E815 Collaboration, A. Vaitaitis et al., Search for neutral heavy leptons in a high-energy neutrino beam, Phys.Rev.Lett. 83 (1999) 4943-4946, [hep-ex/9908011].

[587] CHARM Collaboration, F. Bergsma et al., A Search for Decays of Heavy Neutrinos in the Mass Range (0.5-2.8) GeV, Phys. Lett. B 166 (1986) 473.

[588] CHARM II Collaboration, P. Vilain et al., Search for heavy isosinglet neutrinos, Phys.Lett. B343 (1995) 453-458.

[589] CMS Collaboration, V. Khachatryan et al., Search for heavy Majorana neutrinos in $\mu^{ \pm} \mu^{ \pm}+$jets events in proton-proton collisions at $\sqrt{s}=8 \mathrm{TeV}$, arXiv:1501.05566.

[590] J. Orloff, A. N. Rozanov, and C. Santoni, Limits on the mixing of tau neutrino to heavy neutrinos, Phys.Lett. B550 (2002) 8-15, [hep-ph/0208075].

[591] NOMAD Collaboration, P. Astier et al., Search for heavy neutrinos mixing with tau neutrinos, Phys.Lett. B506 (2001) 27-38, [hep-ex/0101041].

[592] S. Dell'Oro, S. Marcocci, and F. Vissani, New expectations and uncertainties on neutrinoless double beta decay, Phys. Rev. D 90 (2014) 033005.

[593] G. Feinberg and M. Goldhaber, Microscopic tests of symmetry principles, Proc. Nat. Aca. Sci. USA 45 (1959), no. 8 1301-1312.

[594] B. Pontecorvo, Superweak interactions and double beta decay, Phys. Lett. B26 (1968) 630-632.

[595] J. Barea, J. Kotila, and F. Iachello, $0 \nu \beta \beta$ and $2 \nu \beta \beta$ nuclear matrix elements in the interacting boson model with isospin restoration, Phys.Rev. C91 (2015), no. 3034304.

[596] A. Faessler, G. Fogli, E. Lisi, V. Rodin, A. Rotunno, and F. Simkovic, Overconstrained estimates of neutrinoless double beta decay within the QRPA, J. Phys. G 35 (2008) 075104. 
[597] J. Barea, J. Kotila, and F. Iachello, Nuclear matrix elements for double- $\beta$ decay, Phys. Rev. C 87 (Jan, 2013) 014315.

[598] J. Kotila and F. Iachello, Phase-space factors for double- $\beta$ decay, Phys. Rev. C 85 (Mar, 2012) 034316 .

[599] S. Kovalenko, Z. Lu, and I. Schmidt, Lepton Number Violating Processes Mediated by Majorana Neutrinos at Hadron Colliders, Phys. Rev. D 80 (2009) 073014.

[600] S. Pascoli, M. Mitra, and S. Wong, The Effect of Cancellation in Neutrinoless Double Beta Decay, Phys.Rev. D90 (2014) 093005, [arXiv: 1310.6218].

[601] J. Lopez-Pavon, S. Pascoli, and C.-f. Wong, Can heavy neutrinos dominate neutrinoless double beta decay?, Phys.Rev. D87 (2013), no. 9 093007, [arXiv:1209.5342].

[602] A. Merle and V. Niro, Influence of a keV sterile neutrino on neutrinoless double beta decay: How things changed in recent years, Phys.Rev. D88 (2013), no. 11 113004, [arXiv:1302.2032].

[603] D. Gorbunov and I. Timiryasov, Testing $\nu M S M$ with indirect searches, arXiv:1412.7751.

[604] N. Berger, A Novel experiment searching for the lepton flavour violating decay $\mu \rightarrow$ eee, J.Phys.Conf.Ser. 408 (2013) 012070, [arXiv:1110.1504].

[605] COMET Collaboration, E. V. Hungerford, COMET/PRISM muon to electron conversion at J-PARC, AIP Conf.Proc. 1182 (2009) 694-697.

[606] COMET Collaboration, A. Kurup, The COherent Muon to Electron Transition (COMET) experiment, Nucl.Phys.Proc.Suppl. 218 (2011) 38-43.

[607] R. Sawada, 2012. talk at the Neutrino 2012 conference, Tokyo: http://meg.icepp.s.u-tokyo.ac.jp/docs/talks/JPS/2012s/sawada_jps2012s.pdf.

[608] A. Abada, C. Biggio, F. Bonnet, M. Gavela, and T. Hambye, Low energy effects of neutrino masses, JHEP 0712 (2007) 061, [arXiv:0707.4058].

[609] S. Blanchet, T. Hambye, and F.-X. Josse-Michaux, Reconciling leptogenesis with observable mu to e gamma rates, JHEP 1004 (2010) 023, [arXiv:0912.3153].

[610] T. Cheng and L.-F. Li, $\mu \rightarrow e \gamma$ in Theories With Dirac and Majorana Neutrino Mass Terms, Phys.Rev.Lett. 45 (1980) 1908.

[611] A. Ilakovac and A. Pilaftsis, Flavor violating charged lepton decays in seesaw-type models, Nucl.Phys. B437 (1995) 491, [hep-ph/9403398].

[612] X. Chu, M. Dhen, and T. Hambye, Relations among neutrino observables in the light of a large $\theta_{13}$ angle, JHEP 1111 (2011) 106, [arXiv:1107.1589].

[613] SINDRUM II Collaboration. Collaboration, C. Dohmen et al., Test of lepton flavor conservation in $\mu \rightarrow e$ conversion on titanium, Phys.Lett. B317 (1993) 631-636.

[614] MEG Collaboration, J. Adam et al., New constraint on the existence of the $\mu^{+} \rightarrow e^{+} \gamma$ decay, Phys.Rev.Lett. 110 (2013) 201801, [arXiv:1303.0754].

[615] SINDRUM Collaboration, U. Bellgardt et al., Search for the Decay $\mu^{+} \rightarrow e^{+} e^{+} e^{-}$, Nucl.Phys. B299 (1988) 1.

[616] COMET Collaboration, Y. Cui et al., Conceptual design report for experimental search for lepton flavor violating mu- - e-conversion at sensitivity of $10^{* *}(-16)$ with a slow-extracted bunched proton beam (COMET), .

[617] T. Hambye, CLFV and the origin of neutrino masses, Nucl.Phys.Proc.Suppl. 248-250 (2014) 13-19, [arXiv: 1312.5214].

[618] A. Abada, C. Biggio, F. Bonnet, M. Gavela, and T. Hambye, $\mu \rightarrow e \gamma$ and $\tau \rightarrow l \gamma$ decays in the fermion triplet seesaw model, Phys.Rev. D78 (2008) 033007, [arXiv:0803.0481]. 
[619] A. Dolgov, Neutrinos in cosmology, Phys.Rept. 370 (2002) 333-535, [hep-ph/0202122].

[620] S. H. Hansen and Z. Haiman, Do we need stars to reionize the universe at high redshifts? Early reionization by decaying heavy sterile neutrinos, Astrophys.J. 600 (2004) 26-31, [astro-ph/0305126].

[621] G. M. Fuller, C. T. Kishimoto, and A. Kusenko, Heavy sterile neutrinos, entropy and relativistic energy production, and the relic neutrino background, arXiv:1110.6479.

[622] M. Shaposhnikov, Baryon Asymmetry of the Universe in Standard Electroweak Theory, Nucl.Phys. B287 (1987) 757-775.

[623] M. Shaposhnikov, Structure of the High Temperature Gauge Ground State and Electroweak Production of the Baryon Asymmetry, Nucl.Phys. B299 (1988) 797.

[624] S. Nasser and N. Turok, Z condensation and standard model baryogenesis, hep-ph/9406270.

[625] G. R. Farrar and M. Shaposhnikov, Baryon asymmetry of the universe in the standard electroweak theory, Phys.Rev. D50 (1994) 774, [hep-ph/9305275].

[626] M. Gavela, P. Hernandez, J. Orloff, O. Pene, and C. Quimbay, Standard model CP violation and baryon asymmetry. Part 2: Finite temperature, Nucl.Phys. B430 (1994) 382-426, [hep-ph/9406289].

[627] P. Huet and E. Sather, Electroweak baryogenesis and standard model CP violation, Phys.Rev. D51 (1995) 379-394, [hep-ph/9404302].

[628] K. Kajantie, M. Laine, K. Rummukainen, and M. E. Shaposhnikov, Is there a hot electroweak phase transition at $m(H)$ larger or equal to $m(W)$ ?, Phys.Rev.Lett. 77 (1996) 2887-2890, [hep-ph/9605288].

[629] K. Rummukainen, M. Tsypin, K. Kajantie, M. Laine, and M. E. Shaposhnikov, The Universality class of the electroweak theory, Nucl.Phys. B532 (1998) 283-314, [hep-lat/9805013].

[630] F. Csikor, Z. Fodor, and J. Heitger, Endpoint of the hot electroweak phase transition, Phys.Rev.Lett. 82 (1999) 21-24, [hep-ph/9809291].

[631] E. W. Kolb and M. S. Turner, Grand Unified Theories and the Origin of the Baryon Asymmetry, Ann.Rev.Nucl.Part.Sci. 33 (1983) 645-696.

[632] A. Dolgov, NonGUT baryogenesis, Phys.Rept. 222 (1992) 309-386.

[633] A. G. Cohen, D. Kaplan, and A. Nelson, Progress in electroweak baryogenesis, Ann.Rev.Nucl.Part.Sci. 43 (1993) 27-70, [hep-ph/9302210].

[634] M. Trodden, Electroweak baryogenesis, Rev.Mod.Phys. 71 (1999) 1463-1500, [hep-ph/9803479].

[635] A. Riotto and M. Trodden, Recent progress in baryogenesis, Ann.Rev.Nucl.Part.Sci. 49 (1999) 35-75, [hep-ph/9901362].

[636] M. Dine and A. Kusenko, The Origin of the matter - antimatter asymmetry, Rev.Mod.Phys. 76 (2003) 1, [hep-ph/0303065].

[637] W. Buchmuller, Baryogenesis: 40 Years Later, arXiv:0710.5857.

[638] A. G. Cohen, A. De Rujula, and S. Glashow, A Matter - antimatter universe?, Astrophys.J. 495 (1998) 539-549, [astro-ph/9707087].

[639] E. W. Kolb and S. Wolfram, Baryon Number Generation in the Early Universe, Nucl.Phys. B172 (1980) 224.

[640] J. N. Fry, K. A. Olive, and M. S. Turner, Higgs Bosons and the Evolution of Baryon Asymmetries, Phys.Rev. D22 (1980) 2977.

[641] T. Prokopec, M. G. Schmidt, and S. Weinstock, Transport equations for chiral fermions to order $h$ bar and electroweak baryogenesis. Part 1, Annals Phys. 314 (2004) 208-265, [hep-ph/0312110]. 
[642] T. Prokopec, M. G. Schmidt, and S. Weinstock, Transport equations for chiral fermions to order h-bar and electroweak baryogenesis. Part II, Annals Phys. 314 (2004) 267-320, [hep-ph/0406140].

[643] S. Davidson, E. Nardi, and Y. Nir, Leptogenesis, Phys. Rept. 466 (2008) 105-177, [arXiv:0802.2962].

[644] A. D. Sakharov, Violation of CP Invariance, c Asymmetry, and Baryon Asymmetry of the Universe, Pisma Zh. Eksp. Teor. Fiz. 5 (1967) 32-35.

[645] Super-Kamiokande Collaboration, H. Nishino et al., Search for Proton Decay via $p \rightarrow e+$ pi0 and $p \rightarrow m u+$ piO in a Large Water Cherenkov Detector, Phys.Rev.Lett. 102 (2009) 141801, [arXiv: 0903.0676].

[646] G. 't Hooft, Symmetry breaking through Bell-Jackiw anomalies, Phys. Rev. Lett. 37 (1976) 8-11.

[647] V. Kuzmin, V. Rubakov, and M. Shaposhnikov, On the Anomalous Electroweak Baryon Number Nonconservation in the Early Universe, Phys.Lett. B155 (1985) 36.

[648] K. Kajantie, M. Laine, K. Rummukainen, and M. E. Shaposhnikov, The Electroweak phase transition: A Nonperturbative analysis, Nucl.Phys. B466 (1996) 189-258, [hep-lat/9510020].

[649] M. Joyce and T. Prokopec, Turning around the sphaleron bound: Electroweak baryogenesis in an alternative postinflationary cosmology, Phys.Rev. D57 (1998) 6022-6049, [hep-ph/9709320].

[650] A. Tranberg, A. Hernandez, T. Konstandin, and M. G. Schmidt, Cold electroweak baryogenesis with Standard Model CP violation, Phys.Lett. B690 (2010) 207-212, [arXiv:0909.4199].

[651] I. Affleck and M. Dine, A New Mechanism for Baryogenesis, Nucl.Phys. B249 (1985) 361.

[652] M. Fukugita and T. Yanagida, Resurrection of grand unified theory baryogenesis, Phys.Rev.Lett. 89 (2002) 131602, [hep-ph/0203194].

[653] W. Buchmuller, P. Di Bari, and M. Plumacher, Leptogenesis for pedestrians, Annals Phys. 315 (2005) 305-351, [hep-ph/0401240].

[654] T. Hambye and G. Senjanovic, Consequences of triplet seesaw for leptogenesis, Phys.Lett. B582 (2004) 73-81, [hep-ph/0307237].

[655] T. Hambye, M. Raidal, and A. Strumia, Efficiency and maximal CP-asymmetry of scalar triplet leptogenesis, Phys.Lett. B632 (2006) 667-674, [hep-ph/0510008].

[656] T. Hambye, Y. Lin, A. Notari, M. Papucci, and A. Strumia, Constraints on neutrino masses from leptogenesis models, Nucl.Phys. B695 (2004) 169-191, [hep-ph/0312203].

[657] W. Fischler and R. Flauger, Neutrino Masses, Leptogenesis, and Unification in the Absence of Low Energy Supersymmetry, JHEP 0809 (2008) 020, [arXiv: 0805.3000].

[658] W. Buchmuller and M. Plumacher, Neutrino masses and the baryon asymmetry, Int.J.Mod.Phys. A15 (2000) 5047-5086, [hep-ph/0007176].

[659] G. Giudice, A. Notari, M. Raidal, A. Riotto, and A. Strumia, Towards a complete theory of thermal leptogenesis in the SM and MSSM, Nucl.Phys. B685 (2004) 89-149, [hep-ph/0310123].

[660] M. Beneke, B. Garbrecht, M. Herranen, and P. Schwaller, Finite Number Density Corrections to Leptogenesis, Nucl.Phys. B838 (2010) 1-27, [arXiv:1002.1326].

[661] A. Salvio, P. Lodone, and A. Strumia, Towards leptogenesis at NLO: the right-handed neutrino interaction rate, JHEP 1108 (2011) 116, [arXiv:1106.2814].

[662] A. Anisimov, D. Besak, and D. Bodeker, Thermal production of relativistic Majorana neutrinos: Strong enhancement by multiple soft scattering, JCAP 1103 (2011) 042, [arXiv:1012.3784].

[663] M. Laine and Y. Schroder, Thermal right-handed neutrino production rate in the non-relativistic regime, JHEP 1202 (2012) 068, [arXiv:1112.1205]. 
[664] M. Laine, Thermal right-handed neutrino production rate in the relativistic regime, JHEP 1308 (2013) 138, [arXiv:1307.4909].

[665] D. Besak and D. Bodeker, Thermal production of ultrarelativistic right-handed neutrinos: Complete leading-order results, JCAP 1203 (2012) 029, [arXiv:1202.1288].

[666] M. Beneke, B. Garbrecht, C. Fidler, M. Herranen, and P. Schwaller, Flavoured Leptogenesis in the CTP Formalism, Nucl.Phys. B843 (2011) 177-212, [arXiv:1007.4783].

[667] M. Garny, A. Kartavtsev, and A. Hohenegger, Leptogenesis from first principles in the resonant regime, Annals Phys. 328 (2013) 26-63, [arXiv:1112.6428].

[668] T. Frossard, M. Garny, A. Hohenegger, A. Kartavtsev, and D. Mitrouskas, Systematic approach to thermal leptogenesis, Phys.Rev. D87 (2013) 085009, [arXiv:1211.2140].

[669] P. Di Bari and L. Marzola, SO(10)-inspired solution to the problem of the initial conditions in leptogenesis, Nucl.Phys. B877 (2013) 719-751, [arXiv:1308.1107].

[670] L. Covi, E. Roulet, and F. Vissani, CP violating decays in leptogenesis scenarios, Phys.Lett. B384 (1996) 169-174, [hep-ph/9605319].

[671] S. Davidson, J. Garayoa, F. Palorini, and N. Rius, CP Violation in the SUSY Seesaw: Leptogenesis and Low Energy, JHEP 0809 (2008) 053, [arXiv:0806.2832].

[672] K. Hamaguchi, H. Murayama, and T. Yanagida, Leptogenesis from $N$ dominated early universe, Phys.Rev. D65 (2002) 043512, [hep-ph/0109030].

[673] S. Davidson and A. Ibarra, A Lower bound on the right-handed neutrino mass from leptogenesis, Phys.Lett. B535 (2002) 25-32, [hep-ph/0202239].

[674] J. Martin, C. Ringeval, and V. Vennin, Observing the Inflationary Reheating, Phys.Rev.Lett. 114 (2015) 081303, [arXiv:1410.7958].

[675] W. Buchmller, V. Domcke, K. Kamada, and K. Schmitz, The Gravitational Wave Spectrum from Cosmological B - L Breaking, JCAP 1310 (2013) 003, [arXiv:1305.3392].

[676] Planck Collaboration, P. Ade et al., Planck 2013 results. XXII. Constraints on inflation, Astron.Astrophys. 571 (2014) A22, [arXiv:1303.5082].

[677] M. Y. Khlopov and A. D. Linde, Is It Easy to Save the Gravitino?, Phys.Lett. B138 (1984) 265-268.

[678] J. R. Ellis, J. E. Kim, and D. V. Nanopoulos, Cosmological Gravitino Regeneration and Decay, Phys.Lett. B145 (1984) 181.

[679] J. R. Ellis, D. V. Nanopoulos, and S. Sarkar, The Cosmology of Decaying Gravitinos, Nucl.Phys. B259 (1985) 175.

[680] M. Kawasaki, K. Kohri, T. Moroi, and A. Yotsuyanagi, Big-Bang Nucleosynthesis and Gravitino, Phys.Rev. D78 (2008) 065011, [arXiv:0804.3745].

[681] A. Pilaftsis, CP violation and baryogenesis due to heavy Majorana neutrinos, Phys.Rev. D56 (1997) 5431-5451, [hep-ph/9707235].

[682] J. Liu and G. Segre, Unstable particle mixing and CP violation in weak decays, Phys.Rev. D49 (1994) 1342-1349, [hep-ph/9310248].

[683] M. Flanz, E. A. Paschos, and U. Sarkar, Baryogenesis from a lepton asymmetric universe, Phys.Lett. B345 (1995) 248-252, [hep-ph/9411366].

[684] V. Kuzmin, Cp violation and baryon asymmetry of the universe, Pisma Zh.Eksp.Teor.Fiz. 12 (1970) 335-337.

[685] A. Pilaftsis, Resonant CP violation induced by particle mixing in transition amplitudes, Nucl.Phys. B504 (1997) 61-107, [hep-ph/9702393]. 
[686] A. Pilaftsis and T. E. Underwood, Resonant leptogenesis, Nucl.Phys. B692 (2004) 303-345, [hep-ph/0309342].

[687] A. Pilaftsis, Resonant tau-leptogenesis with observable lepton number violation, Phys.Rev.Lett. 95 (2005) 081602, [hep-ph/0408103].

[688] A. Pilaftsis and T. E. Underwood, Electroweak-scale resonant leptogenesis, Phys.Rev. D72 (2005) 113001, [hep-ph/0506107].

[689] T. Endoh, T. Morozumi, and Z.-h. Xiong, Primordial lepton family asymmetries in seesaw model, Prog.Theor.Phys. 111 (2004) 123-149, [hep-ph/0308276].

[690] A. Pilaftsis, Radiatively induced neutrino masses and large Higgs neutrino couplings in the standard model with Majorana fields, Z.Phys. C55 (1992) 275-282, [hep-ph/9901206].

[691] Y. Grossman, T. Kashti, Y. Nir, and E. Roulet, Leptogenesis from supersymmetry breaking, Phys.Rev.Lett. 91 (2003) 251801, [hep-ph/0307081].

[692] G. D'Ambrosio, G. F. Giudice, and M. Raidal, Soft leptogenesis, Phys.Lett. B575 (2003) 75-84, [hep-ph/0308031].

[693] R. Gonzalez Felipe, F. Joaquim, and B. Nobre, Radiatively induced leptogenesis in a minimal seesaw model, Phys.Rev. D70 (2004) 085009, [hep-ph/0311029].

[694] K. Turzynski, Degenerate minimal seesaw and leptogenesis, Phys.Lett. B589 (2004) 135-140, [hep-ph/0401219].

[695] G. C. Branco, A. J. Buras, S. Jager, S. Uhlig, and A. Weiler, Another look at minimal lepton flavour violation, $l_{i} \rightarrow l_{j \gamma}$, leptogenesis, and the ratio $M_{\nu} / \Lambda_{L F V}$, JHEP 0709 (2007) 004, [hep-ph/0609067].

[696] M. Shaposhnikov, The nuMSM, leptonic asymmetries, and properties of singlet fermions, JHEP 0808 (2008) 008, [arXiv:0804.4542].

[697] M. Fukugita and T. Yanagida, Baryogenesis Without Grand Unification, Phys. Lett. B174 (1986) 45.

[698] A. Pais and O. Piccioni, Note on the Decay and Absorption of the Theta0, Phys.Rev. 100 (1955) 1487-1489.

[699] P. Bhupal Dev, P. Millington, A. Pilaftsis, and D. Teresi, Flavour Covariant Transport Equations: an Application to Resonant Leptogenesis, Nucl.Phys. B886 (2014) 569-664, [arXiv:1404.1003].

[700] P. Bhupal Dev, P. Millington, A. Pilaftsis, and D. Teresi, Kadanoff-Baym Approach to Flavour Mixing and Oscillations in Resonant Leptogenesis, Nucl.Phys. B891 (2015) 128-158, [arXiv: 1410.6434].

[701] B. Garbrecht and M. Herranen, Effective Theory of Resonant Leptogenesis in the Closed-Time-Path Approach, Nucl.Phys. B861 (2012) 17-52, [arXiv:1112.5954].

[702] T. Asaka and H. Ishida, Flavour Mixing of Neutrinos and Baryon Asymmetry of the Universe, Phys.Lett. B692 (2010) 105-113, [arXiv: 1004.5491].

[703] T. Asaka, S. Eijima, and H. Ishida, Kinetic Equations for Baryogenesis via Sterile Neutrino Oscillation, JCAP 1202 (2012) 021, [arXiv:1112.5565].

[704] L. Canetti, M. Drewes, and M. Shaposhnikov, Sterile Neutrinos as the Origin of Dark and Baryonic Matter, Phys.Rev.Lett. 110 (2013), no. 6 061801, [arXiv:1204.3902].

[705] M. Drewes, The Phenomenology of Right Handed Neutrinos, Int.J.Mod.Phys. E22 (2013) 1330019, [arXiv:1303.6912].

[706] M. Drewes, Theoretical Status of Neutrino Physics, arXiv:1502.06891.

[707] L. Canetti, M. Drewes, and M. Shaposhnikov, Matter and Antimatter in the Universe, New J.Phys. 14 (2012) 095012, [arXiv:1204.4186]. 
[708] B. Shuve and I. Yavin, Baryogenesis through Neutrino Oscillations: A Unified Perspective, Phys.Rev. D89 (2014) 075014, [arXiv: 1401.2459].

[709] M. Drewes and B. Garbrecht, Leptogenesis from a GeV Seesaw without Mass Degeneracy, JHEP 1303 (2013) 096, [arXiv:1206.5537].

[710] L. Canetti, M. Drewes, and B. Garbrecht, Probing leptogenesis with GeV-scale sterile neutrinos at LHCb and Belle II, Phys.Rev. D90 (2014) 125005, [arXiv:1404.7114].

[711] B. Garbrecht, More Viable Parameter Space for Leptogenesis, Phys.Rev. D90 (2014) 063522, [arXiv: 1401.3278].

[712] E. Graverini, Heavy neutral leptons in ship, Talk given at 2nd SHiP workshop at CERN (September 2014) (2014).

[713] A. Abada, V. De Romeri, S. Monteil, J. Orloff, and A. Teixeira, Indirect searches for sterile neutrinos at a high-luminosity Z-factory, arXiv:1412.6322.

[714] A. Anisimov, W. Buchmuller, M. Drewes, and S. Mendizabal, Leptogenesis from Quantum Interference in a Thermal Bath, Phys.Rev.Lett. 104 (2010) 121102, [arXiv:1001.3856].

[715] A. Anisimov, W. Buchmller, M. Drewes, and S. Mendizabal, Quantum Leptogenesis I, Annals Phys. 326 (2011) 1998-2038, [arXiv:1012.5821].

[716] M. Drewes, S. Mendizabal, and C. Weniger, The Boltzmann Equation from Quantum Field Theory, Phys.Lett. B718 (2013) 1119-1124, [arXiv:1202.1301].

[717] B. Garbrecht, F. Gautier, and J. Klaric, Strong Washout Approximation to Resonant Leptogenesis, JCAP 1409 (2014), no. 09 033, [arXiv:1406.4190].

[718] D. Boyanovsky, Nearly degenerate heavy sterile neutrinos in cascade decay: mixing and oscillations, Phys.Rev. D90 (2014), no. 10 105024, [arXiv:1409.4265].

[719] D. Boyanovsky, Spacetime evolution of heavy sterile neutrinos in cascade decays, Nucl.Phys. B888 (2014) 248-270, [arXiv:1406.5739].

[720] M. Endo and T. Yoshinaga, Lepton Universality Test of Extra Leptons using Hadron Decay, arXiv: 1404.4498.

[721] M. Shaposhnikov, Is there a new physics between electroweak and Planck scales?, arXiv:0708. 3550 .

[722] J. Lesgourgues and S. Pastor, Massive neutrinos and cosmology, Phys.Rept. 429 (2006) 307-379, [astro-ph/0603494].

[723] S. Tremaine and J. E. Gunn, Dynamical role of light neutral leptons in cosmology, Phys. Rev. Lett. 42 (1979) 407-410.

[724] A. Boyarsky, O. Ruchayskiy, and D. Iakubovskyi, A Lower bound on the mass of Dark Matter particles, JCAP 0903 (2009) 005, [arXiv:0808.3902].

[725] D. Gorbunov, A. Khmelnitsky, and V. Rubakov, Constraining sterile neutrino dark matter by phase-space density observations, JCAP 0810 (2008) 041, [arXiv:0808.3910].

[726] A. Dolgov and S. Hansen, Massive sterile neutrinos as warm dark matter, Astropart.Phys. 16 (2002) 339-344, [hep-ph/0009083].

[727] T. Asaka, M. Laine, and M. Shaposhnikov, Lightest sterile neutrino abundance within the nuMSM, JHEP 0701 (2007) 091, [hep-ph/0612182].

[728] M. Laine and M. Shaposhnikov, Sterile neutrino dark matter as a consequence of nuMSM-induced lepton asymmetry, JCAP 0806 (2008) 031, [arXiv: 0804.4543].

[729] K. Petraki and A. Kusenko, Dark-matter sterile neutrinos in models with a gauge singlet in the Higgs sector, Phys.Rev. D77 (2008) 065014, [arXiv:0711.4646]. 
[730] A. Boyarsky, D. Iakubovskyi, and O. Ruchayskiy, Next decade of sterile neutrino studies, Phys.Dark Univ. 1 (2012) 136-154, [arXiv:1306.4954].

[731] P. B. Pal and L. Wolfenstein, Radiative Decays of Massive Neutrinos, Phys.Rev. D25 (1982) 766. Revised Version.

[732] V. D. Barger, R. Phillips, and S. Sarkar, Remarks on the KARMEN anomaly, Phys.Lett. B352 (1995) 365-371, [hep-ph/9503295].

[733] K. Abazajian, G. M. Fuller, and W. H. Tucker, Direct detection of warm dark matter in the X-ray, Astrophys. J. 562 (2001) 593-604, [astro-ph/0106002].

[734] J. den Herder, A. Boyarsky, O. Ruchayskiy, K. Abazajian, C. Frenk, et al., The Search for decaying Dark Matter, arXiv:0906.1788.

[735] A. Boyarsky, A. Neronov, O. Ruchayskiy, and M. Shaposhnikov, Constraints on sterile neutrino as a dark matter candidate from the diffuse x-ray background, Mon.Not.Roy.Astron.Soc. 370 (2006) 213-218, [astro-ph/0512509].

[736] A. Boyarsky, J. Lesgourgues, O. Ruchayskiy, and M. Viel, Lyman-alpha constraints on warm and on warm-plus-cold dark matter models, JCAP 0905 (2009) 012, [arXiv:0812.0010].

[737] A. Boyarsky, A. Neronov, O. Ruchayskiy, and M. Shaposhnikov, Restrictions on parameters of sterile neutrino dark matter from observations of galaxy clusters, Phys.Rev. D74 (2006) 103506, [astro-ph/0603368].

[738] A. Boyarsky, A. Neronov, O. Ruchayskiy, M. Shaposhnikov, and I. Tkachev, Where to find a dark matter sterile neutrino?, Phys.Rev.Lett. 97 (2006) 261302, [astro-ph/0603660].

[739] C. R. Watson, J. F. Beacom, H. Yuksel, and T. P. Walker, Direct X-ray Constraints on Sterile Neutrino Warm Dark Matter, Phys.Rev. D74 (2006) 033009, [astro-ph/0605424].

[740] A. Boyarsky, J. Nevalainen, and O. Ruchayskiy, Constraints on the parameters of radiatively decaying dark matter from the dark matter halo of the Milky Way and Ursa Minor, Astron.Astrophys. 471 (2007) 51-57, [astro-ph/0610961].

[741] A. Boyarsky, D. Iakubovskyi, O. Ruchayskiy, and V. Savchenko, Constraints on decaying Dark Matter from XMM-Newton observations of M31, Mon.Not.Roy.Astron.Soc. 387 (2008) 1361, [arXiv:0709.2301].

[742] M. Loewenstein and A. Kusenko, Dark Matter Search Using XMM-Newton Observations of Willman 1, Astrophys.J. 751 (2012) 82, [arXiv:1203.5229].

[743] C. R. Watson, Z.-Y. Li, and N. K. Polley, Constraining Sterile Neutrino Warm Dark Matter with Chandra Observations of the Andromeda Galaxy, JCAP 1203 (2012) 018, [arXiv:1111.4217].

[744] S. Riemer-Sorensen, S. H. Hansen, and K. Pedersen, Sterile neutrinos in the Milky Way: Observational constraints, Astrophys.J. 644 (2006) L33-L36, [astro-ph/0603661].

[745] S. Riemer-Sorensen and S. H. Hansen, Decaying dark matter in Draco, arXiv:0901.2569.

[746] S. Riemer-Sorensen, K. Pedersen, S. H. Hansen, and H. Dahle, Probing the nature of dark matter with Cosmic X-rays: Constraints from Dark blobs and grating spectra of galaxy clusters, Phys. Rev. D76 (2007) 043524, [astro-ph/0610034].

[747] A. Boyarsky, O. Ruchayskiy, and M. Markevitch, Constraints on parameters of radiatively decaying dark matter from the galaxy cluster 1E0657-56, Astrophys.J. 673 (2008) 752-757, [astro-ph/0611168].

[748] M. Loewenstein and A. Kusenko, Dark Matter Search Using Chandra Observations of Willman 1, and a Spectral Feature Consistent with a Decay Line of a $5 \mathrm{keV}$ Sterile Neutrino, Astrophys.J. 714 (2010) 652-662, [arXiv:0912.0552]. 
[749] A. Boyarsky, O. Ruchayskiy, D. Iakubovskyi, M. G. Walker, S. Riemer-Sorensen, et al., Searching for Dark Matter in X-Rays: How to Check the Dark Matter origin of a spectral feature, Mon.Not.Roy.Astron.Soc. 407 (2010) 1188-1202, [arXiv:1001.0644].

[750] S. Horiuchi, P. J. Humphrey, J. Onorbe, K. N. Abazajian, M. Kaplinghat, et al., Sterile neutrino dark matter bounds from galaxies of the Local Group, Phys.Rev. D89 (2014) 025017, [arXiv: 1311.0282].

[751] M. Loewenstein, A. Kusenko, and P. L. Biermann, New Limits on Sterile Neutrinos from Suzaku Observations of the Ursa Minor Dwarf Spheroidal Galaxy, Astrophys.J. 700 (2009) 426-435, [arXiv:0812.2710].

[752] A. Kusenko, M. Loewenstein, and T. T. Yanagida, Moduli dark matter and the search for its decay line using Suzaku X-ray telescope, Phys.Rev. D87 (2013) 043508, [arXiv: 1209.6403].

[753] N. Mirabal, Swift observation of Segue 1: constraints on sterile neutrino parameters in the darkest galaxy, Mon.Not.Roy.Astron.Soc. 409 (2010) 128, [arXiv:1010.4706].

[754] H. Yuksel, J. F. Beacom, and C. R. Watson, Strong Upper Limits on Sterile Neutrino Warm Dark Matter, Phys.Rev.Lett. 101 (2008) 121301, [arXiv:0706.4084].

[755] A. Boyarsky, D. Malyshev, A. Neronov, and O. Ruchayskiy, Constraining DM properties with SPI, Mon.Not.Roy.Astron.Soc. 387 (2008) 1345, [arXiv:0710.4922].

[756] A. Boyarsky, J. W. den Herder, A. Neronov, and O. Ruchayskiy, Search for the light dark matter with an X-ray spectrometer, Astropart.Phys. 28 (2007) 303-311, [astro-ph/0612219].

[757] R. Essig, E. Kuflik, S. D. McDermott, T. Volansky, and K. M. Zurek, Constraining Light Dark Matter with Diffuse X-Ray and Gamma-Ray Observations, JHEP 1311 (2013) 193, [arXiv: 1309.4091].

[758] P. Bode, J. P. Ostriker, and N. Turok, Halo formation in warm dark matter models, Astrophys.J. 556 (2001) 93-107, [astro-ph/0010389].

[759] A. Boyarsky, J. Lesgourgues, O. Ruchayskiy, and M. Viel, Realistic sterile neutrino dark matter with keV mass does not contradict cosmological bounds, Phys.Rev.Lett. 102 (2009) 201304, [arXiv:0812.3256].

[760] U. Seljak, A. Makarov, P. McDonald, and H. Trac, Can sterile neutrinos be the dark matter?, Phys.Rev.Lett. 97 (2006) 191303, [astro-ph/0602430].

[761] M. Viel, J. Lesgourgues, M. G. Haehnelt, S. Matarrese, and A. Riotto, Can sterile neutrinos be ruled out as warm dark matter candidates?, Phys.Rev.Lett. 97 (2006) 071301, [astro-ph/0605706].

[762] M. Viel, G. D. Becker, J. S. Bolton, and M. G. Haehnelt, Warm dark matter as a solution to the small scale crisis: New constraints from high redshift Lyman-forest data, Phys.Rev. D88 (2013) 043502, [arXiv: 1306.2314].

[763] P.-H. Gu, Large Lepton Asymmetry for Small Baryon Asymmetry and Warm Dark Matter, Phys.Rev. D82 (2010) 093009, [arXiv:1005.1632].

[764] A. Boyarsky, J. Franse, D. Iakubovskyi, and O. Ruchayskiy, Checking the dark matter origin of $3.53 \mathrm{keV}$ line with the Milky Way center, arXiv:1408.2503.

[765] S. Riemer-Sorensen, Questioning a $3.5 \mathrm{keV}$ dark matter emission line, arXiv:1405.7943.

[766] M. E. Anderson, E. Churazov, and J. N. Bregman, Non-Detection of X-Ray Emission From Sterile Neutrinos in Stacked Galaxy Spectra, arXiv:1408.4115.

[767] A. Boyarsky, J. Franse, D. Iakubovskyi, and O. Ruchayskiy, Comment on the paper "Dark matter searches going bananas: the contribution of Potassium (and Chlorine) to the $3.5 \mathrm{keV}$ line" by T. Jeltema and S. Profumo, arXiv:1408.4388. 
[768] T. Jeltema and S. Profumo, Reply to two comments on "dark matter searches going bananas the contribution of potassium (and chlorine) to the $3.5 \mathrm{kev}$ line", arXiv:1411.1759.

[769] E. Carlson, T. Jeltema, and S. Profumo, Where do the $3.5 \mathrm{keV}$ photons come from? A morphological study of the Galactic Center and of Perseus, JCAP 1502 (2015) 009, [arXiv: 1411.1758].

[770] T. E. Jeltema and S. Profumo, Dark matter searches going bananas: the contribution of Potassium (and Chlorine) to the $3.5 \mathrm{keV}$ line, arXiv:1408.1699.

[771] E. Bulbul, M. Markevitch, A. R. Foster, R. K. Smith, M. Loewenstein, et al., Comment on "Dark matter searches going bananas: the contribution of Potassium (and Chlorine) to the $3.5 \mathrm{keV}$ line", arXiv: 1409.4143.

[772] D. Malyshev, A. Neronov, and D. Eckert, Constraints on $3.55 \mathrm{keV}$ line emission from stacked observations of dwarf spheroidal galaxies, Phys.Rev. D90 (2014) 103506, [arXiv:1408.3531].

[773] F. Bezrukov, D. Gorbunov, and M. Shaposhnikov, Late and early time phenomenology of Higgs-dependent cutoff, JCAP 1110 (2011) 001, [arXiv:1106.5019].

[774] A. Boyarsky, S. Eijima, O. Ruchayskiy, and M. Shaposhnikov, In preparation, .

[775] S. Gninenko, D. Gorbunov, and M. Shaposhnikov, Search for GeV-scale sterile neutrinos responsible for active neutrino oscillations and baryon asymmetry of the Universe, Adv.High Energy Phys. 2012 (2012) 718259, [arXiv:1301.5516].

[776] F. Bezrukov, D. Gorbunov, and M. Shaposhnikov, On initial conditions for the Hot Big Bang, JCAP 0906 (2009) 029, [arXiv:0812.3622].

[777] J. Garcia-Bellido, D. G. Figueroa, and J. Rubio, Preheating in the Standard Model with the Higgs-Inflaton coupled to gravity, Phys.Rev. D79 (2009) 063531, [arXiv:0812.4624].

[778] F. Bezrukov, J. Rubio, and M. Shaposhnikov, Living beyond the edge: Higgs inflation and vacuum metastability, arXiv:1412.3811.

[779] M. Shaposhnikov and D. Zenhausern, Scale invariance, unimodular gravity and dark energy, Phys.Lett. B671 (2009) 187-192, [arXiv:0809.3395].

[780] M. Shaposhnikov and D. Zenhausern, Quantum scale invariance, cosmological constant and hierarchy problem, Phys.Lett. B671 (2009) 162-166, [arXiv:0809.3406].

[781] K. Babu and R. Mohapatra, Is There a Connection Between Quantization of Electric Charge and a Majorana Neutrino?, Phys.Rev.Lett. 63 (1989) 938.

[782] C. Froggatt and H. B. Nielsen, Standard model criticality prediction: Top mass 173 +- 5-GeV and Higgs mass 135 +- 9-GeV, Phys.Lett. B368 (1996) 96-102, [hep-ph/9511371].

[783] G. Isidori, V. S. Rychkov, A. Strumia, and N. Tetradis, Gravitational corrections to standard model vacuum decay, Phys.Rev. D77 (2008) 025034, [arXiv:0712.0242].

[784] J. Ellis, J. Espinosa, G. Giudice, A. Hoecker, and A. Riotto, The Probable Fate of the Standard Model, Phys.Lett. B679 (2009) 369-375, [arXiv:0906.0954].

[785] J. Elias-Miro, J. R. Espinosa, G. F. Giudice, G. Isidori, A. Riotto, et al., Higgs mass implications on the stability of the electroweak vacuum, Phys.Lett. B709 (2012) 222-228, [arXiv:1112.3022].

[786] CMS Collaboration, CMS, Combination of the CMS top-quark mass measurements from Run 1 of the $L H C$, .

[787] CMS Collaboration, V. Khachatryan et al., "Combined mass and couplings of the higgs boson at cms." Talk at CERN https://indico.cern.ch/event/360238/, 2015.

[788] F. Bezrukov and M. Shaposhnikov, Why should we care about the top quark Yukawa coupling?, arXiv:1411.1923. 
[789] J. Espinosa, G. Giudice, and A. Riotto, Cosmological implications of the Higgs mass measurement, JCAP 0805 (2008) 002, [arXiv:0710.2484].

[790] K. J. Bae, K. Choi, E. J. Chun, S. H. Im, C. B. Park, et al., Peccei-Quinn NMSSM in the light of 125 GeV Higgs, JHEP 1211 (2012) 118, [arXiv:1208.2555].

[791] R. Peccei and H. R. Quinn, Constraints Imposed by CP Conservation in the Presence of Instantons, Phys.Rev. D16 (1977) 1791-1797.

[792] F. Wilczek, Problem of Strong $p$ and $t$ Invariance in the Presence of Instantons, Phys.Rev.Lett. 40 (1978) 279-282.

[793] S. Weinberg, A New Light Boson?, Phys.Rev.Lett. 40 (1978) 223-226.

[794] E. Witten, Some Properties of O(32) Superstrings, Phys.Lett. B149 (1984) 351-356.

[795] J. P. Conlon, The QCD axion and moduli stabilisation, JHEP 0605 (2006) 078, [hep-th/0602233].

[796] P. Svrcek and E. Witten, Axions In String Theory, JHEP 0606 (2006) 051, [hep-th/0605206].

[797] A. Arvanitaki, S. Dimopoulos, S. Dubovsky, N. Kaloper, and J. March-Russell, String Axiverse, Phys.Rev. D81 (2010) 123530, [arXiv:0905.4720].

[798] B. S. Acharya, K. Bobkov, and P. Kumar, An M Theory Solution to the Strong CP Problem and Constraints on the Axiverse, JHEP 1011 (2010) 105, [arXiv: 1004.5138].

[799] M. Cicoli, M. Goodsell, and A. Ringwald, The type IIB string axiverse and its low-energy phenomenology, JHEP 1210 (2012) 146, [arXiv: 1206.0819].

[800] A. Broggio, E. J. Chun, M. Passera, K. M. Patel, and S. K. Vempati, Limiting two-Higgs-doublet models, JHEP 1411 (2014) 058, [arXiv:1409.3199].

[801] M. J. Duncan, N. Kaloper, and K. A. Olive, Axion hair and dynamical torsion from anomalies, Nucl.Phys. B387 (1992) 215-238.

[802] S. Mercuri, Peccei-Quinn mechanism in gravity and the nature of the Barbero-Immirzi parameter, Phys.Rev.Lett. 103 (2009) 081302, [arXiv:0902.2764].

[803] O. Castillo-Felisola, C. Corral, S. Kovalenko, I. Schmidt, and V. E. Lyubovitskij, Axions in gravity with torsion, arXiv: 1502.03694.

[804] N. Arkani-Hamed, S. Dimopoulos, and G. Dvali, The Hierarchy problem and new dimensions at a millimeter, Phys.Lett. B429 (1998) 263-272, [hep-ph/9803315].

[805] L. Randall and R. Sundrum, A Large mass hierarchy from a small extra dimension, Phys.Rev.Lett. 83 (1999) 3370-3373, [hep-ph/9905221].

[806] F. Wilczek, Axions and Family Symmetry Breaking, Phys.Rev.Lett. 49 (1982) 1549-1552.

[807] C. D. Carone, Pseudo-Familon Dark Matter, Phys.Lett. B707 (2012) 529-533, [arXiv:1201.1205].

[808] J. Jaeckel, A Family of WISPy Dark Matter Candidates, Phys.Lett. B732 (2014) 1-7, [arXiv: 1311.0880].

[809] SuperCDMS Collaboration, R. Agnese et al., Search for Low-Mass Weakly Interacting Massive Particles Using Voltage-Assisted Calorimetric Ionization Detection in the SuperCDMS Experiment, Phys.Rev.Lett. 112 (2014), no. 4 041302, [arXiv:1309.3259].

[810] SuperCDMS Collaboration, R. Agnese et al., Search for Low-Mass Weakly Interacting Massive Particles with SuperCDMS, Phys.Rev.Lett. 112 (2014), no. 24 241302, [arXiv:1402.7137].

[811] CRESST-II Collaboration, G. Angloher et al., Results on low mass WIMPs using an upgraded CRESST-II detector, Eur.Phys.J. C74 (2014), no. 12 3184, [arXiv:1407.3146].

[812] ATLAS Collaboration, G. Aad et al., Search for Invisible Decays of a Higgs Boson Produced in Association with a Z Boson in ATLAS, Phys.Rev.Lett. 112 (2014) 201802, [arXiv:1402.3244]. 
[813] CMS Collaboration, S. Chatrchyan et al., Search for invisible decays of Higgs bosons in the vector boson fusion and associated ZH production modes, Eur.Phys.J. C74 (2014), no. 8 2980, [arXiv: 1404.1344].

[814] M. Freytsis and Z. Ligeti, On dark matter models with uniquely spin-dependent detection possibilities, Phys.Rev. D83 (2011) 115009, [arXiv:1012.5317].

[815] K. R. Dienes, J. Kumar, B. Thomas, and D. Yaylali, Overcoming Velocity Suppression in Dark-Matter Direct-Detection Experiments, Phys.Rev. D90 (2014) 015012, [arXiv:1312.7772].

[816] J. March-Russell, J. Unwin, and S. M. West, Closing in on Asymmetric Dark Matter I: Model independent limits for interactions with quarks, JHEP 1208 (2012) 029, [arXiv:1203.4854].

[817] J. Redondo, Bounds on Very Weakly Interacting Sub-eV Particles (WISPs) from Cosmology and Astrophysics, arXiv:0810.3200.

[818] J. Jaeckel and A. Ringwald, The Low-Energy Frontier of Particle Physics, Ann.Rev.Nucl.Part.Sci. 60 (2010) 405-437, [arXiv: 1002.0329].

[819] D. Cadamuro and J. Redondo, Cosmological bounds on pseudo Nambu-Goldstone bosons, JCAP 1202 (2012) 032, [arXiv:1110.2895].

[820] J. Hewett, H. Weerts, R. Brock, J. Butler, B. Casey, et al., Fundamental Physics at the Intensity Frontier, arXiv:1205.2671.

[821] J. Jaeckel, M. Jankowiak, and M. Spannowsky, LHC probes the hidden sector, Phys.Dark Univ. 2 (2013) 111-117, [arXiv:1212.3620].

[822] K. Mimasu and V. Sanz, ALPs at Colliders, arXiv:1409.4792.

[823] A. Payez, C. Evoli, T. Fischer, M. Giannotti, A. Mirizzi, et al., Revisiting the SN1987A gamma-ray limit on ultralight axion-like particles, JCAP 1502 (2015), no. 02 006, [arXiv:1410.3747].

[824] M. Millea, L. Knox, and B. Fields, New Bounds for Axions and Axion-Like Particles with keV-GeV Masses, arXiv:1501.04097.

[825] A. Belyaev, N. D. Christensen, and A. Pukhov, CalcHEP 3.4 for collider physics within and beyond the Standard Model, Comput.Phys.Commun. 184 (2013) 1729-1769, [arXiv:1207.6082].

[826] C. Bobeth, T. Ewerth, F. Kruger, and J. Urban, Analysis of neutral Higgs boson contributions to the decays $\bar{B}\left(s^{)} \rightarrow \ell^{+} \ell^{-}\right.$and $\bar{B} \rightarrow K \ell^{+} \ell^{-}$, Phys.Rev. D64 (2001) 074014, [hep-ph/0104284].

[827] P. Ball and R. Zwicky, New results on $B \rightarrow$ pi, K, eta decay formfactors from light-cone sum rules, Phys.Rev. D71 (2005) 014015, [hep-ph/0406232].

[828] LHCb Collaboration, R. Aaij et al., Differential branching fraction and angular analysis of the $B^{+} \rightarrow K^{+} \mu^{+} \mu^{-}$decay, JHEP 1302 (2013) 105, [arXiv:1209.4284].

[829] J. Blumlein, J. Brunner, H. Grabosch, P. Lanius, S. Nowak, et al., Limits on neutral light scalar and pseudoscalar particles in a proton beam dump experiment, Z.Phys. C51 (1991) 341-350.

[830] M. Duffy, G. Fanourakis, R. Loveless, D. Reeder, E. Smith, et al., Neutrino Production by 400-GeV/c Protons in a Beam-dump Experiment, Phys.Rev. D38 (1988) 2032.

[831] J. L. Feng, Naturalness and the Status of Supersymmetry, Ann.Rev.Nucl.Part.Sci. 63 (2013) 351-382, [arXiv: 1302.6587].

[832] V. A. Mitsou, Experimental status of particle and astroparticle searches for supersymmetry, J.Phys.Conf.Ser. 447 (2013) 012019, [arXiv: 1304.1414].

[833] N. Craig, The State of Supersymmetry after Run I of the LHC, arXiv:1309.0528.

[834] F. Domingo and U. Ellwanger, Updated Constraints from B Physics on the MSSM and the NMSSM, JHEP 0712 (2007) 090, [arXiv:0710.3714]. 
[835] R. Ball, C. Coffin, H. R. Gustafson, I. Leedom, L. W. Jones, et al., Supersymmetry Mass and Lifetime Limits From a Proton Beam Dump Experiment, Phys.Rev.Lett. 53 (1984) 1314.

[836] D. Choudhury, H. K. Dreiner, P. Richardson, and S. Sarkar, A Supersymmetric solution to the KARMEN time anomaly, Phys.Rev. D61 (2000) 095009, [hep-ph/9911365].

[837] H. K. Dreiner, S. Heinemeyer, O. Kittel, U. Langenfeld, A. M. Weber, et al., Mass Bounds on a Very Light Neutralino, Eur.Phys.J. C62 (2009) 547-572, [arXiv:0901.3485].

[838] H. K. Dreiner, O. Kittel, and U. Langenfeld, Discovery potential of radiative neutralino production at the ILC, Phys.Rev. D74 (2006) 115010, [hep-ph/0610020].

[839] H. Dreiner, C. Hanhart, U. Langenfeld, and D. R. Phillips, Supernovae and light neutralinos: SN1987A bounds on supersymmetry revisited, Phys.Rev. D68 (2003) 055004, [hep-ph/0304289].

[840] H. K. Dreiner, J.-F. Fortin, J. Isern, and L. Ubaldi, White Dwarfs constrain Dark Forces, Phys.Rev. D88 (2013) 043517, [arXiv:1303.7232].

[841] L. Calibbi, J. M. Lindert, T. Ota, and Y. Takanishi, Cornering light Neutralino Dark Matter at the LHC, JHEP 1310 (2013) 132, [arXiv:1307.4119].

[842] L. Calibbi, J. M. Lindert, T. Ota, and Y. Takanishi, LHC Tests of Light Neutralino Dark Matter without Light Sfermions, JHEP 1411 (2014) 106, [arXiv: 1410.5730].

[843] R. Cowsik and J. McClelland, An Upper Limit on the Neutrino Rest Mass, Phys.Rev.Lett. 29 (1972) 669-670.

[844] S. Gershtein and Y. a. Zeldovich, Rest Mass of Muonic Neutrino and Cosmology, JETP Lett. 4 (1966) 120-122.

[845] P. Hut, Limits on Masses and Number of Neutral Weakly Interacting Particles, Phys.Lett. B69 (1977) 85.

[846] K. Sato and M. Kobayashi, Cosmological Constraints on the Mass and the Number of Heavy Lepton Neutrinos, Prog.Theor.Phys. 58 (1977) 1775.

[847] D. Hooper and T. Plehn, Supersymmetric dark matter: How light can the LSP be?, Phys.Lett. B562 (2003) 18-27, [hep-ph/0212226].

[848] G. Belanger, F. Boudjema, A. Pukhov, and S. Rosier-Lees, A Lower limit on the neutralino mass in the MSSM with nonuniversal gaugino masses, hep-ph/0212227.

[849] D. Albornoz Vasquez, G. Belanger, and C. Boehm, Revisiting light neutralino scenarios in the MSSM, Phys.Rev. D84 (2011) 095015, [arXiv:1108.1338].

[850] G. Blanger, G. Drieu La Rochelle, B. Dumont, R. M. Godbole, S. Kraml, et al., LHC constraints on light neutralino dark matter in the MSSM, Phys.Lett. B726 (2013) 773-780, [arXiv:1308.3735].

[851] S. Scopel, N. Fornengo, and A. Bottino, Embedding the 125GeV Higgs boson measured at the LHC in an effective MSSM: Possible implications for neutralino dark matter, Phys.Rev. D88 (2013), no. 2 023506, [arXiv: 1304.5353].

[852] J. M. Lindert, F. D. Steffen, and M. K. Trenkel, Direct stau production at hadron colliders in cosmologically motivated scenarios, JHEP 1108 (2011) 151, [arXiv:1106.4005].

[853] B. Allanach, A. Dedes, and H. Dreiner, $R$ parity violating minimal supergravity model, Phys.Rev. D69 (2004) 115002, [hep-ph/0309196].

[854] H. K. Dreiner, C. Luhn, H. Murayama, and M. Thormeier, Baryon triality and neutrino masses from an anomalous flavor U(1), Nucl.Phys. B774 (2007) 127-167, [hep-ph/0610026].

[855] H. K. Dreiner, C. Luhn, and M. Thormeier, What is the discrete gauge symmetry of the MSSM?, Phys.Rev. D73 (2006) 075007, [hep-ph/0512163]. 
[856] L. E. Ibanez and G. G. Ross, Discrete gauge symmetries and the origin of baryon and lepton number conservation in supersymmetric versions of the standard model, Nucl.Phys. B368 (1992) $3-37$.

[857] H. K. Dreiner, An Introduction to explicit R-parity violation, Adv.Ser.Direct.High Energy Phys. 21 (2010) 565-583, [hep-ph/9707435].

[858] E. J. Chun and H. B. Kim, Nonthermal axino as cool dark matter in supersymmetric standard model without R-parity, Phys.Rev. D60 (1999) 095006, [hep-ph/9906392].

[859] B. Allanach, A. Dedes, and H. K. Dreiner, Bounds on R-parity violating couplings at the weak scale and at the GUT scale, Phys.Rev. D60 (1999) 075014, [hep-ph/9906209].

[860] E. Perazzi, G. Ridolfi, and F. Zwirner, Signatures of massive sgoldstinos at $e+e$ - colliders, Nucl.Phys. B574 (2000) 3-22, [hep-ph/0001025].

[861] N. Arkani-Hamed, M. Dine, and S. P. Martin, Dynamical supersymmetry breaking in models with a Green-Schwarz mechanism, Phys.Lett. B431 (1998) 329-338, [hep-ph/9803432].

[862] E. Cremmer, S. Ferrara, L. Girardello, and A. Van Proeyen, Yang-Mills Theories with Local Supersymmetry: Lagrangian, Transformation Laws and SuperHiggs Effect, Nucl.Phys. B212 (1983) 413.

[863] J. R. Ellis, K. Enqvist, and D. V. Nanopoulos, NONCOMPACT SUPERGRAVITY SOLVES PROBLEMS, Phys.Lett. B151 (1985) 357.

[864] A. Brignole, F. Feruglio, and F. Zwirner, Aspects of spontaneously broken $N=1$ global supersymmetry in the presence of gauge interactions, Nucl.Phys. B501 (1997) 332-374, [hep-ph/9703286].

[865] J. L. Lopez and D. V. Nanopoulos, Experimental consequences of no scale supergravity in light of the CDF e e gamma gamma event, Phys.Rev. D55 (1997) 4450-4462, [hep-ph/9608275].

[866] D. Gorbunov, Light sgoldstino: Precision measurements versus collider searches, Nucl.Phys. B602 (2001) 213-237, [hep-ph/0007325].

[867] D. S. Gorbunov and A. V. Semenov, CompHEP package with light gravitino and sgoldstinos, hep-ph/0111291.

[868] J. R. Ellis, K. Enqvist, and D. V. Nanopoulos, A Very Light Gravitino in a No Scale Model, Phys.Lett. B147 (1984) 99.

[869] G. Giudice and R. Rattazzi, Theories with gauge mediated supersymmetry breaking, Phys.Rept. 322 (1999) 419-499, [hep-ph/9801271].

[870] S. Dubovsky, D. Gorbunov, and S. V. Troitsky, Gauge mechanism of mediation of supersymmetry breaking, Phys.Usp. 42 (1999) 623-651, [hep-ph/9905466].

[871] M. Nowakowski and S. D. Rindani, Astrophysical limits on gravitino mass, Phys.Lett. B348 (1995) 115-120, [hep-ph/9410262].

[872] S. Demidov and D. Gorbunov, SUSY in the sky or a keV signature of sub-GeV gravitino dark matter, Phys.Rev. D90 (2014) 035014, [arXiv:1404.1339].

[873] A. Brignole and A. Rossi, Flavor nonconservation in goldstino interactions, Nucl.Phys. B587 (2000) 3-24, [hep-ph/0006036].

[874] D. A. Dicus, S. Nandi, and J. Woodside, Unusual $Z^{0}$ decays in supersymmetry with a superlight gravitino, Phys.Rev. D43 (1991) 2951-2955.

[875] S. Demidov and D. Gorbunov, Flavor violating processes with sgoldstino pair production, Phys.Rev. D85 (2012) 077701, [arXiv:1112.5230].

[876] LHCb Collaboration, R. Aaij et al., Search for rare $B_{(s)}^{0} \rightarrow \mu^{+} \mu^{-} \mu^{+} \mu^{-}$decays, Phys.Rev.Lett. 110 (2013) 211801, [arXiv: 1303.1092]. 
[877] HyperCP Collaboration, H. Park et al., Evidence for the decay Sigmat $\rightarrow$ p mu+ mu-, Phys.Rev.Lett. 94 (2005) 021801, [hep-ex/0501014].

[878] D. Gorbunov and V. Rubakov, On sgoldstino interpretation of HyperCP events, Phys.Rev. D73 (2006) 035002, [hep-ph/0509147].

[879] S. Demidov and D. Gorbunov, More about sgoldstino interpretation of HyperCP events, JETP Lett. 84 (2007) 479-484, [hep-ph/0610066].

[880] Belle Collaboration, H. Hyun et al., Search for a Low Mass Particle Decaying into $\mu^{+} \mu^{-}$in $B^{0} \rightarrow K^{* 0} X$ and $B^{0} \rightarrow r h o^{0} X$ at Belle, Phys.Rev.Lett. 105 (2010) 091801, [arXiv:1005.1450].

[881] KTeV Collaboration, E. Abouzaid et al., Search for the Rare Decays $K_{L} \rightarrow \pi^{0} \pi^{0} \mu^{+} \mu^{-}$and $K_{L} \rightarrow \pi^{0} \pi^{0} X^{0} \rightarrow \pi^{0} \pi^{0} \mu^{+} \mu^{-}$, Phys.Rev.Lett. 107 (2011) 201803, [arXiv:1105.4800].

[882] D. A. Dicus and P. Roy, Restrictions on Gravitino Mass From Chiral Scalar and Pseudoscalar Production, Phys.Rev. D42 (1990) 938-940.

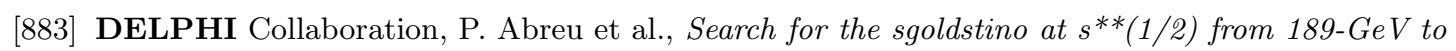
202-GeV, Phys.Lett. B494 (2000) 203-214, [hep-ex/0102044].

[884] D. Dicus, S. Nandi, and J. Woodside, Collider Signals of a Superlight Gravitino, Phys.Rev. D41 (1990) 2347.

[885] D. A. Dicus and S. Nandi, New collider bound on light gravitino mass, Phys.Rev. D56 (1997) 4166-4169, [hep-ph/9611312].

[886] E. Perazzi, G. Ridolfi, and F. Zwirner, Signatures of massive sgoldstinos at hadron colliders, Nucl.Phys. B590 (2000) 287-305, [hep-ph/0005076].

[887] D. Gorbunov and N. Krasnikov, Prospects for sgoldstino search at the LHC, JHEP 0207 (2002) 043, [hep-ph/0203078].

[888] S. Demidov and D. Gorbunov, LHC prospects in searches for neutral scalars in $p p \rightarrow$ gamma gamma + jet: SM Higgs boson, radion, sgoldstino, Phys.Atom.Nucl. 69 (2006) 712-720, [hep-ph/0405213].

[889] K. Astapov and S. Demidov, Sgoldstino-Higgs mixing in models with low-scale supersymmetry breaking, JHEP 1501 (2015) 136, [arXiv:1411.6222].

[890] D. Gorbunov, V. Ilyin, and B. Mele, Sgoldstino events in top decays at LHC, Phys.Lett. B502 (2001) 181-188, [hep-ph/0012150].

[891] D. Gorbunov and V. Rubakov, Kaon physics with light sgoldstinos and parity conservation, Phys.Rev. D64 (2001) 054008, [hep-ph/0012033].

[892] K. Hsieh, Pseudo-Dirac bino dark matter, Phys.Rev. D77 (2008) 015004, [arXiv:0708.3970].

[893] L. M. Carpenter, P. J. Fox, and D. E. Kaplan, The NMSSM, anomaly mediation and a Dirac bino, hep-ph/0503093.

[894] G. Belanger, K. Benakli, M. Goodsell, C. Moura, and A. Pukhov, Dark Matter with Dirac and Majorana Gaugino Masses, JCAP 0908 (2009) 027, [arXiv:0905.1043].

[895] A. De Simone, V. Sanz, and H. P. Sato, Pseudo-Dirac Dark Matter Leaves a Trace, Phys.Rev.Lett. 105 (2010) 121802, [arXiv:1004.1567].

[896] M. R. Buckley, D. Hooper, and J. Kumar, Phenomenology of Dirac Neutralino Dark Matter, Phys.Rev. D88 (2013) 063532, [arXiv:1307.3561].

[897] L. Hall and L. Randall, U(1)-R symmetric supersymmetry, Nucl.Phys. B352 (1991) 289-308.

[898] G. D. Kribs, E. Poppitz, and N. Weiner, Flavor in supersymmetry with an extended R-symmetry, Phys.Rev. D78 (2008) 055010, [arXiv:0712.2039]. 
[899] G. D. Kribs and A. Martin, Supersoft Supersymmetry is Super-Safe, Phys.Rev. D85 (2012) 115014, [arXiv: 1203.4821].

[900] C. Frugiuele, T. Gregoire, P. Kumar, and E. Ponton, 'L=R' $-U(1)_{R}$ Lepton Number at the LHC, JHEP 1305 (2013) 012, [arXiv: 1210.5257].

[901] Y. Morita, H. Nakano, and T. Shimomura, Neutrino Mass and Proton Decay in a $U(1)_{R}$ Symmetric Model, PTEP 2013 (2013) 053B02, [arXiv:1212.4304].

[902] S. Chakraborty and S. Roy, Higgs boson mass, neutrino masses and mixing and keV dark matter in an $U(1)_{R}$ - lepton number model, JHEP 1401 (2014) 101, [arXiv:1309.6538].

[903] E. Bertuzzo, C. Frugiuele, T. Gregoire, and E. Ponton, Dirac gauginos, R symmetry and the 125 GeV Higgs, arXiv: 1402.5432.

[904] P. Diener, J. Kalinowski, W. Kotlarski, and D. Stckinger, Higgs boson mass and electroweak observables in the MRSSM, JHEP 1412 (2014) 124, [arXiv:1410.4791].

[905] S. Chakraborty, A. Datta, and S. Roy, $h$ in $U(1)_{R}$-lepton number model with a right-handed neutrino, JHEP 1502 (2015) 124, [arXiv:1411.1525].

[906] P. J. Fox, A. E. Nelson, and N. Weiner, Dirac gaugino masses and supersoft supersymmetry breaking, JHEP 0208 (2002) 035, [hep-ph/0206096].

[907] A. E. Nelson, N. Rius, V. Sanz, and M. Unsal, The Minimal supersymmetric model without a mu term, JHEP 0208 (2002) 039, [hep-ph/0206102].

[908] K. Benakli, M. D. Goodsell, and A.-K. Maier, Generating mu and Bmu in models with Dirac Gauginos, Nucl.Phys. B851 (2011) 445-461, [arXiv:1104.2695].

[909] M. Heikinheimo, M. Kellerstein, and V. Sanz, How Many Supersymmetries?, JHEP 1204 (2012) 043, [arXiv: 1111.4322].

[910] K. Benakli, M. D. Goodsell, and F. Staub, Dirac Gauginos and the 125 GeV Higgs, JHEP 1306 (2013) 073, [arXiv:1211.0552].

[911] K. Benakli, M. Goodsell, F. Staub, and W. Porod, Constrained minimal Dirac gaugino supersymmetric standard model, Phys.Rev. D90 (2014), no. 4 045017, [arXiv:1403.5122].

[912] S. Ipek, D. McKeen, and A. E. Nelson, CP Violation in Pseudo-Dirac Fermion Oscillations, Phys.Rev. D90 (2014), no. 7 076005, [arXiv:1407.8193].

[913] M. D. Goodsell and P. Tziveloglou, Dirac Gauginos in Low Scale Supersymmetry Breaking, Nucl.Phys. B889 (2014) 650-675, [arXiv:1407.5076].

[914] CMS Collaboration, Search for new physics in monojet events in pp collisions at sqrt(s) $=8 \mathrm{TeV}$, Tech. Rep. CMS-PAS-EXO-12-048, CERN, Geneva, 2013.

[915] O. Buchmueller, M. J. Dolan, S. A. Malik, and C. McCabe, Characterising dark matter searches at colliders and direct detection experiments: Vector mediators, JHEP 1501 (2015) 037, [arXiv: 1407.8257].

[916] B. S. Acharya, G. Kane, S. Watson, and P. Kumar, A Non-thermal WIMP Miracle, Phys.Rev. D80 (2009) 083529, [arXiv:0908.2430].

[917] I. Antoniadis, A. Delgado, K. Benakli, M. Quiros, and M. Tuckmantel, Splitting extended supersymmetry, Phys.Lett. B634 (2006) 302-306, [hep-ph/0507192].

[918] K. Benakli, L. Darm, M. D. Goodsell, and P. Slavich, A Fake Split Supersymmetry Model for the 126 GeV Higgs, JHEP 1405 (2014) 113, [arXiv:1312.5220].

[919] E. Dudas, M. Goodsell, L. Heurtier, and P. Tziveloglou, Flavour models with Dirac and fake gluinos, Nucl.Phys. B884 (2014) 632-671, [arXiv:1312.2011].

[920] P. J. Fox, G. D. Kribs, and A. Martin, Split Dirac Supersymmetry: An Ultraviolet Completion of Higgsino Dark Matter, Phys.Rev. D90 (2014), no. 7 075006, [arXiv:1405.3692]. 
[921] G. Arcadi, Y. Mambrini, and F. Richard, Z-portal dark matter, JCAP 1503 (2015) 018, [arXiv: 1411.2985].

[922] A. Djouadi, A. Falkowski, Y. Mambrini, and J. Quevillon, Direct Detection of Higgs-Portal Dark Matter at the LHC, Eur.Phys.J. C73 (2013), no. 6 2455, [arXiv:1205.3169].

[923] N. Zhou, Z. Khechadoorian, D. Whiteson, and T. M. Tait, Bounds on invisible Higgs boson decay

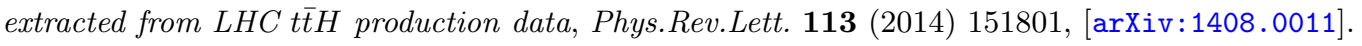

[924] N. Cartiglia, Measurement of the proton-proton total cross section at 2, 7, 8 and 57 TeV, arXiv: 1303. 2927.

[925] A. Ibarra, A. Ringwald, and C. Weniger, Hidden gauginos of an unbroken U(1): Cosmological constraints and phenomenological prospects, JCAP 0901 (2009) 003, [arXiv:0809.3196].

[926] A. Arvanitaki, N. Craig, S. Dimopoulos, S. Dubovsky, and J. March-Russell, String Photini at the LHC, Phys.Rev. D81 (2010) 075018, [arXiv:0909.5440].

[927] J. Polchinski, Dirichlet Branes and Ramond-Ramond charges, Phys.Rev.Lett. 75 (1995) 4724-4727, [hep-th/9510017].

[928] T. W. Grimm, T.-W. Ha, A. Klemm, and D. Klevers, The D5-brane effective action and superpotential in N=1 compactifications, Nucl.Phys. B816 (2009) 139-184, [arXiv:0811.2996].

[929] OPAL Collaboration, G. Abbiendi et al., Search for chargino and neutralino production at $s^{* *}(1 / 2)=192-G e V$ to 209 GeV at LEP, Eur.Phys.J. C35 (2004) 1-20, [hep-ex/0401026].

[930] M. Baryakhtar, N. Craig, and K. Van Tilburg, Supersymmetry in the Shadow of Photini, JHEP 1207 (2012) 164, [arXiv:1206.0751].

[931] CMS Collaboration, CMS, Precise determination of the mass of the Higgs boson and studies of the compatibility of its couplings with the standard model, .

[932] H. Dreiner, S. Grab, D. Koschade, M. Kramer, B. O'Leary, et al., Rare meson decays into very light neutralinos, Phys.Rev. D80 (2009) 035018, [arXiv:0905.2051].

[933] Belle Collaboration, K.-F. Chen et al., Search for $B \rightarrow h\left(^{*}\right)$ nu anti-nu decays at Belle, Phys.Rev.Lett. 99 (2007) 221802, [arXiv:0707.0138].

[934] BaBar Collaboration, B. Aubert et al., A search for the decay $B^{+} \rightarrow K^{+} \nu \bar{\nu}$, Phys.Rev.Lett. 94 (2005) 101801, [hep-ex/0411061].

[935] C. Cheung, J. T. Ruderman, L.-T. Wang, and I. Yavin, Kinetic Mixing as the Origin of Light Dark Scales, Phys.Rev. D80 (2009) 035008, [arXiv:0902.3246].

[936] E. Chun, J. E. Kim, and H. P. Nilles, Axino mass, Phys.Lett. B287 (1992) 123-127, [hep-ph/9205229].

[937] E. Chun and A. Lukas, Axino mass in supergravity models, Phys.Lett. B357 (1995) 43-50, [hep-ph/9503233].

[938] J. E. Kim and M.-S. Seo, Mixing of axino and goldstino, and axino mass, Nucl.Phys. B864 (2012) 296-316, [arXiv:1204.5495].

[939] L. Covi and J. E. Kim, Axinos as Dark Matter Particles, New J.Phys. 11 (2009) 105003, [arXiv:0902.0769].

[940] S. Dittmaier, G. Hiller, T. Plehn, and M. Spannowsky, Charged-Higgs Collider Signals with or without Flavor, Phys.Rev. D77 (2008) 115001, [arXiv:0708.0940].

[941] M. Arana-Catania, S. Heinemeyer, M. Herrero, and S. Penaranda, Higgs Boson masses and B-Physics Constraints in Non-Minimal Flavor Violating SUSY scenarios, JHEP 1205 (2012) 015, [arXiv: 1109.6232].

[942] DONuT Collaboration, K. Kodama et al., Final tau-neutrino results from the DONuT experiment, Phys.Rev. D78 (2008) 052002, [arXiv:0711.0728]. 
[943] OPERA Collaboration, N. Agafonova et al., Observation of tau neutrino appearance in the CNGS beam with the OPERA experiment, PTEP 2014 (2014), no. 10 101C01, [arXiv:1407.3513].

[944] C. H. Albright and C. Jarlskog, Neutrino Production of $m+$ and e+ Heavy Leptons. 1., Nucl.Phys. B84 (1975) 467.

[945] S. Kretzer and M. H. Reno, Tau neutrino deep inelastic charged current interactions, Phys.Rev. D66 (2002) 113007, [hep-ph/0208187].

[946] I. Schienbein, V. A. Radescu, G. Zeller, M. E. Christy, C. Keppel, et al., A Review of Target Mass Corrections, J.Phys. G35 (2008) 053101, [arXiv:0709.1775].

[947] S. Kretzer and M. H. Reno, Target mass corrections to electroweak structure functions and perturbative neutrino cross-sections, Phys.Rev. D69 (2004) 034002, [hep-ph/0307023].

[948] Y. S. Jeong and M. H. Reno, Tau neutrino and antineutrino cross sections, Phys.Rev. D82 (2010) 033010, [arXiv: 1007.1966].

[949] M. H. Reno, Electromagnetic structure functions and neutrino nucleon scattering, Phys.Rev. D74 (2006) 033001, [hep-ph/0605295].

[950] HERA-B Collaboration, I. Abt et al., Measurement of D0, D+, D+(s) and $D^{*}+$ Production in Fixed Target 920-GeV Proton-Nucleus Collisions, Eur.Phys.J. C52 (2007) 531-542, [arXiv:0708.1443].

[951] E789 Collaboration, M. Leitch et al., Nuclear dependence of neutral D meson production by 800-GeV/c protons, Phys.Rev.Lett. 72 (1994) 2542-2545.

[952] P. Nason, S. Dawson, and R. K. Ellis, The Total Cross-Section for the Production of Heavy Quarks in Hadronic Collisions, Nucl.Phys. B303 (1988) 607.

[953] P. Nason, S. Dawson, and R. K. Ellis, The One Particle Inclusive Differential Cross-Section for Heavy Quark Production in Hadronic Collisions, Nucl.Phys. B327 (1989) 49-92.

[954] M. L. Mangano, P. Nason, and G. Ridolfi, Heavy quark correlations in hadron collisions at next-to-leading order, Nucl.Phys. B373 (1992) 295-345.

[955] M. Cacciari, M. Greco, and P. Nason, The P(T) spectrum in heavy flavor hadroproduction, JHEP 9805 (1998) 007, [hep-ph/9803400].

[956] M. Cacciari, S. Frixione, and P. Nason, The $p(T)$ spectrum in heavy flavor photoproduction, JHEP 0103 (2001) 006, [hep-ph/0102134].

[957] B. A. Kniehl and G. Kramer, Charmed-hadron fragmentation functions from CERN LEP1 revisited, Phys.Rev. D74 (2006) 037502, [hep-ph/0607306].

[958] ZEUS Collaboration, H. Abramowicz et al., Measurement of charm fragmentation fractions in photoproduction at HERA, JHEP 1309 (2013) 058, [arXiv: 1306.4862].

[959] R. Nelson, R. Vogt, and A. Frawley, Narrowing the uncertainty on the total charm cross section and its effect on the $J / \psi$ cross section, Phys.Rev. C87 (2013) 014908, [arXiv:1210.4610].

[960] C. Lourenco and H. Wohri, Heavy flavour hadro-production from fixed-target to collider energies, Phys.Rept. 433 (2006) 127-180, [hep-ph/0609101].

[961] H.-L. Lai, M. Guzzi, J. Huston, Z. Li, P. M. Nadolsky, et al., New parton distributions for collider physics, Phys.Rev. D82 (2010) 074024, [arXiv:1007.2241].

[962] T. Gaisser, Cosmic rays and particle physics. Cambridge University Press, 1990.

[963] G. Barr, T. Gaisser, and T. Stanev, Flux of Atmospheric Neutrinos, Phys.Rev. D39 (1989) 3532-3534.

[964] S. I. Dutta, M. H. Reno, I. Sarcevic, and D. Seckel, Propagation of muons and taus at high-energies, Phys.Rev. D63 (2001) 094020, [hep-ph/0012350]. 
[965] E. Bugaev, T. Montaruli, Y. Shlepin, and I. A. Sokalski, Propagation of tau neutrinos and tau leptons through the earth and their detection in underwater / ice neutrino telescopes, Astropart.Phys. 21 (2004) 491-509, [hep-ph/0312295].

[966] L. Pasquali and M. H. Reno, Tau-neutrino fluxes from atmospheric charm, Phys.Rev. D59 (1999) 093003, [hep-ph/9811268].

[967] E. Bugaev, A. Misaki, V. A. Naumov, T. Sinegovskaya, S. Sinegovsky, et al., Atmospheric muon flux at sea level, underground and underwater, Phys.Rev. D58 (1998) 054001, [hep-ph/9803488].

[968] D. Gross and F. Wilczek, Asymptotically Free Gauge Theories. 1, Phys.Rev. D8 (1973) 3633-3652.

[969] D. Gross and F. Wilczek, ASYMPTOTICALLY FREE GAUGE THEORIES. 2., Phys.Rev. D9 (1974) 980-993.

[970] LHeC Study Group Collaboration, J. Abelleira Fernandez et al., A Large Hadron Electron Collider at CERN: Report on the Physics and Design Concepts for Machine and Detector, J.Phys. G39 (2012) 075001, [arXiv: 1206.2913].

[971] D. Boer, M. Diehl, R. Milner, R. Venugopalan, W. Vogelsang, et al., Gluons and the quark sea at high energies: Distributions, polarization, tomography, arXiv:1108.1713.

[972] M. L. Mangano, S. Alekhin, M. Anselmino, R. Ball, M. Boglione, et al., Physics at the front end of a neutrino factory: A Quantitative appraisal, hep-ph/0105155.

[973] S. Alekhin, J. Blümlein, and S. Moch, Parton Distribution Functions and Benchmark Cross Sections at NNLO, Phys.Rev. D86 (2012) 054009, [arXiv:1202.2281].

[974] S. Moch, J. Vermaseren, and A. Vogt, The Three loop splitting functions in QCD: The Nonsinglet case, Nucl.Phys. B688 (2004) 101-134, [hep-ph/0403192].

[975] A. Vogt, S. Moch, and J. Vermaseren, The Three-loop splitting functions in QCD: The Singlet case, Nucl.Phys. B691 (2004) 129-181, [hep-ph/0404111].

[976] W. van Neerven and E. Zijlstra, Order alpha-s**2 contributions to the deep inelastic Wilson coefficient, Phys.Lett. B272 (1991) 127-133.

[977] E. B. Zijlstra and W. L. van Neerven, Order alpha-s**2 QCD corrections to the deep inelastic proton structure functions F2 and F(L), Nucl. Phys. B383 (1992) 525-574.

[978] E. B. Zijlstra and W. L. van Neerven, Order alpha-s**2 correction to the structure function F3 (x, $Q^{* * 2)}$ in deep inelastic neutrino - hadron scattering, Phys. Lett. B297 (1992) 377-384.

[979] S. Moch and J. Vermaseren, Deep inelastic structure functions at two loops, Nucl.Phys. B573 (2000) 853-907, [hep-ph/9912355].

[980] J. Vermaseren, A. Vogt, and S. Moch, The Third-order QCD corrections to deep-inelastic scattering by photon exchange, Nucl.Phys. B724 (2005) 3-182, [hep-ph/0504242].

[981] S. Moch, M. Rogal, and A. Vogt, Differences between charged-current coefficient functions, Nucl.Phys. B790 (2008) 317-335, [arXiv:0708.3731].

[982] S. Moch, J. A. M. Vermaseren, and A. Vogt, Third-order QCD corrections to the charged-current structure function $F_{3}$, Nucl. Phys. B813 (2009) 220-258, [arXiv:0812.4168].

[983] E. Laenen, S. Riemersma, J. Smith, and W. L. van Neerven, Complete O (alpha-s) corrections to heavy flavor structure functions in electroproduction, Nucl. Phys. B392 (1993) 162-228.

[984] E. Laenen, S. Riemersma, J. Smith, and W. van Neerven, O(alpha-s) corrections to heavy flavor inclusive distributions in electroproduction, Nucl.Phys. B392 (1993) 229-250.

[985] H. Kawamura, N. Lo Presti, S. Moch, and A. Vogt, On the next-to-next-to-leading order QCD corrections to heavy-quark production in deep-inelastic scattering, Nucl.Phys. B864 (2012) 399-468, [arXiv:1205.5727]. 
[986] I. Bierenbaum, J. Blümlein, and S. Klein, Mellin Moments of the $O\left(\alpha^{3}(s)\right)$ Heavy Flavor Contributions to unpolarized Deep-Inelastic Scattering at $Q^{2} \gg m^{2}$ and Anomalous Dimensions, Nucl.Phys. B820 (2009) 417-482, [arXiv:0904.3563].

[987] J. Ablinger, A. Behring, J. Blmlein, A. De Freitas, A. von Manteuffel, et al., The 3-loop pure singlet heavy flavor contributions to the structure function $F_{2}\left(x, Q^{2}\right)$ and the anomalous dimension, Nucl.Phys. B890 (2015) 48-151, [arXiv:1409.1135].

[988] T. Gottschalk, Chromodynamic corrections to neutrino production of heavy quarks, Phys.Rev. D23 (1981) 56.

[989] M. Glück, S. Kretzer, and E. Reya, The Strange sea density and charm production in deep inelastic charged current processes, Phys.Lett. B380 (1996) 171-176, [hep-ph/9603304].

[990] J. Blümlein, A. Hasselhuhn, P. Kovacikova, and S. Moch, $O\left(\alpha_{s}\right)$ Heavy Flavor Corrections to Charged Current Deep-Inelastic Scattering in Mellin Space, Phys.Lett. B700 (2011) 294-304, [arXiv:1104.3449].

[991] M. Buza and W. van Neerven, O (alpha-s**2) contributions to charm production in charged current deep inelastic lepton - hadron scattering, Nucl.Phys. B500 (1997) 301-324, [hep-ph/9702242].

[992] J. Blümlein, A. Hasselhuhn, and T. Pfoh, The $O\left(\alpha_{s}^{2}\right)$ Heavy Quark Corrections to Charged Current Deep-Inelastic Scattering at large Virtualities, Nucl.Phys. B881 (2014) 1-41, [arXiv:1401.4352].

[993] S. Alekhin, J. Blümlein, L. Caminada, K. Lipka, K. Lohwasser, et al., Determination of Strange Sea Quark Distributions from Fixed-target and Collider Data, arXiv:1404.6469.

[994] M. Brucherseifer, F. Caola, and K. Melnikov, On the NNLO QCD corrections to single-top production at the LHC, Phys.Lett. B736 (2014) 58-63, [arXiv:1404.7116].

[995] S. Alekhin, J. Blümlein, and S. Moch, The ABM parton distributions tuned to LHC data, Phys.Rev. D89 (2014), no. 5 054028, [arXiv:1310.3059].

[996] P. Jimenez-Delgado and E. Reya, Dynamical NNLO parton distributions, Phys.Rev. D79 (2009) 074023, [arXiv:0810.4274].

[997] A. Martin, W. Stirling, R. Thorne, and G. Watt, Parton distributions for the LHC, Eur.Phys.J. C63 (2009) 189-285, [arXiv:0901.0002].

[998] R. D. Ball, V. Bertone, S. Carrazza, C. S. Deans, L. Del Debbio, et al., Parton distributions with LHC data, Nucl.Phys. B867 (2013) 244-289, [arXiv:1207.1303].

[999] J. Gao, M. Guzzi, J. Huston, H.-L. Lai, Z. Li, et al., The CT10 NNLO Global Analysis of QCD, Phys.Rev. D89 (2014) 033009, [arXiv:1302.6246].

[1000] H1 and ZEUS Collaboration Collaboration, F. Aaron et al., Combined Measurement and QCD Analysis of the Inclusive e+- p Scattering Cross Sections at HERA, JHEP 1001 (2010) 109, [arXiv:0911.0884].

[1001] HERAPDF Collaboration. https://www.desy.de/h1zeus/combined_results/index.php?do=proton_structure.

[1002] L. Harland-Lang, A. Martin, P. Motylinski, and R. Thorne, Parton distributions in the LHC era: MMHT 2014 PDFs, arXiv:1412.3989.

[1003] The NNPDF Collaboration, R. D. Ball et al., Parton distributions for the LHC Run II, arXiv: 1410.8849

[1004] NuTeV Collaboration, M. Goncharov et al., Precise measurement of dimuon production cross-sections in muon neutrino Fe and muon anti-neutrino Fe deep inelastic scattering at the Tevatron, Phys.Rev. D64 (2001) 112006, [hep-ex/0102049].

[1005] NOMAD Collaboration, O. Samoylov et al., A Precision Measurement of Charm Dimuon 
Production in Neutrino Interactions from the NOMAD Experiment, Nucl.Phys. B876 (2013) 339-375, [arXiv: 1308.4750].

[1006] A. Kayis-Topaksu, G. Onengut, R. van Dantzig, M. de Jong, R. Oldeman, et al., Measurement of charm production in neutrino charged-current interactions, New J.Phys. 13 (2011) 093002, [arXiv:1107.0613].

[1007] G. Bozzi, L. Citelli, and A. Vicini, PDF uncertainties on the $W$ boson mass measurement from the lepton transverse momentum distribution, arXiv:1501.05587.

[1008] Technical proposal: A facility to search for hidden particles (SHiP) at the SPS, SHiP collaboration, tech.rep. (2015).

[1009] NNPDF Collaboration, R. D. Ball et al., Reweighting NNPDFs: the W lepton asymmetry, Nucl.Phys. B849 (2011) 112-143, [arXiv:1012.0836].

[1010] R. D. Ball, V. Bertone, F. Cerutti, L. Del Debbio, S. Forte, et al., Reweighting and Unweighting of Parton Distributions and the LHC W lepton asymmetry data, Nucl.Phys. B855 (2012) 608-638, [arXiv: 1108.1758].

[1011] A. Kusina, T. Stavreva, S. Berge, F. Olness, I. Schienbein, et al., Strange Quark PDFs and Implications for Drell-Yan Boson Production at the LHC, Phys.Rev. D85 (2012) 094028, [arXiv: 1203.1290].

[1012] European Muon Collaboration, J. Aubert et al., The ratio of the nucleon structure functions $F 2_{n}$ for iron and deuterium, Phys.Lett. B123 (1983) 275.

[1013] K. Kovarik, I. Schienbein, F. Olness, J. Yu, C. Keppel, et al., Nuclear corrections in neutrino-nucleus DIS and their compatibility with global NPDF analyses, Phys.Rev.Lett. 106 (2011) 122301, [arXiv:1012.0286].

[1014] N. Armesto, Nuclear shadowing, J. Phys. G32 (2006) R367-R394, [hep-ph/0604108].

[1015] S. J. Brodsky and H. J. Lu, Shadowing and Antishadowing of Nuclear Structure Functions, Phys. Rev. Lett. 64 (1990) 1342.

[1016] D. F. Geesaman, K. Saito, and A. W. Thomas, The nuclear EMC effect, Ann. Rev. Nucl. Part. Sci. 45 (1995) 337-390.

[1017] D. de Florian, R. Sassot, P. Zurita, and M. Stratmann, Global Analysis of Nuclear Parton Distributions, Phys.Rev. D85 (2012) 074028, [arXiv:1112.6324].

[1018] I. Schienbein, J. Yu, K. Kovarik, C. Keppel, J. Morfin, et al., PDF Nuclear Corrections for Charged and Neutral Current Processes, Phys.Rev. D80 (2009) 094004, [arXiv:0907.2357].

[1019] K. J. Eskola, H. Paukkunen, and C. A. Salgado, EPSO9 - A New Generation of NLO and LO Nuclear Parton Distribution Functions, JHEP 04 (2009) 065, [arXiv:0902.4154].

[1020] M. Hirai, S. Kumano, and T. H. Nagai, Determination of nuclear parton distribution functions and their uncertainties at next-to-leading order, Phys. Rev. C76 (2007) 065207, [arXiv:0709.3038].

[1021] I. Schienbein et al., Nuclear PDFs from neutrino deep inelastic scattering, Phys. Rev. D77 (2008) 054013, [arXiv: 0710.4897].

[1022] S. A. Kulagin and R. Petti, Global study of nuclear structure functions, Nucl.Phys. $\mathbf{A 7 6 5}$ (2006) 126-187, [hep-ph/0412425].

[1023] H. Paukkunen and C. A. Salgado, Compatibility of neutrino DIS data and global analyses of parton distribution functions, JHEP 07 (2010) 032, [arXiv:1004.3140].

[1024] S. A. Larin and J. A. M. Vermaseren, The alpha-s**3 corrections to the Bjorken sum rule for polarized electroproduction and to the Gross-Llewellyn Smith sum rule, Phys. Lett. B259 (1991) $345-352$. 
[1025] S. Moch, S. Weinzierl, S. Alekhin, J. Blümlein, L. de la Cruz, et al., High precision fundamental constants at the TeV scale, arXiv:1405.4781.

[1026] S. Alekhin, J. Blümlein, and S. Moch, Higher order constraints on the Higgs production rate from fixed-target DIS data, Eur.Phys.J. C71 (2011) 1723, [arXiv:1101.5261].

[1027] E. Paschos and L. Wolfenstein, Tests for neutral currents in neutrino reactions, Phys.Rev. D7 (1973) 91-95.

[1028] S. Davidson, S. Forte, P. Gambino, N. Rius, and A. Strumia, Old and new physics interpretations of the NuTeV anomaly, JHEP 0202 (2002) 037, [hep-ph/0112302].

[1029] K. S. McFarland and S. Moch, Conventional physics explanations for the NuTeV sin**2theta(W), hep-ph/0306052.

[1030] S. Kretzer, QCD aspects of the NuTeV anomaly, hep-ph/0405221.

[1031] E. A. Paschos, Precise ratios for neutrino nucleon and neutrino nucleus interactions, hep-ph/0204090.

[1032] A. Blondel, P. Bockmann, H. Burkhardt, F. Dydak, A. Grant, et al., Electroweak Parameters From a High Statistics Neutrino Nucleon Scattering Experiment, Z.Phys. C45 (1990) 361-379.

[1033] CHARM Collaboration Collaboration, J. Allaby et al., A Precise Determination of the Electroweak Mixing Angle from Semileptonic Neutrino Scattering, Z.Phys. C36 (1987) 611.

[1034] K. Fujikawa and R. Shrock, The Magnetic Moment of a Massive Neutrino and Neutrino Spin Rotation, Phys.Rev.Lett. 45 (1980) 963.

[1035] R. E. Shrock, Electromagnetic Properties and Decays of Dirac and Majorana Neutrinos in a General Class of Gauge Theories, Nucl.Phys. B206 (1982) 359.

[1036] Particle Data Group Collaboration, C. Amsler et al., Review of Particle Physics, Phys.Lett. B667 (2008) 1-1340.

[1037] W. J. Marciano and Z. Parsa, Neutrino electron scattering theory, J.Phys. G29 (2003) 2629-2645, [hep-ph/0403168].

[1038] G. Domogatsky and D. Nadezhin, Modern theory of star evolution and experiments of $f$. reines on antineutrino-electron scattering detection, Yad.Fiz. 12 (1970) 1233-1242.

[1039] G. Radel and R. Beyer, Neutrino electron scattering, Mod.Phys.Lett. A8 (1993) 1067-1088.

[1040] A. Beda, V. Brudanin, V. Egorov, D. Medvedev, V. Pogosov, et al., Gemma experiment: The results of neutrino magnetic moment search, Phys.Part.Nucl.Lett. 10 (2013) 139-143.

[1041] LSND Collaboration, L. Auerbach et al., Measurement of electron - neutrino - electron elastic scattering, Phys.Rev. D63 (2001) 112001, [hep-ex/0101039].

[1042] DONUT Collaboration, R. Schwienhorst et al., A New upper limit for the tau - neutrino magnetic moment, Phys.Lett. B513 (2001) 23-29, [hep-ex/0102026].

[1043] L. Maiani, New Spectroscopy with Charm and Beauty Multiquark States, arXiv:1404.6618.

[1044] BaBar Collaboration, B. Aubert et al., Search for the charmed pentaquark candidate Theta(c)(3100)0 in e+ e-annihilations at $s^{* *}(1 / 2)=10.58-G e V$, Phys.Rev. D73 (2006) 091101, [hep-ex/0604006].

[1045] DELPHI Collaboration, J. Abdallah et al., Search for Pentaquarks in the Hadronic Decays of the $Z$ Boson with the DELPHI Detector at LEP, Phys.Lett. B653 (2007) 151-160, [arXiv:0708.0415].

[1046] G. De Lellis, A. Guler, J. Kawada, U. Kose, O. Sato, et al., Search for charmed pentaquarks in high energy anti-neutrino interactions, Nucl.Phys. B763 (2007) 268-282.

[1047] W. J. Marciano, T. Mori, and J. M. Roney, Charged Lepton Flavor Violation Experiments, Ann.Rev.Nucl.Part.Sci. 58 (2008) 315-341. 
[1048] Y. Kuno and Y. Okada, Muon decay and physics beyond the standard model, Rev.Mod.Phys. 73 (2001) 151-202, [hep-ph/9909265].

[1049] A. Czarnecki, W. Marciano, and K. Melnikov, Muon electron conversion in nuclei, AIP Conf.Proc. 549 (2000) 938-941.

[1050] T. Kosmas, G. Leontaris, and J. Vergados, Lepton flavor nonconservation, Prog.Part.Nucl.Phys. 33 (1994) 397-448, [hep-ph/9312217].

[1051] SINDRUM II Collaboration, W. H. Bertl et al., A Search for muon to electron conversion in muonic gold, Eur.Phys.J. C47 (2006) 337-346.

[1052] SINDRUM II Collaboration, W. Honecker et al., Improved limit on the branching ratio of mu $\rightarrow$ e conversion on lead, Phys.Rev.Lett. 76 (1996) 200-203.

[1053] DeeMe Collaboration, M. Aoki, A new idea for an experimental search for nu-e conversion, PoS ICHEP2010 (2010) 279.

[1054] Mu2e Collaboration, R. K. Kutschke, The Mu2e experiment at Fermilab, AIP Conf.Proc. 1182 (2009) 718-721.

[1055] COMET Collaboration, Y. Kuno, A search for muon-to-electron conversion at J-PARC: The COMET experiment, PTEP 2013 (2013) 022C01.

[1056] H. Witte, B. Muratori, K. Hock, R. Appleby, H. Owen, et al., Status of the PRISM FFAG Design for the Next Generation Muon-to-Electron Conversion Experiment, Conf.Proc. C1205201 (2012) 79-81.

[1057] T. Kosmas, S. Kovalenko, and I. Schmidt, Nuclear muon- - conversion in strange quark sea, Phys.Lett. B511 (2001) 203, [hep-ph/0102101].

[1058] A. Faessler, T. Kosmas, S. Kovalenko, and J. Vergados, Exotic mu e conversion in nuclei and R-parity violating supersymmetry, Nucl.Phys. B587 (2000) 25-44.

[1059] M. Gonzalez, T. Gutsche, J. C. Helo, S. Kovalenko, V. E. Lyubovitskij, et al., Limits on lepton flavor violation from $\mu^{-}-e^{-}$conversion, Phys.Rev. D87 (2013), no. 9 096020, [arXiv:1303.0596].

[1060] T. Gutsche, J. C. Helo, S. Kovalenko, and V. E. Lyubovitskij, New bounds on lepton flavor violating decays of vector mesons and the Z0 boson, Phys.Rev. D83 (2011) 115015, [arXiv:1103.1317].

[1061] A. Faessler, T. Gutsche, S. Kovalenko, V. E. Lyubovitskij, and I. Schmidt, Scalar meson mediated nuclear mu- - e- conversion, Phys.Rev. D72 (2005) 075006, [hep-ph/0507033].

[1062] M. Achasov, K. Beloborodov, A. Bergyugin, A. Bogdanchikov, A. Bukin, et al., Search for Lepton Flavor Violation Process $e^{+} e^{-} \rightarrow e \mu$ in the Energy Region $\sqrt{s}=984-1060 \mathrm{MeV}$ and $\phi \rightarrow e \mu$ Decay, Phys.Rev. D81 (2010) 057102, [arXiv:0911.1232].

[1063] S. Nussinov, R. Peccei, and X. Zhang, On unitarity based relations between various lepton family violating processes, Phys.Rev. D63 (2001) 016003, [hep-ph/0004153].

[1064] K. Hayasaka, K. Inami, Y. Miyazaki, K. Arinstein, V. Aulchenko, et al., Search for Lepton Flavor Violating Tau Decays into Three Leptons with 719 Million Produced Tau+Tau-Pairs, Phys.Lett. B687 (2010) 139-143, [arXiv: 1001.3221].

[1065] LHCb Collaboration, R. Aaij et al., Searches for violation of lepton flavour and baryon number in tau lepton decays at LHCb, Phys.Lett. B724 (2013) 36-45, [arXiv:1304.4518].

[1066] Belle-II Collaboration, T. Abe et al., Belle II Technical Design Report, arXiv:1011.0352.

[1067] A. Bobrov and A. Bondar, Search for $\tau \rightarrow \mu+\gamma$ decay at Super $c-\tau$ factory, Nucl.Phys.Proc.Suppl. 225-227 (2012) 195-197, [arXiv:1206.1909].

[1068] D. A. Mzhavia and G. Mitselmakher, On the Decays $\tau \rightarrow 3 \mu$ and $\tau \rightarrow 3 \mu 2 \nu$, JINR Rapid Communications 20-86 (1986) 4-9. 
[1069] LHCb Collaboration, LHCb VELO TDR: Vertex locator. Technical design report, .

[1070] T. Sjostrand, S. Mrenna, and P. Z. Skands, PYTHIA 6.4 Physics and Manual, JHEP 0605 (2006) 026, [hep-ph/0603175].

[1071] E. Augé, J. Dumarchez, and J. Trân Thanh Vân, eds., Proceedings, 49th Rencontres de Moriond on Electroweak Interactions and Unified Theories, 2014. 